Index of Generic

Names of Fossil

Plants, 1820-1950

G E O L O G I C A L S U R V E Y B U L L E T I N 1013

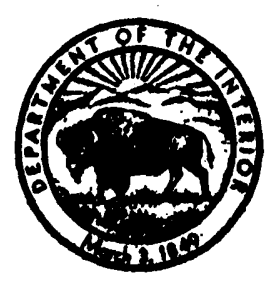





\title{
INDEX OF GENERIC NAMES OF FOSSIL PLANTS 1820-1950
}

\author{
By Henry N. Andrews, Jr.
}

\begin{abstract}
This work comprises an index of generic names of fossil plants, exclusive of the diatoms, that have been published from 1820 through 1950. It is based on the U. S. Geological Survey's working Compendium Index of Paleobotany and its accompanying bibliography. Although several hundred genera have been added by the author, it is realized that the list is not complete, particularly for the past two decades.

An attempt has been made to cite for each genus a type species, or one that is representative. In addition a brief notation is given concerning the age, geographic origin, and taxonomic status of most of the fossils. For some plants this information is further supplemented with such notations as seemed desirable to aid most effectively those persons who may have occasion to seek the type of information that is presented. It may be emphasized that this is not a critical study but is intended rather as an informational source concerning the origin of the respective generic concepts.

The introduction includes a sketch of the historical origin of the Compendium and states in some detail the types of problems encountered in presenting the work in this form. The bibliography presents primarily the full citations of those references indicated in the index.
\end{abstract}

\section{INTRODUCTION}

The Paleobotanical Library of the U. S. Geological Survey, Paleontology and Stratigraphy Branch, includes a card file index and bibliography of flames assigned to fossil plants. ${ }^{1}$ The Index was started in the latter part of the last century and by about 1933 it had developed to a point where it probably represented a compilation of binomials assigned to fossil plants since 1820 that is as nearly complete as is attainable.

After 1933 the indexing was carried on in a somewhat less thorough manner. However, in recognition of the unique importance of this reference source, effort is now being made to bring the Compendium Index up to date and to maintain it thereafter. Because of its importance and especjally because of the little publicity that the index

\footnotetext{
1 Prepared by members of the Paleontology and Stratigraphy Branch, U. S. Geological Survey, see 1952, Availability of U. S. Geological Survey technical fles in Paleontology: Geol. Soc. America Bull., v. 63, no. 5, p. 519-520; Jour. Paleontology, v. 26, no. 3, p. $535-53.6$.
} 
has received, it seems desirable to record something of its origin and development.

Microfilm copies of the complete index and accompanying bibliography are now available for sale on a cost basis. ${ }^{2}$

\section{ACKNOWLEDGMENTS}

The Geological Survey's Paleobotanical Library as well as the Compendium Index and bibliography were initiated chiefly through the efforts of Lester Frank Ward. Ward is well known for his historical and bibliographical works in the field of paleobotany, the most distinguished of these being a "Sketch of paleobotany" in the Fifth Annual Report of the Director of the U. S. Geological Survey, in 1885, and The Geographical Distribution of Fossil Plants, which appeared in the Eighth Annual Report in 1889. Shortly after his appointment as assistant geologist on the U. S. Geological Survey about 1881, Ward began work on a paleobotanical index that was apparently intended to include a citation of all fossil species believed to be of plant origin. This project was initiated, using quarto-size notebooks, and nine of these large volumes eventually were nearly filled. At present they are on file in the Paleobotanical Library.

Frank Hall Knowlton was appointed "aid in Botany" at the Smithsonian Institution in November 1884 and was transferred to the Geological Survey as assistant paleontologist in June 1889. I am not able to appraise Knowlton's influence in the early days of this bibliographic work although we have concrete evidence of his interest in it as well as a practical application in his paper, "A catalogue of the Cretaceous and Tertiary plants of North America," published in 1898. This was followed in 1919 by an amplified edition called "A catalogue of the Mesozoic and Cenozoic plants of North America." 3 A Supplement to that work was issued in 1944 by Robert Smith Lamotte.

David White started working for Lester Ward in My 1886 and was appointed to the Geological Survey the following October. White's contributions to the general progress of the Survey and the science are, of course, well known. ${ }^{4}$ Through David White's efforts, Miss Charlotte H. Schmidt was brought in to carry on the bibliographic work after Lester Ward left the Survey.

The compilation of a large index in notebook form is a cumbersome task and the fact that it was rapidly becoming impossible is evident from a perusal of Ward's first volume, where the entries are excessively crowded. Probably through the interest and planning of Ward, white, and Knowlton this was abandoned and the work trans-

\footnotetext{
See footnote 1.

${ }^{3}$ Knowlton, F. H., 1919, A catalogue of the Mesozoic and Cenozoic plants of North America : U. S. Geol. Survey, Bull. 696.

'Schuchert, Charles, 1936, Biographical memoir of David White: Natl. Acad. Sci. Mem. 9. v. 17, p. $189-221$.
} 
ferred to slips of paper, approximately $2 \frac{1}{2}$ by 8 inches, with an accompanying bibliography recorded on 4 - by 6 -inch cards. This transfer was apparently initiated about 1900. Considerable thought had been devoted to the work from the standpoint of its being a longrange project and with regard to ultimate publication. The first clear-cut evidence of this that I have found is a ten-page letter dated June 23, 1904 (files of the Paleobotanical Library, U. S. Geol. Survey), from chief geologist C. W. Hayes to Lester Ward. Because this letter may be taken as the starting point of the Compendium Index in its present form, it seems significant to record certain facts concerning the official Survey attitude toward the project as stated by Hayes. The instructions deal separately with the Index and bibliography. It was originally intended that the bibliography be published and that it should include all significant references to descriptions and figures of fossil plants.

The bibliography accompanying this present work is strictly subordinate to the Compendium Index and is by no means complete. This, however, need not be regarded as a violation of the original intention stated by Hayes. All concerned will agree that the index of plant names is the important feature of the project, and to reduce the cost of publication a bibliography has been compiled primarily to serve the needs of the index.

Regarding the index, Hayes notes that "Work should be continued upon the Index of Paleobotany along the same general lines as heretofore pursued, but with certain modifications indicated below." The modifications include the following instructions: Each slip should bear a complete reference to the species under consideration; "the type species of each genus should be determined by the currently accepted rules of nomenclature and should also be so marked, either by a conventional sign, or the word 'Type" "; the zone and locality should be given; in addition to the original publication, references should be given in which orthographical or nomenclatural changes are recorded; names of diatoms are to be omitted; and remarks concerning acceptable form of publication by the Geological Survey are included.

Apparently Lester Ward relinquished official charge of the project shortly after the date of the letter mentioned above, for in a second one from C. W. Hayes dated February 9, 1905, David White and F. H. Knowlton were appointed a "special committee to have charge of all Survey bibliographic work in paleobotany" and Miss Charlotte Schmidt was appointed to "continue work on the paleobotanical bibliography under the immediate supervision of this committee." Miss Schmidt continued in this capacity until her retirement on June 6, 1928. She was succeeded by Miss E. M. Thom during the period of December 1928 to July 1933. 
During the years $1933-45$ the progress of the Compendium remained nearly quiescent although additions to the bibliography were made by Roland W. Brown and Charles B. Read. From July 1945 until 1950, under the direction of Dr. Brown, Mrs. Marie Wandel worked part time at bringing the project up to date. It was largely through the continuing interest of Dr. Brown that an active program was ultimately re-established and that continuance of the project was assured by the assigniment to it, in February 1951, of Mrs. Jane Evans. Mrs. Evans is now engaged, under the direction of Sergius H. Mamay, in the difficult task of keeping the index and bibliography up to date and gathering up the omissions of the past 20 years.

Finally, I should like to acknowledge with gratitude the assistance of the library staff of the Missouri Botanical Garden, St. Louis, where it was possible to check a great many of the references.

\section{ORGANIZATION AND SCOPE OF THE COMPENDIUM INDEX}

The Compendium Index consists of about 135,000 slips bearing the names of species of fossil plants or living plants that have been reported in fossil form. The arrangement is alphabetical according to genus, and the species are alphabetically arranged within each genus. Many species are represented by two or more slips corresponding to as many publications in which they are described. Consequently the 135,000 slips do not imply as many species. It is my. estimate that there are about half that many represented.

After the first slip for each genus considered below the following information is recorded for each species: The genus, species, and author or authors; this is followed by an abbreviated bibliographic citation. In general this citation is sufficient to lead one directly to the original source, but I have found an appreciable number in which the abbreviation is too brief, and one must therefore refer to the accompanying bibliography, of some 17,000 references where the title of the article and journal are given more fully. For most species a brief notation regarding the age and geographic location is also given on the slip.

As to the first slip for each genus, we may consider the two "kinds" of genera represented, those based on modern plants and those based on fossil plants. For the genera based on modern plants, only the genus and author are given. If further information is needed for such genera, one must refer to the Index Kewensis or other reference works dealing with living plants. For the genera based on fossil plants, this first slip cites a type species with its bibliographic data. This species is repeated in its respective alphabetical order. More will be said about the type species in the following section. 


\section{PLAN OF THE GENERIC INDEX}

'The present task was undertaken with the knowledge that it probably would not be possible in the immediate future to publish formally the compendium in full, owing to financial reasons and the vast amount of editorial work that would be required. The present Generic Index was therefore prepared to supply basic information concerning the starting date of the names of all fossil genera, which it was felt would serve a useful purpose to working paleobotanists and which could be integrated into a complete index if such proved possible later. 'There are, therefore, several very important points which should be considered by those using this index.

The Generic Index is in no sense a critical treatment. The primary objective has been to cite for each genus a type or representative species that will serve as a much-needed basic reference for paleobotanical taxonomy. Two points may be stressed here. First, it should be noted that no botanical authority stands back of these proposed "types." 'The species cited here are mostly the ones that are, on somewhat casual inspection, presumed to be the types for the genera. For only a few species has critical opinion been available. Many revisions will be necessary because of initial errors or mistakes in subsequent interpretation. Second, the question will certainly be raised concerning the necessity of detailed editing if, indeed, the type species is already noted in the Compendium Index. This second point must be considered in some detail.

It was the intention of the present writer and his associates to cite useful basic information concerning the names for all fossil plant genera. Actually this is not always given in the Compendium Index in the "type slip." The latter in all or nearly all cases leads one to the original citation of the first binomial published for the genus. It is my estimate that from one-quarter to one-third of these are of no real value for the following reasons: In hundreds of cases they are nomina nuda that were followed (but not invariably) by valid descriptions in later publications; in many instances the original descriptions are so brief as to be of no real use; in hundreds of cases no illustrations are given or an illustration only is given. It is of course understood that illustrations are not required for valid publication before 1912; yet reference to an illustration or particular specimens that may be recovered and studied is usually necessary for satisfactory understanding of any fossil.

It therefore became evident, in spite of the genuinely monumental work of Lester Ward and Charlotte Schmidt, that the starting date of each generic name should be checked by reference to the original source. This has been done by the present writer for approximately 90 percent of the genera. The balance were not checked, chiefly because of the extreme rarity of some of the references. 
There are two points in particular, bearing on valid publication, which deserve consideration. First, the concept of a "description" has certainly varied in the minds of different paleobotanists. These range from a short phrase, which may convey little or much, to lengthy ones of many pages. In the present work the term "nom. nud." is applied only where there is no description at all. Second, hundreds of new genera of fossil plants have been created via the "new combination" route. It seems to have been the delight of many paleobotanists of the past to create new names under the slightest pretext. From the standpoint of taxonomy, the fact that new genera have been set up on trivial grounds, is, however, not the most serious problem. The ways in which new combinations have been established are many and equally varied. Sometimes these have been made formally and in the most clear-cut fashion, often accompanied by additional description and illustrations. At the other extreme the generic name has been merely "suggested" as being more suitable for certain species, and it is sometimes difficult to know just how serious the author's intentions were.

Usage of names in the present report has been determined chiefly by the established conventions of the science. That is, it appears that most workers in the field in the past have required but little formality in this respect; they have not deemed it necessary that the author of a new genus (based on a previously described fossil) should cite the original source with any degree of formality. Actually no generic names that are included in the Compendium Index have been omitted.

The information given for each genus in the Generic Index is then, as follows: For the first described species of each genus, the genus, species, and authorship is given, followed by a date, page number, and reference to plate or text figures; this is followed by a brief notation concerning the affinities of the fossil (not recorded in the Compendium Index), the geological horizon and age, and the geographical location. If an illustration did not accompany the first valid description (before 1912), I have also cited the next reference in chronological order where an illustration appears. Other sources of information are also indicated where it was felt they would be useful.

Where a new genus was created by new combination, $I$ have added a citation to the original description of the species. There are thus two "types" of records, examples of which are as follows:

PITYOIDOLEPIS Hollick and Jeffrey, 1909.

Pityoidolepis statenensis Hollick and Jeffrey, 1909, p. 54, pl, 9, figs. 13, 14; cone scale, Coniferales; Cretaceous; rreischerville. Staten Island, N. Y.

In this case we are dealing with a clear-cut new description and the reference may be consulted by referring to Hollick and Jeffrey for the year 1909 in the Bibliography. 
PASSALOSTROBUS Endlicher, 1847.

Passalostrobus tesselatus (Bowerbank) Endlicher, 1847, p. 278. For Cupressinites tesselatus Bowerbank, 1840, p. 63, pl. 10, figs. 26, 27, 30, 31; cone, Coniferales ; Eocene ; Sheppey, Kent, England.

Reference to Endlicher for 1847 in the Bibliography leads to the original source of the genus, whereas reference to Bowerbank for 1840 leads to the original description of the species which is accompanied by illustrations. This second reference is sometimes omitted where the first presents an adequate description with illustration.

There are, necessarily, variations from these two patterns, and I should like to emphasize that my principal effort has been to present pertinent and significant information bearing on the initiation of each genus which will be of use in solving taxonomic problems. To do this, it is at least necessary to emphasize that every valid genus must include a species that is recognized as its nomenclatural type.

\section{PROBLEIMS IN NOMENCLATURE}

The preparation of a work such as the present report reveals the greatness of some workers and the shortcomings of others; it displays the many pitfalls that all may stumble into; and it portrays with startling clarity the real character, or at least certain facets of character, of those who have contributed to or confused their science. It would not be polite to reveal all nor would it be to the point.

As indicated above, the Compendium is by no means complete after 1933 although an effort is now being made to remedy that defect. It seemed most desirable, however, to bring the Generic Index up to date insofar as possible at the time of its publication. The present writer has added names of several hundred genera although he is well aware that there are probably many proposed during the past two decades that are not included. The cooperation of all paleobotanists is invited to achieve this end, and it is planned that such omissions will be included in a later supplement.

There are of course many genera in which a type species means nothing. Two categories come to mind here; there are the dozens of genera based on minute fragments of fernlike foliage that display no significant characters and yield no clew to their affinities; there are also scores of genera based on highly problematical remains which, apparently for lack of a better guess, are called "algae." Undoubtedly many of these latter "genera" do not even represent organic remains.

The authors have clearly stated that some genera are not natural and a type species therefore cannot be established. For example, Bennetticarpus, Harris, T. M., 1932b, is presumed to be a bennettitalean fruit, but its more exact affinities are not known, and Harris (1932b, p. 101) states "Bennetticarpus, not being a natural genus, has no Typespecies." For such genera I have simply cited the first species 
described or one that is especially well described and illustrated. It thus serves, if not as a type species, as an informational source to the original treatment of the genus. Acceptance or rejection of Harris' philosophy in this and like cases is not a concern of the present-work.

The establishment of genera and species is certainly justifiable when the fossil remains are well preserved even though the affinities cannot be determined, but there is no justification for setting up new names when the natural relationships cannot be determined because of poor preservation. Paleobotanists who cannot refrain from bringing such specimens into their laboratories should at least refrain from recording them in print.

Delayed publication of valid names is perhaps the most common cause of nomenclatural confusion. Some instances appear to have been unintentional whereas others clearly have been due to the author's hurry to see his binomials in print or to "stake out a claim" on a particular locality. Regardless of the reason it seems inexcusable. Nearly as bad is the publication of very brief descriptions designed to meet the minimum requirements of the rules until the author may, at his leisure (many years later or never), describe the fossils in proper fashion.

The case of Botrychioxylon may be cited as an example. The generic name was published with a brief account by Dukinfield Henry Scott in 1906; it was referred to in several publications through the next few years (Scott, 1907; Scott, 1909; Bower, 1911), but it was not until 1912 that a full account was given and a specific name applied.

Burserites Berry presents an interesting example. In 1921 Edward Wilbur Berry described Burserites venezuelana n. sp. and in 1924 appeared the description of $B$. fayettensis under the heading of Burserites n. gen. Apparently the latter (original) description was delayed in going to press until after the later described $B$. venezuelana appeared in print. Here it is the clear intention of the author that $B$. fayettensis should serve as the type for the genus and therefore should be accepted as such.

Perhaps the most serious violation of all rules, written and unwritten, is the publication of a generic name for possible future use; a few examples may be cited: Dawson $(1881$, p. 11$)$ proposed the name Isoetoides for spores, compared with Isoetes, with no description or specific name, the name being simply "suggested . . . pending further investigation"; and Berry (1911, p. 242) in discussing the taxonomy of Cladophlebis suggested the possibility of transferring certain species to the new generic entity Aspidiopteris.

In the past, authors have not stated clearly that the genus they are describing is new. In this respect I feel that Miss Schmidt performed an especially. remarkable task in seeking out the original source. 
Many generic names have been duplicated or even triplicated, and again it is to Miss Schmidt's credit that she has been able to sort out the various species described and attribute them to the correct author's genus. Whether or not these are correct in every case in the Compendium Index, I am unable to say. This general problem is further complicated by the propensity of some writers to alter existing generic names very slightly and append their own names to them; the works of Schimper and Meschinelli are noteworthy in this respect. It must of course be remembered that rules of nomenclature were scarcely in existence and certainly not well agreed on at the time when these treatises appeared.

The correction of orthographic errors has resulted in no little confusion in the literature; for example, the multiplicity of spellings in cases such as Lepidofloyos-Lepidophloios-Lepidophloyos and Cardiocarpon - Cardiocarpum-Cardiocarpus may be cited as typical. In most cases the generally accepted spelling has been adopted here. Although it is recognized that the International Rules of Botanical Nomenclature clearly allow correction of unintentional orthographic errors (1935 ed., sec. 13), it is the present writer's opinion that acceptance of the original spelling is preferable unless an especially bad misspelling is involved.

Undoubtedly the description of fragmentary and meaningless scraps of fossil plants has brought more richly deserved criticism to paleobotany than any other shortcomings of the science. Some workers admittedly consider it their duty to give a name to everything including inferior or poorly preserved material that conveys no knowledge to themselves or to others. This practice is not confined to the distant past.

Paleobotanists, particularly those dealing with remains of late Mesozoic and Cenozoic age, have always been concerned with the problem of whether or not to use generic names based on living plants. For example, is a maplelike leaf actually referable to Acer or, for reason of question, should it be assigned to a different genus called Acerites or Aceriphyllum, etc.? Similarly we have Juglandinium Unger, Juglandoxylon Kraus, Juglansoxylon Falqui, and Jugloxylon Stopes and Fujii, all of which are based on woods supposedly comparable with that of modern Juglans.

A very recent practice is the introduction by certain spore and pollen workers of the taxonomic entity "Nov. Sporomorph." Pollens are unique structures but no more deserving of a special taxonomic category than seeds, stems, leaves, or any other organs.

It is perhaps not out of order to comment briefly on the mode of publication of certain of the older works, for example, the monographic contributions of such paleobotanists as Sternberg, Brongniart, 
Ettingshausen, Schimper, Lindley and Hutton, Saporta, Unger, and several others. Many of these were first issued in parts, either independently or in journals, over a period of several years and then bound into large volumes, and they have become available to many paleobotanists only in this latter form. In the "Bibliography" I have tried insofar as possible to give the exact date of publication of the separate parts of such works.

A considerable number of names retained in the Generic Index are based on remains that are probably not of plant origin, or even doubtfully organic. There are also some, such as Palaeoxyris and Vetacapsula, which were once held to refer to plants and now are definitely assigned as animals. It has seemed most expedient, for the benefit of those who are not familiar with such changes and doubtful cases, to include them.

\section{GEOGRAPHIC AND GEOLOGIC NAIMES}

A point of considerable concern to the present writer has been that of citing geographical localities. The Compendium slips, and of course the original publications, bear innumerable place names such as Styria, Bohemia, Hesse, Saxony, Liguria, and many others which no longer appear on modern maps. These could of course be translated into the closest modern political equivalents, but whether these would mean anything in another 50 years is debatable. Most of these old names are readily located on C. S. Hammond's "Historical atlas," which is a small and inexpensive booklet; and, as many of them are smaller and more precisely defined geographical units than the countries into which they have since been incorporated, there seems to be no advantage in bringing them up to date.

Geologic names in this bulletin are those employed in the original sources, and their use here does not imply approval by the Geological Survey.

\section{RECENT BIBLIOGRAPHIC LITERATURE}

An earnest effort has been made to complete this Generic Index through the year 1950. However, as stated above there are undoubtedly some omissions and particularly for the past two decades. As an aid to those readers who may not be well acquainted with the recent literature in this field, it will be useful to cite the more comprehensive bibliographic literature. The publications referred to below will be found cited more fully in the "Bibliography."

In 1921 the National Research Council, Washington, D. C., started to issue annual reports on American paleobotany. These have continued up to the present and now cover paleobotanical work in both North and South America (see American Paleobotany Report, Na- 
tional Research Council). Recent European paleobotany has been thoroughly reviewed in two comprehensive reports (1939-47 and 1948-49) issued by the Paleobotany Department of the Swedish Museum of Natural History under the editorship of Olof H. Selling (see Selling, O. H.). Corresponding reports for Britain have been edited by John Walton for the period 1939-51 (see British Paleobotanists). The Indian reports edited by Birbal Sahni and later by Rajendra Varma Sitholey, cover paleobotanical work in that area for the years 1940-50 (see Indian Paleobotany). The eastern Asiatic area is covered by Oishi's recent "Illustrated catalogue of East-Asiatic fossil plants" (see Oishi, Saburo, 1950). Finally may be mentioned Gothan's list of generic names of fossil plants proposed since 1900. This is not complete for that period, but it does present a very valuable contribution to our bibliographic literature (see Gothan, Walther, 1942b). 



\section{BIBLIOGRAPHY}

Abich, Otto Wilhelm Hermann, 1857, Über das Steinsalz und seine geologische Stellung im russischen Armenien : Acad. imp. sci. St. Petersburg Mém., tome 7, p. 59-150, pls. 1-10.

Achepohl, Ludwig, 1880-84, Das Niederrbeinische Westfalische Steinkohlen Gebirge-Atlas der fossilen fauna und flora : p. 1-8, charts 1-11, 1880; p. 9-72, pls. 0 , i-xxi, 1881; p. 73-108, pls. xxii-xxxiii, 1882; p. 109-160, pls. xxxiv-xli, supp. pls. i-iii, 1883, supp. pl. iv, 1884.

Airoldi, M., 1930, Su di un nuovo genere di Coralinacea fossile dell'oligocene Ligure : R. accad. naz. Lincei Atti, v. 12, p. 681-684, Rome.

1936, Sull' appartenenza alla 'Pelagesite' ad un neuve genre di alga calcarea : R. accad. naz. Lincei Atti, Rend., ser. $6^{n}$, v. 24, p. 12-23.

Allan, J. A., Warren, P. S., and Rutherford, R. L., 1932, A preliminary study of the eastern ranges of the Rocky Mountains in Jasper Park, Alberta : Royal Soc. Canada Trans., 3d ser., v. 26, sec. 4, p. 225-248, pls. 1, 2.

American Paleobotany Reports, National Research Council, 1921, First aunual report: Washington, D. C.

1929-51, Annual Reports 1-21.

1949, Summary of paleobotantical research in the United States and Canada, compiled and published by the Paleobotantical Section of the Botanical Society of America.

Andrä, Carl Justus, 1848, Calloxylon hartigii m. ein fossiles Cupressen-Holz aus der Gegend von Halle: Bot. Zeitung, Band 6, p. 633-638, pl. 5.

1855, Tertiär-Flor von Szakadat und Thalheim in Siebenbürgen: K.-k. geol. Reichsanst. Abh., Band, 2, Abt. 3, p. 1-48, pls. 1-12, Vienna.

1865-69, Vorweltliche Pflanzen aus dem Steinkohlenbirge der preussischen Rheinlande und Westphalens : Teil 1, p. 1-18, pls. 1-5, 1865 ; Teil 2, p. 19-34, pls. 6-10, 1866 ; Teil 3, p. 35-50, pls. 11-15, 1869.

Andreanszky, Gabor, 1949, Reste einer neuen Tertiären Palme aus Ungarn: Hungarica Acta Biol., v. 1, p. 31-36.

Andrews, E. B., 1875, Descriptions of fossil plants from the Coal Measures of Ohio: Ohio Geol. Survey Rept., v. 2, Geology and Palaeontology, pt 2, p. 415-426, pls. 46-53.

Andrews, Henry N., Jr., 1940, A new cupule from the Lower Carboniferous of Scotland : Torrey Bot. Club Bull., v. 67, p. 595-601, figs. 1-3.

- 1941, Dichophyllum moorei and certain associated seeds : Mo. Bot. Garden Annals, v. 28, p. 375-382, pls. 13-15.

- 1942a, Contributions to our knowledge of American Carboniferous floras, I, Scleropteris, gen. nov., Mesoxylon and Amyelon: Mo. Bot. Garden Annals, v. 29 , p. 1-11, pls. 1-4.

1942b, Contributions *** V, Heterangium: Mo. Bot. Garden Annals, v. 29, p. 275-282, pl. 25.

1945, Contributions * VII, Some pteridosperm stems from Iowa: Mo. Bot. Garden Annals, v. 32, p. 323-360, pls. 5-15.

Andrews, Henry N., Jr., and Baxter, Robert W., 1948, Contributions * ${ }^{*}$, An osmundaceous stem from Iowa : Mo. Bot. Garden Annals, v. 35, p. 193-197, pls. 9-10. 
Andrews, Henry N., Jr., and Kern, E. M., 1947, The Idaho Tempskyas and associated fossil plants: Mo. Bot. Garden Annals. v. 34, p. 119-186, pls. 15-27.

Andrews, Henry N., Jr., and Pearsall, C. S., 1941, On the flora of the Frontier formation of southwestern Wyoming: Mo. Bot. Garden Annals. v. 28, p. 165-192, pls. 1-7.

Arber, Agnes, 1910, On the structure of the Palaeozoic seed Mitrospermum compressum (Williamson) : Annals Botany, v. 24, p. 491-509, pls. 37-39.

Arber, Edward Alexander Newell, 1902, Notes on the Binney collection of Coal Measure plants: Cambridge Philos. Soc. Proc., v. 11, p. 281.

1912, A note on some fossil plants from the Kent Coal-field : Geol. Mag., v. 9, p. 97-99, pl. 5, London.

1913, A preliminary note on the fossil plants of the Mount Potts beds, New Zealand, collected by Mr. D. G. Lillie, biologist to Captain Scott's Antarctic Expedition in the 'Terra Nova': Royal Soc. London Proc., v. 86B, p. 344-347, pls. 7,8 .

1914, A revision of the seed impressions of the British Coal Measures: Annals Botany, v. 28, p. 81-108, pls. 6-8.

- 1916, On the fossil floras of the Coal Measures of South Staffordshire: Royal Soc. London Philos. Trans., v. 208B, p. 127-154, pls. 2-4.

- 1917, The earlier Mesozoic floras of New Zealand: New Zealand Geol. Survey Palaeont. Bull. 6, p. 1-80, pls. 1-14.

- 1921, Devonian floras-A study of the origin of the Cormophyta: $100 \mathrm{p}$, Cambridge.

Arber, Ediward Alexander Newell, and Goode, R. H., 1915, On some fossil plants from the Devonian rocks of North Devon: Cambridge Philos. Soc. Proc., v. 18, p. $89-104$, pls. $4,5$.

Arnold, Chester Arthur, 1930, The genus Callixylon from the Upper Devonian of central and western New York: Mich. Acad. Sci. Papers, 1929, v. 11, p. 1-50, pls. 1-19.

- 1935, Some new forms and new occurrences of fossil plants from the Middle and Upper Devonian of New York State: Buffalo Soc. Nat. Sci. Bull., v. 17, p. 1-12, pl. 1.

1937, Observations on fossil plants from the Devonian of eastern North America, III, Gilboaphyton goldringiae, gen. et sp. nov., from the Hamilton of eastern New York : Mich. Univ. Mus. Paleontology, Contr., v. 5, p. 75-79, pl 1.

1939, Observations *** IV, Plant remains from the Catskill delta deposits of northern Pennsylvania and southern New York: Mich. Univ. Mus. Paleontology, Contr., v. 5, p. 271-314, 10 pls.

- 1940, Structure and relationships of some Middle Devonian plants from western New York : Am. Jour. Bot., v. 27, p. 57-62.

1945, Silicified plant remains from the Mesozoic and Tertiary of western

North America : Mich. Acad. Sci. Papers, 7. 30, p. 3-34, pls. 1-10.

1947, An introduction to paleobotany : 433 p., New York, McGraw-Hill Book Co., Inc.

_ 1948, Some cutinized seed membranes from the coal-bearing rocks of Michigan : Torrey Bot. Club Bull., v. 75, p. 131-146, figs. 1-16.

Arnold, Chester Arthur, and Steidtmann, Waldo E., 1937, Pteridospermous plants from the Pennsylvanian of Illinois and Missouri : Am. Jour. Botany, v. 24, p. 644-650, figs. 1-14.

Artis, Edmund Tyrell, 1825, Antediluvian phytology : 24 p., 24 pls., London. 
Bailey, Irving W., 1924, The problem of identifying the wood of Cretaceous and later dicotyledons-Paraphyllanthoxylon arizonense: Annals Botany, v. 38, p. 439-451, pl. 15.

Baily, William H., 1860, On Corynepteris; a new generic form of fossil fern; with observations on the associated plants from the Coal Measures of Glin, County of Limerick: Geol. Soc. Dublin Jour., v. 8, p. 237-241, pl. 21.

Ball, O. M., 1931, A contribution to the paleobotany of the Eocene of Texas: Tex. Agr. and Mech. Coll., Bull., v. 2, p. 1-173, 48 pls.

Bancroft, Helen, 1932a, A fossil cyatheoid stem from Mount Elgon, East Africa : New Phytologist, v. 31, p. 241-253, pls. 9, 10.

- $1982 \mathrm{~b}$, Some fossil dicotyledonous woods from the Miocene (?) beds of East Africa : Annals Botany, v. 46, p. 745-767, pl. 29.

Bancroft, Nellie, 1913, Rhexoxylon africanum, a new Medullosean stem : Linnean Soc: London Trans., v. 8, p. 87-103, pls. 10, 11.

Bandulska, Helena, 1923, A preliminary paper on the cuticular structure of certain dicotyledonous and coniferous leaves from the Middle Eocene flora of Bournemouth : Linnean Soc. London Jour., v. 46, p. 241-270, pls. 20, 21.

Banks, Harlan P., 1944, A new Devonian lycopod genus from southeastern New York: Am. Jour. Botany, v. 31, p. 649-659, figs. 1-25.

Barbour, Erwin Hinckley, 1892, Notes on a new order of gigantic fossils : Nebr. Univ. Studies, v. 1, no. 4, p. 301-323, pls. 1-6.

1898, Chalcedony-lime nuts from the Badlands; Archichicoria siouxensis gen. et sp. nov. : Nebr. State Hist. Soc., Proc. Coll., 2d ser., v. 2, p. 272-273, pl. 5.

Barghoorn, Elso S., and Darrah, W. C., 1938, Horneophyton, a necessary change of name for Hornea: Harvard Univ. Bot. Mus. Leaflets, v. 6, p. 142-144.

Barràde, Joachim, 1852-1911, Systême silurien du centre de la Bohême. (See Fritsch, Anton, 1908.)

Barsanti, Leopoldo, 1903, Contribuzione allo studio della flora fossile di Iano: Soc. toscana sci. nat. Atti, v. 19, p. 1-2.

Bassler, Harvey, 1919, A sporangiophoric lepidophyte from the Carboniferous [Cantheliophorus] : Bot. Gazette, v. 68, p. 73-108, pls. 9-11.

Baxter, Robert W., 1949, Some pteridosperm stems and fructifications with particular reference to the Medullosae: Mo. Bot. Garden Annals, v. 36, p. 287-353, pls. 2-17.

$\longrightarrow 1950$, Peltastrobus reedae, a new sphenopsid cone from the Pennsylvanian of Indiana : Bot. Gazette, v. 112, p. 174-182.

Bayer, Edvin, 1914, Fytopalaeontologicke příspěvky ku Poznáni českých křídových vrster peruckých : Archiv Př́rodov. Výzk. Cech., svazek 25, p. 1-66, Prague.

Beck, Gordon F., 1945, Tertiary coniferous woods of western North America: Northwest Sci. v. 19, p. 89-102.

Beck, Richard, 1883, Das Oligocän von Mittweida mit besonderer Berücksichtingung seiner Flora: Deutsche geol. Gesell. Zeitschr., Band 34, p. 735770, pls. 31, 32 .

- 1920, Über Protothamnopteris baldaufi n. sp. einen neuen verkieselten Farn aus dem Chemnitzer Rotliegenden: Akad. Wiss. Leipzig, Math.-phys. Kl., Abh., Band 36, Heft 5, p. 513-522.

Beede, J. W., 1899, New fossils from the Kansas coal measures: Kans. Univ. Quart., v. 8; p. 123-130.

Behrend, Fritz, 1909, Uber einige Carbonfarne aus der Familie der Sphenopteriden: Preuss. geol. Landesanst. Jahrb., Band 29, p. 645-690, pls. 17, 18.

Bell, W. A., 1922, A new genus of Characeae and new Merostomata from the Coal Measures of Nova Scotia : Royal Soc. Canada Proc. and Trans., 3d ser., จ. 16 , sec. 4 , p. 159-168, pl. 1. 
Bennie, James, and Kidston, Robert, 1886, On the occurrence of spores in the Carboniferous formation of Scotland : Royal Phys. Soc. Proc., v. 9, p. 82-117, pls. 3-6.

Benson, Margaret J., 1904, Telangium scottii, a new species of Telangium (Calymmatotheca) showing structure: Annals Botany, v. 18, p. 161-177, pl. 11.

- 1908, Miadesmia membranacea, Bertrand-a new Palaeozoic lycopod with a seed-like structure : Royal Soc. London Philos. Trans., v. 199B, p. 409-425, pls. $30-37$.

- 1909, Sphaerostoma ovale, n. gen., and Crossotheca grievii, n. spec., an account of the structure and relations of the reproductive organs of Heterangium grievii; Nature, v. 80, p. 239

—_ 1914, Sphaerostoma ovale (Conostoma ovale et intermedium, Williamson), a Lower Carboniferous ovule from Pettycur, Fifeshire, Scotland: Royal Soc. Edinburgh Trans., v. 50, p. 1-15, pls. 1, 2.

- 1918, Mazocarpon or the structural Sigillariostrolus: Annals Botany, v. 32 , p. $569-589$, pls. $17,18$.

1922, Heterotheca grievii the microsporange of Heterangium grievii: Bot. Gazette, v. 74, p. 121-142, pls. 4, 5 .

Berendt, Georg Carl, 1845, Die im Bernstein befindlichen organischen Reste der Vorwelt: 125 p., 7 pls. Berlin.

Berger, H. A. C., 1832, Die Versteinerungen der Fische und Pflanzen im Sandsteine der Coburger Gegend : 29 p., 4 pls., Coburg.

Berger, L. G., 1923, Fossiele Houtsoorten uit het Tertiair van Zuid-Sumatra : Geol.-mijnb. genoot. Nederland en Koloniën Verh., boekdeel 7, p. 143-148.

Berger, Reinholdus, 1848, De fructibus et seminibus ex formatione lithanthracum : Dissert. Inauguralis, Univ. Breslau, 30 p., 3 pls.

Berkeley, M. J., 1848, On three species of mould detected by Dr. Thomas in the amber of East Prussia: Annals and Mag. Nat. History, v. 2, p. 380-383, pls. $11,12$.

- 1849, Brief note on Brachycladium: Annals and Mag. Nat. History, v. 3. p. 78.

Berry, Charles T., 1934, A Talbot cypress swamp at Greenbury Point, Md.: Torreya, v. 34, p. 85-91.

Berry, Edward Wilbur, 1903, The American species referred to Thinnfeldia: Torrey Bot. Club Bull., v. 30, p. 438-445.

- 1905, The flora of the Cliffwood clays: N. J. Geol. Survey Ann. Rept., 1905, p. 135-156, pls. 19-26.

- 1908, A mid-Cretaceous species of Torreya: Am. Jour. Sci., 4th ser., v. 25, p. $382-386$.

- 1909, Contributions to the Mesozoic flora of the Atlantic Coastal Plain, III : Torrey Bot. Club Bull., v. 36, p. 245-264, pls. 18, 18a.

1910a, Contributions *** V; Torrey Bot. Club Bull., v, 37, p. 181-200.

1910b, Contributions * * VI: Torrey Bot. Club Bull., v. 37, p. 503-511.

$1910 \mathrm{c}$, The evidence of the flora regarding the age of the Raritan formation : Jour. Geology, v. 18, p. 252-258.

1911a. Systematic palentology of the Lower Cretaceous deposits of Maryland : Md. Geol. Survey, Lower Cretaceous, p. 173-597.

1911b, Contributions to the Mesozoic flora of the Atlantic Coastal Plain, VII : Torrey Bot. Club Bull., v. 38, p. 399-424, pls. 18, 19.

- 1911c, A Lower Cretaceous species of Schizaeaceae from eastern North America : Annals Botany, v. 25, p. 193-198, pl. 12. 
Berry, Edward Wilbur, 1913, A fossil flower from the Eocene: U. S. Natl. Mus. Proc., v. 45, p. 261-263, pl. 21.

1914a, The Upper Cretaceous and Eocene floras of South Carolina and

Georgia : U. S. Geol. Survey Prof. Paper 84, 200 p., 29 pls.

1914b, Contributions to the Meozoic flora of the Atlantic Coastal Plain, $X$,

Maryland : Torrey Bot. Club Bull., v. 41, p. 295-300.

1915, An Eocene ancestor of the Zapodilla [Eoachras eocenica]: Am.

Jour. Sci., 4tir ser., v. 39, p. 208-213, pl. 1.

1916a, The Upper Cretaceous deposits of Maryland: Md. Geol. Survey,

p. 757-901, pls. $50-90$.

- 1916b, The lower Eocene floras of southeastern North America: U. S.

Geol. Survey Prof. Paper 91, 481 p., 117 pls.

1916c, Remarkable fossil fungi : Mycologia, v. 8, p. 73-79, pls. 180-182.

1917, The physical conditions and age indicated by the flora of the Alum

Bluff formation: U. S. Geol. Survey Prof. Paper 98, p. 41-60, pls. 7-10.

1919a, Upper Cretaceous floras of the eastern Gulf region in Tennessee,

Mississippi, Alabama, and Georgia: U. S. Geol. Survey Prof. Paper 112,

177 p., 32 pls.

1919b, Miocene fossil plants from northern Peru : U. S. Natl. Mus. Proc.,

v. 55, p. 279-294, pls. 14-17.

1921, Tertiary fossil plants from Venezuela: U. S. Natl. Mus. Proc., v. 59 , p. 553-579.

1922a, A new genus of fossil fruits: Am. Jour. Sci., 5th ser., v. 3, p. 251-253.

-1922b, The flora of the Woodbine sand at Arthurs Bluff, Tex. : U. S. Geol.

Survey Prof. Paper 129, p. 153-180, pls. 36-40.

- 1922c, Additions to the flora of the Wilcox group: U. S. Geol. Survey

Prof. Paper 131, p. 1-20, pls. 1-18.

1922d, The flora of the Concepion-Arauco Coal Measures of Chili : Johns

Hopkins Univ. Studies Geology 4, p. 73-132, pls. 1-5.

1922e, Pliocene fossil plants from eastern Bolivia: Johns Hopkins Univ.

Studies Geology 4, p. 146-186, pls. 1-8.

1923, Miocene plants from southern Mexico: U. S. Natl. Mus. Proc., v. 62,

p. 1-27, pls. 1-7.

1924a, The middle and upper Eocene floras of southeastern North Amer-

ica : U. S. Geol. Survey Prof. Paper 92, 206 p., 65 pls.

1924b, A cucurbitaceous fruit from the Tertiary of Texas: Torreya, $\nabla$.

24, p. 5-7.

1924c, A fọsil flower from the Miocene of Trinidad: Am. Jour. Sci., v. 7,

p. 103-108, figs. $1,2$.

- 1925a, Miocene Araceae related to Caladium from Trinidad: Pan-Am.

Geologist, v. 44, p. 38-42, pl. 5 .

- 1925b, A Miocene flora from Patagonia: Johns Hopkins Univ. Studies

Geology 6, p. 163-250, pls. 1-9.

- 1929a, Seeds of a new species of Vitaceae from the Wilcox Eocene of

Texas: Washington Acad. Sci. Jour., v. 19, p. 39-41, flg.

1929b, Early Tertiary fruits and seeds from Belen, Peru : Johns Hopkins

Univ. Studies Geology 10, p. 137-174, pls. 1, 2.

1929c, A revision of the flora of the Latah formation: U. S. Geol. Sur-

vey Prof. Paper 154, p. 225-265, pls. 49-64.

1929d, Tertiary fossil plants from Colombia, South America : U. S. Natl.

Mus. Proc., v. 75. Pub. 2795, p. 1-12, pls. 1-5. 
Berry, Edward Wilbur, 1930, Revision of the lower Eocene Wilcox flora of the Southeastern States, with descriptions of new species, chiefly from Tennessee and Kentucky: U. S. Geol. Survey Prof. Paper 156, p. 1-144, pls. 7-50.

1935, A preliminary contribution to the floras of the Whitemud and Ravenscrag formations: Canada Dept. Mines, Geol. Survey, Mem. 182, p. 1-107, pls. 1-20.

- 1937, A Paleocene flora from Patagonia: Johns Hopkins Univ. Studies Geology 12, p. 33-50, pls. 5-9.

1938, Tertiary flora from the Río Pichileufu, Argentina : Geol. Soc. America Special Paper 12, p. 1-149, pls. 1-56.

- 1939, Fossil plants from the Cretaceous of Minnesota : Washington Acad. Sci. Jour., v. 29, p. 331-336.

Berry, Willard, 1937, Spores from the Pennington coal, Rhea County, Tenn.: Am. Midland Naturalist, v. 18, p. 155-160.

Bertrand, C. E., 1883, Sur le genre Vesquia, Taxinée fossile du terrain aachénien de Tournai : Acad. sci. Paris Comptes Rendus, tome 97, p. 1382-1384.

- 1895, Sur une nouvelle centradesmide de l'époque houillère: Assoc. Français av. sci. Comptes Rendus $23^{\circ}$ sess. [1894], p. 588-593.

- 1898, Les charbons humiques et les charbons de purens: Univ. Lille Travaux et Mém., tome 6, Mém. 21, 218 p., 11 pls.

1907, Les carateristiques due genre Taxospermum de Brongniart: Soc. bot. France Bull., tome 54, p. 213-224.

- 1910, Sur le genre Compsotesta de Ad. Brongniart: Annales Jardin bot. Buitenzorg, supp. $3^{\text {e }}$, p. 187-212, pl. 1 .

1913, Premières observations sur le schiste paraffineux d'Alexinatz, Serbie: Soc. histoire nat. Autun Bull., tome 26, p. 337-358, pls. 3, 4.

Bertrand, C. E., and Renault, Bernard, Pila bibractensis et le boghead d'Autun: Soc. histoire nat. Autun Bull., tome 5, p. 159 253 , pl. 6 .

- 1893, Reinschia australis et premières remarques sur le kerosène shale de la Nouvelle-Galles du Sud: Soc. histoire nat. Autun Bull., tome 6, p. 321425, pls. 4-7.

Bertrand, Paul, 1907, Classification des Zygopteridées d'apres les caracteres de leurs traces ioliaires: Acad. sci. Paris Comptes Rendus, tome 145, p. 775-777. 1909, Etudes sur la fronde des Zygopteridées: 286 p., 16 pls., Lille.

1911, Structure des stipes d'Asterochlaena laxa Stenzel: Soc. géol. Nord Mém, tome 7, p. 55.

1913, Les fructifications de Nevropteridées recueillies dans le terrain houiller du nord de la France: Soc. géol. Nord Annales, tome 42, p. 113-144, pls. 6,7 .

1926, Les gisements a Mixoneura de la region de Saint-Gervais-Chamonix : Soc géol. France Bull., sér. $4^{\circ}$, tome 26, p. 381-388, pl. 19.

Beyschlag, Franz, and Fritsch, Karl, 1900, Das jüngere Steinkohlengebirge und das Rothliegende in der Provinz Sachsen und den angrenzenden Gebieten:

Preuss. geol. Landesanst. Abb., Nene Folge, Heft. 10, p. 1-263.

Bigsby, John J., 1878, Thesaurus Devonico-Carboniferous-The flora and fauna of the Devonian and Carboniferous Periods : 447 p., London.

Billings, Elkanah, 1857, Report of the palaeontologist for the year 1856: Canada Geol. Survey Rept. Progress, 1853-56, p. 247-345.

1865, Palaeozoic fossils: Canada Geol. Survey. v. 1 (1861-65), 426 p. 1872, On some fossils from the Primordial rocks of Newfoundland: Canadian Naturalist, new ser., v. 6, p. 465-479. 
Binney, E. W., 1866, On fossil wood found in calcareous nodules in the lower coal seams of Lancashire and Yorkshire: Manchester Lit. Philos. Soc. Proc.., v. 5, p. 61-64.

1869, Observations on the structure of fossil plants found in the Carboniferous strata Part 1: Palaeont. Soc., p. 1-32, pls. 1-6, London.

1871, Observations * * Part 2: Palaeont. Soc., p. 33-62, pls. 7-12, London.

- 1872a, Observations ***Part 3: Palaeont. Soc., p. 63-96, pls. 13-18, London.

1872b, Notes: Manchester Lit. Philos. Soc. Proc., v. 11, p. 69.

1875, Observations * * Part 4: Palaeont. Soc., p. 97-147, pls. 19-24, London.

Blanckenhorn, Max, 1885, Die fossile Flora des Buntsandsteins und des Muschalkalks der Umgegend von Commern : Palaeontographica, Band 32, p. 117-153, pls. 15-22.

Bleicher, G., and Elinche, P. 1889, Recherches relatives à quelques tufs quaternaires du nord-est de la France: Soc. géol. France Bull., sér. $3^{\circ}$, tome 17, p. $566-602$.

Bode, Hans, 1928, Über eine merkwürdige Pteridophytenfrulktiflkation aus dem oberschlesischen Carbon: Preuss. geol. Landesant. Jahrb., Band 49, p. 245247 , pl. 22.

Bornemann, J. G., 1856, Über organische reste der Lettenkohlengruppe Thüringens : 85 p., 12 pls., Leipzig.

- 1883, Paleontologische aus dem Cambrischen Gebiete von Canalgrande in Sardinia: Deutsche geol. Gesell. Zeitschr., Band 35, p. 270-274.

1886, Die Versteinerunge des Cambrischen Schichtensystems der Insel Sardinien: Nova Acta Leopoldina, Band 51, p. 1-84, pls. 1-33.

1887, Geologische Algenstudien: Preuss. geol. Landesanst. Jahrb., Band 7, p. 116-134, pls. $5,6$.

Borson, Stefano, 1829, Notice sur quelques fossiles de la Tarantaise en Savoye: R. accad. sci. Turin Mem., v. 33 p. 174-182, 1 pl.

Bosc, Louis, 1806, Description du rétéporite ọvoide: Jour. physique, tome 62, p. $433-435$, Paris.

Boucek, Bedřich, 1941, Über neue Algenreste aus dem bohmeischen Silur: Královske Ceské Společnosti Nauk, Tṛ̛ida Matematicko-Přirod., Věstník Čislo 7, p. 1-5.

Boulay, Jean Nicolas, 1876, Le terrain houiller du nord de la France et ses végétaux fossiles: Thesis, Univ. Lille, 74 p., 4 pls.

Boureau, Edouard, 1950a, Etude paléoxylologique du Sahara IX-Sur un Myristicoxylon princeps n. gen., n. sp., du Danien d'Asselar (Sahara soudanais) : Mus. natl. historie nat. Bull., sér., $2^{\circ}$, tome 22, no. 4, p. 523-52S, Paris.

1950b, Ettude paléoxylologique du Sahara XII-Sur un Annoxylon striatum n. gen., n. sp., des couches de Tamoguilel: Soc. géol. France Bull., sér. $5^{\circ}$, tome 20 , nos. $7-9$, p. 393-497, pl. 21 .

Boursault, Henri, 1889, Nouvelles empreintes problematiques des couches boloniennes du Portel (Pas-de-Cạlais) : Soc. géol. France Bull., sér. $3^{\circ}$, tome 17, p. 725-728, figs. $1,2$.

Bower, F. O., 1911, On the primary xylem, and the origin of medullation in the Ophioglossaceae: Annals Botany, v. 25, p. 537-574, pl. 47.

Bowerbank, James S., 1840, A history of the fossil fruits and seeds of the London Clay : 144 p., 17 pls., London. 
Bradley, Wilmot H., 1931, Origin and microfossils of the oil shale of the Green River formation of Colorado and Utah: U. S. Geol. Survey Prof. Paper 168, 58 p., 28 pls.

1929, Fresh-water algae from the Green River formation of Colorado : Torrey Bot. Club Bull., v. 56, p. 421-428, pls. 22, 23.

Braun, Alexander, 1854, Einige Beiträge zur flora der Tertiar-Zeit: Neues Jahrb. p. 138-147, pl. 3 .

Braun, Carl Friedrich Wilhelm, 1840, Verzeichniss der in der Kreis-naturalien. Sammlung zu Bayreuth befindlichen petrefacten: 118 p., 22 pls., Leipzig.

— 1847, Die fossilen Gewächse aus den Granzschichten zwischen dem Lias und Keuper des neu aufgefundenen Pflanzenlagers in dem Steinbruche von Veitlahm bei Culmback : Flora, Band 30, p. 81-87, Regensburg.

1849, Weltrichia eine neue Gattung fossiler Rhizantheen: Flora, Band 7, p. 705-712, Regensburg.

—_ 1854, Beiträge zur Urgeschichte der Pflanzen gesammelt: 15 p., 3 pls., Bayreuth.

Bristow, Henry W., 1862, Catalogue of the fossils of the Isle of Wight: Great Britain Geol. Survey Mem. and Mus. Pract. Geol., Expl. Sheet 10, p. 121-138.

British Paleobotanists, 1939-51, Committee Reports for 1939-47, 1948, 1949, 1950, 1951, Walton, John, ed. : Univ., Glasgow.

Britton, Elizabeth G., and Hollick, Arthur, 1915, A new American fossil moss: Torrey Bot. Club Bull., v. 42, p. 9-10, figs. 1, 2.

Britton, N. L., 1888a, Note on Archaeophyton newberryanum: New York Acad. Sci. Trans., v. 7, p. 89.

$1888 \mathrm{~b}$, On an archaean plant from the White Crystalline limestone of Sussex County, N. J. : New York Acad. Sci. Annals, v. 4, p. 123-124, pl. 7.

Broili, Ferdinand, 1928, Ein Pflanzenrest aus dem Hunsrückschiefern : Bayer.

Akad. Wiss., Math.-naturw. Abt., Heft. 3, p. 191-196, pls. 1, 2.

Brongniart, Adolphe, 1822, Sur la classification et la distribution des végétaux fossiles en général, et sur ceux des terrains de sédiment supérieur en particulier : Mus. histoire nat. Paris Mém., tome 8, p. 203-348.

1823, Observations sur les Fucoides et sur quelques autres plantes marines fossiles: Soc. histoire nat. Paris Mém., tome 1, p. 301-320, pls. 19-21.

- 1825a, Note sur les végétaux fossiles del'oolite a Fourgères de Mamers: Annales sci. nat., sér. $1^{\text {ere }}$, tome 4, p. 417-423, Paris.

- 1825b, Observations sur les vegétaux fossiles renfermes dans les Gres de Hoer en Scanie: Annales sci. nat., sér. 1ere, tome 4, p. 200-219, Paris.

1828a-38, Histoire des végétaux fossiles ou recherches botaniques et géologiques sur les végétaux renfermes dans les diverses couches du globe : v. 1 , p. $1-136,1828$; p. $137-208,1829$; p. 209-248, 1830 ; p. 249-264, 1831?; p. 265288, 1832?; p. 289-336, 1834 ; p. 337-368, 1835?; p. 369-488, 1836 ; vol. 2, p. 1-24, 1837; p. 25-72, 1838 . Plates appeared irregularly, v. 1, pls. 1-166; v. 2, pls. $1-29$.

_ـ 1828b, Prodrome d'une histoire des végétaux fossiles: Dictionnaire sci. nat., tome 57, p. 16-212, Paris.

1828c, Notice sur les plantes d'Armissan près Narbonne: Annales sci. nat.., sêr. $1^{\text {ere }}$, tome 15, p. 43-51, pl. 3, Paris.

1828d, Essai d'une flora du grès bigarre: Annales sci. nat. sés. $1^{\text {oro }}$, tome 15, p. 435-460, Paris.

1845, Végétaux, in Murchison, Roderick I., Verneuil, Edouard, and Keyserling, Alexandre, Géologie de la Russie d'Europe et des montagnes de l'Oural, tome 2, fasc. 3, p. 1-13: London and Paris. 
Brongniart, Adolphe, 1849, Tableau des genrès de végétaux fossiles considérés sous le point de vue de leur classification botanique et de leur distribution géologique: Dictionnaire univ. histoire nat., tome 13, p. 1-127, Paris.

- 1861, Note sur une collection des plantes fossiles recueillies en Grèce: Acad. sci. Paris Comptes Rendus, tome 52, p. 1232-1239.

1874, Les graines fossiles trouvées a l'état silicifié dans le terrain houiller de Saint-Etienne: Annales sci. nat., Botanique, tome 20, p. 234-260, pls. 2123, Paris.

—_ 1875, "Observations": Acad. sci. Paris Comptes Rendus, tome 80, p. 10201022.

1881, Recherches sur les graines fossiles siliciflées: 93 p., 21 pls., Paris.

Brongniart, Alexandre, 1829, Tableau du terrains que composent l'ecorce du globe, ou essai sur la structure de la parties connue de la terre: 435 p., Paris.

Bronn, Heinrich Georg, 1825, System der Urweltlichen Pflanzenthiere durch Giagnose, Analyse und Abbildung der Heschlechter Erlaütert: 47 p., 7 pls., Heidelberg.

1837-38, Lethaea geognostica oder abbildingen und beschreibungen der fur die gebirges-formationen bezeichnendsten versteinerungen: v. 1, p. 1-672, atlas, pls. 1-47, 1837 ; v. 2 , p. $673-1350,1838$.

1848, Index Palaeontologicus oder übersicht der bis Jetzt bekannten Fossilen organismen : 1384 p., Stuttgart.

1858, Beiträge zur triassischen Fauna und Flora der bituminösen Schiefer von Raibl : Neues Jahrb., p. 1-32, 129-144, pls. 1-9.

Brown, Robert, 1851, Some account of Triplosporites, an undescribed fossil fruit: Linnean Soc. London Trans., v. 20, p. 469-475, pls. 22-24.

Brown, Roland W., 1934, The recognizable species of the Green River flora: U. S. Geol. Survey Prof. Paper 185-C, p. 45-68, pls. 8-15.

1935, Some fossil conifers from Maryland and North Dakota; Washington Acad. Sci. Jour., v. 25, p. 441-450.

1946, Alterations in some fossil and living floras: Washington Acad. Sci. Jour., v. 36, p. 344-355.

1950, Cretaceous fish egg capsule from Kansas: Jour. Paleontology, v. 24, p. 594-600.

Brunner, Carl, 1848, Beiträge zur Kenntniss der Schweizerischen Nummuliten und Flysch Formation; Naturf. Gesell., Bern, Mitt., p. 9-22.

Buckland, William, 1828, On the Cycadeoideae, a family of fossil plants found in the Oolite quarries of the Isle of Portland: Geol. Soc. London Trans., 2d ser., v. 2, p. $395-401$; pls. $1-49$ [1829].

- 1836, Geology and mineralogy considered with reference to natural theology : v. 1, 599 p. ; v. 2, 128 p., 69 pls.

Bureau, Edouard, 1886, Etude sur une plante phanerogame (Cymodoceites parisiensis) de l'ordre des Naiadées, que vivait dans les mers d' l'époque éocène: Acad. sci. Paris Comptes Rendus, tome 102, p. 191-193.

1896, Sur quelques Palmiers d'Italie: Mus. histoire nat. Paris. Bull. 4, p. $280-285$.

- 1905, Sur une Lépidodendrée nouvelle du terrain houiller inférieur de Main-et-Loire: Soc. études sci. Angers Bull., tome 35, p. 147-158.

1914, Etudes des gites minéraux de la France, bassin houiller de la basse Loire: tome 1, 433 p., 1910 ; tome 2, 417 p. ; Atlas, 80 pls., 1914.

Bureau, Edouard, and Bureau, Louis, 1908, Excursion du 7 septembre à Oudon, Ancenis, Mésanger, Cop-Choux, Mouzeil : Soc. géol. France Bull., sér. 4e tome 8, p. 641-655. 
Butts, Charles, 1926, The Paleozoic rocks : Ala. Geol. Survey, Special Rept. 14, p. 41-230.

Buvignier, Armand, 1852, Statistique géologique, minéralogique, métallurgique et paléonotologique du Département de la Meuse: 52 p. 32 pls., Paris.

Capellini, Giovanni, 1884, Il cretaceo superiore e il gruppo di Priabona nell'Apennino settentrionale e in particolare nel Bolognese e loro rapporti col Grès de Celles in parte en con gli strati a Clavutina Szaboli : Accad. sci. Ist. Bologna Mem., ser. $4^{\text {a }}$, v. 5, p. 535-550.

Capellini, Giovanni, and Heer, O., 1866, Les Phyllites crétacées du Nebraska: Soc. Helvétique sci. nat., Nouv. Mém., tome 22, no. 1, p. 1-22, pls. 1-4.

Capellini, Giovanni, and Solms-Laubach, Graf, 1892, I Tronchi di Bennettitee dei Musei Italiani : R. accad. sci. Ist. Bologna Mem., ser. 5a, v. 2, p. 1-56, pls. 1-5.

Carpenter, William Benjamin, 1862, Introduction to the study of the Foraminifera : Ray Society, 319 p., London.

Carpentier, Alfred, 1919a, Notes paléophytologiques sur le carbonifère du Bassin de la Basse-Loire : Rev. gén. botanique, tome 31, p. 81-93, pl. 3.

- 1919b, Notes d'excursions et remarques sur le bassin houiller de la BasseLoire: Soc. géol. France Bull. Compte Rendu sommaire, tome 18, sér. $4^{\circ}$, p. 235-247, pls. 3-4.

- 1920, Sur des empreintes de fructifications recueillies dans plusiers gisements carbonifères de l'ouest de la France: Soc. sci. nat. ouest France Bull., sér. $3^{\mathrm{e}}$. tome 6, p. 110-119, pls. 1, 2.

— 1927, La flore wealdienne de Féron-Glageon [Nord] : Soc. géol. Nord Mém., tome 10, p. 1-151, pls. 1-25.

Carruthers, William, 1865, On an undescribed cone from the Carboniferous beds of Airdrie, Lanarkshire: Geol. Mag., v. 2, p. 433-440, pl. 12, London.

1867a, On British fossil Cycadeae: British Assoc. Adv. Sci., Rept. 37th

Mtg., Dundee, 1867 ['Trans.], p. 80 [1868].

1867b, On gymnospermatous fruits from the secondary rocks of Britain:

Jour. Botany British and Foreign, v. 5, p. 1-21, pls. 57-60.

1868, British fossil Pandaneae: Geol. Mag., v. 5, p. 153-156, pl. 9, London.

1869, On Beania, a new genus of cycadean fruit, from the Yorkshire Oolites: Geol. Mag., v. 6, p. 97-99, pl. 4, London.

1870, On fossil Cycadean stems from the secondary rocks of Britain:

Linnean Soc. London Trans., v. 26, p. 675-708, pls. 54-63.

1872, On the histological structure, and affinities of Nematophycus logani,

Carr. (Prototaxites logani Dawson), an alga of Devonian age: Monthly

Micros. Jour., v. 8, p. 160-172, pls. 21, 22.

- 1873, On Traquairia, a radiolarian rhizopod from the Coal Measures:

British Assoc. Adv. Sci., Rept. 42d Mtg., Brighton, 1872 [Trans.], p. 126.

Caspary, Robert, 1856, Les Nymphéacées fossiles: Annales sci. nat., Botanique, sér. $4^{\theta}$, tome 6, p. 199-222, pls. 12, 13, Paris.

- 1872, Einige in Bernstein eingeschlossene fossile Zweige einer untergegangenen Gnetaceen-Gattung: Phys.-ökon. Gesell. Königsberg Schr., Band

13, p. 20, 21.

1881, Einige pflanzliche Abdrücke and Einschlüsse in Bernstein: Phys.-

ökon. Gesell. Königsberg Schr., Band 21, p. 28-30.

-___ 1882, Neue fossile Pflanzen der Blauen Erde : Phys.-ökon. Gesell. Königsberg Schr., Band 22, p. 22-31.

1886, Einige neue Pflanzenreste aus dem samländischen Bernstein : Phys.ökon. Gesell. Königsberg Schr., Band 27, p. 1-9, pl. 1. 
Caspary, Robert, 1888, Einige neue fossile Holzer Preussens nebst pritischen Bemerkungen über die Anatomie des Holzes und die Bezichnung fossiler Holzer : Phys.ökon. Gesell. Königsberg. Schr., Band 28, p. 27-45.

1889, Einige fossile Hölzer Preussens : Abh. geol. Specialkarte Preussen, Band 9, no. 2, p. 113-198, pls. 1-15.

1907, Die Flora des Bernsteins und anderer fossiler Harze des ostpreussischen Tertiärs: Preuss. geol. Landesanst. Abh., Neue Folge, Heft 4, p. 1-181, pls. 1-30, Berlin.

Chachloff, V. A., 1921, La flore du dévonien supérieur de la rive sud-ouest du Lac Balbach : Comité géol. Russie Bull., tome 2, no. 5, p. 1-26.

_ 1924, Les plantes fossiles du bassin houiller d'Irkoutsk: Comité géol. Russie Bull., tome 4, no. 2, p. 1-39, pls. 1-11. .

- 1939, On some remains of older Devonian flora of the Minusinsk depression : Rec. Tomsk State Kubyshev Univ., v. 96, p. 91-96, pls. 1-3, Tomsk. [English suikmary.]

Chandler, Marjorie E. J., 1925, The upper Eocene flora of Hordle, Hants., Part 1: Palaeont. Soc., v. 77, p. 1-32, pls. 1-4, London.

1926, The upper Eocene flora of Hordle, Hants., Part 2 : Palaeont. Soc., v. 78, p. 31-52, pls. 5-8, London.

Chaney, Ralph W., 1944, A fossil cactus from the Eocene of Utah: Am. Jour. Botany, v. 31, p. 507-528, pls. 1-5.

Chapman, Frederick, 1927a, On a new genus of calcareous algae, from the Lower Cambrian?, west of Woollana, South Australia : Royal Soc. South Australia Trans. and Proc., v. 51, p. 123-125, pl. 6.

1927b, Monograph on the Triassic flora of Bald Hill, Bacchus Marsh, Victoria : Natl. Mus. Victoria Mem. 7, p. 121-153, pls. 10-14.

Chiarugi, Alberto, 1933, Legni fossili della Somalia Italiana: Paleontographia Italica, v. 32, supp. 1, p. 97-167, pls. 9-22.

- 1947, Palaeocodium saharianum n. gen., n. sp., nuova Codiacea paleozoica del Deserto Libico: Paleontographia Italica, v. 41, p. 121-130, pl. 9.

Chowdhury, K. Ahmed, 1934, A fossil dicotyledonous wood from Assam : Current Sci., v. 3, p. 255-256.

1936, A fossil dicotyledonous wood from Assam: Annals Botany, v. 50, p. 501-510, pl. 7.

Chowdhury, K. Ahmed, and Ghosh, S. S., 1939, On the anatomy of a fossil dicotyledonous wood from Nailalung, Assam [abs.]: Indian Sci. Cong., 26th, Lahore, Proc., Pt. 3, abs., p. 127.

1946, On the anatomy of Cynometroxylon indicum gen. et sp. nov., a fossil dicotyledonous wood from Nailalung, Assam: Natl. Inst. Sci. India Proc., v. 12, no. 8, p. 435-447, pls. 10, 11, Lahore.

Chowdhury, K. Ahmed, and Tandan, K. N., 1949, Kayeoxylon assamicum, gen. et sp. nov., a fossil dicotyledonous wood from Assam : Natl. Inst. Sci. India Proc., v. 15, no. 2, p. 59-65, pls. 5, 6.

Clarke, J. M., 1885, On Devonian spores: Am. Jour. Sci., v. 29, p. 284-289.

Claypole, E. W., 1878a, On the occurrence of a tree-like fossil plant, Glyptoden. dron, in the upper Silurian (Clinton) rocks of Ohio: Am. Jour. Sci., 3d ser., v. 15, p. 302-304.

$1878 b$, On the occurrence of a fossil tree in the Clinton Limestone: Geol.

Mag., decade 2, v. 5, p. 558-564, London.

1895, Daemonelix or, what?: Am. Geologist, v. 16, p. 113. 
Cockerell, T. D. A., 1909, Eocene fossils from the Green River, Wyo.: Am. Jour. Sci., 4th ser., v. 28, p. 447-448.

1922, A new genus of fossil Liliaceae: Torrez Bot. Club Bull., v. 49, p. 211-213.

— 1924, A genuine fossil Ophioglossum [Tertiary, Wyoming] : Torreya, v. 24, p. 10, 11 .

1925, Plant and insect fossils from the Green River Eocene of Colorado:

U.S. Natl. Mus. Proc., v. 66, p. 1-13, pls. 1, 2.

1926a, The supposed fossil Ophioglossum [O. hastatiforme, Eocene,

Wyoming] : Torreya, v. 26, p. 10, 11.

1926b, An alga from the Eocene of Colorado: Torreya, v. 26, p. 111-112.

Combes, Paul, 1907, Contribution à l'étude de la flore éocène: Soc. géol. France Bull., sér. $4^{\ominus}$, tome 7, p. 28-29, pl. 1.

Condra, G. E., and Elias, M. K., 1945, Bicorbula, a new Permian Bryozoan, probably a Bryozoan-algal consortium: Jour. Paleontology, v. 19, p. 116-125, pls. 13-16.

Conrad, T. A., 1837, First annual report on the geological survey of the third district of the State of New York : N. Y. Geol. Survey Ann. Rept. 1, p. 155-186. 1838, Report on the Palaeontological Department of the Survey: N. Y. Geol. Survey Ann. Rept. 2, p. 107-119.

Conwentz, Hugo, 1880, Die fossilen Hölzer von Karlsdorf am Zobten: Naturiss.

Gesell. Schr. Danzig, Band 4, p. 1-47, pls. 1-8.
1885, Sobre algunos arboles fosiles del Río Negro: Acad. Nac. Cienc. Córdoba Bol., tomo 7, p. 435-456, Buenos Aires.

1886, Die flora des Bernsteins-Band 2, Die Angiospermen des Bernsteins: 140 p., 13 pls., Danzig.

- 1890, Monographie de Baltischem Bernsteinbäume : 151 p., 18 pls., Danzig. Cookson, Isabel C., 1935, On plant remains from the Silurian of Victoria, Australia, that extend and connect floras hitherto described : Royal Soc. London Philos. Trans., v. 225B, p. 127-148, pls. 10-12.

- 1947a, On fossil leaves (Oleaceae) and a new type of fossil pollen grain from Australian brown coal deposits : Linnean Soc. New South Wales Proc., v. 72 , p. 183-197, pls. 8-10.

1947b, Fossil fungi from Tertiary deposits in the southern hemisphere, part I : Linnean Soc. New South Wales Proc., v. 72, p. 207-214, pls. 11-14.

1950, Fossil pollen grains of Proteaceous type from Tertiary deposits in Australia : Australian Jour. Sci. Research, ser. B, v. 3, p. 166-177, pls. 1-3. Cookson, Isabel C., and Duigan, Suzanne L., 1950, Fossil Banksieae from Yallourn, Victoria, with notes on the morphology and anatomy of living species: Australian Jour. Sci. Research, ser. B, v. 3, p. 133-165, pls. 1-9.

Corda, August Joseph, 1840, Diploxylon-Ein neues Geschlecht urweltlicher Pflanzen: Gesell. vaterl. Mus Böhmen Verh., p. 20-26, pl. 1, Prague.

- 1842a, [Arbeiten über fossile Pflanzen] : Böhmen Gesell. Wiss. Ber. Verh., 1841, p. 9-10, Prague.

1842b, Araucaria sternbergii. Wine neue fossile conifere aus dem Rohlensandsteine zu Radnitz in Böhmen: Gesell. vaterl. Mus. Böhmen Verh., p. 63-67, p1. 1, Prague. Berlin. 1845 , Flora Protogaea-Beiträge zur Flora der Vol'welt: 128 p., 60 pls.,

1847, Uber fossile Pflanzenreste: Böhmen Gesell. Wiss. Abh., Band 4, p. 16-18, Prague. 
Corsin, Paul, 1929, Sur les fructifications et la position systématique de Sphenopteris zamioides Paul Bertrand: Soc. géol. Nord Annales, tome 53, p. 222-230, pls. 7-10, Lille.

1948, Reconstitutions de Pécoptéridées: genres Caulopteris Lindley and Hutton, Megaphyton Artis et Hagiophyton nov. gen. : Soc. géol. Nord Annales, tome 67 , p. 6-25, pls. 1-4, Lille.

Cotta, C. Bernhard, 1832, Die Dendrolithen in Beziehung auf ihren inneren Bau : 89 p., 18 pls., Dresden and Leipzig.

1843, Rothenbergia hollebenii: Neues Jahrb., p. 411-412, pl. 2.

Cragin, F. W., 1893, A contribution to the invertebrate paleontology of the Texas Cretaceous : Tex. Geol. Survey Ann. Rept. 4, p. 141-246, pls. 24-46.

Cremer, Leo, 1893, Über die fossilen Farne des Westfälischen Carbons und ihre Bedeutung für eine Gliederung des letzteren : Inaugural Dissert., 49 p., Univ. Marburg.

Crepin, Francois, 1874, Fragments paléontologiques pour servir a la flore du terrain houiller de Belgique: Acad. royale Belgique Bull., tome 38, p. 568-577, pls. 1, 2, Brussels.

1875, Observations sur quelques plantes fossiles des dépôts dévoniens: Soc. royale botanique Belgique Bull., tome 14, p. 214-230, pls. 1-6.

Cribbs, J. E., 1938, A new fossil plant from the Reed Springs formation of southwestern Missouri : Am. Jour. Botany, v. 25, p. 311-321.

1939, Caloxylon ambiguum, gen. et sp. nov., a new fossil plant from the Reed Springs formation of southwestern Missouri: Am. Jour. Botany, v. 26, p. $440-449$.

1940, Structure of fossil stems of pityean affinity from Reed Spring formation of Missouri : Bot. Gazette, v. 101, p. 582-597.

Crie, Louis, 1878, Les Tigillites siluriennes: Acad. sci. Paris Comptes Rendus, tome 86, p. $686-689$.

1885, Contributions a l'étude de la flore oolithique de l'ouest de la France: Acad. sci. Paris Comptes Rendus, tome 101, p. 83-86.

1888, Recherches sur la flore pliocene de Java: Geol. Reichs.-Mus. Leiden Samm., Band 5, p. 1-22, pls. 1-8.

- 1889a, Beiträge zur Kenntnis der fossilen Flora einiger Inseln des Südpacifischen und Indischen Oceans : Palaeontographica Abh., Band 5, p. 77-90, pls. 9-18.

1889b, Paléontologie des Colonies françaises et des pays de protectorat. Exposition paléontologique de Madagascar-Araucarioxylon grandidieri et Helvillea lapparenti: Pub. Exposition Universelle de Paris, 32 p., Paris.

Crookall, Robert, 1930, On some curious fossils from the Downtonian and Lower Old Red Sandstone of Scotland : Royal Soc. Edinburgh Proc., 1929-30, v. 50, p. 175-178, pl.

1931, The genus Lyginorachis Kidston : Royal Soc. Edinburgh Proc., v. 51, pt. 1, p. 27-34, pls. 1-3.

Cuvier, Georges, and Brongniart, Alexandre, 1822, Description géologique des environs de Paris: 428 p., 11 pls., Paris.

Dana, James D., 1849, Description of fossils, in Wilkes, Charles, United States exploring expedition [1838-42] **, v. 10, app. 1, p. 681-730, pls. 12-14, New York. 1863, Manual of Geology, 1st ed. : 798 p., Philadelphia. 
D'Archiac, Adolphe, 1843, Description géologique du Département de l'Aisne : Soc. géol. France Mém., tome 5, pt. 2, p. 129-418, pls. A-J.

_ـ 1847, Extrait d'un Mémoire sur les fossiles des couches à Nummulites des environs de Bayonne et de Dax: Soc. géol. France Bull., sér. $2^{e}$, tome 4, p. 1006-1010.

1850, Description des fossiles du groupe Nummulique recueillis par M. S. P. Pratt et M. J. Delbos aux environs de Bayonne et de Dax: Soc. géol. France Mém., sér. $2^{e}$, tome 3, p. 397-448.

Daugherty, Lyman H., 1934, Schilderia adamanica-A new fossil wood from the petrified forests of Arizona : Bot. Gazette, v. 96, p. 363-366, pl. 5.

1941, The Upper Triassic flora of Arizona : Carnegie Inst. Washington Pub. 526,108 p., 40 pls.

IJavies, David, 1929, Correlation and palaeontology of the Coal Measures in East Glamorganshire: Royal Soc. London Philos. Trans., v. 217B, p. 91-154, pls. 34-40.

Dawson, John William, 1859, On fossil plants from the Devonian rocks of Canada : Geol. Soc. London Quart. Jour., v. 15, p. 477-488.

1860 , On an undescribed fossil fern from the lower Coal-Measures of Nova Scotia : Canadian Naturalist, v. 5, p. 460-461.

1862, On the flora of the Devonian period in northeastern America: Geol. Soc. London Quart. Jour., v. 18, p. 296-330, pls. 12-17.

1863a, Further observations on the Devonian plants of Maine, Gaspé, and New York: Geol. Soc. London Quart. Jour., v. 19, p. 459-469, pls. 18, 19.

1863b, Synopsis of the flora of the Carboniferous period in Nova Scotia: Canadian Naturalist, v. 8, p. 431-457.

1866, On the conditions of the deposition of coal, more especially as illustrated by the Coal formation of Nova Scotia and New Brunswick: Geol. Soc. London Quart. Jour., v. 22, p. 95-169, pls. 5-13.

1871, The fossil plants of the Devonian and Upper Silurian formations of Canada : Geol. Survey Canada, p. 1-92, pls. 1-20.

1873, Note on the fossil plants from British Columbia, collected by $\mathrm{Mr}$

James Richardson in 1872: Geol. Survey Canada, Rept. Progress 1872-73, p. 66-71, pl. 1 .

1878, Notes on some Scottish Devonian plants : Canadian Naturalist, new ser., v. 8, p. 379-389.

1880a, On new Erian (Devonian) plants: Canadian Naturalist, new ser., v. 9, p. $475-476$.

1880b, The chain of life in geological time : $272 \mathrm{p}$., London [1880].

1881a, Palaeontological notes: Canadian Naturalist, new ser., v. 10, p. 1-11.

1881b, Notes on new Erian (Devonian) plants: Geol. Soc. London Quart. Jour., v. 37, p. 299-308, pls. 12, 13.

1883, On the Cretaceous and Tertiary floras of British Columbia and the Northwest Territory: Royal Soc. Canada Ploc. and Trans., v. 1. sec. 4, p. 15-34, pls. 1-7.

1885 , On the Mesozoic floras of the Rocky Mountains region of Canada:

Rnyal Soc. Canada Proc. and Trans., v. 3, sec. 4, p. 1-22, pls. 1-4.

1886 , On the fossil plants of the Laramie formation of Canada: Royal

Soc. Canada Proc. and Trans., v. 4, sec. 4, p. 19-34, pls. 1, 2.

1888 , The geological history of plants : 290 p., New xork.

1889, A new Erian (Devonian) plant allied to Cordaites: Am. Jour. Sci., $3 d$ ser., v. 38, p. 3. 
Dawson, John William, 1890, On fossil plants from the Similkameen Valley and other places in the southern interior of British Columbia : Royal Soc. Canada Proc. and Trans., v. 8, sec. 4, p. 75-91.

1894, On new species of Cretaceous plants from Vancouver Island : Royal Soc. Canada Proc. and Trans., v. 11, sec. 4, p. 53-71, pls. 5-14.

1895, On collections of Tertiary plants from the vicinity of the city of Vancouver, British Columbia: Royal Soc. Canada Proc. and Trans., 2d ser., v. 1, sec. 4, p. 137-151, pls. 5-8.

Deane, Henry, 1902a, Notes on fossil leaves from the Tertiary deposits of Wingello and Bungonia : Geol. Survey New South Wales Rec., v. 7, pt. 2, p. 59-65, pls. $15-17$.

1902b, Notes on the fossil flora of Berwick: Geol. Survey Victoria Rec., v. 1, p. 21-32, pls. 3-7, Melbourne.

1904, Further notes on the Cainozoic flora of. Sentinel Rock, Otway Coast: Geol. Survey Victoria Rec., v. 1, p. 212-216, pl. 20, Melbourne.

1907 , Notes on specimens of fossil leaves from the Warrumbungle Mountains: Geol. Survey New South Wales Rec., v. 8, pt. 3, p. 189-191, pls. 34-36.

Debey, Matthias H., 1848, Übersicht der urweltlichen Pflanzen des Kreidegebirges überhaupt und der Aachener Kreideschicten insbesondere: Naturh. Ver. Preuss. Rheinlande u. Westfalens Sitzungsber., Band 5, p. 113-125.

1849, Entwurf zu einer Geognostisch-Geogenetischen Darstellung del Gegend von Aachen: Deutscher Naturf. Aertzte Amtliche Ber. 25 Vers., p. 269-328, Aachen.

Debey, Matthias H., and Ettingshausen, C., 1859a, Die urweltlichen Thallophyten des Kreidegebirges von Aachen und Maestricht: Akad. Wiss. Wien, Math.naturwiss. Kl., Denkschr., Band 16, p. 131-214, pls. 1-3.

- 1859b, Die urweltlichen Acrobryen des Kreidegebirges von Aachen und Maestricht: Akad. Wiss. Wien. Math.-naturwiss. Kl., Denkschr., Band 17, p. 183-248, pls. 1-7.

Deecke, Wilhelm, 1883, Über einige neue.Siphoneen: Neues Jahrb., Band 1, p. $3-14$, pl. 1.

1901, Über Hexagonaria v. Hag. und Goniolina Roem., Centralbl. Mineralogie, 1901, Heft 15, p. 469-473.

Deevers, Charles L., 1937, Structure of Paleozoic seeds of the Trigonocarpales: Bot. Gazette, v. 98, p. 572-585, figs. 1-36.

Deflandre, Georges, 1932, Litharchaeocystis costata, nov. gen., nov. sp., Chrysophycée marine fossile: Acad. sci. Paris Comptes Rendus, tome 194, p. 12731275.

1947, Calciodinellum nov. gen., première representant d'une famille nouvelle de Dinoflagelles fossiles a theque calcaire: Acad. sci. Paris, Comptes Rendus, tome 224, p. 1781-1782.

Defrance, J. L. M., 1S22, [Larvaire] in Dictionnaire sci. nat. : tome 25, p. 287-288, Paris.

1827, [Réceptaculite] in Dictionnaire sci. nat.: tome 45, p. 5-7, Paris.

Dekay, J. E., 1824, Note on the organic remains termed Bilobites from the Catskill Mountains : Lyceum Nat. History New York Annals, p. 45-49, pl. 5.

Delgado, Joaquin Filippe N., 1886, Jetude sur les Bilobites et autres fossiles des quartzites de la base due systeme silurique du Portugal: 113 p., 42 pls., Lisbon.

Deninger, Karl, 1906, Einige neue Tabulaten und Hydrozoen aus mesozoischen Ablagerungen: Neues Jahrb. Referate 1, 1906, p. 61-70, pls. 5-7. 
Derville, P. Henry, 1931, Les marbres du calcaire carbonifère en Bas-Boulonnais: Thesis, Univ. Strasbourg, 322 p., 24 pls.

De Stefani, T., 1948, Sopra alcune graminaceae del terziario italiano (Oryza sinagrensis $\mathbf{n}$. sp. et Triticoides bianchii $\mathrm{n}$. gen. et $\mathrm{n}$. sp.) : Plinia, v. 1, no. 2.

Dettmer, Friedrich, 1915, Neues zum Fucoidenproblem: Centralbl. Mineralogie, 1915, Heft 9, p. 285-287.

Dietrich, W. O., 1930, Chaetetes polyporus Qu. aus dem oberen Weissen Jura: Paleont. Zeitschr., Band 12, p. 99-109, pls. 2-4.

Dijkstra, J. J., 1949, Megaspores and some other fossils from the Aachenian (Senonian) in South Limburg, Netherlands: Mededelingen geol. Stichling, 1948-49, nieuwe ser., nr. 3, p. 19-32, Heerlen.

D'Orbigny, Alcide, 1842, Voyage dans l'Amérique méridionale: tome 3, pt. 4, Paléontologie, p. 1-188, pls. 1-22, Paris.

1850, Prodrome de paléontologie stratigraphique universelle des animaux mollusques et Rayonnes faisant suite au cours élémentaire de paléontologie et de séologic stratigraphiques : tome $2,427 \mathrm{p}$., Paris.

1851-52. Cours élémentaire de paléontologie et de géologie stratigraphiques : tome 2 , p. 1-258, 1851 ; p. 259-847, 1852.

Dorf, Erling, 1933, A new occurrence of the oldest known terrestrial vegetation, from Beartooth Butte, Wyo.: Bot. Gazette, v. 95, p. 240-257, pls. 5, 6.

- 1938, Upper Cretaceous floras of the Rocky Mountain region, I, Flora of the Lance formation at its type locality, Niobrara County, Wyo.: Carnegie Inst. Washington Pub. 508, p. 1-78, pls. 1-19.

Dotzler, Auguste, 1938, Zur Kenntnis der Oligozänflora des Bayerischen Alpenvorlandes: Palaeontographica, Band 83, Abt. B, p. 1-66, pls. 1-8.

Duden, Hans, 1897, Some notes .on the black slate or Genesee shale of New Albany, Ind.: Ind. Dept. Geology Nat. Res., Ann. Rept. 21, 1896, p. 108-119, pls. 2, 3.

Duncan, P. Martin, 1876, On some unicellular algae parasitic within Silurian and Tertiary corals, with a notice on their presence in Calceola sandalina and other fossils: Geol. Soc. London Quart. Jour., v. 32, p. 205-210, pl. 16.

Dunker, Wilhelm, 1846, Monographie der Norddeutschen Wealdenbildung: 83 p., 21 pls.

Dusen, Per Carl HJalmar, 1899, Über die tertiäre Flora der Magellansländer: Schwedisch Exped. Magellansland. Wiss. Ergebnisse 1895-97, p. 87-107, pls. 8-12, Stockholm.

DuToit, A. L., 1927, The fossil flora of the Upper Karroo Beds: Annals South African Mus., v. 22, p. 289-420.

1932, Some fossil plants from the Karroo System of South Africa: Annals South African Mus., v. 28, p. 369-393, pls. 39-40.

Dutt, C. P., 1916, Pitystrobus macrocephalus, L. and F.-A Tertiary cone showing ovular structures : Annals Botany, v. 30, p. 529ட549, pl. 15.

Dybowski, Władisław, 1877, Die Chaetetiden der 0stbaltischen Silur-Formation:

Russisch-K. mineralog. Gesell. St. Petersburg Verh., ser. 2, Band 14, 1878 [1879], 134 p., 4 pls.

Eckhold, Walter, 1921, Die Hoftüpfel bei rezenten und fossilen Koniferen: Bot.

Inst. Univ. Breslau [2] p.

- 1922, Die Hoftüpfel bei rezenten und fossilen coniferen: Preuss. geol. Landesanst. Jahrb., Band 42, p. 472-505, pl. 8, Berlin. 
Edwards, W. N., 1922, An Eocene Microthyriaceous fungus from Mull, Scotland :

British Mycological Soc. Trans., v. 8, p. 66-72, pl. 8.

1923, On some Tertiary plants from South-East Burma: Geol. Mag., v. 60 , p. 159-165, pl. 5, London.

1931, Fossilium catalogus, Pars 17, Dicotyledones (Ligna), 95 p.; edited by W. Jongmans, Berlin.

1932, Lower Eocene plants from Istria : Annals and Mag. Nat. History, 10th ser., v. 10, p. 213-216, London.

Eichwald, Eduard, 1840, Silurische Schichten-system in Esthland: 210 p., St. Petersburg.

1842, Die Urwelt Russlands durch abbildungen Erlaeutert (1840-42), p. 180-184, pl. 4, St. Petersburg.

1844, Über einige fossile Pflanzen des Kupfer-fuhrenden Sandsteins im

Permischen und Orenburg'schen Gouvernment: Neues Jahrb., p. 142-147.

1846, Geognosy particularly in relation to Russia : 572 p., St. Petersburg.

1851, Paleontologii Rossii : 284 p., 14 pls., St. Petersburg.

1854, Die Grauwackenschichten von Liev- und Esthland : Soc. naturalistes

imp. Moscou Bull., tome 27, pt. 1, no. 1, p. 3-111.

- 1860-68, Lethaea Rossica ou paléontologie de la Russie: tome 1, pt. 1, p. $1-681$, pt. 2 , p. $682-1657,1860$; tome 2 , pt. 1 , p. $1-640,1865-68$, pt. 2 , p. $641-$ 1304, 1868 ; tome 3, p. 1-533, 1863 . Atlas, pt. 1, pls. 1-59, 1859; pt. 2, pls. 1-39, $186 \mathrm{~S}$.

1861, Der Grünsand in der Umgegend von Moskwa: Soc. naturalistes imp. Moscou Bull., tome 34, no. 3, p. 278-313.

Elias, Maxim K., 1931, On a seed-bearing Annularia and on Annularia foliage: Kans. Univ. Sci. Bull., v. 20, p. 115-150, pls. 12-16.

1932, Grasses and other plants from the Tertiary rocks of Kansas and Colorado: Kans. Univ. Sci. Bull., v. 20, p. 333-367.

1942, Tertiary prairie grasses and other herbs from the High Plains: Geol. Soc. America, Special Paper 41, 176 p., pls. 1-16.

1947, Permopora keenae, a new late Permian alga from Texas: Jour. Paleontology, v. 21, p. 46-58.

Ellis, David, 1915, Fossil micro-organisms from the Jurassic and Cretaceous rocks of Great Britain: Royal Soc. Edinburgh Proc., iv. 35, p. 110-132, pls. $1,2$.

1916, On fossil fungi and fossil bacteria : British Assoc. Adv. Sci., Rept. 85th Mtg., Manchester, 1915, p. 729-730.

Elouski [Yelouskiy], V. A., 1930, Microscopical structure of the coal seam Moshchny, Chernogorski mines, Minusinsk Basin: United Geol. Prosp. Service U. S. S. R. Trans., pt. 4, p. 1-40, pls. 1-3.

Emberger, Louis, 1944, Les plantes fossiles dans leurs rapports avec les végetaux vivants, 492 p., Paris.

Emmons, Ebenezer, 1844, The Taconic System-Based on observations in New York, Massachusetts, Maine, Vermont, and Rhode Island: 65 p., 6 pls., Albany.

1856, Geological report of the midland counties of North Carolina: N. C. Geol. Survey, p. 1-347, pls. 1-14.

1857, American geology, containing a statement of the principles of the science with full illustrations of the characteristic American fossils, part 6: 152 p., pls. 3-10, Albany.

$296347-5 n-\cdots-3$ 
Endlicher, Stephano, 1836-40, Genera plantarum : 1483 p., Vienna.

1842, Mantissa botanica sistens genera plantarum supplementum secundum, 114 p., Vienna.

1847, Synopsis Coniferarum, $368 \mathrm{p}$.

Endo, Riuji, 1933, Manchuriophycus, nov. gen. from a Sinian formation of south Manchuria : Japanese Jour. Geology and Geography Trans. and Abs., v. 11, p. $43-48$, pls. $6,7$.

Engelhardt, Hermann, 1870, Flora der Braunkohlenformation im Königreich Sachsen: Preisschriften Gekrönt und Herausgegeben von der Fürstlich Jablonowski'schen Gesellschaft zu Leipzig, no. 16, p. 1-69, pls. 1-15, Leipzig. 1877, Über die fossilen Pflanzen des Süsswassersandsteins von Tschernowitz: Nova Acta Leopoldina, Band 39, Heft. 7, p. 359-392, pls. 20-24.

- 1885, Die Tertiärflora des Jesuitengrabens bei Kundratitz in Nordböhmen: Nova Acta Leopoldina, Band 48, p. 299-308, pls. 8-28.

1888, Über Rossellina congregata Beck sp. eine neue Pilzart aus der Braunkohlenformation Sachsens: Naturwiss. Gesell. Isis Dresden Abh., p. 33-35.

- 1891, Über Tertiärphflanzen von Chile: Senckenberg. naturf. Gesell. Abh., Band 16, p. 629-692, pls. 1-13.

— 1895, Über neue Tertiärpflanzen Süd Amerikas: Senckenberg. naturf. Gesell. Abh., Band 19, p. 1-47, pls. 1-9.

- 1922, Die alttertiäre Flora von Messel bei Darmstadt: Hesse geol. Landesanst. Abh., Band 7, Heft 4, p. 17-128, pls. 1-40, Darmstadt.

Engelhardt, Hermann, and Kinkelin, F., 1908, Oberpliocäne Flora und Fauna des Untermaintales insbesondere des Frankfurter Klärbeckens: Senckenberg. naturf. Gesell. Abh., Band 29, p. 150-306, pls. 22-36.

Erdtman, Gunnar, 1948, Did dicotyledonous plants exist in early Jurassic times?: Geol. Fören. Stockholm Förh., band 70, p. 265-271.

Etheridge, Robert 1891, On the occurrence of microscopic fungi, allied to the genus Palaeochlya Duncan, in the Permo-Carboniferous rocks of New South Wales and Queensland: Geol. Surey New South Wales Rec., v. 2, p. 95-99, pl. 7 .

-1899a, On two additional perforating bodies, believed to be Thallophytic cryptogams, from the Lower Palaeozoic rocks of New South Wales: Australian Mus. Rec., v. 3, p. 121-127.

- $1899 \mathrm{~b}$, On a fern (Blechnoxylon talbragarense), with secondary wood, forming a new genus, from the Coal Measures of the Talbragar district, New South Wales : Australian Mus. Rec., v. 3, p. 135-147, pls. 24-27.

- 1904, An endophyte (Stichus mermisoides) occurring in the test of a Cretaceous bivalve: Australian Mus. Rec., v. 5, p. 255-257, pls. 30-31.

Ettingshausen, Constantin, 1851, Die Tertiär-Floren der Oesterreichischen Monarchie: K.-k. geol. Reichsanst. Abh., Band 2, Heft 1, p. 4-36, pls. 1-5, Vienna.

1852a, Die Steinkohlenflora von Radnitz in Böhmen: K.-k. geol. Reichsanst. Abh., Band 2, Heft 3, p. 1-74, pls. 1-29, Vienna.

1852b, Über Palaeobromelia, ein neues fossiles Pflanzengeschlect: $\mathbf{K} .-\mathbf{k}$. geol. Reichsanst. Abh., Band 1, Heft 1, p. 1-8, pls. 1, 2, vienna.

18j20, Bergründung einiger neuen oder nicht genau bekannten arten Lias und der Oolithflora: K.-k. geol. Reichsanst. Abh., Band 1, Heft 3, p. 1-10, pls. 1-3, Vienna.

- 185̄3, Die Tertiäre Flora von Häring in Tirol : K.-k. geol. Reichsanst. Abh., Band 2, Heft 2, p. 1-118, pls. 1-31, Vienna. 
Ettingshausen, Constantin, 1854, Die Steinkohlenflora von Radnitz in Böhmen:

K.-k. geol. Reichsanst. Abh., Band 2, Heft 3, p. 1-74, pls. 1-29, Vienna.

1858, Die fossile Flora von Koflach in Steiermark : K.-k. Reichsanst., Jahrb,

Band 8, p. 738-756, pls. 2-4, Vienna.

1865, Die Farnkrüuter der Jetztwelt: 298 p., 180 pls., Vienna.

1867, Die Kreideflora von Niederschloena in Sachsen: K. Akad. Wiss.

Sitzungsber., Band 55, Abt. 1, p. 235-264, pls. 1-3, Vienna.

1868a, Die fossile Flora des Tertiär-Beckens von Bilin: K. Akad. Wiss.

Denkschr., Band 28, p. 191-242, Vienna.

1868b, Die fossile Flora der alteren Braunkohlenformation der Wetterau :

K. Akad. Wiss. Sitzungsber., Band 57, Heft 1, p. 807-893, pls. 1-5, Vienna.

1869, Die fossil Flora des Tertiär-Beckens von Bilin: K. Akad. Wiss.

Denkschr., Band 29, p. 1-110, pls. 45-55, Vienna.

1870a, Beiträge zur Kenntniss der Tertiärflora Steiermark: K. Akad.

Wiss. Sitzungsber., Band 60, Abt. 1, p. 17-100, pls. 1-6, Vienna.

1870b, Beiträge zur Kenntniss der fossilen Flora von Radoboj: K. Akad.

Wiss. Sitzungsber., Band 61, Abt. 1, p. 829-906, pls. 1-3, Vienna.

1871, Die fossile Flora von Sagor in Krain: K. Akad. Wiss. Sitzungsber.,

Band 63, Abt. 1, p. 406-413, Vienna.

1872, Die fossile Flora von Sagor in Krain: K. Akad. Wiss. Denkschr.,

Band 32, p. 159-202, Vienna.

1878, Report on phyto-palaeontological investigations generally and on

those relating to the Eocene flora of Great Britain in particular : Royal Soc.

London Proc., v. 28, p. 221-227.

1879, Report on phyto-palaeontological investigations of the fossil flora of

Sheppey : Royal Soc. London Proc., v. 29, p. 388-396.

- 1883, Beiträge zur Kenntniss der Tertiärflora australiens : K. Akad. Wiss.

Denkschr., Band 47, p. 101-148, pls. 1-7, Vienna.

1885, Die fossile Flora von Sagor in Krain: K. Akad. Wiss. Denkschr.,

Band 50, p. 1-56, pls. 28-32, Vienna.

1887a, Beiträge zur Kenntniss der Tertiärflora australiens: K. Akad.

Wiss. Denkschr., Band 53, p. 81-142, pls. 8-15, Vienna.

1887b, Beiträge zur Kenntniss der fossilen Flora Neuseelands : K. Akad.

Wiss. Denkschr., Band 53, p. 143-192, pls. 1-9, Vienna.

1888, Die fossile Flora von Leoben in Steiermark: K. Akad. Wiss.

Denschr., Band 54, p. 261-318, pls. 1-4, Vienna.

1890, Die fossile Flora von Schoenegg bei Wies in Steiermark: K. Akad.

Wiss. Denkschr., Band 57, p. 61-112, pls. 1-4, Vienna.

1891, Die fossile Flora von Schoenegg bei Wies in Steiermark: K. Akad.

Wiss. Denkschr., Band 58, p. 283-304, pls. 5, 6, Vienna.

1893, Über fossile Pflanzenreste aus der Kreiderformation australiens:

K. Akad. Wiss. Sitzungsber., Band 102, p. 126-151, Vienna.

1895, Beiträge zur Kenntniss der Kreideflora australiens: K. Akad. Wiss.

Denkschr., Band 62, p. 1-56, pls. 1-4, Vienna.

Falqui, Guiseppe, 1906, Su alcune piante fossili della Sardegna : 25 p., pl., Cagliarl.

__ 1907, Su alcune piante fossili del miocene inferiore (oligocene) di Zuri:

20 p., Cagliari-Sassari.

Fedin, A. H., 1943, A new genus of fossil conifers, Papaninia involucrata Fedin:

Acad. sci. U. R. S. S. Comptes Rendus, tome 41, no. 8, p. 365-367. [Russian.]

Feistmantel, Karl, 1867, Bemerkung iibereinige interessante Petrefacte aus dem

Steinkohlenbecken von Radnic: K.-Böhmische Gesell. Wiss. Sitzungsber., p.

59-61, Prague. 
1880, Eine neue Pflanzengattung aus böhmischen Steinkohlenschichten:

K.-Böhmische Gesell. Wiss. Sitzungsber., p. 298-303, Prague.

Feistmantel, Ottokar, 1871, Fruchtstande fossiler Pflanzen aus der boehmischen

Steinkohlenformation: K.-Böhmische Gesell. Wiss. Sitzungsber., p. 43-61,

Prague.

- 1874, Studien in Gebiete des Kohlengebirges von Böhmen: K.-Böhmische

Gesell. Wiss. Abh., Band 7, Heft 6, p. 1-175, pls. 1-3, Prague.

1875, Vorbericht über die Perucer Kreideschichten in Böhmen und ihre

fossilen Reste: K.-Böhmische Gesell. Wiss. Sitzungsber., Band 8, p. 254-276,

Prague.

1876a, Contributions towards the knowledge of the fossil flora in India :

Asiatic Soc. Bengal Jour., v. 45, p. 329-382, pls. 15-21.

1876b, Palaeontologische Beiträge, Part I, Über die Indischen Cycadeen-

gattungen Ptilophyllum Morr. und Dictyozamites Aldh.: Palaeontographica,

Band 23, supp. 3, Heft 3, p. 1-24, pls. 1-6.

- 1876c, Versteinerungen der boehmischen Kohlen-ablagerungen, III :

Palaeontographica, Band 23, p. 223-262, pls. 50-58.

1877, On a tree fern stem from the Cretaceous rocks near Trichinopoly

in southern India : Geol. Survey India Rec., v. 10, pt. 3, p. 133-140, pl. 10.

1878, Palaeontologische Beiträge, III, Palaeozoische und mesozoische

Flora des östlichen Australiens : Palaeontographica, Beil. 3, Lief. 3, p. 55-84,

pls. 1-10.

1879, The flora of the Talchir-Karharbari beds : India Geol. Survey Mem.,

Palaeontologia Indica, 12th ser., v. 3, p. 1-48, pls. 1-27.

1880-81, The fossil flora of the Lower Gondwanas, II, The flora of the

Damuda and Panchet Divisions: India Geol. Survey Mem., Palaeontologia

Indica, 12th ser., v. 3, p. 1-77, 1880 ; p. 78-149, 1881.

1886, The fossil flora of the Gondwana System, Part II, The fossil flora of some of the coalfields in western Bengal : India Geol. Survey Mem., Palaeontologia Indica, 12th ser., v. 4, p. 1-71, pls. 1A-12A.

Felix, Johannes, 1882a, Studien über fossile Hölzer: Inaugural Dissert., Univ. Leipzig, $81 \mathrm{p}$., pl.

1882b, Beiträge zur Kenntniss fossiler Coniferen-Hölzer: Bot. Jahrb., Band 3, p. 260-279, pl. 2.

- 1883a, Untersuchungen über fossile Hölzer: Deutsche geol. Gesell.

Zeitschr., Band 35, p. 59-92, pls. 2-4.

1883b, Die fossilen Hölzer Westindiens: Samm. Palaeont. Abh., Teil 1.

Heft 1, p. 1-29, pls. 1-5.

1884, Die Holzopale ungarns in Palaeophytologische hinsicht: Geol. Ungarischen Anst. Mitt. Jahrb., Band 7, p. 1-43, 4 pls.

- 1887a, Untersuchungen über fossile Hölzer, Stïck 3, Deutsche geol.

Gesell. Zeitschr., Band 39, p. 517-528, pl. 25.

- 1887b, Beiträge zur Kenntniss der fossilen Hölzer Ungarns : Geol. Ungarischen Anst. Jahrb., Band 8, p. 145-162, pls. 27-27A. 1894a, Untersuchungen über fossile Hölzer, Stück 4, IV, Hölzer aus dem Kaukasus : Deutsche geol. Gesell. Zeitschr., Band 46, p. 79-110, pls. 8-10. 1894b, Studien über fossile Pilze: Deutsche geol. Gesell. Zeitschr., Band 46, p. 260-280, p1. 19.

Felix, Johannes, and Nathorst, A. G., 1893, versteinerungen aus dem mexicanischen Staat Oaxaca, in Felix, Johannes, and Lenk, Hans, Beitr. Geologie u. Palaontologie der Republick Mexico, Teil 2, p. 39-54, pls. 1-3, Leipzig.

Fenton, Carroll L., 1943, A new Devonian alga from Western Australia: Am. Midland Naturalist, v. 30, p. 112, fig. 1 . 
Fenton, Carroll L., and Fenton, M. A., 1924, The stratigraphy and fauna of the Hackberry stage of the Upper Devonian : Mich. Univ., Mus. Geology, Contr., จ. 1,260 p., 45 pls.

1931, Algae and algal beds in the Belt series of Glacier National Park: Jour. Geology, v. 39, p. 670-686, pls. 1-10.

1937, Cambrian calcareous algae from Pennsylvania: Am. Midland Naturalist, v. 18 , p. $435-441,3$ pls.

1939, Pre-Cambrian and Paleozoic algae: Geol. Soc. America Bull., v. 50, p. 89-114, pls. 1-11.

Feruglio, Egidio, 1942, La flora Liasica del valle del Río Genoa (Patagonia) : Ginkgoales et Gymnospermae Incertae Sedis: Inst. Mus. Univ. Nac. La Plata-Mus. La Plata Notas, tome 7, Paleontogía, no. 40, p. 104-108.

Fiedler, Heinrich, 1857, Die fossilen Fruckte der Steinkohlen-Formation: Nova Acta Leopoldina, Band 26, p. 241-296, pls. 21-28.

Fischer-Ooster, Carl, 1858 Die fossilen Fucoiden der Schweinzer-Alpen, nebst Erörterungen ïber deren geologisches Alter : 73 p., 16 pls., Bern.

Fischer de Waldheim, G., 1826, Notice sur les végétaux fossiles du Gouvernment de Moscou : 23 p., pl., Moscow.

Fitch, Asa, 1850, A historical, topographical, and agricultural survey of the county of Washington: N. Y. State Agr. Soc., Albany, 1849, v. 9, p. 861-863.

Fliche, Paul, 1886, Note sur les flores tertiares des environs de Mulhouse: Soc. Indus. Mulhouse Bull., tome 56, p. 348-362.

1894, Sur des fruits de Palmiers trouvés dans le cénomanien aux environs de Sainte-Menehould: Acad. sci. Paris Comptes Rendus, tome 118, p. 889-890. 1896, Jitudes sur la flore fossile de l'Argonne Albien-Cénomanien: Soc. sci. Nancy Bull., sér. $2^{\circ}$, tome 14, p. 114-306, pls. 1-17.

1899a, Note sur un bois de Vigne des cinerites du Cantal : Soc. géol. France Bull., sêr. $3^{\circ}$, tome 27 , p. 318-321.

- 1899b, Note sur quelques fossiles végétaux de l'oligocène dans les Alpes

Françaises: Soc. géol. France Bull., sér. $3^{\circ}$, tome 27, p. 466-479, pl. 12.

1900, Contribution à la flore fossile de la Haute-Marne: Soc. sci. Nancy Bull., tome 16, p. 11-31, pls. 1, 2.

1903a, Sur les corps problematiques et les algues du trias en Lorraine: Acad. sci. Paris Comptes Rendus, tome 136, p. 827-829.

1903b, Sur les Lycopodenées du trias en Lorraine: Acad. sci. Paris

Comptes Rendus, tome 136, p. 907-908.

1905a. Flore fossile du trias en Lorraine et en Franche-Comté: Soc. sci.

Nancy Bull., tome 6, p. 1-66, pls. 1-5.

1905b, Flore fossile du trias en Lorraine et en Franche-Comté: Soc. sci.

Nancy Bull., tome 6, p. 67-166, pls. 6-15.

1908, Flore fossile du trias en Lorraine et en Franche-Comté: Soc. sci.

Nancy Bull., sér. $3^{\circ}$, tome 9 , p. 167-214, pls. 16-22.

1909, Sur une algue fossile du sinemurien : Acad. sci. Paris Comptes Rendus, tome 148, p. 210-212.

1910, Flore fossile du trias en Lorraine et en Franche-Comté: Soc. sci. Nancy Bull., sér. $3^{\circ}$, tome 11, p. 222-286, pls. 23-27.

Fliche, Paul, and Bleicher, G., 1882, Étude sur la flore de l'oolithe inférieure aux environs de Nancy : Soc. sci. Nancy Bull., sér. $2^{\circ}$, tome 6, p. 54-100.

Florin, Rudolf, 1926, Über einige Blatterabdrücke vom Cyclopteris-typus aus dem Karbon und Perm : Arkiv Botanik, Band 20A, p. 1-19, pls. 1-6.

1927, Preliminary descriptions of some Palaeozoic genera of coniferae: Arkiv Botanik, band 21A, no. 13, p. 1-7. 
Florin, Rudolf, 1929a, Über einige Algan und Koniferen aus dem mittleren und oberen Zechstein: Senckenbergiana, Band 11, p. 241-276, pls. 1-5.

1929b, Palaeozoic conifers : Internat. Cong. Plant Sci. Proc., Ithaca, N. Y., 1926, p. 401-411.

1933, Studien über die Cycadales des Mesozoikums: K. Svenska vetensk. akad. Handl., band 12, p. 1-134, pls. 1-16.

1934, Note on the nomenclature of Palaeozoic conifers: Svensk bot. Tidskr., band 28, p. 468-469.

- 1936a, Die fossilen Ginkgophyten von Franz-Joseph-Land, nebts Erörterunger über vermeintliche Cordaitales mesozoischen Alters-I, Spezieller Teil : Palaeontographica, Band 81, Abt. B, p. 1-173, pls. 11-42.

- 1936b, Die fossilen Ginkgophyten von Franz-Joseph-Land, nebts Erörterungen über vermeintliche Cordaitales mesozoischen Alters-II, Allgemeiner Teil.: Palaeontographica, Band 82, Abt. B, p. 1-72, pls. 1-6.

- 1938, Die Koniferen des Oberkarbons und des Unteren Perms, Erste Lief.: Palaeontographica, Band 85, Abt. B, p. 1-62, pls. 1-30.

- 1940a, On Walkomia n. gen. a genus of Upper Palaeozoic conifers from Gondwanaland: K. Svenska vetensk. akad. Handl., band 18, p. 1-23, pls. 1-4. - 1940b, Die Koniferen des Oberkarbons und des Unteren Perms : Palaeontographica, Band 85, Abt. B, p. 244-363, pls. 151-166.

- 1940c, The Tertiary fossil conifers of south Chile and their phytogeographical significance: K. Svenska vetensk. akad. Handl. 3, no. 2, p. 3-92, pls. 1-6.

1944, Die Koniferen des Oberkarbons und des Unteren Perms : Palaeontographica, Band 85, Abt. B, p. 457-654, pls. 173-186.

- 1951, Evolution in Coräaites and Conifers : Acta Horti Bergiani, Stockholm, v. 15, no. 11, p. 285-388.

Foerste, A. F., 1916, Dictyophlois reticulata gen. et sp. nov.: Torrey Bot. Club Bull., v. 42, p. 675-677, pl. 33 .

- 1923, Notes on Medinan, Niagaran, and Chester fossils: Denison Univ. Bull., v. 20, p. 37-120, pls. 14-15a.

Fontaine, William M., 1883, Contributions to the knowledge of the older Mesozoic flora of Virginia: U. S. Geol. Survey Mon. 6, 144 p., 54 pls.

- 1889, The Potomac or younger Mesozoic flora: U. S. Geol. Survey Mon. 15, 377 p., 180 pls.

Fontaine, William M., and White, I. C., 1880, The Permian or upper Carboniferous flora of West Virginia: Pa. 2d Geol. Survey, Rept. Progress PP. p. 1143, pls. 1-38.

Forbes, Eaward, 1851, Note on the fossil leaves represented in Plates II, III, and IV : Geol. Soc. London Quart. Jour., v. 7, p. 103, pls. 2-4.

Forchhammer, Johan Georg. 1845, On the influence of fucoidal plants upon the formations of the earth: British Assoc. Adv. Sci., Rept. 14th Mtg., York, 1844, p. 155-169.

Fox, C. S., 1931, Coal in India, II, The Gondwana System and related formations : Geol. Survey India Mem., v. 58, p. 1-241, pls. 1-10.

Fraipont, Charles, 1921, Contribution à la paleophytologie du wealdien conifère nouveau du Wealdien Belge Smeystersia minuta (Nov. gen.; Sew. sp.): Soc. geol, Belgique Annales, tome 44, p. M51-53.

Fremont, James C., 1845, Report of the exploring expedition to the Rocky Mountains in the year 1842 and to Oregon and North California: 583 p., Washington. 
Frenguelli, Joaquim, 1942, Contribuciones al conocimiento de la flora del Gondwana superior en la Argentina : Mus. La Plata Notas, tomo 7, Palaeontología, nos. 42-51, p. 265-353.

1943a, Reseña critica de los géneros atribuídos a la "Serie de Thinnfeldia": Revista Mus. La Plata, nueva ser., tomo 2, Paleontología, no. 12, p. 225-342.

1943b, Contribuciones al conocimiento de la flores del Gondwana superior en la Argentina: Mus. La Plata Notas, tomo 8, Paleontologí, nos. 57-60, p. 401-430.

- 1944a, Las especies del género Zuberia en la Argentina: Mus. La Plata Anales, nueva ser., Paleontología, sec. B, Paleobotánica, no. 1, p. 1-30, pls. 1-13.

1944b, Contribuciones al conocimiento de la flora del Gondwana superior en la Argentina: Mus. La Plata Notas, tomo 9, Paleontologia, nos. 64-68, p. 377-420.

- 1950, Addendo a la flora del Gondwana superior en la Argentina: Rev. Assoc. Geol. Argentina, tomo 5, no. 1, p. 15-30, pls. 1, 2.

Frentzen, Kurt, 1916, Die Flora des Buntsandsteins Badens: Baden Geol. Landesanst. Mitt. 8, p. 63-162, pls. 10-23.

1931, Studies über fossilen Hölzer der Sammelgattung Dadoxylon Endl.:

Heidelberger Alad. Wiss. Abh., Heft 16, p. 1-93, pls. 1-3.

1932, Beiträge zur Kenntnis der fossilen Flora des südwestlichen Deutschland: Oberrkein. geol. Ver. Jahresber., Neue Folge, Band 21, p. 75-94, pls. 1-3.

Fric, Anton, 1893, Studien in Gebiete der böhmischen der kreideformationPalaeontologische Untersuchungen der einzelnen: Archiv naturwiss. Landesdurchforschung Böhmen, Band 9, no. 1, p. 1-134, Prague.

Fric, Anton, and Bayer, Edwin, 1901, Studien in Gebiete der Böhmischen Kreideformation: Archiv naturwiss. Landesdurchforschung Böhmen, Band 11, p. 1-180, Prague.

Friedrich, Paul, 1883, Beiträge zur Kenntniss der Tertiärflora der Provinz Sachsen: Abh. geol. Specialkarte Preussen, Band 4, Teil 3, p. 159-463, pls. 1-31.

Fritel, P. H., 1904, Plante fossile nouvelle des Grès de Belleu : Le Naturaliste, tome 26, p. $233-235$.

1909, Revision de la Flore fossile des Grès Yprésiens du bassin de Paris : Jour. botanique, sér. $2^{\circ}$, tome 2, p. 101-112.

- 1910, Etude sur les végétaux fossiles de l'étage sparnacien du bassin de Paris: Soc. géol. France Mém., Paléontologie, tome 16, no. 40, p. 4-37, pls. 20-22.

1912 Sur une plante fossile nouvelle des calcaires marneux du Bois d'Asson et sur un fruit de Nympheacés de même gisement: Soc. géol. France Bull., sér. $4^{\circ}$, tome 12, pl. 643-648, pl. 22.

1924, Suite et additions à la révision de la flore cuisienne des grès de Belleu: Soc. géol. France Bull., sér. $4^{\circ}$, tome 24, p. 150-175.

1927, Sur deux fruits nouveaux de la flore aturienne de Provence: Soc. géol. France Compte Rendu, fasc. 10-12, p. 117-119, figs. 1-4.

Fritsch, Anton, 1908, Problematica Silurica, in Barrande, Joachim, 1852-1911, Systême silurien du centre de la Bohême, 28 p. 12 pl., Prague. [Separate.]

Frollo M. M., 1938, Sur un noureau genre de Codiacée du jurassique supérieur des Carpates orientales: Soc. géol. France Bull., sér. $5^{\circ}$, tome 8 p. 268-271. 
Gagel, Curt, 1904, Über einige Bohrergebnisse und ein neues pflanzenfuhrendes Interglazial aus der Gegend von Elmshorn : Preuss. geol. Landesanst. Jahrb., Band 25, p. 246-281, pls. 8-11.

Gallwitz, Hans, and Gothan, Walther, 1939, Tier und Pflanzenversteinerungen aus dem oberdevon von Blosenberg im Vogtland: Preuss. geol. Landesanst. Jahrb., 1938, Band 59, p. 755-768.

Ganju, P. N., 1944, The Jurassic flora of Onthea in the Rajmahal Hills: Nat. Acad. Sci. India Proc., v. 14, sec. B, p. 76-77, pis. $2,3$.

Gardner, John Starkie, 1887, On the leaf beds and gravels of Ardtun, Carsaig, *** in Mull: Geol. Soc. London Quart. Jour., v. 43, p., 270-276.

Gardner, John Starkie, and Ettingshausen, Constantin, 1879, A monograph of the British Eocene flora: Palaeontographical Soc. London, v. 33, p. 1-38, pls. 1-5.

1882, A monograph of the British Eocene flora: Palaeontographical Soc.. London, v. 36, p. 59-86, pls. $12,13$.

Gardner, Nathaniel Lyon, 1924, Two new fossil algae from the Miocene: Acad. Nat. Sci. Philadelphia Proc., v. 75, p. 361-363.

Garwood, E. J., 1914, Some new rock-building organisms from the Lower Carboniferous beds of Westmorland: Geol. Mag., new ser., decade 6, v. 1, p. 265-271, pls. 20, 21, London.

1931, The Tuedian beds of northern Cumberland and Roxburghshire east of the Liddel Water : Geol. Soc. London Quart. Jour., v. 87, p. 97-156, pls. 7-16.

Gasparis, Aurelio de, 1895, Su di una epatica del trias: R. accad. sci., Naples. Rend. v. 1, p. 67-69.

Geinitz, Eugen, 1873, Versteinerungen aus dem Brandschiefer der unteren Dyas vòn Weissig bei Pillnitz in Sachsen : Neues Jahrb., p. 691-704, pl. 3.

Geinitz, Hanns Bruno, 1839-42, Charakteristik der Schichten und Petrefacten des Sächsischböhmischen Kreidegebirges : 116 p., 24 pls., Dresden and Leipzig. - 1854, Darstellung der flora des Hainichen-Ebersdorfer und des Floehaer Kohlenbassins : 80 p., Leipzig.

1855, Die Versteinerungen der Steinkohlenformation in Sachsen : 61 p., 35 pls., Leipzig.

1858, Die Leitpflanzen des Rothliegenden und des Zechsteinbirges oder der permischen Formation in Sachsen : K. polytech. Schule u. K. Baugewerkenschule, Dresden, p. 1-27, pls. 1, 2.

1862, Dyas oder die Zechsteinformation und das Rothliegende: Band 2,

Die Pflanzen der Dyas und Geologisches, p. 131-342, pls. 24-42.

1863, Über zwei neue dyadische Pflanzen: Neues Jahrb., p. 525-530, pls. $6,7$.

1864, Über organischer überreste in dem Dachshiefer von Wurzbach bei Lohenstein : Neues Jahrb., p. 1-9, pls. 1, 2.

1865, Über einige seltene Versteinerungen aus der unteren Dyas und der Steinkohlen-Formation : Neues Jahrb., p. 385-395.

1870, Über eine neue fossile Frucht aus dem Zechsteine und einige Ueberreste aus der Steinkohlenformation; Naturwiss. Gesell. Isis Dresden Sitzungsber., p. 60-65, pl. 1, Dresden.

1880, Nachträge zur Dyas I : K. min. geol. Praehistoriche Mus. Mitt., Dresden, Band 30, p. 1-43, pls. 1-7.

1925, Contribuciones a la paleontolgía de la Republica Argentla-Sobre plantas y animales reticos en las provincias argentinas de la Rioja San Juan y Mendoza. [Translated by G. Bodenbender Anquin: Acad Nac. Cienc. Actas, tomo 8, p. 333-347, pls. 1, 2.] 
Geinitz, Hanns Bruno, and Gutbier, August, 1848-49, Die Versteinerungen des Zechsteingebirges und Rothliegenden oder des permischen Systemes in Sachsen : 26 p., 8 pls., $1848 ; 31$ p., 11 pls., 1849.

Germar, Ernest Friedrich, 1844-53, Die Versteinerungen des Steinkohlengebirges von Weltin und Löbejün im Saalkreise : Teil 1, p. 1-12, pls. 1-5, 1844; Teil 2, p. 13-28, pls. 6-10, 1845 ; Teil 3, p. 29-40, pls. 11-15, 1845; Teil 4, p. 41-48, pls. 16-20, 1846?; Teil 5, p. 61-79, pls. 26-30, 1849; Teil 7, p. 81-96, pls. 31-33, 1851; Teil 8, p. 97-116, pls. 32-40, 1853.

— 1846, Über Omphalomela scabra eine neue Pflanzenversteinerung aus dem Keuper von Badeleben in Thuringen: Palaeontographica, Band 1, p. 26-29.

_ 1852, Sigillaria sternbergi Münst. aus dem bunten Sandstein: Deutsche geol. Gesell, Zeitschr., Band 4, p. 183-189, pl. 8.

Germar, Ernst Friedrich, and Kaulfuss, F., 1831, Über einige merkwürdige Pflanzenabdrücke aus der Steinkohlenformation: Nova Acta Leopoldina, Band 15, pt. 2, p. 219-230, pls. 65-66.

Geyler, H. T., 1877, Über fossile Pflanzen von Borneo: Palaeontographica, supp. 3, p. $61-84$, pls. $11,12$.

- 1879, Palaeontologie Geographie : Bot. Jahresber. [Just], Band 5, p. 775824.

—_ 1883, Reviews of paleobotanical papers: Bot. Jahresber. [Just], 1880, Band 8, p. 174-647.

— 1887a, Über fossile Pflanzen von Labuan: Vega-Exped. Vetenskapliga Arb., band 4, p. 475-507, pls. 32-39, Stockholm.

—_ 1887b, Notiz über eine neuerdings aufgeschlossere Pliocänflora in der Umgebung von Frankfurt a. M.: Bot. Jahrb. [Engler], Band 8, p. 161-164.

Geyler, H. T., and Kinkelin, P. F., 1887, Oberpliocän Flora aus dem Baugruben des Klärbeckens bei Niederrad und der Schleuse bei Höchst a. M.: Senckenberg.. naturf. Gesell. Abh., Band 15, p. 1-47, pls. 1-4.

Glocker, E. F., 1841, Über die Kalkführende Sandsteinformation auf beiden Se1ten der mittleren March, in der Gegend zwischen Kwassitz und Kremsier: Nova Acta Leopoldina, Band 19, Beil. 2, p. 309-334, pl. 4.

Gluck, Hugo, 1912, Eine neue gesteinsbildende Siphonee (Codiacee) aus dem marinen Tertiar von Suddeutschland: Grossh.-Bad. geol. Landesanst. Mitt., Band 7, nt. 1, p. 1-24, pls. 1-4, Heidelberg.

Goebel, F., 1906, Protocalamariaceae Pontonie 1899 : Centralbl. Mineralogie, p. 241-242.

Goeppert, Henricus Robertus, 1834, Über die Bestrebungen der Schlesier, die Flora der Vorwelt zu erläutern-Schlesischen Provinzial-Blattern: 26 p. Breslau.

1836, Die fossilen Farrenkräuter (Systema filicum fossilium) : Nova Acta Leopoldina, Band 17, p. 1-486, pls. 1-44.

- 1837, Übersicht der bis jetzt bekannten Gattungen der fossilen Pflanzen, in Germar, E. F., Lehrbuche der Mineralogie, 2d ed., p. 424-453, Halle.

- 1838, De floribus in statu fossili commentatio: Nova Acta Leopoldina, Band 18, p. 547-572, pls. 41, 42.

1839, Bemerkungen über die als Geschiebe im nördlichen Deutschland vorkommenden Versteinerten : Neues Jahrb., p. 518-521, pl. 8b.

—_ 1840, Über die Verbreitung der fossilen Gewächse in der Steinkohlenformation: Schles. Gesell. vaterl. Kultur Arb. Verh., p. 74-77, Breslau.

- 1841a, Über die fossile Flora der Quadersandsteinformation in Schlesien, als erster Beitrag zur Flora der Tertiürgebilde : Nova Acta Leopoldina, Band 19, Teil 2, p. 99-134, pls. 46-53. 
Goeppert, Henricus Robertus, 1841b, Taxites scalariformis, eine neue Art fossilen

Holzes: Archiv Mineralogie Geognosie, Band 15, p. 727-730, pl. 17, Berlin.

- 1841c-46, Les genres des plantes fossiles: p. 1-70, pls. 1-18, 1841; p.

71-118, pls. 1-18, 1842; p. 119-154, pls. 1-20, 1846, Bonn.

_ 1842a, Fossile Pflanzenreste des Eisensandes von Achen als zweiter

Beitrag zur Flora der Tertiärgebilde : Nova Acta Leopoldina Band 19, Teil 2,

p. 137-160, pl. 54.

- 1842b, Über die fossile Flora der Gypsformation zu Dirschel in ober-

schlesien: Nova Acta Leopoldina, Band 19, Teil 2, p. 369-378, pls. 66-67. 1844, Uebersicht die fossilen Flora Schlesiens, in Wimmer, Friedrich,

Flora von Schlesien, Band 2, p. 157-225, Breslau.

1845, Description des végétaux fossiles recueillis par M. P. de Tchihatcheff en Scherie, in Tchihatcheff Pierre, Voyage scientifique dans l'Altai Oriental et les parties adjacentes de la frontière de la Chine, p. 379-390, pls. 25-35, Paris.

- 1850, Monographie der fossilen Coniferen: Hollandsche Maatsch.

Wetensch. Natuurk. Verh., boekdeel 6, p. 1-286; p. 1-73, pls. 1-58, Leiden.

1851, Úber die Flora des Uebergangsgebirges: Deutsche geol. Gesell.

Zeitschr., Band 3, p. 185-207.

1852a, Beiträge zur Tertiärflora Schlesiens: Palaeontographica, Band 2,

p. 257-285, pls. 33-38.

1852b, Fossile Flora des Übergangsgebirges : Nova Acta Leopoldina, Band

22, p. 1-299, pls. 1-44.

- 1852c, Über die Braunkohlenflora des nordostlichen Deutscshlands :

Deutsche geol. Gesell. Zeitschr., Band 4, p. 484-496.

- 1853a, Über die Tertiär Flora Java's: Neues Jahrb., p. 433-436.

1853b, Über die Bernsteinflora: Monatsh. K. Akad., p. 450-477, Berlin;

Schles. Gesell. vaterl. Kultur, Jahresber. 31, p. 46-62, 1854, Breslau.

1853c, Über die gegenwärtigen Verhaltnisse der Paläontologie in Schlesien,

so wie über fossile Cycadeen: Schles. Gesell. Jubiläums Denkschr. 1853, p.

251-265, pls. 7-10, Breslau.

1855, Die Tertiäre Flora von Schossnitz in Schlesien: 52 p., 26 pls., Gorlitz.

1857, Die Tertiärflora auf der Insel Java: 162 p., 14 pls., Elberfeld.

1859, Über die fossile Flora der Silurischen, der Devonischen und unteren

Kohlenformation: Nova Acta Leopoldina, Band 27, p. 427-606, pls. 34-39.

1863, Die versteinten Hölzer Sachsens: Schles. Gessell. vaterl. Kultur

Jahresber. 40, p. 33-36, Breslau.

1864-65, Die fossile Flora der permischen Formation : Palaeontographica,

Band 12, p. 1-224, pls. 1-40, 1864 ; p. 225-316, pls. 41-64, 1865. 1865a, Über das Vorkommen von Baumfarn in der fossilen Flora, insbeson-

dere in der Kreideformation : Neues Jahrb., p. 395-399.

1865b, Über Aphyllostachys, eine neue fossile Pflanzengattung aus der

Gruppe der Calamarien * * *: Nova Acta Leopoldina, Band. 32, p. 1-16, pl. 1.

1869, Über algenartige Einschlüsse in Diamanten und über Bildung der-

selben: Schles. Gesell. vaterl. Kultur Abh., 1868, Band. 46, p. 61-67, pl. 1, Breslau.

- 1881, Arboretum fossile-Sammalung von Dünnschliren fossiler Coniferen-

Hölzer der polaeozoishen Formation : 6 p., Breslau.

Goeppert, Henricus Robertus, and Stenzel, G., 1881, Die Medulloseae, eine neue

Gruppe der fossilen Cycadeen: Palaeontographica, Band 28, p. 111-128, pls. 14-17. 
Goldenberg, Friedrich, 1855-62, Flora Saraepontana fossilis-Die Pflanzenversteinerungen des Steinkohlengebirges von Saarbrücken: Teil 1, p. 1-38, 1855 ; Teil 2, p. 1-60, 1857 ; Teil 3, p. 1-47, 1862; pls. A, B. 1-16.

Goldring, Winifred, 1924, The Upper Devonian forest of seed ferns in eastern New York: N. Y. State Mus. Bull. 251, p. 50-72, pls. 2-11.

Gordon, W. T., 1912, On Rhetinangium arberi-A new genus of Cycadoflices from the Calciferous Sandstone.series: Royal Soc. Edinburgh Trans, v. 48, p. 813-825, pls. 1-3.

- 1938, On Tetrastichia bupatides-A Carboniferous pteridosperm from East Lothian: Royal Soc. Ediṇburgh Trans., v. 59, p. 351-370, pls. 1-6.

1941, On Salpingostoma dasu-A new Carboniferous seed from East Lothian: Royal Soc. Edinburgh Trans., v. 60, p. 427-464, pls. 1-6.

Gothan, Walther, 1905, Zur anatomie lebender und fossiler Gymnospermum Hölzer : Preuss. geol. Landesanst. Abh., Neue Folge, Heft 44, p. 1-108.

1906, Die fossilen Coniferenhölzer von Senftenberg: Preuss. geol. Landesanst. Abh., Band 46, p. 155-171.

1907, Die fossilen Hölzer von König Karls Land: K. Svenska vetensk. akad. Handl., band 42, p. 1-44, pl. 1 .

1908, Die fossilen Hölzer von der Seymour und Snow Hill Insel : Schwedisch Sudpolar Exped., Wiss. Ergebnisse 1901-03, Band 3, Lief. 8, p. 1-3, pls. 1-2.

1909, Review of papers by Raciborski : Geol. Zentralbl., Band 13, p. 399. 1910, Die fossilen Hölzreste von Spitzbergen: K. Svenska vetensk. akad.

Handl., band 45, p. 1-56, pls. 1-7.

1912, Über die Gattung Thinnfeldia Ettingshausen : Naturh. Gesell. Nürnberg Abh., Band 19, p. 1-14, pls. 13-16.

ــ1913a, Die Oberschlesische Steinkohlenflora, Teil I, Farne und farnähnliche Gewächse (Cycadofilices bezw. Pteridospermen) : Preuss. geol. Landesanst. Abh., Neue Folge, Heft 75, p. 1-278, pls. 1-53.

1913b, Über das angebliche flozführende Rotliegende im oberschlesischen

Steinkohlenbecken: Deutsch. geol. Gesell. Zeitschr., Band 65, Heft 6, p. 281-288.

- 1914, Die unter-liassische (rhatische) Flora der Umgegend von Nürnberg: Naturh. Gesell. Nürnberg Abh., Band 19, p. 1-98, pls. 17-39.

- 1927a, Strukturzeigende Pflanzen aus dem Oberdevon von Wildenfels: Sächs. geol. Landesanst. Abh., Band 3, p. 1-12, pls. 1, 2.

1927b, Fossile Pflanzen aus den Karru-schichten der Urngeburg des Ulugurugebirges in Deutsch-Ostafrika : Palaeontographica, Beil. 7, p. 145152, pls. 18, 19.

- 1928a, Über einige eigentumliche Pflanzenreste aus dem Karbon von Flöha : Natur. Gesell. Chemnitz Ber., p. 1-5, pls. 1-3.

1928b, Bemerkungen zur Alt-Carbonflora von Peru, besonders von Paracas : Neues Jahrb., Band 69, Abt. B, p. 292-299.

— 1929, Die Steinkohlenflora der westlichen paralischen Carbonreviere Deutschlands : Preuss. geol. Landesanst. Inst. Paläobotanik u. Petrographie Brennsteine Arb., Band 1, Heft 1, p. 17-19.

1941, Über die Samen und Pollenorgane von Lonchopteris rugosa: Reichsstelle Bodenforschung Jahrb. 1940, Band 61, p. 278-282, pl. 22.

1942a, Über Palmenwurzelhölzeraus der Braunkohle von Böhlen : Geschiebeforschung Flachlandsgeol. Zeitschr., Band 18, p. 2-14.

1942b, Arbeiten von 1936 bis 1939 und Nachträge-Paläobotanik : Just's

Bot. Jahresber., Band 67, Abt. 1, p. 1-160. 
Gothan, Walther, 1949, Die Unterkarbon-flora der Dobrilugker Tiefbohrungen: Geol. Landesanst. Berlin Abh.y Neue Folge, no. 217, p. 7-31, pls. 1-6.

- 1950, Übereine neue Farnfruktifikation (Waldenburgia corynepteroides, nov. gen. et sp.) des Niederschlesischen Karbons: Bot. Archiv, Band 1, p. 349-353, pl. 1.

Gothan, Walther, Berlin, E. F., and Gimm-Elgersburg, Otto, 1930, Neuere Beobachtungen und Betrachtungen über die Flora des Rotliegenden von Thuringen: Preuss. geol. Landesanst. Inst. Paläobotanik u. Petrographie Brennsteine Arb., Band 2, Heft 1, p. 39-74, pl. 9.

Gothan, Walther, and Nagel, K., 1921, Über einen cedroiden Coniferenzapfen aus dem Unter-Eocän der Greifswalder Oie: Preuss. geol. Landesanst. Jahrb., Band 41, p. 121-131, pl. 8.

Gothan, Walther, and Sze, H. C., 1933, UUber die Pälaozoische Flora der Provinz Kiangsu : Natl. Research Inst. Geology (Acad. Sinica), Mem. 13, p. 1-40, pls. 1-4.

Gothan, Walther, and Zimmerman, F., 1932, Die Oberdevonflora von Liebischen und Bögendorf: Preuss. geol. Landesanst. Inst. Paläobotanik u. Petrographie Brennsteine Arb., Band 2, p. 103-130, 6 pls.

Gottsche, Carl, 1886, Über die im Bernstein eingeschlossenen Lebermoose: Bot. Centralbl., Band 25, p. 122.

Gourlie, William, 1843, Notice of the fossil planets in the Glasgow Geological Museum: Glasgow Philos. Soc. Proc., v. 1, p. 105-108, pl. 2.

Graham, Roy, 1934, Pennsylvanian flora of Illinois as revealed in coal balls, I : Bot. Gazette, v. 95, p. 453-476, pls. 8, 9 .

Grand'Eury, F. Cyrille, 1877, Flore carbonifère du Département de la Loire et du centre de la France: Acad. sci. inst. France Mém., tome 24, p. 1-624, pls. $1-34$.

1879, Feuilles et chatons des Calamodendrées: Assoc: Française av. sci. Comptes Rendus 7th Sess., 1878, p. 578-580.

1890, Geologie et paléontologie du bassin houiller du Gard: 354 p., 22 pls., Saint-Etienne.

_ 1904, Sur les graines des Névropteridées : Acad. sci. Paris Comptes Rendus, tome 139, p. 782-786.

Grandori, Luigia, 1916, Su di un seme mesozoico di pteridosperma e sulle sue affinita con forme palaeozoiche e forme Viventi: Accad. sci. veneto-trentinoistriana Atti, v. 8, p. 107-116.

Grant, C. W., 1840, Memoir to illustrate a geological map of Cutch: Geol. Soc. London Trans., 2d ser., v. 5, pt. 2, p. 289-330, pls. 21-26.

Graves, Louis, 1847, Essai sur la topographie geognostique du Departement de l'Oise : 804 p., Beauvais.

Groves, James, 1924, British Charophyta : Jour. Bot. British and Foreign, v. 62, p. 116.

Groves, James, and Bullock-Webster, G. R., 1924a, A sketch of the geological history of the Charophyta, in The British Charophyta, v, 2, Royal Soc. London, p. 72-90.

Gruiss, Johannes, 1924, Nematophora fascigera gen. nov., eine Devonalga als Vorläufer des Gymnospermen und ihre Beziehungen zu einer neuen Kohlentheorie auf gärphysiologischer Grundlage : 95 p., 6 pls., Berin.

- 1927, Weitener Mitteilungen über die Urhefe Saccharomyces devonicus: Wochenschr. Brauerei, Band 44, Heft 32, p. 365-369.

1928a, Untersuchungen über den Ursprung der Hefe: Wochenschr.

Brauerei, Band 45, Heft 30, p. 341-344, p. 353-357.

1928b, Zur Biologie Devonischer Thallophyten: Palaeobiologica, Band 1, Teil 2, p. 487-517, pls. 39-41. 
Guillard, Achille, 1839, Note sur un végétal fossile des terrains houiller de Rivede-Gier : Annales sci. phys. nat. agriculture, Paris, tome 2, p. 123-130, pls. 3, 4.

Gümbel, C. W., 1859a, Beiträge zur Flora der Vorzeit namentlich des Rothliegendent bel Erbendorf in der bayerischen Obrepfalz: K. bayer. bot. Gesell. Denkschr., Band 4, p. 84-107, pl. 8, Regensburg.

- 1859b, Nachtrag zur Abhandlung-Beiträge zur Flora der Vorzeit * * *

K. bayer. bot. Gesell. Denkschr., Band 4, p. 161, Regensburg.

1871, Die sogenannten Nulliporen (Lithothamnium und Dactylopora) und ihre Bethieligung an der Zusammensetzung der Kalkgesteine: Bayer. Akad. Wiss., Math.-naturwiss. Abt. Abh., Band 11, Teil 1, p. 13-52, pls. 1, 2; Teil 2, p. 231-290, pls. D1-4, Munich.

1879, Geognostische Beschreibung des Fitchtelgebirges mit dem Frankenwalde und dem westlichen Vordlande : Band 3, 698 p., Gotha.

Gupta, K. M., 1936, Leguminoxylon burmense gen. et sp. nov. a dicotyledonous wood from the Tertiary of Burma: Indian Sci. Cong., 23d, Indore, Proc., p. 305 .

Gtirich, Georges, 1906, Les Spongiostromides du Viseen de la Province de Namur: Mus. royal histoire. nat. Belgique Mém., tome 3, p. 1-55, pl. 1-23.

Gutbier, August, 1835, Abdrucke und Versteinerungen des Zwickauer Schwarzkohlengebirges: 80 p., 11 pls., Zwickau.

Hacquaert, Armand I., 1932, A propos des fossiles decouverts dans les roches du systeme du Kundelungu au Katanga (Congo Belge): Acad. royale Belgique Bull., Cl. Sci., sér. $5^{\circ}$, tome 18, p. 256-268.

Hagenow, Karl Friedrich, 1840, Monographie der Rügenschen Kriedeversteinerungen : Neues Jahrb., p. 631-672.

Hagström, O., 1906, Holstia splendens n. g. et n. sp. : Geol. Fören. Stockholm Förh., band 28 , no. 1, p. 90-93, pl. 3 .

Hall, James, 1843, Geology of New York, Part 4: 683 p., 19 pls., Albany.

- 1847, Palaeontology of New York, v. 1 : 338 p., 33 pls., Albany.

1852, Palaeontology of New York, v. $2: 362$ p., 83 pls., Albany.

- 1863, Contributions to palaeontology; principally from investigations made during the years 1861 and 1862 : N. Y. State Cabinet Nat. History, Ann. Rept. 16, p. 19-226, pls. 1-11.

- 1865, Figures and descriptions of Canadian organic remains-Decade II, Graptolites of the Quebec group: Geol. Survey Canada, p. 1-151, pls. A, B, 1-21, Montreal.

1884, Cryptozoon: N. Y. State Mus. Nat. History Ann. Rept. 36, opposite pl. 6.

- 1886, Note on some obscure organisms in the roofing slates of Washington County, N. Y. : N. Y. State Mus. Nat. History Ann. Rept. 39, p. 160, pl. 11.

Halle, Thore G., 1908, Zur Kenntnis mesozoischen Equisetales Schwedens: K. Svenska vetensk. Akad. Handl, band 43, no. 1, p. 1-40, pls. 1-9.

1910, On the Swedish species of Sagcnopteris Presl and on Hydropterangium nov. gen.: K. Svenska vetensk. akad. Handl., band 45, p. 1-15, pls. 1-3.

- 1911a, On the fructification of Jurassic fern leaves of the Cladophlebis denticulata type: Arkiv Botanil, band 10, no. 15, p. 1-10, pls. 1, 2.

- 1911b, Cloughtonia, a problematic fossil plant from the Yorkshire Oolite: Arkiv Botanik, band 10, no. 14, p. 1-6, pls. 1, 2 .

1913, The Mesozoic flora of Graham Land: Schwedisch Sudpolar Exped. Nordenskjold Wiss. Ergebnesse 1901-03, band 3, no. 14, p. 1-123, Stockholm.

- 1916a, Lower Devonian plants from Rüragen in Norway: K. Svenska vetensk. akad. Handl., band 57, p. 1-46, pls. 1-4. 
Halle, Thore G., 1916b, A fossil sporogonium from the Lower Devonian of Röragen in Norway : Bot. Notiser, p. 79-81.

1925, Tingia, a new genus of fossil plants from the Permian of China: Geol. Survey China Bull. 7, p. 1-12, pls. 1, 2.

- 1927, Palaezoic plants from central Shansi: Geol. Survey China, Palaeontologia Sinica, ser. A, v. 2, fasc. 1, p. 1-316, pls. 1-64.

1933, The structure of certain fossil spore-bearing organs believed to belong to pteridosperms: K. Svenska vetensk. akad. Handl., band 12, p. 1-103, pls. 1-15.

1936, Notes on the Devonian genus Sporogonites: Svenska bot. Tidskr., band 30 , p. $613-623$, pls. 3,4 .

Hallier, Ernst, 1865, Untersuchung fossiler Zellenpflanzen: Bot. Zeitung, Band 23, p. 189-191, pl. 9B.

Hancock, A., and Atthey, T., 1869, On some curious fossil fungi from the black shale of the Northumberland coal field: Annals and Mag. Nat. History 4th ser., v. 4, p. 221-228, pls. 9, 10.

Harkness, Robert, 1855, On the anthracitic schists and the fucoidal remains occurring in the Lower Silurian rocks of the south of Scotland: Geol. Soc. London Quart. Jour., v. 11, p. 468-476.

Harris, Thomas Maxwell, 1926, The Rhaetic flora of Scoresby Sound : Meddelelser om Grönland, Bind 68, p. 45-147, pls. 1-13.

1929, Schizopodium davidi gen. et sp. nov.-a new type of stem from the Devonian rocks of Australia: Royal Soc. London Philos. Trans., $\nabla$. B217, p. $395-410$, pls. 91-93.

- 1931a, Rhaetic floras : Biol. Rev., v. 6, p. 133-162.

1931b, The fossil flora of Scoresby Sound, east Greenland, Part 1, Cryptograms (exclusive of Lycopodiales): Meddelelser om Grönland, Bind 85, Nr. 2, p. 1-102, pls. 1-18.

1932a, The fossil flora of Scoresby Sound, east Greenland, Part 2, Description of seed plants incertae sedis, together with a discussion of certain cycadophyte cuticles: Meddelelser om Grönland, Bind 85, Nr. 3, p. 1-112, pls. 1-9.

1932b, The fossil flora of Scoresby Sound, east Greenland, Part 3, Caytoniales and Bennettitales: Meddelelser om Grönland, Bind 85, Nr. 5, p. 1-133, pls. 1-19.

1935, The fossil flora of Scoresby Sound, east Greenland, Part 4, Ginkgoales, coniferales, lycopodiales and isolated fructifications: Meddelelser om Grönland, Bind 112. Nr. 1, p. 1-176, pls. 1-29.

1937, The fossil flora of Scoresby Sound, east Greenland, Part 5, Stratigraphic relations of the plant beds: Meddelelser om Grönland, Bind 112. Nr. 2, p. 1-114.

1938, The British Rhaetic flora: British Mus. Nat. History, p. 1-84, pls. 1-5.

1939, British Purbeck Charophyta : British Mus. Nat. History, p. 1-83, pls. 1-17, London.

1941, Cones of extinct Cycadales from the Jurassic rocks of Yorkshire: Royal Soc. London Philos. Trans., v. B231, p. 75-98, pls. 5, 6.

- 1942a, Notes on the Jurassic flora of Yorkshire: Annals and Mag, Nat. History, 11th ser, v. 8, p. 568-587.

1942b, Wonnacottia, a new Bennettitalean microsporophyll; Annals Botany, new ser., จ. 6, p. 577-592.

Harris, W. H., 1884, Fossil plants in the Sllurian formation near Cardiff: Scl. Gossip, v. 20, p. 28-30, London. 
Hartig, Theador, 1848a, Beiträge zur Geschichte der Pflanzen und zur Kenntniss der norddeutschen Braukohlen-Flora : Bot. Zeitung, Band 6, p. 166-172.

-1848b, Beiträge zur Geschichte der Pflanzen und zur Kenntniss der norddeutschen Braunkohlen-Flora : Bot. Zeitung, Band 6. p. 137-146.

1848c, Beiträge zur Geschichte der Pflanzen und zur Kenntniss der norddeutschen Braunkohlen-Flora : Bot. Zeitung, Band 6, p. 185-190.

Hartung, Wolfgang, 1940, Pflanzenverte aus der Kohlenführenden oberkreide im Zentral-Balkan : Bulgarian Geol. Soc. Rev., 1939, v. 11, p. 95-120, pls 1-11.

Hartung, Wolfgang, and Gothan, W., 1939, Uber eine als Callipteridium gothant Krestew beschriebene Blattform aus der Oberen Kreide Bulgariens : Preuss. geol. Landensanst. Jahrb. 1938, p. 513-525, Berlin.

Haughton, Samuel, 1859, Plates for 1860 article (see Haughton, Samuel, 1860) : Nat. History Rev., v. 6, pls. 38-41, London.

1860, On Cyclostigma, a new genus of fossil plants from the Old Red Sandstone of Kiltorcan, County Kilkenny; and on the general law of phyllotaxis : Nat. History Rev., v. 7, p. 209-222, London.

Heard, Albert, 1927, On Old Red Sandstone plants showing structure, from Brecon (South Wales) : Geol. Soc. London Quart. Jour., v. 83, p. 195-206, pls. 13-15.

Heard, Albert, and Jones, John F., 1931a, A new plant (Thallomia) showing structure from the Downtonian rocks of Llandovery, Carmarthenshire : Geol. Soc. London Quart. Jour., v. 87, p. 551-562, pls. 43-46.

1931b, Eohepatica dyfriensis, a liverwort-like plant from the Lower Downtonian of the Llandovery district; British Assoc. Adv. Sci., Bristol, Rept. 1930, p. 330-331.

Hector, James, 1878, Report of geological explorations during 1877-78: New Zealand Geol. Survey, 1878, p. viii.

1880, Fossil plants: Official Cat. New Zealand Court, Internat. Exhibit, Sydney, 1879, p. 47-50.

1886, Fossil plants: Detailed cat. and guide, Geol. Exhibits, New Zealand Court, Indian and Colonial Exhibition, London, p. 31-32, 60-61.

Heer, Oswald, 1853, Beschreibung der angeführten Pflanzen und Insekten in Escher: Schweizer. Gesell. Naturw., Neue Denkschr., Band 13, p. 115-135, pls. 6-8, Zurich.

1855, Flora Tertiaria Helvetiae: Band 1, 117 p., pls. 1-50, Winterthur.

1856, Flora Tertiaria Helvetiae: Band 2, 110 p., pls. 51-100, Winterthur.

1859, Flora Tertiaria Helvetiae: Band 3, 377 p., pls. 101-157, Winterthur.

1861, Recherches sur le climat et la végétation du pays tertiaire: $220 \mathrm{p}$,

2 pls., Winterthur.

1864-65, Die Urwelt der Schweiz, 1st ed: Teil 1, p. 1-496, pls. 1-10; Teil 2, p. 497-622, pl. 11, Zurich.

1866, Über den versteinerten Wald von Atanekerdluk in Nordgrönland:

Naturf. Gesell. Zürich, Vierteljahrschr., Band 11, p. 259-280.

1868, Die fossile Flora der Polarländer in Flora Fossilis Arctica, Band 1, p. 1-192, pls. 1-50, Zurich.

1869 a, Contributions to the fossil flora of North Greenland, being a descrip-

tion of the plants collected by Mr. Edward Whymper during the summer of 1867, in Flora Fossilis Arctica, Band 2, Heft 4: Royal Soc. London Philos. Trans., v. 159, p. 445-488, pls. 39-56.

1869b, Flora fossilis Alaskana, in Flora Fossilis Arctica, Band 2, no. 2 :

K. Svenska vetensk. akad. Handl., band 8, no. 4, p. 1-41, pls. 1-10.

- 1869c, Beiträge zur Kreide-Flora, I, Flora von Moletein in Mähren : Soc.

Helvétique sci. nat. Nouv. Mém., tome 23, no. 2, p. 1-24, pls. 1-9. 
Heer, Oswald, 1870, Die miocene Flora und Fauna Spitzbergens, in Flora Fossilis Arctica, Band 2, Heft 3: K. Svenska vetensk. akad. Handl., band 8, no. 7, p. 1-98, pls. 1-16.

1871a, Fossile Flora der Bären-Insel, in Flora Fossilis Arctica, Band 2,

Heft 1: K. Svenska vetensk. akad. Handl., band 9, no. 5, p. 1-51, pls. 1-15. 1871b, Beiträge zur Kreide-Flora, II, Kreide-Flora von Quedlinburg: Soc.

Helvétique Sci. nat. Nouv. Mém., tome 24, no. 2, p. 9-10, pl. 1.

1874a, Die Kreide-Flora der arctischen Zone, in Flora Fossilis Arctica,

Band 3, Heft 2 : K. Svenska vetensk. akad. Handl., band 12, no. 6, pl. 1-140, pls. 1-38. 1874b, Übersicht der miocenen Flora der arctischen Zone: 24 p., Zurich. 1874c, Beiträge zur Steinkohlen-Flora der arctischen Zone, in Flora Fossilis Arctica, Band 3, Heft 1: K. Svenska vetensk. akad. Handl., band 12, p. 1-11, pls. 1-6.

1874d, Nachträge zur miocene Flora Grönlands, in Flora Fossilis Arctica,

Band 3, Heft 3 : K. Svenska vetenska. akad. Handl., band 13, p. 1-29, pls. 1-5. - 1874e, Übersicht der miocenen Flora der arctischen Zone, in Flora Fossilis Arctica, Band 3, Heft 4 : p. 1-24, Zurich.

- 1876a, Flora Fossile Helvetiae, Teil I, Die Pflanzen der Steinkohlen Periode, p. 1-60, pls. 1-22, Zurich.

1876b, Beiträge zur fossilen Flora Spitzbergens, in Flora Fossilis Arctica,

Band 4, Heft 1: K. Svenska vetensk. akad. Handl., band 14, p. 1-141, pls. $1-32$.

1876c, Beiträge zur Jura-Flora Ostsibiriens und des Amurlandes, in Flora

Fossilis Arctica, Band 4, Heft 2: Acad. imp. sci. St.-Pétersbourg Mém., v. 22, p. 1-122, pls. 1-31.

1876d, The primaeval world of Switzerland, v. 1. [English translation by

W. S. Dallas, p. 1-393, pls. 1-10.]

1877a, Flora Fossile Helvetiae, Teile II-V, p. 101-182, pls. 23-70, Zurich.

1877b, Über die Pflanzen-Versteinerungen von Audö in Norwegen, in Flora

Fossilis Arctica, Band 4, Heft 3, p. 1-15, pls. 1, 2.

1878a, Die miocene Flora des Grinnell-Landes, in Flora Fossilis Arctica,

Band 5, Heft 1, p. 1-38, pls. 1-9, Zurich.

- 1878b, Beiträge zur fossilen Flora Sibiriens und des Amurlandes in Flora

Fossilis Arctica: Acad. imp. sci. St.-Pétersbourg Mém., tome 25, p. 1-58, pls. 1-15.

- 1878c, Miocene Flora des Insel Sachalin in Flora Fossilis Arctica, Band

5, Heft 3: Acad. imp. sci. St.-Pétersbourg Mém., v. 25, p. 1-61, pls. 1-15.

1878d, Beiträge zur miocenen Flora von Sachalin in Flora Fossilis Arc-

tica, Band 5, Heft 4: K. Svenska vetensk. akad. Handl., band 15, no. 4, p.

1-11, pls. 1-4.

1878e, Über Fossile Pflanzen von Novaja Selmja in Flora Fossilis Arctica,

Band 5, Heft 5: K. Svenska vetensk. akad. Handl., band 15, no. 3, p. 1-6, pl. 1 .

1880a, Nachträge zur Jura-Flora Sibiriens, in Flora Fossilis Arctica, Band

6, Teil 1, Heft 1: Acad. imp. sci. St.-Pétersbourg Mém., v. 27, p. 1-34, pls. 1-9.

1880b, Nachträge fossilen Flora Grönlands, in Flora Fossilis Arctica,

Band 6, Teil 1, Heft 2: K. Svenska vetensk. akad. Handl., band 1S, p. 1-17, pls. 1-6.

1880c, Beiträge zur miocenen Flora von Nord-Canada in Flora Fossilis

Arctica, Band 6, Teil 1, Heft 3, p. 1-17, pls. 1-3, Zurich.

1881, Contributions à la flore fossile du Portugal : 51 p., 28 pls., Zurich. 
Heer, Oswald, 1882, Die Flora der Komeschichten, and Die Flora der Ataneschichten: Flora Fossilis Arctica, Band 6, Teil 2, p. 1-112, pls. 1-47, Zurich. 1883, Die fossile Flora der Polarlander : Flora Fossilis Arctica, Band 7, p. 1-275, pls. 48-110, Zurich.

Heinhold, Max, 1906, Über die Entstehung des Pyropissites: Preuss. geol. Landesanst. Jahrb., Band 27, p. 114-158.

Herlin, Rafael, 1896, Pälaontologisk-växtgeografiska studier i norra Satakunta: Vet. Meddel. Geog. Fören. Finland, Teos 3, p. 177-219, pls. 8, 9, Helsingfors.

Herzer, Herman, 1893a, A new fungus from the Coal Measures [Tuscarawas County, Ohio] : Am. Geologist, v. 11, p. 365-366, pl. 9.

-1893b, A new fungus from the Coal Measures: Am. Geologist, v. 12, p. 289-290, pl. 13.

1893c, A new tree from the Carboniferous rocks of Monroe County, Ohio: Am. Geologist, v. 11, p. 285-286, pl. 6.

1901, Six new species, including two new genera, of fossil plants: Ohio State Acad. Sci., 9th Ann. Rept., p. 22-26, pls. 1-3.

1902, New fossil plants from the Carboniferous and Devonian: Ohio State Acad. Sci., 10th Ann. Rept., p. 40-45, pls. 1-3.

Heydrich, F., 1900a, Eine systematische Skizze fossiler Melobesieae: Deutsche bot. Gesell. Ber., Band 18, p. 79-83.

1900b, Eine neue fossile Alge aus Rukin: Geol. Soc. Tokyo Jour., v. 7, no. 80 , p. 1-2.

Hick, Thomas, 1892a, On a new fossil plant from the Lower Coal-Measures: Linnean Soc. London Jour., Botany, v. 29, p. 86-102, pls. 16, 17.

- 1892b, Supplementary note on a new fossil plant: Linnean Soc. London Jour., v. 29, p. 216.

Hick, Thomas, and Cash, William, 1881, A contribution to the flora of the Lower Coal Measures of the Parish of Halifax, Part III : Yorkshire Geol. Polytech. Soc. Proc., v. 7, p. 400-405, pl. 21.

Hicks, Henry, 1882, Additional notes on the land plants from the Pen-y-glog Slate quarry near Corwen, North Wales: Geol. Soc. London Quart. Jour., v. 38, p. 97-102, pl. 3 .

Hildreth, Samuel Prescott, 1836, Miscellaneous observations made during a tour in May, 1835, to the falls of the Cuyahoga, near Lake Erie: Am. Jour. Sci., v. 31, p. 1-84.

Hirmer, Max, 1927, Handbuch der paläobotanik, Band 1: p. 708, Berlin.

—_ 1933a, Zur Kenntnis der strukturbietenden Pflanzenreste des jüngeren Paläozoikums : Palaeontographica, Band 78, Abt. B, p. 57-113.

1933b, Zur Kenntnis der strukturbietenden Pflanzenreste des jüngeren Paliiozoilkums: Palaeontographica, Band 77, p. 121-140, pls. 17-22.

- Hirmer, Max, and Hoerhammer, Ludwig, 1936, Morphologie, Systematik, und geographische Verbreitung der Fossilen und Rezenten Matoniaceae: Palaeontographica, Band 81, Abt. B, p. 1-70, pls. 1-10.

Hisinger, Wilhelm, 1823, Arteckningar i physik och geognosi under resor ut.1 Sverige och Norrige : Band 3, p. 1-103, pl. 3, Upsala.

- 1837, Lethaea svecica seu Petrificata sveciae, iconibus et characteribus illustrata: 124 p., pls. A-C, 1-39, Stockholm.

Hoeg, Ove Arbo, 1927, Dimorphosiphon rectangulare, preliminary note: Norske vidensk.-akad. Oslo Avh., hefte 4, p. 1-15, pls. 1-3.

- 1931, A psilophyte in South Africa: K. Norske vidensk. selsk. Förh., bind 3, hefte 1, p. 92-94. 
Hoeg, Ore Arbo, 1933, Ordovician algae from the Trondheim area: Norske vidensk.-akad., Oslo, Mat.-naturv. Kl. Skr., bind 1, p. 63-96.

1935, Further contributions to the Middle Devonian flora of western Norway : Norske geol. Tidsskr., bind 15, p. 1-17, pls. 1-4.

1938, Callisphenus graciis, n. gen., n. sp., a fossil alga from the Wenlock of the Oslo region: Norske geol. Tidsskr., bind 17, p. 43-45, pls. 1, 2, Oslo.

1942, The Downtonian and Devonian flora of Spitzbergen: Svalbard Ishava-Unders Skr., no. 83, 228 p., 62 pls.

Hofmann, Elsie, 1932, Epidermisreste und Blattabderücke aus den Braunkohlenlagern des Geiseltales: Nova Acta Leopoldina, Band 1, p. 59-74, pls. 1-14.

Hofmann, Hermann, 1883, Über die fossilen Hölzer aus dem mecklenburgischen Diluvium: Freunde Naturg. Mecklenburg Arch. Verh, Band 36, p. 65-107. 1884a, Untersuchungen über fossile Hölzer: Zeitschr. Naturw., Band 57, p. 156-195.

1884b, Untersuchungen über fossile Hölzer: Inaugural Dissert. Univ. Leipzig, $195 \mathrm{p}$.

Holden, Ruth, 1913, Contributions to the anatomy of Mesozoic conifers, No. 1, Jurassic coniferous woods from Yorkshire: Annals Botany, v. 27, p. 534-545, pls. 39,40 .

1915, On the cuticles of some Indian conifers: Bot. Gazette, v. 60, p. 215-227, pl. 11.

1916, A fossil wood from Burma : Geol. Survey India Rec., v. 47, p 267-272, pl. 29.

Hollick, Arthur, 1893a, Preliminary contribution to our knowledge of the Cretaceous formation of Long Island and eastward : New York Acad. Sci. Trans., จ. 12, p. 222-237.

1893b, A new fossil palm from the Cretaceous formation at Glen Cove, Long Island [Serenopsis kempii] : Torrey Bot. Club Bull., v. 20, p. 168-169, pl. 149.

- 1907, Description of a new Tertiary fossil flower from Florissant, Colo.: Torreya, v. 7, pp. 182-184, figs. 1, 2.

- 1909, A new genus of fossil Fagaceae from Colorado: Torreya, v. 9, p. 1-3. 1910, A new fossil Polypore [Pseudopolyporous carbonicus, Carboniferous, W. Va.] : Mycologia, v. 2, p. 93-94, figs. 1, 2.

1912, Additions to the paleobotany of the Cretaceous formation of Long Island: New York Bot. Garden Bull., จ. 8, p. 154-170, pls. 162-170.

1916, A fossil fern monstrosity [Anomalofilicites monstrosus, Fort Union formation, Dawson County, Mont.] : New York Bot. Garden Mem., v. 6, p. $473-474$, pl. 31 .

1928, Scientific survey of Porto Rico and the Virgin Islands: New York Acad. Sci., v. 7, p. 177-238, pls. 51-88.

1931, Records of Triassic fossils on Staten Island, N. Y., with descriptions of specimens: Staten Island Inst. Arts Sci. Proc., v. 6, p. 5-22, pls. 1-9.

Hollick, Arthur, and JeffreJ, E. C., 1906, Affinities of certain Cretaceous plant remains commonly referred to the genera Dammara and Brachyphyllum: Am. Naturalist, $\nabla .40$, p. 189-216, pls. 1-3.

1900, studies of Cretaceous coniferous remains from Kreischerville, N. Y.: New York Bot. Garden Mem., $\vee$. 3, p. 1-76, pls. 1-2o.

Hollick, Arthur, and Martin, G. C. 1930, The Upper Cretaceous floras of Alaska: U. S. Geol. Survey Prof. Paper 159, p. 1-116, pls. 1-86.

Hooker, Joseph, 1852, On the sphaeroidal bodies resembling seeds, from the Ludlow bone beds: Geol. Soc. London Quart. Jour., v. 9, p. 12. 
Hörhammer, Ludwig, 1933, Über die Coniferen-Gattungen Cheirolepis Schimper und Hirmeriella nov. gen. aus dem Rhät-Lias von Franken: Bibliotheca Botanica, Band 27, Heft 107, p. 1-33, pls. 1-7.

Hörich, O., 1915, Phialophloios quadratus, eine neue Lepidophytengattung: Preuss. Geol. Landesanst. Jahrb., Band 35, p. 425-430, 1 pl.

Hosius A., and von der Marck, W., 1880, Die Flora der westfälischen Kreideformation: Palaeontographica, Band 26, p. 125-236, pls. 24-44.

Hoskins, J. Hobart, 1923, A Palaeozoic angiosperm from an American coal ball: Bot. Gazette, v. 75, p. 390-399, pl. 17.

Hoskins, J. Hobart, and Cross, Aureal T., 1943, Monograph of the Paleozoic cone genus Bowmanites (Sphenophyllales): Am. Midland Naturalist, v. 30, p. 113-163; figs. 1-40.

Houlbert, Constant, 1910, Les Bois des Faluns de Touraine: Feuille jeunes naturalistes, tome 40, p. $70-76$, pls. $3-8$.

Hovelacque, Maurice, 1890, Sur la nature végétale de l'Aachenosaurus multidens G. Smets: Soc. Belge géologie Bull., tome 4, p. 59-72, pl. 3.

Howe, Marshall A., 1932a, Chlorotylites, a fossil green alga from Alabama: Torrey Bot. Club Bull., v. 59, p. 219-220, pl. 15.

- 1932b, The geologic importance of the lime-secreting algae, with a description of a new travertine-forming organism: U. S. Geol. Survey Prof. Paper 170 , p. 57-64, pls. 19-23.

Howe, Marshall A., and Hollick, Arthur, 1922, A new American fossil hepatic [Jungermanniopsis from Florissant, Colo.] : Torrey Bot. Club Bull., v. 49, p. 207-209.

Howse, Richard, 1890, Contributions toward a catalogue of the flora of the Carboniferous system of Northumberland and Durham, Part 1, Fossil plants from the Hutton Collection: Nat. History Soc. Northumberland, Durham, and Newcastle Trans., v. 10, p. 19-151.

Ibrahim, Achmen, 1932, Beschreibung von Sporenformen aus Flöz Agir-see, p. 447-449 in Potonie, Robert, Sporenformen aus den Flözen Ägir und Bismarck des Ruhregebietes.

- 1933, Sporenformen des Aegirhorizonts des Ruhr-Reviers: Dissert., Technischen Hochschule, Berlin, 48 p., 8 pls., Würzburg.

Indian Palaeobotany, Paleobotanical reports edited by Birbal Sahni, and R. V. Sitholey : I, 1940, Indian Bot. Soc. Jour., v. 18, p. 201-208; II, 1941, ibid., v. 20, p. 1-9 ; III, 1942, ibid., v. 21, p. 217-224 ; IV, 1943, ibid., v. 22, p. 171182 ; V, 1944, Nat. Acad. Sci., India, Proc., v. 14, p. 67-91; VI, 1948, Indian Bot. Soc. Jour., v. 26, p. 241-273 ; VII, 1950, ibid., v. 29, p. 1-46.

Ishijlma, Wataru, 1943, Kitakamiania, a new type of Cretaceous alga from Japan: Nat. History Soc. Taiwan (Formosa) Trans., v. 33, p. 639-642.

Jaeger, Georg Friedrich, 1827, Uber die Pflanzenversteinerungen welche in dem Bausandstein von Stuttgart vorkommen : 46 p., 8 pls., Stuttgart.

James, Joseph F., 1885, The fucoids of the Cincinnati group: Cincinnati Soc. Nat. History Jour., v. 7, no. 4, p. 151-166, pls. 8, 9.

James, U. P., 1879, Descriptions of newly discovered species of fossils and remarks on others, from the lower and upper Silurian rocks of Ohio : Palaeontologist, no. 3, p. 17-24, Cincinnati.

Janssen, Raymond E., 1940, Some fossil plant types of Illinois : Ill. State Mus. Scl. Papers, v. 1, p. 1-124, pls. 1-28.

Jaworski, Erich, 1926, La Fauna del Lias y Digger de la Cordillera Argentina * * Acad. Nac. Cienc. Córdoba Actas, tomo 9, p. 139-313. 
Jeffrey, Edward C., 1907, Araucariopitys, a new genus of araucarians : Bot. Gazette, v. 44, p. 435-444, pls. 28-30.

1908 , On the structure of the leaf in Cretaceous pines: Annals Botany, v. 22, p. 208-220, pls. 13, 14.

1910, A new araucarian genus from the Triassic: Boston Soc. Nat. History Proc., v. 34, p. 325-332, pls. 31, 32.

Jessen, Knud, and Milthers, V., 1928, Stratigraphical and paleontological studies of interglacial fresh water deposits: Danmarks geol. Unders $\varnothing$ gelse, ser. 2, no. 48 , p. $1-379$, pls. $1-40$.

Johansson, Nils, 1922, Pterygopteris, eine neue Farngattung aus dem Rät Schonens : Arkiv Botanik, band 17, no. 16, p. 1-6, pl. 1.

Johnson, J. Harlan, 1937, Algae and algal limestone from the Oligocene of South Park, Colo.: Geol. Soc. America Bull., v. 48, p. 1227-1236, pl. 1.

1940, Lime-secreting algae and algal limestones from the Pennsylvanian of central Colorado: Geol. Soc. America Bull., v. 51, p. 571-596, pls. 1-10.

1942, Permian lime-secreting algae from the Guadalupe Mountains, $N$. Mex. ; Geol. Soc. America Bull., v. 53, p. 195-226, pls. 1-7.

1943, Geologic importance of calcareous algae, with annotated bibliography : Colo. School Mines Quart., v. 38, no. 1, p. 1-102.

1946, Lime-secreting algae from the Pennsylvanian and Permian of Kansas: Geol. Soc. America Bull., v. 57, p. 1087-1120, pls. 1-10.

Johnson, Thomas, 1912, Forbesia cancellata gen. et sp. nov. : Royal Soc. Dublin Sci. Proc., v. 13, new ser., no. 13, p. 177-183, pls. 13, 14.

1917, Spermolithus devonicus, gen. et sp. nov., and other Pteridosperms from the Upper Devonian beds at Kiltorcan, County Kilkenny : Royal Soc. Dublin Sci. Proc., v. 15, p. 245-254, pls. 11-14.

1937, Notes on the Tertiary flora of Scotland : Edinburgh Bot. Soc. Proc., v. 32, pt. 2, p. 291-340, pls. 10-22.

Johnston, George, 1853, The botany of the Eastern Borders, with the popular names and uses of the plants, and of the customs and beliefs which have been associated with them in Tate, George, Fossil flora of the Mountain Limestone, v. 1, p. 1-289, pl. 13, London.

Johnston, Robert M., 1888, Systematic account of the geology of Tasmania : 408 p., 57 pls., Hobart, Tasmania.

1894, Further contributions to the fossil flora of Tasmania: Royal Soc. Tasmania Papers Proc., 1893, p. 170-178, pls. 1, 2.

1896, Further contributions to the history of the fossil flora of Tasmania, Part II : Royal Soc. Tasmania Papers Proc., 1894-95, p. 57-63, figs. 1-17.

Jones, O. A., and Jersey, N. J., 1947, The flora of the Ipswich coal measuresMorphology and floral succession: Univ. Queensland Papers, Dept. Geol., v. 3, new ser., nos. $3-4,88$ p., 10 pls.

Jongmans, W. J., 1927a, Beschrijving der boring Gulpen: Geol. Bur. Nederlandsche Mijngebied Heerlen Jaarv., p. 54-69.

1927b, Een eigenaardige plantenbank boven Laag B der Mijn Emma: Geol. Bur. Nederlandsche Mijngebied Heerlen Jaarv., p. 47-49.

1930 , On the fructification of Sphenopteris hoeninghausi and its relations

with Lyginodendron oldhamium and Crossotheca schatzlarensis: Geol. Bur, Nederlandsche Mijngebeid Heerlen Jaarv., p. 77-81.

1937, The flora of the Upper Carboniferous of Djambi (Sumatra, Netherlands, Indies) and its possible bearing on the paleogeography of the Carboniferous: Cong. Av. Ettudes Stratigraphie et Géologie Carbonifēre, $2^{\circ}$, Comptes Rendus, tome 1, p. 344-362, Heerlen. 
Jongmans, W. J., and Gothan, Walther, 1925, Beitrïge zur Kenntnis der Flora des oberkarbons von Sumatra: Geol.-mijnb. genoot. Nederland en Koloniën Verh. Geol. ser., boekdeel 8, p. 279-303, pls. 1-5.

- 1935, Die paläobotanischen Ergebnisse der Djambi-expedition 1925 : Jaarb. mijnwezen Nederlish-Indië 1930, p. 72-201, pls. 1-58.

Jurasky, Karl A., 1928, Ein neuer fund von Sciadopitys in der Braunkohle (Sciadopityoxylon wettsteini n. sp.): Senckenbergiana, Band 10, Heft 6, p. 255-264.

Kaiser, P. E. E., 1880a, Ficoxylon bohemicum, ein neues fossiles Laubholz: Zeitschr. gesamt. Naturw., Band 5, p. 309-317.

1880b, Neue fossile Laubhölzer: Bot. Centralbl., Bände 1-2, Heft 16, p. 511-512.

Karpinskiy, Aleksandr Petrovich, 1906, Die Trochilisken: Russia, Comité geol. Mém., nouv. sér., livr. 27, p. 1-159, pls. 1-3, St. Petersburg.

Kawasaki, Shigetaro, 1927-34, The flora of the Heian system : Geol. Survey Korea Bull., v. 6, pt. 1, p. 1-30, pls. 1-14, 1927 ; pt. 2, pls. 16-99, 1931, p. 47-311, pls. 105-110, 1934 ; pt. 3, p. 31-44, pls. 100-104, 1932.

Keeping, Walter, 1881, The geology of central Wales: Geol. Soc. London Quart. Jour., v. 37, p. 141-170.

1882, On some remains of plants, Foraminifera and Annelida in the Silurian rocks of central Wales: Geol. Mag., decade 2, v. 9, p. 485-491, pl. 10, London.

Keferstein, Christian, 1834, Die Naturgeschichte des Erdkorpers in ihren ersten Grundzugen, Theil 2, Die Geologie und Paleontologie: 896 p., Leipzig.

Kellaway, G. A., 1937, Further recent exposures in the Rhaetic and Liassic rocks of the Bristol area: Bristol Naturalists' Soc. Proc., v. 8, no. 2, p. 223-227.

Keller, Robert, 1895, Beiträge zur Tertiärflora des Kantons St. Gallen : St. Gallenischen naturwiss. Gesell., 1894, p. 305-330.

Kern, Ellen M., and Andrews, Henry N., Jr., 1946, Contributions to our knowledge of American Carboniferous floras, IX, Some petritied seeds from Iowa: Mo. Bot. Garden Annals, v. 33, p. 291-304, pls. 18, 19.

Kerner, Fritz, 1916, Alt und ungtertiare Pflanzenreste aus dem obersten CetInatale: K.-k. Geol. Reichsanst. Verh., p. 180-191, Vienna.

Kershaw, E. M., 1910, A fossil solenostelic fern : Annals Botany, v. 24, p. 683-691, pl. 58.

Khvorova, I. V., 1946, On a new genus of algae from the Middle Carboniferous deposits of the Moscow basin: Acad. Sci. U. R. S. S. Comptes Rendus, tome 53 , no. 8, p. 737-739.

Kidston, Robert, 1882, On the fructification of Eusphenopteris tenella Brongn., and Sphenopteris microcarpa Lesquereux: Annals and Mag. Nat. History, 5th ser., v. 10, p. 7-11, pl. 1.

- 1884a, On the fructiflcation of Zeilleria (Sphenopteris) delicatula Sternb. sp. with remarks on Urnatopteris: Geol. Soc. London Quart. Jour., v. 40, p. $590-598, \mathrm{pl} .25$.

1884b, On the fructification of Zeilleria (Sphenopteris) delicatula ***: Annals and Mag. Nat. History, 5th ser., v. 14, p. 294-295.

1886, Catalogue of the Palaeozoic plants in the Department of Geology and Palaeontology, British Museum Natural History : 288 p., London.

1887a, On the fructification of some ferns from the Carbonifierous formation : Royal Soc. Edinburgh Trans., v. 33, p. 137-156, pls. 8, 9 [1888].

$1887 \mathrm{~b}$, On the fossil flora of the Radstock series of the Somerset and Bristol Coal Field (Upper Coal Measures) : Royal Soc. Edinburgh Trans., v. 33 , p. $335-417$, pls. 18-28. 
Kidston, Robert, 1888, On the fructification of two Coal-Measure ferns: Royal Phys. Soc. Edinburgh Proc., v. 9, p. 510-516, pl. 21.

1891, On the fructification and internal structure of Carboniferous ferns in their relation to those of existing genera, with special reference to British Palaeozoic species: Geol. Soc. Glasgow Trans., v. 9, p. 1-56, pls. 1-4.

1894 , On some new species of fossil plants from the Lower Carboniferous rocks of Scotland: Royal Phys. Soc. Edinburgh Proc., v. 12, p. 258-268, pls. 4-6.

1897, On Cryptoxylon forfarense, a new species of fossil plant from the Old Red Sandstone: Royal Phys. Soc. Edinburgh Proc., v. 13, p. 360-363, pls. 8,9 .

1901, Carboniferous lycopods and sphenophylls : Nat. History Soc. Glasgow Trans., v. 6, new ser., p. 25-140.

1903a, The fossil plants of the Carboniferous rocks of Canonbie, Dumfrieshire, and of parts of Cumberland and Northumberland: Royal Soc. Edinburgh Trans., v. 40, p. 741-833, pls. 1-5.

1903b, [Fossil plants from the Campletown Coal Field] : Mem. Geol. Survey, Summary Prog. Geol. Survey United Kingdom for 1902, p. 130-137, London.

1906, On the microsporangia of the pteridosperms, with remarks on their relationships to existing groups : Royal Soc. London Philos. Trans., v. 198B, p. $413-445$, pls. $25-28$.

- 1911, Les végétaux houillers recueillis dans le Hainaut Belge: Mus. royal histoire nat. Belgique Mém., tome 4, p. 1-282, pls. 1-24.

1923a, Fossil plants of the Carboniferous rocks of Great Britain : Great

Britain Geol. Survey Mem., v. 2, pts. 1-3, p. 1-276, pls. 1-68.

1923b, Fossil plants of the Carboniferous rocks of Great Britain : Great

Britain Geol. Survey Mem., v. 2, pt. 4, p. 277-375.

1924, Fossil plants of the Carboniferous rocks of Great Britain : Great

Britain Geol. Survey Mem., v. 2, pt. 5, p. 379-522, pls. 92-122.

1925, Fossil plants of the Carboniferous rocks of Great Britain : Great Britain Geol. Survey Mem., v. 2, pt. 6, p. 525-681, pls. 123-153.

Kidston, Robert, and Gwynne-Vaughan, D. T., 1907, On the fossil Osmundaceae, Part I : Royal Soc. Edinburgh Trans., v. 45, p. 759-780, pls. $1-6$.

1908, On the fossil Osmundaceae, Part II : Royal Soc. Edinburgh Trans., v. 46, p. 213-232, pls. 1-4.

1909, On the fossil Osmundaceae, Part III : Royal Soc. Edinburgh Trans., v. 46 , p. 651-667, pls. 1-8.

- 1910, On the fossil Osmundaceae, Part IV : Royal Soc. Edinburgh Trans., v. 47 , p. $455-477$, pls. $1-4$.

1912, On the Carboniferous flora of Berwickshire, Part I, Stenomyelon tuedianum Kidston: Royal Soc. Edinburgh Trans., v. 48, p. 263-271, pls. 1-4.

Kidston, Robert, and Jongmans, W. J., 1917, A monograph of the Calamites of western Europe, in Jongmans, W. J., Flora of the Carboniferous of the Netherlands and adjacent regions: Rijpsopsporing van Delfstoffen Meded., จ. 1, no. 7, 207 p., 158 pls., Gravenhage.

Kidston, Robert, and Lang, W. H., 1917, On Old Red Sandstone plants showing structure.from the Rhynie chert bed, Aberdeenshire, Part I, Rhynia gwynnevaughani Kidston and Lang: Royal Soc. Edinburgh Trans., v. 51, p. 761-784, pls. 1-10. 
Kidston, Robert, and Lang, W. H., 1920a, On Old Red Sandstone plants showing structure, from the Rhynie chert bed, Aberdeenshire, Part II, Additional notes on Rhynia gwynneavaughani Kidston and Lang; with descriptions of Rhynia major, n. sp., and Hornea lignieri, n. g., n. sp.: Royal Soc. Edinburgh Trans.y v. 52, p. 603-627, pls. 1-10.

1920b, On Old Red Sandstone plants showing structure, from the Rhynie chert bed, Aberdeenshire, Part III, Asteroxylon mackiei Kidston and Lang: Royal Soc. Edinburgh Trans., v. 52, p. 643-688, pls. 1-17.

- 1921, On Old Red Sandstone plants showing structure, from the Rhynie chert bed, Aberdeenshire, Part V, The Thallophyta occurring in the Peat-Bed

* * *: Royal Soc. Edinburgh Trans., v. 52, p. 855-902, pls. 1-10.

1923a, Notes on fossil plants from the Old Red Sandstone of Scotland, I, Hicklingia edwardi K. and L. : Royal Soc. Edinburgh Trans., v. 53, p. 405-407, pl. 53.

1923b, On Palaeopitys milleri M'Nab : Royal Soc. Edinburgh Trans., v. 53, p. $409-417$, pls. $1,2$.

- 1925, Notes on fossil plants from the Old Red Sandstone of Scotland: Royal Soc. Edinburgh Trans., v. 53, p. 603-614, pls. 1, 2.

Kinkelin, Friedrich, 1900, Beiträge zur Geologie der Umgegend von Frankfurt a. M. : Senckenberg, naturf. Gesell., Ber. 1900, p. 121-162.

Kirchheimer, Franz, 1930a, Limnophycus paradoxus nov. gen., eine problematische Alge aus der obermiocänen Kieselgur von Homberg a. d. Ohm (Oberhessen) : Preuss. geol. Landesanst. Jahrb., Band 50, p. 581-591, pl. 35.

- 1930b, Beitrüge zur Kenntnis der Tertiäflora des Vogelsberges und der Wetterau: Ver. Erdkunde Notizblatt, Darmstadt, p. 105-123, pl. 13.

- 1934a, Neue Ergebnisse und Probleme pläobotanischer Braunkohlenforschungen: Braunkohle, Band 33, p. 769-774.

- 1934b, Neue Ergebnisse und Probleme paläobotanischer Braunkohlenforschungen: Braunkohle, Band 33, p. 788-793.

1935, Weitere Mitteilungen über die Früchte und Samen aus deutschen Braunkohlen : Braunkohle, Band 34, p. 289-294.

- 1936a, Bau und botanische Zugehörigkeit von Pflanzenresten aus deutschen Braunkohlen : Bot. Jahrb., Band 67, p. 37-122.

1936b, Beiträge zur Kenntnis der Tertiärflora: Palaeontographica, Band 82, Abt. B, p. 73-141, pls. 7-13.

1936c, Zur Kenntnis der Fruchte rezenter und fossiler Mastixioideen: Bot. Centralbl. Beihefte, Band 55, p. 275-300, pls. 5-8.

- 1936d, Eine Mooskohle aus dem Salzhauser Braunkohlenlager : Zentralbl. Mineralogie, Abt. B, no. 8, p. 337-343.

- 1937, Beiträge zur Kenntnis der Flora des Baltischen Bernstein, I : Bot. Centralbl. Beihefte, Band 57, p. 441-484.

1938a, Umbelliflorae, Cornaceae: Fossilium Catalogus, II, Plantae, Pars 23, p. 1-188.

1938b, Beiträge zur näheren Kenntnis der Mastixioideen Flora des deutschen Mittel-bis Oberoligozäns: Bot. Centralbl. Beihefte, Band 58, p. 303-375, pls. 3-8.

1938c, Palaobotanische Beiträge zur Kenntnis des Alters deutscher Braunkohlenschichten, III : Braunkohle, Band 37, p. 341-347.

Kirchner, Walter C. G., 1898, Contributions to the fossil flora of Florissant, Colo.:

St. Louis (Mo.) Acad. Scl., Trans., v. 8, p. 161-188, pls. 11-15. 
Knoll, F., 1904, "Potamogeton Morloti" Unger, eine Tertiäre Loranthaceae : Bot. Zeitschr., Band 54, p. 64-71, pl. 4.

Knowlton, Frank Hall, 1894, A new fossil hepatic from the lower Yellowstone in Montana [Preissites wardii] : Torrey Bot. Club Bull., v. 21, p. 458-460, pl. 219.

- 1895, Description of a new probematical plant from the Lower Cretaceous of Arkansas [Paleohillia arkansana] : Torrey Bot. Club Bull., v. 22, p. 387390, figs. 1-3.

1898, A catalogue of the Cretaceous and Tertiary plants of North America :

U. S. Geol. Survey Bull. 152, 247 p.

1911, The correct technical name for the 'Dragon Tree' of the Kentish Rag: Geol. Mag., v. 8, p. 467-468, London.

1916, A review of the fossil plants in the United States National Museum from the Florissant Lake beds at Florissant, Colo., with descriptions of new species, and list of type specimens: U. S. Natl. Mus. Proc., v. 51, p. 241-297, pls. 12-27.

- 1917, Geology and paleontology of the Raton Mesa and other regions in Colorado and New Mexico : U. S. Geol. Survey Prof. Paper 101, p. 223-349, pls. 30-113 [1918].

1918, A fossil flora from the Frontier formation of southwestern Wyom-

ing: U. S. Geol. Survey Prof. Paper 108-F, p. 73-108, pls. 28-39.

1919, A catalogue of the Mesozoic and Cenozoic plants of North America :

U. S. Geol. Survey Bull. 696, p. 1-815.

1922a, The Laramie flora of the Denver Basin: U. S. Geol. Survey Prof.

Paper 130, p. 1-175, pls. 1-28.

- 1922b, Revision of the flora of the Green River formation, with descriptions of new species: U. S. Geol. Survey Prof. Paper 131-F, p. 133-176, pls. $36-40$.

- 1926, Flora of the Latah formation of Spokane, Wash., and Coeur

d'Alene, Idaho: U. S. Geol. Survey Prof. Paper 140, p. 17-55, pls. 8-31.

1930, The flora of the Denver and associated formations of Colorado:

U. S. Geol. Survey Prof. Paper 155, 142 p., 59 pls.

Koenig, Charles, 1825, Icones fossilium sectiles : 4 p., 19 pls., London.

Koidzumi, G., 1934, Gigantopteris: Acta Phytotaxonomica et Geobotanica, v. 3, no. 2, p. 112-113, Kyoto.

— 1936, Gigantopteris flora: Acta Phytotaxonomica et Geobotanica, v. 5, no. 2, p. 130-144, Kyoto.

Koninck, de L. G., 1842, Description des animaux fossiles que se trouvent dans le terrain carbonifère de Belgique : Liege.

Kon'no, Enzo, 1020, On genera Tingia and Tingiostachya from the Lower Permian and Permo-Triassic beds in northern Korea : Japanese Jour. Geology and Geography, v. 6, p. 113-147, pls. 23-27.

- 1941, On Neurophyllum koreanicum gen. et sp. nov. from the Lower Permian beds of northern Tyosen (Korea) : Kyusyu Imp. Univ., Faculty Sci. Mem., v. 1, p. 23-41, pls. 1, 2, Japan.

- 1950, On Shirakia kawasake and Shirakiopteris gen. nov.: Geol. Soc. Japan Jour., v. 56, p. 95-97.

Korde, K. B., 1950, Dasycladaceae ie Kembria Tuvy: Acad. sci. u. R. s. s. Comptes Rendus, nouv. sér., tome 73, no. 2, p. 371-374. [Russian.]

Korovin, E. P., 1932, Novyy tretichnyy tip semeystva Proteaceae iz Sredney Aziy. [Genus novum fossile papaeogenum familiae Proteacearum ex Asia Media]: Jour. botanique U. R. S. S, tome 17, nos. 5-6, p. 506-522. 
Kosanke, Robert M., 1950, Pennsylvanian spores of Illinois and their use in correlation : Ill. State Geol. Survey Bull. 74, 218 p.

Krasser, Fridolin, 1889, Über die fossilen Pflanzenreste der Kreideformation in Mähren, Zool.-bot. Gesell. Wien Verh., Band 39, p. 31-35.

1896, Beiträge zur Kenntnis der fossilen Kreideflora von Kunstadt in Mähren: Beitr. Palïonatologie Osterr.-Ungarns u. des Orients, Band 10, Heft 3, p. 113-152, pls. 11-17.

- 1903, Konstantin von Ettingshausen's Studien über die fossile Flora von Ouricanga in Brasilien : Akad. Wiss. Wien Sitzungsber., Band 112, p. 852-860.

1906, Fossile Pflanzen aus Transbaikalien der Mongolei und Mandschurei : Akad. Wiss. Wien Denkschr., Band 78, p. 589-634, pls. 1-4.

- 1916, Studien über die fertile Region der Cycadophyten aus den Lunzer Schichten : Mikrosporophylle und Männliche Zapfen [abs.] : K. Akad. Wiss. Anz., Band 53, p. 335-337.

- 1918, Studien über die fertile Region der Cycadophyten aus den Lunzer Schichten. Mikrosporophylle und Männliche Zapfen: Akad. Wiss. Wien Denkschr., Band 94, p. 489-554, pls. 1-4.

1920, Die Doggerflora von Sardinien: Akad. Wiss. Wien Sitzungsber., Band 129, p. 3-28.

- 1921a, Zur Kenntnis einiger fossiler Floren des unteren Lias der Sukzessionstaaten von Osterreich-Ungarn: Akad. Wiss. Wien Sitzungsber., Band 130, p. 345-373.

1.921b, "Die von Ing. Karl Mandl [Wien] bei Nikolsk-Ussurijsk entdeckten Jurapflanzen" hat folgenden Inhalt: K. Akad. Wiss. Anz., Band 58, p. 219-222.

Krasser, Leo M., 1943, Budingia nov. gen., eine neue Conifere aus dem Zechstein der Wetterau: Oberhess. Gesell. Natur- u. Heilkunde Giessen, Ber., Neue Folge naturwiss. Abt., 1940-43, Bände 20-22, p. 15-19, 1 pl.

Kraus, Gregor, 1866, Einige Bemerkungen über die verkieselten Stämme des frïnkischen Keupers: Wurzburg. Naturw. Zeitschr., Band 6, p. 64-69.

_ 1882, Beiträge zur Kenntniss fossiler Hölzer, I, Hölzer aus den Schwefelgruben Sicilens; II, Zur Diagnostik des Coniferenholzes: Naturf. Gesell. Halle Abh., Band 16, p. 79-109, pl. 1.

Krïusel, Richard, 1919a, Die Pflanzen des schlesischen Tertiärs: Preuss. geol Landesanst. Jahrb., Band 38, Heft 2, p. 1-338, pls. 1-26.

1919b, Die fossilen Koniferenhölzer: Palaeontographica, Band 62, p. 185-275.

— 1922, Fossile Hölzer aus dem Tertiär von Sïd-Sumatra: Geol.-mijnb. genoot. Nederland en Koloniën Verh., Band 5, p. 231-287, 7 pls.

- 1924, Archaeoxylon krasseri, ein Pflazenreste aus dem böhmischen Präkambrian : Lotos, Band 72, p. 31-37, pl. 2.

- 1928, Paläobotanische Notizen X, XI: Senckenbergiana, Band 10, p. 247-254, figs. 1-8.

1929, Fossile Pflanzen aus dem Tertiär von Süd-Sumatra: Geol.-mijnb. genoot. Nederland en Kolonien Verh., Geol. ser., Heft 9, p. 1-44, pls. 1-7.

- 1943a, Furcifolium longifolium (Seward) n. comb., eine Ginkgophyte aus dem Solnhofener Jura: Senckenbergiana, Band 26, p. 426-433, figs. 1-6.

- 1943b, Die Ginkgophyten der Trias von Lunz in Nieder-Osterrich und von neue Welt bei Basel : Palaeontographica, Band 87, Abt. B, p. 59-93, pls. 1-15.

- 1948, Sturiella langeri nov. gen. nov. sp., eine Bennettitee aus der Trias von Lunz (Nieder Osterreich) : Senckenbergiana, Band 29, p. 141-149.

1949, Die fossilen Koniferen-Hölzer: Palaeontographica, Band 89, Abt. B, p. 83-203. 
Kräusel, Richard, and Range, P., 1928, Beiträge zur Kenntnis der Karruformation Deutsch-Sudwest-Afrikas : Preuss. geol. Landesanst., Band 20, p. 1-54, pls. 1-11.

Kräusel, Richard, and Weyland, Hermann, 1923, Beiträge zur Kenntnis der Devonflora : Senckenbergiana, Band 5, p. 154-184, pls. 6-9.

- 1926, Beiträge zur Kenntnis der Devonflora, II: Senckenberg. naturf.

Gesell. Abh., Band 40, p. 115-155, pls. 3-17, Frankfurt.

1929, Beiträge zur Kenntnis der Devonflora, III : Senckenberg. naturf.

Gesell. Abh., Band 41, p. 315-360, pls. 1-15, Frankfurt.

1930, Über Pflanzenreste aus dem Devon Deutschlands : Senckenbergiana,

Band 12, p. 217-221.

1932, Pflanzenreste aus dem Devon ; Senckenbergiana, Band 14, p. 185-190. 1933, Die flora des böhmischen Mitteldevons: Palaeontographica, Band

78, Abt. B, p. 1-44, pls. 1-7.

1941, Pflanzenreste aus dem Devon von Nord-Amerika: Palaeontographica, Band 86, Abt. B, p. 1-78, pls. 1-15.

1949, Gilboaphyton und die Protolepidophytales: Senckenbergiana, Band 30 , p. 129-152, 6 pls.

1950, Kritische Untersuchungen zur Kutikularanalyse tertiärer Blätter,

I : Palaeontographica, Band 91, Abt. B, Lief. 1-4, p. 1-32, 19 pls.

Krejči, Jan, 1853, Kounicka skala [Kaunitz rock] : Ziva, v. 1, p. 28, pls. 1, 2.

- 1880, Notiz über die Reste von Landpflanzen in der böhmischen Silurformation; K. Böhmische Gesell. Wiss., Prag Sitzungsber., 1879, p. 201-204.

Krestew, Krestoe, 1928, Über das Carbon des Iskur-Defles in Bulgarien und seine Altersstellung: Preuss. geol. Landesanst. Jahrb., Band 49, p. 551-579.

Kretschetovitsch [Krechetovich], L. M., 1936, stenogrammites pseudocostata, a new fossil red alga from the Jurassic formation of the Gorky district: Moscow, Univ., Paleont. Laboratoria, Problems Paleontology Pub., v. 1, p. 251-262, pl. 1.

Kryshtofovich [Krishtofovich], A. N., 1915, Jurassic plants from Tyrmaflutz, Amur river: Mus. géol. Pierre le Grand Travaux, tome 8, p. 79-124, pls. 1-7. [Russian.]

1927, Some fossil plants from the Jurassic slate of the north Caucasus: Comité géol, Russia, Bull, tome 45, no. 5, p. 597-608, pls. 12, 13, Leningrad. 1929, The oldest angiosperms in the Cretaceous of Asia and other contributions to the Mesozoic flora of the maritime province, Siberia: Am. Jour. Sci., v. 18, p. 519-525.

Kurr, J. Gottlob, 1845, Beiträge zur fossilen Flora der Juraformation wurtembers : 21 p., 3 pls, stuttgart.

Kurtz, Frederic, 1894, Contribuciones a la palaeophytologia Argentina: Mus. La Plata Rev., tomo 6, p. 117-139, pls. 1-4.

1902, Contribucions a la palaeophytologia Argentina, III, Sobre la existencia de una Dakota-Flora en la Patagonia Austro-occidental: Mus.

La Plata Rev., tomo 10, p. 45-59.

- 1921, Atlas de plantes de la Republica Argentina-Obra postuma de acuerdo con los manuscritos del Dr. F. Kurtz: Acad. Nac. Cienc. Cordoba Actas, tomo 7, p. 129-153, pls. 1-27.

Kušta, Jan, 1892, Přispěvky k seznání nejstarších zkamenělin českých a evropskx́sch vỉbee. [Beitrïge zur Kentniss der ältesten Versteinerungen von Böhmen und Europa überhaupt.]: K. Böhmische Gesell. Wiss., Prag, Sitzungsber., 1892, p. 418-424. 1898, Kamenouhelna rasa Anthracochondrus nyranensis: Ziva, จ. 8, p. 220. 
Kutorga, Stephen, 1838, Beiträge zur Kenntnis der organischen Überreste des Kupfersantsteins am westlichen Abhange des Urals: 38 p., 8 pls., St. Petersburg.

Lacoe, R. D., 1884, Catalogue of the Paleozoic fossil plants of North America : [15] p., Pittston, Pa.

Lakowitz, Conrad, 1895, Beiträge zur Kenntnis der Tertiärflora des Ober-Flsass : Abh. geol. Specialkarte Elsass-Lothringen, Band 5, Heft 3, p. 179-359, pls. 5-13, Strasburg.

Lamarck, Jean, 1804, Sur les fossiles des environes de Paris : Mus. natl. histoire nat. Annales, tome 5, p. 349-357, Paris.

- 1807, Explication des planches relatives aux coquilles fossiles des environs de Paris: Mus. natl. historie nat. Annales, tome 9, p. 236, pl. 15, Paris.

1816, Histoire naturelle des animaux sans vertebres: tome 2, p. 1-568, Paris.

Lamouroux, J. V. F., 1816, Histoire des polypiers coralligenes flexibles, vulgairement nommes zoophytes: Caen.

Landgrebe, G., 1842, Über eine im Basalt-Konglomerat des Knüll-Gebirges aufgefundene Frucht, Dryobalanus basalticus, aus der Famile der Kupuliferen : Neues Jahrb., p. 813-816.

Lang, W. H., 1926, Contribution to the study of the Old Red Sandstone flora of Scotland, III, IV, V : Royal Soc. Edinburgh Trans., v. 54, p. 785-799, pls. $1,2$.

1937, On the plant remains from the Downtonian of England and Wales: Royal Soc. London Philos. Trans., v. 227, p. 245-290, pls. 8-14.

1945, Pachytheca and some anomalous early plants: Linnean Soc., London Jour., v. 53, p. 535-552, pls. 22-25.

Lang, W. H., and Cookson, I. C., 1935, On a flora, including vascular land plants, associated with Monograptus, in rocks of Silurian age, from Victoria, Australia : Royal Soc. London Philos. Trans., v. 224B, p. 421-449, pls. 29-32.

Langeron, Maurice, 1899, Contributions à l'étude de la flore fossile de Sézanne: Soc. histoire nat. Autun, tome 12, p. 433-455, pls. 2-5.

1900, Contributions à l'étude de la flore fossile de Sézanne: Soc. histoire nat. Autun, tome 13, p. 333-369, pls. 1-5.

1902, Note sur une empreinte remarquable provenant des Cinerites du Cantal : Soc. histoire nat. Autun, tome 15, p. 85-96, pl. 6.

Lapparent, Albert de, 1883, Traité de géologie: 1280 p., Paris.

Laurent, Louis, 1899, Flore des calcaires de Celas: Mus. histoire nat. Marseille Annales, sér. $2^{\circ}$, tome 1, p. 1-148, pls. 1-14.

1905, Flore des cinerites pliocenes du Pas-de-la-Mougudo et de SaintVincent La Sabie: Mus. histoire nat. Marseille Annales, sér. $2^{\circ}$, tome 9, p. 1-313, pls. 1-20.

Lebesconte, Paul, 1883, Oeurres posthumes de Marie Rouault: 73 p., 20 pls., Paris.

Leckenby, John, 1863, On the sandstones and shales of the oolites of Scarborough, with descriptions of some new species of fossil plants: Geol. Soc. London Quart. Jour., v. 20, p. 74-82, pls. 8-11.

Leclercq, Suzanne, 1936, A propos du Sphenophyllum fertile Scott: Soc. géol. Belgique, Annales, tome 60, p. 170-172.

LeConte, Joseph, 1882, Elements of Geology-A textbook for colleges and for the general reader, 2 ed. : 633 p., New York.

Lee, G. W., 1912, The British Carboniferous Trepostomata : Great Britain Geol. Survey Mem. [Paleontology], I, pt. 3. 
Lemoine, Mme. Paul, 1930, In das Danien der Ausseren Klippensone bei Wein, XI, Palaeontologischer Teil, Algues: Geologie u. Palaeontologie Abh., Neue Folge, Band 17, Heft 5, p. 534-541.

Lesley, J. P., 1880, Sur les limites du terrain carbonifère et du terrain permien en Amérique, d'après l'étude de leurs flores: Internat. Geol. Cong., Paris, 1878, Comptes Rendus, p. 130-135.

Lesquereux, Leo, 1854, New species of fossil plants, from the anthracite and bituminous coal fields of Pennsylvania: Boston Jour. Nat. History, v. 6, p. 409-431.

- 1860, Botanical and palaeontological report on the Geological State Survey of Arkansas: Second report of a geological reconnaissance of the middle and southern counties of Arkansas, p. 308-317, pls. 1-5, Philadelphia.

1861a, On the fossil fruits found in connection with the lignites of Brandon, Vt: Am. Jour. Sci., 2d ser. v. 32, p. 355-362.

$1861 \mathrm{~b}$, On the fossil fruits found in connection with the lignites of Brandon, Vt: Report on the geology of Vermont, v. 2, p. 712-718.

1868, On some Cretaceous fossil plants from Nebraska: Am. Jour. Sci., 2d ser., v. 46, p. 91-105.

1869, On Fucoides in the coal formations: Am. Philos. Soc. Phila. Trans., v. 13, p. $313-328$, pl. 7 .

1870, Report on the fossil plants of Illinois: Ill. Geol. Survey, v. 4, Geology and Palaeontology, pt. 2, p. 377-508, pls. 5-31.

1874, Contributions to the fossil flora of the Western Territories, Part I, The Cretaceous flora: U. S. Geol. and Geog. Survey Terr. Rept., v. 6, p. 1-136, pls. 1-30.

1876a, Paleontology: U. S. Geol. and Geog. Survey Terr., 8th Ann. Rept. p. 271-366, pls. 1-8.

$1876 \mathrm{~b}$, New species of fossil plants from the Cretaceous formation of the Dakota group: U. S. Geol. and Geog. Survey Terr. Bull., v. 1, no. 5, p. 391-400.

1876c, Species of fossil marine plants from the Carboniferous measures : Ind. Geol. Survey, 7th Ann. Rept., p. 134-145, pls. 1, 2.

1877, Land plants, recently discovered in the Silurian rocks of United

States: Am. Philos. Soc. Phila. Proc., v. 17, p. 163-175, pl. 4.

1878a, Contributions to the fossil flora of the Western Territories, Part

II, The Tertiary flora : U. s. Geol. Survey Terr. Rept., v. 7, p. 1-366, pls. 1-65.

- 1878b, On the Cordaites and their related generic divisions in the Carboniferous formation of the United States: Am. Philos. Soc. Phila. Proc., จ. 17, p. 315-355.

1878c, Remarks on specimens of Cretaceous and Tertiary plants secured by the Survey in 1877, with a list of the species hitherto described: U. S. Geol. and Geog. Survey Terr. for 1876, 10th Ann. Rept. p. 481-520. 1879, Atlas to Coal Flora. see Lesquereux, Leo, 1880.

1880 , Description of the coal flora of the Carboniferous formation in

Pennsylvania and throughout the United States: Pa. $2 \mathrm{~d}$ Geol. Survey Rept. Progress, P., v. 1, p. 1-354; v. 2, p. 355-694, pl. 86 opposite p. 544, pl. 87 opposite p. 560. Atlas, 1879, pls. 1-85.

1883, Contributions to the fossil flora of the Western Territories, Part III, The Cretaceous and Tertiary floras: U. S. Geol. Survey Terr. Rept. 8, p. 1-283, pls. 1-59.

1884, Description of the coal flora of the Carboniferous formation in Pennsylvania: Pa. 2d Geol. Survey, Rept. Progress P, v. 3, p. 695-977, pls. 89-111. 
Lesquereux, Leo, 1887, List of recently identified fossil plants belonging to the United States National Museum, with descriptions of several new species:

U. S. Natl. Mus. Proc., v. 10, p. 21-46.

1892, The flora of the Dakota group: U. S. Geol. Survey Mon. 17, p. 1-256, pls. 1-66.

1893, The genus Winchellia: Am. Geologist, v. 12, p. 209-213, pls. 8, 9.

Lesquereux, Leo, and Rogers, Henry Darwin, 1854, New species of fossil plants, from the anthracite and bituminous coal fields of Pennsylvania : Boston Jour. Nat. History, v. 6, p. 409-431.

Leuthardt, F. 1901, Beiträge zur Kenntnis der Flora und Fauna der Lettenkohl von Neuewelt bei Basel: Eclogae Geol. Helvetiae, Band 7, no. 2, p. 125-128.

Levittan, Edwin D., and Barghoorn, Elso S., 1948, Sphenostrobus thompsonii-a new genus of the Sphenophyllales: Am. Jour. Botany, v. 35, p. 350-358, figs. 1-12.

Liburnau, Josef Ritter Lorenz, 1901, Zur deutung der fossilen fucoiden Gattungen Taenidium und Gyrophyllites: Akad. Wiss. Wien Denskchr., Band 70, p. 523-583, pls. $1-4$.

- 1902, Erganzung zur Beschreibung der fossilen Halimeda fuggeri: Akad. Wiss. Wien Sitzungsber., Band 3, Abt. 1, p. 685-712, pls. 1, 2.

Lignier, Octave, 1895, Végétaux de Normandie, II, Contributions à la flore Liasique Ste. Honorine-la-Guillaume (Orne): Soc. linnéenne Normandie Mém., sér. $2^{\circ}$, tome 18, fasc. 2, p. 124-151, pl. 7.

1906, Radiculites reticulatus, radicelle fossile de Sequoinee: Soc. bot. France Bull., sér. 4, tome 6, p. 193-201.

- 1907, Végétaux fossiles de Normandie: Soc. linnéenne Normandie Mém., tome 22 [sér. 2, tome 6], p. 240-332, pls. 17-23.

-1910a, Sur une Calamodendrée Liassique: Assoc. Française, av. scl., Comptes Rendus, 38 sess., Lille, 1909, p. 620-626.

1910b, Calamitomyelon morierei gen. et sp. nov. : Soc. linnéenne Normandie Bull., sér. $6^{\mathrm{e}}$, tome 2, p. 116-128.

- 1913, Un nouveau sporange seminiforme, Mittagia seminiformis, gen. et sp. nov.: Soc. linnéenne Normandie Mém., sér. $2^{\circ}$, tome 8, p. 49-65, pl. 8.

Lilienstern, Rühle H., 1928, Dioonites pennaeformis Schenk, eine fertile Cyadee aus der Lettenkohle: Palaeont. Zeitschr., Band 10, p. 91-107, pls. 5, 6, Berlin.

Lilpop, J., 1937, New plants from the Permocarboniferous rocks in Poland : Acad. Polonaise sci. Bull., sér. B, p. 1-10, pl. 1.

Lindgren, Waldemar, 1896, The age of the auriferous gravels of the Sierra Nevada; with a report on the flora of Independence Hill: Jour. Geology, v. 4, p. 881-906.

- 1911, The Tertiary gravels of the Sierra Nevada of California : U. S. Geol. Survey Prof. Paper 73, 226 p., 25 pls.

Lindley, John, and Hutton, William, 1831-37, The fossil flora of Great Britain; or figures and descriptions of the vegetable remains found in a fossil state in this country : v. 1 , p. $1-48$, pls. $1-14,1831 ;$ p. $49-166$, and v-li, pls. $15-49$, 1832 ; p. $167-218,1833$; v. 2 , p. $1-54$, pls. $80-99$, 1833 ; p. $57-156$, pls. $100-137$, 1834 ; p. $157-206$, pls. $138-156,1835$; v. 3 . p. $1-72$, pls. $157-176,1835$; p. 73-122, pls. 177-194, 1836; p. 123-205, pls. 195-230, 1837.

Lingelsheim, Alexander, 1917, Ein Beiträge zur fossilen Flora Ungarns: K. Ungarische geol. Reichsanst. Jahresber. 1915, p. 545-563.

- 1929, Über ein Koniferenholz aus dem Tertiär der Niederlausitz: Naturf. Gesell. Görlitz Abh., Band 30, Heft 3, p. 103-115.

Lomax, James, 1911, The microscopical examination of coal, and its use in determining the inflammable constituents present therein: Manchester Geol. Min. Soc. Trans., v. 32, p. 122-135. 
Lomnicki, A. M., 1886, Slodkowodny utwór trzeceorzedny na Podulu galicyjskiém : Akad. umietjet, Krakow, Komi. fizjog. Sprawozdania tom 20, p. 48-119, pls. 1-3.

Loose, Friedrich, 1932, Beschreibung von Sporenformen aus Flöz Bismarck, in Potonie, Robert, 1932, p. 449-452.

Lorenz, Theodor, 1904, Aseosomaceae, eine neue familie der Siphoneen aus dem Cambrium von Schantung: Centralbl. Mineralogie 1904, Heft 7, p. 193-194.

Lotsy, J. P., 1909, Vorträge über botanische Stammesgeschichte, Band 2, Cormophyte Zoidogamia : $901 \mathrm{p}$.

Loubière, August, 1929, Etude anatomique et comparée du Leptotesta grand'Euryi n. gen., n. sp. (graine silicifée du Pecopteris pluckeneti Schlotheim) : Rev. gén. botanique, tome 41, p. 593-605, pl. 12, Paris.

- 1930, Sur les graines et les feuilles stipales associées a l'Odontopteris obtusa Brongniart dans le terraine carbonifêre de Carmaux: Rev. gén. botanique, tome 22, p. 321-326, Paris.

Ludwig, Rudolph, 1857, Fossile Pflanzen aus der jungsten Wetterauer Braunkohle : Palaeontographica, Band 5, p 81-109, pls. 16-23.

- 1859, Fossile Pflanzen aus der altestem Abtheilung der RheinischWetterauer Tertiär-Formation: Palaeontographica, Band 8, p. 39-154, pls. 6-61.

1861, Palaeontologie des Urals: Palaeontographica, Band 10, p. 17-36, pls. 3-6.

1863, Zur Palaeontologie des Urals-Pflanzenreste aus der Steinkohlenformation des Urals: Palaeontographica, Band 10, p. 270-275, pl. 46.

Lutz, Josef, 1933, Zur Kulmflora von Geigen bei Hof: Palaeontographica, Band 78, Abt. B, p. 114-157, pls. 15-19.

McCoy, Frederick, 1847, On the fossil botany and zoology of the rocks associated with the coal of Australia: Annals and Mag. Nat. History, 1st ser., v. 20, p. 145-157.

-1874-76, Prodromus of the paleontology of Victoria ; or figures and descriptions of Victorian organic remains: Geol. Survey Victoria, decade 1, p. 1-43, pls. 1-10, 1874; decade 2, p. 1-37, pls. 9-20, 1875; decade 4, p. 1-32, pls. 31-40, 1876, Melbourne.

MacGinitie, Harry D., 1941, A middle Eocene flora from the central Sierra Nevada : Carnegie Inst. Washington Pub. 534 p. 178 p., 47 pls.

McLean, R. C., 1912, A group of rhizopods from the Carboniferous period : Cambridge Philos. Soc. Proc., v. 16, p. 493-513.

M'Nab, W. R., 1870, On the structure of a lignite from the old Red Sandstone: Edinburgh Bot. Soc. Trans., v. 10, p. 312-314.

Mädler, Karl, 1939, Die pliozäne Flora von Frankfurt am Main: Senckenberg. naturf. Gesell. Abh. 446, p. 1-202, pls. 1-13.

Mägdefrau, Karl, 1932, Über Nathorstiana, eine Isoetaceae aus dem Neokom von Quedlinburg a Harz: Bot. Centralbl., Band 49, p. 706-718, pls. 11, 12. 1937, Lebensspuren fossiler "Bohr" organismen: Beitr.-Naturfundlichen Forschung Sudwestdeutschland, Band 2, p. 54-67, pls. 4-6.

1938, Eine Halophyten-Flora aus dem Unterdevon des Harzes: Bot. Centralb1. Belherte, Bana 58, Abt. B, p. 243-251, p1s. 1, 2.

Magnus, P., 1903, Ein ron $\mathrm{P}$. W. Oliver machgewiesener fossiler parasitischer Pilz: Deutsche bot. Gesell. Zeitschr., Band 21, p. 248-250.

Maire. Rene, 1897, Nouveau cycadeospermum de l'oxfordien: Herbier Boissier Bull., tome 5, p. 338-392. 
Mamay, Sergius Harry, 1950, Some American Carboniferous fern fructifications : Mo. Bot. Garden Annals, v. 37, p. 409-476.

Mantell, Gideon, 1833, The geology of the southeast of England : 415 p., London. 1844 , The medals of creation : $\nabla .1$, xxvii p., 456 p., 6 pls., London.

Marck, W., von, der 1863, Fossile Fische, Krebse und Pflanzen aus dem Plattenkalk der jungsten Kreide in Westphalen: Palaeontographica, Band 11, p. 1-40, pls. 1-14.

Marik, V., 1901, Přispevek k floře českeho cenomanu: Česká akad. Rozpravy, tom 10, no 3, p. 1-16, pls. 1, 2, Prague.

Marion, A. F., 1872, Descriptions des plantes fossiles des calcaires marneux de Ronzon: Annales sci. nat., Botanique, tome 14, p. 326-364, pls. 22, 23, Paris.

- 1884, Sur les caractéres d'une conifère tertiare, voisine des Dammarees (Doliostrobus sternbergi) : Acad. sci. Paris Comptes Rendus, tome 99, p. 821-823.

- 1890a, Sur la flore turonienne des Martiques (Bouches-du-Rhone) : Acad. sci. Paris Comptes Rendus, tome 110, p. 1052-1053.

- 1890b, Sur le Gomphostrobus heterophylla, conifère prototypique du permien de Lodeve: Acad. sci. Paris Comptes Rendus, tome 110, p. 892-894.

Marion, A. F., and Laurent, Louis, 1898, Examen d'une Collection de végétaux fossiles de Roumanie: Mus. géologique paléontologique Annuaire 1895, p. 187-227, pls. 1, 2., Bucarest.

Marmora, Albert, 1857, Voyage en Sardaigne, ou description statistique, psysique et politique de cette ile, avec des recherches sur les prodictions naturelles et ses antiquities: tome 1, p. 1-706; tome 2, p. 1-780, Turin.

Martin, William, 1809, Petrificata Derblensia; or figures and descriptions of petrifactions collected in Derbyshire : 28 p., 52 pls., Wigan.

Martius, D. Carolus, 1822, De plantis nonnullis antediluvianis ope specierum inter tropicos viventium illustrandis : K. bayer. bot. Gesell. Denkschr., Band 2, p. 121-147, pls. 2, 3, Regensburg.

1846, Historia naturalis palmarum, v. 1, p. 53-70, pls. 1-3.

Maslen, Arthur J., 1930, The structure of Mesoxylon platypodium and Mesoxyloides : Annals Botany, v. 44, p. 503-533, pls. 25-28.

Maslov [Masloff], V. P., 1929, Some microscopical algae of the Carboniferous limestones of the Donetz Basin: Russia, Comité géol. Bull., tome 48, no. 10, p. 115-138, pls. 70-71, Leningrad.

1937a, On the Paleozoic rock building algae of east Siberia: Moscow, Univ., Paleont. Laboratoria, Problems Paleont. Pub., v. 2-3, p. 314-325, pls. 1-12.

1937b, On the distribution of calcareous algae in east Siberia: Moscow, Univ., Paleont. Laboratoria, Problems Paleont. Pub., v. 2-3, p. 342-348, pls. 1-5.

1939, Contributions to the knowledge of the fossil algae of the U. S. S. R.: Moscow, Univ., Paleont. Laboratorira, Problems Paleont. Pub., v. 5, p. 287290 , pls. 1-2.

Massalongo, Abramo, 1850, Schizzo geognostico sulla valle del progno o Torrente d'Illasi : 77 p., Verona.

1851, Sopra le piante fossili dei terrent terzairi del Vicentino: 263 p., Padova.

1852a, Sapindacearum fossilium monographia: 28 p., pls. 1-6. Verona. 1S52b, Amphoridium, novum lichenum genus: R. ist. veneto sci. Atti, $\mathbf{v}$. 3, p. 172-1S0, 1 pl. [unnumbered].

1852c, Synopsis palmarum fossilium : Lotos, v. 2, p. 193-208, Prague. 1853a, Plantae fossiles novae in formationibus tertiaris: 24 p., Verona. 
Massalongo, Abramo, 1853b, Enumerazione della piante fossili miocene fine ad ora conosciute in Italia: 31 p., Verona.

1853c, De gramineis in statu fossili brevis commentatio: Flora, vol. 11,

p. 129-133, pl. 3, Regensberg.

- 1853d, Sopra un nuove genere di Pandanee fossili della Provincia Veronese : Accad. agri. Verona Mem., v. 29, p. 185-207, pls. 1-4.

1854, Monografia delle Dombeyacee fossili : 25 p., 1 pl., Verona.

1855, Zoophycos novum genus plantarum fossilium : $52 \mathrm{p.}, 3$ pls., Verona.

1856a, Studii paleontologici : 55 p., 7 pls., Verona.

1856b, Descrizione di alcuni Fuchi fossili della calcaria del Monte Spil-

ecco nella Provincia Veronese: Rev. periodica, v. 4, p. 207-233, pls., 1-6, Padova.

1857a, Flora fossili del Monte Colle nella Provincia Veronese: R. ist. veneto sci. Mem., v. 6, p. 557-578, pls. 1-8.

$1857 \mathrm{~b}$, Vorläufige Nachricht überdie neueren palaontologischen Entdeck-

ungen am Monte Bolca : Neues Jahrb., p. 775-778.

1858a, Synopsis florae fossilis Senogalliensis: $136 \mathrm{p}$., Verona.

1858b, Palaeophyta rariora formationis tertiariae agri Veneti: $R$. ist.

veneto sci. Atti, v. 3, p. 720-793.

1858c, Elenco dei modelli di piante fossili : R. ist. veneto sci. Atti, ser.

$3^{\text {a }}$, v. 3, p. 814-816.

- 1858d, Reliquie della flora fossile eocena del Monte Pastello nella Veronese : R. ist. veneto sci. Atti, v. 3, p. 169-186, pls. 1-8.

1858e, Monografia del genere Silphidium: Soc. Italiana sci. Mem., v. 1, ser. $2^{\text {a }}$, p. 105-125, pls. 1-7.

1859a, Specimen photographicum animalium quorumdam plantarumque fossilium : 101 p., 40 pls., Verona.

1859b, Syllabus plantarum fossilium : $179 \mathrm{p}$., Verona.

Massalongo, Abramo, and Lotze, Maritius, 1859, Saggio fotografico di alcuni animali e piante fossili : $101 \mathrm{p} ., 40 \mathrm{pls} .$, Verona.

Massalongo, Abramo, and Scarabelli, Guiseppe, 1857, Sulla flora fossile di Sinigaglia : $52 \mathrm{p}$., Verona. [Plates referred to in this paper. were published in Massalongo, Abramo, and Scarabelli, Guiseppe, 1859.]

- 1859, Studii sulla flora fossile e geologia stratigraphica del Senigalliese: 504 p., 44 pls., Imola.

Matheisen, Fr. J., 1932, Notes on some fossil plants from East Greenland : Meddelelser om Grönland, Bind 85, Hefte 4, p. 1-62, pls. 1-6, Copenhagen.

Matthew, G. F., 1890a, On Cambrian organisms in Acadia: Royal Soc. Canada Proc. and Trans., v. 7, sec. 4, p. 135-162, pl. 5-9.

1890 b, Eozoon and other low organisms in Laurentian rocks of St. John :

New Brunswick Nat. History Soc. Bull. 9, p. 67.

1906a, New species and a new genus of Devonian plants : New Brunswick

Nat. History Soc. Bull., v. 5, p. 393-396, pls. 8, 9.

$1906 \mathrm{~b}$, A review of the flora of the Little River group : Royal Soc. Canada Proc. and Trans., $2 \mathrm{~d}$ ser., . . 12, sec 4, p. 99-130, pls. 1-8.

1910, Revision of the flora of the Little River group, No. II, Descriptioll of the type of Dadoxylon ouangondianum, Dawson: Royal Soc. Canada Proc. and Trans., 3d ser., v. 3, sec. 4, p. 77-113, pls. 1-4.

1913, A new flora in the older Palaeozoic rocks of southern New Brunswick, Canada : Royal Soc. Canada Proc. and Trans., 3d ser., จ. 6, p. 83-99, pls. $1,2$.

Mawson, Douglas, and Madigan, C. T., 1930, Pre-Ordovician rocks of the McDonnell Ranges: Geol. Soc. London Quart. Jour., v. 86, p. 415-428. 
Medlicott, H. B., and Blanford, W. T., 1879, A manual of the geology of India chiefly compiled from the observations of the Geological Survey: v. 1, p. $1-444$; v. 2, p. 445-817, Calcutta.

Mehta, K. R., 1944, Microfossils from a Carbonaceous shale from the Pali beds of the South Rewa Gondwana Basin: Natl. Acad. Sci. India Proc., v. 14, p. 125-141.

Meneghini, C. J., 1857, Paleontologie de l'Ile de Sardaigne: 584 p., pls. A-H, Turin.

Menge, Anton, 1850, Beiträge zur Naturkunde Preussens, I, Geognostische Bemerkungen: Naturf. Gesell. Danzig, Neueste Schr., Band 4, Heft 3, p. 1-36, pls. 1-3.

- 1858, Beiträge zur Bernsteinflora : Naturf. Gesell. Danzig, Neueste Schr., Band 6, Teil 1, Heft 2, p. 1-18.

Menzel, Paul, 1900, Die Gymnospermen der nordböhmischen Braunkohlenformation Teile 1 u. 2 : Naturwiss. Gesell. Isis Dresden Abh., Teil 1, p. 49-68, pls. 2-4; Teil 2, p. 85-110, pl. 5 .

1909, Fossile Pflanzenreste aus den Mungo-Schichten in Kamerun : Preuss. geol. Landesanst. Abh., Band 62, p. 399-404, pl. 2.

1913, Beitrag zur Flora der Niederrheinischen Braunkohlenformation: Preuss. geol. Landesanst. Jahrb., p. 1-98, pls. 1-7.

1920, Über Pflanzenreste aus Basaltluffen des Kamerungebietes: Beitr. geol. Erforschung Deutschen Schutzgebiete, Heft 18, p. 17-32.

Mercklin, Carl Engen von, 1953, Verzeichniss aller in Russland bis jetzt aufgefundener beschriebener, unsbeschriebener oder zweifelhafter fossiler Pflanzen: Acad. imp. sci. St. Petersburg Bull., tome 11, p. 300-305.

- 1856, Palaeodendrologikon Rossicum. Vergleichende Anatomisch-Mikroskopische untersuchungen fossiler Hölzer: 99 p., 20 pls., St. Petersburg.

Meschinelli, Aloysius, 1892, Sylloge fungorum fossilium, in Saccardo, P. A., Sylloge Fungorum, v. 10, p. 741-809.

1889, Studio sulla flora fossili di Monte Piano: Soc. veneto-trentino sci. nat. Atti, Padova.

—_ 1898, Fungorum fossilium omnium : 144 p., 31 pls., Vicetiae.

Meschinelli, Aloysius, and Squinabol, Senofonte, 1893, Flora tertiaria Italica: 575 p., Patavii.

Meunier, Stanislas, 1886, Sur quelques empreintes problematiques des couches boloniennes du Pas-de-Calais : Soc. géol. France Bull., sér. $3^{\circ}$, tome 14, p. 564568, pls. 29-30.

- 1887, Radiophyton sixii: Le Naturaliste, sér. $2^{\circ}$, tome 9, p. 59.

- 1891, Staurophyton bagnolensis: Le Naturaliste, sér. $2^{\mathrm{e}}$, tome 13, p. 134. 1898, Nouvelle plante fossile Eocene : Le Naturaliste, sér. $2^{\circ}$, tome 20, p. 17. 1904, Sur quelques fossiles provenant du Niger: Cong. Soc. Sav. Comptes Rendus, Paris, p. 156-159.

Michelin, Hardouin, 1840-47, Inconographie zoophytologique, description par 10calités et terrains des polypiers fossiles de France et pays environnant: 348 p., 79 pls., Paris.

Miki, Shigeru, 1941, On the change of flora in Eastern Asia since Tertiary Period, (I), The clay or lignite beds in Japan with special reference to the Pimus trifolia beds in Central Hondo: Japanese Jour. Botany, v. 11, p. 237-303, pls. 4-7.

Milanez, Fernando Romans, 1935, Estudo de un dicotyledones fossil de Cretaces: Rodriquesia, tomo 1, no. 2, p. 83-89, pls. 1-3.

Miller, Hugh, 1847, The testimony of the rocks : 502 p., Boston. 
Miller, S. A., 1889, North American geology and palaeontology for the use of amateurs, students, and scientists : 664 p., Cincinnati.

1892, North American geology and palaeontology pendix; p. 665-718, Cincinnati.

Miller, S. A., and Dyer, C. B., 1878, Contributions to palaeontology : Cincinnati Soc. Nat. History Jour., v. 1, p. 24-39, pls. 1, 2.

Miner, Ernest L., 1935, Paleobotanical examinations of Cretaceous and Tertiary coals : Am. Midland Naturalist, v. 16, p. 585-625, pls. 18-24.

Miquel, F. A. W., 1851a, De Quibusdam plantis fossilibus: Wiss. natuurk. wetensch., Amsterdam, Tijdschr., boekdeel 4, p. 265-269.

1851b, Over de rangschikking der fossiele Cycadeae: Wiss. natuurk. wetensch., Amsterdam, Tijdschr., boekdeel 4, p. 205-227.

1853, De fossiele plantem van het krijt: Geol. Kaart Nederlandsche, Verh., p. 1-7, Haarlem.

Morellet, Lucien, 1908, Deux algues Siphonées verticillées du Thanetien de Boncourt (Oise) : Soc. géol. France Bull., sér. $4^{\circ}$, tome 8, p. 96-99.

Morellet, Lucien, and Morellet, Jean, 1913, Les Dasycladacées du tertiaire parisien: Soc. géol. France, Paléontologie Mém. 47, tome 21, p. 1-43, pls. 1-3.

1917, Les Dasycladacées tertiaires de Bretagne et du Cotentin : Soc. géol. France Bull, sér. 4e, tome 17, p. 362-372, pl. 14.

1922, Nouvelle contribution á l'Étude des Dasycladacées tertiaires: Soc. géol. France, Paléontologie Mém. 58, tome 25, p. 1-35, pls. 9, 10.

1939, Tertiary siphoneous algae in the W. K. Parker collection : British Mus. Nat. History, p. 1-55, pls. 1-6.

Morgenroth, Eduard, 1883, Die fossilen Pflanzenreste im Diluvium der Umgebung von Kamenz in Sachsen: Zeitschr. Gesammt. Naturw. Halle, Bànd. 56, p. 271-318, pls. 3, 4 .

Moriere, M., 1888, Note sur une nouvelle Cyadée du Lias: Soc. linnéenne Normandie Bull., sér. $4^{\mathrm{e}}$, tome 1, p. 125-134, pls. 1-3.

Morris, John, 1841, Remarks upon the recent and fossil cycadeae: Annals and Mag. Nat. History, v. 7, p. 110-120.

1854, A catalogue of British fossils, $2 d$ ed. : 372 p., London.

Mougeot, Antoine, 1851, Note sur les végétaux fossiles du Grès Rouge, suivie de leur comparaison avec ceux du Grès Bigarre: Cong. Sci. France, sess. 17e, Nancy, 1850, tome 1, p. 339-350.

1852, Essai d'une flore du nouveau Grès Rouges des Vosges, ou description des végétaux silicifies qui s'y rencontrent: Soc. émulation, Dépt. Vosges, Annales, tome 7 , p. 1-46, pls. 1-5, Epinal.

Mourlon, Michel, 1875, Sur l'étage dévonien des psammites du Condroz en Condroz: Acad. royale Belgique Bull., sér. 2e, tome 39, p. 602-659.

1881, Géologie de la Belgique: tome 2, 392 p., Brussels.

Müller, Albrecht, 1862, Geognostische skizze des Kantons Basel und der Angrenzenden Gebiete nebst Geognostischer Karte in Farbendruck: Carte geol. Suisse Matériaux, Neuchatel, Band 1, p. 1-70.

Mueller, Ferdinand, 1871-82, New vegetable fossils of Victoria: Victoria Dept. Mines, Rept. Min. Surveys and Registraxs 38, p. 47-48, pls. 2-3, 1871; no. 103, p. $39-41$, pls. $4-5,1871$; no. 4, p. 4142, pls. $7-8,1873$; no. 92, p. $41-42$, pl. 6 , 1873 ; no. 84 , p. 41-42, pls. 9-10, 1874; no. 48, p. 41-42, pls. 9-10, 1875 ; no. 10, p. 35 , pl. 14,1878 ; no. 68 , p. $38-40$, pl. 15 ; no. 3 , p. $37-39$, pls. $16-17,1879$; no. 83 , p. $39-40$, pl. 18,1870 ; no. 27 , p. 13-14, pls. 10-20, 1882, Melbourne. 1874, Observations on new regetable fossils of the auriferous drifts, Part 1: Victoria Geol. Survey, p. 1-31, pls. 1-10, Melbourne. 
Mueller, Ferdinand, 1876, Description of fossil plants from the Upper Tertiary auriferous drifts of New South Wales: New South Wales Dept. Mines, Ann. Rept. 1875, p. 124-126, Sydney.

— 1877a-79, Descriptive notes on the Tertiary flora of New South Wales: New South Wales Dept. Mines, Ann. Rept. 1876, p. 178-180, 1877; Annual Rept. 1878, p. 169-172, pls. 3, 4, 1879, Sydney.

- 1877b, Descriptions of fossil fruits in siliceous deposits, Richmond River: Royal Soc. New South Wales Jour. and Proc., v. 10, p. 239, S.ydney.

- 1883, Observations on new vegetable fossils of the auriferous drifts, Part 2: Victoria Geol. Survey, p. 1-23, pls. 11-20, Melbourne.

Mueller, Ferdinand, and Smyth, R. Brough, 1870, Observations on some vegetable fossils from Victoria : Geol. Soc. London Quart. Jour., v. 26, p. 610.

Munier-Chalmas, E., 1877, Observations sur les algues calcaires appartenant au groupe des Siphonées verticillées et confondues avec les Foraminfères : Acad. sci. Paris Comptes Rendus, tome 85, p. 814-817.

Münster, George Graf, 1839-43, Beiträge zur Petrefacten-Kunde : Teil 1, p. 1-125, pls. 1-18, 1839; Teil 5, p. 1-131, pls. 1-15, 1842 ; Teil 6, p. 1-100, pls. 1-9, 1843.

Murchison, Roderick Impey, 1827, On the coal field of Brora in Sutherlandshire, and some other stratified deposits in the north of Scotland: Geol. Soc. London Trans., 2d ser., v. 2, p. 293-326, pls. 1-49 [1829].

1845 , Outline of the geology of the neighborhood of Cheltenham, new ed., revised by James Buckman and H. E. Strickland : 109 p., 12 pls., London.

1851, On the Silurian rocks of the south of Scotland: Geol. Soc. London Quart. Jour., v. 7, p. 139-178, pls. 9-11.

- 1854, Siluria-The history of the oldest fossiliferous rocks and their foundations; with a brief sketch of the distribution of gold over the earth: 523 p., 37 pls., London.

- 1859, Siluria *** 3d ed. : 592 p., 41 pls., London.

Murchison, Roderick Impey, Verneuil, Edouard, and Keyserling, Alexandre, 1845, Géologie de la Russie d'Europe et des Montagnes d'Oural-tome 2, pt. 3, Paléontologie: 504 p., 43 pls., Londôn and Paris.

Musper, Fritz, 1919, Beitrag zur Deutung der Frage des Aufbaus des oberen weissen Jura in Schwaben: Ver. vaterl. Naturkunde Württemberg Jahrb., Band 75, p. 1-18.

Nagel, Karl, 1915, Juglandaceae, in Jongmans, W., ed., Fossilium Catalogus, pars 6, p. 1-87, Berlin.

Nathorst, A. G., 1875, Fossila växter från den stenkolsförande formationen vid Palsjö i Skåne: Geol. Fören. Stockholm Förh., band 2, p. 373-392.

1876, Bidrag till Sveriges fossila flora: K. Svenska vetensk. akad. Handl,, band 14, p. 1-82, pls. 1-16.

1878a, Om floran Skånes Kolförande Bildningar, I, Floran vid Bjuf: Sveriges geol. undersölning, häfte 27, p. 1-52, pls. 1-9.

1878b, Bidrag till Sveriges, fossila flora, II, Floran, vid Hogans och Helsingborg: K. Svenska vetensk. akad. Handl., band 16, p. 1-53, pls. 1-8. 1878c, Beiträge zur fossilen Flora Schwedens-Über einige rhätische

Pflanzen von Palsjö in Schonen: 3 p., 16 pls., Stuttgart. 1886a, Über die Benennung fossiler Dikotylenblätter: Bot. Centralbl., Band 25, p. 52-55.

1886b, Nouvelles observations sur des traces d'animaux et autres phénomènes d'origine purement mécanique decrit comme "Algues fossiles" :

K. Svenska vetensk. akad. Handl., band 21, häfte 14, p. 1-58, pls. 1-5.

1886c, Om floran Skånes Kolförande Bildningar: Sveriges geol. undersökning, ser. C, häfte 85 , p. 85-131, pls. 19-26. 
Nathorst, A. G., 1888, Zur fossilen Flora Japan's : Palaeont. Abh., Band 4, p. 195250 , pls. 1-14.

1890,.Beiträge zur mesozoischen Flora Japan's : K. Akad. Wiss. Denkschr., Band 57, p. 43-60, pls. 1-6, Vienna.

1894, Zur paläozoischen Flora der arktischen zone enthaltend die auf Spitzbergen, auf der Bären-insel und auf Novaja Zemlja von den schwedischen expeditionen entdeckten paläozoischen Pflanzen: K. Svenska vetensk. akad. Handl., band 26, häfte 4, p. 1-80, pls. 1-16.

1897, Zur mesozoischen Flora Spitzbergens: K. Svenska vetensk. akad.

Handl., band 30, häfte 1, p. 1-77, pls. 1-6.

1899, Fossil plants from Franz Josef Land, in the Norwegian North Polar

Expedition 1893-96, Scientific Results, p. 1-26, pls. 1, 2, Christiania.

1902a, Zur oberdevonischen Flora der Bären-insel: K. Svenska vetensk.

akad. Handl., band 36, häfte 3, p. 1-60, pls. 1-14.

1902b, Beiträge Zur Kenntnis einiger mesozoischen Cycadophyten: K.

Svenska vetensk. akad. Handl., band 36 , häfte 4, p. 1-28, pls. 1-3.

1907, Paläobotanische Mitteilungen, 1, 2: K. Svenska vetensk. akad.

Handl., band 42, häfte 5, p. 1-16, pls. 1-3.

1908a, Paläobotanische Mitteilungen, 7, K. Svenska vetensk. akad. Handl.,

band 43 , häfte 8 , p. 1-20, pls. 1-3.

1908b, Paläobotanische Mitteilungen, 3: K. Svenska vetensk. akad.

Handl., band 43, häfte 3, p. 1-12, pl. 1.

- 1908c, Paläobotanische Mitteilungen, 4-6: K. Svenska vetensk. akad.

Handl., band 33, häfte 6, p. 1-32, pls. 1-4.

1909a, Paläobotanische Mitteilungen, 8: K. Svenska vetensk. akad.

Handl., band 45, häfte 4, p. 1-37, pls. 1-8.

1909b, Über die Gattung Nilssonia Brongn. mit besonderer berücksichti-

gung schwedischer Arten: K. Svenska vetensk. akad. Handl., band 43, häfte

12, p. 1-40, pls. 1-8.

1910, Beiträge zur Geologie der Bären-Insel, Spitzbergens und des König-

Karl-Landes: Upsala Univ., Geol. Inst., Bull., v. 10, p. 261-416, pls. 14, 15.

- 1914, Nachträge zur Paläozoischen Flora Spitzbergens-Zur Fossilen

Flora der Polarländer : 110 p., 15 pls., Stockholm.

1915, Zur Devonflora des westlichen Norwegens: Bergens Mus. Aarbok, hefte 9 , p. 1-34, pls. 1-8.

1919, Zwei kleine paläobotanische Notizen: Geol. Fören. Stockholm

Förh., band 41, p. $457-150$.

Naumova, S. N., 1937, Spory i pyltsa uglei SSSR : 17th Internat. Geol. Cong.,

Moscow, Rept., $\nabla .1$, p. 355-366, 1 chart, 1939.

1949, Spory niznego Kembriya [Spores or the Lower Cambrian]: Akad. Nauk SSSR Izv., Ser. geol., no. 4, p. 49-56.

Nehring, Carl Wtlhelm Alfred, 1892, Die Flora des diluvialen Torflagers von

Klinge bei Cottbus: Naturwiss. Wochenschr., Berlin, Band 7, p. 450-457.

Næmejc, Frant, 1928, The Noeggerathiae and Archaeopterides in the Coal Basins of Central Bohemia-Palaeontographica Bohemiae: Ceska Akad. ved a Umeni, Cislo 12, p. 47-82, pls. 1-10, Prague.

1934, On two new sphenopteris fructifications closely allied to Kidston's

Boweria (Resp. Scott's Pteridotheca) with remarks to some of the already

known Palaeozoic fern sporangia: Soc. roy. sci. Bohême Bull. v. 2, p. 1-17. 1937, The Sphenopterides stated in the Permocarboniferous of Central

Bohemia (A preliminary report, Pt. I) : Soc. roy. sci. Bohême Mém., art. 21, p. 1-24.

Neumann, Richard, 1907, Beiträge zur Kenntnis der Kreideformation in Mittel-

Peru : Neues Jahrb, p. 69-130, pls. 1-5. 
Newberry, John S., 1853a, Fossil plants from the Ohio coal basin: Annals Sci., v. 1, p. 106-108, Cleveland.

1853b, New fossil plants from Ohio: Annals Sci., v. 1, p. 116-117, Cleveland.

- 1853c, New species of fossil plants from Ohio: Annals Sci., v. 1, p. 152-153, Cleveland.

- 1873, Descriptions of fossil plants from the Coal Measures of Ohio: Ohio Geol. Survey, v. 1, pt. 2, Palaeontology, sec. 3, p. 359-385, pls. 41-48. 1885, Some peculiar screw-like casts from the sandstones of the Chemung group of New York and Pennsylvania [Spiraxis]: New York Acad. Scl. Trans., v. 3, p. 33-34.

- 1887, The fauna and flora of the Trias of New Jersey and the Connecticut Valley: New York Acad. Sci. Trans., v. 6, p. 124-128.

1888, Fossil fishes and fossil plants of the Triassic rocks of New Jersey and the Connecticut Valley : U. S. Geol. Survey Mon., v. 14, p. 1-95, pls. 1-26. 1895, The flora of the Amboy clays: U. S. Geol. Survey Mon., v. 26, p. 1-137, pls. 1-58.

Newton, E. T., 1875, On "Tasmanite" and Australian "White Coal": Geol. Mag., decade 2 , v. 2, p. 338-342, London.

Nicholson, H. Alleyne, and Etheridge, Robert, 1878, A monograph of the Silurian fossils of the Girvan district in Ayrshire with special reference to those contained in the "Gray Collection": 135 p., 9 pls., Edinburgh and London.

Nilsson, Sven, 1832, Fossila växter funna i Skåne och beskrifne: K. Svenska vetensk. akad. Handl., p. 340-347.

Ogura, Yadzuru, 1927, On the structure and affinities of some fossil tree-ferns from Japan: Tokyo Imp. Univ. Faculty Sci. Jour., sec. 3 Botany, v. 1, p. $351-380$, pls. $2-8$.

-1932a, On the structure of a silicified wood found near "hobashira-ishi" at Najuna near Fukuoka city: Japanese Jour. Botany, v. 6, no. 2, p. 183-190, pl. 4.

$1932 \mathrm{~b}$, On the structure and affinities of some Cretaceous plants from Hokkaido-Second Contribution: Tokyo Imp. Univ., Faculty Sci. Jour., sec. 3, Botany, v. 2, pt. 7, p. 455-483, pls. 22-24.

Oishi, Saburo, 1931a, On Fraxinopsis Weiland and Yabeiella Oishi, nov.: Japanese Jour. Geology and Geography, v. 8, p. 259-267, pl. 26.

- 1931b, A new type of fossil capular organ from the Jido series of Korea : Japanese Jour. Geology and Geography, v. 8, p. 353-356.

- 1939, Notes on some fossil ferns from the Naktong series [Jurassic] of Korea : Hokkaido Imp. Univ., Faculty Sci, Jour., ser. 4, v. 4, p. 308-312, pl. 35.

__ 1950, Illustrated catalogue of East Asiatic fossil plants : 235 p., atlas, 53 pls., Kyoto, Earth Sci. Pub. Soc.

Oishi, Saburo, and Yamasite, Kazuo, 1936, On the fossil Dipteridaceae : Hokkaido Imp. Univ., Faculty Sci. Jour., ser. 4, v. 3, p. 135-184.

Oldham, Thomas, and Morris, John, 1863, The fossil flora of the Rajamal series, Rajmahal Hills, Bengal: India Geol. Surv. Mem., Palaeontologia Indica, ser. 2, pt. 1, p. 1-52.

Oliver, F. W., 1902, On a vascular sporangium from the Stephanian of Grand'Croix: New Phytologist, v. 1, p. 60-67, pl. 1.

1904, On the structure and affinities of Stephanospermum Brongniart, a genus of fossil gymnospermous seeds: Linnean Soc. London Trans., v. 6, 2d ser., p. 361-400, pls. 41-44.

1909, On Physostoma elegans Williamson, an archaic type of seed from the Palaeozoic rocks: Annals Botany, v. 23, p. 73-116, pls. 5-7. 
Oliver, W. R. B., 1928, The flora of the Waipaoa series (Later Pliocene) of New Zealand: New Zealand Inst. Trans. Proc., v. 59, p. 287-303, pls, 44, 45.

1936, The Tertiary flora of the Kaikoria valley, Otago, New Zealand: Royal Soc. New Zealand Trans. Proc., v. 66, p. 284-311.

Ortmann, Karl, 1922, Beitrag zur Kenntnis der tertiären Braunkohlenhölzer Böhmans : Lotos, Band 70, p. 141-181, pls. 5, 6, Prague.

Osborne, F. F., 1931, A possibly biogenic structure in Grenville limestone in Hastings County, Ontario: Canadian Jour. Research, v. 4, p. 570-573, pl. 1.

Otto, Ernst, 1852-54, Additamente zur Flora des Quadergebirges in de Gegend um Dresden und Dippoldiswalde ***: Pt. 1, p. 1-29, 1852 ? ; pt. 2, p. 1-53, 1854.

Pampaloni, Luigi, 1902, I resti organici nel disodile di Melilli in Sicilia: Palaeontographia Italica, v. 8 , p. 121-130, pls. 10, 11.

- 1904, Sopra alcuni ligni silicizzati del Piemonte: Soc. geol. Italiana Boll., v. 22 , p. $535-548$, figs. 1-14.

P'an, C. H., 1937, Notes on Kawasaki and Konno's Rhipidopsis brevicaulis and $R h$. baieroides of Korea with descriptions of similar forms from Yuhsien, Honan: Geol. Soc. China Bull., v. 16, p. 261-269, pls. 1-5.

Parker, W. K. and Jones, T. R., 1860, On the nomenclature of the Foraminifera : Annals and Mag. Nat. History, $3 d$ ser., v. 5, p. 466-477.

Parkinson, James, 1804, Organic remains of a former world: v. 1, 471 p., 9 pls., London.

Paterson, Robert, 1844, Description of Pothocites grantonii, a new fossil veg. etable, from the coal formation: Bot. Soc. Edinburgh Trans., v. 1, p. 45-52. pl. 3.

Patrick, J. Shedden, 1844, On the fossil vegetables of the sandstone of Ayrshire. Annals and Mag. Nat. History, v. 13, p. 283-291.

Pax, Ferdinand, 1906, Beiträge zur fossilen Flora der Karpathen : Bot. Jahrb.. Band 38, p. 272-321, pls. 3, 4 .

Peck, Raymond E., 1934, Late Paleozoic and early Mesozoic Charophyta: Am. Jour. Sci., 5th ser., v. 27, p. 49-55.

1937, Morrison Charophyta from Wyoming: Jour. Paleontology, v. 11. p. $83-90$, pl. 14 .

- 1938, A new family of Charophyta from the Lower Cretaceous of Texas: Jour. Paleontology, v. 12, p. 173-176, pl. 28.

Pelourde, Ferrana, 1908, sur un nouveau type de petiole de fougère fossile: Acad. sci. Paris Comptes Rendus, tome 147, p. 877-879.

Fenhallow, D. P., 1892, Adaitional notes on Devonian plants from Scotland: Canadian Rec. Sci., จ. 5, p. 1-13, pls. 1, 2.

1893a, Notes on Nematophyton crassum: U. S. Natl. Mus. Proc., v. 16, p. 115-118, pls. 15-18.

1893b, Notes on Erian (Devonian) plants from New York and Pennsylvania : U. S. Natl. Mus. Proc., v. 16, p. 105-114, pls. 9-14.

Penny, John S., 1947, Studies on the conifers of the Magothy flora: Am. Jour. Bot., v. 34, p. 281-296.

Peola, Paolo, 1901, Flora del Torgriano di Bognasco: Geol. Centralbl., Band 1, p. 189.

Perkins, George H., 1904, On the lignite or brown coal of Brandon and its fossils :

Vt. State Geologist Rept. 1903-04, p. 153-212, pls. 75-81.

1905, Tertiary lignite of Brandon, Vt., and its fossils : Geol. Soc. America Bull., v. 16, p. 499-516, pls. 85-87.

1906, Fossils of the [Brandon] lignite: Vt. State Geologist Rept., 1905-06, p. $202-230$, pls. $53-58$. 
Peruzzi, D. G., 1881, Osservazioni sui generi Paleodictyon e Paleomeandron dẹi terreni cretacei ed eocenici : Soc. toscana sci. nat. Atti., v. 5, p. 1-8, Pisa.

Peterhans, Emile, 1929, Les algues jurassiques Solenoporella et Pseudochaetetes : Soc. géol. France Bull., sér. $4^{\circ}$, tome 29, p. 2-10, pls. 1, 2.

Petzholdt, Alexander, 1841, De Balano et Calamosyringe, additamenta ad Saxoniae palaeologiam : 34 p., 2 pls., Dresden and Leipzig.

Phillips, John, 1841, Figures and descriptions of the Palaeozoic fossils of Cornwall, Devon, and West Somerset; observed in the course of the Ordnance Geological Survey of that District : 231 p., 40 pls., London.

Phillips, John, and Salter, John William, 1848, Paleontological appendix, in Great Britain Geol. Survey Mem., v. 2, pt. 1, p. 331-386, pls. 1-30.

Pia, Julius, 1912, Neue Studien über die Triadischen Siphoneae Verticillatae: Beitr. Paläontologie Osterr.-Ungarns u. des Orients, Band 25, p. 25-81, pls. 2-8.

1920, Die Siphoneae Verticillatae vom Karbon bis zur Kreide: Zool.-bot. Gesell. Wien Abh., Band 11, p. 1-263, pls. 1-8.

1924, Über einen markwürdigen Landpflanzenreste aus den Nötscher Schichten: Akad. Wiss. Wien Sitzungsber., Band 133, p. 543-558.

1925, Einige neue odor ungenügend bekannte Siphoneae Verticillatae aus dem mitteleuropäischen Malm: Naturh. Mus. Wien Annalen, Band 38, p. 82-88.

1930, Upper Triassic fossils from the Burmo-Siamese frontier-A new Dasycladaceae, Holosporella siamensis nov. gen., nov. sp., with a description of the allied genus Aciculella Pia: Rec. Geol. Survey India, v. 63, p. 177-181, pl. 4.

1937, Die wichtigsten Kalkalgen des Jungspaläozoikums und ihre geologische Bedeutung: Cong. Av. Études Stratigraphie et Géologie Carbonifère, $2^{a}$, Heerlen, 1935, Comptes Rendu, tome 2, p. 765-856.

Picquenard, Ch., 1922, Prodrome de la flora fossile du bassin houiller de Kergogne: Soc. géol. minerolog. Bretagne Bull., tome 2, p. 340-352.

Piedboeuf, J. L., 1887, Über die jungsten Fossilienfunde in de Umgegend von Dusseldorf : Naturw. Ver. Dusseldorf Mitt., Band 1, p. 9-57, pls. 1-3.

Pimenova, N. V. 1929, Materials for the study of Tertiary flora of Korostenschina : Ukrainisches wiss.-forsch. geol. Inst. Abh., Band 3, p. 186-188.

Piton, Louis, 1940, Paléontologie du gisement eocene de Menat (Puÿ-de-Dome) : Thesis, Univ. Clermont, France, $303 \mathrm{p}$.

Platen, Paul, 1908, Untersuchungen fossiler Hölzer aus dem Westen der Vereinigten Staaten von Nordamerika : 55 p., 3 pls., Leipzig.

1909, Die fossilen Wälden am Amethyst Mount im Yellowstone National

Park und die mikroskopische Untersuchung ihrer Hölzer: Prometheus, .v. 20, p. 241-246.

Pocta, Filip, 1889, Über oolithische Gesteine aus der umgebung von Pribram:

K. Böhmische Gesell. Wiss., Prag, Sitzungsber., 1889, p. 421-430.

Pohlig, Hans, 1910, Xylopsaronius-Les première Filicenées caracterisées par la formation du bois: Soc. Belge géologie Bull., tome 24, p. 335-339, Brussels.

Pomel, Auguste, 1846, Note sur le lias de la Moselle et sur quelques gisements de végétaux fossiles : Soc. géol. France Bull., sér. $2^{\circ}$, tome 3, p. 652-655, Paris. 1849, Materiaux pour servir, a la flore fossile des terrains jurassiques de la France: Deutsch. Naturf. Aertzte, Aachen, Amtl. Ber. 25 Vers. p. 332-354.

Post, Tom, and Kuntze, Otto, 1904, Lexicon generum phanerogamarum : xlvii, 714 p., Stuttgart. 
Posthumus, Oene, 1926, Inversicatenales: Fossilium Catalogus, pars 12, 56 p., Berlin.

1931, Catalogue of the fossil remains described as fern stems and petioles, 234 p., Malang, Java.

Potbury, Susan S., 1935, The La Porte flora of Plumas County, Calif. : Carnegie Inst. Washington Pub. 465, p. 31-81, pls. 1-19.

Potonie, Henry, 1890, Über einige Carbonfarne : Preuss. geol. Landesanst. Jahrb., 1889, p. 21-27, pls. 2-5.

- 1891, Note on "einige Pflanzenreste aus dem thüringer Rothliegenden": Deutsche geol. Gesell. Zeitschr., Band 43, p. 978-980.

1893a, Über einige Carbonfarne: Preuss. geol. Landesanst. Jahrb., p. 1-36, pls. 1-4.

1893b, Die Flora des Rothliegenden von Thüringen: Preuss. geol. Landesanst. Abh., Band 9, Teil 2, p. 1-298, pls. 1-24.

1894, Über seine im August 1893 ausgeführte Reise nach den Steinkohlen-

Revieren an der Ruhr, bei Aachen und des Saar-Rhein-Gebietes: Preuss. geol. Landesanst. Jahrb., Band 14, p. xlvi-xlix.

1897-99, Lehrbuch der Pflanzenpalaeontologie : Teile 1, 2, p. 1-208, 1897 ;

Teil 3, p. 209-288, 1898; Teil 4, p. 289-402, 1899, Berlin.

1900, Über die fossilen Filicales in Allgemeinen und die Reste derselben

zweifelhafter Verwandtschaft, in Engler, Adolf, and Prantl, K., Die natürlichen Pflanzenfamilien, I Teil, 4 Abt., p. 743-519, 548-562, 620-621.

- 1902, Cycadoflices, in Engler, Adolf, and Prantl, K., Die natürlichen Pflanzenfamilien, I Teil, 4 Abt., p. 780-798.

- 1903, Über Sphenopteris elegans Brongniart: Naturwiss. Wochenschr., Jena, Band. 19, p. 16.

1906, Abbildungen und Beschreibungen fossiler Pflanzen-Reste, Lief IV, Nrs. 61-80 : Preuss. geol. Landesanst.

1910, Abbildungen und Beschreibungen fossiler Pflanzen-Reste, Lief VII, Nrs. 121-140 : Preuss. geol. Landesanst.

Potonie, Henry, and Bernard, C., 1903, Flore devonienne de l'étage $\mathbf{H}$ de Barrande: Supp. to Système silurien du centre de la Bohême by J. Barrande, 68 p., Prague.

Potonie, Henry, and Gothan, W., 1909, Palaeoweichselia, in Potonie, Henry, Abbildungen und Beschreibungen fossiler Pflanzen-Reste, Lief 6, Nr. 116, p.

1-4, Preuss. geol. Landensalt.

Potonie, Robert, 1931a, Zur mikroskopie der Braunkohlen : Braunkohle; Band 30,

p. 554-556.

1031b, Zur milkroskopio dor Braunkohlen_Tertiäre Blütenstaubformen: Braunkohle, Band 30, p. 325-333.

1932, Sporenformen aus den flözen Ägir und Bismarck des Ruhrgebietes: Neues Jahrb., Band 67, Abt. B, p. 438-454, pls. 14-20.

1934, Zur Mikrobotanik der Kohlen und ihrer Verwandten-Zur Morphologie aer rossilen Pollen und sporen: Preuss. geol. Landesanst. Inst. Paläobotanik u. Petrographic Brennsteine Arb., Band 4, p. 5-126.

Potonle, Fobert, and Gelletich, J., 1933, Über Pteridophyten-Sporen einer eocïnen Braunkohle aus Dorog in Ungarn: Gesell. naturf. Freunde Berlin Sitzungsber., Jahrg. 1932, Heft 3, p. 517-528.

Potonie, Robert, Thomson, Paul W., and Thiergart, Friedrich, 1950, Zur Nomenklatur und Klassifikation der neogenen Sporomorphae (Pollen und Sporen) : Geol. Jahrb., Hannover, Band 65, p. 35-70. 
Potonie, Robert, and Venitz, H., 1934, Zur Mikrobotanik des Miozänen Humodils der Niederrheinischen Bucht: Preuss. geol. Landesanst. Inst. Paläobotanik u. Petrographic Brennsteine Arb., Band 5, p. 5-54.

Presl, Corolo B., 1847, Supplementum Tentaminis Pteridographiae continens genera et species ordinum dictorum Marattiaceae, Ophioglossaceae, Osmundaceae * * * K. Böhmische Gesell. Wiss., Prag, Abh., Band 4, p. 261-379.

Principi, Paolo, 1921a, Nuovo contributo allo studio Tallofite, Pteridofite, Gimnosperme e Monocotiledoni fossili del giacimento oligocenico di Santa Guistina Sassello in Liguria: Servire Descrizione Carta Geologica Italia Mem. v. 7, p. 1-87, pls. 1-7..

1921b, Synopsis della flora oligocenica di Salcedo e Chiavon : Soc. ligustica, Sci. nat. geog. Atti, v. 31, p. 73-106, Genova.

1926, La flore oligocenica di Chiavon e Salcedo: Servire Descrizione Carta Geologica Italia Mem., v. 10, p. 5-131, pls. 1-11.

Quenstedt, Fredrich August, 1867, Handbuch der Petrefaktenkunde-Die fossilen Pflanzen, 2d ed., p. 838-923, pls. 80-86, Tübingen.

Raciborski, Maryjan, 1889, O obecnym stanie mych Badań flory kopalnej ogniotrwalych glinek krakowskich: Akad. umiejet. Kraków, Kom. flzyjog., Sprawozdania tom 23, częsć 2, p. 129-140.

1890, Über die Osmundaceen und Schizaeaceen der Juraformation: Bot. Jahrb., Band 13, p. 1-8, pl. 1.

1891a, Beiträge zur Kenntnis der rhaetischen Flora Polens: Internat. Acad. Sci. Bull. 10, p. 375-379, Cracow.

— 1891b, Flora retycka Pólnocnego stoku gór swietokrzyskich [Über die rhätische Flora am Nordabhange des polnischen Mittelgebirges]: Akad. umiejet. Wydzial Matematyczno-Przyrod., Rozprawy, ser. 2, tom 3, p. 292326, Cracow.

— 1894, Flora kopalna ogniotrwałych glinek Krakowshich : Akad. umiejet. Wydzial Matematyczno-Przyrod., Pam. 18, p. 141-243, pls. 6-27, Cracow.

1909, Über eine fossile Pangiumart aus dem Miozän Java's: Internat. Acad. Sci. Bull., February, p. 280-284, Cracow.

Radforth, N. W., 1938, An analysis and comparison of the structural features of Dactylotheca plumosa Artis sp. and Senftenbergia ophiodermatica Goeppert sp.: Royal Soc. Edinburgh Trans., v. 59, p. 385-396, pls. 1, 2.

- 1939, Further contributions to our knowledge of the fossil Schizaeaceae; genus Senftenbergia: Royal Soc. Edinburgh Trans., v. 59, p. 745-761, pl. 1.

Raineri, Rita, 1922, Alghe sifonee fossili della Libia : Soc. Italiana sci. nat. Atti, v. 61, p. 72-86, pl. 3 .

Ranzani, Camillo, 1839, De tribus vegetabilibus fossilibus: Novi commentaril acad. sci. inst. Bononiensis, Bonon., v. 3, p. 383-394, pl. 35, Bologna.

Rao, A. R., 1943a, Nipaniostrobus, a new genus of Dacrydium-like seed-bearing cones, and other silicifled plants from the Rajmahal Series: Nat. Acad. Scl. India Proc., v. 13, p. 113-133, pls. 1-6.

- 1943b, Jurassic spores and sporangia from the Rajmahal Hills, Bihar: Nat. Acad. Sci. India Proc., v. 13, p. 181-197, pls. 1-4.

— 1947, Nipanioruha granthia gen. et sp. nov., a new petrifled coniferous shoot from the Rajmahal Hills, Bihar: Indian Bot. Soc. Jour., M. O. P. Iyengar. Commerative V., 1946, p. 389-397, pls. 1-6.

Rao, A. R., and Pia, Julius, 1936, Fossil algae from the uppermost Cretaceous beds (The Niniyur Group) of the Trichinopoly district, S. India: Geol. Survey India Mem., new ser., v. 21, Mem. 4, p. 1-49, pls. 1-6. 
Rasky, Klara, 1945, Fossile Charophyten-Fruchte: Naturwiss. Mon. 2, Naturw. Mus., Budapest, 74 p., 3 pls.

Read, Charles B., 1934, A flora of Pottsville age from the Mosquito Range, Colo.: U. S. Geol. Survey Prof. Paper 185-D, p. 79-91, pls. 16-18.

1936a, The flora of the New Albany shale, Part 1, Diichnia kentuckiensis, a new representative of the Calamopityeae: U. S. Geol. Survey Prof. Paper 185-H, p. 149-155, pls. 30-33.

- 1936b, A Devonian flora from Kentucky: Jour. Paleontology, v. 10, p. 213-227, pls. 28-29.

1938, Some Psilophytales from the Hamilton group in western New York: Torrey Bot. Club Bull., v. 65, p. 599-606.

1939, The evolution of habit in Tempskya: Lloydia, v. 2, p. 63-72.

1946, A Pennsylvanian florule from the Forkston coal in the Dutch Mountain outlier, northeastern Pennsylvania: U. S. Geol. Survey Prof. Paper 210-B, p. 17-28, pls, 1, 2.

Read, Charles B., and Brown, Roland W., 1937, American Cretaceous ferns of the genus T'empskya: U. S. Geol. Survey Prof. Paper 186-F, p. 105-129, pls. $28-43$.

Reagan, Albert B., 1932, Some geological notes of the Upper Cretaceous of Black Mesa, Ariz.: Kans. Acad. Sci. Trans., v. 35, p. 232-252.

Reed, Clyde F., 1947, The phylogeny and ontogeny of the pteropsida : Soc. Broteriana Bol., v. 21, p. 71-197, pls. 1-5.

Reed, Fredda D., 1946, On Cardiocarpon and some associated plant fragments from Iowa coal fields : Bot. Gazette, v. 108, p. 51-64.

Rege, Rosina, 1921, Note su alcuni del carbonifero della Cina: Soc. Italiana sci. nat. Atti, v. 59, p. 183-196.

Reichenbach, H. G. Ludwig, 1836, Ein Leitfaden bei Beschaung der Schatze desselben": 64 p., Leipzig.

Reid, Clement, 1898, On Limnocarpus, a new genus of fossil plants from the Tertiary deposits of Hampshire: Linnean Soc. London Jour., v. 33, p. 464-466.

Reid, Clement, and Groves, J., 1916, Preliminary report on the Purbeck Characeae : Royal Soc. London Proc., ser. B, v. 89, p. 252-256, pl. 8.

Reid, Clement, and Reid, Eleanor M., 1910, The lignite of Bovey Tracey : Royal Soc. London Philos. Trans., v. B201, p. 161-178, pls. 15, 16.

1915, The Pliocene floras of the Dutch-Prussian border: Netherlands, Rijksopsporing van Delfstoffen Meded. 6, 177 p., 20 pls., Hague.

Reid, Eleanor M., 1920, Recherches sur quelques graines pliocenes du Pont-de Gail (Cantal) : Sọc. géol. France Bull., sér. $4^{\circ}$, tome 20, p. 48-87, pls. 3, 4.

1924, Nouvelle recherches sur les graines du Pliocene inférieur du Pont-deGail (Cantal) : Soc. géol. France Bull., sér. $4^{\circ}$, tome 23, p. 308-354, pls. 10, 11.

1930, Tertiary fruits and seeds from Saint Tudy: Soc. géol. minérolog. Bretagne Bull., tome 8, p. 36-62, pls. 1-3.

1933, Note on some fossil fruits of Tertiary age from Colombia, South Americà : Rev. géographie physique et géologie dynamique, tome 6, fasc. 3, Paris. p. 209-214, p1. 14.

Reid, Eleanor M., Chandler, Marjorie E. J., and Groves, James, 1926, Catalogue of Cainozoic plants in the department of Geology, British Museum Natural History-The Bembridge Flora : 206 p., 11 pls., London.

Reinsch, Paulus Friedrich, 1880, Entdeckung neuer pflanzlicher Gebilde in der Steinkohle und im Anthrazit: Bot. Centralbl., Bände 1-2, I Gratis-Beil, Heft 2, p. 1-18, pls. 1, 2.

1881, Neue Untersuchungen über die Mikrostruktur der Steinkohl des Carbon, der Dyas and 'Trias: ' 124 p., 56 pls., Leipzig. 
Reinsch, Paulus Friedrich, 1884, Micro-palaeophytologia formationis carboniferae : v. 1, p. 1-79, pls. 1-66; v. 2, p. 1-54, pls. 67-95, Erlangen and London.

Renault, Bernard, 1869, títude de quelques végétaux silicifiés des environs d'Autun: Annales sci. nat., Botanique, sér. 5, tome 12, p. 161-190, pls. '3, 14, Paris.

— 1872, Végétaux silicifiés d'Autun-Observations sur la structurè du Dictyoxylon: Acad. sci. Paris Comptes Rendus, tome 74, p. 1295-1298.

— 1874, Recherches sur les végétaux silicifies d'Autun-Etude du genre Myelopteris: Acad. sci. Paris Comptes Rendus, tome 78, p. 257-260.

___ 1875a, Recherches sur les végétaux silicifiés d'Autun et de Saint-ÉtienneEtude du genre Botryopteris: Acad. sci. Paris Comptes Rendus, tome 80, p. 202-206.

-1875b, Recherches sur les végétaux silicifiés d'Autun et de Saint-ÉtienneÉtude du genre Botryopteris: Annales sci. nat., Botanique, sér. $5^{\mathrm{e}}$ tome 1, p. 220-240, Paris.

1875c, Recherches sur les végétaux silicifiés d'Autun-fitude du genre Myelopteris: Acad. sci. Paris Mém., tome 22, no. 10, p. 1-28, pls. 1-6.

- 1879, Structure comparée de quelques tiges de la flore carbonifère: Mus. histoire nat. Paris, Nouv. Archives, tome 2, p. 213-348, pls. 10-17.

— 1881, Cours de botanique fossile : tome 1, 184 p., pls. A, 1-20, Paris.

1882, Cours de botanique fossile: tome 2, 194 p., pls. 1-24, Paris.

1883a, Cours de botanique fossile: tome 3, 241 p., pls. A, 1-35, Paris. 1883b, Deuxième note pour servir a l'histoire de la formation de la houille: Acad. sci. Paris Comptes Rendus, tome 97, p. 1019-1021.

1885, Cours de botanique fossile: tome 4, 232 p., pls. A-D, 1-21, Paris. 1890, Sur une nouvelle Lycopodiacée houillere [Lycopodiopsis derbyi]:

Acad. sci. Paris Comptes Rendus, tome 110, p. 809-811.

- 1891, Communication: Soc. histoire nat. Autun Bull., tome 4, p. 498-501. 1892a, Sur un nouveau genre de tige permo-carbonifère, le G. Retinodendron rigolloti: Acad. sci. Paris Comptes Rendus, tome 115, p. 339-341.

$1892 \mathrm{~b}$, Note sur un nouveau genre de gymnosperme fossile: Soc. histoire nat. Autun Bul., tome 5, p. 152-157, pl. 5.

- 1893, Bassin houiller et permien d'Autun et d'Epinac, flore fossile, pt. 2, atlas, pls. 28-89. See Renault, B. 1896a.

1894, Sur quelques nouveaux parasites des Lépidodendrons: Soc. histoire nat. Autun, Procès Verbaux des Seances 1893, p. 168-178.

1895a, Sur quelques Micrococcus du Stephanien, terrain houiller superieur: Acad. sci. Paris Comptes Rendus, tome 120, p. 217-220.

1895b, Sur quelques bacteries des temps primaires: Soc. histoire nat. Autun Bull., tome 7, p. 433-468.

- 1895c, Chytridinées fossiles du Dinantien [Cilm] : Rev. mycologique, tome 17, p. 158-161, pls. 154-155, Toulouse.

- 1895d, Parasites des écorces de Lépidodendrons: Le Naturaliste, sér. $2^{\mathrm{e}}$, tome 9 , p. 77-78.

1896a, Bassin houiller et permien d'Autun et d'Epinac, flore fossile, pt. 1, text, 578 p.: Études des gites minéraux de la France, Fasc. IV ; Atlas, 1893, Paris.

$1896 \mathrm{~b}$, Sur le genre Metacordaite: Soc. histoire nat. Autun Bull., tome 9, p. 90-104, figs. 1-10.

1898, Sur les organismes des Cannels: Mus. histoire nat. Paris Bull. 4, p. 204-209, figs. 1-6.

1899, Sur quelques micro-organisms des combustibles fossiles: Soc. industrie min. St.-Ćtienne Bull., tome 13, p. 865-1161. 
Renault, Bernard, 1900, Sur un nouveau genre de tige fossile: Soc. histoire nat. Autun Bull., tome 13, p. 405-424, pls. 1-10.

1901a, Sur quelques fougéres heterosporées: Soc. histoire nat. Autun, Comptes Rendus, tome 14, p. 207-209.

1901b, Sur quelques cryptogames heterosporées: Soc. histoire nat. Autun, Comptes Rendus, tome 14, p. 339-352.

- 1902, Note sur quelques micro et macrospores fossiles: Soc. histoire nat. Autun Bull., tome 15, p. 97-115, pls. 6-12.

Renault, Bernard, and Bertrand, C. E., 1885, Grilletia spherospermii, Chytridacée fossile du terrain houiller supérieur : Acad. sci. Paris Comptes Rendus, tome 100, p. 1306-1308.

Renault, Bernard, and Roche, A., 1898, Étude sur la constitution des lignites et les organisms qu'ils renferment: Soc. histoire nat. Autun Bull., tome 11, p. 201-239, pls. 11-13.

Renault, Bernard, and Zeiller, R., 1884a, Sur un nouveau genre graines du terrain houiller supérieur: Acad. sci. Paris Comptes Rendus, tome 99, p. 56-58.

$1884 \mathrm{~b}$, Sur un nouveau genre de fossiles végetaux: Acad. sci. Paris Comptes Rendus, tome 98, p. 1391-1394.

- 1885, Sur un nouveau type de Cordaitée: Acad. sci. Paris Comptes Rendus, tome 100 , p. $867-869$.

- 1888, Etudes sur le terrain houiller de Commentry-Flore fossile, Pt. 1 : Soc. industrie min. St.-Étienne Bull., tome 2, 366 p., 42 pls.

1890, Etudes sur le terrain houiller de Commentry-Flore fossile, Pt. 2 : Soc. industrie min. St.-Etienne Bull., tome 4, p. 369-742; Atlas, pls. 43--75.

Renger, Karel, 1866, Předvěké rostlinstro křidorého ritvaru đeského: Ziva, p. 113-141, pl. 1, Prague.

Renier, Armand, 1931, Contribution a l'étude de la bordure méridionale du bassin houiller de Charleroi et de la Basse-Sambre: Soc. Belge géol. Bull., tome 41, p. 268-338, pls. 4, 5 .

Renner, C. W. O., 1907, Teichosperma, eine Monokotylenfrucht aus dem Tertiär ägyptens : Beitr. Osterr. Ungarns Orients, Vienna, Band 20, p. 217-220.

Resi, Otto M., 1921, Erläuterungen zu dem Blatte Donnersberg der geognostischen Karte von Bayern : 320 p.

- 1923, Kalkalgen und Seesinterkalke aus dem rheinpfalzischen Tertiär: Geognostichen Jahresber., Munich, Band 36, p. 103-130, pls. 3-5.

Reuss, August Emil, 1845-46, Die Versteinerungen der böhmischen Kreideformation, Teil 1, p. 1-58, pls. 1-13, 1845; Teil 2, p. 1-80, pls. 14-45, Stuttgart.

1848, Die fossilen Polyparien des Wiener Tertiärbeckens: Haidinger's Naturwiss. Abh., Band 2, Teil 1, p. 1-109, pls. 1-11, Vienna.

ـ_ 1854, Beiträge zur geognostischen Kenntniss Mährens: Geol. Reichsanst. Jahrb., Band 5, p. 660-765, Wien.

Richon, C., 1885, Notice sur quelques Spheriacées nouvelles: Soc. bot. France Bull., tome 32, supp., p. viii-xii.

Rlchter, P. B., 1899, Über Neocompflanzen der Kelb'schen Sandgrube bei Quedlinburg : Deutsche geol. Gesell. Zeitschr., Verh., Band 51, p. 39-41.

—_ 1904, Über die Kreidepflanzen der Umgebung Quadlinburgs, Teil 1: Beilage zum Programm des konigl-Gymnasiums zu Quedlinburg, p. 3-20. pls. $1,2$.

1905, Über die Kreidepflanzen der Umgebung Quedlinburgs, Teil 2: Beilage zum Programm des konigl-Gymnasiums zu Quedlinburg, p 1-19, pls. 1-4. 
Richter, J. B.. 1906-09, Beiträge zur flora der unteren Kreide Quedlinburgs, Teil 1, Die Gattung Hausmannia, p. 1-27, pls. 1-7, 1906; Teil 2, Die Gattung Nathorstiana, p. 1-12, pls. 8-13, 1909.

Rode, K. P., 1933, A note on fossil angiospermous fruits from the Deccan Intertrappean beds of Central Provinces : Current Sci., v. 11, p. 171-172.

Roehl, E., 1869, Fossile Flora der Steinkohlen Formation Westphalens : 191 p., 31 pls., Cassel.

Roemer, Ferdinand, 1852, Die Kreidebildungen von Texas und ihre organischen Einschlüsse, 100 p., 11 pls., Bonn.

1880, Lethaea geognostica oder Beschreibung und Abbildung der fiir die Gebirges Formation bezeichnendsten Versteinerungen, Teil 1, Lethaea Palteozoica: 324 p., Stuttgart.

1885, Lethaea erratica oder Aufzählung und Beschreibung der in der norddeutschen Ebene vorkommenden Diluvial-Geschiebe nordischer Sedimantär-Gesteine: Palaeont. Abh., Heft 5, p. 1-173, pls. 24-34, Berlin.

Roemer, Friedrich Adolph, 1860, Beitrïge zur geologischen Kenntniss des nordwestlichen, Harzgebirges: Palaeontographica, Band 9, Teil 4, p. 1-46, pls. 1-12.

Rogers, Henry Darwin, 1858, The geology of Pennsylvania, a government survey, v. 2 : 1045 p., Philadelphia.

Romanowski [Romanovskij], Gennadi Danilovich, 1890, Materialien zur Geologie von Turkestan, Teil III [Historical geology of a paleontological character of the sedimentary formations of western Thian-Schan and of the valleys of Turan], 165 p., 21 pls., St. Petersburg.

Rossmassler, E. A., 1840, Beiträge zur Versteinerungskunde, Erste Heft, Die Versteinerungen des Braunkohlensansteins aus der Gegend von Altsattel in Böhmen : 142 p., 12 pls., Dresden and Leipzig.

Rothpletz, August, 1880, Die Flora und Fauna der Culformation bei Hainichen in Sachsen : Bot. Centalbl., Bände 1-2, p. 1-40, pls. 1-3.

1890, Über Sphaerocodium Bornemanni, eine neue fossile Kalkalge aus den Raibter Schichten der Ostalpen: Bot. Centralbl., Band 41, p. 9.

1891, Fossile Kalkalgen aus den Familien der Codiaceen und der Corallineen: Deutsche geol. Gesell. Zeitschr., Band 43, p. 295-321, pls. 15-17.

1896, Über Flysch-Fucoiden und einige andere fossile Algen, sowie über liasische, Diatomeen führende Hornschwämme: Deutsche geol. Gesell. Zeitschr., Band 48, p. 854-914, pls. 22-24.

1908, Über Algen und Hydrozoen in Silur von Gotland und Oesel: K. Svenska vetensk.-akad. Handl., band 43, häfte 5, p. 1-25, pls. 1-6.

1913, Über die Kalkalgen, Spongiostromen und einige andere fossilien aus dem Obersilur Gottlands: Sveriges geol. undersökning Afh., ser. C, häfte 10, p. 1-57, pls. 1-10.

Rouault, Marie, 1850, Note preliminaire sur une nouvelle formation decouverte dans le terrain silurien inférieur de la Bretagne: Soc. géol. France Bull., tome 7, p. 724-744.

Royle, J. Forbes, 1833-40, Illustrations of the botany and other branches of natural history of the Himalayan Mountains and of the flora of Cashmere: lxxviii, 472 p., 96 pls., London.

Ruedemann, Rudolf, 1909, Some marine algae from the Trenton limestone of New York : N. Y. State Mus. Bull. 133, no. 453, p. 194-210, pls. 1-3.

1912, The Lower Siluric shales of the Mohawk Valley: N. Y. State Mus. Bull. 162, p. 1-123, pls. 1-10.

1925, The Utica and Lorraine formations of New York, Part 2, Systematic paleontology : N. Y. State Mus. Bull., no. 262, p. 1-140, pls. 1-13. 
Ruedemann, Rudolf, 1931, Some new Middle Cambrian fossils from British .... Columbia :.U. S. Natl. Mus. Proc., v. 79, no. 2893, p. 1-18, pls. 1-7.

Ruprecht, F. J., 1866, Über eine mikroskopische Süsswasser Alge, als Bestandtheil gewisser Mergel des Gouvernements Wjatka : Acad. imp. sci. St. Petersburg Bull., v. 9, 35-43.

Ryba, Franz, 1904, Beiträge zur Kenntniss des Cannelkohlenflotzes bei Myran: K.-k. Geol. Reichsanst. Jahrb., Band 53, p. 351-372, pls. 15-17, Vienna.

Saccardo, P. A., 1892. See Meschinelli, Aloysius, 1892.

Sacco, Federico, 1888, Note di paleoicnologia Italiana: Soc. Italiana sci. nat. Atti, v. 31, p. 151-190, pls. 1, 2.

Sadebeck, Alexander, 1886, [Fossil plants] : Bot. Centralbl., Band 25, p. 121-123. Sahni, Birbal, 1919, On an Australian specimen of Clepsydropsis : Annals Botany, v. 33, p. 81-92, pl. 4.

1920, Petrified plant remains from the Queensland Mesozoic and Tertiary formations: Queensland Geol. Survey Pub. 267, p. 7-38, pls. 1-5.

-_ 1928, On Clepsydropsis australis, a zygopterid tree-fern with a Tempskyalike false stem, from the Carboniferous rocks of Australia: Royal Soc. London Philos. Trans., v. 217B, p. 1-37, pls. 1-6.

- 1930, On Asterochlaenopsis, a new genus of Zygopterid tree ferns from western Siberia : Royal Soc. London Philos. Trans., v. 218b, p. 447-470, pls. 49-51.

1931, Revisions of Indian fossil plants, Part II, Coniferales : Palaeontologia Indica, v. 11, p. 51-124, pls. 7-15.

- 1932a, Homoxylon rajmahalense, gen. et sp. nov., a fossil angiospermous wood devoid of vessels from the Rajmahal hills, Behar: Palaeontologia Indica, v. 20, Mem. 2, p. 1-19, pls. 1, 2.

1932b, On the genera Clepsydropsis and Cladoxylon of Unger, and on a new genus Austroclepsis:. New Phytologist, v. 31, p. 270-278.

-1932c, On the structure of Zygopteris primaria (Cotta), and on the relations between the genera Zygopteris, Etapteris, and Botrychioxylon: Royal Soc. London Philos. Trans., v. 222B, p. 29-45, pls. 6-8.

— 1932d, A petrified Williamsonia from the Rajmahal Hills, India: Palaeontologia Indica, new ser., v. 20, Mem. 3, p. 1-19, pls. 1-3.

- 1933, A fossil pentalocular fruit from Pondicherry, South India: India Geol. Survey Rec., v. 66, p. 430-437, pl. 25.

1934, The silicified flora of the Deccan Intertrappean series, Part II,

Gymnospermous and angiospermous fruits: Indian Sci. Cong., 21st. Bombay, Proc. p. 317-318.

1938, Recent advances in Indian palaeobotany : Lucknow Univ. Studies, Faculty Sci., no. 2; 102 p.

- 1940, The Deccan Traps-An episode of the Tertiary Era: Indian Sci. Cong., 27th, Madras, Proc., pt. 2, p. 3-20, pls. 1-3.

1943, Indian silicified plants, 2, Enigmocarpon parijai, a silicified fruit

from the Deccan, with a review of the fossil history of the Lythraceae : Indian Acad. Sci. Proc., v. 17, p. 59-96, pls. 2-6.

1944, Genus Viracarpon Sahni : Natl. Acad. Sci. India Proc., v. 14, sec. B, p. $80-82$, p1. 3 .

1948, The Pentoxyleae - A new groun of Jurassic gymnosperms from the Rajmahal hills of India : Bot. Gazette, v. 110, p. 47-80.

Sahni, Birbal, and Rao, A. R., 1931, On some Jurassic plants from the Rajmahal hills: Asiatic Soc. Bengal Jour. and Proc., v. 27, p. 183-208, pls. 11-16.

1933, On some Jurassic plants from the Rajmahal hills: Asiatic Soc.

Bengal Jour. and Proc., 1931, v. 27, p. 183-208, pls. 11-16. 
Sahni, Birbal, and Rao, A. R., 1934, Rajmahalia paradoxa gen. et. sp. nov. and other Jurassic plants from the Rajmahal hills: Indian Acad. Sci. Proc., v. 1, p. $258-269$, pls. $35-37$.

- 1935, Further observations on Rajmahalia paradoxa: Indian Acad. Sci. Proc., v. 1, p. 710-713, pls. 67, 68.

Sahni, Birbal, and Rao, H. S., 1943, A silicified flora from the Intertrappean cherts round Sausar in the Deccan: Natl. Acad. Sci. India Proc., v. 13, p. $36-75$, pls. $1-8$.

Sahni, Birbal, and Rode, K. P., 1937, Fossil plants from the Intertrappean beds of Mohgaon Kalan, in the Deccan, with a sketch of the geology of the Chhindwāra district: Natl. Acad. Sci. India Proc., v. 7, p. 165-174.

Sahni, Birbal, and Sitholey, R. V., 1943, Paleobotany in India IV-Progress report for 1942 : Indian Bot. Soc. Jour., v. 22, nos. 2-4, p. 171-182, pls. 6-9.

Sahni, Birbal, and Srivastava, B. P., 1934, The silicified flora of the Deccan Intertrappean series, Part III, Sausarospermum fermori gen. et. sp. nov.: Indian Sci. Cong. 21st., Bombay, Proc. p. 318.

Sahni, Birbal, and Surange, K. R., 1944, On the structure and affinities of Palmoxylon sahnii Rode: Natl. Acad. Sci. India Proc., v. 14, sec. B, p. 83-85.

Salfeld, Hans, 1908, Ein neues fossiles Frankraut aus dem Solnhofer lithographischen Schiefer: Centralbl. Mineralogie, 1908, no. 13, p. 385-386.

- 1909, Beitrïge zur: Kenntnis jurassischer Pflanzenreste aus Norddeutschland : Palaeontographica, Band 56, p. 1-34, pls. 1-6.

Salmon, Ernest S., 1903, Cercosporites sp. a new fossil fungus: Jour. Botany, British and Foreign, v. 41, p. 127-130, London.

Salter, J. W., 1860, On the fossils from the High Andes collected by David Forbes: Geol. Soc. London Quart. Jour., v. 17, p. 62-72, pls. 1-5.

- 1.861, Note on the fossils found in the Worcester and Hereford Railway cuttings : Geol. Soc. London Quart. Jour., v. 17, p. 161-162.

_ـ 1864, On some points in ancient physical geography illustrated by fossils from a pebble bed at Budleigh Saiterton, Devonshire: Geol. Mag., v. 1, p. 5-12, London.

Salter, J. W., and Etheridge, Robert, 1880, On the fossils of North Wales: Geol. Survey Great Britain Mem., v. 3, p. 331-611, pls. 1-26.

Sandberger, Fridolin, 1849, Tertiär Bildungen Eisenerze fossile Pflanzen im Westerwald : Neues Jahrb., p. 447-448.

- 1866, Pflanzen aus dem Rothliegenden des badischen Schwarzwaldes: Wurzburg. Naturw: Zeitschr. Band 6, p. 74-77.

Sang, John, 1885, Fossil algae at Kirkcaldy: Sci. Gossip, v. 21, p. 213.

Saporta, Gaston, 1862, Etudes sur la végétation du sud-est de la France a l'époque tertiaire: Annales sci. nat., Botanique, sér. $4^{\circ}$, tome 71, p. 191-311, pls. 1-14.

_- 1863, tudes sur la végétation du sud-est de la France a l'époque tertiaire : Annales sci. nat., Botanique, sér. $4^{\circ}$, tome 19, p. 6-124, pls. 1-11.

1865, La végétation du sud-est de la France a l'époque tertiaire: Annales sci. nat., Botanique, sér. $5^{\circ}$, tome 3, p. 1-152, pls. 1-8.

1867, lítudes sur la végétation du sud-est de la France a l'époque tertiaire: Annales sci. nat., Botanique, sér. $5^{\circ}$, tome 8, p. 1-136, pls. 1-15.

1868, Prodrome d'une flore fossile des travertines anciens de Sézanne: Soc. géol. France Mém., sêr. 2, tome 8, p. 289-436, pls. 1-15.

1872a-73, Paléontologie française ou description des fossiles de la France, plantes jurassiques: tome 1 ; Algues, equisetacées, characées, fougères, $\mathbf{p}$. $1-432,1872$; p. 433-506, 1873; Atlas, pls. 1-60, 1872 ; pls. 61-70, 1873, Paris. 
Saporta, Gaston, 1872b, Sur une determination plus precise de certains genres de conifères jurassiques par l'observation de leurs fruits: Acad. sci. Paris Comptes Rendus, tome 74, p. 1053-1056.

_ 1873a, Rtudes sur la végétation du sud-est de la France a l'époque tertiaire: Annales sci. nat., Botanique, sér. $5^{\circ}$ tome 18, p. 23-146, pls. 1-18. 1873b, Notice sur les plantes fossiles du niveau des lits a Poissons de Cerin : Annales soc. agriculture et histoire nat., tome 5, p. 87-236, pls. 14-15, Lyon and Paris.

- 1873c-75, Paléontologie française ou description des fossiles de la France, plantes jurassiques-tome 2, Cycadées, p. 1-222, pls. 1-26 [71-96], 1873; p. $223-288$, pls. $27-48$ [97-118], 1874 ; p. $289-352$, pls. $49-58$ [119-128], 1875; Paris.

1875a, Sur la decouverte de deux types nouveaux de conifères dans les schistes permiens de Lodeve (Herault) : Acad sci. Paris Comptes Rendus, tome 80, p. 1017-1022.

1876-84, Paléontologie française ou description de fossiles de la France, plantes jurassiques-tome 3 , Conifères ou aciculariées, 672 p., 97 pls. [129-226].

1878a, Observations sur la nature des végétaux reunis dans le groupe des Noeggerathia; type des Noeggerathia expansa et cuneifolia de Brongniart: Acad. sci. Paris Comptes Rendus, tome 86, p. 864-873.

$1878 \mathrm{~b}$, Sur une nourelle découverte de plantes terrestres siluriennes dans les schistes ardoisiers d'Angers, due à M. L. Crie: Acad. sci. Paris Comptes Rendus, tome 87, p. 767-771.

1879, Le monde des plantes avant l'apparition de l'homme: 146 p., Paris. 1881, Súr la presence supposee des Proteacées d'Australie dans la flore de l'Europe ancienne : Acad. sci. Paris Comptes Rendus, tome 92, p. 1130-1133. 1882, A propos des algues fossiles: 80 p., pls. 1-9, Paris.

1884, Les organisms problematiques des anciennes mers : 102 p., Paris.

1885, Sur un type végétal nouveau provenant du corallien d'Auxey (Côte-d'Or) : Acad. sci. Paris Comptes Rendus, tome 100, p. 1440-1443.

1886-91, Paleontologie française ou description des fossiles de la France, plantes jurassiques-tome 4, Types Proangiospermiques et supplément final : 548 p., 74 pls. [227-300].

- 1887, Nouveaux documents delatifs aux organismes problematiques des anciennes mers : Soc. géol. France Bull., sér. $3^{\mathrm{e}}$, tome 15, p. 286-302, pls. 3-7. 1888, Dernieres adjonctions à la flore fossile d'Aix-en-Provence: Annales sci. nat., Botanique, sér. $7^{\circ}$, tome 7, p. 1-104, pls. 1-10.

- 1889, Dernieres adjonctions a la flore fossile d'Aix-en-Provence: Annales sci. nat., Botanique, sér. $7^{\mathrm{e}}$, tome 10, p. 1-192, pls. 1-20.

1890, Le Nelumbium provinciale: Soc. géol. France Mém. Paléontologie, Mém. 5, p. 1-10, pls. 12-14.

1893a, Sur les rapports de l'ancienne flore avec celle de la region Provencale actualle: Soc. bot. France Bull., sér. $2^{\mathrm{e}}$, tome 40, sess. extraordinaire, 1893, 1st pt., p. $x$-xxxvi, pls. $1,2$.

- $1893 \mathrm{~b}$, Revue des traveaux de paléontologie végétale : Rer. gén. botanique, tome 5, p. 119-125.

1894, Flore fossile du Portugal : 288 p., 39 pls., Lisbon.

Saporta, Gaston, and Marion, A. F., 1873, Essai sur l'état de la végétation a l'époque des Marnes Heersiennes de Gelinden: Acad. royale Belgique Mém. cour. et sav. étrang., tome 37 , no. 6, p. 1-94, pls. 1-12, Brussels.

1878, Revision de la flore Heersienne de Gelinden: Acad. royale Belgique Mém. cour. et sav. etrang., tome 41 , no. 3, p. 1-112, pls. 1-14, Brussels. 
Saporta, Gaston, and Marion, A. F., 1881, L'évolution du règne végétal-Les Oryptogames, 238 p., Paris.

-_ 1885, L'évolution du règne végétal-Les Phanerogames: tome 1, p. 1-249; tome 2, p. 1-247, Paris.

Sauveur, J. J. D., 1848, Végétaux fossiles des terrains houillers de la Belgique: Acad. royale sci. Belgique, pls. 1-69.

Savi, Paolo, 1843, Sopra i carboni fossili dei terreni mioceni maremme Toscane: 78 p., 2 pls., Pisa.

Savi, Paola, and Meneghini, C. J., 1851, Considetazione sulla geologia della Joscana : 245 p., Firenze.

Schafhäutl, Karl Emil, 1851, Geognostische Untersuchungen des südbayerischen Alpengebirges : 207 p., 44 pls.,. Munich.

1863, Siid-Bayerns Lethaea Geognostica-Der Kressenberg und die südlich von ihm gelegenen Hochalpen geognostisch betrachtet in ihren Petrefacten: 487 p., 86 pls., Leipzig.

Schauroth, Karl, 1852, Über die im Coburger mittleren Keupersandstein gefundene Pflanzensteinerung: Deutsche geol. Gessell. Zeitschr., Band 4, p. 538-544.

Schemel, Mart P., 1950, Cretaceous plant mircofossils from Iowa : Am. Jour. Bot., v. 37, p. 750-753.

Schenk, August, 1864a, Beiträge zur Flora der Vorwelt: Palaeontographica, Band 11, p. 296-308, pls. 46-49.

1864b, Beiträge zur Flora des Keupers und der rhätischen Formation: Näturf. Gesell. Bamberg Ber., Band 7, p. 51-142, pls. 1-8.

1865-67, Die fossile Flora der grenzschitchten des Keupers und Lias Frankens : p. $1-32$, pls. $1-5,1865$; p. $33-96$, pls. $6-15$, 1866 ; p. $97-128$, pls. $16-35,1867$, Weisbaden.

1866, Bemerkungen über einige Pflanzen der Lettenkohle und des Schilfstandsteines: Wurzburg. Naturw. Zeitschr., Band. 6, p. 49-63.

1869, Beiträge zur Flora Vorwelt: Palaeontographica, Band 19, p. 1-34, pls. 1-7.

- 1871, Beiträge zur Flora Vorvelt-Die Flora der nordwestdeutschen Wealden-formation: Palaeontographica, Band 19, p. 203-266, pls. 22-43.

1880, Über fossile Hölzer aus der Libyschen Wüste: Bot. Zeitung, Band 38 , p. 658-661.

1882, Die von den Gebrüdern Schlagenweit in Indien gesammelten fossilen

Hölzer : Bot. Jahrb., Band 3, p. 353-358.

1883a, Fossile Hölzer : Paleontographica, Band, 30, p. 1-19, pls. 1-4.

1883b, Review of "Pflanzen aus der Steinkohlenformation" : Neues Jahrb., Band 2, p. 258-259.

1883c, Pflanzen aus der Steinkohlen Formation, in Richthofen, Ferdinand, China, v. 5, no. 2, p. 211-269, pls. 30-54.

1887, Fossile Pflanzen aus der Albourskette : Bibliotheca Botanica, Heft 6, p. 1-12, pls. 1-9.

1888, Fossile Hölzer aus Ostasien und Aegypten : K. Svenska vetensk.-akad. Handl., band 14, p. 1-24.

Schimper, W. P., 1837, Observations in Voltz' "Notice sur le grès bigarre de la Carriere de Soultz-les-Bains": Soc. mus. histoire nat. Strasbourg Mém., tome. 2, p. 9-14.

1869-74, Traité de paléontologie végétale ou la flore du monde primitif: tome 1, p. 1-740, pls. 1-56, 1869; tome 2, p. 1-522, pls. 57-84, 1870; p. 523-968, pls. 85-94, 1872; tome 3, p. 1-896, pls. 95-110, 1874.

Schimper, W. P., and Mougeot, Antoine, 1844, Monographie des plantes fossiles du grès bigarre de la chaine des Vosges : 83 p., 40 pls., Leipzig.

$296347-55-6$ 
Schimper, W. P., and Schenk, August, 1879-90, Zittel's Handbuch der Palaeontologie, Teil II, Palaeophytologie : Lief. 1, p..1-152, 1879 ;. Leif. 2, p. 153-232, 1880 ; Lief. 3 , p. 233-332, 1884 ; Lief. 4, p. 333-396, 1885 ; Lief. 5, p. 397-492, 1887 ; Lief. 6, p. 493-572, 1888 ; Lief. 7, p. 573-668, 1889; Lief. 8, p. 669-764, 1889 ; Lief. 9, p. 765-958, 1890, Leipzig.

Schindehütte, G., 1907, Die Tertiärflora des Basalttuffes vom Eichelskopf bei Homberg: Preuss. geol. Landesanst. Abh., Neue Folge, no. 54, p. 1-81, pls. 1-13.

Schlotheim, E. F., 1804, Beschreibung merkwurdiger Kräuter-Abdrücke und Pflanzen-Versteinerungen, Ein Beitrag zur Flora der Vorwelt @. 68 p., 14 pls., Gotha.

- 1820, Die Petrefactenkunde auf ihrem jetzig Standpunkte durch die Beschreibung seiner Sammlung versteinerter und fossiler Überreste des Thier und Pflanzenreichs der Vordwelt erläuter: lxii, 437 p., Gotha.

- 1822-23, Nachträge zur Petrefactenkunde : 100 p., pls. 1-21, 1822 ; 114 p., pls. $22-37,1823$, Gotha.

1832, Merkwürdige Versteinerungen aus der Petrefactensammlung : 40 p., 37 pls., Gotha.

Schmalhausen, Johannes, 1879, Beiträge zur Jura-Flora Russlands: Acad. imp. sci. St.-Pétersbourg Mém., tome 27, p. 1-96, pls. 1-16.

___ 1883, Beiträge zur Tertiärflora Sïd-West Russlands: Palaeont.'Abh., Band 1, p. 285-335, pls. 28-41, Berlin.

1894, Über devonische Pflanzen aus dem Donetz-Becken : Russia, Comité géol. Mém., tome 8, p. 1-36, pls. 1, 2, St. Petersburg.

Schmid, E. E., 1853, Die organischen Reste des Muschelkalkes im Saalthale bei Jens : Neues Jahrb., p. 9-30.

Schmid, E. E., and Schleiden, M. J., 1846, Die geognostischen Verhaltnisse des Salthales bie Jena: 72 p., 5 pls., Leipzig.

— 1855, Über die Natur der Kieselhölzer : 42 p., 3 pls., Jena.

Schonfeld, Eric, 1930, Über zwei neue Laubhölzer aus dem Miozän: Senckenbergiana, Band 12, p. 111-126.

Schonfeld, Georg, 1947, Hölzeraus dem Tertiär von Kolumbien : Senckenberg. Naturf. Gesell. Abh. 475, p. 1-53, pls. 1-5.

Schopf, James M., 1936a, The paleobotanical significance of plant structure in coal : Ill. Acad. Sci. Trans., v. 28, p. 106-110.

- 1936b, Spores characteristic of Illinois coal No. 6 : Ill. Acad. Sci. Trans., v. 28, p. $173-176$.

1938a, Spores from the Herrin (No. 6) coal bed in Illinois: Ill. Geol. Survey Rept. Inv. 50, p. 1-55, pls. 1-8.

1988b, Two new lycopod seeds from the Illinois Pennsylvanian: Ill. Acad. Sci. Trans., v. 30, p. 139-146.

1941a, Contributions to Pennsylvanian paleobotany-Mazocarpon oedipternum, sp. nov., and sigillarian relationships: Ill. Geol.. Survey Rept. Inv. 75, p. 1-40, pls. 1-6.

1941b, Contributions to Pennsylvanian paleobotany-Notes on the Lepidocarpaceae: Am. Midland Naturalist, v. 25, p. 548-563.

1948, Pteridosperm male fructifications-American species of Dolerotheca, with notes regarding certain allied forms: Jour. Paleontology, v. 22, p. 681724.

Schopf, James M., Wilson, L. R., and Bentall, Ray, 1944, An annotated synopsis of Paleozoic fossil spores and the definition of generic groups: I11. Geor. Survey Rept. Inv. 91, p. 1-66, pls. 1-3. 
Schroeter, Carl Joseph, 1880, Untersuchung über Fossile Hölzer aus der arctischen Zone, in Heer, Oswald, Flora Fossilis Arctica, Band 6, Teil 1, Heft 4, p. 1-38, pls. 1-3, Zurich.

Schubert, R. J., 1907, Vorläufige Mitteilung über Foraminiferen und Kalkalgen aus dem dalmatischen Karbon: K.-k. geol. Reichsanst. Verh., p. 211-214, Vienna.

— 1908, Zur Geologie des österreichischen Velebit: K.-k. geol. Reichsanst. Jahrb., Band 58, pt. 2, p. 345-386, pl. 16, Vienna.

Schultes, Richard E., and Dorf, Erling, 1938, A sphenopsid from the Lower Devonian of Wyoming: Harvard Univ. Bot. Mus. Ieaflets, v. 7, p. 21-33.

Schultz, Charles Bertrand, 1942, A review of the Daimonelix problem: Nebr. Univ. Studies in Sci. and Technology no. 2, p. 1-30.

Schulze, Erwin, 1887, Über die Flora der subhercynischen Kreide: Zeitschr. Naturw., Halle, Band 60, p. 440-470.

Schuster, Julius, 1908, Über ein fossiles Holz aus dem Flysch des Tegernseer Gebietes: : Geog. Jahresber, Oesterreich, Band 19, p. 139-152, pl. 2.

1910, Über Nicolien und Nicolien ähnliche Holzer: K. Svenska vetensk. akad. Handl., band 45, no. 6, p. 1-18, pls. 1-3.

1911a, Paleozane Rebe der Griefswalder Oie: Deutschen bot. Gesell. Ber., Band 29, p. 540-544, pl. 20.

- 1.911b, Weltrichia und die Bennettitales : K. Svenska vetensk. akad. Handl., band 46 , no. 11, p. 1-57, pls. 1-7.

- 1931, Über das Verhältnis der systematischen Gliederung der geographischen Verbreitung und der paläontologischer Entwicklung der Cycadeceen: Bot. Jahrb., Band 64, p. 165-256, pls. 4-11.

Schwager, Conrad, 1883, Die Foraminiferen aus den Eocaenablagerungen der libyschen Wuste und Aegyptens: Palaeontographica, Band 30, teil 2, p. 1-75, pls. 24-29.

Scott, Dukinfield Henry, 1897a, On the structure and affinities of fossil plants from the Palaeozoic rocks: Royal Soc. London Proc. v. 62, p. 166-168.

$1897 \mathrm{~b}$, On Oheirostrobus, a new type of fossil cone from the Calciferous Sandstone : Royal Soc. London Proc., v. 60, p. 417-424.

1898a, On the structure and affinities of fossil plants from the Palaeozoic rocks, II, On Spencerites, a new genus of Lycopodiaceous cones from the Coal Measures: Royal Soc. London Philos. Trans., v. 189B, p. 83-106, pls. 12-15.

$1898 \mathrm{~b}$, On the structure and affinities of fossil plants from the Palaeozoic rocks, I, On Cheirostrobus : Royal Soc. London Philos. Trans., v. 189B, p. 1-34, pls. 1-6.

1899, On the structure and affinities of fossil plants from the Palaeozoic rocks, III, On Medullosa anglica : Royal Soc. London Philos, Trans., v. 191B, p. 81-126.

1900a, Note on the occurrence of a seed-like fructification in certain

Palaeozoic lycopods : Royal Soc. London Proc., v. 67, p. 306-309.

1900b, Studies in fossil botany, 1st ed. : 533 p., London.

1901, On the structure and affinities of fossil plants from the Palaeozoic rocks, IV, The seed-like fructifications of Lepidocarpon: Royal Soc. London Philos. Trans., v. 194B, p. 291-333.

$\therefore \quad 1902$, On the primary structure of certain Palaeozoic stems with the Dadoxylon type of wood: Royal Soc. Edinburgh Trans., v. 40, p. 331-365, pls. 1-6.

1905a, On a new type of Sphenophyllaceous cone from the Lower Coal Measures: Royal Soc. London Philos. Trans., v. 198B, p. 17-39, pls. 3-5. 
Scott, Dukinfield Henry. 1905b, The sporangia of Stauropteris oldhamia Binney : New Phytologist, v. 4, p. 114-120.

1906i, On the structure of some Carboniferous ferns: Royal Micros. Soc.

Jour., 1906, p. 518-520.

1906b, On Sutcliffia insignis, a new type of Medulloseae from the Lower

Coal Measures : Linnean Soc. London Trans., v. 7, p. 45-68, pls. 7-10.

1907, The present position of Palaeozoic botany, in Lotsy, J. P. ed., Progressus rei Botanicae, $\nabla$. 1, 139-217, Jena.

- 1908, Studies in fossil botany, 2d ed. : v. 1, 363 p., London.

1909, Studies in fossil botany, $2 d$ ed. : v. 2, 683 p., London.

1912, On Botrychioxylon paradoxum, sp. nov. a Palaeozoic fern with secondary wood: Linnean Soc. London Trans., v. 7, p. 373-389, pls. 37-41.

1917, The Heterangiums of the British Coal Measures : Linnean Soc. London Jour., Botany, v. 44, p. 59-105, pls. 1-4.

1919, On the fertile shoots of Mesoxylon and an allied genus: Annals Botany, v. 33, p. 1-21, pls. 1-3.

— 1920, Studies in fossil botany, 3d ed. : v. 1, 434 p., London.

_ـ 1923, Studies in fossil botany, 3d ed. : v. 2, 446 p., London.

1925, Fossil plants of the Calamopitys type from the Carboniferous rocks

of Sctoland : Royal Soc. Edinburgh Trans., v. 53, p. 569-596, pls. 1-6.

1930, Cladites bracteatus, a petrified shoot from the Lower Coal Measures: Annals Botany, v. 44, p. 333-348, pls. 17, 18.

Scott, Dukinfield Henry, and Jeffrey, E. C., 1914, On fossil plants, showing structure, from the base of the Waverley shale of Kentucky : Royal Soc. London Philos. Trans., ser. B, v. 205, p. 315-373, pls. 27-39.

Scott, Dukinfield Henry, and Maslen, A. J., 1907, The structure of the Palaeozoic seeds Trigonocarpus parkinsoni Brongniart and Trigonocarpus oliveri sp. nov.: Annals Botany, v. 21, p. 89-134, pls. 11-14.

- 1910, On Mesozylon, a new genus of Cordaitales-Preliminary note : Annals Botany, v. 24, p. 236-239.

Scott, Rina, 1908, On Bensonites fusiformis, sp. nov., a fossil associated with Stauropteris burntislandica, P. Bertrand, and on the sporangia of the latter: Annals Botany, v. 22, p. 683-687, pl. 34.

1911, On Traquairia: Annals Botany, v. 25, pp. 459-467, pls. 39, 40.

Sedgwick, Adam, 1848, On the organic remains found in the Skiddaw Slate, with some remarks on the classification of the older rocks of Cumberland: Geol. Soc. London Quart. Jour., v. 4, p. 216-225.

Seely, H. M., 1904, The Stromatoceria of Isle La Matte, Vt.: vt. State Geologist Rept. 1903-04, p. 144-152.

Sellards, E. H., 1900, A new genus of ferns from the Permian of Kansas: Kans. Univ. Quart., v. 9, p. 179-189, pls. 37-42.

1903, Codonotheca, a new type of spore-bearing organ from the Coal Measures: Am. Jour. Sci., 4th ser., v. 16, p. 87-95, pl. 8.

Selling, Olof H., 1939-47, Report on European Paleobotany : Swedish Mus. Nat. History, Paleobot. Dept., p. 1-121, Stockholm [1948].

-1948-49, Report on European Plaeobotany: Swedish Mus. Nat. History, Paleobot. Dept., p. 1-65, Stockholm [1950].

Seward, Albert C., 1894a, Catalogue of the Mesozoic plants in the Department of Geology, British Museum Natural History-The Wealden Flora, Part I Thallopbyta-Pteridophyta: 179 p., 10 pls. British Mus. Nat. History, London. $1894 \mathrm{~b}$, Note on Catalogue of the Mesozoic plants * * * : Geol. Mag., decade 4, v. 1, p. 384, London. 
Seward, Albert C., 1895, Catalogue of the Mesozoic plants in the Department of Geology, British Museum-The Wealden Flora, Part II, Gymnospermae: 259 p., 20 pls., British Mus. Nat. History, London.

-1896a, Notes on the geological history of monocotyledons : Annals Botany, v. 10, p. 205-220, pl. 14.

1896b, An extinct plant of doubtful affinity : Sci. Progress, v. 5, p. 427-438. 1896c, A new species of conifer, Pinites ruffordi, from the English Wealden

formation: Linnean Soc. London Jour., v. 32, p. 417-425, pls. 2, 3.

1898, Fossil plants : v. 1, 452 p., Cambridge.

1899, Notes on the Binney Collection of Coal Measure plants : Cambridge

Philos. Soc. Proc., v. 10, p. 137-174, pls. 3-7.

1900, Catalogue of the Mesozoic plants in the British Museum-The

Jurassic Flora, I, The Yorkshire coast: 341 p., 21 pls., British Mus. Nat.

History, London.

- 1904, Catalogue of the Mesozoic plants in the British Museum-The

Jurassic Flora, II, Liassic and Oolitic floras of England : 192 p., 18 pls., British

Mus. Nat. History, London.

1910, Fossil plants, v. 2 : 624 p., Cambridge.

1911a, New genus of fossil plants from the Stormberg series of Cape

Colony : Geol. Mag., v. 8, p. 298-299, pl. 14, London.

1911b, The Jurassic flora of Sutherland: Royal Soc. Edinburgh Trans.,

v. 47, p. 643-709, pls. 1-10.

1912, Mesozoic plants from Afghanistan and Afghan-Turkistan: Geol.

Survey India Mem., Palaeontologia Indica, new ser., v. 4, Mem. 4, p. 1-57, pls. 1-7.

- 1913, A contribution to our knowledge of Wealden floras: Geol. Soc.

London Quart. Jour., v. 69, p. 85-114, pls. 11-14.

1914, Antarctic fossil plants: British Mus. Nat. History, Terra Nova

Exped. Rept., Geology, v. 1, p. 1-49, pls. 1-8.

1917, Fossil plants : v. 3, 656 p., Cambridge.

1919, Fossil plants : v. 4, 542 p., Cambridge.

1925, Notes sur la flore crétacique du Groenland: Soc. géol. Belgique, $50^{\circ}$

Livre jubilaire, 1874-1924, tome 1, fasc. 1, p. 228-263, pls. A-C [1925?]

[Translated by S. Leclercq.]

1.926, The Cretaceous plant-bearing rocks of western Greenland: Royal

Soc. London Philos. Trans., ser. B. v. 215B, p. 57-174, pls. 4-12.

1931, Fossil plants from the Bokkeveid and Witteberg beds of South Africa: Geol. Soc. London Quart. Jour., v. 88, p. 358-368, pls. 23, 24.

Seward, Albert C., and Conway, Verona M., 1935a, Additional Cretaceous plants from western Greenland: K. Svenska vetensk. akad. Handl., band 15, p. 1-51, pls. 1-6.

1935b, Fossil plants from Kingigtok and Kagdlunguak, West Greenland : Cophenhague Univ., Mus. minéralogie et géologie, Comm. paleont. 55, 41 p., 5 pls.

Seward, Albert C., and Sahni, B., 1920, Indian Gondwana plants, a revision : Paleontologia Indica, v. 7, p. 1-54, pls. 1-7.

— Postscript to "Indian Gondwana Plants" : Geol. Mag., v. 63, p. 288, London.

Seward, Albert C., Thomas, H. H., and others, 1913, The investigation of the Jurassic flora of Yorkshire: British Assoc. Adv. Sci. Rept. 82d Mtg., Dundee, 1912, p. 294-295.

Shimakura, Misaburo, 1936, Preliminary report on some Cretaceous plants from Karahuto: Geol. Soc. Japan Jour., p. 869-875, pl. 48.

1937, A petrified wood dredged from the bottom off the coast of Tobisima, Yamagata-Ken : Geol. Soc. Japan Jour., v. 44, p. 697-702. 
Shrock, R. R., and Twenhofel, W. H., 1939, Silurian fossils from northern Newfoundland: Jour. Paleontology, v. 13, p. 241-266, pls. 27-30.

Shukla, V. B., 1944, On Sahnianthus, a new genus of petrified flowers from the Intertrappean beds at Mohgaon Kalan in the Deccan and its relation with the fruit Enigmocarpon parijai Sahni from the same locality : Nat. Acad. Sci. India Proc., v. 14, p. 1-39, pls. 1-8.

Silvestri, Alfredo, 1911, Sulla vera natura dei "Palaeodictyon": Soc. geol. Italiana Boll., v. 30, p. 85-107, pls. 6, 7, Rome.

Simionescu, Ioan, 1926, Sur quelques fossiles rares, dans le trias et le crétacé inférieur de Roumanie: Acad. Roumaine Sec. ci. Bull., tome 10, p. 102-109.

- 1940, Sur quelques algues mesozoigues de Roumanie: Acad. Roumaine Sec. sci. Bull., tome 22, no., 8 p. 1-8, 3 pls.

Sinnott, Edmund W., 1909, Paracedroxylon, a new type of Araucarian wood: Rhodora, v. 11, p. 165-173, pls. 80, 81.

Sismonda, Eugene, 1859, Prodrome d'une flore tertiaire du Piémont: Accad. sci. Turin, Mem. ser. $2^{a}$, v. 18, p. 519-547, pls. 1-4.

Smets, Gerard, 1888, Aachenosaurus multidens, reptile fossile des sables d'Aix-laChapelle : 23 p., 1 pl., Hassett.

Smith, George O., and White, David, 1905, The geology of the Perry Basin in southeastern Maine: U. S. Geol. Survey Prof. Paper 35, p. 1-92, pls. 1-6.

Smith, John, 1896, On the discovery of fossil microscopic plants in the fossil amber of the Ayrshire coal-field : Geol. Soc. Glasgow Trans., v. 10, p. 318-322, pl. 7 .

Smith, Worthington G., 1877, A fossil Peronospora : Gardeners Chronicle, v. 8, p. 499-500.

- 1884, Diseases of field and garden crops, chiefly such as are caused by fungi : 353 p., London.

Solms-Laubach, Graf, 1896, Über die seinerzeit von Unger beschriebenen strukturbietenden Pflanzenreste des Unterculm von Saalfeld in Thïringen: Preuss. geol. Landesanst. Abh., Neue Folge, Heft 23, p. 1-100, pls. 1-5.

- 1897, Über Medullosa leukarti: Bot. Zeitung, Band 55, p. 175-292, pIs. 5, 6. 1899, Beschreibung der Pflanzenreste von La Ternera: Neues Jahrb., p. 593-609, pls. 13, 14.

- 1913, Tietea singularis-Ein neuer fossiler Pteridinenstamm aus Brasilien : Zeitischr. Botanik, Band 5, p. 673-700, pls. 6,7.

Sordelli, Ferdinando, 1873, Descrizione di alcuni avanzi regetali delle argille plioceniche Lombardi : Soc. Italiana sci. nat. Atti, v. 16, p. 350-429, pls. 4a-7a.

__ 1896, Flora fossilis insubrica_Studi sulla vegetazione di Lombardia : 298 p., 1-43 pls., Milan.

Spegazzini, Carlos, 1924a, Sobre algunas impresiones vegetales eocénicas de Patagonia : Mus. Nac. Historia Nat. Buenos Aires Comun., tomo 2, p. 95-107. 1924b, Coniferales fosiles Patagonicas : Soc. Cient. Argentina Anales, tomo 98, p. 125-139.

Spencer, James, 1882, Researches in fossil botany : British Assoc. Adv. Sci., Rept. 51st Mtg., 1881, p. 627-628.

Spitz, Albrecht, and Dyhrenfurth, G., 1915, Monographie der Engadiner Dolomiten zwischen Schuls, Scarfs und dem Stilfserjoch: Beitr. geol. Karte Schweiz, Neve Folge, Band 44.

Sprengel, Antonlo, 1828, Commentatio de Psarolithis, ligni fossilis fenere: $42 \mathrm{p}$, 1 pl., Halae.

Squinabol, Senofonte, 1888 , Contribuzioni alla flora fossile dei terreni terziarii della Liguria : Soc. geol. Italiana Boll., v. 6, p. 545-559, pls. 15-19, Rome.

1890, Alghe e pseudoalghe fossile Italiane: Soc. ligustica sci. nat. Atti, v. 1, p. 166-199, pls. 5-12, Genova. 
Squinabol, Senofonte, 1891a,. Note sur quelques types de Monocotyledonées de Sainte-Justine et de Sassello: Soc. géol. France Bull., sér. $3^{\circ}$, tome 19, p. 771782 , pls. $16,17$.

1891b, Contribuzioni alla flora fossile dei terreni terziarii della Liguria, I, Alghe: $p . i-x \dot{x} \nabla$, pls. A-E, Genova.

- 1892, Contribuzioni alla flora fossile dei terreni terziarii della Liguria, IV, Monocotiledoni : p. 1-104, pls. 19-30, Genova.

- 1900 , Sulla vera natura delle Helminthoida: Soc. veneto-trentino sci. nat. Atti ser. $2^{\mathrm{a}}$, v. 4, p. 33-39, figs. 1-3, Padova.

Srivastava, B. P., 1935, On some silicifled plant remains from the Rajmahal series of India : Indian Sci. Cong., 22d, Calcutta, Proc., p. 285.

1937, Studies on some silicified plant remains from the Rajmahal series: Indian Sci. Cong., 24th, Hyderabad, Proc., p. 273-274.

1944, Silicified plant remains from the Rajmahal Hills : Natl. Acad. Sci. India Proc., v. 14, sec. B, p. 73-76.

— 1946, Silicified plant remains from the Rajmahal series of India: Natl. Acad. Sci. India Proc., v. 15, p. 185-211.

Stache, Guido, 1855, De Casuarinis nunc viventibus et fossilibus nonnulla: Inaugural Dissert., 42 p., Univ. Vratislaviae.

- 1872a, Alghe fosseli mioceniche di Cerenaica: Nuova Notarisia, v. $35, \mathrm{p}$. $28-46$.

— 1872b, Über neue characeenreste aus der oberen Abtheilung der liburnischen Stufe bei Pisino in Istrien: K.-k. geol. Reichsanst., Verh., 1872, p. 316-317, Vienna.

- 1880, Die liburnische Stufe : K.-k. geol. Reichsanst., Verh., 1880, p. 195-209, Vienna.

1889, Die liburnische Stufe und deren Grenzhorizonte : K.-k. geol. Reichsanst., Abh., Band 13, p. 1-170, pls. 1-6, Vienna.

Stanton, T. W., and Knowlton, F. H., 1897, Stratigraphy and paleontology of the Laramie and related formations in Wyoming: Geol. Soc. America Bull., v. S, p. 127-156.

Staub, Móricz, 1887, Die aquitanische Flora des Zsilthales im Comitate Hunyad : K. Ungarische geol. Anst. Jahrb., Band 7, p. 223-417, pls. 18-44, Budapest.

Steere, William C., 1946 Cenozoic and Mesozoic bryophytes of North America in Symposium on paleobotanical taxonomy: Am. Midland Naturalist, v. 36, p. 298-324, pls. $1,2$.

Stefani Carlo, 1901, Flore carbonifère e permiane della Toscana: Ist. di studi superiori pratici Perfezionamento, Florence, 212 p., 14 pls.

Steininger, Johann, 1840-41, Geognostische Beschreibung des Landes zwischen der untern Saar und dem Rheine: 150 p., pls. $1-5,1840$; pt. 2, 50 p., 5 unnumbered pls., 1841.

Steinmann, Gustav, 1880, Zur Kenntniss fossiler Kalkalgen (Siphoneen) : Neues Jahrb., p. 130-140, pl. 5.

1899, Über fossile Dasycladaceen vom Cerro Escamela, Mexico: Bot. Zeitung, Band 57, p. 137-154. 1903, Einführung in die Paläontologie : 466 p., Leipzig.

1911, Über Gymnosolen ramsayi, eine coelenterate von der Halbinsel Kanin: Soc. geog. Finland (Fennia) Bull., teos 31, no. 4, p. 18-23, pl. 3. 1929, Geologie von Peru, 448 p., 9 pls., Heidelberg.

Steinmann, Gustav, and Elberskirch, W., 1929, Neue bemerkenswerte Funde in ältesten Unterdevon des Wahnbachtales bei Siegburg: Naturh. Ver. preuss. Rheinlande u. Westfalens Situngsber., p. 1c-74c; pls. 1, 2. 
Stenzel, Carolus Gustavus, 1872, Über fossile Palmenhölzer: Schles. Gesell. vaterl. Kultur Jahresber. 49, 1871, p. 71-72, Breslau.

1886, Rhizoderidron oppoliense Goeppert: Schles. Gesell. vaterl. Kultur Jahresber. 63, p. 1-30, pls. 1-3, Breslau.

1889, Die Gattung Tubicaulis Cotta: K. Mineralog.-geol. prähistorischen Mus. Dresden, Band 8, p. 1-50, pls. 1-7.

Sternberg, Grafen Kașpar, 1820-38, Versuch einer Geognostischen Botanischen Darstellung der Flora der Vorwelt: Band 1, Teil 1, p. 1-24, 1820; Teil 2, p. 1-33, 1822; Teil 3, p. 1-39, 1823; Teil 4, p. 1-48, 1825; Band 2 ,Teile 5, 6, p. 1-80, 1833; Teile 7, 8, p. 81-220, 1838; Tentamen, p. i-vlii, 1825, Leipsic and Prague.

1837, Beschreibung von Huttonia spicata, einer neuen fossilen Pflanzen: Gesell. vaterl. Mus. Böhmen Verh., Prague, p. 37, 69, pl. 1.

Sterzel, J. T., 1880, Über Scolecopteris elegans Zenger und andere fossile Reste aus dem Hornstein von Altendorf bei Chemnitz: Deutsche geol. Gesell. Zeitschr., Band 32, p. 1-18.

- 1881, Palaontologischer charakter der oberen Steinkohlenformation und des Rothliegenden im erzgebirgischen Becken: Naturwiss. Gesell. 7 Ber., Chemnitz, p. 155-270.

- 1883, Über Dicksonites pluckeneti Schloth. sp.: Bot. Centralbl., Cassel, Band 13, p. 282-287, 313-319, pl. 6.

- 1895, Die Flora des Rotliegenden von Oppenau in badischen Schwarzwalde: Grossh.-bad. geol. Landesanst. Mitt., Heidelberg, Band 3, p. 261-353, pls. 8-11.

1907, Die Karbon und Rotliegendenfloren im Grotzherzogtum Baden: Grossh.-bad. geol. Landesanst. Mitt., Heidelberg, Band 5, p. 347-892, pls. 14-68.

Stiehler, August Wilhelm, 1857, Beiträge zur Kenntniss der vorweltlichen Flora des Kreidegebirges im Harze: Palaeontographica, Band 5, p. 47-70, pls. 9-11.

__ 1861, Synopsis der Pflanzenkunde der Vorwelt-Die gamopetalen angiospermen Dicotyledonen der Vorwelt : 196 p., Quedlinburg.

Stizenberger, Ernst, 1851, Übersicht der Versteinerungen des Grossherzogthums Baden : 144 p., Freiburg.

Stockmans, François, 1936, Végétaux éocènes des environs de Bruxelles: Mus. royal histoire nat. Belgique Mém. 76, 56, p., 3 pls., Brussels.

- 1946a, Tour d'horizon paléobotanique en Belgique: Soc. naturalistes Belges Bull., 1946, nos. 7, 8, p. 1-6.

- 1946b, Végétaux de l'assise des Sables d'Aix-la-Chapelle récoltes en Belegique: Mus. royal histoire nat. Belgique Mém. 105, 51 p., 4 pls.

- 1945, végetax du dévonien supérieur de la Belgique: Mus. royal histoire nat. Belgique Mém. 110, 85 p., 14 pls.

Stokes, Charles, and Webb, Phillip Barker, 1824, Description of some fossil vegetables of the Tilgate forest in Sussex: Geol. Soc. London Trans., v. 1, p. $423-426[421-424]$.

Stolley, Ernst 1893, Über silurische Siphoneen : Neues Jahrb., p. 135-146, pls. 7, 8. 1896, Untersuchungen über Coelosphaeridium, Cyclocrinus, Mastopora und verwandte Genera des Silur: Archiv anthrop. geol. Schleswig-Holstein, Kiel and Liepzig, Band 1, p. 177-282.

1925, Die Psilophyten: Naturh. Gesell. Hannover, Jahresb. 18, p. 39-96.

Stopes, Marie C., 1911, The name of the 'Dragon Tree' : Geol. Mag., v. 8, p. 168 169 , London.

1912, Petrifactions of the earliest European angiosperms: Royal Soc. London Philos. Trans., v. 203, p. 75-100, pls. 6-8. 
Stopes, Marie C., 1913, Catalogue of the Mesozoic plants in the British MuseumThe Cretaceous flora, Part 1, Bibliography, algae and fungi : 281 p.. 2 pls., London, British Mus. Nat. History.

1914, The "Fern Ledges" Carboniferous flora of St. John, New Brunswick: Canada Dept. Mines Geol. Survey Mem. 41, 142 p., 25 pls.

1915, Catalogue of the Mesozoic plants in the British Museum-The Cretaceous flora, Part 2, Lower Greensand (Aptian) plants of Britain : 360 p., 32 pls., London, British Mus. Nat. History.

- 1916, An early type of the Abietineae(?) from the Cretaceous of New Zealand: Annals Botany, v. 30, p. 111-125, pl. 4.

Stopes, Marie C., and Fujii, K., 1909, Studies on the structure and affinities of Cretaceous plants : Geol. Mag., decade 5, v. 6, p. 557-559, London.

- 1910, Studies on the structure and affinities of Cretaceous plants: Royal Soc. London Philos. Trans., v. 201, p. 1-90, pls. 1-9.

Strzelecki, Paul Edmund, 1845, Physical description of New South Wales and Van Diemen's Land : 1-462 p., London.

Stur, Dionys Rudolf Josef, 1863, Bericht über die geologishe Übersichtsaufrahme des südwestlichen Siebenbürgen im Sommer 1860 : K.-k. geol. Reichsanst. Jahrb., Band 13, p. 33-120, Vienna.

- 1873, Vorkommen einer Palmenfrucht-Hulle Lepidocaryopsis westphaleni

n. g. et sp. in Kreide-Sandstein der Peruzer-Schichten bei Kaunitz in Böhmen :

K.-k. geol. Reichsanst. Verh., p. 1-3, Vienna.

1875, Beiträge zur Kenntniss der Flora der Vorwelt-Die Culm-Flora,

Teil 1, Die Culm-Flora des mährisch-schlischen Dachschiefers: K.-k. geol.

Reichsanst. Abh., Band 8, p. 1-106, pls. 1-17, Vienna.

- 1877, Beiträge zur Kenntniss der Flora der Vorwelt-Die Culm-Flora,

Teil 2, Die Culm-Flora der Ostrauer und Waldenburger Schichten : K.-k. geol.

Reichsanst. Abh., Band 8, p. 107-472, pls. A-C, 18-44, Vienna.

— 1882, Die Silur-Flora der Etage H-h, in Böhmen: K. Akad. Wiss.

Sitzungsber., Band 84, Abt. 1, p. 330-391, pls. 1-5, Vienna.

-1883, Zur morphologie und systematik der Culm und Carbonfarne: $\mathbf{K}$.

Akad. Wiss. Sitzungsber., Band 88, p. 633-846, Vienna.

- 1885, Die obertriadische Flora der Lunzer-Schichten und des bituminösen

Schiefers van Raibl : K. Akad. Wiss. Sitzungsber., Band 91, Teil 1, p. 93-103, Vienna.

- 1888a, Die Iunzer Flora in den "older Mesozoic beds of the coal field of eastern Virginia": K.-k. geol. Reichsanst. Verh., Heft 10, p. 203-217, Vienna.

1888b, Über die Flora der feuerfesten Thone von Grojec in Galizien : K.-k. geol. Reichsant. Verh., 1888, p. 106-108, Vienna.

Suzuki, Y., 1910, On the structure and affinities of two new conifers and a new fungus from the Upper Cretaceous of Hokkaido (Yezo) : Bot. Mag., v. 24, p. 181-196, pls. 7, Tokyo.

Sze, Hsing-Chien, 1930, Zu Schenks publikationen über die Ostasiatische Permokarbon-Flora, Teil 1: Natl. Research Inst. Geology (Acad. Sinica), Mem. 9, p. 1-31, pl. 1, Shanghai.

1931, Beiträge zur liasischen Flora von China : Natl. Research Inst. Geology (Acad. Sinica), Mem. 12, p. 1-85, Shanghai.

— 1933, Fossile Pflanzen aus Shensi, Szechuan und Kueichow: China, Geol. Survey, Palaeontologia Sinica, v. 1, p. 1-32, pls. 1-6.

- 1943, Plagiozamiopsis podozamioides, n. g. et sp., a new Permian plant from the Gigantopteris coal series: Sci. Record, Acad. Sinica, v. 34, p. 511517. 
Sze, Hsing-Chien, 1945, The Cretaceous flora from the Pantou series in Yungan, Fukien: Jour. Paleontology, v. 19, p. 45-59.

1949, Die mesozoische Flora aus der Hsiangchi Kohlen Serie in Westhupeh: China, Geol. Survey, Palaeontologia Sinica, new ser. A; no. 2, p. 1-71, pls. 1-15.

Tasche, H., 1854, Salzhausen mit besonderer Rücksicht auf die geognostischen Verhältnisse seine Umgegend : Oberrhessiche Gesell. Natur-u. Heilkunde, Ber. Band 4, p. 72-127, Giessen.

Tate, Ralph, 1867, On some secondary fossils from South Africa : Geol. Soc. London Quart. Jour., จ. 23, p. 139-173, pls. 5-8.

Tate, Ralph, and Blake, J. F., 1876, The Yorkshire Lias: 475 p., 19 pls., London.

Tchihatchef, Pierre, 1863, Note sur deux nouveaux genres de bois fossile recueillis dans les environs de Constantinople: Acad. sci. Paris Comptes Rendus, tomé 56 , p. 516-518.

- 1866, Asie Mineure, Palaeontologie Flore tertiaire moyenne (by Unger, Franz) : p. 319-325, pl. 17, Paris.

Tchirkova, N. T., 1937a, Contribution nouvelle à la flore carbonifère inférieure du versant oriental de l'Oural : Moscow, Univ., Paleont. Laboratoria, Problems Paleontology Pub., v. 2-3, p. 236-247.

1937b, Contribution sur l'extension du continent de l'Angaride: Moscow, Univ. Paleont. Laboratoria, Problems Paleontology Pub., v. 2-3, p. 202-203.

Teixeira, Carlos, 1947, Nouvelles recherches et revision de la flore de Cercal [Portugal] : Brotéria, Sér. ciénc. nat., Pôrto, v. 43, p. 5-14, pl. 1.

- 1948, Flora mesozoica portuluesa : Portugal, Serviços geol., pt. 1, 118 p., $45 \mathrm{pls}$.

Tenison-Woods, J. E., 1884, On the fossil flora of the coal deposits of Australia : Linnean Soc. New South Wales Proc., v. 8, p. 37-167.

Termier, Henri, and Termier, Geneviève, 1950, La flore eifelienne de Dechra Ait Abdallah (Maroc central) : Soc. géol. France Bull., sér. 5e, tome 20, p. 206208 , figs. $49-52$.

Theron, H., 1900, Note sur les gisements de phosphate de Chaux du pic de Cabrieres-Faune et flore des Lydiennes noires: Soc. études sci. nat. Beziers Bull., tome 22, p. 105-112.

Thiergart, Friedrich, 1940, Die Mikropalaontologie als Pollenanalyse in Dienst der Braunkohlenforschung: Brennstoffgeologie Schr., Stuttgart, Band 13, p. 48 , pl. 14.

1949, Der. Stratigraphische wert mesozoischer Pollen und Sporen: Palaeontographica, Band 89, Abt. B, p. 1-34, pls. 1-5.

Thiselton-Dyer, w. T., 1872, On some coniferous remains from the lithographic stone of Solenhofen: Geol. Mag., v. 9, p. 193-196, p1. 5, London.

Thomas, H. Hamshaw, 1912, Stachypteris hallei, a new Jurassic fern : Cambridge Philos. Soc. Proc., v. 16, pt. 7, p. 610-614, p1. 4.

1914 , On some new and rare Jurassic plants from Yorkshire-Eretmophyllum, a new type of Ginkgoalian leaf: Cambridge Philos. Soc. Proc., v. 17, p. 256-262, pls. 6,7 .

1915, OnWiltiamsoniella, a new type of Bennettitalean flower: Royal Soc. London Philos. Trans., v. 207B, p. 113-148, pls. 12-14.

1921, An Ottokaria-like plant from South Africa : Geol. Soc. London Quart. Jour., v. 77, p. 285-288.

1925, The Caytoniales, a new group of angiospermous plants from the Jurassic rocks of Yorkshire : Royal Soc. London Philos. Trans., v. 213, p. 299363 , pls. 11-15. 
Thomas, H. Hamshaw, 1930. Further observations on the cuticle structure of Mesozoic Cycadean fronds: Linnean Soc. London Jour., v. 48, p. 389-415, .jpls. 20, 21.

1931, The early evolution of the angiosperms: Annals Botany, v. 45, p. 647-672.

1933, On some Pteridospermous plants from the Mesozoic rocks of South Africa: Royal Soc. London Philos. Trans., v. 222B, p. 193-265.

Torell, Ottone, 1867, Bidrag till Sparagmitetagens geognosi och paleontologi: Lund, Univ., Acta, 1867, 40 p, 3 pls.

1869, Petrificata suecana formationis cambricae: Lund, Univ., Acta, จ. 6, pt. 3, p. 1-14.

Torrey, Ray Ethan, 1921, T'elephragmoxylon and the origin of wood parenchyma : Annals Botany, v. 35, p. 73-77, pl. 3.

1923, The comparative anatomy and phylogeny of the coniferales, Part 3 , Mesozoic and Tertiary coniferous woods: Boston Soc. Nat. Hist. Mem., v. 6, p. 41-106, pls. 8-15.

Toula, Franz, 1883, Geologische Untersuchungen in westlichen Theile des Balkan und in den angrenzenten Gebieten: K. Akad. Wiss. Sitzungsber., Band 88, p. 1279-1344, pls. 1-9, Vienna.

- 1900, Über den marinen Tegel von Neudorf an der March in Umgarn:

Ver. nat. Heilkunde Presburg Verh., Neue Folge, Band 11, p. 1-30.

Trabucco, Giacomo, 1900, Fossili, stratigrafia ed eta dei terreni del Casentino (Toscana) : Soc. geol. Italiana Boll., v. 19, p. 698-720, pl. 11.

Trauth, Friedrich, 1918, Das Eozänvorkommen bei Radstadt im Pongan, II, Ṕalaeontologischer Teil, Thallophyta by Julius Pia: K. Akad. Wiss. Denkschr., Band 95, p. 209-213, pl. 1, Vievna.

Trautschold, Hermann Adolfovich, 1870, Der Klin'sche Sandstein: Soc. imp. naturalistes Moscou Nouv. Mém., tome 13, p. 191-236, pls. 18-22.

1874, Etwas aus dem tertiären Sandstein von Kamüschin: Soc. imp. naturalistes Moscou Bull., tome 48, pt. 2, p. 128-132.

1881, Über Aroides crassispatha Kutorga: Soc. imp. naturalistes Moscou Bull., tome 55, pt. 2, p. 122-125.

-Turutanova-Ketova, A. I., 1930, Jurassic flora of the Chain Kara-Tau (Tian Shan) : Mus. géol. travaux, Acad. sci. U. R. S. S., tome 6, p. 131-172, pls. 1-6.

Tutin, T. G., 1932, A Cretaceous Gleicheniaceous fern from Western Greenland: Annals Botany, v. 46, p. 503-508, pl. 16.

Tuzson, Janos, 1908, Adatok Magyarország fosszilis flórájához II. (Additamenta ad floram fossilem Hungariae): Novenytani Kozlem, Budapest, kötet 7, sazám 1, p. 1-4, pls. 1, 2.

1909, Anatomische Bestimmung der fossilen Hölzer des Balatonsees: Math. u. Nat. Ber. Ungarn, Band 24, p. 376.

1911, Monographia der fossilen Pflanzenreste der Balatonseegegend: Resultante Wiss. Erforschung Balatonsees, Band 4, Teil 1, p. 1-63, pls. 1, 2. 1914, Beiträge zur fossilen Flora Ungarns : K. Ungarischen geol. Reichsanst. Jahrb., Budapest, Band 21, p. 233-262, pls. 13-21.

Twenhofel, W. H., 1919, Pre-Cambrian and Carboniferous algal deposits: Am. Jour. Sci., v. 48, p. 339-352.

Ulke, Titus, 1938, A new genus and species of fossil alga : Torreya, v. 38, p. 57-62. Ulrich, Edward Oscar, 1886, Descriptions of new Silurian and Devonian fossils in Contributions to American Palaeontology, v. 1, p. 3-35, pls. 1-3, Cincinnati. 1904, Fossils and age of the Yakutat formation [Alaska], in Harriman Alaska Exped., v. 4, Geology and Paleontology, p. 125-146, pls. 11-21. 
Unger, Franz, 1839a, Reisenotizen vom Jahre 1838: Steiermarkischen Zeitschr., Neue Folge, Band 5, p. 75-128.

1839b, Geognostische Bemerkungen über die Badelhöhle bei Peggau: Șteiermarkischen Zeitschr., Neue Folge, Band 5, p. 5-16 [1838].

- 1841-47, Beiträge zur Flora der Vorwelt-Chloris Protogaea : Teil 1, p. 116, pls. 1-5, 1941 ; Teile 2 , 3, p. 17-44, pls. 6-15, 1842 ; Teile 4, 5, p. 45-93, pls. 16-25, 1843; Teile 6, 7, p. 6, 7, p. xxv-cx, pls. 26-35, 1945; Teile 8-10, p. 94-150, pls. $36-50,1947$.

1842a, Synopsis lignorum fossilium plantarum acramphibryarum, in Endlicher, Stephano, Genera plantarum Supp. 2, app. p. 10-102.

1842b, Über die Untersuchung fossiler Stämme holzartiger Gewächse:

Neues Jahrb., p. 149-178.

1845, Synopsis plantarum fossilium : 330 p., Leipzig.

1848, Die fossile Flora von Parschlug: Steiermarkischen Zeltschr., Neue Folge, Band 9, p. 26-62.

1849, Einige interessante Pflanzenabdrücke aus der königl Petrefactensammlung in München : Bot. Zeitung, Band 7, p. 345-353, pl. 5.

1850a, Genera et species plantarum fossilium: $627 \mathrm{p}$., Vienna.

1850b, Blätterabdrucke aus dem Schwefelflotze von Swoszowice in Galicien : Haidinger, Wilhelm, Naturwiss. Abh., Band 3, Teil 1, p. 121-128, pls: 13, 14, Vienna.

1851, Die fossile Flora von Sotzka: K. Akad. Wiss. Denkschr., Band 2, p. 131-197, pls. 22-68, Vienna.

1852, Iconographia plantarum fossilium-Abbildungen und Beschreibungen fossiler Pflanzen: K. Akad. Wiss. Denkschr., Band 4, p. 73-118, pls. 24-25, Vienna.

1854a, Ein fossiles Farnkraut: K. Akad. Wiss. Denskchr., Band 6, p. 137151, pls. 1-4, Vienna.

1854b, Zur Flora des Cypridinenschiefers : K. Akad. Wiss. Sitzungsber., Band 12, p. 595-600, Vienna.

___ 1854c, Die fossile Flora von Gleichenberg: K. Akad. Wiss. Denkschr., Band 7, p. 157-184, Vienna.

- 1856, Beitrag zur Palaeontologie des Thüringer Waldes, Teil II, Schiefer und Sandstein Flora: K. Akad. Wiss. Denkschr., Band 11, p. 139-186, pls. 1-13, Vienna.

1858a, Der versteinerte Wald bei Cairo und einige andere Lager verkieselten Holzes in Ägypten: K. Akad. Wiss. Sitzungsber., Band 23, p. 209-233, pls. 1-3, Vienna.

1858b, Über fossile Pflanzen des Süsswasser-Kalkes und Quarzes : K. Akad.

Wiss. Denkschr., Band 14, p. 1-12, pls. 1-3, Vienna.

1860-65, Sylloge plantarum fossilium-Sammlung fossiler Pflanzen besonders aus der Tertiar-Formation: K. Akad. Wiss. Denkschr.; Band 19, p. 1-48, pls. 1-21, 1860; Band 22, p. 1-36, pls. 1-12, 1863; Band 25, p. 1-76, pls. 1-24, 1865, vienna.

1862, Wissenschaftiche Ergebnisse einer Reise in Griechenland und in den Ionischen Inseln: 213 p., Vienna.

1864, Fossile Pflanzenreste aus Neu-Seeland-Novara Expedtion, Geologischer Theil, Band 1, Theil 2, Palaontologie, p. 1-13, pls. 1-5.

1870, Anthracit-Lager in Kärnthen: K. Akad. Wiss. Sitzungsber., Band 60 , p. 777-792, pls. 1-3, Vienna.

Vaffier, Adolph, 1901, Etude géologique et paléontologique du carbonifère inférieur du Maconnais: Lyon Univ., Annales, nouv. sér., tome 1, fasc. 7, p. 1-159, pls. 1-12. 
Vanuxem, Lardner, 1842, Geology of New York, Part III, comprising the survey of the third geological district: $306 \mathrm{p}$., Albany.

Varma, Chandra Prakash, 1950, Un nouveau genre d'Algue calcaire (Dasycladaceae) des "Ranikot beds" (Paléocène) dans le Salt Range du Pendjab. [A new genus of calcareous alga (Dasycladaceae) from the Ranikot Beds (Paleocene) of the Punjab Salt Range] : Current Sci., v. 19, no. 7, p. 207208, Bangalore.

Vater, Henrich, 1884, Die fossilen Hölzer der Phosphoritlager des Herzogthums Braunschweig: Deutsche geol. Gesell. Zeitschr., Band 35, p. 783-853, pls. 27-29.

Velenovsky, Josef, 1882, Vorläufiger Bericht über die dicotyledon Pflanzen der böhmischen Kreideformatton : K. Böhmische Gesell. Wiss., Prag, Sitzungsber., p. 212-219.

— 1883, Die Flora der böhmischen Kreideformation: Beitr. Paläontologie Oesterr.-Ungarns u. des Orients, Band 3, Heft 1, p. 1-22.

— 1884, Die Flora der böhmischen Kreideformation: Beitr. Paläontologie Oesterr.-Ungarns u. des Orients, Band 3, p. 49-61, pls. 16-23.

1885a, Die Gymnospermen der böhmischen Kreideformation : 34 p., 13 pls., Prague.

- 1885b, Die Flora der böhmischen Kreideformation: Beitr. Paläontologie Oesterr.-Ungarns u. des Orients, Band 5, p. 62-75, pls. 24-31.

- 1887, Neue Beiträge zur Kenntnis der Pflanzen des böhmischen Cenomans :

K. Böhmische Gesell. Wiss., Prag, Sitzungsber., 1886, p. 632-645.

_ـ 1889, Kretena Ceskeho Cenomanu: K. Böhmische Gesell. Wiss., Prag, Abh., Band 3, p. 1-75.

Velenovsky, Josef, and Viniklar, Ladislav, 1926, Flora cretacea bohemiae : Czechoslovakia, Statni Geol. Ustav, Ceskoslovenské Repub., Rozpravy č́st 1, p. 1-54, pis. 1-7.

1927, Flora cretacea bohemiae : Czechoslovakia, Statni Geol. Ustav, Ceskoslovenské Repub., Rozpravy cást 2, p. 1-51, pls. 8-17.

1929, Flora cretacea bohemiae: Czechoslovakia, Statní Geol. Ustav, Ceskoslovenské Repub., Rozpravy cást 3, p. 1-31, pls. 17-22.

Viguier, Rene, 1907a, Sur quelques nouvelles plantes du travertin de Sézanne: Acad. sci. Paris Comptes Rendus, tome 145, p. 604-606.

- 1907b, Sur l'organisation et la position systématique du genre Sezanella Mun.-Ch.: Acad. sci. Paris Comptes Rendus, tome 144, p. 1003-1005.

Visiani, Roberto, 1858, Piante fossili della Dalmazia : R. ist. veneto Mem., v. 7, p. $423-455$, pls. $1-6$.

- 1864, Palmae pinnatae tertiariae: R. ist. veneto Mem., v. 11, p. 435-460, pls. 13-23.

- 1869, Di due nuovi generi di piante fossili: Nouvo gior. bot. Italiano, v. 1, p. 236-237, Florence.

1875, Di alcuni generi di piante fossili : R. ist. veneto, Mem., v. 18, p. $457-466$, pls. $19-26$, Venice.

Visiani, Roberto, and Massalongo, A., 1856, Flora de terreni terziarii di Novale nel Vicentino: R. accad. Sci. Torino Mem., v. 17, p. 199-243, pls. 1-13.

Viriani, Vino, 1833, Sur les restes de plantes fossiles trouvés dans les gypses tertiarires de la Stradella, près Paris: Soc. géol. France Mém., tome 1, p. 129-134, pls. 9, 10.

Volkmann, G. A., 1720, Silesia subterranea : Leipzig.

Vologdin, Alexander G., 1939, Middle Cambrian Archaeocyatha and algae from the South Urals: Moscow, Univ., Paleont. Laboratoria, Problems Paleontology Pub., v. 5, p. 245-276, pls. 1-12. 
Vologdin, Alexander G., 1948, Calcareous algae from the Breccia Zone called Kara-Taou : Akad. Nauk SSSR Izv., Ser. biol., no. 1, p. 83-91, 2 pls. [Russian.]

Walcott, Charles D., 1883, The Utica slate and related formations of the same geological horizon: Albany Inst. Trans., v. 10, p. 1-38, pls. 1, 2.

- 1899, Pre-Cambrian fossiliferous formations: Geol. Soc. America Bull., v. 10 , p. $199-244$, pls. $22-28$.

- 1914, Cambrian geology and paleontology, III, No. 2, Pre-Cambrian Algonkian algal flora: Smithsonian Misc. Coll., v. 64, no. 2, p. 77-157, pls. 4-23.

- 1919, Cambrian geology and paleontology, IV, No. 5, Middle Cambrian algae: Smithsonian Misc. Coll., v. 67, no. 5, p. 217-261, pls. 43-59.

- 1931, Addenda to descriptions of Burgess shale fossils: Smithsonian Misc. Coll., v. 85, p. 1-46, pls. 1-23.

Walkon, A. B., 1921, On Nummulospermum, gen. nov., the probable megasporangium of Glossopteris: Geol. Soc. London Quart. Jour., v. 77, p. 289-296, pl. 21. 1925, Notes on some Tasmanian Mesozoic plants: Royal Soc. Tasmania Papers and Proc., 1924, p. 73-89.

1932, Fossil plants from Mount Piddington and Clarence Siding: Linnean Soc. New South Wales Proc., v. 57, pts. 3, 4, p. 123-126.

Walton, John, 1923, On Rhexoxylon Bancroft-a Triassic genus of plants exhibiting a lianetype of vascular organization: Royal Soc. London Philos. Trans., v. 212 B, p. $79-109$, pls. 5,6 .

1925a, Carboniferous Bryophyta, II, Hepaticae: Annals Botany, v. 39, p. 563-572, pl. 13 .

1925b, On some South African woods: Annals South African Mus., v. 22, p. 1-26, pls. 1-3.

1940, An introduction to the study of fossil plants: $188 \mathrm{p}$. , London.

1949a, Calathospermum scotic um - an ovuliferous fructification of Lower Carboniferous age from Dunbartonshire: Royal Soc. Edinburgh Trans., v. 61, p. 719-728, pls. 1-3.

1949b, On some Lower Carboniferous Equisetineae from the Clyde area: Royal Soc. Edinburgh Trans., v. 61, p. 729-736, pls. 1, 2.

IValton, John, and Wilson, J. A. R., 1932, On the structure of Vertebraria: Royal Soc. Edinburgh Proc., v. 52, p. 200-207, pls. 1, 2.

Wanklyn, B. A., 1869, Description of some new species of fossil ferns from the Bournemouth Leaf beds : Annals and Mag. Nat. History, 4th ser., v. 3, p. 1012, pl. 1.

Warburg, Otto, 1897, Zwei neue fossile Phanerogamen Gattungen von der Insel Bangla: Jaarb. mijnwezen Nederlandsche Oost-Indië, boekdeel 26, p. 229234, pl. 4, Amsterdam.

Ward, Lester F., 1899, The Cretaceous formation of the Black Hills as indicated by the fossil plants: u. s. Geol. Survey 19th Ann. Rept., pt, 2, p. 523-712, pls. 57-172.

1900a, Status of the Mesozoic floras of the United States: U. s. Geor. Survey 20th Ann. Rept., pt. 2, p. 213-430, pls. 21-179.

-1900b, Description of a new genus and twenty new species of fossil cycadean trunks from the Jurassic of wyoming: Washington Acad. Sci. Proc., V. 1, p. 253-300, pls. 14-21.

1905, Status of the Mesozoic floras of the United States (second paper): U. S. Geol. Survey Mon., v. 48, pt. 1, 616 p. ; pt. 2, 119 pls.

-_ 1915, Remarks on an undescribed vegetable organism from the Fort Union group of Montana: Glimpses of the cosmos, v. 4, p. 149-150.

Watelet, Adolphe, 1866, Descriptions des plantes fossiles du bassin de Paris: 264 p., 60 pls., Paris. 
Watson, D. M. S.; 1906, On a "Fern" synangium from the Lower Coal Measures of Shore, Lancashire: Royal Micros. Soc. London Jour., p. 1-3, pls. 1-3.

1909 , On Mesostrobus, a new genus of lycopodiaceous cones from the Lower Coal Measures, with a note on the systematic position of Spencerites: Annals Botany, v. 23, p. 379-397, pl. 27.

Weber, C. A., 1891, Über zwei Torflager im Bette des Nord-Ostsee-Canales bei Grünenthal: Neues Jahrb., Band 2, p. 62-85.

1892, Über Cratopleura holsatica, eine interglacial Nymphaeacee, und ibre Beziehungen zu Holopleura Victoria Casp. sowie zu recenten Nymphaeaceen : Neues Jahrb., Band 1, p. 114-137, pls. 4, 5.

Weber, Carl Otto, 1851, Die Tertiärflora der Niederrheinischen Braunkohlenformation: Palaeontographica, Band 2, p. 117-236, pls. 18-25.

Webster, Clement L., 1920, Observations on some marine plants of the Iowa Devonian, with descriptions of new genera and species: Am. Midland Naturalist, $\nabla$. 6, p. 286-289.

Weigelt, Johannes, 1930, Über die vermutliche Nahrung von Protorosaurus und über einen körperlich erhaltenens Fruchtstand von Archaeopodocarpus germanicus aut. : Leopoldina, Band 6, p. 269-280, pls. 1-5.

Weiss, Christian Ernst, 1869-72, Fossile der jungsten Steinkohlenformation und des Rothliegenden im Saar-Rhein-Gebiete: Teil 1, p. 1-100, pls. 1-12, 1869; Teil 2, Fefte 1, 2, p. 101-212, pls. 13-20, 1871; Teil 2, Heft 3, p. 213-250, 1872.

1870a, Studien über Odontopteriden: Deutsche genl. Gesell. Zeitschr., Band 22, p. 853-888, pls. 20-21a.

1870b, [Originale und lithographirte Tafeln eines neuen fossien Coniferen-Typus aus dem untern Rothliegenden]: Naturh. Ver. preuss. Rheinlande $u$. Westphalens, Verh. Band 27, p. 47-48.

1876, Beiträge zur fossilen Flora, Teil 1, Steinkohlen-Calamarien : Abh. geol. Specialkarte Preussen, Band 2, p. 1-149, pls. 1-18.

— 1884a, Beitrüge zur Culm-Flora von Thüringen : K. preuss. geol. Landesanst. Jahrb., p. 6-100, pls. 11-15.

1884b, Beiträge zur fossiles Flora, Teil 3, Steinkohlen-Calamarien: Abh. geol. Specialkarte Preussen, Band 5, p. 1-204, pls. 1-27.

1885a, Zur Flora der ältesten Schichten des Harzes: K. preuss. geol. Landesanst. Jahrb., Band 5, p. 148-180, pls. 5-7, Berlin.

- 1885b, Review of paper by Zeiller, René : Neues Jahrb., Band 1, p. 491-492. 1889, Fragliche Lepidodendronreste im Rothliegenden und jüngeren Schichten : K. preuss. geol. Landesanst. Jahrb., Band 9, p. 159-165, Berlin.

Weiss, Christian Ernst, and Sterzel, J. T., 1893, Die Sigillarien der preussischen Steinkohlen und Rothliegenden Gebiete, Teil 2: K. preuss. geol. Landesanst. Abh., Neue Folge, Heft 2, p. 1-255, pls. 1-28.

Weiss, F. E., 1904a, A mycorhiza from the Lower Coal Measures : Annals Botany, v. 18, p. 255-265, pls. $18,19$.

—_ 1904b, A probable parasite of Stigmarian rootlets: New Phytologist, v. 3, p. 63-68.

Wessel, Phillip, and Weber, Otto, 1855, Neuer Beiträge zur Tertiärflora der niederrheinischen Braunkohlenformation: Palaeontographica, Band 4, p. 111-168, pls. 20-30.

Wethered, Edward, 1884, On the structure and formation of coal: Annals and Mag. Nat. History, 5th ser., v. 13, p. 299-300.

1886, On the structure and organisms of the lower limestone shales, Cas:boniferous limestone and upper limestones of the Forest of Dean: Geol. Mag., decade 3, v. 3, p. 529-540, pls. 14, 15, London. 
Weyland, Hermann, 1925, Die Flora des Elberfelder Mitteldevons in ihrer Bedeutung für die Kenntnis der gesamten Devonflora: Naturwiss. Ver. Elberfeld Jahrbesber., Heft 15, p. 33-47, figs. 1-15.

1938a, Beiträge zur Kenntnis der rheinischen Tertiärflora, II, Erste Ergänzungen und Berichtigungen zur Flora der Blätterkohle und des Polierschiefers von Rott im Siebengebirge: Palaeontographica, Band 83, Abt. B, p. 67-122, pls. 9-14.

1938b, Beiträge zur Kenntnis der rheinischen Tertiärflora, III, Zweite Ergänzungen und Berichtigungen zur Flora der Blätterkohle und des Polierschiefers von Rott im Siebengebirge: Palaeontographica, Band 83, Abt. B, p. 123-171, pls. 15-23.

1943, Beiträge zur Kenntnis der rheinischen Tertiärflora, VI, Vierte Ergänzungen und Berichtigungen zur Flora der Blätterkohle und des Polierschiefers von Rott im Siebengebirge: Palaeontographica, Band 87, Abt. B, p. 94-136, pls. 16-26.

1948, Beiträge zur Kenntnis der rheinischen Tertiärflora, VII, Füfte Ergünzungen und Berichtigungen zur Flora der Blätterkohle und des Polierschiefers von Rott im Siebengebirge: Palaeontographica, Band 88, Abt. B, p. 94-136, pls. 18-23.

Weyland, Hermann, and Budde, E., 1932, Fährten aus dem Mitteldevon von Elberfeld: Senckenbergiana, Band 14, p. 259-273.

Wherry, Edgar T., 1916, Two new fossil plants from the Triassic of Pennsylvania : U. S. Natl. Mus. Proc., v. 51, p. 327-329, pls. $29,30$.

White, David, 1898, Omphalophloios, a new lepidodendroid type: Geol. Soc. America Bull., v. 9, p. 329-342, pls. 20-23.

1899, Fossil flora of the Lower Coal Measures of Missouri: U. S. Geol. Survey Mon., v. 37, 467 p., 73 pls.

1902, Description of a fossil alga from the Chemung of New York, with remarks on the genus Haliserites Sternberg: N. Y. State Mus. Bull. 52, p. 593605 , pls. $3,4$.

1904, The seeds of Aneimites: Smithsonian Misc. Coll., v. 47, p. 322-331, pls. $47-48$.

- 1908, Fossil flora of the Coal Measures of Brazil : Comm. Estudos des Minas de Carvao de Pedra do Brazil, p. 339-617, pls. 5-14, Rio de Janeiro.

- 1928, Study of the fossil floras in the Grand Canyon, Ariz. : Carnegie Inst. Washington Year Book 27, p. 389-390.

1929, Flora of the Hermit shale, Grand Canyon, Ariz.: Carnegie Inst. Washington Pub. 405, 221 p., 1-51 pls.

1937, Fossil flora of the Wedington sandstone member of the Fayettevilie shale [Ark.] : U. S. Geol. Survey Prof. Paper 186-B, p. 13-40.

White, David, and Stadnichenko, Taisia, 1923, Some mother plants of petroleum in the Devonian black shales: Econ. Geology, v. 18, p. 238-252, pls. 5-9.

White, I. C., 1906, Geology of South Brazil : Science, new ser., v. 24, p. 377-379.

Whitfield, R. P., 1894, On new forms of marine algae from the Trenton limestone, with observations on Buthograptus laxus; Am. Mus. Nat. History Bull., v. 6, p. 35̃1-358, pl. 11 .

1902, Notice of a new genus of marine algae, fossil in the Niagara shale: Am. Mus. Nat. History Bull., v. 16, p. 399-400, pl. 53. ।

Whitford, A. C.. 1916. A description of two new fossil fungi: Nebr. Geol. Survey, V. 7, p. 85-92, figs. 1-13.

Wicher, Carl A., 1934, Über Abortiverscheinungen bei fossilen Sporen und ihre phylogenetische Bedeutung: Preuss. geol. Landesanst. Inst. Paläobotanik u. Petrographie Brennsteine, Band 5, p. 87-95. 
Wieland, G. R., 1928, [Cycadeoidea] : Carnegie Inst. Washington Year Book 27, p. $390-391$.

1929a, The world's two greatest petrified forests: Science, v. 69, p. 60-63. $1929 \mathrm{~b}$, Antiquity of the angiosperms : Internat. Cong. Plant Sci., Ithaca,

N. Y., Proc. 1926, p. 429-456, pls. 1-5.

1930, A reef-forming phormidioid alga: Am. Jour. Sci., v. 19, pp. 27-31.

1934, Fossil cycads, with special reference to Raumeria reichenbachiana

Goeppert sp. of the Zwinger of Dresden: Palaeontographica, Band 79B, p. $85-130$, pls. $9-20$.

1935, The Cerro Cuadrado petrified forest: Carnegie Inst. Washington Pub. 449 , p. 1-180, pls. 1-33.

Williams, Henry S., 1887, On the fossil faunas of the Upper Devonian-the Genesee section, New York: U. S. Geol. Survey Bull. 41, v. 6, p. 1-123 [485603], pls. 1-4.

Williams, R. S., 1930, Pleistocene mosses from Minneapolis, Minn.: Bryologist, v. 33, p. 33-36, pl. 5 .

Williamson, William Crawford, $1869 \mathrm{a}$, On the structure and affinities of some exogenous stems from the Coal-measures: Monthly Micros. Jour., v. 2, p. 66-72, pl. 20.

$-1869 \mathrm{~b}$, On the structure of the woody zone of an undescribed form of Calanite: Manchester Lit. Philos. Soc. Mem., v. 24, p. 155-183, pls. 1-5.

-1870 , Contributions toward the history of Zamia gigas Lindley and Hutton:

Linnean Soc. London Trans., v. 26, p. 663-674, pls. 52-53.

$-1871 a$, On the organization of the fossil plants of the Coal-measures, Part 1, Calamites: Royal Soc. London Philos. Trans., v. 161, p. 577-510, pls. 13-19.

1871b, On the organization of Volkmannia dawsoni, an undescribed verticillate strobilus from the Lower Coal Measures of Lancashire: Manchester Lit. Philos. Soc. Mem., 3d ser., v. 25, p. 28-40, pls. 1-3 [1876]. [Separate, 1871 ; volume, 1876.]

1871c, On the structure of the woody zone of an undescribed form of calamite : Manchester Lit. Philos. Soc. Mem., 3d ser., v. 4, p. 155-183, pls. 1-5. 1872, Notice of further researches among the plants of the Coal-measures: R.oyal Soc. London Proc., v. 20, p. 435-438.

1873, On the organization of the fossil plants of the Coal-measures, Part 4, Dictyoxylon, Lyginodendron, and Heterangium: Royal Soc. London Philos. Trans., v. 163, p. 377-408, pls. 22-30.

1874a, On the organization of the fossil plants of the Coal-measures, Part 6. Ferns : Royal Soc. London Philos. Trans., v. 164, p. 675-703, pls. 51-58.

1874b, On the organization of the fossil plants of the Coal-measures, Part 5, Asterophyllites: Royal Soc. London Philos, Trans., v. 164, p. 41-81, pls. 1-8. 1875 , On the organization of the fossil plants of the Coal-measures, Part 7 , Myelopteris, Psaronius, and Kaloxylon: Royal Soc. London Proc., จ. 23, p. $452-455$.

-1876a, On the organization of the fossil plants of the Coal-measures, Part 8, Ferns and gymnospermous stems and seeds : Royal Soc. London Proc., v. 25, p. 69-73.

1876b, On some fossil seeds from the Lower Carboniferous beds of Lancashire: British Assoc. Adv. Sci., Bristol, Rept. 45th Mtg., 1875, p. 159-160.

1877, On the organization of the fossil plants of the Coal-measures, Part 8, Ferns (continued) and gymnospermous stems and seeds : Royal Soc. London Philos. Trans., v. 167, p. 213-270, pls. 5-16.

$296347-55-7$ 
Williamson, William Crawford, 1878, On the organization of the fossil plants of the Coal-measures, Part 9: Royal Soc. London Philos. Trans., v. 169, p. 319364, pls. 19-25.

1880, On the organization of the fossil plants of the Coal-measures, Part 10, including an examination of the supposed radiolarians of the Carboniferous rocks: Royal Soc. London Philos. Trans., v. 171, p. 493-539, pls. 14-21.

1881, Helophyton williamsonis: Nature, v. 25, p. 124.

1883, On the organization of the fossil plants of the Coal-measures, Part 12 : Royal Soc. London Philos. Trans., v. 174, p. 459-475, pls. 27-34.

Wilson, L. R., 1943, Elater-bearing spores from the Pennsylvanian strata of Iowa : Am. Midland Naturalist, v. 30, p. 518-523, figs. 1-6.

Wilson, L. R., and Coe, E. A., 1940, Descriptions of some unassigned plant microfossils from the Des Moines series of Iowa : Am. Midland Naturalist, v. 23, p. 182-186, pl. 1 .

Wilson, W. J., 1913, A new genus of dicotyledonous plant from the Tertiary of Kettle River, British Columbia : Victoria Memorial Mus. Bull. 1, p. 87-88.

Wintle, S. H., 1870, A vegetable impression in shale: Royal Soc. Tasmania Monthly Notices, 1869, p. 2.

Witham, Henry T. M., 1833, The internal structure of fossil vegetables found in the Carboniferus and Oolitic deposits of Great Britain : 84 p., 16 pls., Edinburg.

Wodehouse, R. P., 1933, Tertiary pollen 2, The oil shales of the Eocene Green River formation: Torrey Bot. Club. Bull., v. 60, p. 479-524.

Wolff, Herbert, 1934, Mikrofossilien des pliozänen Humodils der Grube Freigerricht bei Dettingen a. M.: Preuss. geol. Landesanst. Inst. Paläobotanik $\mathfrak{u}$. Petrographie Brennsteine, Arb., Band 5, p. 55-86.

Wood, Alan, 1941, The Lower Carboniferous calcareous algae Mitcheldeania Wethered and Garwoodia gen. nov.: Geologists Assoc. London Proc., v. 52, pt. 3, p. 216-226, pls. 13-15.

1943, The algal nature of the genus Koninckopora Lee, its occurrence in Canada and Western Europe: Geol. Soc. London Quart Jour., v. 98, p. 205220 , pls. 8-10.

Wood, Horatio C., 1861a, Contributions to the Carboniferous flora of the United States: Acad. Nat. Sci. Phila. Proc. 1860, v. 12, p. 236-240.

- 1861b, Catalogue of Carboniferous plants in the Museum of the Academy of Natural Sciences, with corrections in synonymy, descriptions of new species * * : Acad. Nat. Sci. Phila. Proc. 1860, v. 12, p. 436-443.

- 1861c, Contributions to the Carboniferous flora of the United States: Acad. Nat. Sci. Phila. Proc. 1860, v. 12, p. 519-522.

Woodward, Henry, 1879, Notes on a collection of fossil shells * * from Sumatra: Geol. Mag., decade 2, จ. 6, p. 385-393, pl. 10, London.

wurm, Adolph, 1925, Geologie von Bayern, Nordbayern, Fichtelgebirge und Frankenwald: Handb. Geologie u. Bodenschätze Deutschland, Band 2, p. 1373, pls. 1-8.

Yabe, Hisakatsu, 1912, Über einige Gesteinbildende Kalkalgen ron Japan und China: Tohoku Imp. Univ. Sci. Rept., Sec. ser. (Geologie), v. 1, p. 1-9, pls. 1, 2, Sendai.

Yabe, Hisakatsu, and Endō, Seido, 1930, Fossil-nora or Mogı, mizen, and its geological significance: Jour. Geography, จ. 42, p. 599-607, Tokyo.

Yabe, Hisakatsu, and Shimakura, M., 1940a, Schizoneuropsis tokudai gen. et sp. noy., a Paleozoic plant from China: Japanese Jour. Geology and Geography, v. 17, p. 177-178, pl. 15.

1940b, Distichopteris heteropinna, gen. et sp. nov., a Paleozoic plant from China: Japanese Jour. Geology and Geography, v. 17, p. 179-180, pl. 16. 
Yabe, Hisakatsu, and Toyama, Shiro, 1928, On some rock-forming algae from the younger Mesozoic of Japan : Tohoku Univ., Sci. Rept., v. 12, no. 1, p. 141152 , pls. 18-23, Sendai.

- 1949, New Dasycladaceae from the Jurassic Torinosu limestone of the Sakawa basin, Sikohu: Japan Acad. Proc., v. 25, no. 5, p. 160-164.

Yasui, Kono, 1928, Studies on the structure of lignite, brown coal, and bituminous coal in Japan: Tokyo Imp. Univ., Faculty Sci. Jour., v. 1, p. 381-468, pls. 9-23.

Yokoyama, Matajiro, 1889, Jurassic plants from Kaga and Echizen: Tokio Imp. Univ., Coll. Sci. Jour., v. 3, pt. 1, p. 1-66, pls. 1-14.

Young, George, and Bird, John, 1822, A geological survey of the Yorkshire coast, describing the strata and fossils occurring between the Humber and the Tees ***: 335 p., 17 pls., Whitby.

Zalessky, Michael D., 1904, Végétaux fossiles du terrain carbonifère du bassin du Donetz: Russia, Comité géol., Mém., nouv. sêr. no. 13, p. 1-128, pls. 1-14, St. Petersburg.

___ 1905, Pflanzenreste aus dem unteren Carbon des Msta Bassins : RussichK. min. Gesell. Verh., Band 42, p. 316-342, St. Petersburg.

___ 1907, Beiträge zur Kenntnis der fossilen Flora des Steinkohlenreviers von Dombrawa : Russia, Comité géol. Mém., ṇouv. sér., no. 33, p. 1-68, pls. 1, 2, St Petersburg.

- 1911a, Etude sur l'anatomie du Dadoxylon tchihatcheff Goeppert sp.: Russia,: Comité géol. Mém., nouv. sér., no. 68, p. 1-29, pls. 1-4, St. Petersburg 1911b, Etudes paleobotaniques, Part I, Structure du rameau du Lepidodendron obovatum Sternberg et note preliminaire sur le Caenoxylon scotti, nov. gen et sp. : 1-16 p.

_ـ 1912, Sur le Cordaites aequalis Güeppert sp. de Sibérie et sur son identité avec la Noeggerathiopsis hislopi Bunbury sp. de la Flore du Gondwana: Russia, Comité géol. Mém., nouv. sér., livr. 86, 43 p., 7 pls., St. Petersburg. 1913, Flore gondwanienne du bassin de la Petchora : Soc. Ouralienne Amis sei., nat., Catherinebourg Bull., tome 33, p. 1-31, pls. 1-4.

- 1915, Histoire naturelle d'un Charbon: Russia, Comité géol. Mém. 139, p. 1-74, pls. 1-12, St. Petersburg.

1917, Sur le sapropelite marin de l'age silurien formé par une algue cyanophycée : Soc. paléont. Russie Annuaire, tome 1, p. 25-42, pls. 2, 3.

1918, Flore paléozoïque de la série d'Angara: Russia, Comité géol.

Mém. 174, p. 1-76, pls. 1-63, St. Petersburg.

1920, Über einen durch eine Zyanalge gebildeten marinen Sapropel silurischen alters (Kucherseit) : Centralbl. Mineralogie, 1920, p. 77-94.

1927a, Flore permienne des limites ouraliennes de l'Angaride: Russia, Comité, géol. Mém. 176, p. 1-50, pls. 1-38, Leningrad.

1927b. Premières observations microscopiques sur le schiste bitumineux dn Volgien inférieur: Soc. géol. Nord Annales, tome 51, p. 65-104, pls. 2-6, Lille.

1928, Observations sur la flore carbonifèrienne du nord du Caucase:

Cong. Stratigraphie Carbonifère, Heerlen, 1927, p. 797-803.

1929a, Observations sur quelques végétaux fossiles nouveaux: Soc. géol.

France Bull., tome 29, sér. $4^{\circ}$, p. 189-198, pls. 16-18.

1929b, [Permian plants from the Soutchan region] : Acad. Sci. U. R. S. S.

Bull., sér. 7, no. 2, p. 123-138. [Russian.]

1929c, Sur les debris nouvelles plantes permiennes: Acad. Sci. U. R. S. S. Bull. 7, p. 677-689. 
Zalessky, Michael D., 1929d, Observations sur les nouveaux specimens Psygmophyilum expansum Brongniart et sur une nouvelle plante fossile Idelopteris elegans n. g. et sp. : Acad. Sci. U. R. S. S. Bull., sér. 8 $^{\ominus}$, p. 717-728.

$1929 \mathrm{e}$, Sur le Syniopteris resterenkoi $\mathrm{n}$. g. et sp. et le Syniopteris demetriana n. g. et sp., nouveaux végétaux permiens: Acad. Sci. U. R. S. S. Bull., sér. $8^{\text {e }}$, p. 729-736.

1930a, Sur les végétaux fossiles nouveaux du carbonifère de l'Oural: Soc. géol. France Bull., sér. $4^{\circ}$, tome, 30, p. 737-741.

1930b, Lower Permian deposits in the Verkhoyansk mountains : Acad. Sci.

U. R. S. S. Bull., sér. $7^{\circ}$, p. 217-222, pls. 1, 2.

1930c, [New Lower Carboniferous plants] : Acad. Sci. U. R. S. S. Bull., sér. $7^{\mathrm{e}}, 3$, p. 223-228. [Russian.]

1930d, Two new fossil plants from the Lower Carboniferous layers of the Donets Basin : Acad. Sci. U. R. S. S. Bull., sér. $7^{\circ}$, p. 229-233.

1930e, [A Lower Carboniferous Sigillaria] : Acad. Sci. U. R. S. S. Bull., sér. $7^{\circ}$, no. 7 , p. $661-663$. [Russian.]

1930f, Sur l'extension de la flore fossile parente de la flore Gondwanienne dans la partie nord de l'Eurasie: Acad. Sci. U. R. S. S. Bull., sér. $7^{\circ}$, p. 913930. [Russian.]

1930g, Observations sur deux végétaux noveaux du dévonien supérier du basin Donetz: Acad. Sci. U. R. S. S. Bull., 1930, p. 1009-1016, pl. 1.

1931a, Structure anatomique du stipe du Petcheropteris splendida $\mathbf{n}$. g. et sp., un nouveau représentant des Osmundacées permiennes: Acad. Scl. U. R. S. S. Bull., sér. $7^{\circ}$, no. 5, p. 705-710, pls. 1, 2.

1931b, Structure anatomique du stipe du Chasmatopteris principalis, n. g. et sp., nouveau représentant des Osmundacées permiennes: Acad. Sci. U. R. S. S. Bull., sér. $7^{\circ}$, no. 5, p. 715-720, pls. $1,2$.

1932, Observations sur l'extension d'une flore fossile voisine de celle de Gondwana dans la partie septentrionale de l'Eurasie: Soc. géol. France Bull. sér. $5^{\mathrm{e}}$, tome 2, p. 109-129.

1933a, Observations sur les végétaux du terrain permien inférieur de l'Oural, II : Acad. Sci. U. R. S. S. Bull., 1933, p. 1093-1103.

1933b, Sur un nouveau végétal du carbonifère inférieur Caragandites rugosus: Acad. Sci. U. R. S. S. Bull., 1933, p. 1383-1385.

1933c, Observations sur trois végétaux nouveaux paléozoĩques: Acad. Scl.

U. R. S. S. Bull., 1933, p. 1386-1390.

1934a, Sur un nouveau végétal dévonien Blasaria sibirica n. g. et n. sp.:

Acad. Sci, U. R. S. S. Bull, p. 235-239.

- 1934b, Observations sur les végétaux permiens du bassin de la Petchora, I : Acad. Sci. U. R. S. S. Bull., 1934, p. 241-290.

1934c, Observations sur les végétaux nouveaux du terrain permien du bassin de Kousnetzk, II : Acad. Sci. U. R. S. S. Bull., 1934, p. 743-776.

1934d, Sur queques végétaux fossiles nouveaux du terrain houlller du Donetz: Acad. Sci. U. R. S. S. Bull., 1934, p. 1105-1117.

- 1935a, Sur un bois nouveau Metacaenoxylon carpentieri Zalessky du ter-

rain permien supérieur du bassin de Kousnetzk : Acad. sci. U. R. S. \$. Bull., no. 5, p. 739-746, 2 pls.

- 1935b, Structure anatomique du stipe d'une nouvelle osmondée du terrain permien du bassin de Kousnetzk : Acad. sci. U. R. S. s. Bull., no. 5, p. 747725 , pls. 1-3.

1936a, Sur quelques plantes nouvelles du systeme anthracolithique du bassin de Kousnetzk: Moscow, Univ., Paleont, Laboratororira, Problems Paleontology Pub., v. 1, p. 223-236. 
Zalessky, Michael D., 1936b, Sur deux nouvelles Lycopodinées permiennes: Moscow, Univ., Paleont. Laboratorira, Problems Paleontology Pub., v. 1, p. 237243.

1936c, Sur la flore triasique de la rivière Sourakai en Bachkirie: Moscow Univ., Paleont. Laboratorira, Problems Paleontology Pub., v. 1, p. 245-250. 1937a, Flores permiennes de la plaine russe ***: Moscow, Univ., Paleont. Laboratoria, Problems Paleontology Pub., จ. 2-3, p. 9-32.

- 1937b, Sur la distinction de l'étage bardien dans le permien de l'Oural et sur sa flore fossile: Moscow, Univ., Paleont. Laboratoriera, Problems Paleontology Pub., v. 2-3, p. 37-101.

- 1937c, Contribution à la flore permienne du bassin de Kousnetzk : Moscow, Univ., Paleont. Laboratoria, Problems Paleontology Pub., v. 2-3, p. 125-142.

__ 1937d, Sur quelques végétaux fossiles noveaux des terrains carbonifère et permien du bassin du Donetz: Moscow, Univ., Paleont. Laboratoria, Problems Paleontology Pub., v. 2-3, p. 155-193.

1937e, Sur deux végétaux nouveaux du dévonien supérieur: Soc. géol. France Bull., sér. $5^{\mathrm{e}}$, tome 7, p. 587-592.

- 1939a, Sur la question de la classification des spores fossile: Moscow, Univ., Paleont. Laboratorira, Problems Paleontology Pub., v. 5, p. 325-327.

1939b, Végétaux permiens du bardien de l'Oural: Moscow, Univ., Paleont. Laboratoriîa, Problems Paleontology Pub., v. 5, p. 329-374.

_ 1944, Über ein neues fossiles Lycopsidum, Tyrgaenina mamillaris n. g., n. sp.: Neues Jahrb. Monatsh., Abt. B, Heft 10, p. 250-259.

- 1948, Über einen neuen Vertetrer der Sphenophylleae, Suvundukia aciculata gen. nov. et. spec. nov.: Neues Jahrb. Monatsh., Abt. B, 1945-48, Hefte 1-4, p. 42-46, 7 figs.

Zeiller, René, 1879, Explication de la carte géologique de la France, Second Partie, Végétaux fossiles du terrain houiller, p. 1-185, pls. 159-176.

- 1883, Fructifications de fougères du terrain houiller: Annales sci. nat., Botanique, sér. $6^{\circ}$, tome 16, p. 177-207, pls. 9-12.

- 1886-88, jétudes des gîtes minéraux de la France--Bassin houiller de Valenciennes, description de la flore fossile : 731, p., 1888 ; Atlas, 96 pls., 1886. 1890, Études des gîtes minéraux de la France-Bassin houiller et permien d'Autun et d'Epinac, Fasc. II, Flore fossile, p. 1-299, pls. 1-27.

1894, Notes sur la flore des couches permiennes de Trienbach (Alsace) : Soc. géol. France Bull., sér. $3^{e}$, tome 22, p. 163-182, pls. $8,9$.

- 1897, Observations sur quelques fougères des dépôts houillers d'Asie Mineure: Soc. bot. France Bull., sér. $3^{\circ}$, tome 4, p. 195-218, pl. 6.

- 1898, Contribution à l'étude de la flore pteridologique des schistes permiens de Lodeve: Mus. histoire nat. Marseille Annales, tome 1, fasc. 1, p. $9-69$, pls. $2-4$.

1899, tutude sur la flore fossile du bassin houiller d'Heraclee: Soc. géol.

France, Mém. Paléontologie, tome 8, fasc. 4, Mém. 21, p. 1-56, pls. 1-4.

1900, Elements de paléobotanique : 421 p., Paris.

1902, Observations sur quelques plantes fossiles des Lower Gondwanas:

India Geol. Survey, Mem., Palaeontologia Indica, v. 2, p. 1-39, pls. 1-7. 1903, Études des gites minéraux de la France, flore fossile des gites de Charbon du Tonkin : 320 p., pls. A-F ; Atlas, 56 pls., 1902, Paris.

1906, Etudes des gites minéreaux de la France, bassin houiller et permien

de Blanzy et du Creusot: 261 p., 51 pls., Paris.

1907, Note sur quelques empreintes végétales des gites de charbon du

Yunnan meridional: Annales Mines, tome 10, p. 472-503, Paris. 
Zenker, Jonathan Carl, 1833a, Beiträge zur Naturgeschichte der Urwelt : 67 p., 6 pls., Jena.

1833b, Folliculites kollennordhemensis, eine neue fossile Fruchtart: Neues Jahrb., p. 177-179.

1837, Scolecopteris elegans Zenker: Linnaea, v. 11, p. 509-512, pl. 10.

Zerndt, Jan, 1930, Megasporen aus einem Flöz in Libiaz (Stéphanien) : Acad. Polonaise sci. Bull. internat., ser. BI, 1930, p. 39-70, pls. 1-8.

1931, Megasporen als Leitfossilien des produktiven Karbons: Acad. Polonaise sci. Bull. internat., ser. A., 1931, p. 165-183, pls. 3-10.

Zigno, Achille, 1853, Sulle cicadee fossili dell'oolite: Riv. periodica, v. 1, p. 345-349, Padua.

1856a-68, Flora fossilis formationis oolithicae-Le piante fossili dell'oolite: v. 1, livr. 1, p. 1-32, pls. 1-6, 1856; livr. 2, p. 33-64, pls. 7-12, 1858; livr. $3-5$, p. $65-223$, pls. $13-25$, 1867 ; v. 2 , livr. 1, p. $1-48$, pls. $26-29,1873$; livr. $2-3$, p. $49-120$, pls. $30-37$, 1881 ; livr. $4-5$, p. $121-203$, pls. $38-42,1885$, Padua.

$1856 \mathrm{~b}$, Sulla flora fossile dell'oolite : $R$. ist. veneto Mem., v. 6, p. 325-339. 1861 , Sopra un nuovo genere di felce fossile : R. ist. reneto sci. Atti, ser. $3^{\mathrm{a}}$, v. 6, p. 574-586, pls. 4-7.

1864, Monografia del genere Dichopteris nuovo genere di felce fossile: R. ist. veneto Mem., v. 12, p. 211-225, pls. 11-13.

1873-85, Flora fossilis formationis oolithicae-Le piante fossili dell'oolite: v. 2 , pt. 1 , p. $1-48$, pls. $26-29,1873$; pt. $2-3$, p. $49-120$, pls. $30-37,1881$; pt. $4-5$, p. 121-203, pls. $38-42,1885$.

Zittle, Karl A., 1879-90, Handbuch der palaeontologie, Band 2, Palaeophytologie. See Schimper, W. P., and Schenk, August, 1879-90.

Zwanziger, Gustav Adolf, 1872, Die Farn oder Zapfen Palmen: Carinthia, v. 62, p. 337-350.

Anonymous [probably Sternberg, Grafen Kaspar], 1827, Review of Artis' Antediluvian Phytology : Flora, Band 1, p. 129-144, Regensburg.

1872, Rapport sur un Mémoire de M. Grand'Eury, intitule, "Flore carbonière de Département de la Loire": Acad. sci. Paris Comptes Rendus, tome 75, p. 391-411. 


\section{GENERIC INDEX OF FOSSIL PLANTS}

This index contains notes on the valid date of establishment of the genus, the type (or a representative) species, and pertinent data pertaining to the latter. For a detailed consideration of the plan of presentation, see "Introduction."

\section{$\mathbf{A}$}

AACHENOSAURUS Smets, 1888.

Aachenosaurus multidens Smets, 1888, pl. 1. See Aachenoxylon multidens (Smets) Hovelacque, 1889, p. 505.

AAOHENOXYLON Hovelacque, 1890.

Aachenoxylon multidens (Smets) Hovelacque, 1890 , p. 60 , pl. 3 ; wood, dicotyledon; Upper Cretaceous; Moresnet, Belglum.

ABAKANIELLA Chachloff, 1939.

Abakaniella devonica Chachloff, 1939, p. 91, pls. $1-3$; Middle Devonian; Minussinsk Bassin, Russia.

$\triangle B E L I E L L A$ Mügdefrau, 1937.'

Abeliella, riccioides Mägdefrau, 1937, p. 60, pl. 5, fig. 1 ; fungus mycellum; Cretaceous ; England.

ABIETIPITES Wodehouse, 1933.

Abietipites antiquus Wodehouse, 1933, p. 491 , figs. 15,16 ; pollen, intermediate Pinus-r'suga; Parachute Creek member, Green River formation, Eocene; Colorado and Utah.

ABIETITES Hisinger, 1837.

Abietites sternbergii (Nilsson) Hisinger, 1837 , p. 110, pl. 34, fig. 3.

ABIETOPITYS Kräusel, 1928.

Abietopitys perforata (Gothan) Krüusel, in Krüusel and Range, 1928, p. 30, pl. 3, flg. 6 ; pl. 4 , flgs. $1-4$; pl. 5, flgs. 3-5 ; coniferous or cordaltean stem; Karroo beds, Permian; German Southwest Africa.

ABIETOXYLON Houlbert, 1910.

Abietoxylon falunense Houlbert, 1910, p. 73, pl. 6 ; contferous wood, compared with Abies; Tertiary; ManthelanBossee-Paulmy, France.

ABIOCAULIS Suzukt, 1910.

Abiocaulis vezoensis Suzuki, 1910, p. 181, pl. 7, fig. 112 ; petrified confferous stem; Upper Cretaceous; Hokkaido, Japan.

$\triangle B R O N I A$ Laurent, 1905.

Abronia bronnii Laurent, 1905, p. 161, pl. 12, fig. 7 ; pl. 13, figs. $1-17$; pl. 14, flgs. $6,7,12$; pl. 15, flgs. 5-7; pl. 16, figs. 5,6 ; fruit, Nyctaginaceae; Pliocene; France.
ACAOIADPHYLLUM Fontaine, 1889. Acaciaephyllum longifolium Fontaine, 1889 , p. 279 , pl. 137 , fig. 6 ; pl. 138 , flgs. 1-3 ; lear, dicotyledon ; Potomac group, Lower Cretaceous; Dutch Gap Canal, $\mathbf{V a}$.

AOACIAPHYLLITES E. W. Berry, 1914.

Acaciaphyllites grevilleoides D. W. Berry, $1914 a$, p. 45, pl. 9, figs. 9, 10 ; leaf, Mimosaceae; Black Creek formation, Upper Cretaceous ; Middendorf, Chesterfleld County, S. C.

AOACIOXYLON Schenk, 1883.

Acacioxylon antigurm Schenk, 1883a, p. 8 ; wood ; Lower Oligocene ; Libyan Desert, Tunisia. Only lllustrated species appears to be Acacioxylon tenas Felix, in Felix and Nathorst, 1893 , p. 49, pl. 3 , figs. 4, 6-8.

ACANTHOOARPUS Goeppert, 1865.

Acanthocarpus xanthioides Goeppert, 1865 a, p. 177 , pl. 26 , flg. 27 ; pl. 28 , figs. 8,9 ; seed ? Permlan ; Braunau, Bohemia.

ACANTHOPHYLLITES Grand'Eury, 1890. Acanthophyllites nicolai Grand'Eury, 1890, p. 262, fig. p. 263 ; Upper Carboniferous ; Molieres and Fontanes, France.

ACANTHOPHYTON Dawson, 1862.

Acanthophyton spinosum Dawson, 1862 , p. 324 , pl. 12, fig. 6 ; psllophyte or fragment of fern rachis; Hamilton group, Devonian; New York.

AOANTHOPTERIS Sze, 1931.

Acanthopteris gothani Sze, 1931, p. 53, pl. 7, figs. 2-4; Jurassic ; Sunchiakou, Jehol province, China.

AOANTHOTRILETES NaUmova, 1949.

Acanthotriletes primigenus Naumova, 1949 , p. 54 , fig. 14 ; Lower Cambrian; USSR.

ACERINIUM Unger, 1842.

Acerinium danubiale Unger, $1842 b$, p. 175 ; wood; Tertiary ; Austria. See also Unger, 1847 (1841-47), p. 136, pl. 44, figs. 9-11.

ACERIPHYLLUM Fontaine, 1889.

Aceriphyllum aralioides Fontaine, 1889 p. 321 , pl. 163 , fig. 8 ; leaf, dicotyledon ; Potomac group, Lower Cretaceous ; "72nd mile post," near Brooke, Va. 
AOERITES Viviani, 1833.

Acerites ficifolia Viviani, 1833, p. 131, pl. 11, fig. 5 ?; leaf, dicotyledon; Tertlary ; Stradella, near Pavia, Italy.

AOHAENITES Alexander Braun, 1851.

Achaenites ungeri Alexander Braun, in Stizenberger, 1851 , p. 83 ; dicotyledon ; Mlocene; Oeningen, Switzerland. See also Braun, Alexander, 1854, p. 147, pl. 3, fig. 18.

ACHLYITES Meschinell, 1898.

Achlyites penetrans (Duncan) Meschinelli, 1898 , p. 10 , pl. 7 , figs. $7-32$; pl. 8 , figs. 1-26 ; fungus, Phycomycete. Meschtnelli erroneously attributes this genus to Nees as a fossil form of Achlya Nees.

ACIOULARIA d'Archlac, 1843.

Acicularia pavantina d'Archiac, 1843, p. 386 , pl. 25, fig. 8a; alga; Eocene; Pisseloup, near Pavant, Dépt, de l'Aisne, France.

$\triangle C T C U L E L L A \mathrm{PIa}, 1927$.

Aciculella bacillum Pia, in Hirmer, 1927, p. 86; Dasycladaceae; Triassic. See also Pia, 1930, p. 180, fig. 1c.

AOIPHYLLA Hector, 1886.

Aciphylla pungens Hector, 1886, p. 61, fig. 24a ; Cretaceous-Tertiary ; Wangopeka, New Zealand.

\section{ACITHECA Schimper, 1879.}

Acitheca polymorpha (Brongntart) Schimper, in Schimper and Schenk, 1879 $(1879-90)$, p. 91 , fig. $66(9-12)$; fertile fern leaflet, Marattiaceae; Upper Carboniferous.

ACLISTOCHARA Peck, 1937.

Aclistochara bransoni Peck, 1937, p. 87, pl. 14, figs. 8-11; oogonium, Characeae ; Morrison formation, Jurassic? ; 18 miles northwest of Rawlins, Wyo.

ACOPHYLLUM Zalessky, 1929.

Acophyllum wolzi Zalessky, 1929a, p. 191, pl. 16 , fig. 1 ; cordaitean? leaf fragment; Carboniferous ; Donets [Donetz], Russia.

ACOROPSIS Conwentz, 1886.

Acoropsis minor Conwentz, 1886, p. 1.2, pl. 1, figs. 14-17 ; inflorescence in amber, Araceae ; early Tertiary ; west Prussia.

ACOXYLON Velenovsky and Viniklar, 1929.

Acoxylon suspectum Velenovsky and Viniklar, 1929, p. 25, pl. 17; fig. 11 ; pl. 20, fig. 1 ; pl. 22 , figs. $1-4$; incertae sedis; Cretaceous ; Slivenec, Bohemia.

ACOZAMITES Zalessky, 1936.

Acozamites elegans Zalessky, 1936c, p. 249, figs. 5,6 ; cycadophyte? follage ; Triassic; left bank river Nakaz, Bachkirie, Russia.

ACREMONITES Pia, 1927.

Acremonites succineus (Caspary) Pia, in Hirmer, 1927, p. 122 ; Mucedinaceae, Fungi Imperfecti ; Eocene. For Acremonium succineum Caspary, 1907, p. 10, pl. 1, flg. 5 .
ACROCARPUS Schenk, 1867.

Acrocarpus cuneatus Schenk, 1867, p. 134, pl. 20, figs. 9-12 ; fern? foliage ; Rhaetic ; Oberwaiz, near Bayreuth, Bavaria. [Caption to plate bears name Acropteris cuneata, apparently a misprint of the generic name.]

ACROCOILA Mueller, 1877.

Acrocoila anodonta Mueller, 1877a (1877$79)$, p. 180 ; Pliocene; Gulgong, Australia.

ACROPTERIS.

See Acrocarpus Schenk.

ACROSTICHIDES Fontaine, 1883.

Acrostichides linnaeaefolius (Bunbury) Fontaine, 1883, p. 25, pl. 6, fig. 3 ; pl. 7, figs. $1-4 ;$ pl. 8 , fig. 1 ; pl. 9 , fig. 1 ; fern foliage ; Mesozoic ; "The Gowry," Black Heath, Va. A slightly emended version of Acrostichites Goeppert.

ACROSTICHITES Goeppert, 1836.

Acrostichites williamsonis (Brongniart) Goeppert, 1836, p. 286 ; fern foliage; Oolite, Jurassic; near Scarborough, England. For Pecopteris villiamsonis Brongnlart, 1828-38, p. 324, pl. 110 , figs. $1,2$.

ACROSTICHOPHYLLUM Velenovsky, 1889. Acrostichophyllum cretaceum Velenovsky, 1889 , p. 28 , pl. 2 , figs. 22,23 ; sterile fern? front fragment; Cretaceous; Vyserovic, Bohemia. [Name only given on page 28; description on page 5 under Acrostichum cretaceum Velenovsky.]

ACROSTICHOPTERIS Fontaine, 1889.

Acrostichopteris longipennis Fontaine, 1889 , p. 107 , pl. 170, fig. 10 ; pl. 171, figs. 5, 7 ; fern foliage ; Potomac group, Lower Cretaceous; Baltimore, Md.

ACROSTIGMA Wood, 1860.

Acrostigma sp. Wood, 1860 , p. 239. [A name suggested by Wood for possible reception of Lepidodendron dubium.]

ACTINIDIOPHYLLUM Nathorst, 1888.

Actinidiophyllum sp. Nathorst, 1888, p. 228 , pl. 10 , fig. 12 ; leaf, dicotyledon; Tertiary; Japan.

ACTINOCARPUS C. F. W. Braun, 1840.

Actinocarpus mysticus C. F. W. Braun, 1840 , p. 105 ; nom. nud.

ACTINOMYCITES Ellis, 1916.

Actinomycites sp. Ellis, 1916, p. 729; fungus; Inferior Oolitic serles, Jurassic; Dunliath, Great Britain.

ACTINOMYCODIUM Zalessky, 1915.

Actinomycodium fioccidum Zalessky, 1915, p. 62 , pl. 2 , flg. 6 ; pl. 3 , figs. $1-6$; pl. 10, figs. 3,4 ; pl. 12, fig. 4 ; Actinomycete; Carbontferous; Russia.

ACTINOPHYLLUM Phillips, 1848.

Actinophyllum plicatum Phillips, in Phillips and Salter, 1848 , p. 386 , pl. 30, fig. 4 ; alga? compared with Acetabulum; Devonian ; near Stoke Edith, Woolhope district, Scotland. 
ACTINOPODIUM Hoeg, 1942.

Actinopodium nathorstii Hoeg, 1942, p. 150 , pls. 59-60; petrifled stem, some simllarity with Schizopodium of Harrls ; Devonian ; Spltzbergen.

ACTINOPORELLA Rainerl, 1922.

Soc. Italiano sci. nat. Attl 1922, v. 61, p. 72, pl. 3, flgs. 12-14 (not seen). Bee also Gothan, 1942b, p. 103.

AOTINOPTERIS Schenk, 1865.

Actinopteris peltata (Goeppert) Schenk, 1865 , p. 23 , pl. 6 , figs. $3-5$; similar to Cuclopteris.

ACTINOSTROBITES Endlicher, 1847.

Actinostrobites globosus (Bowerbank) Endlicher, 1847, p. 273. For Cupressinites globosus Bowerbank, 1840, p. 52, nl. 10, figs. 12-14, 32, 33.

ADELOCERCIS Unger, 1845.

Adelocercis radobojana Unger, 1845, p. 24.5 ; nom. nud.; Leguminosae; Miocene; Radoboj, Croatia.

$\triangle D E L O P H Y T O N$ Renault, 1900.

Adelophyton jutieri Renault, 1900, p. 424, pl. 6 ; pl. 7 , fig. 1 ; petrified lycopod stem ; Carboniferous (Culm) ; Alsace.

ADENANTHEMUM Conwentz, 1886.

Adenanthemum iteoides Conwentz, 1886, p. 92 , pl. 9 , flgs. $15-25$; flower, in amher, Saxifragaceae ; early Tertiary, west Prussia.

ADIANTES Wurm, 1925.

Adiantes sp. Wurm, 1925, p. 189 ; Carbonfferous (CuIm) ; Frankenwald, Germany.

ADIANTIDES Schimper, 1869.

Adiantides nervosus (Brongniart) Schimper, 1869 (1869-74), p, 425. For Sphenopteris nervosus Brongniart, 18.28a-38, p. 174, pl. 66, fig. 2. Based on Adiantitcs Goeppert, although authorshlp is claimed by Schimper.

ADIANTITES Goeppert, 1836.

Adiantites oblongifolius (Brongniart) Geoppert, 1836, p. 227, pl. 21, figs. 4, 5. [This species selected as the type because it is the first described and fllustrated by Goeppert, and because it corresponds with modern usage.]

ADIANTOPHYLLUM Langeron, 1899.

Adiantophyllum reticulatum Langeron, 1899 , p. 435 , pl. 2 , figs. 1,2 ; ginkgophyte? leaf; Eocene; Sézanne, France.

ADROFHYLLUM Zalessky, 1937.

Adrophyllum teschekardense Zalessky, $1937 \mathrm{~b}$, p. 82 , flg. 49 ; leaf fragment, incertae sedis; Permian; Russia.

$A E C I D I T E S$ Debey and Ettingshausen, 1859.

Aecidites stellatus Debey and Ettingshausen, 1859a, p. 212, pl. 3, figs. 2, 3 ; fungus; Cretaceous (Cenomanian); Aachen, Rhenish Prussia.
AENIGMATOPHYLLUM H a r ung and

Gothan, 1939.

Aenigmatophyllum gothani (Krestew) Hartung and Gothan, 1939, p. 520, fig. 1. For Callipteridium gothani Krestew, 1928, p. 577, pl. 39, fig. 1.

AESOULIPHYLLUM Nathorst, 1888.

Aesculiphyllum majus Nathorst, 1888, y. 200 , pl. 1, flg. 3 ; Aesculus-like lear ; Tertiary ; Japan.

AESCULOPHYLLUM Dawson, 1895.

Aesculophyllum hastingsense Dawson, 1895 , p. 149 , pl. 8 , flg. 16 ; leaf fragment compared with Aesculus; Tertiary (Paleocene or Eocene) ; Burrard's Inlet, Vancouver, British Columbia. Uncertain whether or not this is intended as a new genus.

AETHEOTESTA Brongniart, 1874.

Aetheotesta subglobosa Brongniart, 1874, p. 260 , pl. 23 , flgs. 16-18; sillclfled s e e $d$; Carbonfferous ; St.-ftienne, France.

AETHOPHYLLUM Brongnlart, 1828.

Aethophyllum stipulare Brongniart, 1828d, p. 455 , pl. 18 , fig. 1 ; incertae sedis; Sultz-les-Bains, near Strasbourg.

AGARICITES Meschinelli, 1892.

Agaricites wardianus Meschlnell, in Saccardo, 1892 , p. 745 . See also Meschtnelli, 1898, p. 1, pl. 1, figs. 1, 2 ; fungus ; Tertiary; Chiavon, Italy. Meschinell erroneously attributes this genus to Linnaeus, as a fossil form of Agaricus Linnaeus.

AGARITES Saporta, 1890?

Agarites fenestratus Saporta, 1890? (1886-91), p. 314, pl. 276, flgs. 1-4; alga ; Jurassic; Beaune, France.

AGASOPTERIS Zalessky, 1937.

Agasopteris condomana Zalessky, 1937c, p. 140, flg. 24 ; fern follage; Permian; Ossmovsky, Russia.

AGATHOXYLON Hartig, 1848.

Agathoxylon cordaianum Hartig, 1848c, p. 188 ; wood ; Triassic (Keuper) ; Coburg, Germany.

AGAVITES Abich, 1857.

Agavites araratica Abich, 1857, p. 138 , pl. 9, figs. 1-3; Miocene; Russian Armenia.

AGAVITES Visiani, 1869.

Agavites prisca Visiani, 1869, p. 237. See also Visiani, 1875, p. 465, pl. 25.

$\triangle G N O P H Y T O N$ Massalongo, 1850.

Agnophyton aristatum Massalongo, 1850, p. 29 ; alga ; Eocene; Monte Bolca, Italy.

AGNOTOCAULON Fliche, 1910.

Agnotocaulon mervillense Fllche, 1910, p. 252 , pl. 25, flgs. 3, 4 ; stem compression, incertae sedis; Triassic; Meurthe-etMoselle, Vosges, France. 
AGROSTIDIUM Massalongo, 1853.

Agrostidium priscum Massalongo, 1853c, p. 130, pl. 3, figs. 1a-b ; Eocene ; Chlavon, Italy.

AILANTHIPITES Wodehouse, 1933.

Ailanthipites berryi Wodehouse, 1933, p. 612, fig. 44 ; pollen, Simarubaceae ; Parachute Creek member, Green River formation, Eocene; Colorado and Utah.

AILANTHOPHYLLUM Dawson, 1890.

Ailanthophyllum incertum Dawson, 1890, p. 88 , fig. 25 ; leaf; Tertiary ; Tranquille River, British Columbia.

AILANTOIDITES Thomson, 1950.

Ailantoidites sp. Thomson, in Potonie, Robert, Thomson, Paul W., and Thlergart, Friedrich, 1950 , p. 58 ; nom. nud., pollen, Simarubaceae ; Miocene ; ChattAquitan, Germany.

AIPTERIS Zalessky, 1939.

Aipteris speciosa Zalessky, 1939b, p. 348, fig. 27 ; fernllke follage; Permian; Karanaiera, USSR.

AJUGINUCULA Reid and Chandler, 1926.

Ajuginucula 8mithii Reid and Chandler, 1926 , p. 127 , pl. 8 , flgs. 17,18 ; nutlet, Labiatae ; Oligocene ; Isle of Wight, England.

ALANGIOPHYLLUM Potbury, 1935.

Alangiophyllum petiocaulum Potbury, 1935, p. 79, pls. 15-19 ; leaf, Cornaceae?; upper Eocene ; La Porte, Plumas County, Callf.

ALASITES P. H. Fritel, 1923.

Reference not located. Cited in Gothan, 1942 b, p. 104

ALATAMPULLA Miner, 1935. Alatampulla bartlettii Miner, 1935, p. 600 , pl. 18, fig. 20 ; winged seed; Upper Cretaceous; Amisut, Disko Island, Greenland.

ALATISPORITES Ibrahim, 1933.

Alatisporites pustulatus Ibrahim, 1933, p. 32 , pl. 1 , fig. 12 ; spore, Carboniferous

ALBERTIA Schimper, 1837.

Albertia latifolia Schimper, 1837, p. 13. See also Schimper and Mougeot, 1844, p. 17, pl. 22; Triassic; Soultz-les-Bains, Alsace.

ALBUCASTRUM Massalongo, 1859.

Albucastrum perianthiodieum Massalongo, $1859 a$, p. 59 , pl. 23, fig. 1 ; fruit, Lillaceae: Iocene; Italy.

ALOBORNEITES Langeron, 1899.

Alchorneites mallotoides Langeron, 1899, p. 452 , pl. 4 , fig. 1 ; leaf, compared with Alohornea and Mallotus; Eocene; Sezanne, France.

ALCTCORNOPTERIS KIAston, 1887.

Alcicornopteris convoluta Eldston, 1887a, p. 152 , p1. 8, figs. 11-15; fern ? follage; Calciferous Sandstone serfes, Lower Carboniferous ; Berwickshire, Scotland.
ALCYONIDIOPSIS Massalongo, 1856. Alcyonidiopsis longobardiae Massalongo, 1856a, p. 48 , pl. 7 , figs. $1,2$.

ALCYONIUM Hisinger, 1823.

Alcyonium sp. Hisinger, 1823, p. 89, pl. 3 ; Sllurian (?) ; Christiania, Norway.

\section{ALECTORURUS Schimper, 1869.}

Alcctorurus circinnatus (Brongniart) Schimper, 1869 (1869-74), p. 203. For F'ucoides circinnatus Brongniart, 1828a38 , p. 83 , pl. 3 , fig. 3 ; alga ? Silurian ; Lake Wenern, Kinnakulle, Sweden.

ALETES Ibrahim, 1933.

Aletes sp. Ibrahim, 1933 , p. 37. Only Information given is "Sporen ohne Jede Dehiszensmarke."

\section{ALETHOPTERIS Sternberg, 1825}

Alethopteris lonchiticus (Schlothelm) Sternberg, 1825 (1820-38, tentamen), p. xxi ; fernlike foliage, probably Pteridosperm ; Carboniferous ; Saarbruck, Germany. Sternberg refers to pl. 1, fig. 22, of Schlotheim, 1804; latter is Filioites lonchitious Schlotheim. Sternberg gives spelling as lonchitidis but Brongniart uses lonchiticus; see Brongnlart, 1828a-38, p. 275, pl. 84, fgs. 1-7.

ALGACITES Schlotheim, 1822.

Algacites orobiformis Schlotheim, 1822, p. 43. For Carpolithes orobiformis Schlotheim, 1820, p. 419, pl. 27, fig. 2 ; Permain ; Ilmenau, Prussian Saxony.

ALGITES Seward, 1894

Algites valdensis Seward, 1894a, p. 4, pl. 1 , fig. 1 ; alga ; Wealden ; Ecclesbourne, near Hastings, England.

ALISMACITES Saporta, 1862.

Alismacites lancifolius Saporta, 1862, p. 228 ; leaf, compared with Alisma; Tertiary; France.

ALISMAPHYLLITES Knowlton, 1917.

Alismaphyllites crassifolium Knowlton 1917 , p. 286, pl. 55, fig. 1 ; leaf, Alismaceae?; Raton formation, Eocene; Trinidad, Colo.

ALISMAPHYLLUM E. W. Berry, 1911

Alismaphyllum victormasoni (Ward) $\mathbf{E}$. W. Berry, 1911a, p. 452, pl. 79, fig. 5 ; leaf, Alismaceae; Patapsco formation, Lower Cretaceous; White House Bluff, Va.

ALISPORITES Daugherty, 1941.

Alisporites opti Daugherty, 1941, p. 98 pl. 34, fig. 2 ; spore, incertae sedis; Chinle formation, Triassic; Arizona.

ALLANTODTOPSIS Knowlton and Maxon, 1919.

Allantodiopsis erosa (Lesquereux) Knowlton and Maxon, in Knowlton, 1919, p. 61. For Pteris erosa Lesquereux, 1878a, p. 53 , pl. 4 , fig. 8 ; pinnule fragment ; Tertlary ; near Trinidad, N. Mex. 
ALLICOSPERMUM Harris, 1935.

Allicospermum øystum Harris, 1935, p. 121, pl. 9, figs. 1-10, 13, 18 ; gymnosperm seed; late Triassic; Scoresby Sound, east Greenland.

ALLOASTEROPHYLLITES Geyler, 1879.

Alloasterophyllites densifolius (Grand'Eury) Geyler, 1879, p. 795. For Asterophyilites densifolius Grand'Eury, 1877, p. 300, pl. 32, flg. 2; Upper Carboniferous; Sagnat Midi, Peron, France.

ALIJOTOPTERIS Henry Potonie, 1897.

Alloiopteris quercifolia (Goeppert) Henry Potonie, 1897 (1897-99), p. 139, fig. 132. This appears to be valid date although name (Aloipteris) introduced by Potonle, 1894, p. xIvili.

ALLOXYLON Zalessky, 1927.

Allosylon primordiale Zalessky, 1927a, p. 45 , pl. 28, flgs. 1-10 ; coniferous wood ; Permian; Aktyubinsk district, Tourgai province, Russia.

ALMARGEMIA Florin, 1933.

Almargemia dentata (Heer) Florin, 1933, p. 101, pl. 16 , figs. 1-7; cycadophyte leaf ; Cretaceous (Aptian) ; Almargem, Portugal.

$\triangle L N I P H Y L L U M$ Nathorst, 1886.

Alniphyllum sp. Nathorst, 1886a, p. 53 ; nom. nud.

ALNIPOLLENITES Robert Potonie, 1934.

Alnipollenites verus Robert Potonie, 1934, p. 58 , pl. 2, figs. $13,17,18,25,26$; pl. 6 , flg. 28; pollen, Betulaceae; Tertiary (Braunkohle). See also Potonie, Robert, and Venitz, H., 1934, p. 25.

ALNITES Hisiuger, 1837.

Alnitcs friesii (Nilsson) Hisinger, 1837, p. 112 , pl. 34 , fig. 8 .

ALNITES Deane, 1902.

Alnites latifolia Deane, 1902a, p. 63, pl. 15, fig. 4 ; leaf fragment compared with Alnus; Tertiary; Wingello, New South Wales.

ALNOPHYLLUM Staub, 1887.

Alnophyllum reussii (Ettingshausen) Staub, 1887, p. 267. For Alnites reussii Ettingshausen, 1853, p. 39, pl. 31, figs. 13-17; leaf, Betulaceae; Tertiary; Haering, Tirol [Tyrol], Austria.

ALNOXYLON Fellx, 1884.

Alnoxylon vasculosum Felix, 1884, p. 10, pl. 1, fig. 1 ; wood ; Tertiary ; Medgyazo, Hungary.

ALOIOFTERIS.

See Alloiopteris Henry Potonla.

ALOITES Visiani, 1869.

Aloites italica Visiani, 1869, p. 237 ; Tertiary ; Sostizzo, Italy.

ALSINITES Cockerell, 1925.

Alsinites revelatus Cockerell, 1925, p. 7, pl. 1, fig. 2 ; plants with flowers, Alsinaceae; Eocene; Roan Creek opposite Salt: Wash, Colo.
ALSOPHILINA Dormitzer, 1853.

Alsophilina kauniciana Dormitzer, in Krejæ̌l, 1853, p. 28, pl. 1 ; Cretaceous ; Kaunitz, Bohemia. See also Potonle, Henry, 1899, p. 67; and Posthumus, 1931.

ALSOPHILITES HIrmer, 1927.

Alsophilites polonica (Raciborski) Hirmer, 1927 , p. 641 ; fertile foliage, Cyatheaceae ; Jurassic ; Cracow, Poland.

AMADOKIA Zalessky, 1931.

Acad. scl. U. R. S. S. Bull. 1931b, p. 577 ; Lycopidiales; Upper Devonian (not seen). See also Gothan, 1942b, p. 104.

AMANSITES Brongniart, 1849.

Amansites dentata Brongniart, 1849, p. 58. For F'ucoides dentatus Brongniart, 1828 (1828a-38), p. 70 , pl. 6, figs. 9-12 ; graphtolite?; Ordovician( ?); Pointe Levi, near Quebec, Canada.

AMBAROXYLON Houlbert, 1910.

Ambaroxylon lecointrae Houlbert, 1910, p. 74 , pl. 7 ; wood compared with Liquidambar; Tertlary ; Manthelan-BosséePaulmy, France.

AACBERITES Lomax, 1911.

Amberites sp. Lomax, 1911, p. 128, pl. 5, figs. 18,19 ; a name applied to ambercolored bodies in coal; Arley coal seam (and others), Upper Carboniferous; Atherton, Lancashire, England.

AMDRUPIA Harris, 1932.

Amdrupia stenodonta Harris, 1932a, p. 29, pl. 3, fig. 4; gymnosperm leaf; Lepidopteris zone, Rhatic; Scoresby Sound, east Greenland.

AMEGHINOITES Spégazzini, 1924.

A meghinoites desiderata Spegazzinl, 1924a, p. 102 , fig. p. 103 ; leaf, dicotyledon; Eocene; Patagonia.

AMESONEURON Goeppert, 1852.

Amesoneuron noeggerathiae Goeppert, $1852 a$, p. 264, pl. 33, flg. 3a ; fragment of palm leaf ; Tertlary ; Germany.

AMICDOPHYLLUM.

Error for Ancistrophyllum, in Eritsch, 1908, p. 23.

AMMATOPHYLLUM Zalessky, 1936.

Ammatophyllum uninervium Zalessky, 1936a, p. 223 ; Carbontferous ; Kuznets Basin, Russia.

AMMATOPSIS Zalessky, 1937.

Ammatopsis mira Zalessky, 1937b, p. 78, fig. 44; shoot bearing long slender leaves, Coniferales ; Permian; Russia.

AMOMOOARPUM Brongniart, 1828.

Amomocarpum depressum Brongniart, 1828b, p. 137. Apparently first lllustrated species is Amomocarpum affine Sahni, 1938, p. 67, 99, figs. 6, 7.

AMOMOPHYLLUM Watelet, 1866.

Amomophyllum tenua Watelet, 1866, p. 78, pl. 17, figs. 3, 4 ; leaf fragments, ZIngiberaceae?; Tertlary; Vervins, Erance. 
AMPELOCISSITES E. W. Berry, 1929. Ampelocissites lytlensis E. W. Berry, 1929 a, p. 39 , fig. 1 ; seed, Vitaceae ; Wilcox group, Eocene; near Lytle, Atascosa County, Tex.

AMPELOPHYLLITES Knowlton, 1919.

Ampelophyllites attenuatus (Lesquereux) Knowlton, 1919, p.. 67. For Ampelophyllum attenuatum Lesquereux, $1876 \mathrm{~b}$, p. 396. See also Lesquereux, 1876a, p. 354, pl. 2, fig. 3 .

AMPELOPHYLLUM Massalongo, 1859.

Ampelophyllum noeticum Massalongo, $1859 \mathrm{a}$, p. 89 , pl. 37 , figs. 1,2 ; leaf and infructescence, Vitaceae ; Eocene ; Italy.

AMPELOPHYLLUM Lesquereux, 1876.

Ampelophyllum frmus Lesquereux, $1876 \mathrm{~b}$, p. 396 ; leaf ; Cretaceous.

AMPELOXYLON Fliche, 1899.

Ampeloxylon cineritarum Fliche, 1899a, p. 321; wood; Pliocene; Pas de la Mougudo, France. See also Laurent, 1905, p. 210, pl. 17, fig. 11.

AMPHIBENNETITES Fliche, 1896.

Amphibennetites bleicher Fliche, 1896, p. 163 , pl. 14 , fig. 1 ; pl. 5 , flg. 2 ; cycadophyte cone; Cretaceous (Albien); Revigny, France. See also Seward, 1917 , p. 418.

AMPHIBRYOPHYLLUM Debey, 1881.

Amphibryophyllum carinatum Debey, in Mourlon, 1881, p. 133 ; nom. nud.

AMPHITOA Pomel, 1849.

Amphitoa ambigua (Brongniart) Pomel, 1849 , p. 353. For Oulmites ambiguus Brongniart, in Cuvier and Brongniart, 1822 , p. 558, pl. 8, flg. 6; Eocene; Grignon, France.

AMPHITOITES Desmarest, 1822.

Amphitoites parisiensis Desmarest, in Cuvier and Brongniart, 1822, p. 234, pl. 8, fig. 10.

AMPHORIDIUM Massalongo, 1852.

Amphoridium baldense Massalongo, 1852b, p. 177, figs. 1-5 [unnumbered plate]; lichen?; Jurassic ; Monte Baldi, Italy.

AMPHORISPERMUM Harris, 1932.

Amphorispermum ellipticum Harris, 1932b, p. 15, fig. 4 ; seed, Caytoniales ; Lepidopteris bed, Rhaetic; Scoresby Sound, east Greenland.

AMYDROSTROBUS Harris, 1935.

Amydrostrobus groenlandicus Harris, 1935 , p. 148 , pl. 29 ; male cone, some resemblance to Pinus; Dictyophyllum bed, Lepidopteris zone, Rhaetlc; Scoresby Sound, east Greenland.

AMPELON Williamson, 1874.

Amyelon radicans Willamson, $1874 \mathrm{~b}$, p. $67-68$, pl. 7 , fig. 46 ; pls. 8,9 ; root, Cordaitales; Carboniferous; England. Williamson (1872, p. 436) introduced name Amyelon but no specific designa- tion ; in this reference he refers the fossil previously described by himself as Dictyoxylon radicans to Amyelon.

AMYLOXYLON Hartig, 1848.

Amyloxylon huttonii Hartig, 1848a, p. 170 ; wood ; Tertiary ; Germany.

$A M Y L O X Y L U M$.

In Post and Kuntze, 1904, for Amyloxylon Hartig.

ANABACAULUS Emmons, 1857.

Anabacaulus duplicatus Emmons, 1857, p. 26, fig. 6 ; Permian( ?) ; Chatham County, N. C.

ANABARA Vologdin, Chernyskev, Kiparisova, 1937.

Anabara plana Vologdin, Chernyskev, and Kiparisova. This reference not checked; reported by J. H. Johnson, 1943, as follows: Vologdin, A., Chernyskev, B. B., and Kiparisova, D. D., Palaeontology of the Soviet Arctic: Arctic Inst. Trans., v. 91 , p. 1-255; alga; Silurian; Soviet Arctic.

ANABATHRA Witham, 1833.

Anabathra pulcherrima Witham, 1833, pl. 8, flgs. 7-12, pl. 16, fig. 7 ; Allenbank, Berwickshire, Scotland.

ANACARDIOPHYLLUM Ettingshausen, 1870.

Anacardiophyllum dubium Ettingshausen, $1870 a$, p. 90 ; leaf, Anacardiaceae ; Miocene ; Moskenberg, Styria.

ANAOARDIOXYLON Felix, 1882.

Anacardioxylon spondiaeforme Fellx, 1882a, p. 70; Tertiary ; Antiqua, West Indies. See also Felix, 1883a, p. 16, pl. 2, figs. 7, 9 .

ANACARDITES Saporta, 1861.

Anacaraites spectabilis Saporta, in Heer, 1861 , p. 149 ; leaf, Anacardiaceae ; Tertiary. First illustrated specles: Anocardites alnifolius Saporta, 1862, p. 201, pl. 2, fig. 1.

ANACHOROPTERIS Corda, 1845.

Anachoropteris pulchra Corda, 1845, p. 86 , pl. 56 ; petiole with involuted vascular strand; Upper Carboniferous ; Radnitz, Bohemia. See also Posthumus, 1931 .

ANARTHROCANNA Goeppert, 1845.

Anarthrocanna deliquescens Goeppert, 1845 , p. 379 , pl. 25 ; Upper Carboniferous ; village of d'Afonino, Siberia.

ANCHICODIUM J. H. Johnson, 1946.

Anchicodium funile J. H. Johnson, 1946, p. 1100 , pl. 2 , fig. 8 ; pl. 3, flg. 4 ; pl. 7, fig. 1 ; alga, Codiaceae; Wakarusa limestone and Auburn shale of Kansas usage, Pennsylvanian; Kansas.

ANOISTROPBYLLUA Goeppert, 1841. Ancistrophyllum stiomariaeforme Goep. pert, 1841 (1841c-46), p. 67, pl. 17, figs. 1-3; Lonchopteris-like follage; Devonian ; Landshut, Silesia. 
A.NDRIANIA Braun, 1848.

Andriania baruthina Braun, in Münster, 1843, p. 45, pl. 9, figs. 1, 2 ; Lower Lias (Lower Jurassic) ; Theta near Bayreuth, Bavaria. Andriania polycarpa Braun, 1840, p. 101 ; nom. nud.

ANDROLEPIS Nathorst, 1902.

Androlepis ambigua Nathorst, 1902b, p. 6, pl. 1 , figs. 12,13 ; fragment of cycadophyte microsporophyll ; Rhaetic ; Palsjo, Sweden.

$\triangle N D R O M E D I T E S$ Ettingshausen, 1851.

Andromedites paradoxus Ettingshausen, 1851 , p. 19, pl. 3, fig. 10 ; leaf, Erica ceae ; Tertiary ; Vindobonam, Austria.

ANDROSTACHYS Grand'Eury, 1877.

Androstachys frondosus Grand'Eury, 1877, p1. 17, fig. 3. [This generic name is apparently a mistake for Schizostachys. The binomial Schizostachys frondosus Grand'Eury appears in the text, p. 201, and refors to the figure noted above. See discussion by Schopf, 1948, p. 687.]

ANDROSTROBUS Schimper, 1870.

Androstrobus zamioides Saporta, in Schimper, 1870 (1869-74) p. 199, pl. 72 , figs. 1-3 ; cycad cone simllar to Dioon and Zamia; Jurassic (Bathonian); Etrochey, France.

ANDROVETTIA Hollick and Jeffrey, 1909.

Androvettia statenensis Hollick and Jeffrey, 1909 , p. 22 , pls. $3,7,28,29$; coniferous "leaves"; Cretaceous; Krelscherville, Staten Island, N. $\mathbf{Y}$.

ANEIMIDIUM Schimper, 1869.

Aneimidium mantelli (Dunker) Schímper, 1869 (1869-74), p. 486, pl. 31, fig. 13 ; fern frond fragments, supposed simllarity to Aneimia; Wealden; Borgloh, northern Germany.

ANEIAITES (Dawson) Ettingshausen, 1865. Aneimites obtusolobus (Naumann) Ettingshausen, 1865 , p. 249 . For Odontopteris obtusiloba Naumann, in Geinitz and Gutbler, 1849 (1848-49), p. 14, pl. 8, figs. 9-11. [The generic name originally suggested by Dawson, Aneimites acadica Dawson, 1860 , p. 461 , but used as a subgenus.]

$\triangle N E U R O P H Y T O N$ Kräusel and Weyland, 1923.

Aneurophyton germanicum Kräusel and Weyland, 1923, p. 172, pl, 7, figs. 4-7 ; pl. 8 , figs. $6-13$; pl. 9, figs. $14-19$; Psilophytales; Devonian; Germany.

ANGARIDIUM Zalessky, 1933.

Angaridium bardense Zalessky, 1933a, figs. 4-6; ginkgophyte? follage; Permian; Irroutaia Katouchka, Russia.

ANGARODENDRON Zalessky, 1918.

Angarodendron obrutchevi Zalessky, 1918, p. 54 , pl. 13 , fig. 5 ; pl. 62 ; pl. 63 ; lycopod? stem impression; Carboniferous ; Bedoby village; Kirghises Steppes, Russia.
ANGAROPTERIDIOM Zalessky, 1937.

Angaropteridium cardiopteroides (Schmalhausen) Zalessky, in Tchirkova, 1037, p. 218; figs. 10-15; fernlike pinnules; Permian; Petite Boukon, Russia. [The generic name prevlously mentioned, Zalessky, 1930 b, p. 218 ; nom. nud.]

ANGAROPTERIS Chachlow and Pollak, 1036.

Neues Jahrb., 1936, Bell.-Band 76, Abt. B, p. 334 ; Pteridospermae ; Permian (not seen). See also Gothan, 1942b, p. 105.

ANGIODENDRON Elchwald, 1860.

Angiodendron orientale Elchwald, 1860 , p. 263 , pl. 19, fig. 9 ; stem cast, Incertae sedis ; Carboniferous; Kaschkabasch, near Artinsk, Russla.

ANGIOPTERIDIUM Schimper, 1869.

Angiopteridium muensteri (Goeppert) Schimper, $1869(1869-74)$, p. 603, pl. 35, figs. 1-6; fern leaf, Marattlaceae ; Rhaetic; Bayreuth and Bamberg, $\mathrm{Ba}$ varia; Steierdorf, Hungary. See note under Marattiopsis.

ANGIOSPERMOPHYTON Hoskins, 1923.

Angiospermophyton americanum Hoskins, 1923, p. 397, figs. 1-13 ; petrified medullosan petiole; Coal No. 5, Pennsylvanlan ; Harrisburg, Ill.

ANGIOTHEOA Schimper, 1879.

Angiotheca angiotheca (Grand'Eury) Schimper, in Schimper and Schenk, $1879(1879-90)$, p. 91, flg. 66.

ANISOPHYLLUM Lesquereux, 1874.

Anisophyllum semialatum Lesquereux, 1874 , p. 98 , pl. 6, figs. 1-5; leaf, dicotyledon; Cretaceous; near Beatrice, Gage County, Nebr.

ANKYROPTERIS Stenzel, 1889.

Ankyropteris brongniarti (Renault) Stenzel, 1889 , p. 29 ; coenopterid fern ; Permian ; Autun, France. For Zygopteris brongniarti Renault, 1869 , p. 164, pls. 8-6. See also Renault, 1883, p. 101, pl. 16, fg. 1 ; and Posthumus, 1931.

ANNALEPIS Fliche, 1910.

Annalepis zeilleri Fliche, 1910, p. 272, pl. 27, figs. 3-5; lycopod cone scales?; Triassic ; Meurthe-et-Moselle, Vosges, France.

ANNONOXYLON Boureau, 1950.

Annonoxylon striatum Boureau, 1950b, p. 393, pl. 21, figs. 1, 2 ; Eocene ; Sahara, Africa.

ANNULARIA Sternberg, 1822.

Annularia spinulosa Sternberg, 1822 (1820-38), p. 32 , pl. 19 , fig. 4 ; articulate stem with foliage; Carboniferous.

ANNULARIOPSIS Zeiller, 1903.

Annulariopsis inopinata Zelller, 1903, p. 132, pl. 35, figs. 2-7 ; Annularia-like follage; Carboniferous; Tonkin and numerous other localities, see p. 137. 
ANNULARITES Halle, 1927.

Annularites ensifolius Halle, 1927, p. 19, pls. $1-4$; follage, Equisetales; Upper Shlhhotse series, Permian; central Shansi, China.

ANOEOTOMERIA Saporta, 1865.

Anoectomeria brongniartii Saporta, 1865, p. 125 , pl. 7, fig. 1 ; rhizome?, Nymphaeaceae; Tertiary; St.-Jean-de-Garguer, France.

ANOMALOFILIOITES Hollick, 1916.

Anomalofilicites monstrosus Hollick, 1916 , p. 474 , pl. 31 ; fern frond with abnormal pinnae; Fort Union formation, Eocene ; Kern Ranch, Dawson County, Mont.

ANOMALOPHYCUS Fenton and Fenton, 1937.

Anomalophycus compactus Fenton and Fenton, 1937, p. 438, pl. 3, figs. 1, 2; calcareous alga; Allentown formation, Cambrian; Port la n d, Northampton County, Pa.

ANOMALOPHYLLITES Watelet, 1866.

Anomalophyllites tricarinatus Watelet, 1866 , p. 100 , pl. 28 , fig. 105 ; leaf fragments, Palmaceae?; Tertiary; Belleu, France.

ANOMALOXYLON Fellx, 1887.

Anomaloxyion vicentinum Felix, 1887a, p. 627, pl. 25, fig. 8; wood; Tertiary ; Monte Grumi near Castelgomberto, Italy.

ANOMALOXYLON Gothan, 1910.

Anomaloxylon magnoradiatum Gothan, 1910 , p. 11, pl. 1, fig. 9-11; pl. 2, figs. 2,3 ; coniferous wood; Jurassic; Green Harbor, Spitzbergen.

ANOMASPIS Hollick and Jefirey, 1909. Anomaspis tuberculata Hollick and Jeffrey, 1909 , p. 49 , pls. $10,25,26$; coniferous cone scales; Cretaceous ; Kreischerville, Staten Ysland, N. Y.

ANOMOPTERIS Brongniart, 1828.

Anomopteris mougeotii Brongniart, $1828 \mathrm{~b}$, p. 69 ; fern foliage. See also Brongniart, $1832(1828 \mathrm{a}-38)$, p. 258, pls. 79-81.

ANOMORRHOEA Eichwald, 1844.

Anomorrhoea flscheri Elchwald, 1844, p. 144; stem, Osmundaceae; Permian (Zechstein); Orenbouxg. Russia. See also Eichwald, 1860 (1860-68), p. 102, pl. 4, figs. 3, 4; Kidston and GwynneVaughan, 1908, p. 216; Posthumus, 1931.

ANOMOZAMTTES Sehimper, 1870.

Anomozamites inconstans (Goeppert) Schimper, $1870(1869-74)$, p. $140 ; \mathrm{cy}-$ cadophyte foliage; Rhaetic; Bayreuth Bavaria. For Pterophyllum inconstans Goeppert ; first? lllustration in Schenk, 1867 (1865-67), p. 171, pl. 37, figs, 5-8.
ANONASPERMUM Ball, 1931.

Anonaspermum reidi Ball, 1931, p. 121, pl. 20, flgs. 5, 9, 11, 13 ; seeds, Anonaceae ; Yegua formation, Eocene; Turkey Creek, Brazos County, Tex.

ANOTOPTERIS Schimper, 1869.

Anotopteris distans (Presl) Schimper, $1869(1869-74)$, p. 471 , pl. 33, figs. 1 , 2 ; fern foliage; Triassic (Keuper); Stuttgart.

ANTARCTICOXYLON Seward, 1914.

Antarcticoxylon priestleyi Seward, 1914, p. 17, pls. 4-8; gymnosperm stem; Priestley glacier, Antarctica.

ANTEVSIA Harris, 1937.

Antevsia zeilleri (Nathorst) Harris, 1937, p. 35 ; pteridosperm microsporangiate organ; Lepidopteris zone, Rhaetic; Scoresby Sound, east Greenland. For Antholithus zeilleri Nathorst, 1908c, p. 20 , pl. 2 , figs. 59,60 ; pl. 4 .

ANTHERANGIOPSIS Nathorst, 1902.

Antherangiopsis rediviva Nathorst, $1902 \mathrm{~b}$ p. 20 , pl. 1 , figs. 22,23 ; cycadophyte microsporophylls; Rhaetic; Bjuf, Sweden.

A.NTHIOOOLADUS Zalessky, 1937.

Anthicocladus fimbriatus Zalessky, 1937b, p. 81 , fig. 48 ; pteridosperm male inflorescence ?; Permian ; Matveyevo, USSR.

ANTHITES Schimper, 1874.

Anthites gaudini (Heer) Schimper, 1874, p. 419 ; flower, dicotyledon; Tertiary ; near Lausanne, France.

ANTHOCARPUS Grand'Eury, 1877.

Anthocarpus botryoides Grand'Gury, 1877, p. 521 ; nom. nud.

ANTHOCEPHALE Bayer, 1893.

Anthocephale bohemica Bayer, in Fric, 1893 , p. 132, fig. 193; Cretaceous (Senonian) ; Priessen, Bohemia.

ANTHODIOPSIS Goeppert, 1864.

Anthodiopsis beinertiana Goeppert, 1864, p. 85; Unver Carbonlferous; Sllesia?. See also Goeppert, in Quenstedt, 1867. ๖. 912, pl. 86, fig. 57 .

ANTHOLITHES Brongniart, 1822.

Antholithes liliacea Brongniart, 1822, p. 320 , pl. 14, fig. 7 ; a small "bud-like" impression showing no fertile parts and of unknown affinity. A "type" specles nere seems to be of little significance because of the wide aiversity of fosglls assigned to the genus. For example, compare Antholithus noeggerathi Renault, in Renault and Zeiller, 1888, p. 593 , pl. 67 , fig. 6 ; Antholithes amisas Heer, 1868, p. 139, pl. 23, fig. 12 ; Antholithus arberi Thomas, 1925, p. 327, pl. 14, flgs. 33-40.

ANTHOLITHUS.

See Anthollthes Brongniart. 
ANTHOMYOES Gruss, 1930.

Wochenschr. Braueri, 1930, Band 42, p. 15 ; fungus; Tertiary (not seen). See also Gothan, 1942b, p. 105.

ANTHOPHYOUS Pledboeuf, 1887.

Anthophycus dechenianus (Goeppert) Piedboeuf, 1887, p. 56, pl. 3.

ANTHOPHYLLITES.

Anthophyllites devonicus (probably error for Antholithes devonicus Dawson), in Le Conte, 1882, p. 330, fig. 385.

ANTHOTYPOLITHES Schlotheim, 1820.

Anthotypolithes ranunculiformis Schlotheim, 1820 , p. 423 ; described as ranunculaceous flower?; Permian; Frankenberg, Hesse.

ANTHRACOCHONDRUS KuSta, 1898.

Anthracochondrus nyranensis Kusta, 1898, p. 220; Permian; Nyran, Bohemia. See also Kusta, in Ryba, 1904, p. 352, pl. 17, figs. 1, 2.

ANTHRACOMYCES Renault, 1898.

Anthracomyces cannellensis Renault, 1898, p. 205, figs. 1-3 ; fungus mycellum? in cannel coal; Carboniferous; several localities cited.

ANTHRACOPORELLA Pia, 1920.

Anthracoporella spectabilis Pia, 1920, p. 15, pl. 1, figs. 7-11; alga, Siphoneae Verticllatae; Carbontferous.

ANTHROPHYOPSIS Nathorst, 1878.

Anthrophyopsis nilssoni Nathorst, $1878 \mathrm{a}$, p. 43 , pl. 7 , fig. 5 ; pl. 8 , flg. 6 ; cycadophyte leaf fragment; Rhaetic; Bjuf, Sweden.

APACHEA Daugherty, 1941.

A pachea arizonica Daugherty, 1941, p. 55, pl. 9, fig. 2 ; sterile frond, Dipteridaceae ; Chinle formation, Upper Trlassic; Arizona.

APALOXYLON Renault, 1892.

Apaloxylon rochei Renault, 1892a, p. 157, pl. 5 ; cordaitean stem; Carboniferous; Autun, France.

APEIBOPSIS Heer, 1859.

Apeibopsis gaudini Heer, 1859, p. $40, \mathrm{pl}$. 118, flgs. 24-26 ; fruits, Tillaceae ; Tertiary ; Lausanne, Switzerland.

APHLEBIA Presl, 1838.

Aphlebia acuta (Germar and Kaulfuss) Presl, in Sternberg, 1838 (1820-38), p. 112. For Fucoides acutus Germar and Kaulfuss, 1831, p. 230, pl. 66, fig. 7 ; Carboniferous; Germany.

APELEBIOOARPUS Stur, 1877.

Aphlebiocarpus schutzei Stur, 1877, p. 304, pl. 27, figs. 1-6 ; fern foliage with associated sporangia; Lower Carboniferous (Culm); Altwasser, Silesia.
APHLEBIOPTERIS Gothan, and Zimmerman, 1932.

Aphlebiopteris boegendorfana Gothan and Zimmerman, 1932, p. 107, pl. 15, fig. 1 ; Upper Devonlan; Upper Bögendorf, S1lesia.

APHRALYSIA Garwood, 1914.

Aphralysia carbonaria Garwood, 1914, p. 269 , pl. 21, figs. 3, 4 ; rock-bullding alga ; Lower Carbonlferous ; Ravenstonedale, Westmoreland, England.

APHYLLOPTERIS (Nathorst) Arnold, 1939. Aphyllopteris delawarensis Arnold, 1939, p. 292 , pl. 10, figs. 2,3 ; Incertae sedis, Devonian ; 4 miles north of Port Jervis, N. Y. Aphyllopteris sp. Nathorst, 1915 , p. 14 , pls. $4,5,7$.

APHYLLOSTAOHYS Goeppert, 1865.

Aphyllostachys jugleriana Goeppert, 1865a, p. 14, pl. 1 ; articulate cone infructescence; Lower Jurassic (Lias); Hannover, Germany.

APHYLLUM Artis, 1825.

A.phyllum cristatum Artis, 1825, p. 16, pl. 16 ; lycopod stem impression ; Carboniferous ; Banktop, Yorkshire, England.

APHYLLUM Unger, 1856.

Aphyllum paradoxum Unger, 1856, p. 175, pl. 11, figs. $1-4$; incertae sedis ; Upper Devonian; Saalfeld, Thuringia. Earller citation: Unger, 1854, p. 589 ; nom. nud.

APICULATASPORITES Ibrahim, 1933.

Apiculatasporites spinulistratus Loose, in Ibrahim, 1933, p. 37. No figures given; Ibrahim refers to Neues Jahrb, 1932, Beil.-Band 67, Abt. B, p. 451, pl. 18.

APICULATISPORITES Ibrahim, 1933.

Apiculatisporites aculeatus Ibrahim, 1933, p. 23, pl. 6, fig. 57 ; spore ; Carboniferous. [Generic concept based on Triletes VI in Bennle and Kidston, 1886, p. 109 , pl. 3, figs. $6 \mathrm{a}-\mathrm{c}$.]

APIDIUM Stolley, 1896.

Apidium kräusei (Klesow) Stolley, 1896, p. 261, figs. $46,99$.

APLOPHLEBIS (Brongniart) Meneghini, 1857.

Aplophlebis arborescens (Schlotheim) Meneghini, 1857, p. 108, pl. D, fig. V5.

APLUDOPHYTON Massalongo, 1859.

Apludophyton scleroides Massalongo, 1859 b, p. 22 ; nom. nud.

\section{APOOINOPHYLLUM.}

See Apocynophyllum Unger.

APOCYNOCARPUM Ettingshausen, 1887.

Apocynocarpum sulcatum Ettingshausen, 1887, p. 119, pl. 13, fig. 11 ; Apocynaceae; Eocene; Vegetable Creek, near Emmaville, New South Wales. 
APOOYNOPHYLLUM Unger, 1850.

Apocynophyllum seyfriedii Braun, in Unger, 1850a, p. 433. Apparently first 11lustrated species is Apocynophyllum lanceolatum Unger, $1850 \mathrm{~b}$, p. 125 , pl. 14, fig. 14 ; leaf, Apocynaceae ; Miocene ; Radoboj, Croatia. Cited earler as Apocinophyllum, Unger, 1845, p. 230 ; nom. nud.

APOOYNOSPERMUM Reld and Chandler, 1926.

Apocynospermum striatum Reld and Chandler, 1926, p. 118, pl. 8, fig. 3 ; seed, Apocynaceae; Bembridge beds, lower Oligocene; Isle of Wight, England.

$\triangle P O R O X Y L O N$ Unger, 1856.

Aporoxylon primigenium Unger, 1856, p. 181 , pl. 13, figs. $3-11$; stem of cordaltean? affinities; Upper Devonian; Saalfeld, Thuringla. [Binominal first cited in Unger, 1854 ; nom. nud.]

APTERALETES Zalessky, 1939.

Apteraletes Zalessky, 1939a, p. 326 ; nom. nud.

APTEROMONOLETES Zalessky, 1939

Apteromonoletes Zalessky, 1939a, p. 326 ; nom. nud.

APTEROSTROBUS Gothan and Nagel, 1921.

Apterostrobus cedroides Gothan and $\mathrm{Na}-$ gel, 1921, p. 131, pl. 8 ; cone ; Coniferales ; Eocene.

APTEROTRILETES Zalessky, 1939.

$s$

Apterotriletë8 Zalessky, 1939a, p. 326; nom. nud.

APTIANA Stopes, 1912.

Aptiana radiata Stopes, 1912, p. 84 , pls. 6-8; wood, numerous suggestions as to affinity, see Edwards, 1931, p. 20 ; Lower Cretaceous (Aptian); Isle of Wight, England.

ARACEAEITES Fritel, 1910.

Araceaeites parisiense Fritel, 1910, p. 29, pl. 22, fig. 1 ; spadix, Araceae?; Paleocene; Meudon, Vanves, France.

ARACEOPHYLLUM Kräusel, 1929.

Araceophyllum engleri Kräusel, 1929, p. 13, pl. 4, flgs. 3, 4 ; leaf fragment ; Araceae; Tertlary (Pliocene?) ; Sungi Tjaban, South Sumatra.

ARACHNOXYLON Read, 1938.

Arachnoxyton kopf (Arnold) Read, 1938, p. 602, figs. 4, 5 ; petrified stem, Psilophytales: Tully pyrites, Devonian; 1 mile east of Gooding's Landing, Canandaigua Lake, N. Y.

ARAEIS Stenzel, 1872.

Araeis axonensts (Watelet) Stenzel, 1872, p. 71. For Palmacites anonensis Watelet, 1866, p. 103, pl. 30, flg. 3 ; Eocene; Quincy-sous-le-Mont, France.

ARALIAOEA Velenovsky, 1882.

Araliacea propinqua Velenorsky, 1882, p. 217 ; nom. nud.
ARALIACITES Saporta, 1865.

Araliacites cordatus Saporta, 1865, p. 48 ; leaf, Arallaceae; Tertiary ; France.

ARALIAECARPUM Menzel, 1913.

K. preuss. geol. Landesant., Jahrb., 1913, Band 34, p. 9, pl. 1, fig. 19. ; Arallaceae ; lower Miocene (not seen). See also Gothan, 1942b, p. 106.

ARALIAEPHYLLUM Fontaine, 1889.

Araliaephyllum obtusilobum Fontaine, 1889 , p. 317 , pl. 163 , figs. 1,4 ; pl. 164 , fig. 3 ; leaf ; Potomac group, Lower Cretaceous; near Brooke, V.a.

ARALIANTHEA Massalongo, 1893.

Aralianthea brongniarti Massalongo, in Meschinelli and Squinabol, 1893, p. 403. For Fucoides obtusus Brongnlart, $1828 a-38$, p. 60 , pl. 8 , fig. 4 ; inflorescence, Araliaceae; Eocene; Monte Bolca, Italy. [Name given previously as Aralianthea brongniarti Massalongo, 1857 b, p. 777, nom. nud.]

\section{ARALINIUM Platen, 1908.}

Aralinium excellens Platen, 1908, p. 59 ; wood, early Tertiary; California.

ARALIOPHYLLUM Ettingshausen, 1868.

Araliophyllum dubium Ettingshausen, 1868b, p. 867. For Quinguefolium sp. Ludwig, 1859, p. 145, pl. 58, fig. 8 ; leaf, dicotyledon?; Miocene ; Muenzenberg, Hesse. [Unger, 1865 (1860-65), p. 72, refers to Araliophyllum denticulatum Ettingshausen but apparently the name had not been published.]

$A R A L I O P S I S$ Saporta and Marion, 1878.

Araliopsis cretacea (Newberry) Saporta and Marion, 1878, p. 78. For Sa8safras cretaceum Newberry, in Dana, 1863 , p. 471 , fig. 746 ; see also Lesquereux, 1874 , pl. 11, flgs. 1,2 ; pl. 12 , fig. 2 ; leaf, Arallaceae ; Cretaceous ; Blackbend Hills, Nebr.

ARALIOPSIS E. W. Berry, 1911.

Araliopsis cretacea (Newberry) E. W. Berry, 1911b, p. 413 ; leaf, compared with modern Sassafras; Upper Cretaceous; Bull Mountain, Cecil County, Md.

ARALIOPSOIDES E. W. Berry, 1916.

Araliopsoides breviloba E. W. Berry, 1916a, p. 878, pl. 86, fig. 2 ; leaf, Arallaceae; Raritan formation, Upper Cretaceous ; Bull Mountaln, Cecll County, Ma.

ARALIPHYLLUM Nathorst, 1888.

Araliphyllum raumanni Nathorst, 1888 , $p$ 219 , pl. 20, fig. 10 ; leaf, dicotyledon;

Mlocene; Miogamura, Iyo. province, Japan.

ARALITES Goeppert, 1854.

Aralites lanceus Goeppert, 1854, D. 130 ; Miocene; Bodenheim, Hesse; nom. nud. 
ARANETZIA Zalessky, 1934.

Aranetzia splendens Zalessky, 1934b, p. 271, flgs. 46-48; sphenopterid follage ; Permian; Pechora [Petchora] basin, Russia.

ARAUCARIOCAULON Lignier, 1907.

Araucariocaulon breveradiatum Lignier, 1907 , p. 290 , fig. 2 ; petrified stem, compared with Araucarioxylon; Upper Cretaceous (Cenomanian); Dives, France.

ARAUCARIOPHLOIOS Lignier, 1907.

Araucariophloios breveradiatum. Lignier, 1907, p. 291. 'For Araucariocaulon breveradiatum Lignier, 1907, p. 290, pl. 19, figs. 33-43.

ARAUOARIOPITYS Jeffrey, 1907.

Araucariopitys americana Jeffrey, 1907, p. 435, pls. $28-30$; araucarian wood; Cretaceous; Staten Island, N. $\mathbf{Y}$.

ARAUOARIOPSIS Caspary, 1888.

Araucariopsis macractis Caspary, 1888, p. 45. For illustrations, see Caspary, 1889 , p. 193 , pl. 14, figs. $16-20$.

ARAUCARIOSTROBUS Krasser, 1921.

Araucariostrobus mandiii Krasser, 1921b, p. 221; Jurassic; Nokolsk-Ussurysk, Russia.

ARAUCARIOXYLON Kraus, 1870.

Araucarioxylon carbonaceum (W1tham) Kraus, in Schimper, 1870 (1869-74), p. 381. For Pinites carbonaceus Witham, 1833, p. 73, pl. 11, figs. 6-9 ; Carboniferous; England.

ARAUCARITES Presl, 1838.

Araucarites goepperti Presl, in Sternberg; 1838 (1820-38), p. 204, pl. 39, flg. 4; cone, Coniferales ; Tertiary ? ; Tirol.

ARBERIA David White, 1908.

Arberia minasica David White, 1908, p. 537 , pl. 8 , figs. 8-10; regarded as inflorescence of Gangamopteris; "PermoCarbonlferous"; near Minas, Santa Catharina, Brazil. [Thls binomial previously published as Arberia minasica I. C. White, 1906 , p. 379 ; nom. nud.]

ARBVTITES Ettingshausen, 1868.

Arbutites euri Ettlngshausen, 1868a, p. 236 , pl. 39 , fig. 14 ; leaf, Ericaceae; Miocene ; Priesen, Bohemia.

ARCELLITES Miner, $193 \overline{\text { s. }}$

Arcellites disciformis Miner, 1935, p. 600, pl. 20, figs. $61,64-66$; incertae sedis; Upper Cretaceous; Skansen, Disko Island, Greenland.

ARCHAEOCALAMITES Stur, 1875.

Archaeocalamites radiatus (Brongniart) stur, 1875, p. 2, pl. 1, figs. 3-8; pls. $2-4 ;$ pl, 5, flgs. 1,2 ; articulate stems bearing fliform dichotomous leaves; Carboniferous (Culm) ; Altendorf, Mohradorf, Germany.

$296347-55-8$
AROHAEOLITHOTHAMNIUM Rothpletz, 1891.

Archaeolithothamnium nummulitioum (Gümbel) Rothpletz, 1891, p. 316, pl. 17, fig. 5 ; alga, Corallinaceae; Eocene. Correct genotype?

ARCHAEOMNIUM Britton, 1926.

Archaeomnium patens Britton, in Knowlton, 1926, p. 24, pl. 8, figs. 1, 2 ; moss, Mniaceae; Latah formation, Miocene; Spokane, Wash.

ARCHAEOPHYTON Britton, 1888.

Archaeophyton newberryanum Britton, 1888 a, p. 89 ; plant ?; Archaean; Sussex County, N. J. For full description, see Britton, 1888b, p. 123.

ARCHAEOPITYS Scott and Jeffrey, 1914.

Archaeopitys eastmanii Scott and Jeffrey, 1914 , p. 345 , pl. 38, figs. 17-19 ; petrlfled cordaitean stem; base of Waverley shale, Mississippian; Kentucky.

AROHAEOPODOCARPUS Weigelt, 1930.

Archaeopodocarpus germanicus Weigelt, 1930 , p. 269 , pl. 1 , figs. $2-7$; pl. 3, flg. 1 .

ARCHAEOPTERIDIUM Kidston, 1923.

Archaeopteridium tschermaki (Stur) Kidston, $1923 a$, p. 182 , pls. $40,41,43$; follage of Archaeopteris type; Onl Shale group, Carboniferous Limestone, Lower Carboniferous; Scotland.

ARCHAEOPTERIS Dawson, 1871.

Archaeopteris hibernica (Forbes) Dawson, 1871 , p. 48 ; "fern," at least one species of which has been demonstrated to be heterosporous; 8ee Arnold, 1939; Devonlan. For Cyclopteris hibernica Forbes, in Murchison, 1854, p. 255, fig. 51.

ARCHAEORRHIZA Torell, 1869.

Archaeorrhiza tuberosa Torell, 1869, p. 7 ; Cambrian ; Lugnas, Sweden.

AROHAEOSIGILLARIA Kidston, 1901.

Archaeosigillaria vanuxemi (Goeppert) Kidston, 1901 , p. 39 ; sigtllarian stem, sald to lack lateral parichnos scar; Carboniferous. For Sigillaria vanuxem Goeppert, 1852 b, p. 249 . For lllustration, see Dawson, 1862 , p. 307, pl. 12, fig. 7 ; and Kidston in Linnean Soc. London Jour., Botany, 1886, v. 21, p. 560 , pl. 18.

AROHAEOSIGILLARIOPSIS Gothan, 1928. Archaeosigillariopsis serotina Gothan, $1928 \mathrm{a}$, p. 1 , pl. 1 , figs. $1-4$; pl. 2 ; lycopod stem impression; Carboniferous; Flöha, Saxony.

AROHAEOTHRIX Kidston and Lang, 1921. Archaeothrix oscillatoriformis Fidston and Lang, 1021, p. 875, pl. 8, figs. 89, 90 ; slender unbranched flaments, Cyano. phyceae?; Devonian; Muir of Rlyynie, Aberdeenshire, Scotland. 
AROHAEOXYLON Krïusel, 1924.

Archaeoxylon krasseri Kräusel, 1924, p. 31 , pl. 2 ; fragment of pteridophyte? stem showing cells with bordered pits; pre-Cambrian?; Bohemla.

ARCHAEOZOON Matthew, 1890.

Archaeozoon acadiense Matthew, 1890b, p. 67 ; plant ?; Laurentian ; Green Head, St. John, New Brunswick, Canada.

ARCHAGARICON Hancock and Atthey, 1869.

Archagaricon bulbosum Hancock and Atthey, 1869 , p. 226, pl. 10 ; fungus ; Cramlington Black Shale, Upper Carbonfferous; Cramlington, Newsham, Northumberland, England.

ARCHAMPHIROA Steinmann, 1926.

Archamphiroa jurassica Steinmann, in Jaworski, 1926, p. 139, figs. 1a, b ; alga ; Jurassic; Arroyo Negro, Argentina.

ARCHIHICORIA Barbour, 1898.

Archihicoria siouxensis Barbour, 1898, p. 272 , pl. 5 ; petrified kernel of fruit, compared with Hicoria; Miocene; Badlands of Hat Creek basin, Sioux County, Nebr.

ARCTOBAIERA Florin, 1936.

Arctobaiera flettii Florin, $1936 \mathrm{~b}$, p. 119 , pls. $26-31$; pl. 32, figs. 1-6 ; structurally preserved ginkgophyte foliage; Jurassic; Franz Joseph Land.

ARCTODENDRON Nathorst, 1919.

Arctodendron kidstonii Nathorst, 1919, p. 457. For Dictyodendron kidstonit Nathorst, 1914, p. 72 , pl. 8, figs. 1-4; p1. 9 , flgs. $1-8 ;$ pl. 12, figs. 11-20; pl. 13, figs. 32-36.

ARCTOPODIUM Unger, 1856.

Arctopodium insigne Unger, 1856, p. 177, pl. 12, figs. 1,2 ; regarded as identical with Cladoxylon (see discussion in Seward, 1917, p. 200); Upper Devonian ; Saalfeld, Thuringia. See also Posthumus, 1931.

ARCTOSTAPHYLOIDES KIrchhelmer, 1936. Arctostaphyloides globula (Menzel) Kirchheimer, 1936b, p. 117, pl. 12, figs. 12a-g ; fruit, Ericaceae; Tertiary (Braunkohle) ; Salzhausen, Germany.

ARCTOXYLON Kräusel, 1949.

Arctoxylon magnoradiatum (Gothan) Kräusel, 1949, p. 112, 186 ; coniferous wood; Lower Cretaceous or Jurassic.

AROYOPTERIS Zalessky, 1936.

Arcyopteris asiatica Zalessky, 1936a, p. 224, fe. 1 . cern or ptertalosperm; age; Carboniferous; Russia.

ARDISTOPHYLLUM Geyler, 1887.

Ardisiophyllum sp. Geyler, 1887a, p. 497, pl. 36, figs. 1-3; leaf fragments, Myrsinaceae?; Eocene; Labuan, Borneo.
ARECIPITES Wodehouse, 1933.

Arecipites punctatus Wodehouse, 1933, p. 497 , fig. 22 ; pollen, Arecaceae ; Parachute Creek member, Green River formation, Eocene; Colorado and Utah.

ARECITES Squinabol, 1892.

Arecites trabucci Squinabol, 1892, p. 71, pl. 28, fig. 5 ; leaf, Araceae ; Tertiary ; Santa Giustina, Italy.

ARECOPSIS Fritel, 1927.

Arecopsis communis Fritel, 1927, p. 118 , fig. 1b; Upper Cretaceous; Faveau, Provence, France.

ARGOPHYLLITES Deane, 1902.

Argophyllites levis Deane, 1902a, p. 62, pl. 17, fig. 2 ; leaf fragment, compared with Argophyllum; Tertiary; Wingello, New South Wales.

ARISAEMITES Knowlton, 1896.

Arisaemites sp. Knowlton, in Lindgren, 1896, p. 889 ; Miocene; Independence Hill, Placer County, Calif.

ARISTOLOCHIAEPHYLLUM Fontaine, 1889.

Aristolochiaephyllum crassinerve Fontaine, 1889 , p. 322 , pl. 160, figs. 3-6; leaf ; Potomac group, Lower Cretaceous ; near Brooke, Va.

ARISTOLOCHITES Heer, 1866.

Aristolochites dentata Heer, in Capellin! and Heer, 1866, p. 18, pl. 2, flgs. 1, 2 ; Upper Cretaceous; Tekamah, Nebr.

ARISTOLOCHOPSIS Kuntze, 1904.

Aristolochopsis Kuntze, in Post and Kuntze, 1904, p. 44.

ARISTOPHYCOS Massalongo, 1858.

Aristophycos agardhianus Massalongo, 1858b, p. 745 ; alga ; Tertiary ; Italy.

ARISTOPHYCUS Miller and Dyer, 1878.

Aristophycus ramosum Miller and Dyer, 1878 , p. 4 , pl. 4 , flg. 2 ; probably not of plant origin ; Cincinnati group, silurian; Cincinnati, Ohio.

$\triangle R N O L D E L L A$ Read, 1936.

Arnolaella minuta Read, 1936a, p. 221 , figs. 3, 4; petrified petiole, Pityeae; Upper Devonian; Junction City, Boyle County, Ky.

AROIDES Kutorga, 1838.

Aroides crassipatha Kutorga, 1838, p. 24. See Saporta and Marion, 1885, p. 231, figs. $100 \mathrm{~b}, 100 \mathrm{c}$; seed ?; Permian, West Ural Mts, Russia.

ARONITES Heer, 1855.

Aronites dubius Heer, 1855, p. 98, pl. 48, As. 5 ; leaf fragment, Araceae?; TerAg. 5; leaf fragme

ARONIUM Rttingshausen, 1870.

Aronium extinctum Ettingshausen, 1870b, p. 872 , pl. 1 , fig. 32 ; root?; Aracere; Miocene; Radoboj, Croatia. 
ARPEXYLON Williamson, 1872.

Arpexylon simplex Williamson, 1872, p. 438, flg. 1 ; coenopterid petiole ; Calciferous Sandstone series, Lower Carboniferous; Burntisland, Scotland. See also Hirmer, 1927, p. 495 ; and Posthumus, 1931.

A RTH RARIA Blllings, 1872.

Arthraria antiquata Blllings, 1872, p. 467, fig. 2; plant?; Sllurian; Great Bell Island, Newfoundland, Canada.

A.RTHROCLADION Sauveur, 1848.

Arthrocladion rhodii Sauveur, 1848, p. 2, pl. 65 ; fragment of decorticated stem?; Carbontferous ; Belgium; nom. nud.

ARTHRODENDROMYELON LIgnier, 1910. Arthrodendromyelon morierei Lignier, $1910 a$, p. 626 ; articulate stem cast?; Lower Jurassic (Llas) ; St.-Honorinela-Guillaume, France.

ARTHRODENDRON Scott, 1900.

Arthrodendron sp. Scott, $1900 \mathrm{~b}$, p. 32. This name, proposed by Scott, was first introduced by Seward, 1898, p. 301, for a calamitean stem described by Williamson, 1871c. Seward, however, used the name as a subgenus.

ARTHRODENDRON Ulrich, 1904.

Arthrodendron diffusum Ulrich, 1904, p. 138 , pl. 14 , figs. $1-3$; alga, possibly related to Cymopolia and Corallina: Yakutat formation, Lower Jurassic (Lias) ; Poglbshi Island, opposite village of Kodiak, Alaska.

ARTHROON Renault, 1894.

Arthroon rochei Renault, 1894, p. 178; see also Renault, 1896 , p. 435 , figs. 85 , 86 ; arthropod eggs or possibly of fungus origin ?, parasite in Lepidodendron; Upper Carboniferous ; Esnost and Combres, France.

-ARTHROPHYCUS Hall, 1852.

Arthrophycus harlani (Conrad) Hall, 1852, p. 5, pls. 1, 2; incertae sedis, worm tracks?; Medina sandstone, Sllurian; Rochester, N. Y.

ARTHROPITYOSTACHYS Renault, 1896.

Arthropityostachys borgiensis Renault, 1896 a, p. 133 , pl. 61 , figs. $1-4$; calam1tean cone ; Upper Carboniferous; Borgis, France.

ARTHROPITYS Goeppert, 1864.

Arthropitys bistriata (Cotta) Goeppert, 1864 , p. 185 , pls. 32,33 ; calamitean stem; Permian; Chemitz, Germany.

ARTHROPORELLA Stolley, 1893.

Arthroporella catenularia Stolley, 1893, p. 145, pl, 7, figs. 9-10; siphonaceous alga ; Upper Silurian; Holstein, Kiel, Prussia.

ARTHROSTIGMA Dawson, 1871.

Arthrostigma gracile Dawson, 1871, p. 41, pl. 13, psilophyte; Devonian; Gaspé, Canada.
ARTHROT AXITHS.

See Athrotaxites Unger.

ARTHROTAXOPSIS.

Arthrotaxopsis grandis Fontaine; this name given in a list in Allan, Warren, and Rutherford, 1932, p. 243, apparently a mistake for Athrotaxopsis.

ARTISIA Sternberg, 1838.

Artisia transversa (Artis) Sternberg, 1838 (1828-38i), p. 192, pl. 53, figs. 7-9 ; cordaitean pith cast; Upper Carbonfferous; England. [The binomial Artisia interrupta first appears in Anonymous, 1827 , p. 134 (this is undoubtedly by Sternberg) and is a name given for Sternbergia transversa Artis, 1825, pl. 8.]

ARTOCARPIDIUM Unger, 1851.

Artocarpidium integrifolium Unger, 1851, p. 166 , pl. 35 , figs. 3,4 ; leaf fragment, Artocarpaceae ; Tertiary ; Sotzka, Styria.

ARTOCARPOIDES Saporta, 1865.

Artocarpoides perampla Saporta, 1865, p. 46. Apparently first illustrated species : Artocarpoides conocephaloidea Saporta, 1868, p. 356, pl. 6, fig. 6 ; leaf, Artocarpaceae ; Eocene, Sezanne, France.

ARTOCARPOPHYLLUM Crie, 1889.

Artocarpophyllum damesii Crie, 1889a, p. 90 ; nom. nud.

ARTOCARPOPHYLLUM Dawson, 1894.

Artocarpophyllum occidentale $\mathrm{D}$ a w $80 \mathrm{n}$, 1894 , p. 60 , pl. 12 , fig. 51 ; pl. 13 , fig. 52 ; leaf fragment, incertae sedis; Cretaceous; Vancouver Colliery, Nanalmo, Vancouver Island.

ARTOPHYOUS J. H. Johnson, 1940.

Artophycus columnaris J. H. Johnson, 1940 , p. 589 , pl. 7 , fig. 1 ; alga, probably Cyanophyceae; Weber formation, Pennsylvanian; Trout Creek Pass, Chaffee County, Colo.

ARUNDINARITES Saporta, 1862

Arundinarites restiaceus Saporta, 1862, p. 296 [142] ; Tertiary ; France.

ARUNDINITES Otto, 1854.

Arundinites wohlfarthi Otto, 1854 (185254 ), p. 27 , pl. 4 , fig. 2 ; pl. 7, figs. 1-5 ; stem fragments, Incertae sedis; Cretaceous (Quadersandstein) ; Paulsdorf, Saxony.

ASCHEMONIA Dettmer, 1915.

Aschemonia gigantea Dettmer, 1915, p. 287 , fig. p. 285 ; Incertae sedis ; Cretaceous (Cenomanian); Weissen Berge, near Prague, Bohemia.

ASCLEPIADITES MacGinitie, 1941.

Asclepiadites laterita MacGinitie, 1941, p. 157 , pl. 44, flg. 6 ; leaf, Asclepladaceae ; Eocene; You Bet, Nevada County, Calif.

ASCOSOMA Lorenz, 1904.

Ascosoma phaneroporata Lorenz, 1904, p. 194 ; alga, Siphonaceae ; Cambrian ; Shantung, China. 
ASKISIELLA Chachlof, 1939.

Askisiella ramosa Chachlof, 1939, p. 91, pls. 1-3 ; Middle Devonian ; Minussinsk Bassin, Russia.

ASOLANUS Wood, 1861.

Asolanus ornithicnoides Wood, 1861a, p. 238, pl. 4, fig. 1 ; Pennsylvanian ; Milnes mine, St. Clair, Pa.?

ASOLENOXYLON Renault, 1883.

Asolenoxylon sp. Renault, 1883b, p. 1019 ; nom. nud.

ASPASIA Stefanl, 1901.

Aspasia amplectens Stefani, 1901, p. 75, pl. 11, figs. 1-4; incertae sedis ; Lower Permian; Monte Vignale, Italy.

ASPIDIARIA Presl, 1838.

Aspidiaria schlotheimiana Presl, in Sternberg, 1838 (1820-38), p. 131, pl. 68, fig. 10 ; partly decorticated Lepidodendron.

ASPIDIOIDES Jaeger, 1827.

Aspidioides stuttgardiensis Jaeger, 1827, p. 32 , pl. 8 , fig. 1 ; sterile fern frond; Triassic; Stuttgart, Württemberg.

ASPIDION Zalessky, 1937.

Aspidion decemnervium Zalessky, $1937 \mathrm{~b}$, p. 80 , fig. 46 ; incertae sedis; Permian; Matveyevo, USSR.

ASPIDIOPHYLLUM Lesquereux, 1876.

Aspidiophyllum trilobatum Lesquereux, $1876 \mathrm{a}$, p. 361 , pl. 2, figs. 1, 2 ; leaf, incertae sedis; Cretaceous ; south of Fort Harker, Kans.

ASPIDIOPSTS Henry Potonie, 1893.

Aspidiopsis coniferoides Henry Potonle, 1893 a, p. 242 , pl. 1 , flg. 8 ; pl. 26 ; stem impression, incertae sedis; Permian (Rothliegendes) ; Manebach-Kammerberg, Germany.

ASPIDIOPTERIS E. W. Berry, 1911.

Nom. nud.; a name suggested by Berry, E. W., 1911a, p. 242 , for possible reception of certain specles of Oladophlebis.

ASPIDITES Colla, 1829.

Aspidites flibmas Colla, in Borson, 1829, p. 181.

ASPIDITES Goeppert, 1836.

Aspidites dentatus Goeppert, 1836, p. 355, pl. 21, figs. 7, 8.

ASPLENIOIDES Koenig, 1825.

Asplenioides obtusum Koenig, 1825, pl. 16, fig. 199 ; no description ; fernlike foliage.

ASPLENIOPTERIS Sternberg, 1825.

Aspleniopteris difformis Sternberg, 1825 (1820-38), Tentamen, p. $x \Sigma i$, pl. 24, fig. 1 ; fern? foliage; Tertiary (Braunkohle); Bohemia.

ASPLENIOPTERIS Fontaine, 1889

Aspleniopteris pinnatifial Fontaine, 1889, p. 118 , pl. 22, figs. 1-3, 6, 7 ; fern follage ; Potomac group, Lower Cretaceous; Fredericksburg, Va.
ASPLENIPHYLLUM Hartung, 1940.

Aspleniphyllum foerstert (Debey and Ettingshausen) Hartung, 1940, p. 98 ; pl. 1 , figs. 1-3, 5, 6 ; pl. 2, flgs. 6, 7 ; Upper Cretaceous; Prince Boris mine, Bulgaria.

ASPLENITES Colla, 1829.

Asplenites trichomanes Colla, in Borson, 1829, p 33.

ASPLENITES Goeppert, 1836.

Asplenites heterophyllus Goeppert, 1836, p. 278 , pl. 18, fig. 1 ; fertile fern follage ; Charlottenbrunn, Silesia.

ASSEIBOPSIS.

Error for Apeibopsis, in Peola, 1901, p. 189.

ASTELIAEPHYLLUM Squinabol, 1892.

Asteliaephyllum italicum Squinabol, 1892, p. 52, pl. 20, fig. 1 ; leaf, Draceneae ; Tertiary; Santa Giustina, Italy.

ASTEROCALAMITES (Schimper) Zelller, 1879.

Asterocalamites 8crobiculatus (Schlotheim) Zeiller, 1879 , p. 17, pl. 159, fig. 2 ; articulate stem cast, vascular strands not alternating at node ; Carboniferous; France.

ASTEROCALAMITOPSIS Gothan, 1949.

Asterocalamitopsis sphenophylloides Gothan, 1949, p. 18, pl. 3, figs. 7-9 ; pl. 4, figs. 1-4; articulate stem and foliage impression; Lower Carboniferous ; Dobrilugk, Germany.

ASTEROOALYX Ettingshausen, 1888.

Asterocalys stiriacus Ettingshausen, 1888, p. $281, \mathrm{pl} .3$, figs. 1-4; leaf and inflorescence?; Dioscoreae ; Miocene; Münzenberg, Styria.

ASTEROCARPUS Goeppert, 1836.

Asterocarpus sternbergii Goeppert, 1836, p. 188 , pl. 6 , figs. 1-3; fertile fern foliage, Marattiaceae?

ASTEROCELASTRU'S Velenoviky and Viniklar, 1926.

Asterocelastrus cretaceous Velenovsky and Yiniklar, 1926, p. 50, pl. 1, fig. 11 ; fruit, compared with Pterocelastrus; Cretaceous; Utruby, Bohemia.

ASTEROCHLAENA Corda, 1845.

Asterochlaena cottai Corda, 1845, p. 81.

For Tubicaulis ramosus Cotta, 1832,

p. 23 , pl. 3 , figs. 1-3; origin unknown. See also Goeppert, $1864-65$, p. 41, pl. 8 , fig. 1 ; pl. 9, flg. 1 ; and Posthumus, 1931.

ASTEROCHLAENOPSIS Sahn1, 1930.

Asterochlaenopsis kirgisica (Stenzel) sahn1, 1930, p. 461; tree fern, alled to Asterochlaena and "Clepsydropsis" australis; age unknown, possibly Per. mian; near Pawlodar on river Irtisch, near Akmolinsk, Kirges Steppes, west Siberia. 
ASTEROOYOLITES Romansk1, 1890.

Asterocyclites sp. Romansk1, 1890, p. 144, pl. 19, fig. 3a ; Lower Jurassic; ThlanSchan, Turkistan.

ASTIRODENDRON Eichwald, 1846.

Asterodendron issedonum Eichwald, 1846, p. 562. . See also Eichwald, 1851, p. 252, pl. 14, figs. 4-9.

ASTERODISOUS Zalessky, 1937.

Asterodiscus disparis Zalessky, $1937 \mathrm{~b}$, p. 78 , flg. 45 ; lobed cupulellke organ; Permian; Russia.

ASTEROPHRAGMIUM Reinsch, 1880.

Asterophragmium superbum Reinsch, 1880, p. 7, pl. 2, figs. 4, 5 ; Upper Carboniferous; Saarbruck, Rhenish Prussia.

ASTEROPHYCUS Lesquereux, 1876.

Asterophycus coxii Lesquereux, $1876 \mathrm{a}, \mathrm{p}$. 139 , pl. 2, figs. 1,2 ; incertae sedis; Carboniferous ; near New Harmony, Ind.

ASTEROPHYLLITES Brongniart, 1822.

Asterophyllites radiatus Brongniart, 1822, p. 235 , pl. 2, fig. 7 ; foliage ; Carboniferous.

ASTEROPHYLLOSTAOHYS Schimper, 1880. Asterophyllostachys binneyana Schimper, in Schimper and Schenk, 1880 (1879$90)$, p. 169,173 , fig. $128(2)$; calamite cone, Upper Carboniferous.

ASTEROPHYLLUM Schimper, 1869.

Asterophyllum furcatum (Lindley and Hutton) Schimper, 1869 (1869-74) p. 34.5. For Solenites furcatus Lindley and Hutton, 1831-37, pl. 209.

ASTEROPTERIS Dawson, 1880.

Asteropteris noveboracensis Dawson, $1880 a$, p. 476 ; stem, Cladoxyleae; Devonian; New York. See also Dawson, 1881 b, p. 299 , pl. 12, flgs. 1-9.

ASTEROSOMA Otto, 1854.

Asterosoma radiciforme Otto, 1854 (1852$54)$, p. 15, pl. 2, fig. 4; pl. 3, flgs. 1,2 ; described as "algae dubiae," probably not plant; Cretaceous (Quadersandstein) ; Königsteln, Saxony.

ASTEROTHECA Presl, 1845.

Asterotheca sternbergii (Goeppert) Presl, in Corda, 1845, p. 89. For Asterocarpus sternbergii Goeppert, 1836 , p. 188, pl. 6, figis. 1-4; fertile frond, Marnttiaceae; Carboniferous.

ASTEROTHYRITES Cookson, 1947.

Asterothyrites sinuatus Cookson, 1947, p. 209 , pl. 12, fig. 8 ; mycelium and ascomata, Microthyriaceae ; Oligocene-Miocene; Yallourn and Hazelwood, Victoria.

ASTEROXYLON KIdston and Lang, 1920.

Asteroxylon mackiei Kidston and Lang, 1920b, p. 664, pls. 1-17 ; Psilophytales; Old Red Sandstone, Devonian; Muir of Rhynle, Aberdeenshire, Scotland.
ASTRAPAEITES Langeron, 1899

Astrapaeites pumicosus Langeron, 1899, p. 448 , pl. 4 , flg. 2 ; leaf, compared with Dombeya and Astrapaea; Eocene; Sezanne, France.

ASTROOARYOPSIS Fliche, 1896.

Astrocaryopsis sanctaemanehildae Fliche, 1896 , p. 276 , pl. 13 , figs. 5 , 6 ; seed, incertae sedis; Upper Cretaceous (Cenomanian) ; Ste.-Manehould, France.

ASTROOHARA Stache, 1880.

Astrochara liburnica Stache, 1880, p. 201 ; nom. nud.

ASTROCLADIUM Braun, 1840.

Astrocladium lineare Braun, 1840, p. 94 ; nom. nud.

ASTROCUPULITES Halle, 1927.

Astrocupulites acuminatus Halle, 1927, p. 219 , pl. 48, figs. 10, 11 ; "Inflorescence"bearing cupules; Lower Shihhotse series, Permian ; Ch'en-chla-yu, central Shansi, China.

ASTROMYELON Williamson, 1883.

Astromyelon williamsonis (Cash and Hicks) Williamson, 1883, p. 463, pl. 27 ; petrifled calamite root; Hallfax beds, Upper Carbontferous; England.

ASTROPOLITHON Dawson, 1878.

Astropolithon hindil Dawson, 1878, p. 83. See also Dawson, 1888, p. 30, fig. 9.

ATACTOXYLON Hartig, 1848.

Atactoxylon linkii Hartig, 1848a, p. 171; wood; Tertiary; Wetterau, Ratzeburg, Germany.

ATHROTAXIDIUM Menzel, 1900.

Athrotaxidium bilinicum Menzel, 1900, p. 97, pl. 5, figs. 13-16; Oligocene ; Preschen, Bohemia.

ATHROTAXITES Unger, 1849.

Athrotaxites lycopodioides Unger, $1849, \mathrm{p}$. 346 , pl. 5 , figs. 1,2 ; follage-bearing shoots and cones, Coniferales; Jurassic ; Solenhofen, Bavaria.

ATHROTAXOPSIS Fontaine, 1889.

Athrotaxopsis grandis Fontaine, 1889, p. 240 , pls. 114, 116, 135 ; foliage and cones, believed to be related to Athrotaxis; Potomac group, Lower Cretaceous ; Fredericksburg, Va.

ATOPOCHARA Peck, 1938.

Atopochara trivolvis Peck, 1938, p. 174, pl. 28, figs. 8-12; oogonlum, Charophyta; Trinity group, Lower Cretaceous ; Irion County, Tex.

ATRACTYLIOPSIS Pia, 1937 ?

Atractyliopsis sp. Pla, 1937, p. 829 ; Dasycladaceae; Paleozolc.

ATTALEINITES Tuzson, 1914.

Attaleinites apiculata Tuzson, 1914, p. 246, pl. 16, flg. 1 ; fragment of infructesence, Palmaceae?; Oligocene; Palvolgy valley near Budapest, Hungary. 
$\triangle U E R B A C H I A$ Trautschold, 1870.

Auerbachia echinata Trautschold, 1870, p. 228 , pl. 22 , fig. 3 ; incertae sedis ; Wealden; Tarjuchina-Berg, Russia.

AULACOLEPIS Ettingshausen, 1895.

Aulacolepis rhomboidalis Ettingshausen, 1895 , p. 12 , pl. 1 , fig. 10 ; seed, Conlferales; Upper Cretaceous; Station Oxley, Australia. See also Ettingshausen, 1893, p. 147 ; nom. nud.

AULACOPHYCOS Massalongo, 1859.

A generic name proposed for Palaeophyous simplex Hall, apparently intended as Aulacophyco simplex (Hall) Massalongo, in Massalongo and Scarabelli, 1859, p. 82.

AULACOPHYCUS Eichwald, 1860.

Aulacophycus costatus Eichwald, 1860, p. 50, pl. 1, fig. 1 ; incertae sedis; CarbonIferous ; Tarkhansk, Alta1, Russia.

AULACOPTERIS Corda, 1847.

Aulacopteris sackii Corda, 1847, p. 17 ; nom. nud.

AULACOPTERIS Grand'Eury, 1877.

Aulacopteris vulgaris Grand'Eury, 1877, p. 125 , pl. 12 ; fernlike foliage; Carboniferous; Loire, France. See also Posthumus, 1931.

AULACOTHEOA Halle, 1933.

Aulacotheca elongata (Kidston) Halle, 1933 , p. 30 , pl. 7 ; figs. 7,9 ; pteridosperm microsporangiate organ; Lower Yorkian, Carboniferous ; Calderbank near Airdrie, Scotland.

AULACOXYLON Combes, 1907.

Aulacoxylon sparnacense Combes, 1907, p. 28 , pl. 1, figs. 1-3; wood, dicotyledon; Eocene.

AULARTHROPHYTON Massalongo, 1857.

Aularthrophyton foromosum Massalongo, 1857 a, p. 570 , pl. 1 , figs. 1,4 ; pl. 2, figs. 1,2 ; pl. 3 , figs. 1,3 ; pl. 4, figs. 1 , 2 ; pl. 5 , figs. 1,3 ; pl. 8 , figs. $1-3$; 1 certae sedis ; Eocene ; Monte Colle, Italy.

AULOPHYCUS Fenton and Fenton, 1939.

Aulophycus repens Fenton and Fenton, 1939 , p. 104 , fig. 5 ; pl. 7 , figs. 1,2 ; calcareous alga ; Cambrian; head of Death Canyon, Teton Mts., Wyo.

AUSTRELLA Dana, 1849.

Austrella rigida Dana, 1849, p. 720, pl. 14, figs. 7, 8 ; Carboniferous : Newcastle, New South Wales.

AUSTROCLEPSIS Sahni, 1932.

Austroclepsis australis (Osborn) Sahnl, $1932 b$, p. 274. For Ankyropteris australis Osborn, in Sahni, 1919, p. 82, pl, 4 ; Zygopterid fern ; Carbontferous; Australia. See also Sahni, 1928; Sahn1, 1932.

AUTOPHYLLITES Grand'Eury, 1890.

Autophyllites furcatus Grand'Eury, 1890, p. 225 , pl. 17, figs. 9-19; articulate stem with follage; Carboniferous; St.Etienne, France.
AUTUNIA Krasser, 1921.

Autunia milleryensis (Renault) Krasser. 1921a, p. 20. For Cycadospadio milleryensis Renault, 1896, p. 329, pl. 73, figs. 1-7.

AZOLLOPHYLLUM Penhallow, 1890.

Azollophyllum primaevum Penhallow, in Dawson, 1890 , p. 77, fig. 2 ; compared with Azolla caroliniana but apparently poorly preserved; Miocene; Stump Lake, British Columbia.

\section{$\mathbf{B}$}

BACCA Engelhardt, 1922.

Bacca diospyroides Engelhardt, 1922, $p$. 77, pl. 23, fig. 17 ; lower Tertiary ; Messel near Darmstadt, Hesse.

BACCHARITES Saporta, 1881.

Baccharites aquensis Saporta, 1881, p. 1132. For Lomatites aquensis Saporta, 1862 , p. 253 , pl. 7 , fig. 10 ; Ollogocene; Aix, Provence, France.

BACCITES Zenker, 1833.

Baccites cacaoides Zenker, 1833 , p. 10 , pl. 1, figs. 4-8; seed or fruit?; Tertiary (Braunkohle); Altenburg, Germany.

BACHASUPTERIS Zalessky, 1937.

Bachasupteris lobata Zalessky, $1937 \mathrm{~b}$, p. 589, figs. 4-7 ; fern pinnule fragments ; Upper Devonian; near Bakhtcha and Grande Karakouba, Donets Basin, Russia.

BACILLARITES Karl Feistmantel, 1867.

Bacillarites problematicus Karl Feistmantel, 1867, p. 59; Pennsylvanian; Radnitz, Bohemia. See also Gelnitz, 1870, p. 63 , pl. 1, fig. 12 .

BACILLITES Meschinelli, 1898.

Bacillites permiensis (Renault and Bertrand) Meschinelli, 1898, p. 67, pl. 19, fig. 12; Schizomycete, in coprolite; Permian ; France.

BACTRITES E. W. Berry, 1924.

Bactrites pandanifoliolus E. W. Berry, 1924 b, p. 52, pl. 7, figs. 1-6 ; leaf fragment, Palmaceae ; Lisbon formation, Eocene; near Newton, Newton County, Miss.

BACTRYLLIUM Heer, 1853.

Bactryllium canaliculatum Heer, 1853, p. 125, pl. o, fig. E; Upper Triassic (Keuper) ; Val Gorno, Austria.

BAIERA Braun, 1843.

Baiera dichotoma Braun, in Münster, 1843, p. 20, p1. 12, figs. 1-5; deeply dissected, apparently ginkgophyte leaf; Bayreuth and strullendorf, Bavaria.

BAIERELLA Robert Potonie, 1933.

Preuss. geol Landesanst. Inst. Paläobotanik u. Petrograhie Brennstelne, 1933, Arb., Band 3, p. 249 ; Gymnospermae; Lower Jurassic (not seen). See also Gothan, $1942 b$, p. 108. 
BAIERIDIUM Gothan and Gimm, 1930.

Baieridium alphlebiaeforme Gothan and Gimm, 1930, p. 62-63, pl. 9, figs. 4-6 ; Carboniferous; Linderberg near Ilmenau, Germany.

BAIEROPSIS Fontaine, 1889.

Baieropsis expansa Fontaine, 1889, p. 207, pls. 89-92 ; ginkophy te follage ; Potomac group, Lower Cretaceous; Fredericksburg, Va.

BAJERA Sternberg, 1825.

Bajera Scanica Sternberg, 1825 (1820-38). Tentamen, p. xxvli1, pl. 47, fig. 2 ; in certae sedis.

BALANITOCARPUM Menzel, 1913.

Balanitocarpum ovatum Menzel, 1913, p. 36, pl. 4, fig. 15 ; fruit, Zygophyllaceae ; Tertiary (Braunkohle); Germany.

BALANTI'TES Goeppert, 1836.

Balantites martii Goeppert, 1936, p. 337, pl. 37 , figs. 5, 6 ; sterile fern follage; Waldenburg, Silesia.

BALIOSTICHUS Sternberg, 1833.

Baliostichus ornatus Sternberg, 1833 $(1820-38)$, p. 31 , pl. 25, fig. 3 ; defoliated twig, Coniferales?; Opper Jurassic ; Solenhofen, Bavaria.

BAMBUSITES Ettingshausen, 1887.

Bambusites arthrostylinus Ettingshausen, 1887 a, p. 95 , pl. 9 , figs. 1, 1a ; leaf, Gramineae?; Eocene; Vegetable Creek, Australia.

$B A M B U S I U M$ Unger, 1845.

Bambusium sepultum Unger, 1845 (1841$47)$, p. 128 , pl. 40 , figs. 1,2 ; incertae sedis ; Tertiary ; Radohoj, Croatia.

BANARA PHYLLUM E. W. Berry, 1937.

Banaraphytlum ovatum E. W. Berry, 1937, p. 46 , pl. 9 , fig. 1 ; leaf, Flacourtiaceae ; Paleocene; Cerro Funes, between Chubut and Santa Cruz, Patagonia.

BANISTERIOPHFLLUM Ettingshausen, 1886.

Banisteriophyllum australiense Ettingshausen, 1886 , p. 125 , pl. 14 , fig. 13 ; leaf, Malpighiaceae; Eocene, Tingha, Australla.

BANKSICARPVS Velenovsky and Viniklar, 1927.

Banksicarpus cretaceus Velenovsky and Viniklar, 1927, p. 44, pl. 10, figs. 4, 5; infructescence, compared with Banksia; Cretaceous ; Vyserovice, Bohemia.

BANKSIEAEIDITES Cookson, 1950.

Banksieaeidites minimus Cookson, 1950, p. 169, pl. 1, figs. 8, 9 ; pollen, compared with Banksia and Dryandra; Tertiary (Oligocene-Miocene?); Yallourn and Yallourn North, Victorfa.

BANKSIEAEPHXLLUM Cookson, 1950.

Banksieaephyllum angustum Cookson, 1950 , p. 146, pl. 1, figs. 1-10 ; mummified leaves, Proteaceae; Oligocene (Brown coal); Yallourn and Yallourn North, Victoria.
BANKSIOXYLON Crie, 1889

Banksioxylon australe Crie, 1889a, p. 78 ; nom. nud; Pleistocene, Australia.

BANKSIPHYLLUM Velenovsky, 1889.

Bunksiphyllum pusillum Velenovsky, 1889, p. 53.

BANKSITES Saporta, 1861.

Banksites integer Saporta, in Heer, 1861, p. 138 ; Eocene ; St. Zacharie, Provence, France. See also Saporta, 1863, p. 68, pl. 8, fig. 7 .

BARAGWANATHIA Lang and Cookson, 1935.

Baragwanathia longifolia Lang and Cookson, 1935 , p. 425 , pls. 29-31 ; lycopod, leafy shoots with sporangia; Lower Ludlow, Stlurian; Australia.

BARAKARIA Seward and Sahni, 1920.

Barakaria dichotoma (Feistmantel) Seward and Sahni, 1920, p. 16, pl. 3, fig. 29 ; foliage, some resemblance to Schizoneura; Barakar beds, Lower Gondwana ; Auranga coalfield, India.

BARDELLA Zalessky, 1937.

Bardella splendida Zalessky, 1937b, p. 76, fig. 43 ; shoots bearing leaves, Conlferales ; Permian ; Kroutala Katouchka, Russia.

BARDIA Zalessky, 1933.

Acad. sci. U. R. S. S. Bull., 1933a, p. 284 ; Pteridospermae; Permian (not seen). See also Gothan, 1942b, p. 108.

BARDOCARPUS Zalessky, 1937.

Bardocarpus aliger Zalessky, 1937b, p. 87, fig. 56 ; winged seed; Permian; Matveyevo, USSR.

BARINOPHYTON David White, 1905.

Barinophyton richard8oni David White, in Smith and White, 1905 , p. 65, pl. 4, figs. 5-8; fertile fern? frond; Upper Devonian ; I'erry, Maine.

BARINOSTROBUS Kräusel and Weyland, 1941.

Barinostrobus spicatus (Dawson) Kräusel and Weyland, 1941, p. 51, pl. 13, flgs. 10, 11 ; cone?, incertae sedis; Upper Devonian; Perry, Maine.

BARRANDEINA Stur, 1882.

Barrandeina dusliana (Krejđ̄i) Stur, 1882, p. 362 , pl. 3, flgs. 3,4 ; pl. 5 ; psilophyte ; Devonian (Etage H-h); near Srbsko, Bohemia.

BARREALIA Frenguelli, 1942.

Barrealia dichotoma Frenguelli, 1942, p. 281, flg. 1, pl. 1, fig. 1 ; leaf, Matoniaceae?; Triassic; Argentina.

BARSASSIA Zalessky, 1933.

Bar8as8ia ornata Zalessky, 1933c, p. 1387, fig. 1 ; mummifled stem, Psilophytales ? Upper Devonian; Kuznets [Kousnetzk], Russia. 
BASSANIA Gasparis, 1895.

Bassania keuperiana Gasparis, 1895, p. 69, figs. a, b ; Üpper Triassic (Keuper) ; Bayreuth, Bavaria.

BATHYPTERIS Eichwald, 1860.

Bathypteris rhomboidea Eichwald, 1860, p. 96 , pl. 4 , figs. 1,2 ; stem, Osmundaceae ; Bjelebel, Orenbourg, Russia. See also Posthumus, 1931.

BATODENDRON Landsborough, 1844.

Batodendron sp. Landsborough, in Patrick, 1844, p. 290 ; nom. nud.

BATODENDRON Chachloff, 1921.

Batodendron sp. Chachlofi, 1921, p. 19 , figs. 23-25 ; Upper Devonlan ; Lake Balbach, Siberia.

BAUHINITES Seward and Conway, 1935.

Bauhinites groenlandica Seward and Conway, 1935 , p. 25, fig. 21 ; leaf, compared with Bauhinia glauca Wall, Leguminosae ; Cretaceous ; Greenland.

BEANIA Carruthers, 1869.

Beania gracilis Carruthers, 1869, p. 98, pl. 4 ; infructescence, Cycadales; Jurassic ; Gristhorpe, Yorkshire, England. For recent discussion and assoclated parts, see Harris, 1941.

BEANIOPSIS Ganju, 1944.

Beaniopsis rajmahalensis Ganju, 1944, p. 76, pl. 2, figs. 15,16 ; fig. 2 ; seed-bearing cone resembling Beania, probably Cycadaceae ; Jurassic ; Onthea, Rafmahal Hills, India.

BEATRICEA Billings, 1857.

Beatricea nodulosa Billings, 1857, p. 344 ; incertae sedis; Lower Silurlan; Anticostl at Wreck Point, Canada.

BEAUPREAIDITES Cookson, 1950.

Beaupreaidites elegansiformis Cookson, 1950 , p. 168 , pl. 1 , figs. $2-4$; pollen, compared with Beauprea elegans; Tertiary (Oligocene-Miocene?) ; many 10calities, southeastern Australia.

BECHERA Sternberg, 1825.

Bechera ceratophylloides Sternberg, 1825 (1820-38), Tentamen, p. xxx, pl. 35, fig. 3 ; roots, or poorly preserved articulate stem and leaf remalns; Upper Carboniferous; Swina, Bohemia.

BEGKETTIA Reid and Chandler, 1933.

Beckettia mastixioides Reld and Chander, 1933 , p. 456 , pl. 25 , figs. $28-36$; endocarp, Cornaceae ; London Clay, Eocene ; Sheppey, Kent, England.

BECKLESIA Seward, 1895.

Beclclesia anomala Seward, 1895, p. 179, pl. 14, figs. 2, 3; follage, incertae sedis ; Wealden; Ecclesbourne, near Hastings, England.

BEDHEIMIA Schuster, 1933.

Beitr. Geologie Thüringen, 1933, Band 3, p. 239 ; Lycopodiales; Keuper (not seen). See alao Gothan, $1942 b$, p. 108.
BEINERTIA Goeppert, 1836.

Beinertia : gymnogrammoides Goeppert, 1836 , p. 273 , pl. 16 , figs. 4 , 5 ; sterile fern follage ; Charlottenbrunn, Sllesia.

BELEMNOPTERIS Ottokar Feistmantel, 1876.

Belemnopteris voodmasoniana Ottokar Feistmantel, 1876, p. 371, pl. 20, flgs. 1, 2 ; fern? follage; Damuda series Gondwana system; Ranlganj, India.

BELENOPHYLLUM Zalessky, 1928.

Belenophyllum aericulum Zalessky, 1928, p. 801 ; nom. nud. ; Lower Carboniferous; North Caucasus.

BELENOPTERIS Zalessky, 1930.

Belenopteris ivanovi Zalessky, 1930f, p. 928 ; nom. nud.

BELIDOXYLON Hartig, 1848.

Belidoxylon acerosa (Unger) Hartig, 1848 b, p. 138. For Peuce acerosa Unger, 1841 (1841-48), p. 14, pl. 3, figs. 1-44; Miocene; Wurmberg, Styria.

$B E L O D E N D R O N$ Debey, 1848.

Belodendron nesii Debey, 1848, p. 121 ; nom. nud.

BELONODENDRON Marck, 1863.

Belonodendron densifolium Marck, 1863, p. 80, pl. 13 , figs. 8,9 .

BELTINA Walcott, 1899.

Beltina danai Walcott, 1899, p. 239, pls. 25-27; considered by Walcott to be crustacean but by others to be alga (8ee Fenton and Fenton, 1931, p. 686); Greyson shales, Algonkian ; Deep Creek Canyon, near Glenwood, Mont.

BELZUNGIA Morellet, 1908.

Belzungia borneti Morellet, 1908, p. 97, fig. 2; siphonaceous alga; Eocene (Thanetien); Boncourt, France.

BEMBERGIA Caspary, 1881.

Bembergia pentatrias Caspary, 1881, p. 29 ; Tertiary; Samland, Baltic Prussia.

BENIZIA Debey and Ettingshausen, 1859.

Benizia calopteris Debey and Ettingshausen, 1859b, p. 216, pl. 5, figs. 13-17 ; fertlle fern frond fragment; Upper Cretaceous ; Aachen, Rhenish Prussia.

BENNETTICARPUS T. M., Harris, 1932.

Bennetticarpus oxylepidus T. M. Harris, 1932b, p. 101, pl. 14, figs. 1-6, 11; fruit, Bennettitales; Lepidopteris bed, RhaetoLianots; Scoresby Sound, east Greenland.

BENNETTISTEMON Harris, 1932.

Bennetistemon amblum Harris, $1932 \mathrm{~b}$, p. 98 , pls. 11, 12 ; microsporophyll, Bennettitales ; Lepidopteris bed, RhaetoLiassic; Scoresby Sound, east Greenland.

BENNETTITACEARUM Gothan, 1914.

Bennettitacearum sp. Gothan, 1914, p. 132, pl. 27, fig. 5 ; cycadophyte cone fragment ; Rhaetic ; Wasserstuhl, near Rollhofen, Bavaria. 
BENNETTITANTHUS Turutanova-Ketova, 1930.

Bennettitanthus masculinus TurutanovaKetova, 1930, p. 151, pl. 5, fig. 38 ; Jurassic, southwest Turkistan.

BENNETTITES Carruthers, 1870.

Bennettites saxbyanus Carruthers, 1870, p. 698, pl. 57; cycadophyte trunk; Wealden; Brook Point, Isle of Wight, England.

BENNETTITOLEPIS Florin, 1933.

Bennettitolcpis dactylota (Harris) Florin, 1933, p. 34. For Cycadospadix dactylota Harrfs, 1932b, p. 97, pl. 10, figs. 1, 2 ; megasporophyll, Bennettitales; Lepidopteris bed, Rhaeto-Llassic; Scoresby Sound, east Greenland.

BENSONIA Buckman, 1845.

Bensonia ovata Buckman, in Murchison, 1845 , p. 93; "a parallel veined (aquatic?) endogen"; Stonesfleld slate ; Sevenhampton Common, England.

BENSINITES Rina Scott, 1908.

Bensonites fusiformis Rina Scott, 1908, p. 683 , figs. 1-7; sporangia; Lower Carboniferous ; Burntisland, Scotland.

BENSTEDTIA (Seward) Knowlton, 1911.

Benstedtia benstedi (König) Knowlton, 1.911 , p. 468 ; coniferous stem fragment ; Lower Greensand, Cretaceous; Kent, Jingland. The generic name was assigned by Seward, 1896a, but no species designated; the taxonomy is reviewed by Knowlton, 1911, although Stopes, 1911 , criticizes his treatment on the grounds that it "is not a recognizable ispecies."

BENTHAMIPHYLLUM Velenovsky, 1889.

Benthamiphyllum dubium Velenovsky, 1889 , p. 58. For Benthamia dubia Velenovsky, 1887, p. 11, pl. 7, fig. 4; Upper Cretaceous; Vyserovice, Czechoslovakia.

BERBERIDIPHYLLUM Dusen, 1899.

Berberidiphyllum reflexum Dusen, 1.899 , p. 106 , pl. 8 , fig. 11 ; leaf fragment, compared with Berberis buxifolia Lamarck; Oligocene ; Río Guillermo, Chile.

BERENDTIA Goeppert, 1845.

Berendtia primuloides Goeppert, in Berendt, 1845 , p. 80 , pl. 5, figs. 21-26; staminate flower, dicotyledon; Miocene; Prussia.

BERGERIA Presl, 1838.

Bcrgeria acuta Presl, in Sternberg, 1838 (1820-38), p. 184, pl. 48, fig. 1a; impression of Lepidodendron leaf cushion ; Carboniferous ; Bohemia.

BER.AIOPHYTON Kurtz, 1902.

Bergiophyton insigne Kurtz, 1902, p. 211 ; nom. nud.

BERGIOPTERIS Kurtz, 1921.

Bergiopteris insignis Kurtz, 1921, p. 149 ; "Permo-Carbonlferous"; Argentina.
BERNETTIA Gothan, 1914.

Bernettia inopinata Gothan, 1914, p. 58, pl. 27 , figs. $1-4$; pl. 34 , fig. 3 ; cycadophyte? cone; Rhaetic; Nurnberg.

BERNOULLIA Heer, 1876.

Bernoullia helvetica Heer, $1876 \mathrm{a}$, p. 88 , pl. 38, figs. 1-6 ; fertlle fern follage ; Triassic; Switzerland.

BERRIOCHLOA Elias, 1932.

Berriochloa glabra (Berry) Elias, 1932, p. 347 , pl. 28 , figs. $13-16$; pl. 29 , fig. 1 ; grass fruit, Hordeae?; near base of Ogallala formation, upper Mlocene-lower Pliocene; Wallace County, Kans.

BERRYA Knowlton, 1930.

Berrya racemosa Knowlton, 1930 , p. 134, pl. 41, figs. 4, 5 ; raceme of frults, incertae sedis ; Denver formation, Upper Cretaceous ; Golden, Colo.

$B E R W Y N I A$ Hicks, 1882.

Berwynia carruthersi Hicks, 1882, p. 100 , pl. 3 ; arborescent lycopod stem; S1lurian; North Wales.

BETULAEPOLLENITES Robert Potonle, 1934.

Betulaepollenites microexcelsus Robert Potonie, 1934, p. 58, pl. 2, figs. 22, 27 ; pollen, Betulaceae; Miocene (Braunkoule).

BETULINIUM Unger, 1842.

Betulinium tenerum Unger, 1842a, p. 101 ; wood, incertae sedis; Tertlary; Mecklenburg, Austria, See also Unger 1847 (1841-47), p. 118, pl. 34, figs. 8-10.

BETULIPHYLLUM Dusen, 1899.

Betuliphyllum patagonicum Dusen, 1899 , p. 102 , pl. 10 , figs. 15,16 ; leaf, $\mathrm{Be}$ tulaceae?; Oligocene; Punta Arenas, Chile.

BETULITES Goeppert, 1838.

Betulites 8alzhausensis Goeppert, 1838, p. 567, pl. 42 ; staminate inflorescence; Miocene; Salzhausen, near Nedda, Wetterau, Hesse.

BETULOIDITES Thlergart, 1950.

Betuloidites sp. Thlergart, in Potonle, Robert, Thomson, Paul W., and Thiergart, Friedrich, 1950, p. 52, pl. C, flg. 17 ; pollen, Betulaceae; nom. nud.

BETULOXYLON KaIser, 1880.

Betuloxylon oligocenicum Kaiser, $1880 \mathrm{~b}$, p. 511 ; Betulaceae; Oligocene.

BEVOCASTRIA Garwood, 1931.

Bevocastria conglobata Garwood, 1931, p. 141, pl. 12, figs. 1-3; alga; Tuedian, Lower Carboniferous; Hole of Lyne, northern Cumberland, England.

BIARMELLA Zalessky, 1939.

Biarmella triloba Zalessky, 1939b, p. 353, flg. 32 ; fern? pinnule fragment ; Permian; Tchekarda, USSR. 
BIARMOBAIERA Zalessky, 1939.

Biarmobaiera uralensis Zalessky, 1939b, p. 361, fig. 40 ; ginkgophyte? leaf fragment ; Permian ; 'lchekarda, USSR.

BIARMODENDRON Zalessky, 1939.

Biarmodendron foliosum Zalessky, 1939b, p. 368 , fig. 51 ; foliage twig, Coniferales ; Permian; Matreyevo, USSR.

BIARMOPTERIS Zalessky, 1937.

Biarmopteris pulchra Zalessky, 1937b, p. 47, fig. 11 ; incertae sedis ; Permian; near village of Matveyevo, USSR.

BIOARPELLITES Perkins, 1904.

Bicarpellites grayana Perkins, 1904, p. 190 , pl. 78 , fig. 69 ; fruit; Tertlary ; Brandon, Vt.

BICORBULA Condra and Elias, 1945.

Bicorbula arizonica Condra and Elias, 1945 , p. 118 , pl. 13 , figs. $1-8$; pl. 14 , figs. $1-3$; pl. 15, figs. $1-7$; pl. 16, figs. 1 , 2 ; bryozoan with algal association; Kaibab formation, middle Permian ; east of Jacob's Lake, Ariz,

BIDENTITES Heer, 1859.

Bidentites antiquus Heer, 1859, p. 6, pl. 101, fig. 20 ; seed, Compositae ; Tertlary ; Oeningen, Switzerland.

BIGNONICAPSULA E. W. Berry, 1930.

Bignonicapsula formosa $\mathrm{E}$. W. Berry, 1930, p. 132 , pl. 43 , fig. 3 ; large capsule containing winged seeds, Bignoniaceae; Wilcox group, lower Eocene; a quarter of a mile east of Denmark, Madison County, Tenn.

BIGNONIOPHYLLUM Ettingshausen, 1870.

Bignoniophyllum getoniaeformis Ettingshausen, 1870 b, p. 881 , pl. 1 , flgs. 6,7 ; leaf, Bignoniaceae; Miocene; Radoboj, Croatia.

BIGNONIPHYLLUM Velenotsky, 1889.

Bignoniphyllum cordatum Velenovsky, 1889, p. 54.

BIGNONITES Saporta, 1861.

Bignonites palaeospermus Saporta, in Heer, 1861, p. 147 ; seed, Blgnonlaceae ; Tertiary. Apparently first illustrated species is Bignonites americanus Berry, 1925 b, p. 176, pl. 2, fig. 12.

BIGNONOIDES E. W. Berry, 1923.

Bignonoides orbicularis E. W. Berry, 1923, p. 25 , pl. 3 , fig. 4 ; seeds, Bignoniaceae ; Miocene; Palomares, Saravia estate, Caxaca, Mexico.

BILIGNEA Fidston, 1923.

Bilignea solida Kidston, in Scott, 1923, p. 134 ; pteridosperm ? stem ; Carboniferous; Ayrshire, Scotland. See also Scott. 1925 , p. 579, pl. 3, figs. 22-37; pl. 4 ; pl. 5, flg. 35.

BILLARDIERITES Caspary, 1882.

Billardierites longistylus Caspary, 1882, p. 24 ; flower, in amber, Pittosporaceae; Miocene; Samland, Baltic Prussia. See also Conwentz, 1880 , p. 80 , pl. 8, figs. 16-19.
BILOBITES Dekay, 1824.

Bilobites rugosa (D'Orbigny) Saporta, 1879 , p. 164 , fig. 1 ; incertae sedis ; Stlurian. Not specifically named in Dekay, 1824.

BIORBIA Elias, 1932.

Biorbia rugosa (Berry) Elias, 1932, p. 350, pl. 29 ; nutlets, Borraginaceae ; Ogallala beds, early Plfocene; Wallace County, Kans., Yuma County, Colo.

BIOTOCALAMITES Grand'Eury, 1877.

Biotocalamites sp. Grand'Eury, 1877, p. 332 ; nom. nud.

BJUVIA Florin, 1933.

Bjuvia simplex Florin, 1933, p. 50, pl. 1, fig. 3 ; pl. 2 , figs. 4-7; pl. 3, figs. 4-8; cycadophyte leaf ; Rhaetic ; Bjuv, Sweden.

BLASARIA Zalessky, 1934.

Blasaria siberica Zalessky, 1934a, figs. 1, 2 ; lycopod leaf base impression; Devonian; Russia.

BLASTOLEPIS Zigno, 1885.

Blastolepis otozamites Zigno, 1885, p. 174, pl. 42 , fig. 9 ; cycadophyte seed; lower Oolite, Middle Jurassic; Salaorno Valley near Rovere di Velo, Italy.

BLASTOPHRAGMIUM Relnsch, 1880.

Blastophragmium elegans Relnsch, 1880, p. 6 , pl. 1 ; pl. 2 , fig. 1 . See also Reinsch, 1881 , p. 113 , pl. 47 , figs. $1-7$; pl. 48 , figs. $1-5$; pl. 49, figs. $1-3$; Upper Carboniferous; Saarbruck, Rhenish Prussia, etc.

BLA STOPHYCUS Miller and Dyer, 1878.

Blastophycus diadematus Miller and Dyer, 1878 , p. 24 , pl. 1 , figs. 1,2 ; plant ?: Upper Ordovician; Cincinnati, Ohio.

BLECHNOXYLON Etheridge, 1899.

Blechnoxylon talbragarense Etheridge, $1899 b$, p. 135 ; partly petrified fern stem with leaves attached showing development of secondary wood; "PermoCarboniferous"; between Gulgong and Cockabutta Hill, county of Bligh, New South Wales.

BLOSENBERGIA Gothan, 1939.

Blosenbergia gallwitziana Gothan, in Gallwitz and Gothan, 1939 , p. 763 , pl. 49, figs. 9-19; ly copod or psilophyte? stem impression; Upper Devonian; Blosenberg, Vogtland, Saxony.

BOCKSCHIA Goeppert, 1836

Bockschia flabellata Goeppert, 1836, p. 176, pl. 1, figs. 1, 2; fertile fern frond: Waldenburg, Silesia.

BOEGENDORFIA Gothan and Zimmerman. 1932.

Boegendorfla semiarticulata Gothan and Zimmerman, 1932, p. 110, pl. 13, figs. 2,3 ; pl. 15 , fig. 6 ; pl. 17 , figs. 3,4 ; Upper Devonian; Upper Bögendorf, Silesia. 
BOLBOPODIUM Saporta, 1874.

Bolbopodium pictaviense Saporta, 1874 $(1873 c-75)$, p. 258 , pl. 118, fig. 2 ; cycad stem ; Jurassic (Oxfordian); Montanaise, near Poitiers, France.

BOLIVIANA Salter, 1860.

Boliviana melocactus Salter, 1860, p. 71, pl. 5, fig. 9 ; incertae sedis ; Silurian?; Illimani, Bolivia.

BOLONIA Meunier, 1886.

Bolonia lata Meunler, 1886, p. 567, pl. 30, fig. 8 ; plant? ; Upper Jurassic ; Pas-deCalais, France.

BOMBACIPHYLLUM Engelhardt, 1891. Bombaciphyllum opacum Engelhardt, 1891 , p. 669 , pl. 8 , fig. 9 ; leaf, Malvaceae ; Tertiary ; Caronel, Chile.

BOMBACITES E. W. Berry, 1916.

Bombacites formosus $\mathrm{E}$. W. Berry, 1916a, p. 289 , pl. 75, fig. 1 ; leaves, Bombaceae ; Lagrange formation, Wilcox group, lower Eocene; Puryear, Henry County, Tenn.

BOMBACOPHYLLUM Velenovsky, 1889.

Bombacophyllum argillaceum Velenovsky, 1889 , p. 39.

BONAVENTUREA Debey and Ettingshausen, 1859.

Bonaventurea cardinalis Debey and Ettingshausen, $1859 \mathrm{~b}$, p. 203 , pl. 3 , figs. 2-9 ; fern frond and spores; Upper Cretaceous; Aachen, Rhenish Prussia.

BORNIA Sternberg, 1825.

Bornia equisetiformis (Schlotheim) Sternberg, 1825 (1820-38), Tentamen, $p$. xxviii. See Schlotheim, 1804, pl. 2, fig. 3. First illustrated after 1820 in Steiniger, 1841 (1840-41), fig. 13.

BOROLDAIPHYCUS Vologdin, 1948.

Boroldaiphycus borovikovi Vologdin, 1948, p. 83 , pl. 1 ; alga ; Devonian; Russia.

BOROVICZIA Zalessky, 1905.

Boroviczia karpinskii Zalessky, 1905, p. 331, figs. 19-23 ; seeds ; Lower Carboniferous; Russia. See Seward, 1917, p. 358.

BORRAGINITES Heer, 1859.

Borraginites myosotiflorus Heer, 1859, p. 17, pl. 103, fig. 19 ; flower, Borraginaceae ; Tertiary ; Oeningen, Switzerland.

BOSTRICHOPHYTON Squinabol, 1890.

Bostrichophyton pantanellii Squinabol, 1890 , p. 183 , pl. 7 , fig. 5 ; alga ? Tertiary; Vallata, Valle del Tresinaro, Italy.

BOSWORTHIA Walcott, 1919.

Bosworthia simulans Walcott, 1919, p. 241 , pl. 57, fig. 31 ; pl. 58, flg. 1 ; alga ; Burgess shale, Stephen formation, Middle Cambrian ; 1 mile northeast of Burgess Pass, above Field, British Columbla.
BOTHRODENDRON Lindley and Hutton, 1833.

Bothrodendron punctatum Lindley and Hutton, 1833, p. 1, pl. 81 ; stem compression; High Main coal seam, Carboniferous ; Jarrow Colliery, England.

BOTHROSTROBUS (Nathorst) Zalessky, 1904.

Bothrostrobus olryi (Zeiller) Zalessky, 1904, p. 46, 107, pl. 6, figs. 4, 4a, 11, 12 ; cone of Bothrodendron; Upper Carboniferous; Marihaye, Belgium. Seo also Nathorst, 1894, p. 43 ; Seward, 1910 , p. 262.

BOTRYCHIOPSIS Kurtz, 1894.

Botrychiopsis weissiana Kurtz, 1894, p. 121 , pl. 1 ; fern? foliage ; "Permo-Carboniferous" ; Retamito, San Juan province, Argentina.

BOTRYCHIOXYLON D. H. Scott, 1912.

Bothrychioxylon paradoxum D. H. Scott, 1912 , p. 373 , pls. $37-41$; coenopteris fern stem with secondary wood; Lower Coal Measures, Upper Carboniferous; Lancashire, England. The generic name was first given by Scott, 1906, p. 518, with a very brief description; later references were made as follows: Scott, 1907, p. 181 ; Scott, 1909, p. 318, 344 ; Bower, 1911, p. 546; however, it was not until 1912 that Scott assigned a specific name, described the fossil in detail, and presented illustrations. See also Posthumus, 1931.

BOTRYOCOCCITES C. E. Bertrand, 1898.

Botryococcites largae C. E. Bertrand, 1898, p. 182 , pl. 5 , fig. 30 a ; pl. 11, figs. $127-$ 132 ; Oligocene; Bois d'Asson, France. BOTRYOCONUS Goeppert, 1864.

Botryoconus goldenbergi Goeppert, 1864 , p. 152 ; inflorescence, Cordaitales ; Upper Carboniferous. See also Grand'Eury, 1877, p. 279, pl. 33.

BOTRYOPTERIS Renault, 1875.

Botryopteris forensis Renault, 1875a, p. 202 ; petrified fertile frond, Coenopteridales; Upper Carboniferous ; St.Etienne, France. See also Renault, 1875 b, p. 227 , pl. 8 ; pl. 9, figs. 4, 7 ; pl. 11, fig. 20 ; Posthumus, 1931.

BOTRYTITES Meschinelli, 1892.

Botrytites similis (Menge and Goeppert) Meschinelli, in Saccardo, 1892, p. 789. For Botrytis similis Menge and Goeppert, in Goeppert, 1853, p. 453.

BOTTGERIA Crie, 1889.

Bottgeria multiradiata Crie, 1889b, p. 19 ; nom. nud. According to information received by Prof. $L$. F. Ward from Zeiller, all the specimens (of Bottgeria, Feistmantelia, Martinia, and Taenioxylon) of species discussed in this paper by Crie were lost, none of them having been described or figured. 
BOUEINA Toula, 1883.

Boueina hochstetteri Toula, 1883, p. 1318 , pl. 6, figs. 10a-c; pls. 7-9; Middle Jurassic (Oolite); Pirot near Sofla, Bulgaria.

BOULAYA (Carpentler) Halle, 1933.

Boulaya fertilis (Kidston) Halle, 1933, p. 25 , pl. 6, figs. $4-9$; text fig. 6 ; pteridosperm microsporangiate organ; Westphalten, Carbontferous ; France, Germany, Holland, England. The genus created by Carpentier, 1925, but no specific entity assigned.

BOWERBANKELLA Reid and Chandler, 1933.

Bowerbankella tiliacoroidea Reld and Chandler, 1933, p. 153, pl. 3, figs. 34-41; endocarp, Menispermaceae; London Clay, Eocene; Sheppey, Kent, England.

BOWERBANKIA Debey, 1849.

Bowerbankia attenuata Debej, 1849, p. 299 ; nom. nud.

BOWERIA Kidston, 1911.

Boweria schatzarensis (Stur) Kidston, 1911, figs. 5, 6 ; fern frond fragment, intermediate between botryopterid and modern leptosporangiate ferns?; Upper Carboniferous; Belgium.

BOWMANITES Binney, 1871. Bonomanites cambrensis Binney, 1871, p. 59, pl, 10, figs. 1-3; cone, Sphenophyllales; Lower Coal Measures, Upper Carboniferous; near Pontypool, South Wales. See also Hoskins and Cross, 1943.

BRACHYBACULITIES Gruss, 1928.

Palaeobiologica, 1928, Band 1, p. 514; alga; Devonian (not seen). See also Gothan, 1942b, p. 110.

BRACHYCARPHIUM Berkeley, 1849.

Brachycarplium thomasinum Berkeley, 1849 , p. 78. A name substituted for the earlier invalid name Brachycladium thomasinum Berkeley, 1848, p. 382.

BRACHYCLADITES Meschinelli, 1802.

Brachycladites thomasinus (Berkeley) Meschinell, in Saccardo, 1892, p. 790. See also Meschinelli, 1898, p. 81, pl. 22, figs. 9, 10.

BRAOHYCLADIUM Berkeley, 1848.

Brachycladium thomasinum Berkeley, 1848 , p. 382 , pl. 11 , fig. 2 ; fungus, compared with Botrytis, in amber; East Prussia. See Brachycarphium.

BRACHYDACTYLUS Reis, 1923.

Brachydactylus radialis Reis, 1923, p. 113, pl. 3, flgs. 7-9 ; pl. 4, fig. 9 ; Tertiary ; Rhenish Prussia.

$B R A C H Y O X Y L O N$ Hollick and Jeffrey, 1909.

Brachyoxylon notabile Hollick and Jeffrey, 1909 , p. 54, pls. 13, 14; araucarian wood ; Cretaceous ; Kreischerville, Staten Island, N. $\mathbf{Y}$.
BRAOHYPHYLLUM Brongniart, 1828.

Brachyphyllum mamillare Brongniart, 1828 b, p. 109 ; twig and follage, Coniferales; Jurassic (Oolitic).

BRACHYRUSCUS Cockerell, 1922.

Brachyruscus alleni Cockerell, 1922, p. 213, flg. 1 ; pistillate flower, Liliaceae ; Miocene ; Florissant, Colo.

BRANDONIA Perkins, 1904.

Brandonia globulus Perkins, 1904, p. 192, pl. 78, flgs. 73, 74; fruit; Tertiary; Brandon, vt.

BRASENIOPSIS Saporta, 1894.

Braseniopsis venulosa Saporta, 1894, p. 192, p1. 34, figs. 1-4 ; leaf, Nymphaceae ; Mesozoic ; Portugal.

BRAVARDIA Hauthal, 1902.

Bravardia mendozensis Hauthal, in Kurtz, 1902 , p. 57 ; nom. nud.

$B R E D A E A$ Goeppert, 1857.

Bredaea moroides Goeppert, 1857, p. 56, pl. 1, figs. 6,7 ; petrified wood, incertae sedis; Tertiary; Java.

BRESCIPIY YLUM Velenovsky, 1889.

Bresciphyllum cretaceum Velenovsky, 1889, pl. 5, figs. 2, 3; dicotyledonous leaf compared with Brescia formosa; Upper Cretaceous (Cenomanian); Lidic, Bohemia.

BRETONIA Bertrand and Hovelacque, 1892. Bretonia hardingheni Bertrand and Hovelacque, in Bertrand and Renault, 1892, p. 243, pl. 7, figs. 32-34; Carboniferous ; Autun, France.

BRIARDINA (Munier-Chalmas) Morellet and Morellet, 1922.

Briardina archiace Munier-Chalmas, in Morellet and Morellet, 1922, p. 26 (type species?) ; Eocene ; Gaas, France. [The generic name appears as nom. nud. in Munier-Chalmas, 1877 , p. 817.$]$

BRIGHTONIA Harris, 1932.

Brightonia arota Harris, 1932b, p. 119, pl. 19, microsporophyll, incertae sedis; Lepidopteris zone, Rhaetic; Scoresby Sound, east Greenland.

BRITTSIA David White, 1899.

Brittsia problematica David White, 1899 , p. 98 , pl. 47 ; figs. $1-5$; pl. 48 , figs. $1-3$; fernlike roliage; Pennsylvanian; 3/2 miles southeast of Clinton, Mo.

BROECKELLA (Munier-Chalmas) Morellet and Morellet, 1922.

Broeckella belgica Munier-Chalmas, in Morellet and Morellet, 1922, p. 22, pl. 10, figs. 56-57, alga, Dasycladaceae ; Eocene (Montien) ; Mons, Belglum.

BRÖGGERIA Nathorst, 1915.

Bröggeria norvegica Nathorst, 1915, p. 21, pl. 3, figs. 5-7; pl. 4, flgs. 4-9; Devonian; Norway. 
BROMELIANTHUS Massalongo, 1859.

Bromelianthus heuflerianus Massalongo, 1859 a, p. 62 , pl. 36 , flgs. 2,3 ; flower, Bromellaceae?; Eocene ; Italy.

BROMELITES Schmalhausen, 1884.

Bromelites dolinski Schmalhausen, 1884, p. 296, pl. 30, fig. 7; Eocene; Kiev, Russia.

BRONGNIARTITES Unger, 1845.

Brongniartites graecus Unger, 1845, p. 264 ; wood ; Tertiary ; Lestos, Greece.

BRONG:NIARTITES Zalessky, 1927.

Brongniartites salicifolius (Fischer) Zalessky, 1927a, p. 39 , pl. 9 , fig. 1 ; pl. 10 , figs. $1-3$; pl. 11 , fig. 1 ; pl. 12, fig. 2 ; leaf, Incertae sedis; Permian; Belebei district, Urals, Russia.

BRONNITES Unger, 1842.

Bronnites antiquensis Unger, 1842, p. 102 ; wood ; Tertlary ; Antigua, West Indes.

BRUKMANNIA Sternberg, 1825.

Brukmannia tenuifolia Sternberg, 1825 (1820-38), Tentamen, p. xxix, pl. 19, fig. 2 ; Asterophyllites-like follage shoot ; Carboniferous ; Radnitz, Bohemia.

BRUNSWICKIA Wherry, 1916.

Brunswickia dubia Wherry, 1916, p. 329, pl. 30 ; leaves, incertae sedis ; Brunswick formation, Triassic; three-quarters of a mile south of Sellersville station, Bucks County, $\mathrm{Pa}$.

BRYACITES C. F. W. Braun, 1840.

Bryacites lignitarum C. F. W. Braun, 1840 , p. 94 ; nom. nud. Braun attributes this genus to Brongniart.

BRYASTERITES Reinsch, 1881.

Bryasterites sp. Reinsch, 1881, p. 105, pl. 44, fig. 1 ; pl. 45, figs. $1-3$; Permian; Stockheim, Wurttemberg.

BRYOCARPUS Debey, 1849.

Bryocarpus monostachys Debey, 1849, p. 299 ; nom. nud.

$B U B U L O I A$ Massalongo, 1857.

Bubulcia globifera (Sternberg) Massalongo, $1857 \mathrm{~b}$, p. 777 . For Sargassites globifera Sternberg, 1833 (1820-38), p. 36 , pl. 10 , fig. 1 .

BUCHERIA Dorf, 1933.

Bucheria ovata Dorf, 1933, p. 246, figs. 0-17; Psllophytales; Lower Devonian; Beartooth Butte, Wyo.

BUCINELLA Fucini, 1936.

Reference not seen. See Gothan, 1942b, p. 110.

BUCKLA.NDIA Presl, 1825.

Bucklandia anomala (Stokes and Webb) Presl, in Sternberg, 1825 (1820-38), Tentamen, p. xxxili. For Olathraria anomala Stokes and Webb, 1825, p. 423 ; cycadophyte trunk; Wealden; Sussex, Fingland. See also Seward, 1917, p. 675.

BUDINGIA Krasser, 1943.

Budingia sp. Krasser, 1943, p. 15, 1 pl. ; - Upper Permian; Wetterau, Germany.
BURIADIA Seward and Sahni, 1920.

Buriadia heterophylla (Feistmantel) Seward and Sahnl, 1920, p. 12, pl. 2, flgs. 20-25 ; Voltzia-like shoots, but with b1furcated leaves; Karharbart beds, "Permo-Carboniferous"; Burladi, India.

BURSERICARPUM Reid and Chandler, 1933.

Bursericarpum angulatum Reld and Chandler, 1933, p. 275, pl. 11, figs. 8-10 ; fruit, Burseraceae; London Clay, Eocene ; Sheppey, Kent, England.

BURSERITES E. W. Berry, 1924.

Burserites fayettensis E. W. Berry, 1924a, p. 175 , pl. 41, figs. 7,8 ; leaf, Burseraceae; Fayette sandstone, Eocene; Sabine Parish, La. [This description clearly bears the inscription "n. gen." and it seems evident that the species was intended as the genotype. However (apparently owing to delay in publication of the above) another species was described earlier : $B$. venezuelana Berry, 1921 , p. 574 , pl. 107, fig. 7 ; leaf, Burseraceae; Teritary ; Betijoque, State of Trujillo, Venezuela.]

BURTINIA Endlicher, 1845.

Burtinia faujasii Endlicher, in Unger, $1845(1841-47)$, p. Ixxi. Apparently first illustration is in Weber, 1851, p. 45, pl. 1, flg. 7 ; palm fruit?; Tertiary. See also Endlicher, 1837 (1836-40), p. 257.

BUTHOTREPHIS Hall, 1847.

Buthotrephis gracilis Hall, 1847, p. 62, pl. 21, fig. 1 ; alga ; Trenton limestone, Middle Ordovician; Jacksonburgh and Middleville, Herkimer County, N. $\mathbf{Y}$.

BUTOMITES Velenovsky, 1889.

Butomites cretaceous Velenovsky, 1889, p. 25, pl. 3, figs. 10-13, 15; Upper Cretaceous; Vidovic, Bohemia.

BYSMATOSPERMUM Harris, 1935.

Bysmatospermum macrotrachelum Harris, 1935, p. 132 , pl. 29 ; seed, Bennettitales? Lepidopteris zone, Rhaetlc; Scoresby Sound, east Greenland.

BYTHOCLADUS Whitfield, 1894.

A name suggested by Whitfleld, 1894, p. 353 , as belng more approprlate than Buthograptus but no specific entity assigned.

BYTHOTREPHIS.

An emended spelling for Buthotrephis Hall, in Elchwald, 1860 (1860-68), p. 56.

\section{$\mathbf{0}$}

OACTITES Martius, 1822.

Cactites giganteus Martius, 1822, p. 189 ; Carboniferous; silesia.

CADIOSPORA Kosanke, 1950.

Oadiospora magna Kosanke, 1950, p. 50, pl. 16, fig. 1 ; spore; LaSalle coal bed, Pennsylvanian; Bureau County, Ill. 
OAENODENDRON Zalessky, 1918.

Caenodendron primaevum Zalessky, 1918, p. 54 , pl. 13, figs. $1-4$; ly copod stem impression ; Carboniferous; Kouou-Tchekou Basin, Russia.

OAENOMYCES E. W. Berry, 1916.

Caenomyces laurinea E. W. Berry, 1916b, p. 162 , pl. 88 , fig. 4 ; fungus, $\mathrm{Py}-$ renomycetes?; Wilcox group, lower Eocene ; Oxford Gully, Lefayette County, Miss.

OAENOPTERITES Goeppert, 1836.

Caenopterites volkmanni Goeppert, 1836 , p. 23. Goeppert refers to Volkmann, 1720 , pl. 12 , fig. 5. Also described as Sphenopteris volkmanniana Goeppert, 1834, p. 12. See also Goeppert, 1836, p. 267.

OAENOXYLON Zalessky, 1911.

Oacnoxylon 8cotti Zalessky, 1911, p. 13, figs. 1-4; petrified cordaitean stem; Permian; Russia. See also Seward, 1917, p. 293 ; Scott, 1923, p. 283 ; Zalessky, 1927, p. 44.

OAESALPINIOXYLON Schenk, 1890.

Caesalpinioxylon quirogae Schenk, in Schimper and Schenk, 1890 (1879-90), p. 901 , fig. 432 ; wood; Tertiary?; western Sahara on the coast near Huissi Aissa.

CAESALPINIOXYLON Kräusel, 1922.

Caesalpinioxylon palembangense Kräusel, 1922 , p. 247 , pl. 2 , fig. 1 ; pl. 3 , figs. 1,2 ; pl. 7, figs. 6, 11 ; wood, Caesalplneae ; Tertiary ; Sumatra.

UAESALPINITES Suporta, 1802.

Caesalpinites dispersus Saporta, 1862, p. 289 ; leaf, compared with Caesalpinia bahamensis; Tertiary; Aix, Provence, France. See also Saporta, 1873, p. 125, pl. 18, fig. 30.

OAESALPINIUM Schleiden, 1855.

Gaesalpinium triolienso Schleiden, in Schmid and Schleiden, 1855 ; wood, said to resemble that of Caesalpinia echinata; Oligocene: :Tyrol. Apparently first illustrated species: Caesalpinium oweni (Carruthers) Schuster, 1910, p. 8, pl. 2, figs. 10-12.

OALADIOSOMA E. W. Berry, 1925.

Caladiosoma miocenica E. W. Berry, 1925a, p. 38, pI. 5; leaf fragment, compared with Caladium and Xanthosoma, Aracene; Miocene; Trinidad, British West Indies.

CALAMARIOPHYLLUM Hirmer, 1927.

Calamariophyllum lingulatum (Germar)

Hirmer, 1927, p. 452; articulate stem impression; Carboniferous. For Equisetites lingulatus Gelmar, 1845, p. 27, pl. 10.
CALAMARIOPSIS Henry Potonie, 1902. Calamoriopsis Henry Potonle, 1902, p. 797, no specific name assigned. This genus established for Calamopsis Solms, 1896, because of the earlier use of that name by Heer, 1859.

OALAMITEA Cotta, 1832.

Calamitea striata Cotta, 1832, p. 67, pl. 14 ; pl. 15, figs. 1,2 ; petrified culamite stem ; Permian ; Chemnitz, Germany:

CALAMITES Schlotheim, 1820 .

Calamites cannaeformis Schlotheim, 1820, p. 398 , pl. 20, fig. 1 ; pith cast; Upper Carboniferous; Manebach, Wettin, Sax. ony. See also Seward, 1898, p. 295 ; and Kidston and Jongmans, 1917.

CALAMITINA C. E. Weiss, 1876.

Calamitina göpperti (Ettingshausen) C. E. Weiss, 1876, p. 127, pl. 17 ; calamitean stem; Carboniferous.

CALAMITOMYELON Lignier, 1910.

Calamitomyelon morierei Lignier, $1910 \mathrm{~b}$, p. 128, calamitean stem; Lower Jurassic (Middle Lias) ; St. Honorine-la-Guillaume, France.

OALAMOCLADUS Schimper, 1869.

Calamocladus longifolius (Brongniart) Schimper, 1869, p. 323, pl. 22, figs. 1-4; calamite foliage.

CALAMODENDREA Grand'Eury, 1877.

Calamodendrea rhizobola Grand'Eury, 1877 , p. 296 , pl. 31 ; calamitean roots ; Carboniferous; Treve, Loire, France.

CALAMODENDROFLOYOS Grand'Eury, 1877.

Calamodendrofloyos cruciatus (Sternberg). Grand'Eury, 1877, p. 203, pl. A, fig. 0; cortex of Calamodendron; Carbonlferous; France.

CALAMODENDRON Brongniart, 1849.

Calamodendron striatum Brongniart, 1848, p. 50 ; petrifed calamitean stem; Carboniferous. First? lllustration in Mougeot, 1852 , p. 32 , pl. 5, figs. 1-4. See also Goeppert, 1864 (1864-65), p. 180, pls. 30, 31 .

CALAMODENDROPHYLLUM Grand'Eury, 1879.

Calamodendrophyllum bifurcatum Grand' Eury, 1879, p. 579 ; calamitean follage : Upper Carboniferous; Vendee, France.

CALAMODENDROSTACHYS Renault, 1890. Calamodendrostachys aubius Renault, in Renault and Zeiller, 1890, p. 471, pl. 55, fgs. 3-6; articulate cone impression; Carbonfferous; Commentry, France.

\section{CALAMODENDROXYLON Grand'Eury,} 1877.

Calamodendroxylon striatum (Cotta) Grand'Eury, 1877, p. 291; wood of a calamite?; Carboniferous; Porchere, Loire, France. 
OALAMOPHLOIO Đ. A. N. Arber, 1916. Calamophloios rugosus Đ. A. N. Arber, $19: 16$, p. 141 , pl. 8 , flg. 0 ; calamitean stem Impression; Red Clay serles. Transition Coal Measures, Upper Carboniferous; Granville Pit, old Hill, South Staffordshire, England.

OALAMOPHYCUS Lesquereux, 1877.

Calamophycus septus Lesquereux, 1877, p. 165; Lower Helderberg sandstone, Lower Devonian; Michigan.

OALAMOPHYLLITES Grand'Eury, 1877. Calamophyllites communis Grand'Eury, 1877 , p. 39 . See also Calamophyllites sp. Grand'Eury, 1869, p. 708. First llustrated specles appears to be Calanophyllites geinitzii Grand'Eury, 1890, p. 208, pl. 14, fig. 1. Articulate pith impression ; Carboniferous.

CALAMOPHYTON Kräusel and Weyland, 1025.

Calamophyton primaevum Krïusel and Weyland, in Weyland, 1925, p. 43, fig. 12; Calamophytaceae; upper Middle Devonian; northwest Germany. See also Kräusel and Weyland, 1926.

OALAMOPITUS Williamson, 1869.

Oalamopitus sp. Willlamson, $1869 \mathrm{~b}$, p. 174. See also Williamson, 1871a, p. 506, pl. 23, fig. 1 ; and Williamson, 1871c; petrified calamite stem; Upper Carbonlferous; England. See Arthrodendron Scott, 1900a; no specific name ever assigned to this fossil. Only specific name assigned to this (invalid) genus appears to be: Calamopitus parrani Grand'Eury, 1890, p. 211, pl. 14, tigs. 6-8.

OALANOPITYS Unger, 1856.

Calamopitys 8aturni Unger, 1856, p. 160, pl. 3 , fig. 7 ; petrified stem, Calamopityeae ; Upper Devonian ; Saalfeld, Thuringla. See also Calamopitys saturni Unger, 1854b, p. 599 ; nom. nud.

CALAMOPSIS Heer, 1859.

Calamopsis bredana Heer, 1859 , p. 169 , pl. 149 ; palm leaf ; Miocene ; Oeningen, Switzerland.

OALAMOPTERIS Unger, 1856.

Calamopteris debilis Unger, 1856, p. 158, pl. 2, figs. 1-7 ; petiole, Calamopltyeae ; Upper Devonian; Saalfeld, Thuringia. See also Calamopteris debilis Unger, 1854, nom. nud ; and Posthumus, 1931.

CALAMORRIIZA Grand'Eury, 1877.

A name to which Grand'Eury, 1877, p. 26, assigned roots that apparently belonged to the Calamites; no specific entities mentioned.

CALAMOSPORA Schopf, Wllson, and Bentall, 1944.

Calamospora hartungiana Schopf, in Schopf, Wilson, and Bentall, 1944, p. 51, fig. 1 ; spore; middle McLeansboro for- mation, Pennsylvanian ; Salt Fork of Vermilion River northwest of Fairmound, Vermilion County, IIl.

OALAMOSTAOHYS Schimper, 1869.

Calamostachys typica Schimper, 1869 (1869-74), p. 328, pl. 23 ; calamite cone.

CALAMOSYRINX Petzholdt, 1841.

Calamosyrins zwickaviensis Petzholdt, 1841 , p. 28, pl. 2 ; sigillarian stem compression ; Upper Carboniferous ; Zwickau, Saxony.

OALAMOSYRINX Unger, 1856.

Calamosyrinx devonica Unger, 1856, p. 159, pl. 3, figs. 1-6 ; petiole, Calamopityeae; Upper Devonian; Saalfeld, Thuringia. See also Calamosyrino devonica Unger, 1854 ; nom. nud.

OALAMOXYLON Corda, 1838.

Calamoxylon cycadeum Corda, in Sternberg, 1838 (1820-38), p. 195, pl. 54, figg. 8-13 ; stele fragment of arborescent lycopod ?; Carboniferous; Radnitz, Bohemla.

CALATHELLA Florin, 1929.

Calathella kräuseii Florin, 1929a, p. 255, pl. 3, figs. 8-10; pl. 4, flgs. 6-9; alga, Siphonocladales; upper Zechsteln, Permian ; Oberhessen, Büdingen, Germany.

CALATHIOPS Goeppert, 1865.

Calathiops beinertiana Goeppert, 1865a, p. 268 , pl. 64 , flgs. $4-6$; pteridosperm cupulate or microsporangiate? organ; Permian ; near Rothwaltersdorf, Silesia.

CALATHOSPERMUM Walton, 1940.

Calathospermum scoticum Walton, 1940, p. 132 , fig. 110 ; large pteridosperm cupule containing numerous seeds ; Lower Carboniferous; Kilpatrick Hills, Scotland. For full treatment, see Walton, 1949.

CALATOLOIDES E. W. Berry, 1922.

Calatoloides eocenicum E. W. Berry, 1922a, p. 253 , fig. 1 ; fruit, Icacinaceae; Wilcox group ; Eocene; Freestone County, Tex.

CALCIDELETRIX Mïgdefrau, 1937.

Calcideletrix flexuosa Mägdefrau, 1937, p. 57, pl. 4 , fig. 4 ; fruit ? Cretaceous ; Misburg near Hannover, Germany.

CALCIODINELLUM Deflandre, 1947.

Calciodinellum operosum Deflandre, 1947, p. 1781, figs. 1-6 ; Dinoflagellate; Sahelien d'el Medhi, Oranie, Algerla.

CALCIPHYTON Kusta, 1892.

Calciphyton praecambri Kusta, 1892, p. 418, fig. p. 420.

OALCISPHAERA Williamson, 1880.

Calcisphaera laevis Williamson, 1880, p. 521 , pl. 20, fig. 70 ; plant?; Carbontferous; Rhylymwyn, near Mold, Flintshire, England.

CALLEOPHYLLUM Zalessliy, 1939.

Calleophyllum lobatum Zalessky, 1939a, p. 370 , flg. 53 ; incertne sedis ; Permlan ; Matveyevo, Krasnaia Glinka, USSR. 
CALLIGONOPSIS Massalongo, 1859.

Calligonopsis strumphsioides Massalongo, 1859b, p. 55. For Casuarina strumphsioides Massalongo, $1857 \mathrm{~b}$, p. 778.

CALLIPITY\& Harris, 1935.

Callipitys leptoderma Harris, 1935, p. 110 , pls. 19, 21 ; cone, Coniferales; Thaumatopteris zone, Rhaetic; Scoresby Sound, east Greenland.

OALLIPTERIDIUM C. E. Weiss, 1870.

Callipteridium sullivanti (Lesquereux) C. E. Weiss, $1870 a$, p. 876 , pl. 21 , figs. 1-3 ; fernllke follage; Carbonlferous.

OALLIPTERIS Brongniart, 1849.

Callipteris conferta (Sternberg) Brongniart, 1849 , p. 66. For Neuropteris conferta Sternberg, 1820-38, p. 75, p1. 22, fig. 5 ; fernlike follage ; Carboniferous ; Ottendorf, Silesia.

CALLISPHENUS Hoeg, 1938.

Callisphenus gracilis Hoeg, 1938, p. 43, pls. 1,2 ; alga, probably Dasycladaceae ; Wenlock, Silurian; east side island of Kommersoy, near Holmestrand, Oslo Fjord, Norway.

OALLISTEMOPHYLLUM Ettingshausen, 1853.

Callistemophyllum verum Ettingshausen, 1853 , p. 83 , pl. 27 , figs. 11,12 ; leaf, Myrtaceae; Tertiary ; Haering, Tirol, Austria.

OALLITHAMNOPSIS Whitfield, 1894.

Callithamnopsis fruticosa (Hall) Whitfield, 1894, p. 354, pl. 11, flgs. 4-8; alga; Trenton group, Ordovician; Platteville, Wis.

OALLITRITHS Endlicher, 1847.

Callitrites brongniartii Endlfcher, 1847, p. 274. For Equisetum brachyodon Brongniart, 1882, p. 329 , pl. 16, fig. 3 ; coniferous follage and cones; Eocene; near Paris, France. See also Callitrities brongniartii Endlicher in Goeppert, 1850 , p. 179 , pl. 17, figs. 9-12.

GALLITROXYLON Hartig, 1848.

Callitrooylon ayckei (Goeppert) Hartig, 1848a, p. 140. For Taxites aycket Goeppert, 1840 , p. 77 , and 1841 a, p. 730 , pl. 17, flgs. 10-12 ; wood; Tertiary ; Germany.

OALLIXYLON Zalessky, 1911.

Oallixylon triflievi Zalessky, 1911, p. 29, pl. 4, flgs. 1-3; cordaitean wood with bordered pits of tracheids characterist1cally grouped; Devonian. See also Arnold, 1930.

OALLORITES Fiore, 1932.

Soc. naturalisti Napoll Boll., 1932, v. 43, p. 153 ; fungi; Eocene (not seen). See Gothan, $1942 b$, p. 111.

CALLOXYLON Andra, 1848.

Calloxylon hartigii Andra, 1848, p. 633, pl. 5, figs. 7-12 ; coniferous wood ; Tertlary ; Bruckdorf, Saxony.
CALOPTERIS Corda, 1845.

Calopteris dubia Corda, 1845, p. 88, pl. 19, figs. 1b, 3 ; petiole; Upper Carboniferous; Radnitz, Bohemia. See also Hirmer, 1927, p. 540 ; and Posthumus, 1931.

CALOTHRICITES C. E. Bertrand, 1913.

Calothricites alexinatzia C. D. Bertrand, 1913 , p. 357, pl. 4, flgs. 1-16; alga, Cyanophyceae?; Tertiary ; Alexinatz, Serbia.

CALVARINUS Reld and Reid, 1910.

Calvarinus reticulatus Reid and Reid, 1910, p. 169 , pl. 15, flgs. 18-20; nutlet, Boraglnaceae ; Upper Ollgocene ; Bovey Tracey, Devon, England.

CALYOITES.

See Calycithes, Massalongo. Original spelling was Calycithes, but Massalongo and other authors adopted Calycites.

CALYCITHES Massalongo, 1850.

Calycithes pentasepalus Massalongo, 1850, p. 72. Apparently first species illustrated is Calycites lythroides Visiani and Massalongo, 1856, p. 242, pl. 13.

OALYCOCARPUS Goeppert, 1850.

Calycocarpus thujoides Goeppert, 1850, p. 180, pl. 18, fig. 5; Thuja-like frult; Upper Carboniferous; Charlottenbrunn, Silesia.

CALYCOPHYSOIDES Berry, 1924.

Calycophsoides balli Berry, 1924b, p. 6, figs. 1, 2; human artifact; Foard County, Tex. See also Berry, in Torreya, v. 37, p. 108.

CALYMMATOTHECA Stur, 1877.

Calymmatotheca stangeri Stur, 1877, p. 151 , pls. 8,9 ; stem, follage, cupulate organs, Pteridospermae ; Carboniferous (Culm) ; Hruschau, Witkowitz, Moravia. See also Zelller, 1883, p. 182.

CALYPTOPHYCUS J. H. Johnson, 1940.

Calyptophycus verrucius J. H. Johnson, 1940 , p. 590 , pl. 10 , figs. $1-3$; calcareous alga, probably Cyanophyceae; Weber formation, Pennsylvanian; Mule Shoe Gulch, Park County, Colo.

CAMASIA Walcott, 1914.

Camasia 8pongiosa Walcott, 1914, p. 115, pl. 9 , figs. 1,2 ; pl. 12 , figs. 1,$2 ;$ pl. 20 , figs. 2-6; alga, Cyanophyceae?; Beltian series, Alkonkian ; 8 miles west of White Sulphur Springs, Meagher County, Mont.

CAMBROPORELLA Korde, 1950.

Cambroporella tuvensis Korde, 1950, p. 371, fgs. 1-3; alga, Dasycladaceae; Lower Cambrian ; Russia.

CAMPOXYLON Hartig, 1848.

Campoxylon hoedlianum (Unger) Hartig, 1848a, p. 138; wood ; Tertiary ; Germany. For Peuce hoedliana Unger, 1839 , p. 13 ; and 1842 (1841-47), p. 26, pl. 10 , figs. $1-4$. 
CAMPTERONEURA Debey, 1849.

Campteroneura paradoxa Debey, 1849, p. 299 ; nom. nud.

CAMPTOPHYLLUM Nathorst, 1875.

Camptophyllum 8chimperi Nathorst, 1875. p. 389. See al8o Nathorst, 1876 , p. 69 , pl. 16, figs. 13-16; Rhaetic ; Palsjo, Sweden.

CAMPTOPTERIS Presl, 1838.

Camptopteris münsteriana Presl, in Sternberg, 1838 (1820-38), p. 168, pl. 33, fig. 9 ; leaf impression, dicotyledon?

CAMPYLOPHYLLUM Gothan, 1914.

Campylophyllum hormanni Gothan, 1914, p. 53 , pls. $31-33,39$; cycadophyte? foliage ; Rhaetic ; Nürnberg, Germany.

CAMPYLOSPERMUM Chandler, 1925.

Campylospermum hordwellensis Chandler, 1925 , p. 16 , pl. 1, figs. $6 a-c$; fruit, Araceae ; Upper Eocene ; Hordle, Hampshìre, England.

OANCELLOPHYCUS Saporta, 1872.

Cancellophycus liasinus Saporta, 1872a73 , p. 135 , pl. 5, alga ; Jurassic ; Digne, France.

CANNOPHYLLITES (Brongniart) Nilsson, 1832.

Cannophyllites septentrionalis Nilsson, 1832 , p. 346, pl. 1, fig. 9 ; Lower Cretaceous; Hoganas, Sweden. See also Cannophyllites virletii Brongniart, 1828 ; nom. nud.

CANTHELIOPHORUS Bassler, 1919.

Cantheliophorus linearifolius. (Lesquereux) Bassler, 1919, p. 97, pl. 9, figs. 1, 2 , 8-10; pl. 11, figs. 34-37 ; lycopod cone scale and sporangium (probably Lepidocarpon) ; coal B8, Pennsylvanian; Boston mine, Pittston, Luzerne County, Pa. See also Schopf, 1941b, p. 559.

OANTHIDIOM Unger, 1850.

Oanthidium radobojanum Unger, $1850,1 i$. 429 ; Rubiaceae ; Croatia.

CANTIA Stopes, 1915.

Cantia arborescens Stopes, 1915 , p. 260 , pls. 26-28 ; wood, dicotyledon; Folkestone beds, Lower Greensand, Cretaceous ; near Ightham, Kent, England.

OANTIOARPUM Reid and Chandler, 1933. Canticarpum celastroides Reid and Chandler, 1933 , p. 320 , pl. 14, figs. 29-33 ; fruit, Celastraceae; London Clay, Eocene ; Minster, Kent, England.

CANTICARYA Reid and Chandler, 1933.

Canticarya sheppeyensis Reid and Chand ler, 1933 , p. 258 , pl. 10, figs. $1-5$; fruit, Rutaceae; London Clay, Eocene; Sheppey, Kent, England.
CANTISOLANUM Reid and Chandler, 1933. Cantisolanum daturoides Reld and Chandler, 1933, p. 484 , pl. 28, figs. 10-12 ; fruit, Solanaceae; London Clay, Eocene; Sheppey, Kent, England.

CANTITILIA Reid and Chandler, 1933.

Cantitilia polysperma Reid and Chandler, 1933 , p. 393, pl. 20, figs. 4-11 ; fruit, Tiliaceae; London Clay, Eocene; Sheppey, Kent, England.

CAPPARIDIUM Kuntze, 1904.

Capparidium Kuntze, in Post and Kuntze, 1904, p. 98.

CAPPARIDOCARPUS Berry, 1924.

Capparidocarpus sphericus Berry, 1924a, p. 166, pl. 55, figs. 4-9.; fruit, Capparidaceae?; Lagrange formation, Eocene; Hickman, Fulton County, Ky.

CAPPARIDOXYLON Schenk, 1883.

Capparidoxylon geinitzi Schenk, 1883a, p. 12 , pl. 1, figs. 3,4 ; wood ; Oligocene?; near Cairo, Egypt.

CAPPARITES E. W. Berry, 1919.

Capparites cynphylloides E. W. Berry, 1919a, p. 95, pl. 22, fig. 1 ; leaf, Capparidaceae; Tuscaloosa formation, Opper Cretaceous; Shirleys Mill, Fayette County, Ala.

CAPRIFOLIIPITES Wodehouse, 1933.

Caprifoliipites viridifluminis Wodehouse, 1933, p. 518, fig. 54; pollen, Capr1foliaceae; Parachute Creek member, Green River formation, Eocene; Colorado and Utah.

CAPSULOCARPUS E. W. Berry, 1939.

Capsulocarpus dakotensis E. W. Berry, 1939 , p. 332 , figs. $1-4$; podlike capsule, Bignoniaceae?; Cretaceous; half a mlle north of Springfield; Brown County, Minn.

OARACUBOXYLON Zalessky, 1930.

Caracuboxylon bakhasuense Zalessky, $1930 \mathrm{~g}$, p. 1011, pl. 1; petrified stem, Cordaitales ; Devonian ; Donets, Russia.

CARAGANDITES Zalessky, 1933.

Caraganaites rugosus Zalessky, 1933b, p. 1385, fig. 1 ; incertae sedis; Lower Carboniferous ; Karaganda, Russia.

OARATOHETOPTERIS Zalessky, 1932.

Soc. geol. France Bull, 1932, sêr. 5, tome 2, p. 322, fig. 10 ; pteridophyte ; Permian ; Russia (not seen). See Gothan, $1942 \mathrm{~b}, \mathrm{p} .112$.

CARBONACARPA, John Smith, 1896.

Carbonacarpa annandalensis John Smith, 1896 , p. 321, pl. 7, figs. 20-23; incertae sedis; Upper Carboniferous ; Annandale, near Kilmarnock, Scotland. 
OARDIOCARPON Brongniart, 1828.

First valld description appears to be Cardiocarpon acutum Lindley and Hutton, 1833 (1831-37), p. 209, pl. 76; seed casts; Carboniferous; England. Brongniart, $1828 \mathrm{~b}$, p. 87 , lists five species but all nom. nud; later Brongniart, 1881 , p. 37, described petrified species. See Seward, 1917, p. 334. Variously spelled as Cardiocarpum and Cardiocarpus, the latter being adopted by Brongniart, 1881, and by most recent writers.

CARDIOGLOSSUM Koidzumi, 1934.

Cardioglossum antiquum (Kawasaki) Koldzum1, 1934, p. 113: For Gigantopteris antiqua Kawasaki, 1932 (1929$34)$, p. 34, pl. 100 , figs. 2,3 ; Jido series, Lower Permian; Tae-dong, Korea.

CARDIONEURA Zalessky, 1934.

Cardioneura amadoca Zalessky, 1934d, p. 1108, figs. 4-6; neuropterid foliage; Donets, Russia.

CARDIOPTERIDIUM Nathorst, 1914.

Cardiopteridium spetsbergense Nathorst, 1914 , p. 16 , pl, 1 , figs. $9-15$; pl. 8 , figs. 5,6 ; pl. 9, figs. 14-26; fernllke foliage ; Paleozoic; Spitzbergen.

CARDIOPTERIS Schimper, 1869.

Cardiopteris polymorpha (Goep pe $\mathrm{r}$ ) Schimper, 1869 (1869-74), p. 452; Neuropteris-like foliage; lowermost Carboniferous. For Cyclopteris polymorpha Goeppert, .1859, p. 502, pl. 38, figs. $5 a, 5 b$.

OARNOCONITES Srivastava, 1944.

Carnoconites compactum Srivastava, 1944 , p. 75 , pl. 2 , fig. 12 ; female cone of Pentoxylon; Jurassic ; Santal Parganas District, Behar, India. Brlef description with no specific name and no lllustrations in Srivastava, 1935, p. 285. See also Srivastava, 1946, p. 204, pl, 5, figs. 46-68. For full consideration of Pentoxyleae, see Sahni, 1048.

OAROLITES Spegazzini, 1924.

Carolites patagonica Spegazzini, 1924a, p. 100, fig. 101; lear, dicotyledion: Eocene; Patagonia.

oAROLOPTERIS Debey and Ettingshausen, 1859.

Carolopteris aquensis Debey and Ettingshausen, 1850 b, p. 206, pl. 3, figs. 20-27; fern pinnules; Upper Cretaceous; Aachen, Rhenish Prussia.

CARPANNULARIA Elias, 1931.

Carpannularia americana Elias, 1931, p. 118 , pls. 12,13 ; pl. 14 , figs. $1,3,4$; pl. 15; Annularia-like follage shoots with seeds sald to be attached; lower Pennsylvanian; near Clinton, Henry County, Mo.

OARPANTHOLITES.

See Carpantholithes.
CARPANTHOLITHES Goeppert, 1838.

Carpantholithes berendtii Goeppert, 1838, p. 571 , pl. 42 , figs. 36,37 ; flower ; Miocene; Danzig, Baltic Prussia.

CARPENTERELLA (Munier-Chalmas) Morellet and Morellet, 1922.

Carpenterella jonesi Morellet and Morellet, 1922, p. 20, pl. 1, figs. 77-80 ; Dasycladaceae; Eocene; Beynes, France. [Carpenterella first cited in Munter-Chalmas, 1877 , p. 817 ; nom. nud.]

CARPENTERIANTHUS Borsuk, 1935.

USSR, Central Geol. et Prosp. Inst. Trans., 1935 , v. $37 \mathrm{~A}$, p. 21 ; Hydrangeaceae; Tertlary (not seen). See Gothan, 1942b, 112.

CARPENTIERIA Němejc and Augusta, 1934. Carpentieria marocana Nemejc and $\mathrm{Au}$. gusta, 1934, p. 1, figs. 1a, b.

CARPINIPHYLLUM Nathorst, 1888.

Carpiniphyllum pyramidale (Goeppert) Nathorst, 1888, p. 217, pl. 8, figs. 1-3, 6-8; leaf, dicotyledon; Tertiary ; Japan.

CARPINITES Goeppert and Berendt, 1845.

Carpinites dubius Goeppert and Berendt, in Berendt, 1845, p. 85, pl. 4, figs. 29-31 ; pistillate ament?, Fagaceae; Miocene; Baltic Prussia.

CARPINOXYLON Vater, 1884.

Carpinoxylon compactum Vater, 1884, p. 848 , pl. 29 , figs. 28,29 ; wood ; Cretaceous (Lower Cenonian); Helmstedt, Brunswick.

CARPITES Schimper, 1874.

Carpites pruniformis (Heer) Schimper, 1874 , p. 421 ; seed, incertae sedis ; Miocene ; Oeningen, Switzerland. For Carpolithes pruniformis Heer, 1859, p. 139, pl. 141, figs. $18-30$; pl: 68 , fig. 5 b.

CARPODIUM Zalessky, 1934.

USSR, Central Sci. Geol. Research Inst. Geol. Survey Sec., 1934, p. 12 ; Gymnospermae; Upper Carboniferous (not seen). See Gothan, 1942b, p. 112.

CARPOLITHES Schlotheim, 1820.

Many species of fossil seeds based on impressions, compressions, and casts have been assigned to Carpolithus of Linnaeus and Carpolithes of Schlotheim. As Carpolithes is a repository for seeds and supposed seeds from almost every geological horizon that cannot be assigned to a natural plant group, a type species can hardly be of significance. For further discussion, see Seward, 1917, p. 364, 497.

CARRADORITES Massalongo, 1859.

Carradorites eseri (Unger) Massalongo, in Massalongo and Scarabelli, 1859, p. 91. Specific name spelled "escheri" by. Massalongo but is for Caulerpites eseri Unger, $1850 \mathrm{a}, \mathrm{p}_{3} 3$, 
CARYAEPOLLENITES Robert Potonie, 1934. Caryaepollenites simplex Robert Potonle, in Potonie, Robert, and Venitz, H., 1934, p. 21, pl. 2, figs. 28-30 ; pollen, Juglandaceae; Mlocene; Oberlausitz, Germany.

OARYOJUGLANS Kirchhelmer, 1936.

Caryojuglans quadrangula Kirchheimer, $1936 a$, p. 82 , pl. 12 , figs. $36 a-1$; fruit, Juglandaceae; Tertiary (Braunkohle) ; Borna and Meuselwitz, Germany.

CARYOTISPERMUM Reid and Chandler, 1933.

Caryotispermum cantiense Reld and Chandler, 1933, p. 104, pl. 1, figs. 11, 12 ; seed, Palmae; London Clay, Eocene; Sheppey, Kent, England.

CASEA Newberry, 1853.

Oasea membranacea Newberry, 1853, p; 106 ; compared with Cyclopteris; Pennsylvanian; Middlebury, Ohio.

CASSIOPHYLLUM Geyler, 1887.

Cassiophylum sp. Geyler, 1887a, p. 504, pl. 39 , figs. 7,8 .

CASSIOXYLON Felix, 1882.

Cassioxylon anomalum Fellx, 1882a, p. 69 ; wood; 'Tertiary ; Antigua, West Indies. Seé Fellx, 1883, p. 15, pl. 2, figs. 3, 5.

CASTALIITES Hollick, 1930:

Castalittes ordinarins Hollick, 1930 , p. 76, pl. 41, fig. 7 ; leaf, Nymphaceae; Upper. Cretaceous; Willians coal mine, Yukon River.

CASTANEOIDITES Robert Potonie, 1950.

Castaneoilites exactus Robert Potonie, in Potonie, Robert, Thomson, Paul W., and Thiergart, Friedrick, 1950 , p. 56, pl. B, fig. 30 ; pollen, Fagaceae; Pllocene, Chatt-Aquitan, Germany.

OASTELLINIA Massalongo, 1852.

Castellinia macrocarpa Massalongo, 1852c, p. 206 ; Eocene;. Monte Bolca, Italy.

OASUARINITES Schlotheim, 1820.

Casuarinites equisetiformis Schlothelm, 1820 , p. 397 , for lllustrations Schlotheim refers to his 1804,' pl. 2, flg. 3 ; Asterophyllites-type follage; Upper Carboniferous ; Wettin, Manebach, Saxony:

OASUARINITES Goeppert and Stache, 1855. Casuarinites iugleri Goeppert and Stache, 1855 , p. 42 ; inflorescence, Casuarinaceae ?; Upper Trfassic (Keuper) ; Enger, Prussia.

CASUAROXYLON Goeppert and Stache, 1855.

Casuaroxylon anglia Goeppert and Stache, in' Stache, 1855, p. .42; locality and horizon unknown.

CAT'E'NARIA Sternberg, 1825.

Catenaria decora Sternberg, 1826 (182038), Tentamen, p. xxv, $\therefore$ pl. 52, flg. 1 ; articulate? stem; Carboniferous.
CATHAYSIOPTERIS Koldzuml, 1034.

Cathaysiopteris woitei (Halle) Koldzumi, 1934, p. 113. For Gigantopteris whitei Haile, 1927, p. 173, pl. 47, figs. 1-9; Lower Shihhotse series, Lower Permian; Central Shansi, China.

CATHISPERMUM Reld and Chandler, 1933. Cathispermum pulchrum Reid and Chandler, 1933 , p. 317 , pl. 14, figs. 23-28; fruit, Celastraceae; London Clay, Eocene; Sheppey, Kent, England.

CACDALPHYLLUMI Achepohl, 1883.

Caudaephyllum longifolium Achepohl, 1883 , p: 115 ; calamitean roots ?; Upper Carboniferous; Westphalia.

$C A U D E X$ Lesquereux, 1883.

Caudex spinosus Lesquereux, 1883, p. 91. For Caulinites spinosa Lesquereux, 1874, p. 115 ; stem, incertae sedis ; Cretaceous ; near Fort Harker, Kans.

CAULERPIDES Schimper, 1869.

Caulerpides pyramidalis (Sternberg) Schimper, 1869 (1869-74), p. 160. For Caulerpites pyramidalis Sternberg, 1833 (1820-38), p. 21, pl. 6, fg. 2. Justtfcation for Schimper's claim to this genus is not clear, for it is admittedly based on Sternberg's Caulerpites.

CAULERPITES (Brongniart). Sternberg, 1833.

Caulerpites lycopodioides (Brongnlart) Sternberg, $1833(1820-38)$, p. 20 . For Fucoides lycopodioides Brongniart, 1828 (1828a-38), p. 72 , pl. 9, fig. 3 .

CAULINITES Brongniart, 1828.

Caulinites parisiensis (Deslarest) Brongnlart, $1828 \mathrm{~b}$, p. 115 , leaf, monocotyledon. see also Cuvier and Brongniart, 1822 , p. 234, pl. 8, fig. 10.

CAULOMATITES C. F. W. Braun, 1847.

Caulomatites zamites C. F. W. Braun, 1847 , p. 85 ; nom. nud.

OÁULOMORPHA Saporta, 1886-91.

Caulomorpha locardi Saporta, 1886-91, p. 83 , pl. 236 , fig. 2 ; stem impression, incertae sedis; Jurassic (Kimmerldigian) ; Orbagnoux, France.

CAULOPSIS Gothan and Hartung, 1949.

Caulopsis punctata Gothan and Hartung, in Gothan, 1949, p. 27, pl. 3, figs. 4-6.

CAULOPTERIS Lindley and Hutton, 1832. Caulopteris primaeva Lindley and Hutton, 1832 (1831-37), p. 121, pl. 42 ; treefern trunk impression; Upper Carboniferous; Radstock, near Bath, England. See also Posthumus, 1931.

CAULOXYLON Cribbs, 1939.

Cauloxylon ambiguum Cribbs, 1939, p. 440 , figs. 1-24; petrified cordaitean stem ; Reeds Spring limestone, Mississipplan; Missouri. 
OAXTONIA Reid and Chandler, 1933.

Caxtonia glandulosa Reid and Chandler, 1933 , p. 265 , pl. 10, figs. 17-19 ; carpel, Rutacene?; London Clay, Eocene; Minster, Kent, England.

OAYEUXIA Frollo, 1938.

Cayeuxia moldavica Frollo, 1938, p. 269 , pl. 1 ; calcareous alga; Upper Jurassic ; eastern Carpathians.

OAYTONANTHUS Harris, 1937.

Caytonanthus arberi (Thomas) Harris, 1937 ; p. 40 ; microsporangiate organ, Caytoniales; Jurassic; Cayton Bay, Yorkshire, England. For Antholithus arberi Thomas, 1925, p. 327, pl. 14.

CAYTONIA Thomas, 1925.

Caytonia sewardi Thomas, 1925, p. 315, pls. 12, 13, 15 ; seed-bearing organ, Caytoniales; Middle Estuarine series, Middle Jurassic; Cayton Bay, Yorkshire, England.

CEDRELOPHYLLUM Deane, 1902.

Cedrelophyllum antiqua Deane, 1902a, p. 63 , pl. 15, fig. 1 ; leaf, Meliaceae ? Tertiary; Wingello, New South Wales.

CEDRELOSPERMITES Saporta, 1894.

Cedrelospermites venulosus Saporta, 1894, p. 98 , pl. 16, fig. 21 ; winged seed, dicotyledon ; Cretaceous ; Quinta-doLeiriao, Portugal.

CEDRELOSPERMUM Saporta, 1889.

Cedrelospermum aquense Saporta, 1889, p. 93 , pl. 18, fig. 11 ; winged weed, Cedrelaceae ; Tertiary ; Aix, Provence, France.

CEDRIPITES Wodehouse, 1933.

Cedripites eocenicus Wodehouse, 1933, p. 490, fig. 13 ; Cedrus-like pollen ; Parachute Creek member, Green River formation, Eocene; Colorado and Utah.

CEDRITES R. W. Brown, 1935.

Cedrites primevus R. W. Brown, 1935, p. 445 , fig. 11 ; mold of cone, possibly related to Cedrus; Lower Cretaceous; bank of Anacostia River, three-quarters of a mile beyond the District of Columbia line, Maryland,

ODDROTDITES Tulergart?, 1950.

Cedroidites sp. Thiergart, in Potonie, Robert, Thomson, Paul W., and Thiergart, Fredrich, 1050, p. 47.

CEDROPHLOIOS Fliche, 1896.

Cedrophloios breicheri flche, 1890, p. 258, pl. 12 , fig. 4 ; pl. 14, fig. 3 ; petrifled conlferous bark? Cretaceous (Albian); Villotte, France.

CEDROSTROBUS Stopes, 1915.

Oedrostrobus leckenbyi (Carr $\mathrm{u}$ the $\mathrm{r}$ s) stopes, 1015, p. 143, tg. 39 ; cone, Conlferales; Lower Greensand, Cretaceous ; Shanklin, Isle of Wight, England.
OEDROXYLON Kraus, 1870.

Cedroxylon withami Kraus, in Schimper, 1870 (1869-74), p. 370 ; Carboniferous ; England. For Peuce withami Lindley and Hutton, 1831-33, p. 73, pls. 23, 24.

CELASTRINANTHIUM Conwentz, 1886.

Celastrinanthium hauchecornei Conwentz, 1886 , p. 76 , pl. 8 , figs. $10-13$; fruit, in amber, Celastraceae; early Tertiary : West Prussia.

OELASTRINITES Saporta, 1865.

Celastrinites venulosus Saporta, 1865, p. 52 ; leaf, Celastraceae ; Tertiary ; France. See also Saporta, 1868, p. 412, pl. 36, figs. 12, 13.

CELASTRIN OXYLON Schenk, 1888.

Celastrinoxylon affine Schenk, 1888, p. 21 ; wood ; Tertiary ; Egypt ; nom. nud.

CELASTROCARPUS E. W. Berry, 1930.

Celastrocarpus eocenicus E. W. Berry, 1930 , p. 97 , pl. 25, figs. 26-29 ; capsule, Celastraceae; Wilcox group, Eocene : Saulsbury station, Hardeman County, Tenn.

CELASTROPHYLLUM Goeppert, 1854.

Celastrophyllum attenuatum Goeppert, 1854 , p. 52, pl. 14, fig. 89 ; leaf, Celastraceae; Tertiary; Java. See also Celastrophyllum attenuatum Goeppert, 1853 , p. 435 ; nom. nud.

OELLULOXYLON Dawson, 1881.

Celluloxylon primaevum Dawson, 1881b, p. 302. See also Dawson, in Penhallow, 1893a, p. 115, pl. 15, fig. 1 ; pl. 17 , figs. 5,6 ; pl. 18 , flgs. 7,8 ; pl. 16 , fig. 4 . Earliest reference: Dawson, $1880 a$, p. 476 ; nom. nud.; Devonian; New York.

CELTIDOPHYLLUM Krasser, 1896.

Celtidophyllum praeaustrale Krasser, 1896, p. 130 , pl. 16, figs. 8-14 ; leaf, Ulmaceae ; Cretaceous ; Kunstadt. This appears to be the correct citation for the type species although the presentation is confused. The caption to flgures bears the name Celtidophyllum cretaceum. Earlier. Krasser (1.889) gave the name Celtiophyllum cretaceum as a nom. nud. ; in his "Register," 1896 , p. 151, he inafoates that oeitiophyllum=celtidophyllum.

\section{OELTIOPHYLLUM.}

See Oeltidophyzlum Erasser.

OELTITIS Tuzson, 1909.

Celtitis kleinii Tuzson, 1909, p. 376 ; P1ocene; Balaton Lake, Sumeg, Hungary.

OELYPHINIA Mueller, 1871.

Celyphinia mccoyi Mueller, 1871 (187182 ), p. 40 , pl. 5 .

CENANGITES Meschinelli, 1892.

Genangites piri (Ludwig) Mesch1nelli, in Saccardo, 1892, p. 775 ; fungus, Discomycete; central Germany. See al8o Meschinelil, 1898, p. 50, pl. 15, fig. 32. 
CEPHALOPTERIS Nathorst, 1914.

Cephalopteris mirabilis Nathorst, in Bureau, 1914, p. 23 , pl. 1 bis, figs. 3, 4, 4a ; microsporangiate organ, Pteridospermeae ; Upper Devonian ; Ancenis, France. Earlier given as Cephalopteris mirabilis Nathorst, 1910, p. 277 ; nom. nud.

CEPHALOTAXITES Heer, 1883.

Cephalotaxites insignis Heer, 1883, p. 10, pl. 53, fig. 12 ; fertile (seed) shoot, Coniferales; Upper Cretaceous ; Patoot, Greenland.

CEPHALOTAXOPSIS Fontaine, 1889. Cephalotaxopsis magnifolia Fontaine, 1889 , p. 236 , pls. $104-108$; foliage-bearing twigs, Coniferales; Potomac group, Lower Cretaceous; Fredericksburg, Va.

CEPHALOTAXOSPERMUM F. W. BerTy, 1910.

Cephalotaxospermum carolinianum E. W. Berry, 1910a, p. 187 ; fruit, Taxaceae ; Black Creek formation, Upper Cretaceous; Hale County, Ala.

CEPHALOTHEOA Nathorst, 1902.

Cephalotheca mirabilis Nathorst, 1902a, p. 15 , pl. 1, figs. 18-35; fern? (sporangial clusters borne on under side of rachis near junction with stem) ; Upper Devonian; Bear Island, Norway.

OERAMITES Liebmann, 1845.

Oeramites hisingeri Liebmann, in Forchhammer, 1845 , p. 162 ; alga, Rhodophyceae; Silurian; Bornbolm and Scania, Sweden.

OERAMITES Massalongo, 1859.

Ceramites 8phacelarioides Massalongo, $1859 \mathrm{~b}, \mathrm{p} .11$. For Monemites sphacelarioides Massalongo, 1850, p. 24.

CERATONIOPHYLLUM Ktrchheimer, 1930. Ceratoniophyllum schottleri KIrchheimer, 1930 b, p. 113, pl. 13, figs. 2a-d.

CERATOPHYCUS Schimper, 1879.

Ceratophycus bicornia Schimper, in Schimper and Schenk, 1879 (1879-90), p. 59; alga, Cylindritiae.

CERATOPHYLLITES Unger, 1845.

Ceratophyllites faujasii (Brongniart) Unger, 1845 (1841-47), p. 77. For A8terophyllites faujasii Brongniart, 1822, p. 306 ; Eocene ; Roche-Sauce, Vivarais, France.

OERATOSTROBUS Velenovsky, 1885.

Ceratostrobus sequoiaephyllus Velenovsky, 1885 a, p. 24 , pl. 12 , figs. 14-16 ; follage shoot and fragmentary cone; Cretaceous ; Lipenec, Bohemia.

OERATOZAMITES Meschinelli, 1889.

Ceratozamites vicetinus Meschinell1, 1889, p. 9, pl. 6, figs. 1, 2.

CERCOSPORITES Salmon, 1903.

Cercosporites sp. Salmon, 1903, p. 128, figs. 6-9 ; fungus, Demaliaceae, Fung1 Imperfecti ; Miocene; Melllli, Italy.
CERCOSPORITES Stopes, 1913.

Cercosporites coriococcus (Bayer) Stopes, 1913 , p. 276, flg. 24 ; fungus, Hyphomycetes; Perucer Beds, Upper Cretaceous ;

$\longrightarrow$ Vyserovic and Kounic, Bohemia.

CHAETHOMITES Pampaloni, 1902.

Chaethomites intricatus Pampaloni, 1902, p. 127, pl. 10, fig. 11 ; fungus perithecium; Miocene?; Sicily.

CHABAKSVIA Vologdin, 1939.

Chabakovia ramosa Vologdin, 1939, p. 256, pl. 2 , fig. 4 ; pl. 12 , ftg. $3 a$; pl. 11, figs. 1, 2, 3a; small dendritic thallus, compared with Epiphyton; Middle Cam. brian: South Urals.

CHAETOCLADUS Whitfleld, 1894.

Chaetocladus plumula Whitfield, 1894, p. 356, pl. 11, figs. 11-13; marine alga ; Trenton group, Ordovician; Platteville, Wis.

C.FAETOPHORITES Fliche, 1886.

Chaetophorites tertiarius Fliche, 1886, p. 353 ; Oligocene; Riedisheim near Mulhouse, France.

CHAETOSPHAERITES Felix, 1894.

Chaetosphaerites bilychnis Fellx, 1894a, p. 272 , pl. 19, fig. 4 ; fungus spores, compared with Chaetosphaeria; Eocene ; Perekeschkul near Baku, Transcaucasia. Meschinelli, 1898 , p. 17 , erroneously attributes this genus to Tulasne.

CHAMAECYPARITES Endlicher, 1847.

Chamaecyparites hardtii (Goeppert) Endlicher, 1847, p. 277. For Cupressites hardtii Goeppert, 1837, p. 429 ; Oligocene; Bavaria. For lliustrations, вee Ettingshausen, 1851, p. 157, pl. 23, fig. 18.

OHANGARNIERA Saporta. 1885.

Changarniera inquirenda Saporta, 1885, p. 1442 ; leaf, "proangiosperm" ; Jurassic (Corallian) ; Auxey, France. See also Saporta, 1889 (1886-91), p. 246, pl. 265, figs. 1-3; pl. 266, figs. 1, 2.

CHANSITHEOA Rege, 1920.

Chansitheca palaeosilvana Rege, 1920, p. 193 , pl. 9, figs. 6,7 ; fertile fern follage; Carboniferous.

CHARACEITES Tuzson, 1914.

Characeites verrucosa Tuzson, 1914, p. 234, pl. 13, fig. 1 ; oogonium, Charophyta ; Eocene; Estergom, Hungary.

CHARAXIS Harris, 1939.

Charaxis durlstonense Harris, 1939, p. 67, pI. 16, fig. 10 ; vegetative organs, Characeae; Purbeck beds, Jurassic; Dorset, England. Harris lists six other species as new combinations with the comment: "As this is probably an artificial genus, it would be meaningless to select a type species."

OHARPENTIERIA Unger, 1845.

Charpentieria nivium Unger, 1845 (184147), p. xc; wood, Pliocene; Lemberg, Galicia, Austria. 
CHASMATOPTERIS Zalessky, 1931. Chasmatopteris principalis Zalessky, 1931b, p. 715 , pls. 1,2 ; petrifled stem, Osmundaceae ; Permian; Russia.

CHAUVINIOPSIS Saporta, 1872.

Chauviniopsis pellati Saporta, 1872a-73, p. 119 , pl. 8, fig. 2; alga; Jurassic; Maninghen, near Wimille, France.

CHEILANTHITES Goeppert, 1836.

Cheilanthites mantellii (Brongniart) Goeppert, 1836, p. 231 ; sphenopterid foliage ; Carboniferous; Tilgate Forest, Sussex, England. For Sphenopteris mantelli Brongniart, 1828a-38, p. 170, pl. 45, figs. 3-7.

CHEILOLEPTITES Saporta, 1861.

Cheiloleptites dispersus Saporta, in Heer, 1861 , p. 151 ; fern ; Tertiary ; nom. nud.

C.HEIROLEPIS Schimper, 1870.

Cheirolepis mïnsteri (Schenk) Schimper, 1870 (1869-74), p. 248; coniferous twigs; Rhaetic ; near Bayreuth, Bavaria.

CHEIROSTROBUS Scott, 1897.

Cheirostrobus pettycurensis Scott, $1897 \mathrm{~b}$, p. 421 ; petrified articulate cone ; Calciferous Sandstone series, Lower Carboniferous; Pettycur, near Burntisland, Scotland. See also Scott, 1898b, pls, 1-6.

CHELEPTERIS Corda, 1845.

Chelepteris voltzii (Schimper and Mougeot) Corda, 1845, p. 76. For Camlopteris. voltzii Schimper and Mougeot, 1844 , p. 65 , pls. $30-31$; Triassic (Gres Bigarre) ; Gottenhausen, Alsace-Lorraine. See also Posthumus, 1931.

CHENOPODITES Saporta, 1889.

Chenopodites helicoides Saporta, 1889, ‥ 26, pl. 17, figs. 6, 7 ; seeds, Chenopodiacene; Tertiary ; Aix, Provence, Irance.

CHINLEA Daugherty, 1941.

Chinlea campii Daugherty, 1941, p. 45, pl. 4, fig. 4; stem, Osmundaceae; Chinle formation, Triassic; Arizona.

CHIROPTERIS Kurr, 1858.

Chiropteris digitata Kurr, in Bronn, 1858, p. 143, pl. 12; leaf, incertae sedis; Lettenkohlen-Sandstein, Triassic.

CHITOSPERMUM Harris, 1935.

Ohitospermum stereococcus Harris, 1935, $\mathrm{p}$ : 134, pl. 29; seed, incertae sedis; Lepidopteris zone, Rhaetic; Scoresby Sound, east Greenland.

CHLAMIDOSTACHYS White, 1937.

chlamidostachys chesterianus $\mathrm{Wh}$ it e, 1937 , p. 38 , pl. 8 , figs. $11,17-19,21$; cone impression, Sphenophyllum type?; Kidd Hollow, 3.2 miles southwest of Prairie Grove, Ark.

CHLAMYDOCARPUS Goeppert, 1864.

Chlamydocarpus palmaeformis Goeppert, 1864 , p. 150 , pl. 27, fig: 17 ; seed ; Permian; near Braunau, Bohemia.
CHLOEPHYCUS Miller and Dyer, 1878.

Chloephycus plumosum Miller and Dyer, 1878 , p. 3 , pl. 4 , fig. 1 ; incertae sedis; Cincinnati group, Silurian; Cincinnati, Ohio.

CHLORELLOPSIS Reis, 1923.

Chiorellopsis coloniata Reis, 1923, p. 107, pl. 3 , figs. $1,2,9$; pl. 4 , figs. $3-6$; pl. 5, figs. 2-6.

CHLOROTYLITES Howe, 1932.

Chlorotylites berryi Howe, 1932a, p. 220, figs. 1-3 ; silicified alga, Chlorophyceae ; Sucarnooche clay, lower Eocene; Sumter County, Ala.

CHOFFATIA Saporta, 1894.

Choffatia francheti Saporta, 1894, p. 150, pl. 24 , fig. 8 ; pl. 26 , flgs. $19-22$; plant of Salvinia-like habit; Cretaceous; Portugal.

CHONDRIDES Schimper, 1869.

Chondrides furcatus (Brongniart) Schimper, 1869 (1869-74), p. 168, pl. 3, fig. 8.

CHONDRITES Sternberg, 1833.

Chondrites targionii (Brongniart) Sternberg, 1833 (1820-38), p. 25. For Fucoides targionii Brongniart, 1828a38 , p. 56, pl. 4, figs. 2-6 ; alga?; England.

CHONDROPHYTON Saporta and Marion, 1885.

Chondrophyton dissectum Saporta and Marion, 1885 , p. 120 , fig. 126 ; leaf, dicotyledon.

CHONDROPOGON Squinabol, 1890.

Chondropogon inorosolense Squinabol, 1890 , p. 181 , pl. 11, fig. 3 ; alga?; Tertiary ; Morosolo, Italy.

CHORDITES Fliche, 1905.

Chordites lebruni Fliche, 1905a, p. 50, pl. 4, fig. 1; alga, Phaeophyceae?; Triassic (upper Muschelkalk) ; Meurtheet-Moselle, France. Briefly described but no species cited in Fliche, 1903.

CHORDOPHYLLITES Tate, 1876.

Chordophyllites cicatricosus Tate, in Tate and Blake, 1876, p. 474 , pl. 14, fig. 9: incertae sedis; Lower Jurassic (Lias) ; old Nab, Staithes, Yorkshire, England.

OHORIONOPTERIS Corda, 1845.

Chorionopteris gleichenioides Corda, 1845, p. 90 , pl. 54, figs. 10-16; petrified fern pinnules with synangia; Carboniferous ; Radnitz, Bohemia. Sce also Posthumus, 1931.

CHROOCOCCITES Reinsch, 1881.

Chroococcites sp. Reinsch, 1881, p. 48, pl. 11 , figs. $11-28$; pl. $7 \mathrm{c}$, figs. $3,5,6$; Upper Carboniferous: Mittelbexbach. Bavaria.

CHRYSODIOPTERIS Saporta, 1894.

Chrysodiopteris marchantiaeformis Saporta, 1894, p. 41, pl. 4, figs. 9, 17 ; fern foliage ; Jurassic ; Cabanas-de-Torres, -Portugal. 
CHRYSOTHECA Miner, 1935.

Chrysotheca diskoensis Miner, 1935, p. 590, pl. 18, figs. 1-10 ; perianth ?, Jungermanniales; Upper Cretaceous; Amisut, Disco Island, Greenland.

OHUARIA Walcott, 1928.

Chuaria sp. Walcott, in White, 1928, p. 389 ; alga ; pre-Cambrian ; Grand Canyon, Ariz.

CIBOTIOCAULIS Ogura; 1927.

Cibotiocaulis tateiroae Ogura, 1927, p. 364, pl. 3, figs. $13-15$; pl. 7 ; petrified treefern stem, Cyatheaceae?; Lower Kyongsang formation, Jurassic; Syong-jye Gun, North Kyong-sang Do, Korea.

OIOATRICOSISPORITES Robert Potonle and Gelletich, 1933.

Cicatricosisporites dorogensis Robert Potonie and Gelletich, 1933, p. 522, pl. 1, figs. 1-5 ; Eocene ; Dorog, Hungary.

CINCHONIDIUM Unger, 1850.

Cinchonidium racemosum Unger, $1850 \mathrm{a}$, p. 430 ; fruit, Rubiaceae; Miocene; Radoboj, Croatia. See also Unger, 1865 (1860-65), p. 11, pl. 3, figs. 1, 2, 6 .

CINGULARIA C. E. Welss, 1871.

Cingularia typica C. E. Weiss, 1871, p. 138, pl. 14, fig. 4 ; fragment of articulate cone; Upper Carboniferous; Steinbachstollen, Rhenish Prussia. See also Renault, 1882 , p. 144 , and later works.

CINNAMOMIPHYLLUM Nathorst, 1888

Cinnamomiphyllum sp. Nathorst, 1888, p. 9 , pl. 1, figs. 7-11 ; leaf, dicotyledon ; Tertiary ; Japan.

OINNAMOMOPHYLUM Kräusel and Weyland, 1950.

Cinnamomophyllum (Cinnamomum) scheuchzeri (Heer) Krïusel and Weyland, 1950 , p. 68 , pl. 11 , fig. 7 ; pl. 16, figs. $1-6$; pl. 17, figs. 1, 4-6 ; Tertiary ; Regis mine near Altenburg, Germany.

CINNAMOMOIDES Seward, 1925.

Cinnamomoides newberryi (Berry) Seward, 1925, pl. C, fig. 29 ; Cretaceous; Atanlkerdluk, Greenland.

OIRCIDOXYLON Platen, 1908.

Circidoxylon zirkeli Platen, 1908, p. 139, pl. 2, figs. 5, 6 ; wood ; Tertiary ; Nebraska.

OIRCOPOROXYLON Kräusel, 1949.

Circoporoxylon goepperti (Conwentz) Kräusel, 1949, p. 115. For Glyptostroboxylon goepperti Conwentz, 1885, p. 445; lower Oligocene; Katapuliche, Argentina. See also Kräusel, 1919b, p. 211.

CIRRATRIRADITES L. R. Wilson and Coe, 1940.

Cirratriradites maculatus L. R. Wilson and Coe, 1940, p. 183, pl. 1, fig. 7 ; spore; Des Moines group, Pennsylvanian ; Green County coal mine, Franklin Township, Green County, Iowa.
CISSITES Debey, 1866

Cissites aceroides Debey, in Capellint and Heer, 1866, p. 11, pl. 2, flg. 5.

CISSOPHYLLUM Ettingshausen, 1887.

Cis8ophyllum malvernicium Ettingshausen, $1887 \mathrm{~b}$, p. 171 , pl. 5, fig. 8 ; leaf frag ment, Ampelideae; Eocene; Malvern Hills, New Zealand.

CISTELITES Heer, 1878.

Cistelites sachalinensis Heer, $1878 \mathrm{c}$, p. '69, pl. 15, fig. 12 ; nom. nud; Mlocene Island of Sachalin, Mgratsch, Siberia.

CISTINOCARPUM Conwentz, 1886.

Cistinocarpum roemeri Conwentz, 1886, p. 59 , pl. 6, figs. 10-15; fruit, in amber, Cistaceae; early Tertiary; west Prussia.

CISTOCARPUM Menzel, 1913.

Oistocarpum decemvalvulatum Menzel, 1913 , p. 49 , pl. 5, fig. 5 ; capsule, Cistaceae; Tertiary (Braunkohle); Germany.

CITROPHYLLUM E. W. Berry, 1909.

Citrophyllum aligerum (Lesquereux) E. W. Berry, 1909, p. 258, pl. 18a, figs. 1-8; leaf, compared with Citrus; Raritan formation, Upper Cretaceous ; South Amboy, N. J.

CLADIOCARYA Reid and Chandler, 1926.

Cladiocarya foveolata Reid and Chandler, 1926 , p. 77 , pl. 4 , figs. 22,23 ; fruit, Cyperaceae; Bembridge beds, Oligocene ; Isle of Wight, England.

CLADISCOTHALLUS Renault, 1896.

Cladiscothallus keppeni Renault, 1896a, p. 554, figs. 146, 147 ; alga?; Upper Devonian or Lower Carboniferous ; Ryazan and Tula, Russia.

CLADISCUS Grand'Eury, 1877.

Cladiscus schnorrianus (Gelnitz) Grand' Eury, 1877, p. 382. Generic name given but no species cited in Anonymous, 1872, p. 403.

CLADITES D. H. Scott, 1930.

Cladites bracteatus D. H. Scott, 1930, p. 342, figs. 1-12 ; petrified shoot, Cordaitales?; Lower Coal Measures, Upper Carboniferous; Shore, Littleborough, Lancashire, England.

CLADOCEDROXYLON Fellx, 1882.

Cladocedroxylon auerbachi (Ludwig) Felix, 1882b, p. 265, pl. 2, fig. 5. For Pinus auerbachi Ludwig, 1863, p. 275, pl. 46, figs. 5-7 ; Permian; Lithwinsk, etc., Perm [Molotov], Russia.

CLADOCUPRESSOXYLON Fellx, 1882.

Cladocupressoxylon protolariw Fellx, $1882 a$, p. 46 ; coniferous wood ; Oligocene.

CLADOPHLEBIDIUM Sze, 1931.

Cladophlebidium wongi Sze, 1931, p. 4, pl. 2, fig. 4 . 
OLADOPHLEBIS Brongniart, 1849.

Cladophlebis albertsii (Dunker) Brong niart, 1849, p. 107. For Neuropteris albertsii Dunker, 1846, p. 8, pl. 7, fig. 6 ; fernlike follage; Wealden?; Germany.

CLADOPHORITES Rels, 1923.

Cladophorites dubius Reis, 1923, p. 116, pl. 5, figs. 14, 15 .

CLADOSPORITES Felix, 1894

Cladosporites bipartitus Felix, 1894a, p. 276 , pl. 19, fig. 1 ; fungus conidia, compared with Cephalothecium and Cladosporium; Eocene; Perekeschkul near Baku, Transcaucasia. This genus erroneously attributed to Link by Meschinelli, 1898, p. 80 .

CLADOSTROBUS Zalessky, 1918.

Cladostrobus lutugini Zalessky, 1918, p. 7, pl. 54, figs. 6, 6a ; incertae sedis ; Carboniferous ; near village Vasskino, Kuznets Basin, Russia.

OLADOTHECA Halle, 1911.

Cladotheca undans (Lindley and Hutton) Halle, 1911a, p. 4, pls. 1, 2 ; fertile fern frond, Osmundaceae or Schizaeaceae?; Jurassic; Gristhorpe Bay, Yorkshire, England.

OLADOTHRICINIUM Zalessky, 1915.

Cladothricinium pancratovi $\mathrm{Z}$ a les s k y, 1915 , p. 55 , pl. 10 , figs. 1,2 ; Trichobacterinae? ; Carboniferous ; Russia.

OLADOTHRYX Renault, 1899.

Cladothryx martyi Renault, 1899, p. 894, figs. $3-6,29,30$; pl. 8 , figs. 7,8 ; bacteria ; Pleistocene; Aulne, France.

OLADOXYLON Unger, 1856.

Oladoxylon mirabile Unger, 1856, p. 178, pl. 12, figs. 6,7 ; pteridosperm ?; stems with complex stelar organization; Upper Devonian; Saalfeld, Thuringia. This binomial first used by Unger, 1854a ; nom. nud.

CLASTERIA Dana, 1849.

Clasteria australis Dana, 1849, p. 719, pl. 14, figs. 3-5; Upper Carboniferous ; New South Wales.

CLASTEROSPORITES Pia, 1927.

Clasterosporites eocaenicus (Fritel and Viguier.) Pia, in Hirmer, 1927, p. 123, ber), Cistaceae; early Tertiary; west fig. 113; Dematiaceae, Fungi Imperfecti, in rhizome of Equisetum noviodunense; Eocene.

OLATHRARIA Brongniart, 1822.

Clathraria brardii Brongniart, 1822, p. 222 , pl. 12 , fig. 5 ; sigillarian stem frag. ment; Upper Carboniferous: Terrasson. Dépt. Dordogne, France.

CLATHROPHYLLUM Heer, 1862.

Clathrophyllum meriani (Brongniart) Heer, in Müller, Albrecht, 1862, p. 54. See also Heer, 1864-65, p. 54, pl. 2, fig. 10 ; Upper Triassic (Keuper); Rütihard, Switzerland.
CLATHROPODIUM Saporta, 1873-75.

Clathropodium trigeri Saporta, 1873c-75, p. 288 , pl. 122 , figs. $1-3$; petrifled cycadophyte trunk; Jurassic; Sarthe, France.

CLATHROPTERIS Brongniart, 1828.

Clathropteris meniscioides Brongniart, 1828 b, p. 62, fern foliage ; Lower Jurassic (Lias) ?; Scania, Sweden. See also Brongniart, 1828-38, p. 380, pl. 134.

CLAUSENISPERMUM Reid and Chandler, 1933.

Clausenispermum aubium Reld and Chandler, 1933, p. 264, pl. 10, figs. 15, 16 ; seed, Rutaceae; London Clay, Eocene; Sheppey, Kent, England.

CLAVATOR Reid and Groves, 1924.

Clavator reedii Groves, 1924, p. 116; Characeae; Purbeck beds, Jurassic; Dorset, England. See also Clavator sp. Reid and Groves, 1916, p. 252, pl. 8.

CLAVIJOPSIS Schindehutte, 1907.

Clavijopsis staubi Schindehutte, 1907, p. 62 , pl. 12, figs. 2a-c.

OLEMIENTSIELLA Elias, 1942.

Clementsiella laminarum (Cockerell) Elias, 1942, p. 103, pl. 4, figs. 3, 4; grass fruit; upper Oligocene or lower Miocene ; Florissant, Colo.

CLEPSYDROPSIS Unger, 1856.

Clepsydropsis antiqua Unger, 1856, p. 165, pl. 7 , figs. 1-13; coenopterid fern petiole; Upper Devonian; Saalfeld, Thuringia. This binomial given by Unger, 1854 ; nom. nud. For later accounts, see Hirmer, 1927 ; Sahni, 1928, 1932b.

CLETHRAECARPUM Menzel, 1913.

Clethraecarpum asepalum Menzel, 1913, $\mathrm{p}$. 55, pl. 5, figs. 27, 28 ; fruit, Clethraceae ; Tertiary (Braunkohle); Germany.

CLEVDA Crie, 1889.

Clevea americana Crie, 1889 b, p. 23 ; nom. nud.

CLIMACTOPHYTON Steinmann and Elberskirch, 1929.

Climaciophyton trifoliatum Steinmann and Elberskirch, 1929 , p. 49 , pl. 2, fig. 3.

CLOSTEROXYLON Hartig, 1848.

Closteroxylon lindleyanum Hartig, 1848a, p. 170; wood; Tertiary ; Germany.

CLOUGHTONIA Halle, 1911.

Cloughtonia rugosa Halle, 1911b, p. 2, pls. 1-2 ; cycadophyte? leaflets ; Jurassic (Midale Nistuarinc shnles): Cloushton Wyke, Yorkshire, England.

CLUSIAPHYLLUM E. W. Berry, 1930.

Clusiaphyllum eocenicum E. W. Berry, 1930 , p. 113, pl. 18, fig. 2 ; leaf fragment, Guttiferae; Wilcox group, Eocene ; Nevada County, Ark. 
CLYPEINA Michelin, 1845.

Clypeina marginoporella Michelin, 1845 (1840-47), p. 177, pl. 46, fig. 27; alga, Dasycladaceae ; Upper Cretaceous; near d'Etampes (Seine-et-Oise), France.

COCOOLOBITES Visiani, 1858.

Coccolobites mas8alongiana Visiant, 1858, p. 440, pl. 4, fig. 1; Eocene; Monte promina, Italy.

UOCOOLOBITES E. W. Berry, 1916.

Coccolobites cretaceus N. W. Berry, 1916a, p. 830 , pl. 68 , fig. 1 ; Jeaf, Polygonaceae; Magothy formation, Upper Cretaceous; Grove Point, Cecil County, Md. See also Berry, 1914b, p. 298 ; nom. nud.

COCCOPLASMIUM Reinsch, 1881.

Coccoplasmium sp. Reinsch, 1881, p. 31, pl. 7, flgs. 3-10; pl. 7a, figs. 1-3 ; Upper Carboniferous; Mittelbronn, Wüttemberg.

OOCOULITES Heer, 1874.

Cocculites kanii Heer, 1874 b, p. 21 ; Menlspermaceae ; Mlocene; Greenland ; nom. nud.

OOCCULOPHYLLUM Velenovsky, 1889.

Oocculophyllum cinnamomeum Velenovsky, 1889 , p. 54. For Cocculus cinnamomeus Velenovsky, 1885a, p. 65, pl. 8, figs. 1621 ; Upper Cretaceous; Lipenec, Bohemia.

COCHLIOCARPOS VIsianl, 1858.

Cochliocarpus scorpiuroides Visiani, 1858, p. 44, p1. 2, fig. 6 ; Eocene ; Monte Promina, Italy.

OOOITES Bronn, 1838.

Cocites sp. Bronn 1838 (1837-38), p. 861; palm? fruits.

OOCOOPSIS Fliche, 1896.

Cocoopsis zeilleri Fliche, 1896, p. 271, pl. 12 , figs. 5,6 ; pl. 13 , flgs. 1,2 ; seed, Palmaceae; Cretaceous; Argers and Chaudefontaine, near Ste. Menehould, France. See also Cocoopsis sp. Wiche, 1894, p. 889 .

CODITES Sternberg, 1833.

Codites serpentinus Sternberg, 1833 (1820-38), p. 20 , pl. 3 , flg. 1 ; Incertae sedis ; Jurassic ; Solenhofen, Bavaria.

CODONOPHORA Massalongo, 1857.

Codonophora turbinata (Brongntart) Massalongo, $1857 \mathrm{~b}$, p. 778 . For Fucoides turbinatus Brongniart, $1823, \mathrm{p} .314, \mathrm{pl}$. 20, fig. 1 ; Eocene; Monte Bolca, Italy.

OODONOPHYCUS Fenton and Fenton, 1939.

Codonophycus austinii Fenton and Fenton, 1939, p. 113, pl. 11, flgs. 1-3; alga; Madison formation, Mississippian; Horse Creek, Bald Mtn. quadrangle, Big Horn Mts., Wyo.

CODONOPHXTON Nothorst, 1902.

Codonophyton epiphyticum Nathorst, 1902 a, p. 45, pl. 8 , tigs. 1,2 ; pl. 13, flgs. 9-15; incertae sedis ; Upper Devonian; Bear Island, Norway.
OODONOSPERMUM Brongniart, 1874.

Codonospermum anomalum Brongniart, 1874 , p. 258 , pl. 23 , figs, 9-12 ; sillelfied seed; Carbontferous; St.-fitienne, France.

CODONOTHECA Sellards, 1903.

Codonotheca caduca Sellards, 1908, p. 90, pl. 8 ; pteridosperm microsporanglate organ; Pennsylvanian; Mazon Creek, III.

OOELOSPHAERIDIUM Roemer, 1885.

Coelosphaeridium cyclocrinophilum Roemer, 1885, p. 57, pl. 27, fig. 1.

\section{DOENOXYLON.}

Brror in Seward, 1917, p. 293, for Caenooylon, Zalessky.

COLAOITES Reinsch, 1881.

Colacites sp. Reinsch, 1881 , p. 70 , pl. 16, fig. 1 ; pl. 16a, figs. 6-8; Dpper CarbonIferous; Zwickau, Saxony.

COLEOPHYLLITES Grand'Eury, 1877.

Coleophyllites zeaeformis Grand'Eury, 1877 , p. 39 , calamitean follage? ; Carboniferous; Beraudiere, Lolre, France. For Poacites zeaeformis Schlothelm, 1820 , p. 416, pl. 26, 6g. 2.

COLLENELLA J. H. Johnson, 1942.

Collenella guadalupensis J. H. Johnson, 1942 , p. 212 , pl. 7 , flg. 3 ; lime-secreting alga; Yates sandstone, Permian; south side Dark Canyon, Guadalupe Mts., N. Mex.

COLLENIA Walcott, 1914.

Collenia undosa Walcott, 1914, p. 113, pl. 13, figs. 1,2 ; pl. 14, flgs. 1, 2 ; alga ; Beltian series, Algonkian; 8 miles west of White Sulphur Springs, Meagher County, Mont.

OOLOMBRICARPUM E. M. Reld, 1933.

Colombicarpum biloculare E. M. Rela, 1933, p. 212 , pl. 14, figs. $10-13$; fruit, Anacardiaceae ; Tertiary ; Colombla.

COLPODEXYLON Banks, 1944.

Colpodexylon deatsii Banks, 1944, p. 651, figs. $1-15,17,19,21,24,25$; lycopod with lobed xylem strand and threeforked leaves; Delaware River flags, lower Upper Devonian; 1 mile southeast of Pond Eddy, Sullivan County, N. $\mathbf{Y}$.

COLPOSPERMUM Renault, 1890.

Colpospermum sulcatum Renault, in Renault and Zeiller, 1890, p. 653 , pl. 72 , figs. 63-66 ; seed ; Carboniferous ; Commentry, France.

COLPOXYLON Brongniart, 1849.

Colpoxylon aeduense Brongniart, 1848, p. 109. See also Renault, 1880, p. 78, pl. 11, fig. 8; and Renault, 1896, p. 299 ; petrified stem, Medulloseae; Permian: Autun, France.

COLUMNARIA Sternberg, 1825.

Columnaria intacta Sternberg, 1825, (1820-38), Tentamen, p. xxv. 
COLYMBETES Stopes, 1915.

; Colymbetes edwardsi Stopes, 1915 , p. 314, pIs. 31, 32 ; petrified cycadophy te trunk ; Lower Greensand, Cretaceous ; locality unknown.

COLYMBOXYLON Hartig, 1848.

No species assigned but apparentiy intended as Colymboxylon cretacea (Corda) Hartig, 1848a, p. 140. For Peuce cretacea (Corda) Endlicher, 1847, p. 296. For Pinus cretacea Corda, in Reuss, $1845-46$, p. 91 , pl. 47 , figs. 1-6.

COMATES Reinsch, 1881.

Comates sp. Reinsch, 1881, p. 92, pl. 31a, figs. 8-10; Upper Carboniferous; England.

COMBRETACINIUM Felix, 1894.

Combretacinium quisqualoides Felix, 1894a, p. 90 , pl. 10 , fig. 1 ; compared with Quiaqualis pubescens; Sumgait series, Eocene; Caucasus.

COMBRETANTHITES E. W. Berry, 1913.

Combretanthites eocenica E. W. Berry, 1913 , p. 262, pl. 21 ; flower, Combretaceae; Wilcox group, Eocene ; Grand Junction, Fayette County, Tenn.

COMBRETIPHYLLUM Menzel, 1909.

Combretiphyllum acuminatum Menzel, 1909 , p. 402 , pl. 2, fig. 7 ; leaf fragment, Anonaceae or Moraceae?; lower Tertiary ; Kamerun, Africa.

COMEPHYLLUM Emmons, 1857.

Comephyllum cristatuum Emmons, 1857, p. 128 , fig. 97 ; incertae sedis ; Triassic ; Chatham County, N. C.

COMIA Zalessky, 1934.

Comia pereborensis Zalessky, 1934b, p. 268, figs. 44, 45 ; fernlike foliage ; Permian; Pechora basin, Russia.

COMIPTERIDIUM Zalessky, 1934.

Comipteridium dobroljubovae Zalessky, $1934 \mathrm{~b}$, p. 253 , $\mathrm{ng}$. 22 ; Tern?; rrond fragment; Permian; Pechora basin, Russia.

COMMELINACITES Caspary, 1881.

Oommelinacites dichorisandroides Caspary, 1881. p. 29.

COMPSOPTERIS Zalessky, 1934.

Compsopteris adzvensis Zalessky, 1934b, p. 264 , figs. 38,39 ; alethopterld follage; Permian; Pechora basin, Russia.

COMPSOTESTA (Brongniart) Bertrand, 1010.

Compsotesta brongniarti Bertrand, 1910, p. 189, pl. 14 ; petrified seed; Carboniferous; Grand Croix, France.

COMPSOXYLON Zalessky, 1927.

Compsoxylon monteverdei Zalessky, 1927a, p. 46 , pl. 29 , figs. $8-10$; Permian; Samara, southeast Russia.
COMPTONIOPTERIS Marion, 1890.

Comptoniopteris provincialis Marion, 1890 , p. 1053; Polypodiaceae ; Cretaceous ; Martigues, France. First species lllustrated : Comptoniopteris cercalina Saporta, 1894, p. 129, pl. 26, fig. 24.

COMPTONIPHYLLUM Nathorst, 1888.

Comptoniphyllum : naumanni Nathorst, 1888 , p. 202 , pl. 18 , flg. 2 ; leaf, compared with Myrica; Miocene; Moriyoshimura, Senbokugori, Ugo province, Japan.

COMPTONITES Hisinger, 1837.

Comptonites antiquus (Nilsson) Hisinger, 1837, p. 111. See also Stur, 1863, p. 57, fig. 7.

COMPTOSPERMUM Grand'Eury, 1877.

Comptospermum jarense (Brongniart) Grand'Eury, 1877, p. 184 ; seed ; Carboniferous ; France.

CONCHOCARYON Mueller, 1879.

Conchocaryon 8mithii Mueller, 1879 (1871$82)$, p. 39 , pl. 17 , figs. 4, 5 ; Pliocene; Gulgong, Australia.

CONCHOPHYLLUM Schenk, 1883.

Conchophyllum richthofeni Schenk, 1883c, p. 223 , pl. 42 , figs. 21-26 ; follage shoots, Corlaitales?; Carboniferous; Kai-ping in Tshilt, China.

\section{CONCHOPTERIS.}

Probably error for Lonchopteris, in Bristow, 1862, p. 20.

CONCHOTHECA Mueller, 1873.

Conchotheca rotundata Mueller, 1873 (1871-82), p. 41, pl. 6, figs. 9-11; Pliocene; Nintingbool, Victoria, Australia.

CONOHYOPHYCUS Saporta, 1872.

Conchyophycus marcignyans Saporta, 1872a-73, p. 151, pl. 11 ; alga ; Jurassic ; Marcigny-sous-Thil, France.

CONDRUSIA Stockmans, 1946.

Condrusia rumex Stockmans, 1946a, p. 1, fig. 2 : Unper Devonian: Belgium. For full account, see Stockmans, 1948, p. 57, pl. 11, figs. 4-12.

CONDYLITES Thistleton-Dyer, 1872.

Condylites squamatus 'Thistleton-Dyer, 1872 , p. 195 , p1. 5, fig. 7 ; coniferous twig?; Jurassic ; Solenhofen, Bavaria.

CONFERVITES Brongniart, 1828.

Confervites thoreaeformis Brongniart, $1828(1828 a-38)$, p. 86, pl. 9 bls, flgs. 34 ; alga?; Tertiary; Monte Bolca, near Verona, Italy.

CONFERVOIDES Jaeger, 1827.

Confervoides arenaceus Jaeger, 1827, p. 34, pl. 8, fig. 2; alga?; Upper Triassic (Keuper) ; Ilsfeld, Wïrttemberg. 
CONIFERITES Unger, 1839.

Coniferites lignitum Unger, 1839b, p. 13 ; Miocene; Peggan, Styria. Apparently only species illustrated is Coniferites? verticillatus Tate in Johnston, 1853, p. 309 , pl. 13, figs. 8, $8 \mathrm{a}$; articulate stem impiession?; Upper Carboniferous; Lammerton, England. Doubtful that these two species are closely related.

CONIFEROCAULON Fliche, 1900.

Coniferocaulon colymbeaeforme Fliche, 1900 , p. 16, figs. 1-3 [unnumbered plate」; stem, Coniferales ; Cretaceous ; France.

CONIFEROMYLLON Fliche, 1908.

Coniferomyelon conchylianum Fliche, 1908, p. 211, pl. 18, figs. 2-3 ; stem cast, Coniferales?; Triassic ; Meurthe-etMoselle, France.

CONIFEROXYLON G. F. Beck, 1945.

Coniferoxylon krausei (Felix) G. F. Beck, 1945 , p. 94 ; a genus established for "anomalous" coniferous wood.

CONIOPTERIS Brongniart, 1849.

Coniopteris murrayana Brongniart, 1849, p. 75. For Pecopteris murrayana Brongniart, $1828 \mathrm{a}-38$, p. 358 , pl. 126, figs. 15 ; fernlike foliage; Jurassic; Scarborough, Yorkshire, England.

CONITES Sternberg, 1823.

Conites bucklandi Sternberg, 1823 (1820$38)$, p. 39 , pl. 30 ; cone, Coniferales?

CONNARACANTHIUM Conwentz, 1886.

Connaracanthium roureoides Conwentz, 1886 , p. 104, pl. 10 , figs. $17-21$; Inflorescence (in amber), Connaraceae; early ''ertiary; West Prussia.

CONNAROPPHYLLUM Ettingshausen, 1903.

Connarophyllum crassinervium Ettingshausen, in Krasser, 1903, p. 858; nom. nud.

CONOCARPITES E. W. Berry, 1919.

Conocarpites formosus E. W. Berry, 1919a, p. 127 , pl. 28 , fig. 9 ; leaf, Combretaceae; Tuscaloosa formation, Upper Cretaceous; Glen Allen, Fayette County, Ala.

CONOPHOROIDES Koenig, 1825.

Conophoroides anthemis Koenig, 1825, pl. 16, flg. 200 ; no description ; later transferred to Lepidostrobus anthemis (Koenig) Kidston, 1886, p. 197.

DONOPHYTON Maslov, 1937.

Conophyton lituus Maslov, 1937b, p. 344, pl. 4, figs. 2, 3; calcareous alga?; Lower Cambrian; Aldan River, western Baikal, USSR.

OONOSPERMITES Ettingshausen, 1867.

Conospermites hakeaefolius Ettingshausen, 1867 , p. 254 , pl. 3 , figs. 4,12 ; leaf, Froteaceae; Upper Cretaceous; Niederschoena, Saxony.

OONOSPERMOPHYLLUM Velenovsky, 1889.

Conospermophyllum hakeaefolium Velenovsky, 1889 , p. 53.
CONOSTICHUS Lesquereux, 1876.

Conostichus ornatus Lesquereux, 1876a, p. 142 , pl. 1 , fig. 6 ; incertae sedis ; Pennsylvanian; Indiana.

CONOSTOMA Williamson, 1876.

Conostoma oblonga Williamson, 1876a, p. 71 ; seed; Upper Carboniferous; Oldham, England. See also Williamson, 1877 , p. 268 , pl. 12 , figs. $80,81,86$.

CONSTANTINIUM Unger, 1863.

Constantinium proteoides Unger, in Tchlhatchef, 1863, p. 517 ; wood, Proteaceae ; Tertiary; Lake Derkos, Thrace. See also Tchihatchef, 1866 , p. 322 , pl. 17, figs. $1,2$.

CONVALLARITES Brongniart, 1828.

Convallarites evecta Brongniart, 1828d, p. 455, pl. 19 ; articulate stem and leaves?; Triassic; Sultz-les-Rains, near Strasbourg.

COOKSONIA Lang, 1937.

Cooksonia pertoni Lang, 1937, p. 250, pl. 8, figs. 4-19; pl. 9, figs. 20-27; small leafless plant, Psilophytales?; Downtonian, Devonian; Perton Quarry, Saltwells, South Pembrokeshire, England.

COPIAPAEA Solms-Laubach, 1899.

Copiapaea plicatella Solms-Laubach, 1899, p. 594, pl. 13, figs. 8-11 ; leaf fragments ; Rhaetic; La Tenera, Chlle.

COPPERIA Walcott, 1914.

Copperia tubiformis Walcott, 1914, p. 110, pl. 19, figs. 1-3; alga; Newland limestone, Algonkian ; 8 miles west of White Sulphur Springs, Meagher County, Mont.

COPROSMAEPHYLLUM Deane, 1904.

Coprosmaephyllum ovatum Deane, 1904, p. 212 , pl. 20 , figs. $1-3$; leaf, compared with Coprosma; Tertiary; Sentinel Rock, Otway Coast, Victoria.

COPROSMITES Hector, 1880.

Coprosmites oblongifolia Hector, 1880, p. 49 ; nom. nud.

CORALLINITES Unger, 1847.

Corallinites arbuscula Unger, 1847 (184147 ), p. 127 , pl, 39 , fig. 6 ; alga ?; Jurassic; Pechgraben near Weiher, Austria.

CORAPHYTON Steinmann and Elberskirch, 1929.

Coraphyton problematicum Steinmann and Elberskirch, 1929 , p. C59, fig. 22, pl. 2, figs. 9, 10; Lower Devonian; Wahnbachtals near Sleburg, Germany.

CORCHORITES Ettingshausen and Gardner, 1879.

Corchorites quadricostatus Ettingshausen and Gardner, in Ettingshausen, 1879, p. 395 ; nom. nud.

CORCHORITES Deune, 1902.

Corchorites crenulata Deane, 1902a, p. 62, pI. 17, fig. 1 ; leaf, compared with Corchorus cunninghamii; Tertiary ; Wingello, New South. Wales. 
CORDAIANTHOPSIS Fliche, 1910.

Cordaianthopsis minieri Fliche, 1910, p. 267, pl. 27, fig. 2 ; inflorescence, Cordaltales?; Triassic; Haute-Saone, Vosges, France.

GORDAIANTHUS Grand'Eury, 1877.

Cordaianthus gemmifer Grand'Eury, 1877, p. 228 , pl. 26 , figs. 4-7; inflorescence, Cordaitales ; Carboniferous ; France.

CORDAICARPON H. B. Geinitz, 1862.

Cordaicarpon cordai H. B. Geinitz, 1862, p. 150. For Carpolithes cordai Geinitz, 1855 , p. 41 , pl. 2 , figs. 7-16 ; seed compressions, thought by Geinitz to be seed of Cordaites principalis; Upper Carboniferous; Zaukerode, Saxony. See also Seward, 1917, p. 334, 338. Spelling Cordaicarpus adopted by many later writers.

CORDAICARPUS

See Cordaicarpon.

CORDAICLADUS Grand'Eury, 1877.

Cordaicladus subschnorrianus Grand'Eury, 1877 , p. 243 , pl. 28 , figs. 1 , 2 ; Cordaite stem cast ; Carboniferous ; France.

CORDAIFLOYOS Grand'Eury, 1877.

Cordaifloyos sp. Grand'Eury, 1877, p. 250 ; stem impression, Cordaltales; Carboniferous; France.

CORDAIOPSIS Renault, 1896.

Cordaiopsis elliptica Renault, 1896a, p. 344 , pl. 86, figs. 12,13 ; vegetative bud, Cordaitales ? Carboniferous ; Les Chevrots, France.

CORDAIPHLOEUM Grand'Eury, 1877.

Cordaiphloeum sp. Grand'Eury, 1877, p. 509 ; nom. nud.

OORDAISPERMUM Renault, 1881.

Cordaispermum gutbieri (Geinitz) Renault, 1881 , p. 103 , pl. 14 , fig. 7 ; seed, Cordaitales; Upper Carboniferous ; St. ftienne, France. See also Seward, 1917, p. 335.

CORDAISTROBUS Lesquereux, 1878.

Cordaistrobus grand'euryi Lesquereux, $1878 b$, p. 328; Pennsylvanian; Cannelton, Beaver County, Pa. See arso Lesquereux, 1879 , pl. 82 , figs. 3 , $4 a$.

CORDAITANTHUS ottokar Felstmantel, 1876.

Coraaitanthus communis Ottokar Feistmantel, 1876 c, p. 272 , pl. 61, figs. $1-4$; inflorescence, Cordaitales.

CORDAITES Unger, 1850.

Cordaites borassifolia (Sternberg) Unger, 1850a, p. 277. For Flabellaria borassifolia Sternberg, 1822 (1820-38), p. 32, pl. 18 ; follage; Upper Carboniferous; Swina, Bohemia. [Flabellaria borassifolia later was changed to $P y$ chnophyllum borassifolia by Brongniart (1849) after Corda had shown that it was not a palm. Thus both Pychnophyllum and Cordaites are based on the same specimen and Seward, 1917, p. 223 , notes: "It has recently been proposed to revive the forgotten designation Pychnophyllum, but the reasons given are hardly likely to induce botanists to discard the familiar generic name which perpetuates the memory of Corda."]

CORDAIXYLON Grand'Eury, 1877.

Cordaixylon sp. Grand'Eury, 1877, p. 257. First? illustrated account is for Cordaioxylon credneri Morgenroth, 1883, $x$. 306 , pls. 3, 4. Note misspelling of generic name here.

COREMATOCLADUS Ruedemann, 1909.

Corematocladus densa Ruedemann, 1909, p. 206, pl. 3, figs. 1-5; alga, Florideae?; Trenton limestone, Ordovician; Glen Falls, N. Y.

CORMARAUCARIOXYLON Lignier, 1907.

Cormaraucarioxylon crasseradiatum $\mathrm{Lig}$ nier, 1907 , p. 305 ; pl. 20, figs. 53-57; pl. 21 , figs. $62-64,69$; pl. 23 , fig. 82 ; coniferous wood; Upper Jurassic (Oxfordian ) ; Trouville, France.

CORMOCEDROXYLON Felix, 1882.

Cormocedroxylon jurense (Roulller and Fahrenkohl) Felix, 1882b, p. 264 ; coniferous wood ; Jurassic ; Khorochovo, Russia.

CORMOCORDAITES Grand'Lury, 1890.

Cormocordaites sp. Grand'Eury, 1890, p. 314, pl. 7, fig. 11; partly petrifled cordaitean stem; Upper Carboniferous; St.-Etienne, France.

\section{CORMOOUPRESSINOXYLON}

Cormocupressinoxylon ucranicum, in Hofmann, $1884 \mathrm{~b}$, p. 171. Mistake? or emended spelling for Cormocupressoxylon ucranicum (Goeppert) Felix, 1882b, p. 267.

CORMOCUPRESSOXYLON Felix, 1882.

Cormocupressoxylon protolarix Felix, $1882 a$, p. 46 ; coniferous wood ; Oligocene.

OORNOPHYLLUM Newberry, 1895.

Cornophyllum vetustum Newberry, 1895, p. 119, pl. 19, fig. 10 ; leaf, Cornaceae; Cretaceous; Woodbridge, N. J.

CORNOXYLON Conwentz, 1882.

Cornoxylon erraticum Conwentz, 1882, p. 157, wood; Plelstocene (erratic derived from an earlier formation); Holstein. See also Conwentz, in Vater, 1884, p. 846, pl. 29, fig. 27.

OORNUCARPUS E. A. N. Arber, 1914

Cornucarpus acutum (Lindley and Hutton) I. A. N. Arber, 1914 , p. 89 , pl. 6, fig. 14 ; platyspermic seed; Carboniferous. 
CORONELIA Florin, 1940.

Coronelia molinae Florin, 1940c, p. 20, pl. 3 , figs. $3-10$; pl. 4, figs. $1-8$; pl. 5, flgs. 1-4 ; Eocene; Coronel, Dept. Coronel, Chile.

OORTIOITES Rossmassler, 1840.

Corticites lenticellosus Rossmassler, 1840, p. 41, pl. 12, fig. 56 ; Miocene; Altsattel, Bohemia.

CORYDOPODIUM Derville, 1931.

Corydopodium pruvosti Derville, 1931, p. 63, pl. 5, flgs. 17,18 ; pl. 6, flgs. 20-24 ; pl. 7 , figs. $25-28$; pl. 9, figs. 33,34 ; alga, Myxophyceae ; Carboniferous ; BasBoulonnais, France.

CORYLIPOLLENITES Robert Potonie, 1934. Corylipollenites coryphaeus Robert Potonie, 1934, p. 53, pl. 2, fig. 10 ; pollen, Betulaceae; Miocene.

OORYLITES J. S. Gardner, 1887.

Corylites macquarrii (Forbes) J. S. Gardner, 1887 , p. 290 , pl. 15, flg. 3 ; Coryluslike leaf; Miocene; Atanekerdluk, Isle of Mull, Scotland.

CORYLOIDITES Thiergart, 1950.

Coryloidites sp. Thlergart, in Potonfe, Robert, Thomson, Paul W., and Thtergart, Friedrich, 1950, p. 53, pl. C, flg. 18 ; pollen, Betulaceae ; Pliocene ; Lippe ; no description.

OORYLOPSITES Mathiesen, 1932.

Oorylopsites groenlandicus Mathiesen, 1932 , p. 16, figs. 5-10; wood, compared with Corylopsis; early Tertiary; Cape Dalton, east Greenland.

OORYNECARPUS C. F. W. Braun, 1840.

Corynecarpus grandis C: F. W. Braun, 1840 , p. 105 , nom. nud.

OORYNEPTERIS Baily, 1860.

Corynepteris stellata Baily, 1860, p. 238, pl. 21, figs. 1a-c; fragment of fertlle fern-like frond; Carboniferous; Ballygiltenan Lower, near Glin, County Limerick; Ireland.

CORYNOPHYLLITES. Zalessky, 1937.

Corynophyllites setiformis Zalessky, $1937 \mathrm{~b}$, p. 43 , fig. 6 ; stem-bearing filiform follage, Equisetales ; Permian; Russia.

COSELEYA Kidston, 1914.

Coseleya glomerata Kidston, 1914, p. 97, pl. 5, figs. $4,4 a, 5,6$; pl. 10 , fig. 4 ; fertile frond fragment, Pteridospermae? ; "Ten-foot Ironstone Measures," Upper Carboniferous; Cosely near Dudley, Stafiordshire, England.

COSTARITES Debey, 1848.

Costarites undulatus Debey, 1848, p. 115 ; nom. nud.

COTTAEA Goeppert, 1836.

Cottaea danaeoides Goeppert, 1836, p. 452 ; Upper Triassic (Keuper); Stuttgart, Württemberg. For illustrations Goeppert refers to Jaeger, 1827, pl. 7, fig. 6. See also Posthumus, 1931.
COTTAITES Unger, 1842.

Cottaites lapidariorum Unger, $1842 \mathrm{~b}, \mathrm{p}$. 176; wood, Leguminoseae; Tertiary; Gleichenberg, Styria. See also Unger, 1854 b, p. 182 , pl. 7, figs. 1-8. This species removed to Ulminium by Edwards, 1931, leaving type specles (?) as Cottaites robustior Unger, 1842b, p. 176.

ORANMERIA Reid and Chandler, 1933.

Cranmeria trilocularis Reld and Chandler, 1933 , p. 424 , pl. 22, flgs. 22-28 ; fruit, Lythraceae?; London Clay, Eocene; Minster, Kent, England.

ORASPEDOSPERMA Zalessky, 1937.

Craspedosperma bardacanum Zalessky, 1937b, p. 87, fig. 58, seed; Permlan; Matveyevo, USSR.

ORASSULITES \& Laurent, 1899.

Crassultes sp. Laurent, 1899 , p. 145 , pl. 14, flgs. $31,31 \mathrm{a}$; stem and follage compared with Sedum; Tertiary; Celas, France.

CRATOPLEURA Weber, 1892.

Cratopleura holsatica Weber, 1892, p. 128, pls. 4, 5 ; seed, Nymphaeaceae; Interglacial.

OREDNERIA Zenker, 1833.

Oredneria integerrima Zenker, 1833a, p. 17, pl. 2, fig. F ; leaf, dicotyledon ; Upper Cretaceous ; Blankenburg, Germany.

CREMATOPTERIS Schimper and Mougeot, 1844.

Crematopteris typica Schimper and Mougeot, 1844, p. 74, pl. 35; Triassic; Soultz-les-Bains, Alsace.

OREPIDOPTERIS Presl, 1838.

Crepidopteris marginata (Brongniart) Presl, in Sternberg, 1838 (1820-38), p. 119 ; alethopterid follage. See also Brongniart, 1834 (1828-38), pl. 87, tig. 2.

ORETOVARIUM Stopes and Fuj11, 1910.

Oretovarium japonicum Stopes and Fujil, 1910 , p..7.0, pl. 9, figs. 58-60; anglosperm ovary compared with Aletris (Lilfaceae); Upper Cretaceous; Hokkaldo, Japan. See Stopes and Fufli, 1909, p. 559 ; nom. nud.

ORINANTHUS Massalongo, 1859.

Crinanthus fenzlianum Massalongo, 1859a, p. 61, pl. 36, flg. 1 ; frult?, Llliaceae ; Eocene; Italy.

ORINITES Tate, 1853.

Crinitcs lanceolata Tate, in Johnston, 1853 , p. 304 , pl. 13, fig. 6 ; leaf? fragment, incertae sedis ; Upper Carboniferous ; England.

ORINOPHYLLUM Achepohl, 1883.

Crinophyllum sp. Achepohl, 1883, p. 96, pl. 32, fg. 12 ; calamitean roots?; Upper Carbonfferous; Westphalla. 
CROMYODENDRON Presl, 1838.

Cromyodenäron radnicense Presl, in Ștèrnberg, 1838 (1820-38), p. 193.

For Scitaminites musaeformis Sternberg, 1825 (1820-38), Tentamen, p. xxxvi, pl. 5, figs. $2 \mathrm{a}-\mathrm{b}$.

CROSSOCHORDA Schimper, 1879.

Crossochoirda scotica (MacCoy) Schimper, in Schimper and Schenk, 1879 (1879$90)$, p. 52, fig. 40 ; alga, Chordophyceae?; Silurian."

CROSSOTHECA Zelller, 1883.

Crossotheca crepini Zelller, 1883, p. 181, pl. 9, figs. 1-9; pteridosperm microsporanglate organ ; Carboniferous. "For recent detailed consideration of the genus, see Kiḍston, $1923 \mathrm{~b}$, p. 326.

CROSSOTOLEPIS Fliche, 1899.

Crossotolepis perroti Fliche, 1899b, p. 474, pl. 12, fig. 2 ; seed cone, Coniferales ; Ollgocene ; near Embrun, France.

Crossozamia Pomel, 1849.

Crossozamia moreana Pomel, 1849; p: 343 ; cycadophyte leaf ; Jurassic; St. Mihiel, France.

CROTONOPHYLLUM Velenovsky, 1889.

Crotonophyllum cretaceum Velenovsky, 1889 , p. 20 , pl. 5, figs. $4-11$; leaf, compared with Croton (Euphorbiaceae); Upper Cretaceous; Vyserovic, Bohemia.

CROWELLA Reid and Chandler, 1933.

Crowella globosa (Bowerbank) Reid and Chandler, 1933 , p. 216, pl. 7, figs. 6-11; fruit, Lauraceae; London Clay, Eocene; Sheppey, England.

CRUZIANA d'Orbigny, 1842.

Cruziana rugosa (Cordier) d'Orbigny, 1842 , p. 30 , pl. 1, fig. 1.

CRYPTOCARYOIDES E. W. Berry, 1937.

Cryptocaryoides mariasantisimensis E. W. Berry, 1937, p. 47, pl. 6, fig. 3 ; leaf, compared with Cryptocarya, Aniba (Lauraceae) ; Paleocene; Cerro Funes, between Chubut and Santa Cruz, Patagonia.

ORYPTOMERIOPSIS Stopes and FUJ11, 1910. Cryptomeriopsis antiqua Stopes and Fujii, 1910 , p. 52 , pl. 1 , fig. 11 ; pl. 6 , figs. $35-$ 41: coniferous shoot. compared with Cryptomeria ('Taxodiaceae); Upper Cretaceous; Hokkaido, Japan. Earlier citation: Stopes and Fujii, 1909, p. 559, nom. nud.

CRYPTOMERITES Brongniart, 1849.

Cryptomerites ulmanni (Bronn) Brongniart, 1849, p. 123. For Cupressites ulmanni Bronn, 1837 (1837-38), p. 42, pl. 8 , fig. 5 ; coniferous seeds.

CRYPTOPHYLLITES R. M. Johnston, 1888. Cryptophyllites tasmanica R. M. Johnston, 1888, pl. 22, fig. 13; Carboniferous?; Campania, Mt. Wellington; Tasmania.
CRYPTOPLASMIUM Reinsch, 1881.

Cryptoplasmium sp, Reinisch, I881, p. 36, pl. $8 a$, figs. 9,10 ; Middle Triassie; Rothenburg, Franconia.

CRYPTOTHECIUM Hübener, 1851.

Cryptothecium antediluvianum Hübener, in Weber, 1851, p. 228 ; moss; Ollgocene; Wohlscheid, Rhenish Prussia.

CRYPTOXYLON Kidston, 1897.

Cryptoxylon forfarense Kidston, 1897, p. 361 , pls. 8,9 ; stem, incertae sedis; Lower Old Red Sandstone, Devonian; Reswallie, near Forfar, Scotland.

CRYPTOZOON Hall, 1884.

Cryptozoon proliferum Hall, 1884, pl. 6, description on unnumbered page opposite pl. 6; alga?; Greenfield, Saratoga County, N. Y.

CTENIDIOPSIS Raciborski, 1894.

Ctenïdiopsis grojecensis Raciborski, 1894, p. 204 , pl. 19, figs. 4-7.

CTENIDIUM Heer, 1881.

Ctenidium integerrimum Heer, 1881; p. 17, pl. 16, figs. 4-11; cycadophyte frond; Cretaceous ; Almargem, Portugal.

CTENIS Lindley and Hutton, 1834.

Ctenis falcata Lindley and Hutton, 1834 (1831-37), p. 63, pl: 103 ; cycadophyte leaf ; Jurassic ; Gristhorpe Bay, Yorkshire, England.

CTENOPHYLLUM Schimper, 1870.

Ctenophyllum braunianum (Goeppert) Schimper, 1870 (1870-72), p. 143; cycadophyte follage ; Rhaetic; Bayreuth, Silesia. For Pterophyllum braunianum Goeppert, 1844, p. 134. See also Schenk, 1867 (1865-67), p. 164; pl. 38, figs. 1-10.

CTENOPSIS E. W. Berry, 1911.

Ctenopsis latifolia (Fontaine) E. W. Berry, 1911a, p. 349, pl. 55, figs. 1, 2 ; foliage, Bennettitales; Patuxent formation, Lower Cretaceous ; Fredericksburg, va.

OTENOPTERIS Saporta, 1872.

Ctenopteris cycadea (Brongniart) Saporta, 1872a-73, p. 355, pl. 40, figs. 2-5; pl. 41, figs. 1, 2 ; cycadophyte leaves ; Jurassic; Moselle, France.

CTENOZAMITES Nathorst, 1886.

Ctenozamites cycadea (Brongniart) $\mathrm{Na}$ thorst 1886 c, p. 122 . For illustrations, see Schenk, 1887, p. 5, p1. 3, figs. 1116 ; pl. 4 , fig. $18 ;$ pl. 6 , fig. $30 ;$ pl. 7 , fig. 36.

OUCUBALITES Goeppert, 1838.

Cucubalites goldfussii Goeppert, 1838, p. 570, pl. 42, fig. 3 ; flower'; Miocene ; Röttgen, near Bonn, Rhenish Prussia. 
CUCUMITES Bowerbank, '1840.

Cucumites variabilis Bowerbank, 1840, p. 91, pl. 13, figs. 1-34 ; fruit, Cucurbttaceae; London Clay, Eocene; Sheppey, Kent, England.

OUCURBITARIOPSIS Richard Beck, 1882. Cucurbitariopsis congregata Richard Beck, 1882 , p. 752 ; fungus; Oligocene; Brandis, near Lelpzig.

OUCURBITES E. W. Berry, 1929.

Cucurbites compressus E. W: Berry, $1929 \mathrm{~b}$, p. 168 , pl. 3 , figs. 14,15 ; seed, Cucurbltaceae; Tertiary ; Belen, Peru.

CULGOWERIA Florin, 1936.

Culgoweria mirabilis Florin, $1936 \mathrm{~b}, \mathrm{p} .133$, pl. 33, figs. 3-12 ; pl. 34 ; pl. 35 , figs. 1 , 2.; petrified ginkgophy te foliage; Franz Joseph Land.

CULMITES Brongniart, 1822.

Culmites nodosus Brongniart, 1822, p. 215, pl. 12, fig. 1 ; articulate? stem cast; Eocene; near Paris, France.

CUNEATOPTERIS Henry Potonie, 1903.

Cuneatopteris elegans (Brongniart) Henry Potonie, 1903, p. 16. For Sphcnopteris elegans Brongniart, 1829 (1828a-38), p. 172 , pl. 53, figs. $1,2$.

CUNNINGHAMIOSTROBUS Stopes and Fujil, 1910.

Cunninghamiostrobus yabariensis Stopes and Fuj1l, 1910, p. $52, \mathrm{pl}, 5$, figs, $27-34$; petrified cone, compared with Cunninghamia (Taxodiaceae); Upper Cretaceous, Hokkaido, Japan. Earlier citation : Stopes and Fujii, 1909, p. 559, nom. nud.

CUNNINGHAMITES Presl, 1838.

Cunninghamites oxycedrus Presl, in Sternberg, $1838(1820-38)$, p. 203, pl. 48, fig. 3 ; pl. 49 , fig. 1 ; coniferous shoots ; Lower Cretaceous; Saxony. See discussion by Seward, 1919, p. 433.

CUPANITES Schimper, 1874.

Cupanites miocenicus (Ettingshausen) Schimper, $1874(1869-74)$, p. 170; leaves, Sapindaceae?; near Vienna, Austrla. For Oupanoides miocenicus Ettingshausen, 1851 , p. 22, pl. 5, fig. 1.

CUPANOIDES Bowerbank, 1840.

Cupanoides lobatus Bowerbank, 1840, p. 69, pl. 2, figs. 1, 2 ; capsule; London Clay, Eocene ; Sheppey, Kent, England.

CUPRESSINANTHUS Caspary, 1886.

Cupressinanthus polysaccus Caspary, 1886, p. 6 ; male cone, Coniferales; Tertiary ; Samland, Baltic Prussia. See also Caspary, 1907, p. 122, pl. 21.

CUPRESSINITES Bowerbank, 1840.

Cupressinites globosus Bowerbank, 1840, p. 52 , pl. 10, figs, 12-14, 32, 33 ; cones, resembling Cupressus (Cupressaceae); London Clay, Eocene; Sheppey, Kent, England.
CUPRESSINOCAULON.

Probably error for Cupressinoxylon, in Tasche, 1854, p. 92.

CUPRESSINOCLADUS Seward, 1919.

Cupressinocladus salicornoides (Unger) Seward, 1919, p. 307, fig. 752 ; coniferous twigs ; Tertiary.

CUPRESSINOSTROBUS Penny, 1947.

Cupressinostrobus delawarensis Penny, 1947 , p. 285 , figs. $4,6,7,17$; seed cones, Coniferales; Magothy formation, Upper Cretaceous; Deep Cut, west of Summit Brldge, Del.

CUPRESSINOXYLON Goeppert, 1850

Oupressinoxylon subaequale Goeppert, 1850, p. 202 , pl. 27, figs, 1-5 ; conlferous wood ; Tertiary. Of the species described by Goeppert, this is the first which is in any way adequately fllustrated.

CUPRESSITES Brongniart, 1828.

Cupressites hulmanni Brongniart, 1828b, p. 109 . See Bronn, '1837 (1837-38), p. 42 , pl. 8 , fig. 5 ; leafy contferous twig and cone?

CUPRESSOXYLON Kraus, 1870.

Cupres8oxylon ucranicum (Goeppert) Kraus, in Schimper, 1870 (1869-74), p. 374 ; coniferous wood; Cretaceous; Ukraine. For Cupressinoxylon ucranicum Goeppert, 1850 , p. 201, pl. 26, figs. 1-4.

CUPULICARPUS Velenovsky and Viniklar, 1929.

Cupulicarpus fechtneri Velenovsky and Vinlklar, 1929, p. 28, pl, 21, flgs, 4, 5; Castanea-1lke "cupule"; Cretaceous ; Slivenec, Bohemia.

CUPULINA (Kidston) Paul Bertrand, 1913. Cupulina filicoides Kidston, in Bertrand, Paul, 1913, p. 135 ; nom. nud.

CURIONIA Sordelli, 1896.

Curionia triumpilina Sordelli, 1896, p. 31, pl. 7 , fig. 3 ; incertae sedis; Permian; Colombine, Val Trompia, Italy.

CUSSONIPHYLLUM Velenovsky, 1889.

Oussoniphyllum partitum Velenoviky, 1889 , p. 22 , pl. 5 , fig. 1 ; leaves, compared with Oussonia specata (Araliaceae) ; Upper Cretaceous; Bohdankov, Bohemia.

CYATHEITES Goeppert, 1836.

Cyatheites 8chlotheimii Goeppert, 1836, p. 320. For Pecopteris cyathea Brongniart, 1828a-38, p. 307, pl. 101, figs. 1-4 ; pecopterid foliage; Carboniferous ; St. Etienne, France.

OYATHEOPTERIS Schimper, 1869

Cyatheopteris tessellata (Schimper and Mougeot) Schimper, 1869 (1869-74), p. 704 ; tree fern stem, Cyatheaceae? For Caulopteris tessellata Schimper and Mougeot, 1844, p. 64, pl. 29. See also Posthumus, 1931 . 
CYATHOCARPUS C. E. Weiss, 1869.

Oyathocarpus arborescens (Schlotheim) C. E. Weiss, 1869 , p. 84 . For Filicites arborescens Schlotheim, 1820, p. 404 ; see also Schlotheim, 1832, p. 7, p1. 8, fig. 13.

OYATHOCAULIS Ogura, 1927.

Cyathocaulis naktongensis Ogura, 1927, p. 352 , pl. 2 ; pl. 3, figs. 7-12 ; pls. 4-6 ; petrified tree fern stem, Cyatheaceae?; Lower Kyong-sang formation, Upper Jurassic ; Chhil-Kok Gun, North Kyongsang Do, Korea.

CYATHODENDRON Arnold, 1945.

Cyathodendron texanum Arnold, 1945, p. 24, pls. 3-6; petrifled tree fern, Cyatheaceae; probably from Fayette formation, lower upper Eocene; 10 miles north of Roma, Starr County, Tex.

CYATHOIDES E. W. Berry, 1922.

Cyathoides thyrsopterioides E. W. Berry, 1922d, p. 119, pl. 1, figs. 1-3 ; fern frond fragments, Cyatheaceae; Tertiary ; Chile.

OYATHOPHYCUS Walcott, 1883.

Oyathophycus reticulatus Walcott, 1883, p. 18, pl. 2, fig. 16 ; Utica slate, Silurian; Trenton, Oneida County, N. Y.

OYATHORACHIS Ogura, 1927.

Oyathorachis fujiiana Ogura, 1927, p. 368, pl. 8 ; petrified tree fern petioles, $\mathbf{C y}$ atheaceae?; Upper Cretaceous ; Yubari and Ikushumbets, Ishikari province, Hokkaido, Japan.

CYATHOTRACUS Watson, 1906.

Cyathotrachus altus Watson, 1906, p. 3, pls. 1-3 ; Upper Foot mine, Upper Carboniferous; Shore, England.

OYOADANGIUM Ogura, 1932.

Cycadangium compactum Ogura, 1932b, p. 455 , pl. 22, figs. 1-4; cycadophyte sporangia on sporophyll; Cretaceous; Hokkaido, Japan.

CYCADEA Capellini and Solms-Laubach, 1892.

Oycadea imolensis Capellini and SolmsLaubach, 1892, p. 42; Lower Cretaceous ; Imolene, Italy.

\section{OYCADEACITES Morris, 1841 ?}

Cycadeacites columnaris (Presl) Morris, 1841, p. 115. For Cycadites columnaris Presl, in Sternberg, 1820-38, p. 194, pl. 47, figs. 1-6. [It 18 dimcult to determine whether Morris actually uses the generic designation Cycadeacites (which he attributes to Presl). The name appears as a page heading in Sternberg, 1820-38, p. 194, but Oycadites is actually employed in the binomials listed. Morris lists as page heading both Cycadeacites and Cycadites, but his binominals which follow are cited as " $O$. columnaris" etc.]
OYOADELLA Ward, 1900.

Cycadella reedii Ward, 1900 b, p. 264, pl. 15 ; petrified cycadophyte trunk ; Jurassic; Freezeout Hills, Carbon County, Wyo.

OYCADEOCARPUS Dawson, 1873.

Cycadeocarpus columbianus Dawson, 1873, p. 69 , pl. 1 ; petrified cycad seed; Lower Cretaceous or Jurassic ; Skidegate Channel, Queen Charlotte Islands, British Columbia. Dawson also describes and figures petrified petioles and leaves as "probably belonging to the same species."

OYCADEOIDEA Buckland, 1828.

Cycadeoidea megalophylla Buckland, 1828, p. 397 , pls. $47,48(1829)$; petrified cycadeoid trunk; Jurassic ; Isle of Portland, England.

CYCADEOMYELON Saporta, 1873-75.

Cycadeomyelon hettangense Saporta, $1873 c-75$, p. 333 , pl. 119 , fig. 5 ; cycadophyte? stem; Jurassic; Hettange near Metz, France.

CYCADEORACHIS Stopes, 1915.

No specific name given; listed as "Pseudogenus," Stopes, 1915, p. 53, fig. 15; cycadophyte rachis; Lower Greensand, Cretaceous; Kentish Rag, Maldstone, England.

CYOADEOSPERMUM Saporta, 1874.

Cycadeospermum hettangense Saporta, 1874 (1873c-75), p. 238, pl. 116, fig. 6 ; cycad? seed; Jurassic (upper Llas); Hellange, France.

OYCADEOSTROBUS Carruthers, 1867.

Cycadeostrobus ovatus Carruthers, $1867 \mathrm{~b}$, p. 6 , pl. 57 , flgs. 1,2 ; cycad cone cast ; Wealden; Brook Point, Isle of Wight, England.

OYCADINOCARPUS Schimper, 1870.

Cycadinocarpus leuperianus (Schenk) Schimper, 1870 (1869-74), p. 208, pl. 72 ; cycad seed?; near Würzburg.

CYCADINOCARPUS Renault, 1896.

Cycadinocarpus augustodensis (Brongniart) Renault, 1896a, p. 385 , pl. 85, figs. 1-4; sillicified seed; Cordesse, Dracy-Salnt-Loup, France.

OYCADITES Sternberg, 1825.

Cycadites nilsoni Sternberg, 1825 (182038), Tentamen, p. xxxil, pl. 47; cycadophyte frond; Cretaceous; Hör, Sweden. According to Seward, 1917, p. 558, the specimens on which Sternberg's genus was based have been shown to be referable to other genera, and, "As employed by Brongniart and other authors Cycadites stands for fossil fronds agreeing in habit with the pinnate leaves of recent specles of Cycas * * the presence of a single median in the linear pinnae is generally regarded as an essential feature." 
OYCADITES Buckland, 1836.

Oycadites megalophyllus Buckland 1836, p. 497 , p1. 60 ; petrifled cycadophyte trunk ; Isle of Portland, England.

OYOADIUM Gullard, 1839.

Cycadium cyprinopholis Guillard, 1839, p. 129, pl. 3, Carboniferous; Mines of Rivede-Gler, France.

OYCADOCARPIDIUM Nathorst, 1886.

Oycadocarpidium erdmanni Nathorst, 1886 c, p. 91 , pl. 26, flgs. $15-20$; cycad megasporophyll ; Rhaetic ; Bjuf, Sweden.

CYOADOOAULUM Frentzen, 1932.

Cycadocaulum rhaeticum Frentzen, 1932, p. 86, pl. 2, fig. 3 ; Rhaetlc ; Swabla, Nürtingen, Germany.

OYCADOCEPHALUS Nathorst, 1902.

Oycadocephalus sewardi Nathorst, $1902 \mathrm{~b}$, p. 7, pl. 1, figs. 7-10; cycadophyte cone compression; Rhaetic ; Bjuf, Sweden.

CYOADOFILIX Kuntze, 1904.

Oycallofilix, Kuntze, in Post and Kuntze, 1904, p. 156.

OYCADOLEPIS Saporta, 1873-75.

Cycadolepis villosa Saporta, 1873c-75, p. 201 , pl. 114, flg. 4 ; cycadophyte bud scale?; Jurassic; Orbagnoux, France.

CYOADOPHYCOS Massalongo, 1859.

Apparently intended as Cycadophycos pteroides (Sternberg) Massalongo, in Massalongo and Scarabelli, 1859, p. 91. For Caulerpites pteroides Sternberg, 1820-38, p. 21, pl. 24, fig. 5.

OYOADOPHYLLUM Bornemann, 1856.

Cycadophyllum elegans Bornemann, 1856, p. 73, pl. 6, figs. 9-13; Upper Triassic (Keuper); Johannisthales near Mulhausen, Prussia.

OYCADOPITES Wodehouse, 1933.

Cycadopites sp. Wodehouse, 1933 , p. 484, figs. $1-3$; cyad pollen; Parachute Creek member, Green River formation, Eocene ; Colorado and Utah.

OYCADOPSIS Debey, 1848.

Cyadopsis aquisgranensis (Goeppert) Debey, 1848 , p. 140 . For Pinites aquisgranensis Goeppert, 1842b, p. 151, pl. 54, figs. 1-17; Upper Cretaceous (Senonlan) ; near Kunraad, Belgium.

OYCADOPTERIS Zigno, 1853.

Cycadopteris ungeri Zigno, 1853, p. 349. Apparently first specles lllustrated is Cycadopteris brauniana Zigno, 1861, p. 580 , pls. 4-6 ; fern? follage; Middle Jurassic (Oolite); Monte Pernigotti, Italy.

OYCADOPTERIS Schimper, 1869.

Cycadopteris leckenbyi (Bean) Schlmper, 1869 (1869-74), p. 487 ; cycadophyte follage; Jurasslc; Scarborough, England. For Ctenis leckenbyi Bean, in Leckenby, 1863, p. 78, pl. 10, fig. 1. $296347-55-10$
CYOADORACHIS Saporta, 1873.

Oycadorachis armata Saporta, 1873a, p. 121. See also Saporta, 1874 (1873c-75), p. 196 , pl. 117, flg. 1 ; fragment of cycadophyte rachis; Jurassic ; Armalle, near Belley, France.

OYCADOSPADIX Schimper, 1870.

Cycadospadix hennocquei ( $\mathrm{P} 0 \mathrm{~m} \mathrm{e} 1)$ Schimper, 1870 (1869-74), p. 207, pl. 72 ; Cyca8-l1ke megasporophyll; Lower Jurassic (Lias) ; Moselle, France.

CYCADOXYLON Renault, 1879.

Oycadoxylon fremyi Renault, 1879, p. 283, p1. 14, figs. 9-16 ; cycadlike wood ; Permian; France.

OYOLANTHODENDRON Sahni and Surange, 1944.

Cyclanthodendron sahnii (Rode) Sahn1 and Surange, 1944, p. 84, figs. 3-8 ; petri. fied stem, Cyclanthaceae; Deccan Intertrappean sertes, Eocene; Mohgaon Kalan, India.

OYCLOCARPON Goeppert and Fiedler, 1857. Oyclocarpon nummularium Goeppert and Fiedler, in Fledler, 1857, p. 292, pl. 28, fig. 47 ; seed?; Carboniferous; Saarbrucken.

CYCLOCARPUS C. F. W. Braun, 1840.

Cyclocarpus radiatus C. F. W. Braun, 1840 p. 96 ; nom. nud.

CYCLOCLADIA Lindley and Hutton, 1834.

Cyclocladia major Lindley and Hutton, 1834 (1831-37), p. 137, pl. 130 ; lycopod stem impression?; Bensham coal seam, Upper Carbonlferous ; Jarrow Colliery, England.

CYCLODENDRON Krüusel, 1928.

Cyclodenaron leslii (Seward) Krüusel, in Kräusel and Range, 1928, p. 21, pl. 1, fig. 3-10; lycopod stem compression; Karroo beds, Permian; German Southwest Africa.

OYCLOIS Stenzel, 1872

Cyclois varians (Corda) Stenzel, 1872, p. 72. For Palmacites varians Corda, 1846, p. 87, pl, 47, figs. 7-9; Upper Cretaceous (Cenomanian); Kutschiln near Bilin, Bohemia.

OYOLOITES Gruss, 1928.

Palaeobiologica, 1928, Band 1, p. 516 ; alga ?; Devonian (not seen). See also Gothan, 1942b, p. 117.

CYCLOPITYS Schmalhausen, 1879

Cyclopitys nordenskioldi (Heer) Schmalhausen, 1879 , p. 41 , pl. 1 , flg. $4 \mathrm{~b}$; pl. 2 , fig. $1 \mathrm{c}$; pl. 5 , figs. $2 d, 3 b, 6 b, 10$; articulate foliage; Permian ; Russia.

CYOLOPTERIS Brongniart, 1830.

Cyclopteris reniformis Brongniart, 1830 (1828a-38), p. 216, pl. 61, flg. 1 ; fern. like pinnule; Carboniferous. 
CYCLOSPERMUḾ Seward, 1917.

Cyclospermum tenuis (Brongniart) Seward, 1917, p. 341. For Cyclocarpus nummularis Brongniart, 1881, pl. 4.

CYCLOSTIGMA Haughton, 1860.

Cyclostigma kiltorkense Haughton, 1860, p. 222 ; for illustration, see Haughtion, 1859 , pl. 40, fig. 1 ; decorticated lycopod stem; Upper Devonian; Kiltorcan, County Kilkenny, Ireland. See also Seward, 1910, p. 251.

CYCLOTHECA Kidston, 1888.

Cyclotheca biseriata Kidston, 1888, p. 515, pl. 21 , figs. 10-12 ; sporangla, Marattia. ceae ; shales above "Killorgue" coal, Upper Carboniferous; Ellismuir, Bailleston, Lanarkshire, Scotland.

CYCLOZAMIA Pomel, 1849.

Cyclozamia insignis Pomel, 1849, p. 345 ; cycadophyte leaf; Jurassic; Seyssel, France. The use of this binomial is vague ; the description is headed " $\mathrm{Zam}$ ites insignis ou Cyclozamia insignis Pom."

CYLINDRITES Goeppert, 1841.

Cylindrites spongioides Goeppert, 1841a, p. 115, pl. 46, flgs. 1-5; Cretaceous ; near Bunzlau, Silesia.

CYLINDROPLASMIUM Reinsch, 1881.

Cylindroplasmium sp. Reinsch, 1881, p. 44 , pl. $10 \mathrm{~b}$, flg. 1 ; pl: $10 \mathrm{c}$, fig. 3 ; Silurian; Illinois.

CYLINDROPODIUM Saporta, 1873-75:

Cylindropodium liasinum Saporta, 1873c75 , p. 268 , pl. 118 , fig. 3 ; pl. 119 , figs. 1,2 ; pl. 124 , figs. 3,4 ; cycadophyte trunk; Jurassic ; near Lunéville, France.

CYMODOCEITES Bureau, 1886.

Cymodoceites parisiensis (Brongniart) Bureau, 1886, p. 192 . See Squinabol, 1900 , p. 44 , pl. 5 , fig. 2 ; Naladaceae; Eocene ; Arthon, France.

CYMOGLOSSA Schimper, 1869.

Cymoglossa goepperti (Morris) Schimper, 1869 (1869-74), p. 553; pecopteridlike foliage ; Orenbourg, Russia. For Pecopteris goepperti (Morris) Brongniart, 1845 , pl. A, Ags. $2 a-c$; pl. F, figs. $1 a-c$, 1 e.

CYNAROCEPHALUS Kerner, 1916.

Cynarocephalus schuberti Kerner, 1916, p. 190 ; Tertiary ; Cetina Valley, Italy.

CYNOMETROXYLON. Chowdhury and Ghosh, 1946.

Cynometroxylon indicum Chowdhury and Ghosh, 1946, p. 435, pls. 10, 11; wood, compared with Oynometra (Caesalpinioideae) ; Upper Miocene; Naialung, Assam, India. Prellminary note in Chowdhury and Ghosh, 1939.

CYPARISSIDIUM Heer, 1874.

Cyparissidium gracile Heer, 1874a, p. 74, pl. 17 , figs. $5 \mathrm{~b}, 5 \mathrm{c}$; pls. $19,20,21$; cones and foliage-bearing shoots, Taxodiaceae ; Cretaceous; Kome, Greenland.
OYPERACITES Schimper, 1870 :

Cyperacites aubius (Heer) Schimper, 1870 (1869-74), p. 413. For Cyperites dubius Heer, 1855 , p. 75 , pl. 27, flg. 8; Cyperaceae.; Tertiary ; Oeningen, Switzerland.

OYPERITES Lindley and Hutton, 1832.

Oyperites bicarinata Lindley and Hutton, $1832(1831-37)$, p. 123 , pl. 43 ; lycopod leaf ; Carboniferous; Leebotwood Coal Pit, England.

CYPEROCARPUS Fax, 1906.

Cyperocarpus uncinatus $\mathrm{Pax}, 1906$, p. 279, pl. 4, figs. 10,11 ; fruit, Cyperaceae.

CYPEROCAULON 'Lingelsheim, 1917.

Cyperocaulon paxianum Lingelsheim, 1917, p. 545, figs, 1-3; Tertiary; Monte Szentgyorgy near Tapelcza, Hungary.

CYPHOPTERIS Presl, 1838.

Cyphoteris punctulata (Brongniart) Presl, in Sternberg, 1838 (1820-38), p. 121; alethopterid foliage. See also Brongniart, 1828-38, pl. 93, figs. 1, 2.

CYPSELITES Heer, 1859.

Cypselites naegelii Heer, 1859, p. 2, pl. 101, fig. 1 ; fruit, Compositae; Tertiary ; Oeningen, Switzerland.

CYRRHITES. Heer, 1859.

Cyrrhites oeningensis Heer, 1859, p. 136, pl. 140, fig. 55 ; incertae sedis ; Tertiary ; Oeningen, Switzerland.

OYSTIPHYCUS Herzer, 1901.

Cystiphycus latifrons Herzer, 1901, p. 23, fig. 1; "fucoid"; Carboniferous ; Marietta, Ohio.

CYSTORRHIZA Massalongo, 1859.

Cystorrhiza pillularioides Massalongo, 1859 , p. 20 ; Marsileaceae; Eocene; Monte Bolca, Italy ; nom. nud.

CYSTOSEIRITES Sternberg, 1833.

Cystoseirites partschii Sternberg, 1833 (1820-38), p. 35 , pl. 11, fig. 1; alga, some resemblance to Sargassum?; Miocene ; Szakadat, Transylvania.

CYSTOSEIRITES C. F. W. Braun, 1840.

Cystoseirites lancifolius C. F. W. Braun, 1840 , p. 93 ; nom. nud.

CYSTOSPORITES Schopf, 1938.

Cystosporites breretonensis Schopf, 1938a, p. 40 , pl. 1 , figs. 10,11 ; pl. 3 , fig. 5 ; pl. 8, figs. 1-4; spore ; Carbondale formation, Pennsylvanian; Illinois.

CZEKANOWSKIA Heer, 1876.

Czekanowskia setacea Heer, 1876c, p. 68, pl. 5, figs. 1-7; pl. 6, figs. 1-6; pl. 10, fig. 11 ; pl. 12, fig. 5b; pl. 13, fig. 10c; fasicles of fillform leaves, Ginkgophyte; Jurassic.

\section{D}

DACRYDITES Marik, 1901.

Dacrydites incertus Marik, 1901, p. 10, pl. 1, fig. 20 ; Cretaceous (Cenomanian) ; Slivenec, Bohemia. 
DACOTYLODISCUS Rénault, 1899.

Dactylodiscus triangularis Renault, 1899 , p. 977 , pl. 17, fig. 12 ; Tertiary ; Asson, France.

DACTYLOIDITES Hall, 1886.

Dactyloidites bulbosus Hall, 1886 , p. 160 , pl. 11 ; marine alga?; Paleozoic; Middle Granville, Washington County, N. Y.

DACTYLOPHYCUS Miller and Dyer, 1878. Dactylophyous tridigitatum Miller and Dyer, 1878 , p. 1, pl. 3, fig. 2 ; incertae sedis ; Cincinnati group, Silurian ; Cincinnati, Ohio.

DACTYLOPHYLLUM Read, 1934.

Dactylophyllum johnsoni Read, 1934, p. 91 , pl. 18, figs. 2, 3 ; leaf of Baiera type ; Weber(?) formation; lower Pennsylvanian; Bed 17 of Evans Peak section, Mosquito Range, Colo.

DACTYLOPORA. Lamarck, 1838.

Dactylopora cylinaracea Lamarck, in Bronn, 1838 , p. 885 , pl. 35 , figs. $27 \mathrm{a}$, $27 \mathrm{~b}$; alga, Dasycladaceae; Lower Eocene; Versailles, France.

DACTYLOPORELLA Guembel, 1871.

Dactyloporella cylindracea (DeFrance) Guembel, 1871 , p. 263 , pl. D, figs. $9 a$, $9 \mathrm{~b}$.

DACTYLOPORUS Herzer, 1893.

Dactyloporus archaeus Herzer, 1893b, p. 289 , pl. 13 ; fungus, Polyporaceae?; Carboniferous; Tuscarawas County, Ohio.

DACTYLOPTERIS Goeppert, 1852.

Dactylopteris stiehleriana Goeppert, $1852 \mathrm{~b}$, p. 166, pl. 13, fig. 6 ; fernlike? foliage.

DACTYLOTHEOA Zeller, 1883.

Dactylotheca dentata (Brongniart) Zeiller, 1883 , p. 184 , pl. 9 , figs. $12-15$; fertile fern frond; Carboniferous. For recent discussion, see Radforth, 1938, 1939.

DACTYOLEPIS Hollick and Jeffrey, 1909.

Dactyolepis cryptomerioides Hollick and Jeffrey, 1909, p. 52, pl. 10, figs. 12, 13 ; cone scales, Coniferales; Cretaceous ; Kreischerville, Staten Island, N. Y.

DADOXYLON Endlicher, 1847.

Dadoxylon withami (Lindley and Hutton) Endlicher, 1847, p. 298. For Pinites withami Lindley and Hutton, 1831-37, p. 9, pl. 2 ; Upper Carboniferous ; Craigleith, Scotland. See also Frentzen, 1931.

DAEDALEITES Meschinelli, 1892.

Daedaleites queroinus (Massalongo) Meschinelli, in Saccardo, 1892, p. 747 ; fungus in oak wood; Quarternary ; Italy. See also Meschinelli, 1898, p. 6.

DAEDALUS Roualt, 1850.

Daedalus newtoni Roualt, 1850, p. 737 ; Silurian; Brittany.
DAIMONELIX Barbour, 1892.

Daimonelix circumaxilis Barbour, 1892, p. 314 , pls. 1,3 ; fig. 10 ; a problematical fossil considered by some authors to be of plant origin; Miocene ; near Harrison, Sioux County, Nebr. For recent review, see Schultz, 1942.

DALBERGIOPHYLLUM Ettingshausen, 1886.

Dalbergiophyllum affine Ettingshausen, 1886 , p. 134 , pl. 151 , flgs. 21,22 ; leaf, Papilionaceae; Eocene; Vegetable Creek, New South Wales.

DALBERGITES Kuntze, 1904.

Dalbergites Kuntze, in Post and Kuntze, 1904 , p. 162.

DALBERGITES E. W. Berry, 1916.

Dalbergites ellipticifolius 10. W. Berry, 1916 b, p. 247, pl. 54, fig. 10 ; leaf, Papilionaceae; Grenada formation, lower Eocene; Grenada, Grenada County, Miss.

DALIOSTROBUS.

Daliostrobus sternvergit; probably error for Doliostrobus, in Henry Potonte, 1893 b, p. 223

DALYIA Walcott, 1919.

Dalyia racemata Walcott, $1919, \mathrm{p} .237, \mathrm{pl}$. 55 , fig. 4 ; pl. 56, flg. 1 ; alga, Rhodomelaceae; Stephen formation, Middle Cambrian; Burgess Pass fossil quarry, above Field, British Columbia.

DAMMARITES Presl, 1838.

Dammarites albens Presl, in Sternberg, 1838 (1820-38), p. 203, pl. 52, flgs. 11, 12 ; cone, Contferales; Cretaceous (Cenomanian); Neubldschow, Bohemia.

DAMMAROPHYLLUM Velenovsky, 1889.

Dammarophyllum striatum Velenovsky, 1889 , p. 7. For Podozamites striatus Velenovsky, 1885, p. 10, pl. 2, fig. 8; Upper Cretaceous; Liebenau, Bohemia.

DANAEIDES Schimper, 1869.

Danaeides asplenioides (Goeppert) Schimper, 1869 (1869-74), p. 616. For Danaeites asplenioides Goeppert, 1836, p. 380, pl, 19, figs. 4,5 ; fertile fernlike follage; Carboniferous; Charlottenbrunn, Silesia.

DANAEITES Goeppert, 1836.

Danaeites asplenioides Goeppert, 1836, p. 380 , pl. 19 , figs, 4 , 5; fertile fernlike follage; Carboniferous ; Charlottenbrunn, Sllesia.

DANAEOPSIS Heer, 1864.

Danaeopsis marantacea Heer, in Schenk, $1864 a$, p. 303 , pl. 48 , fig. 1.

DAPHNITES Ettingshausen, 1867.

Daphnites goepperti Ettingshausen, 1867, p. 253, pl. 2, fig. 8 ; leaf, Daphnoideae?; Cretaceous; Aigen, Austria. 
DAPHNOGENE Unger, 1845.

Daphnogene cinnamomeifolia (Brongniart) Unger, 1845, p. 227. For Phyllites cinnamomeifolia Brongniart, 1828b, p. 209. See also Unger, 1851, p. 168, pl. 39, figs. 7-9; leaf, dicotyledon ; Miocene; Radoboj, Croatia.

DAPHNOPHYLLUM Heer, 1869.

Daphnophyllum fraasii Heer, 1869c, p. 17, pl. 6, figs. 1, 2 ; Cretaceous (Cenomanian); Moletein, Moravia.

DASYOLADITES Fucini, 1936.

Reference not seen. See Gothan, $1942 \mathrm{~b}$, p. 117.

DASYPHYLLUM Nathorst, 1886.

Dasyphyllum rigidum Nathorst, 1886c, p. 112 , pl. 26, figs. 1-5; incertae sedis; Rhaetic; Bjuf, Sweden. See also Dasyphyllum rigidum Nathorst, 1878, p. 12 ; nom. nud.

DASYPORELLA Stolley, 1893.

Dasyporella silurica Stolley, 1893, p. 139, pl. 8 , figs. 1-6; siphonaceous alga; SiIurian.

DAUBREEIA Zeiller, 1888.

Daubreeia pateraeformis (Germar) Zelller, in Renault and Zeiller, 1888, p. 10, pl. 41 , flg. 1 ; cyclopterid leaflet; Carboniferous; Commentry, France.

DAVALLITES Dawson, 1883.

Davallites richardsoni Dawson, 1883 , p. 25 , pl. 5, figs. $18,18 a, 18 b$; fertile fern follage; Upper Cretaceous; Protection Island, British Columbia. This specles appears to be the first described. Earliest reference is Davallites delicatulus Braun, 1840 , p. 96 ; this species and five others nom. nud.

DAVIDOIDEA Thomas Johnson, 1937.

Davidoidea hebridica Thomas Johnson, 1937 , p. 330 , pl. 21, fig. 3 ; leaf, Nyssaceae; Tertiary ; Scotland.

DAVISELLA Reid and Chandler, 1933.

Davisella ehretioides Reid and Chandler, 1933 , p. 483 , pl. 28 , figs. 6-9 ; Boraginaceae ; London Clay, Eocene; Harefleld, Middlesex, England.

DAWSONITES Halle, 1916.

Dawsonites arcuatus Halle, 1916, p. 24, pl. 3, figs. 1-9; pl. 4, figs. 18-21; psllophyte; Lower Devonian; Röragen, Norway.

DEBEYA Miquel, 1853.

Debeya serrata Miquel, 1853, p. 38, pl, 1, fig. 1 ; leaf, Artocarpeae? (Moraceae) ; Upper Cretaceous (Senonian); near Kunraad, Belgium.

DECAGONOCARPUS Renault, 1800.

Decagonocarpus olivaeformis Renault, in Renault and Zeiller, 1890 , p. 651, pl. 72, fig. 56 ; seed ; Carboniferous ; Commentry, France.
DEOAPLATYSPERMUM Reld and Chandler, 1933.

Decaplatyspermum bowerbanki Reld and Chandler, 1933, p. 256, pl. 9, figs. 2329 ; fruit, Linaceae?; London Clay, Eocene; Sheppey, Kent, England.

DECHENIA Goeppert, 1842.

Dechenia euphorbioides Goeppert, 1842 (1841-46), p. 77 , pl. 3, fig. 1 ; incertae sedis; Devonian; Landshut, Silesia.

DELESSERITES Sternberg, 1833.

Delesserites lamourouxii ' (Brongniart) Sternberg, 1833 (1820-38), p. 32. For F $u$ co $i$ de 8 lamourouxii Brongniart, 1828a-38, p. 64, pl. 8, fig. 2.

DELESSERITES Ruedemann, 1925.

Delesserites salicifolia Ruedemann, 1925, p. 8, pl. 1, fig. 2; alga ?; Utica shale, Ordovician; New York.

DELGADOA Heer, 1881.

Delgadoa occidentalis Heer, 1881 , p. 6, pl. 6, figs. 4-8; pl. 7 ; fern?, compared with Jamesonia imbricata Hooker; Jurassic ; San Pedro near Cintra, Portugal.

DELGADOPSIS Saporta, 1894.

Delgadopsis rhizostigma Saporta, 1894, p. 141 , pls. 23, 25, 26 ; leaf, incertae sedis ; Cretaceous ; Portugal.

DELTOIDOSPORA Miner, 1935.

Deltoidospora hallii Miner, 1935, p. 618, pl. 24, figs. 7, 8 ; spore, Gleicheniaceae?; Kootenal formation, Lower Cretaceous; Cascade County, Mont.

DELTOLDPIS Harris, 1942.

Deltolepis credipota Harris, 1942a, p. 573, figs. 3, 4 ; bud scale, referred to Androlepis and Beania; Middle Estuarine, Jurassic; Cayton Bay, Yorkshire, Eng. land.

DEMETRIA Zalessky, 1930.

Demetria amadoca Zalessky, 1930d, p. 231, pl. 1, fig. 8 ; lycopod stem similar to Lepidodendron; Lower Carboniferous; Staro-Beshev, Donets, Russia.

DENDRACTIS Reis, 1.923.

Denaractis brevis Rels, 1923, p. 111, pl. 3 , figs. 2-4?, 5, 6 ; pl. 4, figs. 5, 6 ; Tertiary; Rhenish Bavaria.

DENDRAENA Nermejc, 1934.

Dendraena pinnatilobata NXmejc, 1934 , p. 3, figs. 1, 2; figs. 7-12 [unnumbered plate]; sphenopterid foliage-bearing sporangia; Carboniferous; Central Bohemia.

DENDROPHYCUS Lesquereux, 1884

Dencrophycus desorii Lesquereux, 1884, p. 699 , pl. 88, fig. 1 ; marine alga ; No. 11 Mauch Chunk shale, Pennsylvanian; bluffs of the Susquehanna above Pittston, $\mathbf{P a}$. 
DENDROPLASMIUM Reinsch, 1881.

Dendroplasmium sp. Reinsch, 1881, p. 30, pl. 3, figs. 1-5 ; Upper Carboniferous; Zwickau, Saxony.

DENDROPTERIDIUM Bancroft, 1932.

Dendropteridium cyatheoides Bancroft, 1932 a, p. 251 , pls. 9,10 ; petrifled stem, Cyatheaceae; late Tertiary ; near Butandiga, Mount Elgon, British East Africa.

DENSOSPORITES Willard Berry, 1937.

Densosporites covensis Willard Berry, 1937, p. 157, fig. 11 ; spore ; Pennington coal, Mississippian; Cranmore Cove, Rhea County, Tenn.

DEPAZITES Meschinelli, 1892.

Depazites acericola (Saporta) Meschinelli, in Saccardo, 1892, p. 785 . See also Meschinelli, 1898, p. 71, pl. 20, fig. 3 ; fungus, Sphaeropsideae.

DERBYELLA David White, 1908.

Derbyella aurita David White, 1908, p. 545 , pl. 9 , flgs. $1,1 \mathrm{a}, 2,2 \mathrm{a}, 3$; reproductive organs of Gangamopteris obovata?; "Permo-Carboniferous"; northeast of Minas, Brazil. Earller citation : Derbyella aurita I. C. White, 1906, p. 379 ; nom. nud.

DERMATOPHYLLITES Goep pert and Berendt, 1845.

Dermatophyllites stilligerus Goeppert and Berendt, 1845, p. 76, pl. 5, flgs. 48-50 ; leaf, Ericaceae ; Mlocene; Prussia.

DESMIA Elchwald, 1860.

Desmia flstulosa Eichwald, 1860, p. 101, pl. 18 , figs. 8,9 ; petrified stem, incertae sedis ; Permian ; Kargala, Orenbourg, Russia. Earlier reference: Desmia fistulosa Elchwald, in Mercklin, 1856, p. 82 ; nom. nud. See also Posthumus, 1931.

DESMTOPHYLLUM Lesquereux, 1878.

Desmiophyllum gracile Lesquereux, 1878b, p. 333; Pennsylvanian; Cannelton, Beaver County, Pa. See also Lesquereux, 1879 , pl. 82 , fig. 1.

DESMODITES Unger, 1839.

Desmodites radobojensis Unger, $1839 \mathrm{a}, \mathrm{p}$. 104 ; Miocene ; Radoboj, Croatia.

DESMODOPHYLLUM Unger, 1850.

Desmodophyllum viticinoides Unger, 1850 a, p. 487 ; leaf, Leguminoseae ; Miocene ; Radoboj, Croatia.

DESMOPHLEBIS Brongniart, 1849.

Desmophlebis flexuosa (Goeppert) Brongniart, 1849 , p. 152 ; apparently for Alethopteris flexuosa in Goeppert, 1836, p. 308 , although Goeppert attributes the species to Sternberg (1820-38), who figures Pecopteris flexuosa (Goeppert) on pl. 33, fig. 1 ; fernlike follage; Triassic (Keuper) ; Relndorf near Bamberg, Bavaria.
DESMOPTERIS Stur, 1883.

Desmopteris alethopteroides (Ettingshausen) Stur, 1883, p. 701: For Asplenites alethopteroides Ettingshausen, 1854, p. 41, pl. 19, flg. 5 ; Carboniferous ; Swina near Radnitz, Bohemia.

DEWALQUEA Saporta and Marion, 1873.

Dewalquea haldemiana (Debey) Saporta and Marion, 1873, p. 60, pl. 7, fig. 1; Tertlary?; Haldem, Westphalia.

DIACHAENITES Alexander Braun, 1859.

Diachaenites heeri Alexander Braun, in Heer, 1859, p. 25, pl. 104, fig. 22 ; fruit, Umbelliferae?; Tertiary ; Oeningen, Switzerland. Earlier reference : Stizenberger, 1851 , p. 89 ; nom. nud.

DIOALAMOPHYLLUM Sterzel, 1880.

Dicalamophyllum altendorfense Sterzel, 1880 , p. 13 , pl. 2, figs. $17-21,25,26$; Permian ; Altendorf near Chemnitz, Germany.

DICERAS Velenovsky, 1889.

Diceras cenomanicus Velenovsky, 1889, p. 14, pl. 2, figs. 5-7; shoots and cones, Taxodiaceae ; Cretaceous (Cenoman(an) ; Vyserovic, Bohemia.

DIOERATOSPERMA H. N. Andrews, 1941. Diceratosperna carpenteriana H. N. Andrews, 1941, p. 379, pl. 15, figs. 8-10; platyspermic seed associated with $D i$ chophyllum moorei; Victory Junction member of Stanton limestone, Missouri group; Pennsylvanian; 6 miles northwest of Garnett, Kans.

DICHONEURON Saporta and Marion, 1885. Dichoneuron hoolceri Saporta and Marion, 1885, p. 231, fig. 100a. Earlier reference: Dichoneuron hookeri Saporta, 1878 , p. 872 ; nom. nud.

DICHOPHLEBIS.

Error for Dicrophlebis, in Bigsby, 1878, p. 375.

DICHOPTERIS Zigno, 1864.

Dichopteris visianica Zigno, 1864, p. 218, pl. 11 , figs. $1-3$; pl. 12, fig. 1 ; fern frond, dichotomously branching rachis; Jurasslc (Oolite); Val d'Assa, Vicetína, Italy.

DICHOTOZAMITES E. W. Berry, 1911.

Dichotozamites cycadopsis (Fontaine) $\mathbf{E}$. W. Berry, 1911a, p. 365, pl. 77, figs. 2, 3 ; cycad? foliage; Patapsco formation, Lower Cretaceous; Mt. Vernon near Brooke, Va.

DICKSONIOPSIS E. W. Berry, 1911.

Dicksoniopsis vernonensis (Ward) E. W. Berry, 1911a, p. 237, pl. 27, figs. 3, 4; frond, Cyatheaceae ; Arundel formation, Lower Cretaceous ; Arlington, Md.

DICKSONIOPTERIS Nathorst, 1890.

Dicksoniopteris naumanni Nathorst, 1890 , p. 51, pl. 5, flg. 4 ; sterile fern follage; Mesozolc; Yakiomura, Haginodani, Japan. 
DIOKSONITES Sterzel, 1881.

Dicksonites pluckeneti (Schlotheim) Sterzel, 1881, p. 226 ; Permian ; Lungau, Saxony. See also Sterzel, 1883, p. 282, 318 , pl. 6, figs. 1-6.

DICLIDOCARYA E. Reid, 1920.

Diclidocarya gibbosa E. Reid, 1920, p. 82, pl. 4, figs. $23-25$; seed, family uncertain; Pliocene; Pont-de-Gail, France.

DICOTYLOPHYLLUM Saporta, 1894.

Dicotylophyllum cerciforme Saporta, 1894, p. 147, pl. 26, fig. 14 ; leaf, dicotyledon; Cretaceous ; Portugal.

DICOTYLOPHYLLUM Bandulska, 1923.

Dicotylophyllum stopesii Bandulska, 1923, p. 244,433 , pl. 20 , figs. 1-4; leaf, dicotyledon ; Eocene ; Bournemouth, England.

DICRANITES Klebs, 1907.

Dicranites casparyi Klebs, in Caspary, 1907, p. 52, pl. 7, figs. 42-45 ; Tertiary ; Baltic Prussia.

DICRANOPHYLLUM Grand'Eury, 1877.

Dicranophyllum gallicum Grand'Eury, 1877 , p. 275 , pl. 14, flgs. 8-10; shoot bearing fillform dichotomizing leaves; Carboniferous; St.-Etienne, France.

DICRANOPHYTON Zalessky, 1937.

Paleophytographica, p. 10: Moskva, Akad. Nauk SSSR, 1937 (not seen). See Gothan, 1942b, p. 118.

DICRANOPTERIS Zalessky, 1937.

Dicranopteris regia Zalessky, 1937b, p. 48, figs. 13-15 ; sphenopterid foliage ; Permian ; Matveyevo, Russia.

DICKOIDIOPSIS Frenguelli, 1943.

Dicroidiopsis incisa (Du Toit) Frenguelli, $1943 a$, p. 288 , fig. 22 ; pteridosperm? foliage; Molteno beds, upper Keuper, Triassic; Konings Kroon, Cape Colony.

DICROIDIUM Gothan, 1912.

Dicroidium odontopteroides (Morris) Gothan, 1912 , p. 78 , pl. 16 , fig. 5 ; pteridosperm? follage; Rhaetic; South Africa.

DICROPHLEBIS (Brongniart) Meneghini, 1857.

Dicrophlebis aftnis (Schlotheim) Meneghini, 1857 , p. 108 , pl. D, fig. V4. See also Brongniart, 1849 , p. 74 .

DICROPTERIS Pomel, 1849.

Dicropteris laciniata Pomel, 1849, p. 339 ; fern; Jurassic; St. Mihlel, France.

DIOTIOPHRAGMIUM Reinsch, 1881.

Dictiophragmium sp. Reinsch, 1881, p. 99, pl. 34 , fig. 1 ; pl. 35 , figs. 1,$2 ;$ Upper Carboniferous; Newcastle, England.

DICTUOLITES COUrad, 1838.

Dictuolites beckii Conrad, 1838, p. 113; Silurian; New York. See also Conrad, in Hall, 1843, p. 48, pl, 1, fig. 1.
DICTYOCALAMITES E. A. N. Arber, 1912. Dictyocalamites burri E. A. N. Arber, 1912 , p. 97, pl. 5 , figs $1,3,5$; calamitean stem impression; Upper Carboniferous; Barfreston, Kent coalfield, England.

DICTYOCALLIPTERIDIUM Jongmans and Gothan, 1935.

Dictyocallipteridium sundaicum Jongmans and Gothan, 1935, p. 137, pl. 44, figs. 3, 4 ; fern follage; Upper Carbontferous ; Residentie Djambi, Mengkarang, Sumatra.

DICTYOCORDAITES Dawson, 1889.

Dictyocordaites lacoi Dawson, 1889 , p. 3, fig. [unnumbered] ; cordaitean stem and leaf compression; Upper Devonian ; Meshoppen, Wyoming County, Pa.

DIOTYYODNDRON Landsborough, 1844.

Dictyodendron patrioii Landsborough, in Patrick, 1844, p. 287, pl. 5, fig. 1 ; stem cast ? Carboniferouz ; Ardeer, Ayrshire, England.

DICTYODENDRON Elchwald, 1860.

Dictyodendron leuchtenbergii Eichwald, 1860 , p. 247 , pl. 19, figs. 5, 6 ; pl. 20, figs. 9-11; coniferous wood ; Carboniferous ; Artinsk, Russia.

DICTYODENDRON Nathorst, 1914.

Dictyodendron kidstonii Nathorst, 1914, p. 72 , pl. 8 , figs. $1-4$; pl. 9 , figs. $1-8$; pl. 12 , figs. $11-20$; pl. 13 , figs. $32-36$; stem cast; Paleozolc; Spitzbergen.

DICTYODORA C. E. Weiss, 1884.

Dictyodora liebeana (Geinitz) C. E. Weiss, 1884 a, p. 84 , pl. 11 ; pl. 12, figs. 1-5; plant?; Lower Carboniferous (Culm); Thuringia.

DICTYOPHLOIS Foerste, 1916.

Dictyophlois reticulata Foerste, 1916, p. 675 , pl. 33 ; rhlyophore compared with Stigmaria; Carboniferous; Sample, Breckenridge County, Ky.

DICTYOPHYCUS Ruedemann, 1931.

Dictyophycus gracilis Ruedemann, 1931, p. 1, pls. 1, 2 ; alga ?; Burgess shale, Middle Cambrian; Burgess Pass, near Field, British Columbia.

DICTYOPHYLLUM Lindley and Hutton, 1834.

Dictyophyllum vugosum Lindley and Hutton, 1834 (1831-37), p. 65, pl. 104 ; fern leaf, Dipterinae: Turassic (Oolite); Yorkshire, England.

"DICTYOPHYLLUM" Sve, 1933.

"Dictyophyllum" Sze, 1933, p. 14, pl. 4, figs. 12, 13; leaf fragment, incertae sedis; Paleozoic; Kunngyuen, China. Cited doubtfully as a new genus.

DICTYOPHYTON Fall, 1863

Dictyophyton newberryt Hall, 1883, p. 76, pl. 4, figs. 1-3; Chenung group, Devonian; Cuyahoga Falls, Richfleld, Ohio. 
DICTYOPLASMIUM Reinsch, 1881.

Dictyoplasmium sp. Reinsch, 1881, p. 41, pl. $10 \mathrm{~b}$, figs. 7,$8 ;$ pl. 15 , fig. 3 ; Upper Carboniferous; Zwickau, Saxony.

DICTYOPORUS Mägdefrau, 1937.

Dictyoporus nodusus Mägdefrau, 1937, p. 55, pl. 4, fig. 10 ; .plant?; Cretaceous (Upper Senonian); Misburg near Han. nover.

DICTYOPTERIDIUM Ottokar Feistmantel, 1880.

Dictyopteridium sporiferum Ottokar Feistmantel, 1880 (1880-81), p. 14, pl. 23A, figs. 4-6, 14; Permían; Talchir and Gopalprasad, India.

DICTYOPTERIS Gutbier, 1835.

Dictyopteris brongniarti Gutbier, 1835, $\mathrm{p}$. 63 , pl. 11, figs. 7, 9, 10 ; Upper Carboniferous ; Zwickau, Saxony.

DICTYOSPORITES Fellx, 1894.

Dictyosporites loculatus Felix, 1894a, p. 277 , pl. 19 , fig. 2 ; fungus conidia, compared with Septosporidium; Eocene; Perekeschul, near Baku, Transcaucasia. Meschinelli, 1898, p. 79, erroneously attributes this genus to Corda.

DICTYOTESTA Gothan, 1941.

Dictyotesta lonchopteroides Gothan, 1941, p. 279 , figs. a, b ; described as seed of Lonchopteris rugosa; Carboniferous ; Aachen, Rhenish Prussia.

DICTYOTHALAMUS Goeppert, 1864.

Dictyothalamus schrolliamus Goeppert, 1864 , p. 164 , pl. 24 , figs. $4-6$; pl. 25 , figs. 1-4; microsporangiate inflorescence?; Permian.

DICTYOTITES (Brongniart) Massalongo, 1859.

Dictyotites brongniartii Massalongo, 1859 , p. 51, pl. 14, fig. 1 ; alga ?; Italy.

DICTYOXYLON Williamson, 1869.

Dictyoxylon oldhamium (Binney) Williamson, 1869a, p. 66, pl. 20, figs. 3, 4 ; pteridosperm stem, see Lyginopteris; Carbontferous; England. See also Seward, 1917, p. 38.

DICTYOXYLON Brongniart, 1872.

Dictyoxylon sp. Brongniart, in Renault, 1872 , p. 1295 ; silicifled sigillarian? trunk; Upper Carboniferous; Autun, France.

DICTYOZAMITES (Oldham) Medlicott and Blanford, 1879

Dictyozamites falcatus (Morris) Medlicott and Blanford, 1879 , p. 142, pl. 8, fig. 6. For Dictyopteris falcata Morris, in Oldham and Morris, 1863, p. 38, pl. 24, figs. 1, $1 \mathrm{a}$.

DIDYMOPHYLLON Goeppert, 1841.

Didymophyllon schottini Goeppert, 1841a (1841-46), p. 69; decorticated lycopod stem?; Devonian; Landshut, Silesia.
DIDYMOSORUS Debey and Ettingshausen, 1859.

Didymosorus comptonifolius Debey and Ettingshausen, 1859b, p. 186, pl. 1, figs. 1-5 ; foliage, Gleicheniaceae ; Upper Cretaceous; Aachen, Rhenish Prussia. Earlier citation : Didymosorus comptoni aefolius Debey, 1849 , p. 299 ; nom. nud.

DIDYMOTHECA Goeppert, 1864.

Didymotheca cordata Goeppert, 1864, p. 178, pl. 26, fig. 24 ; pl. 28, figs. 12, 13 ; seed ? Permian; Braunau, Bohemia.

DIEMENIA Ettingshausen, 1887.

Diemenia speciosa Ettingshausen, $1887 a$ p..108, pl. 11, figs. 7-9 ; Ieaf, Lauraceae ; Eocene; Vegetable Creek, near Emmaville, New South Wales.

DIEUNE Mueller, 1874.

Dieune pluriovulata Mueller, 1874, p. 22, pl. 9, figs. 1-4; angiosperm fruit, affinities uncertain ; lower Pliocene; Haddon, Victoria.

DIFURCOSPHENOPHYLLUM Lotsy, 1909. Difurcosphenophyllum fertile (Scott) Lotsy, 1909, p. 526, fig. 350III. For Sphenophyllum fertile Scott, 1905. See also Leclercq, 1936.

DIGITELLA Morellet and Morellet, 1913.

Digitella dactyloporoides Morellet and Morellet, 1913, p, 28, figs. 14-16; alga, Bornetellaceae; Tertiary ; Echampees, near Lizy-sur-Ourcq, France.

DIGITOLITHUS Fritsch, 1308.

Digitolithus rugatus Fritsch, 1908, p. 23, fig. 7 ; Silurian; Vorder-Treban, Bohemia.

DRGONOSPERMUM Renault, 1907.

Digonospermun grilleti Renault, in Bertrand, C. E., 1907, p. 222.

DIICHNIA Read, 1936.

Diichnia kentuchiensis Read, 1936, p. 151, pls. 30-33; petrified stem, Eu-Calamopitys group; Upper Devonian; Ken. tucky.

DILLENIAECARPUN Weyland, 1948.

Dilleniaecarpum rottense Weyland, 1948, p. 138 , pl. 22 , fig. 9 ; figs. $10-12$; infructescence, Dilleniaceae; Tertiary ; Rott, Siebengebirge, Germany.

DILLENITES E. W. Berry, 1916.

Dillenites microdentatus (Follick) E. W. Berry, 1916a, p. 291, pl. 75, fig. 3; p). 77 , fig. 1 ; leaves, Dilleniaceae; Wilcox group, lower Eocene; Coushatta, Red River Parish, La.

DILOGOPTERIS Grand'Eury, 1877.

Dilogopteris orbicularis Grand' Eury, 1877, p. 521 ; nom. nud.

DIMERIPTERIS Schmalhausen, 1894.

Dimeripteris fasoiculata Schmalhausen, 1894 , p. 30 , pl. 1., figs. 10,11 ; Telangium-like microsporangiate organs; Upper Devonian; Donets, Russia. 
DIMORPHOSIPHON Hoeg, 1927.

Dimorphosiphon rectangulare Hoeg, 1927, p. 4 , pls. $1-3$; petrifled alga, Codiaceae ; Middle Ordovician; south of Bergviken. Island of Helgöen, Norway.

DIMORPHOSTROMA Reis, 1921.

Dimorphostroma varians Reis, 1921, p. 313; Tertiary; Rhenish Bavaria. See also Reis, 1923, pl. 4, fig. 12.

DINEURON Renault, 1896.

Dineuron pteroides Renault, 1896a, p. 22, fig. 19 ; coenopterid fern petiole ; Esnost, France. See also Posthumus, 1931.

DIOONIPITES Wodehouse, 1933.

Dioonipites sp. Wodehouse, 1933 , p. 484 , figs. 4, 5; cycad pollen; Parachute Creek member, Green River formation. Eocene; Colorado and Utah.

DIOONITES Miquel, 1851.

Designation of a type species is problematical. Dioonites feneonis (Brongniart) Miquel, 1851b. For Zamia feneonis Brongniart, $1828 \mathrm{~b}, \mathrm{p} .99$, illustrated in Miller, 1857, p. 69, fig. 36. Other species described by Emmons, 1856, 1857; and Schenk, 1871.

DIOONITOCARPIDIUM Lilienstern, 1928.

Dioonitocarpidium pennaeforme (Schenk) Lilienstern, 1928, p. 103, pls. 5, 6 ; fig. 1 ; cycadophyte megasporophyll ; Upper Tr1assic (Keuper); Estenfeld, Bavaria. For Dioonites pennaeformis Schenk, 1864b.

DIOONOPTERIS Goeppert, 1864.

Dioonopteris permica Goeppert, 1864, p. 126, pl. 13, figs. 3, 4 ; leaf fragment; Permian ; Braunau, Bohemia.

DIOSCORITES Saporta, 1863.

Dioscorites resurgens Saporta, 1863, p. 42 , pl. 4, flg. 5 ; leaf, Dioscoreae ; Tertiary ; France.

DIOSCOROIDES Fritel, 1904.

Dioscoroides lyelli (Watelet) Fritel, 1904, p. 233, figs. 1, 2 ; Eocene ; Belleu, Paris, France.

DIOSPYROPHYLLUM Velenovsky, 1889.

Diospyrophyllum provectum Velenovsky, 1889 , p. 50 . For Diospyros provecta Velenovsky, 1884, p. 49, pl. 8, fig. 1-5, 10: Unper Cretaceous; Melntk, noar Llebenau, Bohemla.

DIPHYLLITES Heer, 1883.

Diphyllites membranaceus Heer, 1883, p. 45 , pl. 60, fig. $4 \mathrm{a}$; leaf fragment, Leguminosae; Upper Cretaceous; Patoot, Greenland.

DIPLASIOPHYLLUM Frenguelli, 1943.

Diplasiophyllum hughesi (Feistmantel) Frenguelli, 1943a, p. 299, figs. 23, 24 ; sterile fern? frond; Rhaetic to Keuper ; China, India, South Africa.
DIPLASTEROTHECA Hirmer, 1927.

Diplasterotheca exigua (Renault) Hirmer, 1927 , p. 585 ; fertile pecopterid foliage; Permian; Autun, France. For Pecopteris exigua Renault, 1883, p. 115, pl. 19, figs. 13-18. Hirmer refers to Renault in Zeller, 1890, p. 70-72.

DIPLAZITES Goeppert, 1836.

Diplazites emarginatus Goeppert, 1836, p. 274 , pl. 16, Ag. 12 ; fern pinnules ; Carboniferous.

DIPLOCYMA Stelnmann and Elberskirch, 1929.

Diplocyma elberskirchianum Steinmann and Elberskirch, 1929, p. C57, fig. 21; Lower Devonian; Wahnbachtals near Sieburg, Germany.

DIPLODENDRON Eichwald, 1846.

Diplodendron hastatum Eichwald, 1846, p. 456. See also Eichwald, 1860 , p. 225, pl. 17 , figs. 3,4 ; fern or cycadophyte stem?; upper Paleozoic; mines of Kloutschewsk, Orenbourg, Russia.

\section{DIPLODICTYUM Braun, 1843.}

Diplodictyum obtusilobum Braun, in Münster, 1843 (1839-43), p. 14, pl. 13, flgs. 11, 12 ; Jurassic ; Bayreuth, Bavaria.

DIPLOLABIS Renault, 1896.

Diplolabis forensis Renault, 1896a, p. 14, figs. 6-10 ; coenopterid fern.

DIPLOMASTIXIA K1rchheimer, 1934.

Diplomastixia carinat Kirchhelmer, 1934b, p. 789 , fig. 17 ; fruit, Cornaceae : Tertiary (Braunkohle); Germany.

DIPLOPHAOELUS Corda, 1845.

Diplophacelus arboreus Corda, 1845, p. 87, pl. 55 ; fern petlole; Upper Carboniferous; Radnitz, Bohemia.

DIPLOPHRAGMIUM Reinsch, 1881.

Diplophragmium sp. Reinsch, 1881, p. 102 ; pl. 41 , fig. 6 ; pl. 42 , figs. $1-5$; pl. 43 , figs. $1-5$; Pennsylvanian; Swickau, Saxony.

DIPLOPHYLLUM Velenovgky and Viniklar, 1929.

Diplophyllum cretaceum Velenovsky and Viniklar, 1929, p. 25, pl. 17, fig. $10:$ pl. 19 , fig. 10 ; pl. 20 , fig. 5 ; leaf, Leguminosae?; Cretaceous; Cernikov, Bohemia.

DIPLOPORA Schafhautl, 1863.

Diplopora annulata Schafhautl, 1863, p. 324, pl. 65e, fig. 6 ; alga, Dasycladaceae.

DIPLOPTERTDIUAX Walton, 1931.

Diplopteridium teilinnum (Kidston) Wal. ton, 1931, p. 349, pl. 23; sphenopterid foliage, probably bore Telangium-like fructifications; Lower Carboniferous ; Gwaenysgor, Flintshire, England. 
DIPLOPTEROTESTA Nathorst, 1914.

Diplopterotesta spitzbergensis (Heer) $\mathrm{Na}$ thorst, 1914, p. 36, pl. 15, flgs. 77-82; winged seed; Paleozoic; Robert-Tal, Spitzbergen.

DIPLOSPORITES PIa, 1927.

Diplosporites ovalis (Renault) Pia, in Hirmer, 1927, p. 122 ; Fungi Imperfecti, Mucedinaceae; Oligo c e n e; Asson, France. For Diplosporium ovale Renault, 1899, p. 978, pl. 17, fig. 13.

DIPLOTAXIS Wood, 1861.

A generic name proposed for possible future reception of certain species of Syringodendron, Wood, 1861a, p. 238.

DIPLOTEGIUM Corda, 1845.

Diplotegium brownianum Corda, 1845, p. 112 , pl. 59 , figs. $3-7$; incertae sedis; Upper Carboniferous ; Radnitz, Bohemia.

DIPLOTESTA Brongniart, 1874.

Diplotesta grand'euryana Brongniart, 1874 , p. 261, pl. 21, flgs. 12-14 ; silicifled s e e d ; Carbontferous ; St.-Etienne, France.

DIPLOTHEOA Kidston, 1903.

Diplotheca stellata Kidston, 1903a, p. 131; fructifleation allied to Calymmatotheca Stur; Machrihanish Water, Scotland. See also Kidston, 1906, p. 431, figs. 11a-e.

DIPLOTHMEMA Stur, 187.7.

Diplothmema patentissimum (Ettingshausen) Stur, 1877, p. 128. For Rhodea patentissima Ettingshausen, in Stur, 1875 , pl. 9 ; pteridosperm? foliage ; Carboniferous (Culm); Altendorf.

DIPLOXYLON Corda, 1840.

Diplowylon elegans Corda, 1840, p. 25, pl. 1 ; Upper Carboniferous ; Chomle, Bavaria.

DIPT'ERIPHYLLUM Krasser, 1896.

Dipteriphyllum cretaceum (Velenovsky) Krasser, 1896, p. 123, pl. 15, fig. 7.

DIPTERITES Kuntze, 1904.

Dipterites Kuntze in Post and Kuntze, 1904, p. 179.

DIPTEROCARPACEOPHYLLUM Kräusel, 1929.

Dipterocarpaceophyllum \& $u m a t r e n \& e$ Krüusel, 1929 , p. 33 , pl. 6 , fig. 6 ; leaf fragment, Dipterocarpaceae; Pliocene?; Sungi TJaban, Palembang, South Sumatra.

DIPTEROCARPOPHYLLUM Edwards, 1923.

Dipterocarpophyllum gregoryi Edwards, 1923 , p. 160 , pl. 5, fig. 2 ; leaf, Dipterocarpaceae ; Tertiary ; three-quarters of a mile north of Tichara village, southeast Burma.

DIPTEROCARPOXYLON Holden, 1916.

Diptérocarpoxylon burmense Holden, 1916, p. 271, pl, 29, figs. 1-5; wood, considered to be related to Hopea or Shorea (Dipterocarpaceae); Tertiary ; Burma. See also Edwards, 1931.
DIPTEROSPERMUM Goeppert, 1851.

Dipterospermum bignonioides Goeppert, in Weber, 1851 , p. 223 , pl. 25, fig. 5 ; seed impression, Bignoniaceae; Tertiary.

DISCINITES Karl Feistmantel, 1880.

Discinites bohemicus Karl Feistmantel, 1880 , p. 303, fig. [unnumbered] p. 299 ; Upper Carboniferous; Bohemia.

DISCITES Harris, 1931.

Discites minutus Harris, 1931b, p. 6, pl. 7 ; 11 verwort?; Thaumatopteris zone, Rhaetic; Scoresby Sound, east Greenland.

DISCOPHORITES Heer, 1877.

Discophorites angustilobus Heer, $1877 \mathrm{a}$, p. 145 , pl. 58 , figs. 18,19 ; alga ? Cretaceous; St. Denls, Canton Frelburg, Switzerland.

DISCOPHYOUS Walcott, 1883.

Discophycus typicalis Walcott, 1883, p. 19, pl. 2, figs. 18, 18a ; Utica slate, Silurian; Trenton, Oneida County, N. Y.

DISCOPHYLLUM Hall, 1847.

Discophyllum peltatum Hall, 1847, p. 277 , pl. 75, fig. 3 ; plant or coral?; Hudson River group, Ordovician(?) ; Troy, N. Y.

DISCOPTERIS Stur, 1883.

Discopteris karwinensis Stur, 1883, p. 693 , figs. 17a, 17b; fertlle fern pinnule; Upper Carboniferous; Kattowitz, Sllesia.

DISCOSTACHYS Grand'Eury, 1890.

Discostachys cebennensis Grand'Eury, 1890 , pl. 8 , fig. 2 ; described in text, p. 306 as Androstachys cebennensis Grand'Eury, but this is apparently a mistake. Grand'Eury's use of these names is quite confused; see Anaro. stachys.

DISCOSTROBUS Krasser, 1906.

Discostrobus argunensis Krasser, 1906, p. 628 , pl. 4, figs. 11-14; incertae sedis; Jurassic; Durol on Argun River, Tranbalkal.

DISOMA Zalessky, 1915.

Russia, Comité geol. Mém., nouv. sér., no. 139, p. 30, St. Petersburg; Flagellatae; Permian (not seen). See Gothan, 1942b, 119.

DISPHENOPHYLLUM Lot\$y, 1909.

Disphenophyllum romerii (Solms-Laubach) Lotsy, 1909, p. 525, fig. 349.

DISSOCLADELLA PIa, 1936.

Dissocladella savitriae $P i a$, in Rao and Pia, 1936, p. 15, pl. 1, figs. 4-4; pl. 3, fig. 4 ; alga, Dasycladaceae; Miniyur group, uppermost Cretaceous; Trichlnopoly district, India.

DISTICHOPHYLLITES Dusen, 1899.

Distichophyllites microphyllus Dusen, 1899 , p. 105 , pl. 11, fig. 11 ; small coniferous? follage shoot; Ollgocene; Río Condor, Chille. 
DISTICHOPHYTUM Mägdefrau, 1938.

Distichophytum mucronatum Mägdefrau, 1938, p. 247, pl. 2, fig. 4 ; text fig. 3 ; Psilophytales; Lower Devonian; near Hahnenklee, Germany.

DISTICHÓPTERIS Yabe and Shimakura, 1940.

Distichopteris heteropinna Yabe and Shimakura, 1940b, p. 179 , pl. 16 ; fernlike foliage ; Lungtan coal series, Permian; Lungtan coal mine, Chuyunghsien, Kiangsu, China.

DISTIOHOSTROBUS Velenovsky and Viniklar, 1929.

Distichostrobus pusillus Velenovsky and Viniklar, 1929, p. 30 , pl. 21, figs. 6, 7 ; inflorescence, compared with Myrica; Cretaceous; Slivenec, Bohemia.

DISTRIGOPHYLLUM Heer, 1876.

Distrigophyllum bicarinatum (Lindley and Hutton) Heer, 1876a, p. 39, pl. 17, fig. 10 ; leaf of arborescent lycopod?; Carboniferous ; Switzerland.

DJAMBIOXYLON Kräusel, 1922.

Djambioxylon sumatrense Kräusel, 1922, p. 272 , pl. 2 , fig. 2 ; pl. 5 , fig. 7 ; wood, Sapindaceae?; 'Tertiary ; Sumatra.

DOBROGEITES Simionescu, 1940.

Dobrogeites vinassayi simionescu, 1940, p. 1, 3 pls. ; alga ; Mesozoic ; Rumania.

DODONAEITES Saporta, 1865.

Dodonaeites decaisnei Saporta, 1865, p. 184, pl. 9, fig. 13 ; fruit, Sapindaceae; Miocene ; Armissan, France.

DOLATOPHYCUS Fenton and Fenton, 1937.

Dolatophycus expansus Fenton and Fen. ton, 1937 , p. 437 , pl. 2, figs. 1, 2 ; alga ; Allentown limestone, Cambrian; Raubsville, Northampton County, Pa.

DOLEROPHYLLUM Saporta, 1878.

Dolerophyllum goepperti (Eichwald) Saporta, 1878 a, p. 872 ; Permian ; Russia. For Noeggerathia goepperti Eichwald, 1860 (1860-68), p. 253 , pl. 18, figs. 1-3.

DOLEROPTERIS Grand'Eury, 1877.

Doleropteris cuneata Grand'Eury, 1877, p. 195 , pl. 16 ; fernlike? foliage ; Carboniferous; Loire, France.

DOLEROTHECA Halle, 1933.

Dolerotheca fertilis (Renault) Halle, 1933 , p. 44 , pls. 9 , 10 ; spore-bearing organ, Whittleseyinae; Upper Carboniferous; st.-fitienne, France.

DOLICHITES Unger, 1850.

Dolichites europaeus Unger, $1850 \mathrm{a}$, p. 489 ; Miocene; Radoboj, Croatia. IEarlier citation : Unger, 1839 , p. 104 ; nom. nud. For illustrations, see Unger 1863 (1860-65), p. 25, pl. 6, figs. 6, 7.

DOLIOSTROBUS Marion, 1884.

Doliostrobus sternbergi (Corda) Marion, 1884, p. 823 ; Coniferales; Tertiary ; France. For Araucaria stembergii Corda, 1842b, p. 63, pl. 1.
DOMBEYOPSIS Unger, 1850.

Dombeyopsis lobata Unger, 1850, p. 447. See also Unger, 1848, p. 47 ; nom. nud. DOMBEYOXYLON Schenk, 1883.

Dombeyoxylon aegyptiacum $\mathrm{sch}$ en $\mathrm{k}$, 1883a, p. 13. "Compared by Schenk with the wood of the Sterculiaceae, and especially with the recent genera Ruizia and Guazuma; by Felix with Guazuma; and by Schuster with Eriodendron," Edwards, 1931; Oligocene?; Egypt. See also Schenk, in Schuster, 1910, p. 12 , pl. 3, fig. 18 .

DONEZELLA Maslov, 1929.

Donezella lutugini Maslov, 1929, p. 125, pl. 71, figs. 5-9 ; Carboniferous ; Donets Basin, Irmenski mine, Russia.

DORATOPHYLLUM Harris, 1932.

Doratophyllum astartensis Harris, 1932a, p. 36 , pls. 2, 3 ; cycadophyte leaf; Lepidopteris bed, Rhaetic; Scoresby Sound, east Greenland.

DORYANTHITES Berry, 1911.

Doryanthites cretacea Berry, 1911b, p. 406, leaf, monocotyledon; Black Creek formation, Upper Cretaceous; North Carolina. See also Berry, 1914, p. 108, pl. 17, fig. 3 .

DORYCORDAITES Zeiller, 1888.

Dorycorlaites palmaeformis (Goeppert) Zeiller, 1888 (1886-88), pl. 93, figs. 1, 2 ; cordaitean leaf ; mines of Meurchin, Upper Carboniferous ; Pas-de-Calais, France. Generic name first introduced by Grand'Eury, 1877, p. 214.

DOTHIDITES Meschinelli, 1892.

Dothidites acericola (Heer) Meschinelli, in Saccardo, 1892, p. 771. See also Meschinelli, 1898, p. 44, pl. 14, fig. 15; fungus, on fossil maple leaf. Switzerland.

DRAOAENITES Saporta, 1861.

Dracaenites sepultus Saporta, in Heer, 1861 , p. 144 ; stem impression, Monocotyledon; Oligocene; Aix, Provence, France.

DRACAENOPHYLLUM Massalongo, 1858.

Dracaenophyllum venetum Massalongo, $1858 \mathrm{~b}, \mathrm{p} .792$.

DREPANOLEPIS Nathorst, 1897.

Drepanolepis angustior Nathorst, 1897, p. 21 , pl. 1 , figs. 16,17 ; incertae sedis; Miadle Jurassic; Cape Boheman, Spltzbergen.

DREPANOPHYCUS Goeppert, 1852.

Drepanophycus spinceformis Goeppert. $1852 b$, p. 92 , pl. 41, fig. 1 ; psilophyte stem impression; Devonian; Hackenburg, Hesse.

DREPANOZAMITES Harris, 1932.

Drepanozamites nils8oni (Nathorst) Harris, 1932 , p. 83 , pls. 7,8 ; leaf, incertae sedis ; Lepidopteris zone, Rhaetic; Scoresby Sound, east Greenland. 
DRIMYSOPHYLLUM KIrchbelmer, 1037. Drimysophyllum succineum Kirchheimer, 1937, p. 474, figs. 7, 8; leaf, MagnoHaceae; Tertiary ; Germany.

DRUPA Lesquereux, 1861.

Drupa rhabdosperma Lesquereux, 1861b, p. 716, flg. 150 ; seed, incertae sedis; Tertiary ; Brandon, Vt.

DRYANDROIDES Unger, 1850.

Dryandroides grandis Unger, 1850a, p. 428 ; leaf, Proteaceae ; Eocene ; Sotzka, Styria. See also Unger, 1851, p. 169, pl. 41, figs. 11-14.

DRYANDROPHYLLUM Velenovsky, 1889.

Dryandrophyllum cretaceum Velenovsky, 1889, p. 53.

DRYOBALANOXYLON Berger, 1923.

Dryobalanoxylon tobleri (Kräusel) Berger, 1923 , p. 146 ; wood, dicotyledon; Tertiary ; Sumatra. For Dipterocarpoœylon tobleri Kräusel, 1922, p. 263, pl. 1, flg. 5 ; pl. 2, fig. 6 , etc.

DRYOBALANUS Landgrebe, 1842.

Dryobalanus basalticus Landegrebe, 1842, p. 813 , pl. 11A, figs. 1-3 ; frult, Fagaceae; Oligocene; Hersfeld near Homberg, Hesse.

DRYOPHYLLUM Debey, 1865.

Dryophyllum subcretaceum Saporta, 1865, p. 46 ; leaf, dicotyledon; Eocene ; Sézanne, France. See also Saporta, 1868 , p. 347 , pl. 26 , figs. $1-3$.

DRYOPTERITES E. W. Berry, 1911.

Dryopterites macrocarpa (Fontaine) E. W. Berry, 1911a, p. 261; follage, Polypodiaceae; Patuxent formation, Lower Cretaceous; Dutch Gap, Va. For Aspidium macrocarpum Fontaine, 1889, p. 103 , pl. 17 , fig. 2 .

DRYOXYLON Schleiden, 1853.

Dryoxylon jenense Schlelden, in Schmid, 1853 , p. 28 ; wood, compared with Salix?; Middle Triassic (Lower Muschelkalk); Wogau near Jena, Germany. First? llustrated species: Dryoxylon chitaense Yasui, 1928, p. 438, pl. 19, figs. 78, 79. See also Bancroft, $1932 \mathrm{~b}$.

DUISBERGIA Kräusel and Weyland, 1929.

Duisbergia mirabilis Kräusel and Weyland, 1929 , p. 333 , pls. $9-12$; figs. 18,19 ; Devonian ; near Elberfeld, Germany.

DULAURENSIA E. M. Reid, 1930.

Dulaurensia pulchra E. M. Reld, 1930, p. 52 , pl. 2, figs. 1-11 ; fruit, Epacridaceae ; Tertiary (Eocene?); St. Tudy near Qulmper, France.

DUNSTANIA Reid and Chandler, 1933.

Dunstania ettingshauseni (Gardner) Reld and Chandler, 1933, p. 459, pl. 25, figs. 41-47; endocarp, Cornaceae; London Clay, Eocene; Sheppey, Kent, England.

DUKANIA K1rchhelmer, 1935.

Durania ehrenbergi Kirchheimer, 1935, p. 291, fig. 7 ; seed, Symplocaceae; Ter. tiary ; Konzendorf, Germany.
DURVILLIDES Squinabol, 1888.

Durvillides eocenicus Squinabol, 1888, p. 560 , pl. 14, flgs. 1,2 ; alga, incertae sedis; Eocene; Liguria, Boccadasse, Italy.

DUTOITIA Hoeg, 1931.

Dutoitia pulohra Hoeg, 1931, p. 92, fig. 1 ; Psllophytales; Lower or Middle Devonlan; between Knysna and Port Elizabeth, near Cape Town, South Africa.

DVIN OPTERIDIUM Zalessky, 1937.

Dvinopteridium edemskit Zulessky, 1937a, p. 18, figs. 3,4 ; fern follage ; Permian; Iesiptzevo village, Tantarien, Russiu.

DYOTUOCAULUS Emmons, 1856.

Dyctuocaulus striatus Emmons, 1856, p. 293, pl. 1, fig. 3 ; incertae sedis; Permian; Farmville, N. C.

DYOTHECA Hartung, 1938.

Sachs. geol. Landesanst. Abh., Band 18, p. 92 (not seen). See Gothan, 1942b, p. 120.

DYSTACTOPHYCUS Miller and Dyer, 1878. Dystactophycus mamillanum Miller and Dyer, 1878 , p. 3 , pl. 3 , fig. 4 ; plant?, appears similar to the problematical Conostichus; Cincinnati group, Silurian; near Morrow, Ohio.

E

EBENACITES Saporta, 1861.

Ebenacites rugosus Saporta, in. Heer, 1861, p. 147 ; calyx, Ebenacene ; Eocene ; Aix, Provence, France.

EBENOXYLON Felix, 1882.

Ebenoxylon diospyroides Felix, 1882a, p. 71, fig. 3 ; Tertiary ; Antigua, West Indies.

EBORACIA Thomas, 1911

Eboracia lobifolia (Phillips) Thomas, 1911 , p. 388 , fig. p. 387 ; fertile fern frond; Jurassic; Yorkshire, England.

ECHINOCARPEOPSIS Langeron, 1900.

Echinocarpeopsis fastigata Langeron, 1900 , p. 346 , pl. 2 , fig. 9 ; leaf, compared with Echinocarpus; Eocene; Sézanne, France.

ECHINOCARPUS Emmons, 1857.

Echinocarpus sp. Emmons, 1857, p. 111, fig. 79 ; incertae sedis, described as "dry carpel, or seed vessel"; Triassic ; Haw River, N. C.?

ECHINOSTACHYS Brongniart, 1828.

Echinostachys oblongus Brongniart, 1828a, p. 457 , pl. 20 , fig. 2 ; incertae sedis; Triassic; Sultz-les-Bains, Alsace-Lorraine, France.

ECHINOSTIPES Pomel, 1849.

Echinostipes nidiformis (Brongniart) Pomel, 1849, p. 346. For Mantellia nidiformis Brongniart, 1828, p. 101 . See also Carruthers, 1870 , p. 702 , pl. 63, fig. 1. 
ECHINOSTROBUS Schimper, 1870.

Echinostrobus sternbergii Schimper, 1870 (1869-74), p. 331, pl. 75, figs. 21-24 ; cone-bearing twigs, Conlferales; Jurassic; Solenhofen, Bavaria.

ECHITONIUM Unger, 1839.

Echitonium superstes Unger, 1839, p. 103 ; Miocene ; Radoboj, Croatia.

EDRAXYLON Williamson, 1872.

Edraxylon sp. Williamson, 1872 , p. 438 , fig. 3 ; petiole of Lyginopteris; Upper Carboniferous; Oldham, England. See also Seward, 1917, p. 38, 47.

EHRETIAECARPUM Menzel, 1913.

Ehretiaecarpum parvulum Menzel, 1913, p. 61 , pl. 5, flg. 35 ; fruit, Borraginaceae ; Tertiary (Braunkohle), near Herzogenrath, Germany.

EICHWALDIA Zalessky, 1927.

Eichwaldia biarmica Zalessky, 1927a, p. 40 , pl. 12, fig. 3 ; Permian ; southeast Russia.

EISDENIA Stockmans, 1936.

Eisdenia aacheniana Stockmans, 1946b, p. 23, pl. 1, fig. 1 ; Senonien; Eisden, Belgium.

EISOTHECARYON Mueller, 1877.

Eisothecaryon semiseptatum Mueller, 1877 a $(1877-79)$, no. 68 , p. 178 , pl. 15, figs. 1-5 ; upper Pliocene; Golgong, Australia.

EKSDALIA.

Error for Eskdalia Kidston, in Posthumus, 1931 , p. 106.

ELAEAGNITES Heer, 1870.

Elaeagnites campanulatus Heer, 1870, p. 58 , pl. 12, fig. 11 ; calyx ; Miocene; Cape Staratschin, Spitzbergen.

ELAEOCARPEOPSIS Langeron, 1900.

Elaeocarpeopsis decora Langeron, 1900, p. 347 , pl. 1 , flg. 4 ; leaf, compared with Echinocarpus ; Eocene; Sézanne, France.

ELAEOCARPITES Kuntze, 1904.

Elaeocarpites Kuntze, in Post and Kuntze, 1904 , p. 193.

ELAEODENDROXYLON Platen, 1908.

Elaeodendroxylon polymorphum platen. 1908 , p. 120 ; wood ; Miocene; Amethyst Mtn., Yellowstone Park, Wyo. See also Platen, 1909, p. 245, figs. 157159.

ELAIOIDES Unger, 1850 .

Elaioides fontanesia Unger, 1850 a, p. 432, leaf, Oleaceae; Miocene; Galicia. See also Unger, $1850 \mathrm{~b}, \mathrm{p}$. 125, p1. 1.4, fig. 12.

ELASMOPHYCOS Massalong0, 1859.

Elasmophycos cuneifolius (Kurr) Massalongo, in Massalongo and Scarabelli, 1859 , p. 92. For Laminarites cuneifolius Kurr, 1845, p. 13, pl. 2, fig. 2.
ELATERITES L. R. Wilson, 1943.

Elaterites triferns L. R. Wilson, 1943, p. 523, figs. 1-6 ; spores with elaters; Des Moines group, Pennsylvanian; What Cheer, Keokuk County, Iowa.

ELATIDES Heer, 1876.

Elatides ovalis Heer, $1876 \mathrm{c}$, p. 77 , pl. 14, fig. 2 ; cone, Coniferales; Upper Jurassic; Ust-Balet, Siberia. [In 1876 Heer described Elatides ovalis, E. brandtiana, and $E$. falcata, the first two being based on cones whereas the last was based on a small twig with follage. Nathorst, 1897 , included all of these species under E. curvifolia (Dunker) Nathorst, 1897, p. 35 , pl. 1, figs. $25-27$; pl. 2, figs. 3-5.

ELATOOLADUS Halle, 1913.

Elatocladus heterophylla Halle, 1913, p. 84 , pl. 8 , figs. $12-14,17-25$; coniferous foliage shoots; Jurassic; Hope Bay, Graham Land, Antarctic.

ELATOXYLON Hartig, 1848.

Hartig, 1848b, p. 139 proposed this genus for certain species included in Thujoxy. lon and presumably intended this new combination as Elatoxylon juniperinum (Unger) Hartig. For Thujoxylon juniperinum Unger, $1854 \mathrm{c}$, p. 172, pl. 1, figs. 1-3.

ELEOXYLON Brongniart, 1849.

No new combination actually cited but evidently intended as Eleoxylon acerosum (Unger) Brongniart, 1849. For Peuce acerosa Unger, 1841 (1841-47), p. 14, pl. 3, figs. 1-4; coniferous wood; Miocene; Wurmberg, Styria. Renault, 1885 , p. 166 cites Eleoxylon acerosum (Unger) Brongniart.

ELEUTHEROPHYLLUM Stur, 1877.

Eleutherophyllum mirabile (Sternberg) Stur, 1877 , p. 65 , pl. 18, figs. 1-7 ; articulate stem; Carboniferous (Culm).

ELONGATOSPORITES Willard Berry, 1937.

Elongatosporites reticulatus Willard Berry, 1937 , p. 158 , fig. 12 ; spore; Pennington coal, Mississippian ; Cranmore Cove, Rhea County, Tenn.

ELTOVARIA David White, 1929.

Eltovaria bursiformis David White, 1929, p. 114, pl. 50, fig. 4; pteridosperm? cupule with seeds; Hermit shale, Permian; on Bright Angel Trail, below El Tovar, Ariz.

EMBOLIANT'HEMUM Corda, 1874.

Embolianthemum truncatum Corda, in Feistmantal, Ottokar, 1874, p. 37 ; Upper Carboniferous ; Bras, Bohemia.

NARBOTREIOPHYLXUMT Dusen, 1899.

Embothriophyllum dubium Dusen, 1899 , p. 104, pl. 10, fig. 6 ; leaf compared with Embothrium lanceolatum Ruiz and Pavon ; Oligocene ; Rto de las Minas near Punta Arenas, Chile. 
EMBOTHRIOPSIS Hollick, 1912.

Embothriopsis presagita Hollick, 1912, p. 159 , pl. 165 , fig. 1 ; leaf, Proteaceae; Raritan formation, Upper Cretaceous ; Glen Cove, Long Island, N. Y.

EMBOTHRITES Unger, 1850.

Embothrites borealis Unger, 1850a, p. 428 ; Proteaceae ; Eocene; Sotzka, Styria. See also Unger, 1851, p. 171, pl. 42, figs. 10-12.

EMPLECTOPTERIDIUM Kawasaki, 1934.

Emplectopteridium alatum Kawasaki, 1934 (1927-34), p. 143, pl. 52, figs. 138, 139 ; fern or pteridosperm foliage ; Jido series, Bed D, Carboniferous ; Kaech'ŏn, North Korea.

EMPLECTOPTERIS Halle, 1927.

Emplectopteris triangularis Halle, 1927, p. 122 , pl. 31 ; pteridosperm foliage; Lower Shjhbotse series; Permian; central Shansi, China.

ENANTIOBLASTOS Goeppert and Berendt, 1845.

Enantioblastos viscoides Goeppert and Berendt, in Berendt, 1845 , p. 76, pl. 6, figs. 6, 7 ; fruit, Loranthaceae ; Miocene ; Prussia.

ENANTIOPHYLLITES Goeppert and Berendt, 1845.

Enantiophyllites sendelit Goeppert and Berendt, in Berendt, 1845, p. 79, pl. 5, fig. 57 ; leaves, Leguminosae?; Miocene ; Prussia.

ENOEPHALARTOPSIS Fontaine, 1889.

Encephalartopsis nervosa Fontaine, 1889, p. 174 , pls. 70-72; cycadophyte leaf fragments ; Potomac group, Lower Cretaceous ; Fredericksburg, Va.

ENCOELITES Sternberg, 1833.

Encoelites mertensii Sternberg, 1833 (1820-38), p. 33, pl. 3, flg. 2 ; incertae sedis; Jurassic ; Solonhofen, Bavaria.

ENCOELOOLADIUM Zigno, 1856.

Encoelocladium tortuosum Zigno, 1856 (1856-68), p. 7. For Caulerpites tortuosus Presl, in Sternberg, 1820-38, p. 103 , pl. 29, fig. 1 ; alga ; Jurassic ; Solonhofen, Bavaria.

ENDOCALAMITES Grand'Eury, 1877.

Endocalamites approximatus (Schlothelm) Grand'Eury, 1877, p. 47. For Calamites approximatus Schlotheim, see Brongn1art, 1828-38, p. 133, pl. 24 ; pl. 15, figs. $7,8$.

ENDOGENITES Brongniart, 1822.

Endogenites echinatus Brongniart, 1822, p. 301 , pl. 16, flg. 2 ; cycad? trunk; Eocene; near Solssons, France.

DNDOGENOPHYLLITES MCCOУ, 1870.

Endogenophyllites wellingtonensis $\mathrm{McCoy}$, in Wintle, 1870 , p. 2 ; nom. nud.
ENDOLEPIS Schlelden, 1846.

Endolepis vulgaris Schlelden, in Schmid - and Schleiden, 1846, p. 72, pl. 5, flg. 25.

ENDOSPORITES L. R. Wilson and Coe, 1940.

Endosporites ornatus L. R. Wilson and Coe, 1940 , p. 184 , pls. 1, 2 ; spore ; Des Moines group, Pennsylvanian; What Cheer, Keokuk County, Iowa.

ENDOXYLON Scott, 1925.

Endoxylon zonatum (Kidston) Scott, 1925, p. 579 , pl. 3, flgs. 19-21 ; petrifled stem, Calamopityeae; Carboniferous Limestone series, Lower Carboniferous; Dalry, Ayrshire, Scotland. For Calamopitys zonata Kidston, in Scott, 1923, p. 133.

ENGELHARDTIOIDITES Robert Potonie, 1950.

Engelhardtioidites microcoryphaeus Robert Potonie, in Potonie, Robert, Thomson, Paul W., and, Thiergart, Friedrich, 1950 , p. 51 , pl. B, flg. 8 ; pl. C, flg. 16 ; pollen, Juglandaceae ; Miocene ; ChattAquitan, Germany.

ENIGMOCARPON Sahni and Rode, 1937.

Enigmocarpon parijai Sahni and Rode, 1937 , p. 168 , pl. 1 , figs. 8-15; petrified frult; Lythraceae; Deccan Intertrappean series, Tertiary, probably Miocene; Mohgaon Kalan, 18 miles east of Chhindwara, India. Brief description without 1llustrations in Sahni, 1934, p. 317 ; full description in Sahni, 1943.

ENIGMOPHYTON Hoeg, 1942.

Enigmophyton superbum Hoeg, 1942, p. 88 , pls. $36-40$; Devonian; Planteryggen, Spitzbergen.

ENISEIELLA Maslov, 1939.

Eniseiella asteroides Maslov, 1939, p. 288, pl. 1, flgs. 1-6; pl. 2, figs. 1, 3-5; alga ; lower Paleozolc; North Yenisel, USSR.

ENTOMOLEPIS Saporta, 1865.

Entomolepis cynarocephala Saporta, 1865, p. 55 , pl. 2 , flg. 3 ; cone; Coniferales; Miocene ; Armissan, France.

ENTONEURON Geyler, 1875.

Entoneuron melastomaceum Geyler, 1875, p. 78 , pl. 2 , fig. 3 ; leaf fragment, Menispermaceae; Eocene; Pengaron, Borneo.

ENTOSTROMIUM Reinsch, 1881.

Entostromium sp. Reinsch, 1881, p. 55, pl. 12, figs. 1-9; Upper Carboniferous ; Zwickau, Saxony.

ENYGMATOSTROBUS Kryshtofovich, 1915.

Enygmatostrobus dokturowskyi Kryshtofovich, 1915 , p. 106 , pl. 5, figs. 3-6; Jurassic; Tyrmaflutz, Amur River, S1beria.

EOACHRAS E. W. Berry, 1915.

Eoachras eocenica D. W. Berry, 1915, p. 210, pl. 1 ; seed, compared with Achras zapote (Sapotaceae); Eocene; near Lexington, Holmes County, Miss. 
EOANGIOPTERIS Mamay, 1950.

Eoangiopteris andrewsii Mamay, 1950, p. 440 , pl. 9 ; petrified spore-bearing organs, Marattiaceae; Des Moines group, Pennsylvanian; Urbandale coal mine, Des Moines, Iowa.

EOCLADOPHORA Fucini, 1936.

Reference not seen; cited in Gothan, 1942 b, p. 121.

EOCLATHRUS Squinabol, 1888.

Eoclathrus fenestratus Squinabol, 1888, p. 552, pl. 16, fig. 3 ; alga?; Tertiary; Genoa, Italy.

EOCLEPSYDROPSIS Bertrand, 1909.

A name proposed by Bertrand for a hypothetical early zygopterid; Bertrand, 1909 , p. 256.

EOGLOBELLA Bradley, 1931.

Eoglobella longipes Bradley, 1931, p. 44, pl. 22 , fig. 3 ; alga ?; Green River formation, Eocene; Asphalt Tunnel, Garfield County, Colo.

EOHEPATICA Heard and Jones, 1931.

Eohepatica dyfriensis Heard and Jones, 1931 b, p. 330 ; liverwort, compared with Marchantia; Lower Downtonian, Silurian ?; Llandovery district, Wales.

EOHYPSERPA Reid and Chandler, 1933.

Eohypserpa parsoni Reid and Chandler, 1933 , p. 168 , pl. 4, figs. 13-21 ; fruit, Menispermaceae ; London Clay, Eocene; Sheppey, Kent, England.

EOLIRION Schenk, 1869.

Eolirion primigenium Schenk, 1869, p. 20, pl. 7 , fig. 4 ;'palm leaf; Lower Cretaceous (Urgonian) ; Grodischtz, Austrian Silesia.

EOMASTIXIA Chandler, 1926.

Eomastixia bilocularis Chandler, 1926, p. 37 , pl. 6, figs. $6 a-c$; endocarp, Cornaceae ; upper Eocene ; Hordle, Hampshire, England.

EOPHYTON Torell, 1867.

Eophyton linnaeanum Torell, 1867, p. 36, pl. 2, fig. 3 ; pl. 3, figs. 1-3; Lower Cambrian and Silurian; near Blllingen, Sweden.

EOPTERIS Saporta, 1878.

Eopteris andegavensis Saporta in Crie, 1878 , p. 687 ; Lower Silurian; France. See also Saportn, 1878 b, p. 769 , fig.

EOPUNTIA Chaney, 1944.

Eopuntia douglassii Chaney, 1944, p. .507, pls. 1-5; stem and fruit impressions, Cactaceae; Middle Eocene; eastern Utah.

DOREACHIS Arnold, 1945.

Eorhachis lomarioides Arnold, 1945, p. 11, pl. 2 ; petrified fernlike petiole : Green River formation, Eocene; Eden valley petrified forest, 26 miles east of Farson, Sweetwater County, Wyo.
EORHAMNIDIUM E. W. Berry, 1919.

Eorhamnidium cretaceum E. W. Berry, 1919 a, p. 113 , pl. 28 , fig. 10 ; leaf, Rhamnaceae; Tuscaloosa formation, Upper Cretaceous; Cottondale, Tuscaloosa County, Ala.

EOSPERMATOPTERIS Goldring, 1924

Eospermatopteris textilis (Dawson) Goldring, 1924, p. 68, pls. 2-6 ; tree-fern stem casts; Upper Devonian; Gilboa, N. Y.

EOSTROBILUS Theron, 1900.

Eostrobilus getisii Theron, 1900, p. 112, fig. 109 ; Lower Carboniferous; Cabrtères, France.

EOTAXITES Brongniart, 1875.

Eotaxites sp. Brongniart, 1875, p. 1021 ; leaves, incertae sedis; Upper-Carboniferous ; near Moulins, France.

EOZANTHOXYLON Reld and Chandler, 1933.

Eozanthoxylon glandulosum Reid and Chandler, 1933, p. 263, pl. 10, figs. 13, 14 ; fruit, Rutaceae ; London Clay, Eocene ; Sheppey, Kent, England.

EPHEDRITES Goeppert and Berendt, 1845.

Ephedrites johnianus Goeppert and $\mathrm{Be}$ rendt. in Berendt, 1845 , p. 105, pl. 4, figs. $8-10$; pl. 5 , fig. 1 ; portion of shoot, Ephedraceae?; Miocene; Prussia.

EPHEDROPSIS Velenovsky and Viniklar 1926.

Ephedropsis strobilifera Velenovsky and Viniklar, 1926, p. 44, pl. 4, figs. 5-12; pl. 3, fig. 7 ; seed cone, Taxodiaceae ; Cretaceous; Vyserovice and Lipenec, Bohemia.

DPIMASTOPORA PIa, 1922.

Reference not seen. See Pia, in Zeitschr. Induktive Abstammungs, Band $30, \mathrm{p}$ 63, Berlin. See also Johnson, J. H., 1946 .

EPIPHYTON Bornemann, 1886.

Bpiphyton flabellatum Bornemann, 1886 . p. 16, p1. 1, flgs. 9-10 ; alga ; Cambrian; Cuccuru, near Iglesias, Sardinia.

EPIPOLAIA C. E. Bertrand, 1898.

Epipolaia bowert C. E. Bertrand, 1898, p 179, pl. 11, figs. 119-124; thallophyte, incertae sedis; Carboniferous; Broxburn, Scotland.

EQUIHENIA Meunier, 1886.

Equihenia rugosa Meunier, 1886, p. 567, pl. 29, fig. 4; plant or worm? tracks; Upper Jurassic; Equihen, Pas-de-Calais, France.

EQUISETIDES Schimper, 1869.

Equisetides gifanteus (Lindley and Hut ton) Sch1mper, $1869(1869-74)$, p. 286. See also Lindley and Hutton, 1831-37, pl. 114. 
EQUISETINA Zalessky, 1939.

Equisetina magnivaginata Zalessky, 1939b, p. 329, figs. 1-3; articulate stem fragment; Permian; Matveyevo, Kroutaia Katouchka, USSR.

EQUISETITES Sternberg, 1833.

Equisetites münsteri Sternberg, 1833 (1820-38), p. 43 , pl. 16, figs. 1-5 ; stems with follage and terminal cone of Equisetum-like plant; Upper Triassic (Keuper); Strullendorf near Bamberg, Germany.

EQUISETOSPORITES Daugherty, 1941.

Equisetosporites chinleana Daugherty, 1941 , p. 63 , pl. 34 , fig. 4 ; spore with elaters, Equisetaceae; Triassic; near Holbrook, A riz.

EQUISETOSTACHYS Jongmans, 1927.

Equisetostachys sp. Jongmans, 1927b, p. 48 ; nom. nud.

EREMOPHYLLUM Lesquereux, 1874.

Eremophyllum fimbriatum Lesquereux, 1874 , p. 107 , pl. 8 , fig. 1 ; leaf, dicotyledon ; Cretaeceous; near Decatur, Nebr.

EREMOPTERIS Schimper, 1869.

Eremopteris artemisiaefolia (Sternberg) Schimper, 1869 (1869-74), p. 416, pl. 30 , fig. 4 ; fernlike foliage ; Carboniferous ; Newcastle, England.

ERETMONIA DuToit, 1932.

Eretmonia natalensis DuToit, 1932, p. 381, pl. 40, figs. 9-12 ; staminate sporangiophores of Glos8opteris? ; Beaufort series, Karroo system, Upper Permian ; Bergville, Natal.

ERETMOPHYLLUM Thomas, 1914.

Eretmophyllum pubescens Thomas, 1914, p. 259 , pl. 6 ; leaf, Ginkgoales ; Gristhorpe plant bed, Jurassic; Cayton Bay, Yorkshire, England.

ERICIPHYLLUM Conwentz, 1886.

Ericiphyllum ternatum Conwentz, 1886, p. 114, pl. 11, figs. 11-13; shoot bearing follage, in amber, Erfcaceae; early Tertiary ;"West Prussia.

ERICIPITES Wodehouse, 1933.

Ericipites longisulcatus Wodehouse, 1933, p. 517 , fig. 52 ; pollen, Ericaceae ; Parachute Creek member, Green River formation, Eocene; Colorado and Utah.

ERIOTESTA. Brongniart, 1874.

Eviotesta velutina Brongniart, 1874, p. 256, pl. 23, figs. 4, 5; silicified seed; Carboniferous ; St.-Etienne, France.

ERISIPHITES Pampaloni, 1902.

Erisiphites melilli Pampaloni, 1902, p. 125, pl. 10 , flg. 8 ; fungus perithecia ; Miocene?; Melilli, SIclly.

ERISTOPYHTON Zalessky, 1911.

Eristophyton beinertianum (Goeppert) Zalessky, 1911, p. 27, pl. 3, flgs. 3-6 ; pl. 4, figs. 5, 7 ; petrified cordaitean stem; Lower Carboniferous.
ERNESTIA Florin, 1927.

Ernestia fliciformis (Schlotheim) Florin, 1927, p. 4 ; Coniferales; Lower Permian ; widely distributed in central, western, southern Europe. See also Florin, $1929 \mathrm{~b}, \mathrm{p} .404$. This generic name proved to be preoccupied and was changed to Ernestiodendron, see below.

ERNESTIODENDRON Florin, 1934.

Ernestiodendron fliciforme (Schlotheim) Florin, 1934, p. 469 . For Ernestia fliciformis (Schlotheim) Florin, 1927, p. 4. See also Florin, Rudolf, 1939, Palaeonto. graphica, Band 85, Abt. B, p. 176.

ERVITES Saporta, 1862.

Ervites primaevus Saporta, 1862, p. 287, pl. 14, flg. 9 ; fruit, Leguminosae; Tertiary; Provence, France.

ERYSIPHITES Meschinelli, 1898.

Erysiphites protogaeus (Schmalhausen) Meschinelli, 1898, p. 15, pl. 9, fig. 4; Pyrenomycete; Tertiary.

ERYTHRINOXYLON Falqui, 1907.

Erythrinoxylon latiporosum Falqui, 1907, p. 11 ; wood ; Oligocene; Sardegna, Italy.

ESCALLONITES Kuntze, 1904.

Escallonites Kuntze, in Post and Kuntze, 1904, p. 207.

ESCALONIIPHYLLUM Dusen, 1899.

Escaloniiphyllum sp. Dusen, 1899, p. 102, pl. 11 , fig. 5 ; small leaf fragment compared with Ascallonia serrata Smith; Oligocene; Chile.

ESKDALIA Kidston, 1903.

Eskdalia minuta Kidston, 1903a, p. 750 , pl. 1 , figs. $4-8$; fern ? stem compression; Cementstone serles, Lower Carboniferous; near Holystone, Northumberland.

ETAPTERIS Paul Bertrand, 1907.

Etapteris tubicaulis (Goeppert) Paul Bertrand, 1907 , p. 776 ; coenopterid petiole; Lower Carbontferous; Falkenberg, S1lesia. For Zygoteris tubicaulis Goeppert, $1852 b$, p. 137 , pl. 11 , figs. $1-3$. See also Bertrand, 1909 , p. 72 ; Posthumus, 1931.

ETERODICTYON Peruzzi, 1881.

Eterodictyon textum (Heer) Peruzzl, 1881, p. 8 , pl. 1 , fig. 7 ; incertae sedis.

ETHERIDGEA Ettingshausen, 1893.

Etheridgea 8ubglobosa Ettingshausen, 1893, p. 141. See also Ettingshausen, 1895 , p. 46 , pl. 4, fig. 3 ; frult, Tiliacene; Upper Cretaceous; Ipswich Road, Bahnstation, Australia.

ETTINGSHAUSENIA Stiehler, 1857.

Ettingshausenia cuneifolia (Bronn) Stiehler, 1857, p. 67. For Credneria cuneifolia Bronn, 1838 (1837-38), p. 583, pl, 28, fig. 11; Cretaceous (Cenomanian); Niederschoena, Saxony. 
EUOALYPTOPHYLLUM Fontaine, 1889.

Eucalyptophyllum oblongifolium Fontaine, 1889 , p. 325 , pl. 162 , fig. 4 ; leaf fragment, affinities with Eucalyptus? ; Potomac group, Lower Cretaceous; near Brooke, Va.

EUDAPHNIPHYLLUM Conwentz, 1886.

Eudaphniphyllum nathorsti Conwentz, 1886 , p. 95 , pl. 10, fig. 1 ; leaf, in amber, Thymelaceae; Tertiary; West Prussia.

EUGEINITZIA Hollick and Jeffrey, 1909.

Eugeinitzia proxima Hollick and Jeffrey, 1909 , p. 43 , pls. 10,25 ; cone scales, Coniferales ; Cretaceous ; Kreischerville, Staten Island, N. $\dot{\mathbf{Y}}$.

EUGENIAITES Loublere, 1933.

Soc. geol. France Bull. 1933, sêr., $5^{\ominus}$, tome 3, p. 128; Myrtaceae; Tertiary (not seen). See Gothan, 1942b, p. 122.

EULEPIDOPHLOIOS Sterzel, 1907.

Eulepidophloios laricinus (Sternberg) Sterzel, 1907, p. 730; Carboniferous; Offenburg, Baden. See Sternberg, 1825 (1820-38), Tentamen, p. xiil, pl. 11, figs. 2-4.

\section{EULITHOTHAMNION?}

Eulithothamnion suganum (Rothpletz) Trabucco, 1900, p. 715 , pl. 11, fig. 12 ; alga ; Miocene; Italy. Earliest reference?

EUMUENSTERIA Rothpletz, 1896.

Eumuensteria fagellaria (Sternberg) Rothpletz, 1896, p. 858. For Münsteria flagellaria Sternberg, 1833 (1820-38), p. 32 , pl. 7, fig. 3 ; alga ?; Eocene; Vienna.

EUPECOPTERIS Kidston, 1925.

Eupecopteris bucklandi (Brongniart) Kidston, 1925, p. 554, pls. 120, 122; pecopterid foliage; Radstockian series, Upper Carboniferous; Camerton, England.

BUPHORBIOIDES Weber, 1855.

Euphorbioides prisca Weber, 1855 , p. 155, pl. 30 , fig. 1 ; inflorescence. Euphorbiaceae; Miocene; Rhenish Prussia.

EUPHORBIOPHLOIOS Langeron, 1899.

Euphorbiophloios sezannensis Langeron, 1899 , p. 451 , pl. 5, fig. 4 ; stem impression, Euphorbiaceae?; Eocene ; Sezanne, France.

EUPHORBIOPHYLLUM Ettingshausen 1853.

Euphorbiophyllum stillingioides Ettings. hausen, 1853 , p. 77 , pl. 26, figs. 1, ?; leaf, Euphorbiaceae; Tertiary ; Haering, Austria.

EUPHORBIOSPERMUM Reid and Chandler, 1933.

Euphorbiospermum eocenicum Reid and Chandler, 1933 , p. 290 , pl. 12, figs. 2025 ; seed, Euphorbiaceae; London Clay, Eocene; Minster, Kent, Hngland.
EUPHORBIOTHECA Reid and Chandler, 1933.

Euphorbiotheca sheppeyensis Reid and Chandler, 1933, p. 284, pl. 12, figs. 1-5; fruit, Euphorbiaceae; London Clay, Eocene; Sheppey, Kent, England.

EUPHORBIOXYLON Felix, 1887.

Euphorbioxylon speciosum Felix, 1887a, p. 525 , pl. 25 , figs. $4,6,7$; wood, Euphorbiaceae; Tertiary?; Sabanilla, Colombia.

EUPHORBITES Martius, 1822.

Euphorbites cicatricosus Martius, 1822, p. 141. See also Artis, 1825 , p. 15, pl. 15 ; sigillarian stem compression; Upper Carboniferous ; England.

EUPHORBOCARPUM Knowlton, 1917.

Euphorbocarpum richardsoni Knowlton, 1917 , p. 328 , pl. 96, flgs. 3,4 ; fruit, Euphorbiaceae; R a t o n formation, Eocene; 5 miles south of Aguilar, Colo.

EUPHORIAECARPUM Menzel, 1913.

Euphoriaecarpum litchiforme Menzel, 1913 , p. 43 , pl. 4, figs. 28,19 ; seed, Sapindaceae; Tertiary (Braunkohle); near Herzogenrath, Prussia.

EUPHORIOPSIS Massalongo, 1852.

Euphoriopsis phaetontis Massalongo, 1852 a, p. 14 , pl. 2 , fig. 5 ; leaf, Sapindaceae.

EUPSARONIUS Presl, 1847.

Eupsaronius carbonifer (Corda) Presl, 1847, p. 289. For Psaronius carbonifer Corda, 1845, p: 95 , pl. 28, figs. $1-4$; Psaronius stem; Upper Carboniferous; Radnitz, Bohemia.

EUROTITES Meschinelli, 1892.

Eurotites elegans (Goeppert and Menge) Meschinelli, in Saccardo, 1892, p. 750 . See also Meschlnelli, 1898, p. 15; Pyrenomycete. For Eurotium elegans Goeppert and Menge, in Goeppert, 1853, p. 453.

EORYOYOADOLEPIS Seward, 1917.

Eurycadolepis jenlinsiana (Tate) Seward, 1917 , p. 496; cycad cone scale?; Uitenhage series, Wealden; Cape Colony, South Africa. For Cyclopteris jenkinsiana Tate, 1867 , p. 130 , pl. 6 , fig. 4.

EURYP HYLLUM Ottokar Feistmantel, 1879.

Euryphyllum whittianum Ottokar Felst. mantel, 1879, p. 26, pl. 21, flgs. 1, 1a ; leaf; Karharbari beds, Lower Gondwana; Buriadi, India.

EURYSACIS Schulze, 1887.

Durysacis squamosa (Heer) Schulze, 1887, p. 18. For Ounninghamites squamosus Heer, 1871b, p. 9, pl. 1, figs. 5-7.

WURYSOLENPORA Dietrich, 1930

Eurysolenpora polypora (Quenstedt) Dietrich, 1930, p. 104, pl. 4; plant?; Jurassic. 
IUUSARCOPHYLLUM Zalegsky, 1933.

Eusarcophyllum amadocum Zalessky, 1933 c, p. 1390 , figs. 4,5 ; lycopod stem fragments?; Carboniferous?; Chakhtionki, Donets, Russia.

EUSPHENOPTERIS (Weiss) KIdston, 1882.

Eusphenopteris tenella (Brongniart) Kidston, 1882, p. 7, pl. 1, flgs. 1-5; fertile fernlike frond, referred tentatively to Hymenophyllaceae; Upper Carbontferous ; Yorkshire, England.

EUTHYTHYRITES Cookson, 1947.

Euthythyrites oleinites Cookson, 1947, p. 210 , pl. 12, figs. 12,13 ; ascomata, Microthyriacease ; Oligocene-Miocene ; Yallorn and Hazelwood, Victoria.

EVIOSTACHYA Stockmans, 1948.

Eviostachya hoegi Stockmans, 1948, p. 64, pl. 10, figs. 2-5a; Upper Devonian; Belgium.

EVODIOXYLON Chiarug1, 1933.

Evodioxylon owent (Carruthers) Chlarugi, 1933 , p. 137 , pl. 20 , fig. 2 ; pl. 21 , figs. 1-4; pl. 22, figs. 1-3; dtcotyledonous wood; Miocene and Cretaceous; ScecGure, southern Italian East Africa (Somaliland) and Gargerre, Garseale, northern Italtan East Africa. For Caesalpinioxylon oveni (Carruthers) Edwards, 1931.

BXCIPULITES Goeppert, 1836.

Excipulites neesii Goeppert, 1936, p. 262, pl. 36, fig. 4 ; peritheclal organs on Hymenophyllites foliage; Upper Cretaceous ; Waldenburg, Silesia. Mesh1nelli, 1892, p. 788, erroneously attributes this genus to Fries.

EXOGENITES Fischer de Waldheim, 1826.

Exogenites sp. Fischer de Waldhelm, 1826, p. 18, plate [unnumbered]; Tertiary; near Moscow, Russia.

\section{$\mathbf{F}$}

FABOIDEA Bowerbank, 1840.

Faboidea longinscula Bowerbank, 1840, p. 104 , pl. 15, flgs. 1, 2 ; seed, Legumtnosae?; Eocene ; Sheppy, Kent, England.

WAGITES Goeppert, 1844.

Fagites gypsaceus Goeppert, in Wimmer, 1844 , p. 219 ; nom. nud? Possibly intended as new name for the leaf described as Fagus sylvatica in Goeppert, 1842 b, p. 219 , pl. 67 , flg. 1.

FAGOPHYLLUM Nathorst, 1888.

Fagophyllum gottschei Nathorst, 1888, p. 199, pl. 17, fig. 2 ; leaf, Miocene; Morlyoshlmura, Ugo province, Japan.

FAGOPSIS Hollick, 1909.

Fagopsis longifolia (Lesquereux) Hollick, 1909, p. 2, figs. 1, 2 ; leap, Fagaceae ; Miocene ; Florissant, Colo.
FAGOXYLON Stopes and Fujli, 1910.

Fagoxylon hokkaidense Stopes and Fujll, 1810 , p. 64, pl. 8, figs. 50-53; wood; Upper Cretaceous; Hokkaido, Japan. See also Edwards, 1931.

FANEROTHECA Franguelli, 1944.

Fanerotheca exstans Frenguell, 1944b, p. 393 , pls. 1-4; microsporanglate organ, Pteridospermae; Triassic; Cacheuta, Argentina.

FASCIOULIT'ES Cotta, 1832.

I'ascioulites didymosolen (Sprengel) Cotta, 1832 , p. 47 , pl. 8, figs. 3, 4.

FASCIOSTELOPTERIS Stopes and FuJll, 1910.

Fasciostelopteris tansleil Stopes and Fuj11, 1910 , p. 15, pl. 2, figs. 2, 3 ; dictyostellc fern stem, Cyatheaceae ?; Upper Cretaceous; Hokkatdo, Japan.

FASCI'IES Reinsch, 1881.

Fuscites sp. Reinsch, 1881, p. 34, pl. 7a, figs. $7-10 ;$ pl. 7 b, figs. 3,$4 ;$ pl. 10 , figs. 5-8; Triassic (Keuper); Basel, Switzerland.

FAVULARIA Sternberg, 1825.

Favularia obovata Sternberg, 1825 (182038), Tentamen, p. xili, a genus estab. lished for species which are now included in Sigillaria

FAYOLIA Renault and Zeiller, 1884.

Fayolia dentata Renault and Zeller, 1884b, p. 1393 , figs. 1,2 ; fish egg capsule (described as a plant). For recent discussion of Fayolia and related fossils, see Brown, R. W., 1950.

FEGONIUM Unger, 1847.

Fegonium vasculasum Unger, 1847 (184147 ), p. 103, pl. 27, figs. 7-9 ; Tertiary ; Freystadt, A ustria. Originally described by Unger, $1839 \mathrm{~b}$, as Phegonium; in 1884 Vater introduced Phegonium as "gen. nov." noting that Unger's Phegonium belongs to Plataninium. See discussion in Edwards, 1931, p. 40.

FEILDENIA Heer, 1878.

Felldenia rigida Heer, $1878 \mathrm{a}$, p. 20, pl. 1 , figs. $3-11$; pl. 2, fig. 1 ; pl. 8, fig. 1 ; Miocene; Grinell Land, Arctic Ocean.

FEILDENIOPSIS Fontaine, 1889.

Feildeniopsis crassinervis Fontaine, 1889, p. 205 , pl. 85 , fig. 5 ; leaf fragment, incertae sedis ; Potomac group, Lower Cretaceous; Virginia.

FEISTMANTELIA Crie, 1889.

Feistmantelia americana Crie, 1889b, p. 23 ; nom. nud. See note under Bottgeria.

FEISTMAANTELIA Ward, 1899.

Feismantelia oblonga Ward, 1899, p. 693, pl. 169, flg. 19 ; seed, compared with Arancarites; Lower Cretaceous; Black Hills, S. Dak. 
FEISTMANTELIA Zeiller, 1902.

Feistmantelia bengalensis Zeiller, 1902, p. 34 , pl. 4, figs. 9, 10 ; cupular organ, Pteridospermae ; Lower Gondwana ; Passerabhua, India. See Seward, 1917, p. 140. See also Ottokaria, Zeiller, 1902.

FELIXIA Platen, 1908.

Felixia latiradiata Platen, 1908 , p. 66, pl. 2, figs. 3, 4; wood, Leguminosae ; Miocene-Pliocene ; California:

FERONIA Carpentier, 1927.

Feronia sewardi Carpentier, 1927, p. 27, pl. 4, figs. 1-4; Wealden; Feron, Monfaux, France.

FERUGLIOA Frenguelli, 1944.

Feruglioa samaroides Frenguelli, 1944b, p. 403, text fig. 1 ; pls. 1,2 ; seeds, Corystospermaseae?; Triassic; Chubut, Argentina.

FICHTELITES Unger, 1842.

Fichtelites articulatus Unger, 1842, p. 101 ; wood, Leguminosae; Tertiary ; Austria.

FICOIDITES Artis, 1825.

Ficoidites verrucosus Artis, 1825, p. 10, pl. 10, stigmarian appendage; Carboniferous; near Wentworth, Yorkshire, England.

FICONIUM Ettingshausen, 1883.

Ficonium solandri Ettingshausen, 1883, p. 124, pl. 3, fig. 4 ; leaf, Moraceae ; Eocene ; Dalton near Gunning, Australia.

FICOPHYLLUM Fontaine, 1889.

Ficophyllum crassinerve Fontaine, 1889 , p. 291, pls. 144-148; leaf, dictoyledon; Potomac group, Lower Cretaceous; Fredericksburg, Va.

FICOXYLON KaIser, 1880.

Ficoxylon bohemicum Kaiser, 1880 a, p. 309 ; wood, compared with Ficus cordata; Tertiary; between Kostenblatt and Zettow, Bohemia. Placed in $\mathrm{Fi}$ coxylon tropicum by Edwards, 1931. See F. tropicum (Schleiden) Felix, 1883a, p. 81, pl. 2, fig. 6 .

FILICITES Schlotheim, 1820.

Filicites cyatheus Schlothelm, 1820, p. 403 ; for lllustrations, see Schlothelm, 1804, pl. 7, fig. 11. A genus of miscellaneous fern foliage fragments; a type species seems meaningless because of the diversity of fossils assigned to it; compare, for example, Berry, 1922e, p. 162, pl. 6, fig. 4, and Crepin, 1875, pl. 6.

FIRMIANITES Cockerell, 1909.

Firmianites aterrimus Cockerell, 1909, p. 447, fig. 2 ; capsule, compared with Firmiana; Eocene; Green River, Wyo.

IITTONIA Carruthers, 1870.

Fittonia squamata Carruthers, 1870, p. 690 , pl. 56 ; cycadophyte trunk; Upper Cretaceous: Rıashurch. Isle of Wight, England
FITTONITES Kuntze, 1904.

Fittonites Kuntze, in Post and Kuntze, 1904, p. 236.

FLABELLARIA Sternberg, 1822.

Flabellaria raphifolia Sternberg, 1822 (1820-38), p. 32, pl. 21; palm leaf; Oligocene; Haering, Tirol, Austria. This is selected as type, for the genus has been generally used for palm leaves ; first species described by Sternberg, $F$. borassifolia, however, is a cordaitean leaf. See Seward, 1917, p. 233.

FLABELLICULA Reid and Chandler, 1926.

Flabellicula anglica Reid and Chandler, 1926, p. 141 , pl. 9, figs. 12,13 ; angiosperm fruit; Oligocene; Isle of Wight, England.

FLABELLITES.

Error for Palmacites, in Cuvier and Brongniart, 1822, p. 35.

FLABELLOPHYCOS Squinabol, 1890:

Flabellophycos ligusticus Squinabol, 1890, p. 199 , pl. 12, fig. 1 ; incertae sedis; Tertiary; Italy.

FLEMINGITES Carruthers, 1865.

Flemingites gracilis Carruthers, 1865, p. 438, pl. 12, figs. A1-10 ; lycopod cone; Upper Carboniferous ; Airdrie, Lanarkshire, Scotland.

FLICHEIA Pelourde, 1908.

Flicheia esnostensis Pelourde, 1908, p. 879, fig. 1 ; silicified fern petiole; Lower Carboniferous (Culm); Autun, France. See also Posthumus, 1931.

FLORENTINITES Spegazzini, 1924.

Florentinites arcuta Spegazzini, 1924a, p. 104, figs. 7-10; foliage, monocotyledon?; Eocene; Patagonia.

FLORINITES Schopf, Wilson, and Bentall, 1944.

Florinites antiquus Schopf, in Schopf, Wilson, and Bentall, 1944, p. 58, figs. 4, 5 ; spore; near top of Tradewater group, lower Allegheny, Pennsylvanian; Soap Creek, Davis County, Iowa.

FLORISSANTIA Knowlton, 1916.

Florissantia physalis Knowlton, 1916, p. 270. For a flower (Convolvulaceae?) described, but not named, by Kirchner, 1898, p. 188, pl. 15, fig. 2.

FLOROPTERIS Achepohl, 1883.

Floropteris sp. Achepohl, 1883, p. 91, pl. 29, fig. 3 ; fernlike foliage; Upper Carboniferous; Westphalia.

FOERSTIA David White, 1923.

Foerstia ohioensis David White, in White, David, and Stadnichenko, Talsia, 1923, p. 240 , pl. 5 ; pl. 6, Higs. 1-5; alga? Devonian; near Vanceburg, $\mathrm{Ky}$.

FOLIOPTERIS Achepohl, 1883.

Foliopteris sp. Achepohl, 1883, p. 91, pl. 29, fig. 7; fernlike foliage fragment: Upper Carboniferous; Westphalia. 
FOLIUM Elise Hofmann, 1932.

Folium sectum Elise Hofmann, 1932, p. 61. pl. 1, figs. 1-3 ; cuticular remains ; Tertiary ; Geiseltals, Germany.

FOLLIOULITES Zenker, 1833.

Folliculites kaltennordhemensis Zenker, 1833b, p. 177, pl. 4A ; fruit, Ranunculaceae?; Tertiary (Braunkohle) ; Welmar, Germany.

FONTAINEA Newberry, 1895.

Fontainea grandifolla Newberry, 1895, p. 96 , pl. 45 , figs. $1-4$; leaf, Leguminosae; Raritan formation, Upper Cretaceous; Woodbridge, N. J.

FORALITES Rouault, 1850.

Foralites pomeli Rouault, 1850, p. 743 ; incertae sedis ; Sllurian; Bain, Guichen, etc., in Brittany, France. See also Delgade, 1886, p. 90, pls. 3, 13.

FORBESIA Thomas Johnson, 1912.

Forbesia cancellata Thomas Johnson, 1812, p. 177 , pls. 13,14 ; fern rachis ?; Lower Carboniferous; near Bandon, County Cork, Ireland.

FORCHHAMMERA Goeppert, 1859.

Forchhammera silurica Goeppert, 1859, p. 438, pl. 34, flg. 5; plant?; Lower Silurian; Bornholm, Denmark.

FORSKOHLEANTHIUM Conwentz, 1886.

Forskohleanthium nudum Conwentz, 1886, p. 45 , pl. 4, figs. 20-22 ; flower, in amber, Urticaceae ; Tertiary ; West Prussia.

FORTISIA Visiani, 1858.

Fortisia haidingeriana Vistani, 1858, p. 10, pl. 1, figs. 1-4; fern? pinnules; Eocene; Monte Promona, Italy.

FRAASIA. Unger, 1850.

Fraasia sapindoides Unger, 1850a, p. 457 ; wood, Sapindaceae; Tertlary ; Hungary.

FRACASTORIA Massalongo, 1858.

Fracastoria clavaeformis Massalongo, 1858b, p. 762 ; Eocene; Monte Bolca, Italy. See also Fracastoria megapepo Massalongo, $1857 \mathrm{~b}$, p. 777 ; nom. nud.

FRAENA Rouault, 1850.

Fraena sanctihilaireb Rouault, 1850, p. 731 ; plant ?; Sllurian; Guichen, Brittany, France. See also Saporta, 1884, p. 54, pl. 8, fig. 3.

FRAXINOIDITES Robert Potonle, 1950.

Fraxinoidites sp. Robert Potonle, in Potonie, Robert, Thomson, Paul W., and Thiergart, Friedrich, 1950, p. 62.

$F R A X I N O P S I S$ Wieland, 1929.

Fraxinopsis minor Wieland, 1929b, p. 448, fig. 5b; winged fruits, compared with Fraxinus; Rhaetic; Minas de Petroleo, southwest of Mendoza, Argentina.
FRENELITES Endllcher, 1847.

F'renelites recurvatus (Bowerbank) Endlicher, 1847, p. 273 . For Cupressinites recurvatus Bowerbank, 1840, p. 55 , pl. 10, fig. 19.

FRENELOPSIS Schenk, 1869.

Frenelopsis hoheneggri (Ettingshausen) Schenk, 1869, p. 13, pl. 4, figs. 5-7; p. 5, figs. 1,2 ; pl. 6 , figs. 1-6; pl. 7, fig. 1 ; defollated coniferous shoot?; Cretaceous.

FRICIA Velenovsky, 1885.

Fricia nobilis Velenovsky, 1885a, p. 8, pl. 8, figs. 1-3, 6, 11 .

FRUCTUS Engelhardt, 1877.

Fructus polyspermus Engelhardt, 1877, p. 389 , pl. 21 , fig. 8 ; incertae sedis; Tschernowitz, Bohemia.

FRULLANITES Sadebeck, 1886 ?

Frullanites succini Sadebeck, 1886, p. 121 ; nom. nud. ; moss ; Tertiary ; Prussia.

FRUTICRISTATUM Webster, 1920.

Fruticristatim iowense Webster, 1920, p. 288 ; marine alga ; Devonian; Bloody Run, Floyd County, Iowa.

FUCHSELIA Endlicher, 1847.

Fuchselia 8chimperi Endlicher, 1847, p. 304. For Strobilites laricoides Schimper and Mougeot, 1844, p. 31, pl. 1 ; pl. 16 ; cone, Coniferales; Triassic; Soulz-lesBains, Alsace-Lorraine.

FUCITES (Brongniart) Unger, 1839.

Fucites dubius Unger, 1839, p. 101 ; nom. nud.

FUCOIDES Brongniart, 1823.

Fucoides orbignianus Brongniart, 1823, p. 308, pl. 19, fg. 1; Lower Cretaceous (Neocomian); Island of Alx, near La Rocbelle, France. See also Brongniart, $1828 a-38$, p. 78 , pl. 2 , figs. 6,7 .

FUNGITES Hallier, 1865.

Fungites apoldensis Haller, 1865, p. 191, pl. 9B; mycellum; Tertiary (Braunkohle); Apolda, Germany.

FURCIFOLIUM Kräusel, 1943.

Furcifolium longifolium (Seward) Kräusel, $1943 a$, p. 431 , figs. 1-6; ginkgophyte, Baiera-like foliage attached to slender stems; Jurassic; Solenhofen, Bavaria.

FURCOPORELLA Pia, 1918.

Furcoporella diplopora Pia, in Trauth, 1918 , p. 209 , pl. 1 , figs. 1,2 ; alga, Dasycladaceae ; Eocene ; Radstadt, Austria.

FURCULA Harris, 1932.

Furcula granulifer Harris, 1932a, p. 4, pl. 1 ; leaf, dicotyledon; Rhaetic; Scoresby Sound, east Greenland. 
FUSIDITES Meschinelli, 1898.

Fusidites sp. (Conwentz) Meschinell, 1898 , p. 78 , fungus.

\section{G}

GALLA (Ludwig) Lesquereux, 1892.

Galla quercina Lesquereux, 1892 , p. 58, pl. 7, fig. 2; oak gall?; Cretaceous; Ellsworth County, Kans. Generic name given by Ludwig, 1857 , p. 90 , but no species assigned.

GALLATINIA Walcott, 1914.

Gallatinia pertexa Walcott, 1914, p. 116, pl. 23, figs. 1, 2 ; alga ; Algonkian ; west of Hillsdale Post Office, Gallatin County, Mont.

GANGAMOPTERIS McCoy, 1875.

Gangamopteris angustifolia McCoy, 1875, p. 11 , pl. 12, flg. 1 ; pl. 13, fig. 2 ; large net-velned leaf; Mudgee, New South Wales. For Cyclopteris angustifolia McCoy, 1847, p. 148, pl. 9, figs. 3, 3a.

GANGAMOPTERIOPSIS Zalessky, 1927.

Gangamopteriopsis netchaevi Zalessky, 1927a, p. 41 , pl. 16, figs. $1-5$; pl. 17 ; leaf; Permian; near Voskressensky, Urals, Russia.

GANITROCERA KIrchheimer, 1934.

Ganitrocera holzapfeli Kirchheimer, 1934a, p. 770, fig. 4 ; seed, Cornaceae; Tertiary (Braunkohle) ; Herzogenrath, Germany. See also Kirchheimer, 1936a.

GaRWOODElla Paul, 1938.

Reference not seen; cited in Gothan, 1942b, p. 123.

GARWOODIA Wood, 1941.

Garwoodia gregaria (Nicholson) Wood, 1941 , p. 222, pl. 14, figs. 1,2 ; pl. 15 , figs. 1-4; alga ; Lower Carboniferous; Kershopefoot, Roxburghshire, Scotland.

GASTRIDIOPSIS Massalongo, 1851.

Gastridiopsis elisae Massalongo, 1851, p. 69; alga; Tertiary ; Italy.

GASTROMYCES Ludwig, 1861.

Gastromyces farinosa Ludwig, 1861, p. 32, pl. 6, figs. 3, 3a-c; gasteromycete?; Upper Carboniferous Malowka, Tula, Russia.

GAUDRYA Grand'Eury, 1890.

Gaudrya trivalvis Grand'Eury, 1890, p. 308, pl. 4, fig. 12 ; petrified seed; Upper Carboniferous; st.-ftienne, France.

GAUSSIA Chachloff?, 1934

Reference not seen; cited in Gothan, 1942b, p. 123.

GEASTERITES P1a, 1927.

Geasterites florissantensis (Cockerell) Pia, in Hirmer, 1927, p. 121, fig. 109; Geaster-like Impression, Lycoperdiaceae; Miocene; Florissant, Colo.
GEIETES Stenzel, 1872.

Geietes moussoni (Heer) Stenzel, 1872, p. 71.

GEINITZIA Endlicher, 1847.

Geinitzia cretacea Endlicher, 1847, p. 281. For Sedites rabenhorstii Geinitz, 1842 (1839-42), p. 97, pl. 24, fig. 5 .

GEINITZIELLA Kuntze, 1904.

Geinitziella Kuntze, in Post and Kuntze, 1904, p. 245.

GEINITZITES Fontaine, 1899.

Geinitzites jenneyi Fontaine, in Ward, 1899 , p. 681 ; coniferous twig impression; Lower Cretaceous; Black Hills, S. Dak. This is a "proposed" name; Fontaine describes the new species Geinitzia jenneyi on p. 676 and on p. 681 writes: "As Geinitzia is hitherto known from no strata older than the Younger Cretaceous, it may be found that our plant is an ancestral form of the true Geinitzia. In that case it would be fittingly named Geinitzites jenneyi."

GELEENITES Dijkstra, 1949.

Geleenites fascinus Dijkstra, 1949, p. 26, pl. 2, fig. 11; incertae sedis; South Limburg, Netherlands.

GELIDINIUM Debey and Ettingshausen, 1859.

Gelidinuim trajectomosanum Debey and Ettingshausen, 1859a, p. 199, pl. 3, fig. 6h ; alga ; Cretaceous ; Aachen, Rhenish Prussla.

GELLERA Hollick, 1931.

Gellera paradoxa Hollick, 1931, p. 9, pl. 2 , figs. 1-3; base of stem and roots, fern?; specimen found in terminal moraine; transported from Triassic rock horizon; Arrochar, Staten Island, N. $Y$.

CENOITES Feruglio, 1942.

Genoites patagonica Feruglio, 1942, p. 104, pl. 1, figs. 3,4 ; pls. 5,6 ; Liassic ; Rio Genoa Valley, Patagonia, Argentina.

GEOCARPUS Kinkelin, 1884.

Geocarpus miocaenicus Kinkelin, 1884, p. 256, pl. 3, figs. 14-18; Miocene ; Frankfurt-Niederrad, Prussia.

GEONOMITES Visiani, 1864.

Geonomites saturnia Visiani, 1864, p. 456, pl. 21, palm leaf; Tertiary; Italy.

\section{GEONOMITES Lesquereux, 1878.}

Geonomites goldianus Lesquereux, 1878a, p. 115 , pl. 4 , fig. 9 ; palm leaf; South Mtn., Golden, Colo.

\section{GERMANOPHYTON Hoeg, 1942.}

Germanophyton psygmophylloides (Kräusel and Weyland) Hoeg, 1942, p. 98, fig. 20; stem, with cells of Prototaxites type, bearing large fanshaped leaves; Lower Devonian; Kirchhunden, West- 
phalia. For Prototaxites psygmophylloides Kräusel and Weyland, 1930, Senckenbergiana, Band 12, p. 218.

GERMARIA Presl, 1838.

Germaria elymiformis Presl, in Sternberg, $1838(1820-38)$, p. 188 , pl. 49 , figs. 19 ; cones?, incertae sedis; Rhaetic; Bayreuth, Bavaria.

GETONITES Ettingshausen, 1887.

Getonites wilkinsoni Ettingshausen, 1887, p. 130 , pl. 15 , figs. $11,11 \mathrm{a}, 12$; leaf, Combretaceae; E ocene; Vegetable Creek, near Emmaville, New South Wales.

GIGANTONOOLEA Koidzumi, 1936.

Gigantonoclea lagrelii (Halle) Koidzumi, 1936, p. 138. For Gigantopteris lagrelii Halle, 1927, p. 170 , pl. 46 ; lower Shihotse series, Lower Permian; central Shansi, China.

GIGANTOPTERIS Schenk, 1883.

Gigantopteris nicotianaefolia Schenk, $1883 \mathrm{~b}$, p. 256. For Megalopteris nicotianaefolia Schenk, 1883 b, p. 238, pl. 32 , flgs. $6-8$; pl. 33 , figs. $1-3$; pl. 35 , fig. 6; Upper Carboniferous; Lui-pakou, Hunan province, China.

GIGANTOSPERMUM Jongmans and Gothan, 1935.

Gigantospermum posthumi Jongmans and Gothan, 1935, p. 169, pl. 58, fig. 1 ; Upper Carboniferous; Djambi, Mengkarang, Sumatra.

GIGARTINITES Brongniart, 1849.

Gigartinites obtusus Brongniart, 1849, p. 59. For Fucoides obtusus Brongniart, $1828 \mathrm{a}-38$, p. 60 , pl. 8 , fig. 4 ; alga?; Tertiary; Monte Bolca, near Verona, Italy.

GILBERTINA Ulrich, 1904.

Gilbertina spiralis Ulrich, 1904, p. 141, pl. 18, figs. 1, 2 ; plant?; Yakutat formation, Jurassic (Liassic) ; Pogibshi Island, Alaska.

GILBOAPHYTON Arnold, 1937.

Gilboaphyton goldringiae Arnold, 1937, p. 76, pl. 1; Psilophytales or Lycopodiales?; Middle Devonian ; Gilboa, Schoharie County, N. Y.

GINKGANTHUS Nathorst, 1899.

Ginkganthus sp. Nathorst, 1899, p. 213, pl. 1, figs. 33,49 ; nrlcrosporangiate organ, ginkgophyte; Jurassic ; Franz Josef Land.

GINKGOCLADUS Ettingshausen, 1887.

Ginkgocladus novaezeelandiae Ettingshausen, 1887 b, p. 179 , pl. 7 , fig. 19 ; leaf, Incertae sedis ; Upper Cretaceous; Wangapeka, Nelson, New Zealand.
GINKGODIUM Yokoyama, 1889.

Ginkgodium nathorsti Yokoyamu, 1889, p. 57, pl. 2, fig. 4e; pl. 3, flg. 7 ; pl. 8 ; pl. 9, figs. 1-10 ; Lower Oolite, Jurassic ; Shimamura, Yauagedani, Japan.

GINKGOITES Seward, 1919

Ginkgoites obovata (Nathorst) Seward, 1919 , p. 12 , fig. 632 ; leaf, Ginkgoaceae ; Rhatic; Scania, Sweden.

GINKGOPHYYLUM Saporta, 1875.

Ginkgophyllum grasseti Saporta, 1875b, p. 1018; leaf, ginkgophyte?; Permian; Lodève, France. See also Saporta, 1879, p, 186, fig. 15 .

GINKGOPHYTON Matthew, 1910.

Ginkgophyton leavitti Matthew, 1910, p. 87 , pl. 4 ; ginkgophyte? leaves and associated seeds : Mississippian; Duck Cove, Lancaster, New Brunswick, Canada.

GINKGOPHYTON Zalessky, 1918.

Ginkgophyton sp. Zalessky, 1918, p. 47.

GINKGOPSIS Zalessky, 1918.

Ginlegopsis czekanowstii (Schmalhausen) Zalessky, 1918, p. 57, pl. 22, figs. $1-4$; ginkgophyte leaf?; Mesozolc; Souka, Russia. This generic nume mentioned in Zalessky, 1912, p. 28 (footnote), but no specifle name assigned.

GINKGOSPERMUM Nathorst, 1878.

Ginkyospermum globulare Nathorst, $1878 \mathrm{a}$, p. 12 ; nom. nud.

GIRVANELLA Nicholson and Etherldge, 1878.

Girvanella problematica Nicholson and Etheridge, 1878 , p. 23, pl. 9, fig. 24; Silurian: Girvan District, Ayrshire, Scotland.

GLEDITSCHIACANTHUS Lakowitz, 1895. Gleditschiacanthus alsaticus Lakowitz, 1895 , p. 288 , pl.. 10, fig. 8 ; Oligocene; Brunstatt, Alsace-Lorruine.

GIEDITSCHITES Fritel, 1924

Gleditschites dubium (Watelet) Fritel, 1924 , p. 169 , fig. $20 \mathrm{~A}$; frult, Legumlnosae; Belleu, France.

GLEDITSIOPHYLLUM E. W. Berry, 1910. Gleditsiophyllum triacanthoides E. W. Berry, 1910a, p. 197 ; leaf, Rosales; Cretaceous; $31 / 2$ miles below Denbars Bridge, Tar River, Edgecomb County, N. C. This species apparently never illustrated; first species illustrated: $\sigma$. eocenicum Berry, 1916b, p. 238, pl. 46, figs. 1-7.

GLEICHENIOPSIS Tutin, 1932.

Gleicheniopsis fecunda (Heer) 'Tutin, 1932, p. 503 , pl. $16^{\circ}$; fertile fern frond fragment, Gleicheniaceae; Lower .Cretaceous; Ritenbenk coal mine, Disko Island, Greenland. 
GLEICHENITES Goeppert, 1836.

Goeppert, 1836, p. 181-187, described five species which in no way conform with modern usage, his plants being Carboniferous sphenopterids, neuropterids, etc. The following is suggested as a type species, being one of the first described which clearly conforms with the modern concept: Gleichenites porsildi Seward, 1926 , p. 76 , pl. 6 , figs. $18,19,24,27$, 29-31; pl. 12, figs. 122, 124 ; Gleichenialike frond; Cretaceous; Anglarsuit, Upernivik Island, Greenland. See also Gleichenites coloradensis (Knowlton) Andrews, in Andrews and Pearsall, 1941, p. 174 , pl. 3, figs. 20-22, 24 ; pl. 4, figs. 26, 27, 29; pl. 7. See also Seward, 1910 , p. 351 , and 1926 , p. 69.

GLEICHENOPHYCOS Massalongo, 1884.

Gleichenophycos granulosus Massalongo, in Capellini, 1884, p. 541; Upper Cretaceous; Granaglione, near Bologna, Italy.

GLENOPTERIS Sellards, 1900.

Glenopteris splenden 8 Sellards, 1900, p. 182 , pl. 37 , fig. 1 ; pl. 38 , fig. 1 ; pl. 40 ; fern frond, compared with Protoblechnum Lesquereux; Permian; 31/2 miles south of Banner City, Dickenson County, Kans.

GLEOCAPSOMORPHA Zalessky, 1920.

Gleocapsomorpha prisca Zalessky, 1920, p. 83, figs. 1-3 ; alga; Silurian.

GLOBULINEA Ulke, 1938.

Globulinea giganteus Ulke, 1938, p. 58, pl. 1, fig. 1 ; alga ; Mississippian ; "Washington, D. C." Type specimen on a step of the 16th Street entrance of the Baptist Memorial Church, Washington, D. C. !

GLOCKERIA Goeppert, 1836.

Glockeria marattioides Goeppert, 1836, p. 379 , pl. 39 , figs. 2 , 3 ; fernlike foliage; Upper Carboniferous; Charlottenbrun, Silesia.

GLOEOOAPSITES Zalessky, 1917.

Gloeocapsites sp. Zalessky, 1917, p. 34.

GLOECAPSOMORPHA Zalessky, 1917.

Gloecapsomorpha prisca Zalessky, 1917, p. 36, pl. 2, figs. 4-7; pl. 3, flg. 2 ; Lower Silurian; Petrograd, Russia.

GLOIOCONIS Renault, 1896.

Gloioconis borneti Renault, 1896a, p. 446, flg. 94, pl. 88, flg. 12; alga; Permian; Lally, France.

GLORIOSITES Heer, 1855.

Gloriosites rostratus. Heer, 1855, p. 83 , pl. 30, fig. 6 ; rhizome, Liliaceae?; Tertiary ; Oeningen. Switzerland.

GLOSSIFUNGITES Lomnicki, 1886.

Glossifungites saxicava Lomnicki, 1886, p. 99 , pl. 3, figs. $64 \mathrm{a}, 64 \mathrm{~b}$; Upper Cretaceous; Rukow near Pomorzany, Galicia.
GLOSSOCARPELLITES Perkins, 1905.

Glossocarpellites parvus Perkins, 1905, p. $510, \mathrm{pl}, 86$, fig. 15; fruit; Tertlary ; Brandon, vt.

GLOSSOCHLAMYS Ettingshausen, 1879.

Glossochlamys transmutans Ettingshausen and Gardner, in Gardner and Ettingshausen, 1879 , p. 31 , pl. 3 , fig. 3 ; fern ? leaf ; Eocene ; Bournemouth, England.

GLOSSOPHIUM Massalongo, 1893.

Glossophium eocenum Massalongo, in Meschinelli and Squinabol, 1893, p. 415. See also Glossophium proliferum Massalongo, 1857b, p. 777 ; nom. nud.

GLOSSOPHYCUS Saporta and Marion, 1881.

Glossophycus camillae Saporta and Marion, 1881 , p. 89 , flg. 26 ; alga ?; Triassic ; Cannet, France.

GLOSSOPHYLLUM Krüusel, 1943.

Glossophyllum florini Kräusel, 1943b, p. 61 , pl. 2 , figs. $9-11$; pl. 3 , figs. $6-10$; ginkgophyte leaf; Triassic; Lunz, Austria.

GLOSSOPTERIS (Brongniart) Sternberg, 1825.

Glossopteris browniana Brongniart, 1833 (1828a-38), p. 223, pl. 62. Glossopteris was proposed by Brongniart, 1822, p. 232, as a section of Filicites and given generic rank by Sternberg,.1825 (182038), Tentamen, p. xv ; thus earliest valid binomial would appear to be Glossopteris dubia (Brongniart) Sternberg, 1825 (1820-38), p. xv, but Brongniart's illustration (Brongniart, 1822, p. 232, pl. 2, fig. 4) which Sternberg refers to is doubtful. See also Seward, 1910, p. 496.

GLOSSOPTEROPSIS Zalessky, 1918.

Glossopteropsis angarica Zalessky, 1918, p. 51, pl, 8, figs. 1, 2 ; ginkgophyte leaf?: Permian; Bassin d'Angara, near Irbinskaia, Russia.

GLOSSOZAMITES Schimper, 1870.

Glossozamites oblongifolius (Kurr) Schimper, $1870(1869-74)$, p. 163 , pl. 71 ; cjeadophyte foliage; Lower Jurassic (Lias) ; Wuirttemberg.

GLOTTOPHYLLUM Zalessky, 1912.

Glottophyllum cuneatum Zalessky, 1912, p. 28 (footnote), pl. 5, fig. 4; ginkgophyte leaf?; Carboniferous; Kuznets Basin, Russia. See also Zalessky, 1918, p. 59, pl. 26, fig. 1 .

GLUTOXYLON Chowdhury, 1936.

Glutoxylon assamicum Chowdhury, 1936, p. 508, pl. 7 ; wood, compared with Gluta (Anacardiaceae) ; middle TerGLYPHOSTROMIUM Reinsch, 1881.

Glyphostromium sp. Reinseh, 1881, p. 58, pl. 14, figs. 1-8; Upper Carboniferous: Zwickau, Saxony. 
GLYPTODENDRON Claypole, 1878.

Glyptodendron eatonense Claypole, 1878a, p. 303 ; arborescent lycopod stem Im. pression; Upper Silurian; Clinton near Eaton, Ohto. See also Claypole, $1878 \mathrm{~b}$, p. 559 , fig.

GLYPTOLEPIDIUM (Heer) Sordell, 1896. Glyptolepidium gornense Sordelli, 1896, p. 49 , pl. 10 , figs. 8,9 ; coniferous twigs with follage; Triassic; Gorno, Val Seriana, Italy. Generic name given by Heer, 1876c, p. 72, but no species named.

GLYPTOLEPIS Schimper, 1870.

Glyptolepis keuperiana Schimper, 1870 (1869-74), p. 244 , pl. 76, flg. 1 ; coniferous follage shoots?; Upper Triassic (Keuper) ; near Coburg, Germany.

GLYPTOSTROBITES Brongniart, 1849.

Glyptostrobites acutifolius Brongniart, 1849 , p. 123. Apparently first lllustrated species : $G$. parisiensis Brongnlart, in D'Orbigny, 1852 (1851-52), p. 775, fig. 596. See also Watelet, 1866, p. 116, pl. 31, fig. 3.

GLYPTOSTROBOXYLON Conwentz, 1885.

Glyptostroboxylon goepperti Conwentz, 1885 , p. 445 ; confferous wood; Lower Oligocene; Katapuliche, Argentina. First illustrated specles: $G$. tenerum Prill and Kräusel, in Kräusel, 1919a, p. 264 , pl. 18, fig. 12 ; pl. 20, figs. 6-7, 10.

GNETOPSIS Renault and Zelller, 1884.

Gnetopsis elliptica Renault and Zefller, $1884 a$, p. 57 ; seeds in cupule, Pteridospermae ; Upper Carboniferous ; R1ve-deGler, France. See also Renault, 1885, p. 179, pl. 20, figs. 1-10; pl. 21, figs. $1-6$; pl. 22, figs. 2-4.

GOEPPERTELLA Oishi and Yamasita, 1936. Goeppertella microloba (Schenk) Oishi and Yamasita, 1936, p. 147. For Woodwardites microlobus Schenk, 1865-67, p. 68, pl. 13, figs. 11-13.

GOEPPERTIA Presl, 1838.

Goeppertia polypodioides Presl, in Sternberg, $1838(1820-38)$, p. 121, pl. 50, fig. 1 ; fertlle fern follage fragment; Upper Carboniferous; near Plass, Bohemia.

GOLDENBERGIA Halle, 1933.

Goldenbergia glomerata Halle, 1933 , p. 8 , pl. 1, figs. 1a-19; pl. 3 ; synanglum, probably pteridosperm; Upper Carboniferous; Saarbrucken, Germany.

GOLDSONIA Shrock and Twenhofel, 1939. Goldsonia burntensis Shrock and Twenhofel, 1939, p. 247, pl. 27, figs. 2-4; alga: Pike Arm formation, Silurian; Burnt Island in Goldson Arm, New World Island, Newfoundland.
GOMPHOSTROBUS Marion, 1890.

Gomphostrobus heterophylla Mar10 1890 b, p. 894 ; araucartanlike follage shoots; Permian; Lodeve, France. First illustrated species: $G$. biflus (Geinitz) Zeiller and Potonie, in Potonie, Henry, 1900, p. 620, fig. 387.

GONATOBOTRYTITES PIa, 1927.

Gonatobotrytites primigenius (Caspary) Pia, in Hirmer, 1927, p. 122, fig. 111; Mucedinaceae, Fungi Imperfectl; Eocene ; East Prussia.

GONDWANIDIUM Gothan, 1927.

Senckenberg. naturf. Gesell. Abh., 1927, Band 39, p. 342 ; Pteridospermae ; Permian (not seen). See also Gothan, 1942b.

GONGROSTROMIUM Reinsch, 1881.

Gongrostromium sp. Reinsch, 1881, p. 58, pl. 13a, figs. 1-3 ; Carboniferous ; Mittelbronn, Württemberg.

GONIOLINA d'Orbigny, 1850.

Goniolina hexagona d'Orbigny, 1850, p. 41; Upper Jurassic; Pointe-du-Che, near Rochelle, France. First lllustrated species: $G$. geometrica Buvignier, 1852 , p. 47 , pl. 32 , figs. $36,37$.

GONIOPHYOUS Saporta, 1884.

Goniophycus implexus Saporta, 1884, p. 53, pl. 8, flg. 4 ; Triassic ; Dragulgnan, France.

GONATOSORUS Raciborsk1, 1894.

Gonatosorus nathorstii Raciborsk1, 1894, p. 173, pl. 9, figs. 5-11. See also Gonatosorus sp. Raciborski, 1889, p. 138.

GORDIA Emmons, 1844

Gordia marina Emmons, 1844, p. 24, pl. 2, fig. 2 ; Cambrian; Jackson, Washington County, N. Y.

GOSSLINGIA Heard, 1927.

Gosslingia breconensis Heard, 1927, p. 198, pls. 13-15; petrifled stem, Psllophytales; Senni beds, Lower Devonian; Brecon, South Wales.

GOTHANIA HIrmer, 1933.

Gothania westfalica Hirmer, 1933b, p. 188, pls. 17-22; petrifled inflorescence, Cordaitales; middle Upper Carboniferous ; Germany.

GOTHANIELLA Fucini, 1936.

Reference not seen; cited in Gothan, $1942 b$, p. 124.

GOTHANOPTERIS Koldzumi, 1936.

Gothanopteris bosschana Koldzuml, 1936, p. 136. For Gigantopterls bosschana Gothan and Jongmans, 1935, Jaar. mijnwezen Nederlandish-Indië, 1930, Verh. boekdeel 59, p. 47, flgs. 2-4; Carbonlferous (Stephanian); Djambl, Sumatra. 
GOULDINA J. H. Johnson, 1940.

Gouldina carbonaria J. H. Johnson, 1940, p. 583, pl. 3, fig. 1 ; calcareous alga, Cyanophyceae?; top of Weber formation, Pennsylvanian; Park County, Colo.

GOUPIOXYLON Schonfeld, 1947.

Goupioxylon stutzeri Schonfeld, 1947, p. 19 , pl. 1, figs. $2-9$; pl. 2 , figs. $1-4$; wood, Celastraceae ; Tertiary ; Colombia.

GRACILERECTUS Webster, 1920.

Gracilerectus hackberryensis Webster, 1920 , p. 288 ; marine alga ; lower Hackberry group, Devonian; Mason City, Iowa. See also Gracilerectus delicatus Fenton and Fenton, 1924, p. 21, pl. 1, figs. 9,10 .

GRAMINITES H. B. Geinitz, 1865.

Graminites feistmanteli H. B. Geinitz, 1865 , p. 392 , pl. 3 , fig. 3 ; articulate? stem fragment; Upper Carboniferous ; Bras, Belgium.

GRAMINOPHYLLUM Conwentz, 1886. Graminophyllum succineum Conwentz, 1886 , p. 15 , pl. 1 , figs. $18-24$; flower, in amber, Gramineae; Tertiary ; West Prussia.

GRAMMAEPHLOIOS Harris, 1935.

Grammaephloios icthya Harris, 1935, p. 152 , pls. $23,25,27,28$; leafy shoot, Lycopodiales; Thaumatopteris zone, Rhaetic; Scoresby Sound, east Greenland.

GRAMMATOP'TERIS Renault, 1891.

Grammatopteris rigolloti Renault, 1891, p. 500 ; coenopterid fern; "Permo-CarbonIferous"; France. See also Renault, $1896 a$, p. 46, p1. 30, figs. 9-10; pl. 31, fig. 1. See also Posthumus, 1931.

GRAMMITES Reinsch, 1881.

Grammites sp. Reinsch, 1881, p. 63, pl. 14c, figs. $1-8$; pl. 15, figs. 1-8; Permian; Mittelbexbach, Bavaria.

GRAMMITITES C. F. W. Braun, 1840.

Grammitites humilis C. F. W. Braun, 1840, p. 96 ; nom. nud.

GRAMMOPHYLLUM C. F. W. Braun, 1840. Grammophyllum lineatum C. F. W. Braun, 1840 , p. 100 ; nom. nud.

GRAND'EURYA Stur, 1883.

Grand'Eurya autunensis Stur, 1883, p. 679 figs. $12 \mathrm{a}, 12 \mathrm{~b}$; petrified pinnules bearing marattlaceous sporangia; Permian Autun, France.

GRAND'EURYA Zeller, 1883.

Grand'Eurya coralloides (Gutbier) Zeiller, 1883 , p. 207 ; pl. 12 , figs. $1-6$; fertile fern frond; Upper Carboniferous; France.

GRAND'EURYELLA C. E. Weiss, $1885^{\circ}$.

Grand'Euryella renaulti (Stur) C. E. Weiss, 1885b, p. 492. For Grand'Eurya renaulti Stur, 1883 , p. 678 , fig. 12 c.
GRANULARIA Pomel, 1849.

Granularia schlotheimi Pomel, 1849, p. 333 ; alga ; Lower Jurassic (Lias); Metz. First species illustrated : Granularia linearis Zigno, 1856-68, p. 37, pl. 2, flg. 5 .

GRANULATISPORITES Ibrahim, 1933.

Granulatisporites granulatus Irbahim, 1933 , p. 22, pl. 6, fig. 51 ; spore ; Carboniferous.

GRAPHIOLITES Fritel, 1910

Graphiolites sabaleos Fritel, 1910; p. 12 pl. 20, fig. 12 ; fungus, Basidiomycete?; Upper Paleozoic ; Cessoy, France.

GRAYSONIA.

Mistake for Greysonia, in Butts, 1926, p. 76 and Mawson and Madigan, 1930, p. 426.

GREVILLEOPHYLLUM Velenovsky, 1889. Grevilleophyllum constans Velenovsky, 1889 , p. 53. For Grevillea constans Velenovsky, 1883 , p. 28 , pl. 1, figs. $6-10$; Upper Cretaceous; Jinovic, Bohemia.

GREWIOPSIS Saporta, 1865.

Grewiopsis tiliacea Saporta, 1865, p. 50, leaf, Malvaceae; Eocene; Sézanne, France. See also Saporta, 1868, p. 406, pl. 33, fig. 12.

GREWIOXYLON Schuster, 1910.

Arewioxylon swedenborgii Schuster, 1910, p. 14, pl. 1., figs. 1-4; compared with Dipterocarpoxylon tobleri Kräusel (see Kräusel, 1922, p. 263) ; Tertiary ; East Indies.

GREYSONIA Walcott, 1914

Greysonia basaltica Walcott, 1914, p. 109, pl. 17 , figs. 1,2 ; pl. 18, figs. 1,2 ; alga ? Newland limestone, Algonkian; 8 miles west of White Sulphur Springs, Meagher County, Mont.

GRILLETIA Renault and Bertrand, 1885.

Grilletia spherospermii Renault and Bertrand, 1885 , p. 1306 ; fungus, Chytridlaceae; Upper Carboniferous; GrandCroix, France.

GRIPHOPORELLA PIa, 1915.

Griphoporella curvata (Gumbel) Pia, in Spitz and Dyhrenfurth, 1915, p. 62, pl. 1, fig. 11 ; alga, Siphoneae Verticillatae; Triassic.

GRISTHORPIA Thomas, 1925.

Gristhorpia nathorsti Thomas, 1925, p. 335 , pls. 11, 14, 16; infructescence; Caytoniales; Jurassic; Cayton Bay, Yorkshire, England.

GRUMILEOPHYLLUM Geyler, 1887.

Grumileophyllum attenuatum Geyler, 1887 a, p. 494 , pl. 35 , figs. 4, 5; leaf fragments, Rublaceae?; Eocene; Labuan, Borneo.

GUA.JACITES Massalóngo, 1858.

Guajacites heerii Massalongo, 1858b, p. 767. 
GUEMBELINA (Munier-Chalmas) Morellet and Morellet, 1913.

Guembelina bellovacina Munier-Chalmas in Morellet and Morellet, 1913, p. 38; Hocene; Bracheux, France. Generic name given (nom. nud.) by MunlerChalmas, 1877 , p. 817.

GUILIELMITES Geinitz, 1858.

Guilielmites permianus Geinitz, 1858, p. 19, pl. 2, figs. 6-9 ; incertae sedis; Permlan; Grüna near Chemnitz, Germany.

GLILLIERA Crie, 1885.

Guilliera sarthacensis Crie, 1885, p. 85; cycadophyte cone?; Jurassic (Oolite); Mamers, France.

GULPENIA Gothan and Jongmans, 1927.

Gulpenia limburgensis Gothan and Jongmans, in Jongmans, 1927a, p. 66 ; sphenopterid follage; Upper Carbonlferous ; Limburg, Gulpen mine, Netherlands.

GU'BBIERIA Presl, 1938.

Gutbieria angustoloba Presl, in Sternberg, 1838 (1820-38), p. 116, pl. 33, flgs. 13a-e; fertile fern fragment; Upper Triassic (Keuper); Strahlendorf.

GUT'TIFEROXYLON Krüusel, 1939.

Bayer. Akad. Wiss., Math.-naturwiss. Abh., 1939, Nene Folge, Band 47, p. 93 (not seen, cited in Gothan, 1942b, p. 124).

GYMNOCAULUS Emmons, 1856.

Gymnocaulus alternatus Rmmons, 1856, p. 289 , pl. 1, flg. 4 ; fern ? frond fragment; Permian; Madison, Stokes County, N. C.

GYMNOCODIUM PLa, 1927.

Gymnocodium bellerophontis (Rothpletz) Pla, in Hirmer, 1927, p. 59, flg. 36b ; alga ; Codiaceae; Upper Permian.

GYMNONEUROPTERIS PIa, 1924.

Gymnoneuropteris carinthiaca Pia, 1924, p. 553, pl. [unnumbered] ; coenopterid fern; Carboniferous; Bleiberg, Carinthia. See also Hirmer, 1927, p. 515.

GYMNOSOLEN Steinmann, 1911.

Gymnosolen ramsayi Steinmann, 1911, p. 18 , pl. 3 ; alga? (described as coelenterate). See Hirmer, 1927, p. 37 ; Johnson, J. H., 1943, p. 100.

GYMNOSTROBUS Bureau, 1914.

Gymnostrobus salisburyi Bureau, 1914, p. 165 , pl. 38 , figs. 1,2 ; ly copod cone ? compression; Lower Carboniferous (upper (Culm) ; Tardiviére, France.

GYNOTROCHOXYLON Kräusel, 1939.

Bayer. Akad. Wiss., Math.-naturwiss. Abh. 1939, Neue Folge, Band 47, p. 97 (not seen, cited in Gothan, 1942b, p. 124).

GYROCALAMUS C. E. Welss, 1884.

Gyrocalamus palatinus C. E. Weiss, $1884 \mathrm{~b}$, p. 152, pl. 4, figs. 3, 4 ; Upper Carboniferous; Alben; Rhenish Bavaria.

GYROOHORDA.

See Gyrochorte Heer.
GYROOHORTE Heer, 1865.

Gyrochorte vermicularis Heer, 1865, p. 142, pl, 9, figs. 8, 10. [Name altered to Gyrochorda by Schimper, in Schimper and Schenk, $1879(1879-90)$, p. 51.]

GYRODENDRON Ulrich, 1904.

Gyrodendron emersoni Ulrich, 1904, p. 140, pl. 18, fig. 3 ; pl. 19, figs. 1, 2 ; plant?; Yakutat formation, Lower Jurassic ; Pogibshi Island, opposite village of Kadlak, Alaska.

GYROGONITES Lamarck, 1804.

Gyrogonites medicaginula Lamarck, 1804, p. 356 ; Charophyte; Eocene; near Paris, France. First lllustrated : Lamarck, 1807, p. 236, pl. 15, fig. 7 . First? publication after 1820 : Hirmer. 1927 , p. 89 , fig. 73 . See also comment by Peck, R. E., 1934, p. 52.

GYROPHYLLITES Glocker, 1841.

Gyrophyllites kwassizensis Glocker, 1841, p. 322, fig. p. 322 ; whorl of leaves, equlsetalean affinities?; Cretaceous (Cenomanian); Capellenberg, near Kwassitz, Moravia.

GYROPORELLA Gümbel, 1871.

Gyroporella annulata (Schafhautel) Gümbel, 1871, p. 269, pl. 2, figs. 1a-11 ; alga, Dasycladaceae.

GYROPTERIS Corda, 1845.

Gyropteris cras8a Corda, 1845, p. 86, pl. 54, figs. 1--6 ; fern petiole fragment; Upper Carboniferous; Radnitz, Bohemia. See also Posthumus, 1931.

\section{$\mathbf{H}$}

HAASTIA. Ettingshausen, 1887.

Haastia speciosa Ettingshausen, $1887 \mathrm{~b}$, p. 180 , pl. 8 , flg. 5 ; leaf fragment, Musaceae; Upper Cretaceous ; Pakawau, Nelson, New Zealand.

HADROPHYOUS Fenton and Fenton, 1939. Hadrophycus immants Fenton and Fenton, 1939 , p. 92 , pl. 2, figs. 1-4; pl. 3, flgs. 1, 2 ; alga ; Nash formation, pre-Cambrian; Medicine Bow Mts., Wyo.

HAGENMULLERIA Munier-Chalmas, 1877. Hagenmulleria, Munter-Chalmas, 1877 , p. 817 ; nom. nud.

HAGIOPHYTON Corsin, 1948.

Hagiophyton sp: Corsin, 1948, p. 19, pls. 3,4 ; tree fern ; Westphallan $D$, CarbonIferous; mines domanlales de la Sarre et de la Lorratne, France.

HAI'INGERIA. Krasser, 1916.

Haitingeria krasseri (Schuster) Krasser, 1916, p. 336. For Cycadospadix krasseri Schuster, 1911a, p. 51, pl. 5, fig. 11; Upper Triassic (Keuper) ; Lunz, Austria. 
HAKEITES Saporta, 1861.

Hakeites deletus Saporta, in Heer, 1861, p. 137 ; leaf, Proteaceae ; Eocene; St. Zacharie, France. First spectes illustrated : $H$. major Saporta, 1867, p. 85, pl. 8 , fig. 5 .

HALIMEDITES Liburnau, 1902.

Halimedites saportae Liburnau, 1902, p. 712, pls. 1, 2; alga ; Tertiary ; near Salzburg, Austria.

HALIMEDOPSIS Massalongo, 1859.

Halimedopsis tuna Massalongo, in Massalongo and Scarabelli, 1859, p. 92. For Corallinites tuna Massalongo, $1856 \mathrm{~b}$, $\mathrm{p}$. 232, pl. 3, flg. 2 ; Eocene; Val Grobe, Italy.

HALISERIDES Schlmper, 1869.

Hallserides dechenianus ( $\mathrm{G}$ o e p p e $\mathrm{t}$ ) Schimper, 1869 (1869-74), p. 185, pl. 2, flg. 1 ; alga ?; Lower Devonian.

HALISERITES Sternberg, 1833.

Haliserites reichii Sternberg, 1833 (182038 ), p. 34, pl. 24, fig. 7 ; alga ; Eocene ; Freiberg, Saxony.

HALLEIA Fucinl, 1936.

Reference not seen; cited in Gothan, 1942 b, p. 125.

HALOCHARIS Miquel, 1853.

Halocharis longifolia Miquel, 1853, p. 49, pl. 5, figs. 4-6; leaf, monocotyledon?; Upper Cretaceous; Mt. St. Peter, Limburg, Belglum.

HALOCHLORIS Unger, 1842.

Halochloris cymodoceoides Unger, 1842 (1841-47), p. 55, pl. 18, figs. 1-3 ; incertae sedis; Eocene; Monte Bolca, Italy.

HALONIA Linđley and Hutton, 1833.

Halonia gracilis Lindley and Hutton, 1833 $(1831-37)$, p. 13 , pl. 86 ; Iycopod stem Impression; Upper Carboniferous ; Low Moor, Yorkshire, England.

HALOPHYTIS Sang, 1885.

Halophytis magnum Sang, 1885, p. 213 ; petrified alga, compared with stalk of Laminaria digitata; Upper Carbontferous ; Kirkealdy, Fife, Scotland.

HALOPOA Torell, 1869.

Halopoa imbricata Torell, 1869, p. 7 ; Cambrian ; Lugnas, Sweden.

HALOPTERIS Stur, 1883.

Halopteris typica Stur, 1883 , p. 660 , fig. 8 , fertlle fern pinnules; Upper Carbonif erous; Schlatzlar, Bohemia.

HALORAGIOARYA Reid and Chandler, 1933.

Haloragicarya quadrilocularis Reld and Chandler, 1933, p. 413, pl. 21, fig. 25 ; fruit, Halorigaceae; London Clay, Eocene; Minster, Kent, England.
HALYMENITES Sternberg, 1833.

Halymenites 8chitzleinii Sternberg, 1833 (1820-38), p. 30, pl. 5, fig. 1 ; alga? ; Jurassic ; Solenhofen, Bavaria.

HALYSERITES Sternberg, 1833.

Halyserites reichii Sternberg, 1833 (182038 ), p. 34, pl. 24, fig. 7 ; alga ?; Eocene ; Schoena near Freiberg, Saxony.

HALYSIS Hoeg, 1933.

Halysis moniliformis Hoeg, 1933, p. 86, pl. 7, figs. 1-3 ; alga?; Ordovician ; Vestre Katugleas, Holandet, Norway.

HAMAMELIDANTHIUM Conwentz, 1886.

Hamamelidanthium succineum Conwentz. 1886 , p. 93 , pl. 9 , figs. $26-29$; flower, in amber, Hamamelidaceae; Tertiary ; West Prussia.

HAMAMELIDOXYLON Lignier, 1907.

Hamamelidoxylon renaulti Lignier, 1907, p. 301 ; pl. 19, fig. 44 ; pl. 20, figs. 45-52 ; wood, dicotyledon; Cretaceous (Cenomanian) ; near Vimoutiers, France.

HAMA MELITES Saporta, 1865.

Hamamelites fothergilloides Saporta, 1865, p. 47 ; leaf, Hamamelidaceae ; Eocene ; Sézanne, France. See also Saporta, 1868 , p. 393, pl. 32, fig. 3.

HAPALOPHLOEA Pia, 1937.

Hapalophloea scissa Pia, 1937, p. 834; alga, Chaetangiaceae; Permian ; Guguk Bulat, Sumatra.

HAPALOPTERIS Stur, 1883.

Hapalopteris typica Stur, 1883, p. 660, fig. 8 ; Upper Carboniferous; Schatzlar, Bohemia.

HAPALOXYLON Renault, 1896.

Hapaloxylon rochei Renault, 1896a, p. 861, pl. 76, figs. 1-8; conlferous wood?; Carboniferous.

HAPLOCALAMUS Unger, 1856.

Haplocalamus thuringiacus Unger, 1856, p. 155 , pl. 1, flgs. $1-3$; pl. 4, fig. 12; stem, calamitean affnities?; Devonian Saalfeld, Thuringia. First citation : Unger, 1854 ; nom. nud.

HAPLOGRAPHITES Felix, 1894.

Haplographites cateniger Felix, $1894 a, b$. 274, pl. 19, figs, 5, 6 ; fungus mycelium and conidia?; Eocene ; Perekeschkul near Baku, Transcaucasia. Meschineli, 1898 , p. 81, erroneously attributes this genus to Berkley and Broome.

HAPLOPHRAGMIUM Reinsch, 1881.

Haplophragmium sp. Reinsch, 1881, p. 119, p1. 52a, figs. 1-3; Upper Carboniferous: Zwickall, Saxony,

HAPLOPLECTITES Reinsch, 1881.

Haploplectites sp. Reinsich, 1881, p. 67, pl. 16b, figs. 1-7; pl. 17, figs. 1-8; Upper Carboniferous ; Zwickau, Saxony. 
HAPLOPORELLA Gümbel, 1871. Haploporella eruca (Parker and Jones) Gümbel, 1871, p. 256, pl. D, flgs. 1a-e.

HAPLOSTIGMA Seward, 1932.

Haplostigma irregulare (Schwarz) Seward, 1932, p. 359, pls. 23, 24 ; lycopod? stem ; Bokkeveld series; Middle Devonian; Steytherville, Cape Province, South Africa.

HARLANIA Goeppert, 1851.

Harlania hallit Goeppert, 1851, p. 189. See also Goeppert, 1852 b, p. 98 , pl. 41 , fig. 4.

HARRINGTONIA Frenguelli, 1942.

Harringtonia argentinica (Arber) Frenguelli, 1942, p. 265, pl. 1, figs. 1-3 ; foliage, cycadophyte?; Triassic ; Argentina.

HARTZIA Harris, 1935.

Hartzia tenuis Harris, 1935, p. 42, fg. 20 ; ginkgophyte leaf; Lepidopteris zone, Rhaetic; Scoresby Sound, east Greenland.

HASPIA Kräusel and Weyland, 1929.

Haspia devonica Kräusel and Weyland, 1929, p. 342, pl. 13, figs. 3,4 ; Devonian; near Düsseldorf, Germany.

HASTIMIMA David White, 1908.

Hastimima whitei David White, 1908, p. 589 , pl. 10, figs. $1-4$; pl. 11, figs. $1-10$; "Permo-Carboniferous"; northeast of Minas, Santa Catharina, Brazil. Name cited earlier in White, I. C., 1906, p. 379 ; nom. nud.

HAUERA Unger, 1845.

Hauera americana Unger, 1845, p. 228; wood; Tertiary ; Antigua Island, West Indies. First? illustrated species: $\boldsymbol{H}$. bornensis Engelhardt, 1870, p. 49, pl. 15, figs. 10-13.

HAUSMANNIA Dunker, 1846.

Hausmannia dichotoma Dunker, 1846, p. 12 , pl. 5 , fig. 1 ; pl. 6 , fig. 12 ; incertae sedis ; Wealden ; near Buckenburg, Hannover, Germany.

HAWLEA Corda, 1845.

Hawlea pulcherrima Corda, 1845, p. 90, pl. 57, figs. 7,8 ; fern foliage with partly preserved sporangia; Upper Carboniferous ; Bohemia.

HAYDENIA Seward, 1912.

Haydenia thrysopteroides Seward, 1912, p. 14 , pl. 2 , figs. 26,29 ; fertile fern folfage; Cyatheaceae?; Jurassic ; Ishpushta, Afghanistan.

HEDEIA Cookson, 1935.

Hedeia corymbosa Cookson, 1935, p. 135, pl. 2, figs. 25-33 ; Psilophytales; Silurian; Mount Pleasant, Alexandra, Victoria, Australia.
HEDERAEPHYLLUM Fontaine, 1889.

Hederaephyllum crenulatum Fontaine, 1889 , p. 324 , pl. 162 , fig. 3 ; leaf, compared with Hedera helix; Potomac group, Lower Cretaceous; near Brooke, Va.

HEDEROIDITES Robert Potonie, 1950.

Hederoidites megagertrudae Robert Potonie, in Potonie, Robert, Thomson, Paul W., and Thlergart, Friedrich, 1950, p. 61 , pl. 3 , fig. 54 ; pollen, compared with Hedera; upper Pllocene; Chatt-Aquitan, Germany.

HEDEROPHYLLUM Velenovsky, 1889.

Hederophyllum primordiale (Saporta) Velenovsky, 1889, p. 50. For Hedera primordialis Saporta, 1879 , p. 200, fig. 29 ; Cretaceous (Cenomanian); Vyserovic, Bohemia.

HEDSTROMIA Rothpletz, 1913.

Hed8tromia halimedoides Rothpletz, 1913, p. 17, pl. 3 ; Upper Silurian; Lummelunds near Storbrut, Sweden.

HEDYCHIOPHYLLUM Principi, 1921.

Hedychiophyllum speciosum (Squinabol) Princip1, 1921a, p. 62 ; Oligocene ; Santa Guistina, Liguria, Italy.

HEERIA Stur, 1888.

Heeria lunzensis Stur, 1888a, p. 209 ; nom. nud.

HELENIA Zalessky, 1930.

Helenia inopinata Zalessky, 1930 a, p. 740 , pl. 73 , fig. 1 ; impression of decórticated stem; Carboniferous; Podossinino, Ourals, Russia.

HELENIELLA Zalessky, 1930.

Heleniella bellula Zalessky, 1930e, p. 663 ; Carboniferous; Donets Basin, Russia.

HELENIODENDRON Sze, 1936.

Geol. Soc. China Bull., 1936, v. 15, p. 113 (not seen, cited in Gothan, 1942b, p. 125).

HELICITES Crie, 1889

Helicites atrocarpa Crie, 1889a, p. 17; nom. nud.

\section{HELICODAEMON.}

A name suggested as being more appropriate for the problematical Daemonelix, in Claypole, 1895, p. 113.

HELICTOXYLON Felix, 1882.

Helictoxylon speciosum Felix, 1882a, p, 66 , pl. 1, fig. 1 ; sillclfied liana ; Tertiary ; Antigua, West Indies.

HELIOPHYCUS Miller and Dyer, 1878.

Heliophycus stelliforme Miller and Dyer, 1878 , p. 2 , pl. 3 , fig. 3 ; plant ? Cincinnatl group, Silurian; Cincinnatl, Ohio.

HELIOTROPITES Ettingshausen, 1868

Heliotropites reussi Ettingshausen, 1868a, p. 221, pl. 37, figs. 7-12; seeds and leaf, Asperifoliae; Miocene; Priesen, Bohemia. 
HELLEBORITES Heer, 1870.

Helleborites marginatus Heer, 1870, p. 63, pl. 7, figs. 17-21 ; fruit, Ranunculaceae?; Mlocene; Cape Staratschin, Spitzbergen.

HELLIA Unger, 1839.

Hellia pulchella Unger, 1839, p. 101 ; Miocene; Radoboj, Croatia.

HELMINTHOIDA Schafhautl, 1851.

Helminthoida irregularis Schafhautl, 1851, p. 142 , pl. 9, fig. 10 ; Eocene?; Bavaria.

HELMINTHOIDIOHNITES Fitch, 1850.

Helminthoidichnites tenuis Fitch, 1850, p. 868, fig. [unnumbered]; Cambrian; Middle Granville, N. Y.

HELMINTHOLITHUS Corda, 1842.

Helmintholithus antiquus Corda, 1842, p. 9 ; nom. nud.

HELMINTHOPSIS Heer, 1877.

Helminthopsis magna Heer, 1877a, p. 116, pl. 47, figs. 1, 2 ; marine alga ; Jurassic ; Switzerland.

HELOPHYTON Williamson, 1881.

Helophyton williamsonis (Cash and Hick) Williamson, 1881, p. 124, incertae sedis; Halifax bed, Upper Carboniferous. See also Williamson, 1883, p. 459 .

IIELVIENSIS Lima, 1896.

Helviensis delgadoi Lima, 1896, p. 94, pls. 1-4; Lower Silurian; near Elvas, Portugal.

HEMIONITITES Saporta, 1865.

Hemionitites scolopendrioides Saporta, 1865 , p. 37 , pl. 2 , fig. 5 ; pl. 5, fig. 5a ; fern pinnule; Miocene; Armissan, France.

HEMIPHOENICITES Visiani, 1864.

Hemiphoenicites dantesiana Visiani, 1864, p. 451 , pl. 18 ; palm leaf; Tertiary; Italy.

HEMITELITES Goeppert, 1836.

Hemitelites cibotioides Goeppert, 1836, p. 330 ; pecopterid follage; Carboniferous ; Saarbruck. For Pecopteris hemitelioides Brongniart, 1828-38, p. 314; pl. 108, figs. 1, 2.

HEMITRAPA Miki, 1941.

Hemitrapa trapelloidea Miki, 1941, p. 289 , pl. 7 ; fruit, Hydrocaryaceae; lower Pliocene; central Hono, Japan.

HEPATICITES Walton, 1925.

Hepatioites kidsconi Walton, 1925a, p. 565 , pl. 13, figs. 1-4; leafy liverwort; Middle Coal Measures, Upper Carboniferous; Preesgweene Colliery, Preesgweéne, Shropshire, England.

IIERACLEITES Kinkelin, 1908.

Heracleites mobiusi Kinkelin, 1908, p. 248, pl. 32, fig. 14; Upper Pliocene; Klarbecken, near Nlederrad, Hesse.
HERMITELLA Munier-Chalmas, 1877.

Hermitella sp. Munier-Chalmas, 1877, p. 817 ; nom. nud.

HEROUVALINA Steinmann, 1899.

Herouvalina herouvalensis (Munier-Chalmas) Steinmann, 1899, p. 153 , figs. 20 , 21 ; siphonaceous alga; Eocene; Herouval, France.

HESPERIDOPHYLLUM Massalongo, 1858. Hesperidophyllum senogallensis Massalongo, 1858a, p. 87 , pl. 28 ; fig. 13 ; leaf, dicotyledon; $\mathrm{M}$ i o c e $\mathrm{n}$ e ; Sinigaglia. Name first cited in Massalongo, 1857a, p. 777 ; nom. nud.

HETERANGIUM Corda, 1845.

Heterangium paradoxum Corda, 1845, p. 22 , pl. 16 ; pteridosperm stem ; Carboniferous ; Radnitz, Bohemia.

HETEROCALYX Saporta, 1873.

Heterocalyx ungeri Saporta, 1873a, p. 111, pl. 16, figs. 19-26; calyx, Anacardiaceae ; Tertiary ; France.

HETEROCLADISCOS Ettingshausen, 1887.

Heterocladiscos thujoides Ettingshausen, 1887 a, p. 90 , pl. 8 , figs. 5-7 ; foliage shoot, Cupressineae; Eocene; Vegetable Creek, near Emmaville, New South Wales.

HETEROFILICITES E. W. Berry, 1905.

Heterofilicites ancep E. W. Berry, 1905, p. 154 , pl. 26 ; fertile fern frond fragments ; Cliffwood clays, Cretaceous ; Kinkora, N. J.

HETEROLEPIS E. W. Berry, 1914.

Heterolepis cretaceus E. W. Berry, 1914a, p. 27 , pl. 3 , fig. 3 ; cone scale, cycad or conifer?; Black Creek formation, Upper Cretaceous; Rocky Point, Sumter County, s. C.

HETEROPTERIS Henry Potonte, 1893.

See Potonie, Henry, 1893b, p. 44, 45 ; a new name intended for Sphenopteris essinghii Andrä, 1866 (1865-69), p. 20, pl. 7, figs. 2, 3 ; Upper Carboniferous; Eschweiler, Srarbrucken, R h e n is h Prussia.

HETEROSPORITES Renault, 1901.

Heterosporites mischotheca Renault, 1901a, p. 208 ; nom. nud.

HETEROSPORITES Kuntze, 1904.

Heterosporites Kuntze, in Post and Kuntze, 1904, p. 278.

HETEROTHEOA Benson, 1922

Heterotheca grievii Benson, 1922, p. 122, pls. 4, 5 ; microsporangiate organ attributed to Heterangium; Calciferous Sandstone series, Lower Carboniferous ; Pettycur, Scotland.

HETEROXYLON Hartig, 1848.

Heteroxylon seyferti Hartig, 1848a, p. 169 ; wood ; Tertlary; Germany. 
IIEXAGONARIA Deecke, 1901.

Hexagonaria senonica Deecke, 1901, p. 473, flgs. 1, 2; alga?; Upper Cretaceous (Senonian) ; Rügen, Prussia.

HEXAGONOCARPOS Renault, 1890.

Hexagonocarpus crassus Renault, in Renault and Zeller, 1890, p. 649 , pl. 72, figs. 53-55; seed; Upper Carbontferous ; Commentry, France.

HEXAPTEROCARPUS Carpentier, 1920.

Hexapterocarpus sp. Carpentier, 1920, p. 118 , pl. 1 , fig. 9 ; pl. 2 , flg. 7 ; winged seeds ; Carboniferous (Westphalien); Bassin du Pas-de-Calals, France.

HEXAPTEROSPERMUM Brongniart, 1874.

Hexapterospermum stenopterum Brongniart, 1874, p. 254, pl. 22, flgs. 12, 13 ; sillicified seed ; Carboniferous ; St.Etienne, France.

HIBISCOXYLON Krïusel, 1939.

Bayer. Akad. Wiss., Math.-naturwiss. Abh., 1939, Neue Folge, Band 47, p. 73; Malvaceae; Upper Cretaceous (not seen). See Gothan, 1942b, p. 126.

HICKLINGIA Kidston and Lang, 1923.

Hicklingia edroardi Kidston and Lang, $1023 a$, p. 407 , pl. 53 ; psilophyte ; mid. dle Old Red. Sandstone, Devontan; Hill of Forss, Waas, Caithness, Scotland.

HICOROIDES Perkins, 1904.

Hicoroides angulata Perkins, 1904, p. 183, pl. 76, figs. 28, 32, 33 ; fruit ; Tertiary ; Brandon, Vt.

HIERACIT'ES Saporta, 1861.

Hieracites salyorum Saporta, in Heer, 1861 , p. 146 ; leaf, Chicoraceae ; Eocene ; Aix, Provence, France. See also Saporta, 1862 , p. 262 , pl. 11, fig. 1.

HIEROGAMMA.

Mistake for Hierogramma, in Read, 1936, p. 223.

HIEROGRAMMA Unger, 1856.

Hierogramma mysticum Unger, 1856, p. 172, pl. 8; flgs. 5-10 ; regarded as Identical with Cladoxylon by Bertrand; Upper Devonian; Saalfeld, Thuringia. See also Seward, 1917, p. 200 ; and Posthumus, 1931.

HIGHTEA Bowerbank, 1840.

Hightea elliptica Bowerbank, 1840, p. 32, pl. 8, figs. 7-9 ; frult, Malvaceae ? London Clay, Eocene ; Sheppey, Kent, England.

HILDESHEIMIA Florin, 1936.

Hildesheimia salfeldi (Lipps) Florin, $1936 \mathrm{~b}$, p. 37, pl. 6, flg. 5 ; ginkgophyte ; Cretaceous; Hildsheim, Germany.

HIMANTHALIOPSIS Zalessky, 1915.

Himanthaliopsis sniatkovi Zalessky, 1915, p. 47 , pl. 2 , fig. 5 ; pl. '5, figs. 5-7; 9 ; pl. 12, figs. 5-8; Carbontferous ; Russta.
HIMANTHALITES Fischer-Ooster, 1858.

Himanthalites taeniatus (Kurr) FischerOoster, 1858, p. 54, pl. 3, fig. 4 ; alga? Lower Jurassic (Lias) ; Fallbrach near Blumenstein, Switzerland.

HIMANTITES Meschinelli, 1892.

Himantites alopecurus (Debey and Ettingshausen) Meschinelli, in Saccardo, 1892, p. 801. See also Meschinelli, 1898, p. 95 , pl. 26, figs. 7,8 .

HIMANTOPHYTON Matthew, 1913.

Himantophyton castorense Matthew, 1913, p. 87, pl. 1, psilophyte?; Silurian; New Brunswich, Canada.

HIPPOCRATEITES Kuntze, 1904.

Hippocrateites Kuntze, in Post and Kuntze 1904, p. 282.

HIPPOCRATEOXYLON Hermann Hofmann, 1884.

Hippocrateoxylon favanicum Hermann Hofmann, 1884b, p. 28 ; Tertiary; near: Indramaju, Java. See also Hofmann, 1884.

HIPPODOPHYCUS Hall and Whitfleld, 1872.

Hippodophycus cowlesi Hall and Whit field, 1872, p. 204 ; Devonlan (Chemung); Salamanca, Cattaraugus County, N. Y.

HIPPURIDELLA Edwards, 1932.

Hippuridella stacheana Edwards, 1932, p. 213 , pl. 10 , figs. 1,2 ; compared with Hip $\boldsymbol{p}$ urus (Hippuridaceae) ; Lower Eocene; Gorge of the Folba, Plsino, Central Istria. For Astrocharas Stache, 1872a, p. 316; Astrochara liburnica Stache, 1880, p. 201 ; and Hipuridella Stache, 1889 , p. 87 ; all nom. nud.

HIPPURITES Lindley and Hutton, 1834.

Hippurites gigantea Lindley and Hutton, 1834 (1831-37), p. 87, pl. 114, calamitean stem impression; Upper Carbonlferous; Jarrow Colliery, near Newcastle-upon-Tyne, England.

HIPURIDELLA Stache, 1889.

Hipuridella sp. Stache, 1889 , p: 87 ; nom. nud. See Hippuridella, Edwards.

HIRAEOCARPUM Lakowitz, 1895.

Hiraeocarpum parvulum Lakowltz, 1895, p. 276, pl. 9, fig. 16; Oligocene; Brunstatt, Alsace-Lorraine.

HIRMERIA Fuclni, 1936

Reference not seen ; cited in Gothan, 1942b, p. 126.

HIRMERIELLA Hörhammer, 1933.

Hirmeriella rhatoliassica Hörhammer, 1933 , p. 29 , pls. 5-7 ; seed cone, Conlferales; Rhaetlc; France.

HISINGERA Miquel, 1842.

Hisingera mantellii Mlquel, 1842, p. 62. For Cycadites brongniarti Mantell, 1833, p. 338, fig. 
HOLCODENDRON Quenstedt, 1867.

Holcodendron sp. Quenstedt, 1867, p. 867, pl. 82, fig. 4 ; Lower Keuper.

HOLCOSPERMUM Nathorst, 1914.

Holcospermum dubium Nathorst, 1914, p. 28, pl. 15 , figs. 53,54 ; seed ; Carboniferous ; Spitzbergen.

HOLEOSPERMUM.

Mistake for Holcospermum, in Davies, 1929 , p. 117.

HOLOPLEURA Caspary, 1856.

Holopleura victoria Caspary, 1856, p. 216, pl. 12, figs. 10-22 ; seeds, Nymphaceae; Miocene; Dorneim, Woelfersheim, Hesse.

HOLOSPORELLA PIa, 1930.

Holosporella siamensis Pia, 1930, p. 177, pl. 4, figs. 1-6; alga, Dasycladaceae; Kamawkala limestone, Upper Triassic; Thaungyin River, frontier of Burma and Siam, north of Myawadi.

HOLSTIA Hagstrom, 1906.

Holstia splendens Hagstrom, 1906, p. 90, pl. 3; Pleistocene; Toppeladugard, Sweden.

HOMOXYLON Hartig, 1848.

Homoxylon' blasii Hartig, 1848c, p. 188 ; wood; Upper Cretaceous; Wetterau, Hesse.

HOMOXYLON Sahni, 1932.

Homoxylon rajmahalense Sahnl, 1932a, p. 1, pls. 1, 2 ; wood, compared with modern homoxylous Magnoliaceae; Jurassic; Rajmahal Hills, Behar, India.

HOOLEYA Reid and Chandler, 1926.

Hooleya hermis (Unger) Reid and Chandler, 1926, p. 93, pl. 6, figs. 7-9 ; fruit, Betulaceae; Oligocene; Isle of Wight, England.

HORMOSPORITES Gruss, 1927.

Hormosporites devonicus Gruss, 1927, p. 367, fig. 810 ; alga, Cyanophyceae?; Devonian; Spitzbergen. See also Gruss, 1928b, p. 504, pl. 41, flgs. 21, 22.

HORNEA Kidston and Lang, 1920.

Hornea lignieri Kidston and Lang, 1920a, p. 611, pls. 4-10; petrifled plant, Psilophytales; Old Red Sandstone, Devonian ; Muir of Rhynie, Aberdeenshire, Scotland. See Horneophyton.

HORNEOPHYTON Barghoorn and Darrah, 1938.

Horneophyton lignieri (Kidston and Lang) Barghoorn and Darrah, 1938, p. 142. For Hornea lignieri Kidston and Lang, 1920a, p. 611, pls. 4-10.

HOSTIMELLA.

See Hostinella.

\section{HOSTINELLA Barrande, 1882.}

Hostinella hostinensis Barrande, In Stur, 1882 , p. 352, pl. 3, figs. 1, 2 ; pl. 4 ; branched dichotomizing naked axis, pstlophyte; "Etage H-h," Silurian; Hostin, Srbsko, Bohemia.
HOVENIPHYLLUM Nathorst, 1888.

Hoveniphyllum thunbergi Nathorst, 1888 , p. 232 , pl. 30 , fig. 6 ; leaf, compared with Hovenia dulcis; Plfocene; Yokohama, Kuragigori, Musashi province, Japan.

HSIANGCHIPHYLLUM Sze, 1949.

Hsiangchiphyllum trinerve Sze, 1949, p. 28, pl. 7, fig. 6; pl. 8, fig. 1; Mesozole; Hsiangchi, China.

HUMILIS Roualt, 1850.

Humilis legalli Roualt, 1850, p. 739 ; Silurian; Guichen, Brittany.

HUTTONIA Sternberg, 1837.

Huttonia specata Sternberg, 1837 (1820$38)$, p. 69 , pl. 1 ; Upper Carboniferous ; Radnitz, Bohemia.

HYDATICA Artis, 1825.

Hydatica prostrata Artis, 1825, p. 1, pl. 1 ; Carboniferous; near Wentworth, Yorkshire, Hngland.

HYDNITES Meschinelli, 1892.

Hydnites argillae (Ludwig) Meschinell, in Saccardo, 1892, p. 748. See also Meschinelli, 1898, p. 8, pl. 5, figs. 5-10 ; fungus, Hymenomycete.

HYDRANGEIPHYLLUM Dusen, 1899.

Hydrangeiphyllum affine Dusen, 1899, p. 102 ; leaf, compared with Hydrangea scandens Poeppig; Oligocene; Barancas de Carmen.Sylva, Chile.

RYDROCHARITES Weber, 1855.

Hydrocharites obcordatus Weber, 1855, p. 129 , pl. 30 , fig. 2 ; leaf, Hydrocharideae ; Miocene; Rott, Rhenish Prussia.

HYDROCOTYLOPHYLLUM Teixeira, 1947. Hydrocotylophyllum lusitanicum Teixeira, 1947, p. 11, pl. 1, fig. 5 ; leaf, compared with Hydrocotyle asiatica; Wealden ; Portugal.

HYDROCYTIUM Matthew, 1889.

Hydrocytium silicula Matthew, 1889, p. 146, pl. 6, fig. 2 ; incertae sedis; Cambrian; Nova Scotia.

HYDRODICTYOLITES Elovski, 1930.

Hydrodictyolites carbonis Elovski, 1930, p. 35 , pl. 1, fig. 4 ; Moshchny coal seam, Chernogorski mines, Minusinsk Basin, Siberia.

BYDRODICTYOPSIS Massalongo, 1858.

Hydrodictyopsis prisca Massalongo, 1858a, p. 5. See also Massalongo, 1859, p. 93, pi. 2.

HYDROPTERANGIOM Halle, 1910.

Hydropterangium marsilioides Halle, 1910, p. 11, pl. 2, figs. 1-14; pl. 3, figs. 12-15; sporocarps? of a water fern; lower Rhaetic; Bjuf and Hyllinge, Sweden,

HYENIA Nathorst, 1915.

Hyenia sphenophylloides Nathorst, 1915, p. 22 , pl. 1, figs. 1-5; pl. 2, fig. 1 ; pl. 4, figs. 1-3; articulate; Deronian; Norway. 
HYGROHYPNIDIUM Kirchheimer, 1936. Hygrohypnidium ludwigi K1rchbelmer, 1936d, p. 340, figs. 1-4 ; Tertiary ; Salzhausen, Germany.

HYLOMITES.

Error for Xylomites, in Geinitz, 1925, p. 337

HYMENAEOPHYLLUM Velenovsky, 1889. Hymenaeophyllum primigenium (Saporta) Velenovsky, 1889, p. 51.

AYMENOPHYLLEA C. E. Welss, 1869. Hymenophyllea subalata (Geinitz) C. D. Weiss, 1869, p. 57. For Hymenophyllites alatus Geinitz, part, see Geinitz, H. B., 1855 , p. 18 , pl. 24 , fig. 15 ; pl. 25 , fig. 1.

I YMENOPHYLLITES Goeppert, 1836.

Hymenophyllites quercifolius Goeppert, 1836 , p. 252 , pl. 14 , figs. 1,2 ; fernlike foliage; Carboniferous ; Silesia.

HYMENOPTERIS Stokes and Webb, 1824. Hymenopteris psilotoides Stokes and Webb, 1824, p. 424, pl. 46, fig. 7 ; pl. 47, fig. 2 ; Wealden ; Tilgate Forest, Sussex, England .

HYMENOPTERITES Stokes and Webb, 1824.

Hymenopterites Stokes and Webb, 1824, p. 426 ; nom. nud.

HYMENOTHECA Henry Potonie, 1890.

Hymenotheca beyschlagi Henry Potonie, 1890 , p. 23 , pl. 3 ; pteridosperm ? microsporangiate organ; Upper Carboniferous; Saarbrucken.

HYMENOZONOTRILETES Naumova, 1937.

Hymenozonotriletes triangularis Mehta, 1944 , p. 129 , pl. 1, fig. 1 ; Paleozole; Rewa, India.

HYOSERITES Ettingshausen, 1868.

Hyoserites 8chultzii Ettingshausen, 1868a, p. 206, pl. 35 , fig. 27 ; achene, Compositae; Miocene; Priesen, Bohemia.

HYPHANTAENIA Ferdinand Roemer, 1880.

Hyphantaenia chemungensis (Vanuxem), Ferdinand Roemer, 1880 , p. 126. For Uphantenia chemungensis Vanuxem, 1842 , p. 183 , flg. 50.

HYPHITES Reinsch, 1881.

Hyphites sp. Reinsch, 1881, p. 36, pl. 7b, figs. 5, 8 ; Lower Silurian; Illinols.

HYPHOPLASMIUM Reinsch, 1881.

Hyphoplasmium sp. Reinsch, 1881, p. 40 ; pl. 7b, fig. 8 ; pl. 8a, figs. 1-8; Upper Carboniferous; Mittelbexbach, Bavaria.

HYPHOPTERIS Schimper; 1869.

Hyphopteris radiata Schimper, 1869, p. 365.

HYPNITES Ettingshausen, 1853.

Hypnites haeringianus Ettingshausen, 1853 , p. 27 , pl. 4, fig. 12 ; moss ; Hocene ; Haering, Tirol, Austria.
HYPOOHNITES Meschinelli, 1898.

Hypochnites sp. (Conwentz) Meschinell, 1898 , p. 8 , pl. 6 , figs. $2-5$; fungus, Hymenomycetaceae.

HYPOGLOSSIDIUM Heer, 1874.

Hypoglossidium antiquum Heer, 1874a, p. 129 , pl. 38 , fig. 14 ; leaf, monocotylodon ; Cretaceous, Greenland.

HYPSILOOARPUS Grand'Eury, 1890.

Hypsilocarpus amygdalaeformis (Goeppert and Berger) Grand'Eury, 1880, p. 328, pl. 6, fig. 7 ; seed, Cordaitales?; Upper Carboniferous; Gard, France.

HYRCANOPTERIS Kristofowitsch and Prynada, 1933.

United Geol. Prosp. Service USSR Trans., 1933, no. 336, p. 10 ; Filices; Upper Triassic (not seen). See Gothan, 1942b, p. 126.

HYSTERITES Goeppert, 1846.

Hysterites opegraphoides Goeppert, 1846 (1841-46), p. 145, pl. 14, figs. 1, 2. See Goeppert, 1836, p. xxili ; nom. nud. Meschinelli, 1892, p. 772, erroneously attributes this to Tode.

HYTHIA Stopes, 1915.

Hythia elgari Stopes, 1915 , p. 278, pls. 29 , 30 ; wood, incertae sedis; Lower Cretaceous (Aptian); Kent, England.

\section{I}

IOACINIOARYA Reid and Chandler, 1933. Icacinicarya platycarpa Reid and Chandler, 1933 , p. 345 , pl. 16, flgs. 11-18; endocarp, Icacinaceae; London Clay, Eocene; Sheppey, Kent, England.

IDELOPTERIS Zalessky, 1929.

Idelopteris elegans Zalessky, 1929d, p. 721, fig. 1 ; compared with Prygmophyllum; upper Permian; Siberia.

IDIOPHYLLUM Lesquereux, 1880.

Idiophyllum rotundifolium Lesquereux, 1880 , p. 160 , pl. 23, flg. 11; Carbondale formation, Pennsylvanian; Mazon Creek, Ill.

IEGOSIGOPTERIS Zalessky, 1935.

Iegosigopteris yavorskii Zalessky, 1935a, p. 752 , pls. $1-3$; petrified stem, Osmundaceae ; Russia.

ILLIOIPHYLLUM Velenovsky, 1889.

Illiciphyllum deletum Velenovsky, 1889, p. 54. For Illicium deletum Velenovsky, 1884, p. 51, pl. 3, fig. 5; Upper Cretaceous ; Lipenec, Bohemia.

ILLIOITES Mueller, 1877.

Illicites astrocarpa Mueller, 1877 (1877$79)$, p. 179 ; Pliocene; Gulgong, Australia. See also Mueller, 1879 (1877$79)$, p. 171, pl. 4, flgs. 3 , 4 . 


\section{ILLINIOCARPON Schopf, 1938.}

Illiniocarpon cadyi Schopf, $1938 \mathrm{~b}$, p. 144, pl. 1, figs. 1-3; pl. 2, figs. 11-13, 15; lycopod seedlike organ; coal No. 6, Pennsylvanian; Nashville, Washington County, Ill.

ILLINITES Kosanke, 1950.

Illinites unicus Kosanke, 1950, p. 51, pl. 1, figs. 3, 4 ; spore ; Pennsylvanian ; 10 inch coal bed exposed in Coffee Creek, Wabash County, Ill.

ILSAEPHYTUM C. E. Weiss, 1885.

Ilsaephytum kayseri C. E. Weiss, 1885a, p. 178 , pl. 6, figs. 1, 2 . See also Posthumus, 1931.

IMPARIPTERIS Gothan, 1941.

Palaont. Zeitschr., 1941, Band 22, p. 427 (not seen, cited in Gothan, 1942b, p. 127).

INOOLARIA Herzer, 1893.

Incolaria securiformis Herzer, $1893 \mathrm{a}, \mathrm{p}$. 365 , pl. 9 ; fungus?; Carboniferous ; Tuscarawas County, Ohio.

INDOPOLIA Pla, 1936.

Indopolia satyavanti $\mathrm{Pia}$, in Rao and Pia, 1936 , p. 20 ; pl. 1 , flgs. $1,5-13$; pl. 2 , fig. 4 ; alga, Dasycladaceae ; Miniyur group, uppermost Cretaceous; Trichinopoly district, southern India.

INDÓSTROBUS Sahni, 1931.

Indostrobus bifldolepis Sahni, 1931, p. 80, pl. 13, figs. 54-66 ; petrified cones, allied to Pityostrobus; probably uppermost Cretaceous ; probably from Deccan area, India.

INDOTHECA Sitholey, 1943.

Indotheca 8akesarensis Sitholey, in Sahni, Birbal, and Sitholey, R. V., 1943, p. 174, pl. 8, figs. 27, 28; Triassic ; three-quarters of a mile east of Sari Village, Salt Ridge, India.

INGOPHYLLUM Velenovsky, 1889.

Ingophyllum latifolium Velenovsky, 1889, p. 54. For Inga latifolia Velenovsky, 1884 , p. 55 , pl. 20, flgs. 6,7 ; Upper Cretaceous; Vyserovic, Bohemia.

INIOPTERIS Zalessky, 1934.

Iniopteris sibirica Zalessky, 1934c, p. 760, fig. 20 ; fern foliage; Permian; Kuznets, Russia.

INOLEPIS Heer, 1874.

Inolepis imbricata Heer, $1874 \mathrm{a}, \mathrm{p} .72$, pl. 16, figs. 12-16; pl. 23, figs. 6-8; coniferous twigs; Cretaceous; Kome, Avkrusak, Greenland.

IRIARTITES E. W. Berry, 1919.

Iriartites tumbezensis $\mathrm{E}$. W. Berry, 1919b, p. 285, pl. 14 : leaf, Arecaceae ; Miocene ; Tumbez, Peru.

IRIDINIUM Wessel, 1855.

Iridinium priscum Wessel, in Wessel and Weber, 1855 , p. $129-130$; pl. 20 , flg. 7 ; Irislike leaf; Miocene; Rott, Rhenish Prussia.
IRIDIUM Heer, 1866.

Iridium groenlandicum Heer, 1866, p. 275 ; leaf fragment. referred to Iridaceae; Miocene; Atanekerdluk, Greenland. See also Heer, 1868, p. 97, pl. 3, figs. 10, 11.

IRIDOPTERIS Arnold, 1940.

Iridopteris eriensis Arnold, 1940, p. 57, figs. 1, 5 ; Iridopteridineae, intermediate between Psilophytales and ferns; Tully limestone, Middle Devonian; Erie County, N. Y.

IRITES Lesquereux, 1587.

Irites alaskana Lesquereux, 1887, p. 36 ; leaves, Iridaceae?; Lower Cretaceous; Cape Lisbourne, Alaska. First? illustrated species: Irites grandifolium Principi, 1921a, p. 60, pl. 3, fig. 1.

IRRA WADIOXYLON Gupta, 1936.

Irrawadioxylon burmense (Holden) Gupta, 1936 , p. 305 . For Dipterocarpoxylon burmense Holden, 1916, p. 271, pl. 29 ; Miocene (Irrawadian); Burma.

ISATIDES Saporta, 1889.

Isatides microcarpa Saporta, 1889, p. 87, pl. 9, fig. 3 ; fruit, Cruciferae ; Eocene ; Aix, Provence, France.

ISIOLOPTERIS Zalessky, 1930.

Isiolopteris serrata Zalessky, 1930f, p. 915, fig. 2 ; fernlike follage; Permian; Pechora River basin, $4 \mathrm{~km}$ below Ost-Voy, Russia. See Zalessky, 1934b.

ISOETITES Muenster, 1842.

Isoetites crociformis Muenster, 1842 (1839-43), p. 107, pl. 4, fig. 4 ; Jurassic ; Daiting near Manheim, Bavaria.

ISOETOIDES Wethered, 1884.

Isoetoides Wethered, 1884, p. 300 ; a generic name proposed for spores, compared with Isoetes, found in the "Carmock Chase" coal ; the name is "suggested * * pending further investigations."

ISOETOPSIS Saporta, 1888.

I80etopsis subaphylla Saporta, 1888, p. 28 ; pl. 2, figs. 16-20; I8oetes-like sporophyll with spores; Eocene; Alx, Provence, France.

ISONANDROPHYLLUM Geyler, 1887.

Isonandrophyllum sp. Geyler, 1887a, p. 498 , pl. 33, flg. 9 ; leaf fragment, Sapotaceae ; Eocene ; Labuan, Borneo.

ISSELIA Squinabol, 1891.

Isselia primaeva Squinabol, 1891a, p. 779, pl. 16, fig, 5 ; leaf fragment, monotyledon; lower Miocene; Ste.-Justine, Sassello, France.

ITIERIA Saporta, 1872.

Itieria brongniarti Saporta, 1872a-73, p. 122, pl. 4; alga? Jurassic; Orbagnoux, France.

IUGLANDOXYLON.

Iuglandoxylon wichmanni Hofmann, $1884 \mathrm{~b}$, p. 36 ; probably mistake for Juglandoxylon. 
IVANOVIA Khvorova, 1946.

Ivanovia tenuissima Khvorova, 1946, p. 737, 2 figs ; alga ; middle Carboniferous ; Moscow Basin, USSR.

IXOROPHYLLUM Geyler, 1887.

Ixorophyllum anceps Geyler, 1887a p. 495, pl. 35, figs. 1, 2; leaf fragment, Rublaceae ; Eocene ; Labuan, Borneo.

IXOSTROBUS Raciborski 1891.

Ixostrobus siemiradzkii Raciborsk1, 1891a, p. 378. For Taxites siemiradzkit Raclborski, 1891b, p. 315 , pl. 5, flg. 7 ; cycadophyte microsporangiate cone?; Rhaetic, Poland. See discussion by Harris, 1935, p. 146-147.

\section{J}

JANENSCHIA Gothan, 1927.

Janenschia obscura Gothan, 1927b, p. 146, pl. 18 , figs. $1-5$; pl. 19 , figs. 11,12 ; "Permo-Carboniferous"; Mkumbl, East Africa.

JEANPAULIA Unger, 1845.

Jeanpaulia dichotoma (C. F. W. Braun) Unger, 1845 (1841-47), p. ll. For Baiera dichotoma C. F. W. Braun in Münster 1843 (1839-43) ; Lower Jurassic (Lias); Hinterholz, Austria. Apparently first lllustrated species is Jeanpaulia munsteriana (Presl) Schenk, 1865-67, pl. 11, flgs. 1-13.

JEJOSIGOPTERIS Zalessky, 1937.

Acad. sci. U. S. S. R. Bull. 1937, seer. 7॰, p. 747 (not seen ; cited in Gothan, 1942b, p. 127).

JENKINSELLA Reid and Chandler, 1933.

Jenkinsella apocynoides Reld and Chandler, 1933, p. 481, pl. 28, figs. 1-5 ; fruit, Apocynaceae or Asclepiadaceae ; London Clay, Eocene; Herne Bay, Kent, England.

JIDOPTERIS Koldzumt, 1936.

Jidopteris manchurica (Kawasak1) Koidzumi, 1936, p. 142. For Pecopteridium manchuricum- Kawasak1, 1931 (1927$31)$; pl. 34 , flg. 73 ; intermediate between Pecopteris and Callipteridium; Jido series, Lower Permian; Tayaokou coal mine, Manchuria.

JIRUSIA Bayer, 1914.

Jirusia bohemica Bayer, 1914, p. 23, figs. 12,13 ; cycadophyte leaves.

JODOTELLA Morellet and Morellet, 1913.

Jodotella veslensis Morellet and Morellet, 1913 , p. 29 , pl. 3 , fig. 12 ; alga, Bornetellées, Eocene (Thanetien), Chalonssur-Vesles, France.

JOHANNOPHYTON Matthew, 1910.

Johannophyton discrepans (Dawson) Matthew, 1910, p. 84, pl. 2, flgs. 7-9 ; pl. 3.

$296347-55-12$
JOHNSTONIA Walkom, 1925.

Johnstonia coriacea (Johnston) Walkom, 1925 , p. 79 , figs. 6-8 ; fernlike follage; Mesozoic ; Tasmanta.

JONGMANSIA Reld and Reld, 1915.

Jongmansia cypreaeformis Reid and Reld, 1915 , p. 95 , pl. 8, figs. 14-21; seeds, Anonaceae ; Pliocene; Reuver, Swalmen, Netherlands.

JORDANIA Goeppert and Fiedler, 1857.

Jordania bignonioides Goeppert and Fledler in Fiedler, 1857, p. 289, pl. 28, figs. $36,37,43,44$; seed compression ; Upper. Carboniferous; near Saarbruck, Rhenish Prussia.

JORDANIA Schenk, 1880.

Jordania ebenoides Schenk, 1880, p. 659 ; wood, dicotyledon; Upper Cretaceous ; Libyan Desert. See also Schenk, 1883a, p. 10, pl. 4, flgs. 13, 14 .

JUGLANDICARYA Reid and Chandler, 1933. Juglandicarya lubbocki Reid and Chandler; 1933 , p. 140, pl. 3, figs. 1-4 ; seed, Juglandaceae; Jondon Clay, Eocene ; Sheppey, Kent, England.

JUGLANDINIUMI Unger, 1845.

Juglandinium mediterraneum Unger, 1845, p. 241 ; Tertlary ; Hungary.

JUGLANDIPHYLLUM Nathorst, 1888.

Juglandiphyllum sp. Nathorst, 1888, p. 208, pl. 4, fig. 6 ; leaf, dicotyledon ; Tertiary ; Japan.

JUGLANDIPHYLLUM Fontaine, 1889.

Juglandiphyllum integrifolium Fontaine, 1889 , p. 315 , pl. 157 , flgs. 3, 5, 6 ; leaf compared with Persea and Quercus; Potomac group, Lower Cretaceous; White House Bluff, Va.

JUGLANDITES Sternberg, 1825.

Juglandites nuxtaurinensis (Brongniart) Sternberg, 1825 (1820-38), Tentamen, p. xl. For Juglans nuxtaurinensis Brongniart, 1822, p. 323 , pl. 6, fig. 6 ; Juglans-like endocarp; Mlocene ; Turln, Italy.

JUGLANDOXYLON Kraus, 1882.

Juglandoxylon mediterraneum Kraus, 1882 , p. 91 ; wood ; Miocene; Girgentl, Sicily.

JUGLANSOXYLON Falqui, 1906.

Juglansoxylon zuriensis Falqui, 1906, p. 26, pl. 1, flg. 2 ; lower Mlocene. Bee Edwards, 1931.

JUGLOXYLON Stopes and Fujil, 1910.

Jugloxylon hamaoanum Stopes and Fufll, 1910 , p. 62 , pl. 7 , fig. 48 ; wood, possible affinities with Juglans; Upper Cretaceous ; Hokkaido, Japan.

JUNGERMANNIOPSIS Howe and Hollick, 1922.

Jungermanniopsis cockerellii Howe and Hollick, 1922, p. 208, fig. 1 ; leafy llverwort ; Miocene ; Florissant, Colo. 
JUNGERMANNITES Goeppert, 1845.

Jungermannites neesianus Goeppert, in Berendt, 1845, p. 113, pl. 6, flgs. 34-37 ; liverwort?; Miocene ; Prussia.

JUNGHUHNITES Goeppert, 1854.

Junghuhnites javanicus Goeppert, 1854, p. 54, pl. 2, figs. 11-16; wood, incertae sedis ; Tertiary ; Java.

JUNIPERITES Brongniart, 1828.

Juniperites alienus (Sternberg) Brongniart, $1828 \mathrm{~b}$, p. 108 . For Thuites alienus Sternberg, $1825 \quad(1820-38)$, Tentamen, pl. 45, fig. 1.

JUNIPEROXYLON Houlbert, 1910.

Juniperoxylon turonense Houlbert, 1910, p. 73, pl. 4 ; coniferous wood; middle Eocene; Touraine, France.

JURANYIA Tuzson, 1908.

Juranyia hemiflabellata Tuzson, 1908, p. 1, pl. 1, figs. 1,2 ; pl. 2 , fig. 3 ; leaves, seeds, Palmaceae; Upper Cretaceous; Ruszkabanya, Krasso-Szoreny, Hungary. See also Tuzson, 1914, p. 248.

\section{$\mathbf{K}$}

KAIDAOARPUM Carruthers, 1868.

Kaidacarpum ooliticum Carruthers, 1868, p. 156 , pl. 9, figs. 1-6; described as cast of a fruit (Pandanaceae), later transferred to Araucarites (Seward, 1919, p. 256) ; Jurassic (Oolite) ; Moulton Park Quarries, Kingsthorpe, near Northampton, England.

KАIKOMAKO Hector, 1880.

Kaikomako penantioides Hector, 1880, p. 49 ; nom. nud.

KAIKORAIA W. R. B. Oliver, 1936.

Kaikoraia gracilis W. R. B. Oliver, 1936, p. 301 , fig. 21 ; leaf, Sapotaceae ; Pliocene; Kaikorai Valley, Otago, New Zealand.

KALINAIA Bayer, 1914.

Kaitinaia dekapetala Bayer, 1914, p. 51; Cretaceous; Vyserovic, Bohemia.

KALOXYLON Williamson, 1875.

Kaloxylon hookeri Williamson, 1875, p. 453 ; roots of Lyginopteris ; Upper Carboniferous; Oldham, England. See also Williamson, $1876 a$, p. 23, p1. 5, figs. 2327 ; pl. 4, fig. 29 ; pl. 6, figs. 28, 30-33 ; pl. 7 , figs. 34-38; and Seward, 1917, p. 67.

\section{KALYMMA Unger, 1856.}

Kalymma grandis Unger, 1856, p. 157 , pl. 1 , figs. 4-6; petiole of Calamopitys; Upper Devonian; Saalfeld, Thuringia. Name first clted in Unger, 1854 ; nom. nud. See also Posthumus, 1931.
KAMARASPERMUM Kern, 1946.

Kamaraspermum leeanum Kern, in Kern and Andrews, 1946, p. 296, pl. 19 ; petrified seed with air chamber, Cardiocarpales?; Des Moines group, Pennsylvanian; Urbandale coal mine, Des Moines, Iowa.

KANTIA Pia, 1912.

Kantia philosophi Pia, 1912, p. 45, pl. 4, figs. 17-21; alga, Siphoneae Verticillatae; Triassic; Austria?

KARRERIA (Munier-Chalmas) Morellet and Morellet, 1913.

Karreria zitteli Munier-Chalmas, in Morellet and Morellet, 1913, p. 11, figs. 13-24.

KARSTENIA Goeppert, 1836.

Karstenia omphalostigma Goeppert, 1836, p. 452 , pl. 33 , fig. 1 ; fern rhizome, compared with Polypodium aureum; Upper Carboniferous; Charlottenbrunn, Silesia.

KATADROMOPTERIS Hartung, 1940.

Katadromopteris boncevi Hartung, 1940, p. 101 , pl. 2 , figs. 1,2 ; pl. 3 , figs. $1-4$; fernlike foliage; Upper Cretaceous.

KATANGASIA Maslov, 1937.

Katangasia samoilovi Maslov, 1937a, p. 321, pl. 3, figs. 2, 4 ; rock-building alga ; Silurian ; Russia.

KAYEOXYLON Chowdhury and Tandan, 1949.

Kayeoxylon assamicum Chowdhury and Tandan, 1949 , p. 59, pls. 5, 6 ; petrífied wood, affinities with Kayea, Guttiferae; Upper Miocene; Thallangthu Nadi, A8sam, India.

KECKIA Glocker, 1841.

Keckia annulata Glocker, 1841, p. 319, pl. 4, figs. 1, 2 ; plant?; Cretaceous ; Capellenberge, near Kwassitz, Moravia.

KENTITES Bureau, 1896.

Kentites pratecinensis Bureau, 1896, p. 285, Tertiary ; Pratecini, Italy.

KERAIAPHYLLUM Frentzen, 1932.

Keraiaphyllum suevicum Frentzen, 1932 , p. 83 , figs. 2, 3 ; Rhaetic ; Swabia, Nürtingen, Germany.

KIDSTONIA Zetller, 1897.

Kidstonia heracleensis Zelller, 1897, p. 209, pl. 6, figs. 3, 4 ; fertlle fernlike follage, Osmundaceae or Schizaeaceae?; Upper Carboniferous; Zongouldak, Asia Minor.

KILTORKENSIA Thomas Johnson, 1917.

Kiltorkensia devonica Thomas Johnson, $1917, p$. 250, pl. 12, igs. 3-5; pl. 13, figs. 1-5; Incertae sedis; Upper Devonian; Kiltorcan, County Kllkenny, Ireland.

KINGTHIOPHYLLUM Crie, 1889.

Kingthiophyllum primaevum Crie, $1889 \mathrm{a}$ p. 89 ; nom. nud. 
KINNEYIA Walcott, 1914.

Kinneyia simulans Walcott, 1914, p. 107, pl. 11, fig. 3 ; alga ; Beltian series, Newland limestone, Algonkian; 8 miles west of White Sulphur Springs, Meagher County, Mont.

KIRCHNERIA Alexander Braun, 1854.

Kirchneria decurrens Alexander Braun, 1854 , p. 6, pl. 1 , figs. $1-3$; Triassic (Keuper); Eckersdorf, B avaria. Earlier citation : Braun, C. F. W., 1840, p. 97 ; nom. nud.

KIRKORIA. Zalessky, 1937.

Kirkoria multifida Zalessky, 1937b, p. 83, figs. 51, 52; ginkgophyte? foliage; Permian; above village of Matveyevo, Ourals, Russia.

KIRSTEA Kirchheimer, 1936.

Kirstea zinkeiseni (Gelnitz) Klrchhelmer, $1936 \mathrm{a}$, p. 86 , pl. 12 , flgs. $38 \mathrm{a}-\mathrm{f}$; seed, Magnoliaceae; Tertiary (Braunkohle); Altenburg, Germany.

KITAKAMIANIA Ishijima, 1943.

Kitakamiania eguchib Ishljima, 1843, p. 639, figs. 1, 2 ; alga ; Cretaceous; Japan.

KLIPPSTEINIA Unger, 1845.

Klippsteinia medullaris Unger, 1845 (1841-47), p. Ixxx11i. Illustrated in Unger, $1858 \mathrm{a}$, p. 12 , pl. 3, figs. 8-10; Miocene ; Thal, Germany.

KLOEDENIA Goeppert, 1839.

Kloedenia quercoides Goeppert, 1839, 1. 521, pl. 8b, flgs. 1, 3, 4; wood (placed in Quercinium by Edwards, 1931) ; Crètaceous; Silesia.

KLUKIA Raciborski, 1890.

Klukia exilis (Phillips) Raciborsk1, 1890, p. 6, pl. 1, figs. 17-19; fertlle follage, Schlzaeaceae ; Jurassic ; Yorkshire, Ingland.

KNIGHTIOPHYLLUM Ettingshausen, 1887.

Knightiophyllum primaevum Ettingshausen, $1887 \mathrm{~b}$, p. 185 , pl. 9 , fig. 12 ; leaf fragment, Proteaceae; Upper Cretaceous; New Zealand.

KNIGHTIOPHYLLUM E. W. Berry, 1916.

Knightiophyllum wilcoxianum E. W. Berry, 1916b, p. 208, pl. 35, figs. 1-3 ; leaf, Proteaceae; Lagrange formation, lower Eocene; Puryear, Henry County, Tenn.

KNIGHTITES Saporta, 1861.

Knightites 8alyorum Saporta, in Heer, 1861 , p. 145 ; leaf, Proteaceae ; Eocene ; Alx, Provence, France. See also Saporta, 1862, p. 254, pl. 9, fig. 1.

KNORRIA Sternberg, 1825.

Knorria imbricata Sternberg, 1825 (1820 38) Tentamen, p. xxxvil, pl. 27 ; partly decorticated arborescent lycopod stem; Carboniferous. Seward, 1910, p. 124 notes: "Although it is now a wellestablished fact that fossils bearing the name Knorria are imperfect lepidoden- drofd stems, the use of the term may be convenfently retained for descriptive purposes." In such a case a "type species" can have little significance, for the - size, anatomical detalls, and degree of decortication will result in correspondingly different fossils.

KNORRIPTERIS Henry Potonie, 1899.

Knorripteris mariana Henry Potonle, 1890, p. 68 , fig. 35 ; petrified fern stem, Knorripteridaceae; Triassic; Krappitz, Upper Silesia. See also Hörlck, in Potonie, Henry, 1910, no. 134, p. 1-19.

KNOWLTONELLA E. W. Berry, 1911.

Knowltonella maxoni E. W. Berry, 1911a, p. 235 , pls. 25-27 ; frond fragments, Matonlaceae?; Patapsco formation, Lower Cretaceous; Stump Neck, near Glymont, Md.; near Widewater, Va.

KOHEKOHE Hector, 1880.

Kohekohe dy80xylotdes Hector, 1880, p. 49 ; nom. nud.

KOHLMANNOPTERIS RIchter, 1899.

Kohlmannopteris insignis Richter, 1898, p. 40 ; nom. nud.

KONINCKOPORA (Lee) Alan Wood, 1948.

Koninckopora inflata (de Koninck) Alan Wood, 1943, p. 208, pls. 8-10; alga, Dasycladaceae; Lower Carboniferous; Visé, Belgium. Prevlously described by de Koninck, 1842, as a coral, and by Lee, 1912 , as a bryozoan.

KORAIA Oishi, 1931.

Koraia koraiensis Oish1, 1931a, flgs. 1-3 ; cupule; Jido series, "Permo-Triassic"; near Heijo, Korea.

KOSMOGYRA Stache, 1889.

Kosmogyra superba Stache, 1889, p. 134, pl. 4, figs. $2 \mathrm{a}, 2 \mathrm{~b}$; oogonium, Characeae ; Cretaceous; Divacca, near Triest, Italy.

KOSMOGYRELLA Stache, 1889.

Kosmogyrella carinata Stache, 1889, p. 121, pl. 2, fig. 19 ; oogonlum, Characeae ; Cretaceous ?

KRANNERA Corda, 1866.

Krannera mirabilis Corda, in Renger, 1866, p. 137, pl. 1, flg. 1 ; Cretaceous ; Kaunic, Bohemia.

KRAUSELIA Fucini, 1936.

Reference not seen; cited in Gothan, $1942 b$, p. 128.

KRYSHTOFOVIOHIA Nikitin, 1934.

Acad. sci. U. R. S. S. Bull., tome 7, p. 1078 (not seen, cited in Gothan, 1942b, p. 128).

KÜNSBERGIA Corda, 1847.

Kiinsbergia primaeva Corda, 1947, p. 16 ; Carboniferous.

KURRIA Schenk, 1866.

Kurria digitata Schenk, 1866, p. 53.

KURTZIANA Frenguelli, 1942.

Kurtziana cacheutensis Frenguell, 1942, p. 331, pl. 1 ; fern frond fragment; Triassic; Argentina. 


\section{$\mathbf{L}$}

\section{LACCOPTERIS Presl, 1838.}

Laccopteris elegans Presl, 1838 in Stern. berg (1820-38), p. 115, pl. 32, figs. 8 a, $8 \mathrm{~b}$; fertile fern pinnules, Matoniaceae ; Upper Triassic (Keuper); Steindorf near Bamberg, Bavaria.

\section{LACOEA Read, 1946.}

Lacoea seriata Read, 1946, p. 18, pl. 1, figs. 1-14; probably pteridosperm microsporangiate organ, compared with Dolerotheca and Potoniea; Forkston coal, Pennsylvanian; Dutch Mtn., Pa.

LACONIELLA Krasser, 1920.

Laconiella 8ardinioa Krasser, 1920, p. 16 ; cyadophyte; Jurassic (Dogger) ; Laconi, Sardinla.

LAESTADITES Meschinelli, 1892.

Laestadites nathorstii Meschinelli, in Saccardo, 1892, p. 750. See also Mesch1nelli, 1898, p. 16, pl. 9, flg. 16 ; fungus, Pyrenomycete.

LAEVIGATISPORITES Ibrahim, 1933.

Laevigatisporites laevigatus Ibrahim, 1933 , p. 17 , pl. 6 , flg. 46 ; spore ; CarbonIferous. See also Bennie and Kidston, 1886, p. 107 (Triletes I), pl. 3, figs. 1a, 1b.

LAEFIGATOSPORITES Ibrahim, 1933 .

Laevigatosporites vulgaris Ibrahim, 1933, p. 39 , pl. 2, fig. 16 ; spore; Carboniserous. For Sporonites vulgaris Ibrahim, 1932, p. 448, pl. 15, fig. 16.

LAFFONIA Heer, 1877.

Laffonia helvetica Heer, 1877a, p. 178, pl. 56, figs. 28, 29; Eocene; Beggingen, Switzerland.

LAGENELLA Reid and Chandler, 1933.

Lagenella alata Reld and Chandler, 1933 , p. 497 , pl. 29 , figs. $28-34$; fruit, incertae sedis; London Clay, Eocene ; Minster, Kent, England.

LAGENIASTRUM Renault, 1894.

Lageniastrum macrosporae Renault, 1894, p. 170. See also Renault, $1896 \mathrm{a}$, p. 429 , figs. 81-84 ; alga, Coelastraceae; Lower Carboniferous; Combres near Rigny, France.

LAGENICULA (Kidston) Zerndt, 1931.

Lagenicula glabrata Zerndt, 1931, p. 175 For Triletes glabratus Zerndt, 1930, pl. 8, figs. 38-41; spore; Carboniferous; Labiaz, Poland, Generlc name (Lagenicula) originally given by Kidston, in Bennie and Kidston, 1886, p. 114.

LAGENIOPTERIS Renault, 1883 Lageniopteris obtusiloba Renauit, 1883, p. 131 , pl. 23, figs. 1-8; petrified pecopterid follage; Upper Carboniferous.
LAGENOIDEA Reid and Chandler, 1933.

Lagenoidea trilocularis Reid and Chandler, 1933 , p. 493 , pl. 29 , figs. 1-18; fruit, incertae sedis; London Clay, Eocene; Sheppey, Kent, England.

LAGENOPTERIS Jongmans, 1935.

Reference not seen ; cited in Gothan, 1942b, p. 129.

LAGENOSPERUM Nathorst, 1914.

Lagenospermum nitidulum (Heer) Nathorst, 1914, p. 30, pl. 15, fig. 59 ; seed ; Carboniferous; Spitzbergen.

LAGENOSTOMA Williamson, 1876.

Lagenostoma ovoides Willamson, 1876a, p. 70 ; pteridosperm seed ; Upper Carboniferous; Oldham, England. See also Williamson, 1877, p. 266, pl. 9, flgs. 63-59 ; pl. 10, figs. 60-69, 71, 74-76 ; pl. 11 , figs. $70,72,73,77,78$.

LAGERSTROEMIOXYLON Madler, 1939.

Lagerstroemioxylon durum Madler, 1939, p. 130 , pl. 12, figs. 8-10 ; wood, Lythraceae ; Pliocene ; Schluesenkammer, near Höchst am Main, Germany.

LAGYNOPHORA Stache, 1880.

Lagynophora liburnica Stache, 1880, p. 198 ; Paleocene ; Divacca near Corgnale, Austria-Hungary.

LA HARPIA Heer, 1859.

Laharpia umbellata Heer, 1859, p. 171, p. 147 , figs. 28,29 ; infructescence, Juncaginaceae; Miocene; Oeningen, Switzerland.

LA.MBERTIPHYLLUM Velenov8ky, 1889.

Lambertiphyllum durum Velenovsky, 1889, p. 53. For Lambertia dura Velenovsky, 1883 , p. 5 , pl. 2 , fig. 16 ; Upper Cretaceous ; Lidic, Bohemia.

LAMINARIOPSIS Meunter, 1904.

Laminariopsis africana Meunier, 1904, p. 157, 4 figs. [unnumbered] ; Devonian?; Tienfala, Africa.

\section{LAMINARITES (Brongniart) Sternberg,} 1833.

Laminarites tuberculosus (Brongniart) Sternberg, 1833 (1820-38), p. 35. For Fucoides tuberculosus B r $0 \mathrm{~g} g \mathrm{n}$ I a $\mathrm{t}$, 1828a-38, p. 54, p1. 7, fig. 5 ; alga ; Cretaceous; Isle of Aix, near La Rochelle, France.

LAMINOPSIS Fucini', 1938.

Reference not seen ; cited in Gothan, 1942d, p. 129.

LAMPETIA Koenig, 1825.

Lampetia lucrymabunda Koenig, 1825, p. 2 , pl. 2, fig. 23 ; fruit, referred to Terebinthaceae; Oligocene; Prussia.

\section{LAMPROCARPITES Heer, 1882.}

Lamprocarpites nitidus Heer, 1882 , p. 68 , pl. 8, figs. 10, 12-14 ; frult, Juncagtna. cene; Upper Cretaceous; Uperivil, Greenland. 
I,ANFRANCIA Reld and Chandler, 1933. Lanfrancia subglobosa Reld and Chandler, 1933 , p. 457 , pl. 25 , figs. $37-40$; fruit, Cornaceae; London Clay, Eocene ; Sheppey, Kent, England.

IANGTONIA Reid and Chandler, 1933. Liongtonia bisulcata Reid and Chandler, 1933 , p. 453, pl. 25, figs. 18-27 ; endocàrp, Cornaceae; London Clay, Eocene ; Shèpey, Kent, England.

LARICITES Goeppert, 1850.

Laricites woodwardii Goeppert, 1850, p. 210 ; for illustrations, see Lindley and Hutton, 1837 (1831-37), pl. 226, figs. B1, B2 ; Quarternary ; Pastor Hill, Norfolk Cliffs, England.

LARICOIDITES Robert Potonie, 1950.

Laricoidites magnus Robert Potonie, in Potonie, Robert, Thomson, Paul W., and Thiergart, Friedrich, 1950 , p. 48 , pl. C, figs. 9,10 ; pollen, compared with Larix; lower Miocene; Niederlausitz, Germany.

LARICOPSIS Fontaine, 1889.

Laricopsis longifolia Fontaine, 1889, p. 233 , pls. $102,103,165,168$; coniferous twigs compared with Larix; Potomac group, Lower Cretaceous; Dutch Gap Canal, Va.

LARVARIA, Defrance, 1822.

Larvaria reticulata Defrance, 1822, p. 287 ; plant? middle Eocene; near Parls, France. See also Morellet and Morellet, 1913, p. 14, pl. 14.

LASKO.VSKIA Zalessky, 1939.

Laskovskia fabellata Zalessky, 1939b, p. 357, fig. 35 ; compared with Callipteris; Permian ; Matveyevo, Kroutaia Katouchka, USSR.

LATANITES Massalongo, 1858.

Latanites parisiensis (Brongniart) Massalongo, 1858, p. 11. For Palmacites parisiensis Brongniart, 1822, p. 312, pl. 16, fig. 1. See also Massalongo, 1859, p. 124, pl. 2, fig. 19.

LAURINASTRUM Unger, 1862.

Laurinastrum dubium Unger, 1862, p. 163, flg. 17 ; leaf, Lauraceae ; Eocene ; Kumi, Greece.

LAURINIUM Unger, 1845.

Laurinium xyloides Unger, 1845, p. 228 ; wood; Pllocene; Vicentino, Italy. See also Edwards, 1931.

LAURINOXYLON Fellx, 1883.

Laurinoxylon diluviale (Unger) Felix, 1883a, p. 59, pl. 2, figs. 1, 3; pl, 3, fig. 1 ; wood, dlcotyledon; Mlocene; Medgyi azo, Hungary.

LAURIPHYLLUM Nathorst, 1888.

Lauriphyllum gaudini Nathrost, 1888, p. 203 , pl. 2, flg. 6; leaf, dicotyledon; Tertiary ?; Shimohinokinalmura, Ugo province, Japan.
LAUROCALYX Reld and Chandler, 1933.

Laurocalyx glóbularis Reid and Chandler, 1933 , p. 219 , pl. 7, figs. 12-15 ; fruit, Lauraceae ; London Clay, Eocene ; Sheppey, Kent, England.

LAUROCARPUM Reid and Chandler, 1933.

Laurocarpum sheppeyense Reid and Chandler, 1933, p. 225, pl. 7, flgs. 27, 28 ; endocarp, Lauraceae; London Clay, Eocene ; Minster, Kent, England.

LAUROGENE Ettingshausen, 1854.

Laurogene cretacea Ettingshausen, in Reuss, 1854, p. 740.

LAUROPHYLLUM Goeppert, 1857.

Laurophyllum beilschmiedioides Goeppert, 1857 , p. 45 , pl. 10 , fig. $65 \mathrm{a}$; pl. 11, flgs. 66, 68 ; leaf, Lauraceae ; Eocene; Java.

LAUROXYLON Meschinelli and Squinabol, 1893.

Lauroxylon xyloides (Unger) Meschinell and Squinabol, 1893, p. 303. For Laurinium xyloides Unger, 1845 (1841-47), p. 81.

LEBACHIA Florin, 1938.

Lebachia piniformis (Schlotheim) Florin, 1938 , p. 25 , pls. $1,2,25,26,27,28,39$, 40 ; contferous follage and cones; Permian.

$L E B E P H Y L L U M$ W. J. Wilson, 1913.

Lebephyllum reineckei W. J. Wilson, 1913, p. 88 , pl. 9 , figs. 1,2 ; dicotyledonous leaf compared with Pilea and Urtica; Miocene?; British Columbia.

$L E C K E N B Y A$ Seward, 1894.

Leckenbya valdensis Seward, $1894 \mathrm{~b}$, p. 384. For Nathorstia valdensis Seward, 1894 , p. 145 , pl. 7 , fig. 5 ; pl. 9 , flg. 2 ; fern foliage; Wealden; England.

LECROSIA Florin, 1940.

Lecrosia grand'euryi Florin, 1940b, p. 315, pls. 161-162 ; seed-bearing cone, Coniferales; Upper Stephanlen, Carboniferous; Le Cros near St.-Etlenne, France. Generic name clted earler in Florin, 1927, p. 2 ; and Florin, 1929 b, p. 403.

LECYTHIDOANTHUS E. W. Berry, 1924.

Lecythidoanthus kugleri $\mathbf{D}$. W. Berry, 1924 c, p. 103 , figs. 1,2 ; flower, Lecyth1daceae; Mlocene; Trintdad, British West Indies.

LECYTHIDOPHYLLUM E. W. Berry, 1923. Lecythidophyllum courataroides E. W. Berry, 1923, p. 21, pl. 6, flgs. 3, 4 ; leaf, Lecythidaceae; Mtocene; Palomares, Oaxaca, Mexico.

\section{LEOYTHIOXYLON Milanez, 1935.}

Lecythioxylon brasiliense Milanez, 1935, p. 88, pls. 1-3 ; wood, dicotyledon ; Upper Cretaceous, Brazll. 
LEGUMINOCARPON Goeppert, 1855.

Leguminocarpon arachioides Goeppert, 1855 , p. 40 , pl. 26, fig. 11 ; fruit, Leguminosae; Miocene; Schossnitz, Silesia. Goeppert also uses the spelling Leguminucarpos.

LEGUMINOCARPUM Dotzler, 1938.

Leguminocarpum anceps (Berry) Dotzler, 1938 , p. 41 , pl. 5 , figs. 4,5 ; fruit, Leguminosae; Eocene. This spelling of generic name also used by Massalongo, $1859 \mathrm{~b}$, p. 121, for Leguminocarpon of Goeppert.

LEGUMINOSITES Bowerbank, 1840.

Leguminosites subovatus Bowerbank, 1840 , p. 125, pl. 17, figs. 1, 2 ; seed, Leguminosae ; Eocene ; Sheppey, Kent, England.

LEGUMINOXYLON Gupta, 1936.

Leguminoxylon burmense Gupta, 1936, p. 305 ; wood, Leguminosae ; Burma.

LEIODERMARIA (Goldenberg) Renault, 1896.

Leiodermaria lepidodendrifolia (Brongniart) Renault, 1896a, p. 208, pl. 36, fig. 1 ; lycopod bark impression; Carboniferous ; France.

LEJEUNITES Sadebeck, 1886 ?

Lejeunites dentifolius Sadebeck, 1886, p. 121 ; moss ; Tertiary ; Prussia ; nom. nud.

LEMANIDIUM Massalongo, 1859.

Lemanidium galaxacera Massalongo, in Massalongo and Scarabelli, 1859, p. 92. For Corallinites galaxacera Massalongo, 1856, p. 42, pl. 6, figs. 1, 2.

$L E M O I N E L L A$ Morellet and Morellet, 1913. Lemoinella geometrica Morellet and Morellet, 1913, p. 24, pl. 1, figs. 54, 55; alga, Dasycladaceae; E o c e ne; Grignon, France.

$L E N N E A$ Kräusel and Weyland, 1932.

Lennea schmidti Krüusel and Weyland, 1932, p. 189 ; Devonian; Rönkhausen, Westphalia, Germany.

LENZITITES Meschinelli, 1892.

Lenzitites gastaldii (Heer) Meschinell, 1892, p. 745. For Lenzites gastaldii Heer, in Sismonda, : 1859 , p. 533, pl. 1;

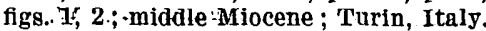

LEPACYOLOTES Emmons, 1856.

Lepacyclotes circularis Emmons, 1856, p. 332 , pl. 3, fig. 4 ; incertae sedis: Triassic ; Ellingtons, N. C.

LEPEOCAULUS Zalessky, 1033

Lepeocaulus aphyllus Zalessky, 1933c, p. 1389, fig. 3 ; lycopod stem impression; Devonian; Novala Zemlia, Russia.

LEPIDANTHIUM Schimper, 1870.

Lepidanthium micrirhombeum Schimper. 1870 (1869-74), p. 200, pl. 72, flg. 24 ; cycadophyte microsporanglate cone?; Rhatic; Veitlahm, near Culmbach, Franconia.
LEPIDIOPSIS Bleicher and Fliche, 1889.

Lepidiopsis tufacea Bleicher and Fliche, 1889 , p. 579 , fig. 1 ; silique? compared with Lepidium salivum; Quarternary; France.

LEPIDOCALAMUS Matthew, 1906.

Lepidocalamus scutiger (Dawson) Matthew, 1906b, p. 117, pl. 4, figs. 1-9; articulate stem; Little River group. Devonian; New Brunswick, Canada.

LEPIDOCARPON Scott, 1900.

Lepidocarpon lomaxi Scott, 1900, p. 309 ; lycopod seed cone; Ganister beds, Lower Coal Measures, Upper Carboniferous; Hough Hill, Stalybridge, Oldham, England. See also Scott, 1901, p. 294, pls. 33-41 ; and Schopf, 1941b.

LEPIDOCARPUS Rothpletz, 1880.

Lepidocarpus ellipsoideus (Goeppert) Rothpletz, 1880, p. 29, pl. 2, fig. $\theta$ For Trigonocarpon ellipsoideum Goeppert, 1852 ; seeds? of doubtful affinity : Carboniferous (Culm) ; Hainichen, Ottendorf and Lerchenberg, Germany.

LEPIDOCARYOPSIS Stur, 1873.

Lepidocaryopsis westphaleni Stur, 1873, p 3 ; Cretaceous; Kaunitz, Bohemia. Only? other species: Lepidocaryopsis rolloti Berry, 1929d, p. 3, pl. 1, fig. 7 ; Guaduas ${ }^{c}$ formation, Tertiary ; Colombla.

LEPIDOCARYTES Kuntze, 1904.

Lepidocarytes, Kuntze, in Post and Kuntze, 1904 , p. 323.

IIEPIDOCLADUS Vaffier, 1901.

Lepidocladus fuisseensis Vaffier, 1901, p. 134, pl. 10, figs. 1a-c; lycopod stem with leaves; Lower Carboniferous; Maconnais, France.

LEPIDOCYSTIS Lesquereux, 1880.

Lepidocystis pectinatus Lesquereux, 1880 , p. 454, pl. 59, fig. 3 ; Lepldocarpaceae? ; Pennsylvanian; near Pittston, $\mathrm{Pa}$.

LEPIDODENDRITES Fliche, 1905.

Lepidodendrites tessellata (Schimper and Mougeot) Fliche, 1905a, p. 144. For Caulopteris tessellata Schimper and Mougeot, 1844, p. 64 , pl. 29 ; incertae sedis ; Triassic ; Ruaux, Vosges, France.

LEPIDODENDRON Sternberg, 1820.

Lepiadenaron aichotomitm Sternberg, 1820 (1820-38), p. 23, pls. 1-3; Upper Carboniferous; Swina, Bohemia. It seems likely that the figures shown on plates 1-3 represent several species; although the second species is the first one described, it would seem that it will serve as a more useful type : Lepidodendron obovatum Sternberg, 1820 (1820-38), p. 23 , pl. 6, fig. 2 ; pl. 8 , fig. 1.

LEPIDODENDROPSIS Lutz, 1933.

Lepidodendropsis hirmeri Lutz, 1933, p. 118, pl. 15, figs. $1-12$; pl. 16, figs. 1 10 ; lycopod stem impression; Carboniferous (Culm) ; Germany. 
LEPIDOFLOYOS Sternberg, 1825.

Lepidofloyos laricinum Sternberg, 1825 (1820-38), Tentamen, p. xill, pl. 23, figs. 2-4; arborescent lycopod stem impression with!horizontally elongate leaf cushions; Carboniferous; Radnitz and Swina, Bohemia. This is original spelling although most modern workers have adopted Lepidophloios.

LEPIDOLEPIS Sternberg, 1823.

Lepidolepis imbricata Sternberg, 1823 $(1820-38)$, p. 39 , pl. 27 ; partly decorticated arborescent lycopod stem.

IEPIDOPHLOIOS.

See note under Lepidofloyos. See also Sterzel, 1907, p. 728; he divides the genus into Eulepidophloios and $\mathbf{S u b}$ lepidophloios.

LEPIDOPHYLLUM Bronguiart, 1828.

Of the species listed by Brongniart, 1828b, the following seems to be the only acceptable one: Lepidophyllum lineare Brongniart, $1828 \mathrm{~b}$, p. 87 . For Poacites carinata Brongniart, 1822, p. 238, pl. 3, fig. 2 ; a long linear leaf ; Carboniferous. Notes on Brongniart's other species may be of interest because of the need of a revision of this genus: $L$. majus Brongniart, a lycopod cone scale which would fall in Lepidostrobophyllum, Hirmer, 1927, p. 231. L. lanceolatum Brongniart, apparently never described by Brongniart; see Riehl, 1869, p. 141, pl. 28 , fig. 10 ; also a Lepidostrobophyllum. $L$. boblayi Brongniart, nom. nud. $L$. trinerve Brongniart, nom. nud.? Lindley and Hutton describe a species under this name which may be Brongniart's; also a Lepidostrobophyllum. Note also that Lepidophyllum is preempted by Cassini for a living Compositae. See Cassini in Sci. soc. Ph1lomatique Bull, 1816, p. 198-200, Paris.

LEPIDOPTERIS Schimper, 1869.

Lepidopteris stuttgartiensis (Jaeger) Schimper, 1869 (1869-74), p. 572, pl. 34 ; fernlike foliage; Upper Triassic (Keuper) ; near Stuttgart. For description of seed-bearing organs, see Thomas, 1933, p. 250 ; Harris, 1932 a, p. 58.

LEPIDOSIGILLARIA. Kräusel and Weyland, 1949.

Lepidosigillaria whitei Kräusel and Weyland, 1949 , p. 148 ; for several fossils previously assigned to Archaeosigillaria and Protolepidodendron; Upper Devonian.

LEPIDOSTROBOPHYLLUM Hirmer, 1927. Lepidostrobophyllum maius (Brongnairt) Hirmer, 1927, p. 193, 231, fig. 213 ; isolated lycopod sporophyll ; Upper Carboniferous ; England.
LEPIDOSTROBUS Brongniart, 1828.

Lepidostrobus ornatus Brongniart, $1828 \mathrm{~b}$, p. 87. See also Lindley and Hutton, 1832 (1831-37), p. 81, pl. 26; lycopod cone (possibly Lepidocarpon); Carboniferous; England.

LEPIDOTRUNCUS Fritach, 1908.

Lepidotruncus fortis Fritsch, 1908, p. 28, pl. 7, figs. 1, 2; Silurian; Chodoun, Bohemia.

LEPIDOXYLON Lesquereux, 1878.

Lepidoxylon anomalum (Brongniart) Lesquereux, $1878 \mathrm{~b}$, p. 334 . See also Lesquereux, 1879, pl. 83, fig. 5 ; pl. 84, fig. 1 ; cordaitean stem impression with leaves attached; Pennsylvanian; Missouri.

LEPROPHRAGMIUM Reinsch, 1881.

Leprophragmium sp. Reinsch, 1881, p. 118, pl. 52, figs. $1-8$; pl. 52a, figs. 8-10 ; Upper Carboniferous; Zwickau, Saxony.

LEPROSPERMUM Heer, 1877.

Leprospermum thurmanni Heer, $1877 a$, p. 133 , pl. 56, figs. 14,15 ; seed, Cycadaceae?; Jurassic ; Delsberg, Switzerland.

LEPTOCARYUM Brongniart, 1874.

Leptocaryum avellanum Brongniart, 1874, p. 248 , pl. 21 , fig. 17 ; silicifled seed; Carboniferous; St.-Etienne, France.

LEPTOLITHOPHYLLUM Airoldi, 1930.

Leptolithophyllum roveretoi Airoldi, 1930, p. 684 ; alga, Corallinacene; Oligocene ; Sassello, Liguria, Italy.

LEPTONEMA John Smith, 1896.

Leptonema tenuis John Smith, 1896, p. 321 , pl. 7 , fig. 9 ; Incertae sedis ; Upper Carboniferous; Annandale, near Kllmarnock, Scotland.

LEPTOPHLOEUM Dawson, 1862.

Leptophloeum rhombicum Dawson, 1862, p. 316 , pl. 12, fig. 8 ; pl. 17, fig. 63 ; lycopod stem; Devonian.

LEPTOPHYCUS Fritsch, 1908.

Leptophycus venosus (Barrande) Fritsch, 1908 , p. 20 , pl. 3, figs. $7-9$; Silurian; Drabov, Bohemia.

LEPTOPHYCUS J. H. Johnson, 1940.

Leptophycus gracilis J. H. Johnson, 1940, p. 586 , pl. 6 , figs. $1-3$; pl. 7 , fig. 3 ; bluegreen or green calcareous alga; South Fork Salt Creek, Park County, Colo.

LEPTOPLASMIUM Reinsch, 1881.

Leptoplasmium sp. Reinsch, 1881, p. 38, pl. $8 b$, figs. 1-5; pl. 7c, figs. 7-12 ; Carboniferous ; Mittelbroun, Württemberg.

LEPTOPTEROPHYLLUM ThOMas, 1930.

Leptopterophyllum nathorsti (Seward). Thomas, 1930 , p. 393 , pl. 20, ffg. 1 ; pl. 21 ; cycadophyte leaf ; Jurassic; Yorkshire, England. 
LEP'OSPERMITES Schmalhausen, 1883. Leptospermites spicatus Schmalhausen, 1883 , p. 319 , pl. 37 , figs. $7 \mathrm{~b}, 7 \mathrm{c}$; pl. 38 , figs. 8-15 ; fruit, compared with Leptospermum, Myrtaceae ; Ollgocene; Wolhyien, Russia.

LEPTOSPERMOCARPUM Menzel, 1913.

Leptospermocarpum herzogenrothense Menzel, 1913, p. 51, pl. 5, figs. 9-16; capsule, Myrtaceae ; Tertiary (Braunkohle); near Herzogenratl, Prussia.

LEPTOSPHAERITES Richon, 1885.

Leptosphaerites lemoinii Richon, 1885 , p. viti, pl. 32 ; fungus compared with Leptosphaeria; Tertiary ; Reims, France. Meschinelli, 1892, p. 751, erroneously attributes this genus to Cesati and $\mathrm{Di}$ Notarius.

LEPTOSTROBUS Heer, 1876.

Leptostrobus laxiflora Heer, 1876c, p. 72, pl. 13, figs. $10-13$; pl. 15, fig. $9 \mathrm{~b}$; seedbearing cones, Taxodiaceae; Jurassic, Siberia.

LEPTOSTKOMIUM Reinsch, 1881.

Leptostromium sp. Reinsch, 1881, .p. 90, pl. 21, figs. 1-6; pl. 22, figs. 1-6; pl. 22a, figs. 1-7 ; Permian ; Stockheim, Württemberg.

LEPTOTESTA Loubiere, 1929.

Leptotesta grand'euryi Loubiere, 1929, p. 594, pl. 12 ; sillicified seed; Carboniferous ; Grand'Crotx, France.

LEPTOTHRICHITES Meschinelli, 1898.

Leptothrichites buccalis (Robin and Lebour) Meschinelli, 1898, p. 70 ; Schizomycete.

LEPTOTHYRIOMYCES Kräusel, 1929.

Leptothyriomyces zonatus Kräusel,.1929, D. 4, pl. 1, figs. 1-3 ; fungus, Leptostromataceae; Tertiary (Upper Miocene?) Anak Slinsing, South Sumatra.

LEPT'OXYLUM Corda, 1845.

Leptoxylum geminium Corda, 1845, p. 21, pl. 15 ; Upper Carboniferous ; Swina, Bohemia.

LESANGEANA (Mougeot) Fliche, 1906? Lesangeuna voltzii (Schimper) Fliche, 1906, p. 164, pl. 13, fig. 3, Earliest cltrition : Lesangeana hassclotii Mougeot, 1851 , p. 346 ; nom. nud. See also $L$. remota Blanckenhorn, 1885, p. 147 ; and Posthumus, 1931.

LESCUkIA Perkins, 1906.

Lescuria attenuata Perkins, 1906, p. 220. pl. 57, figs. 7, 10; Tertiary ; Brandon, vit.

LESCUROPTERIS Schimper, 1869.

Lescuropteris moorii ( $L$ e g g u $\mathrm{r}$ e $\mathrm{x}$ ) Schimper, 1869 (1869-74), p. 465 ; fernlike follage: Pennsylvanian; Greensburg, Pa. For Neuropteris moorit Lesquereux, in Rogers, 1858, p. 860, pl. 19, fig. 1.
LESLEY A Lesquereux, 1880.

Lesleya grandis Lesquereux, 1880, p. 143, pl. 25, flgs. 1-3; Ctlossopteris-like follage ; base of Chester limestone,.Pennsylvanian; Pennsylvania.

LEUOADENDRITES Saporta, 1862.

Leucadendrites sextinctus Saporta, ;1862, p. 249 , p1. 7 , fig. 8 ; leaf, compared with Leucadendron; Tertiary ; France.

LEUOOSPERMITES Saporta, 1861.

Leucospermites denticulatus Saporta, in Heer, 1861, p. 140 ; leaf, Proteaceae ; Eocene; St. Zacharie, France ; nom. nud

LEVEILLEITES Foerste, 1923.

Leveilleites hartnageli Foerste, 1923, p. 62 pls. 4-11; alga?; Medinan formation. Upper Silurian; southern Ontario.

LEYRIDA Reid and Chandler, 1933.

Leyrida bilocularis Reid and Chandler, 1933 , p. 488 , pl. 28 , tigs. $22-32$; endocarp, incertae sedis; London Clay, Eocene; Sheppey, Kent, England.

LIANOPHYCUS Herzer, 1902.

Lianophycus polyfrons Herzer, 1902, p. 41 , pl. 1 ; organic remalns: Carboniferous; Marietta, Ohio.

LIASOPHYCUS Fliche, 1909.

Liasophycus scythothalioides Fliche, 1909, p. 211, alga ; Lower Jurassic (Llas) ; Rimogne, France.

LIASPERMUM Grandori, 1916.

Liaspermum dissectum (Zigno) Grandori, 1916 , p. 108, figs. 2-4 [unnumbered plate] ; seed ?; Jurassic (Lower Oolite) ; Zuliana near Rovere di Velo, Italy.

LIBOCEDRITES Endlicher, 1847.

Libocedrites salicornioides (Unger) Endlicher, 1847, p. 275 . For Thuites sali. cornioides Unger, 1841 (1841-47), p. 11 , pl. 2 , ffgs. $1-4,7$; pl. 20 , fig. 8 ; coniferous foliage shoots; Eocene; Radoboj, Croatia.

LICROPHYCUS Billings, 1862.

Licrophycus ottawaensis Billings, 1862, p. 99, fig. 87 ; alga?; Trenton limestone, Lower Silurian; near Ottawa, Canada.

LILLTA Unger, 1842.

Lillia viticulosa Unger, 1842, p. 178; wood; Tertiary ; Rauca, Hungary. See also Corda, 1845, p. 49, pl. 60, figs. 1-3. LIMNOCARPUS Reid, 1898.

Limnocarpus headonensis (Gardner) Reid 1898 , p. 465, figs. a-d ; frult, compared with Potamogeton; Lower Headon beds, Oligocene; Hordle Cliff, Hampshire, England.

LIMNOPHYCCS Kirchheimer, 1930.

Limnophycus paradoxus Kirchbeimer, .1930a, p. 589, pl. 35; alga, compared with Cutleria, Dictyota, etc.; Upper Pliocene ; Homberg, Germany. 
I.IMNOP'HYLLUM Hosius and Marck, 1880. Limnophyllum primaevum Hosius and Marck, 1880 , p. 183 , pl. 38 , fig. 153 ; leaf, Pisteaceae?; Upper Cretaceous; Westphalia.

IINGUIFOLIUM E. A. N. Arber, 1913. Linguifolium lillieanum E. A. N. Arber, 1913 , p. 346 , pl. 7 , figs. 1,4 ; leaf, resembling Glossopteris; Mt. Pots beds, Rhaeto-Jurassic; Mount Pots, Ashburton County, New Zealand.

LINOPORELLA Steinmann, 1899.

Linoporella capriotica (Oppenhelm) Steinmann, 1899 , p. 149 , fig. 13 ; alga, Dasycladaceae ; Upper Jurassic; Capri.

IINOPTERIS Presl, 1838.

Linopteris gutbieriana Presl, in Sternberg. 1838 (1820-38), p. 167. For Dictyop. teris brongniarti Gutbier, 1835, p. 63 . pl. 11 , figs. $7,9,10$; neuropterid-shaped pinnules with net veination; Carboniferous; Zwickau, Saxony.

LINOSPOROIDEA Keller, 1895.

Linosporoidea populi Keller, 1895, p. 3 pl. 2, fig. 6 ; fungus; Miocene; Herisau. Switzerland.

LIQUIDAMBAROXYLON Felix, 1884.

Liquidambaroxylon speciosum Felix, 1884 p. 24 , pl. 3, figs. $2-4$; pl. 4, fig. 4 ; wood compared with Liquidambar styraciflua Tertiary ; Medgyanzo, Hungary.

IIRIODENDROPSIS Newberry, 1895.

Liriodendropsis simplex Newberry, 1895. p. 83 , pl. 19 , figs. 2,3 ; pl. 53 , figs. $1-4$. 7 ; leaf, Magnoliaceae; Amboy clay. Cretaceous ; Woodbridge, N. J.

LIRIOPHYLLUM Lesquereux, 1878.

Liriophyllum beckwithii Lesquereux. $1878 \mathrm{c}$, p. 482 ; leaf, affinities witl Liriodendron; Cretaceous. See also Lesquereux, 1883, p. 76, pl. 10, fig. 1.

LISTRODIUM Zalessky, 1937.

Listrodium uninervium Zalessky, $1937 \mathrm{~b}$, p. 83 , fig. 50 ; leaf fragment, incertae sedis; Permian; left bank Sylva Rivar near mouth of Tchekarda River, Ourals. Russia.

LISTROPHYLLUM Zalessky, 1934 ?

Listrophyllum uscatense Zalessky, 1934c p. 771 , fig. 35 ; fern pinnule ; Permian : Kuznets Basin, Russia.

LI'THARCHAEOCYSTIS Deflandre, 1932.

Litharchacocystis costata Deflandre, 1932. p. 1273, figs. 1, 2 ; alga, Chrysophyceae ; Kuznets Basin, Russla.

IITHIOTIS Gümbel, 1871.

Lithiotis problematica Gümbel, 1871, p. 48 , pl. 2, figs. 13, 14 ; Lower Jurassic (Liassic) ; near Roveredo, Italy.

LITHOBRYON Ruprecht, 1866.

Lithobryon calcareum Ruprecht, 1866, p. 37; Jurassic: Wjatka, Russia.
LITHOCAOLON Meneghini, .1857.

Lithocaulon minius Meneghini, 1857, p. 550 , pl. H, flg. 7 ; alga ; Tertlary ; Sardinia.

LITHODIOTUON Conrad, 1837.

Lithodietuon beckit Conrad, 1837, p. 167 ; Silurian (Medina sandstone); Medina, N. $\mathbf{Y}$.

LITHOMYXA Howe, 1932.

Lithomyxa calcigena Howe, 1932a, p. 63, pls. 19-23 ; lime-secreting alga ; Recent ; Furnace Creek near Harpers Ferry, W. Va.

LITHOPHYLLODENDRON Musper, 1919.

Lithophyllodendron rubrum Musper, 1919, p. 17, figs. 1-12; "Upper White Jura" ; Schwaben, Württemberg.

LITHOSPERMITES E. W. Berry, 1929.

Lithospermites glabrum E. W. Berry, $1929 \mathrm{~b}$, p. 165, pl. 3, figs. 9-13 ; fruit, Borraginaceae ; Tertiary ; Belen, Peru.

LITHOSTAOHYS Fischer-Ooster; 1858.

Lithostachys alpina Fischer-Ooster, 1858, p. 59 , pl. 3, fig. 1 ; alga ?; Jurassic (Lower Oolite); near Blumenstein, Switzerland.

LI'THOTHAMNISOUM (Rothpletz) Hеydrich, 1900 .

Lithothamniscum nahaense Heydrich, 1900b, fig. 1, pl. 7, figs. 1, 2. Generic name cited in Rothpletz, 1891, p. 311.

LITHOTHAMNITES Saporta, 1882 ?

Lithothamnites croizieri Saporta, 1882, p. 21, pl. 1, fig. 6 ; alga ; Jurassic (Oolite) ; La Rochefoucauld, France.

LITHOXYLON Jaeger, 1827.

Lithoxylon arenaceum Jaeger, 1827, p. 38, pl. 5, flg. 4; stem impression, incertae sedis; Upper Triassic (Keuper) ; Stuttgart.

LI'TSAEOPHYLLUM Deane, 1902.

Litsaeophyllum wingellense Deane, 1902a, p. 64 , pl. 17 , fig. 4 ; leaf, compared with Litsea dealbata Nees (Lauraceae) ; Tertiary; Wingello, New South Wales.

LITSEOPSIS Weyland, 1938.

Litseopsis rottensis Weyland, $1938 \mathrm{~b}, \mathrm{p}$. 141, pl. 19, fig. 1 ; staminate flower, Lauraceae; 'Jertiary ; Rott, Slebengebirge, Germany.

LIVERSIDGEA Mueller, 1877.

Liversidgea oxyspora Mueller, $1877 \mathrm{a}, \mathrm{p}$. 239, figs. 1-5; Pliocene; Richmond River, New South Wales.

LOBATANNULARIA Kawasak1, 1927.

Lobatannularia inequifolia (Tokunaga) Kawasaki, 1927 (1927-34), p. 12, pl. 3A, figs. D, E; pl. 4, flgs. 13-15; pl. 5, figs. $16-22$; pl. 9 , flg. 38 ; pl. 14 , figs. 74,75 ; foliage, intermediate between Annularia and Schizoneura; Jido serles, "PermoCarbonfferous"; Chŏngsŏn, Koren. 
LOBATICARPUM Reid and Chandler, 1933. Lobaticarpum variabile Reid and Chandler, 1933 , p. 314 , pl. 14, flgs. 16-20 ; fruit, Anacardiaceae? ; London Clay, Eocene ; Sheppey, Kent, England.

LOOHMOPHYOUS Debey and Ettingshausen, 1859.

Lochmophycus caulerpoides Debey and Ettingshausen, $1859 \mathrm{a}$, p. 198 , pl. 2, figs. 1-5 ; alga ?; Cretaceous ; Aachen, Rhenish Prussia.

LOCKEIA U. P. James, 1879.

Lockeia siliquaria U. P. James, 1879, p. 17. See James, J. F., 1885, p. 161, pl. 9, flg. 7 ; Lower Sllurian; Kentucky.

LOGANIA Stolley, 1925.

Logania canadensis Stolley, 1925, p. 63 ; Devonian; Campbellton, New Brunswick, Canada.

LOMARITES Hector, 1886.

Lomarites pectinata Hector, 1886, p. 66, flg. 30A ; Jurassic ; Mataura Falls, New Zealand. Cited originally in Hector, 1878 , p. 8 ; nom. nud.

LOMATITES Saporta, 1862.

Lomatites acerosus Saporta, 1862, p. 253 ; leaf, compared with Hakea repanda and Lomatia longifolia (Proteaceae) ; Ollgocene; Aix, Provence, France. See also Saporta, 1873a, p. 52, pl. 9, flg. 20.

LOMATOFLOYOS Corda, 1838.

Lomatofloyos crassicaule Corda, 1838 , in Sternberg (1820-38), p. 206, pl. 66, figs. 10-14; pl. 68, fig. 20 ; arborescent lycopod stem; Carboniferous; Radnitz, Bohemia. Variously spelled in later works as Lomatophloios and Lomatophloyos.

LOM.ATOPHLOIOS.

Sec Lomatofloyos.

LOMATOPHLOYOS.

Sce Lomatofloyos.

LOMATOPTERIS Schimper, 1869.

Lomatopteris jurensis (Kurr) Schimper, $1869(1869-74)$, p. 472, pl. 45, figs. 2-5; fernlike foliage; Upper Carboniferous; Nussplingen, Württemberg.

LOMENTARITES Fliche, 1905.

Lomentarites borneti Fliche, 1905, p. 57, pl. 4, fig. 4 ; pl. 5, fig. $2 \mathrm{~b}$; alga, Rhodophyceae?; Triassic ; Meurthe-et-Moselle, France. Generic name given in Fliche, 1903, p. 828.

LONCHOPTERIS Brongniart, 1836.

Lonchopteris bricil Brongniart, 1836 $(1828 a-38)$, p. 368, pl. 131, figs. $2,3$. First citation: Brongniart, 1828b, p. 60 ; nom. nud.

LOI'ERIA Newberry, 1888.

Loperia simplex Newberry, 1888, p. 93, pl. 25, figs. 1-3; Incertae sedis; Trlassic; Durham, Conn. This binominal cited by Newberry, 1887 , p. 126 ; nom. nud.
LOPHIODENDRON Zalessky. 1936.

Lophiodendron tyrganense Zalessky, $1936 a$, p. 228, fig. 11 ; lycopod leaf bases; Carboniferous ; Russia.

LOPHODERMA Zalessky, 1937.

Lophoderma sibirica Zalessky, 1937c, p. 126, fig. 2 ; lycopod leaf base impression; Permian; Kuznets Basin, Russia.

LORANTHAOITES Conwentz, 1886.

Loranthacites succineus Conwentz, 1886, p. 135 , pl. 13, figs. 6,7 ; stem fragment, in amber, Loranthaceae ; early Tertiary ; West Prussia.

LORANTHOPHYLLUM Unger, 1864.

Loranthophyllum griselinia Unger, 1864, p. 8 , fig. 13 ; leaf, Loranthaceae?; Tertiary ; Manganui, New Zealand.

LOXOPTERIS Pomel, 1846.

Loxopteri adiontoides Pomel, 1846, p. 652; fern follage; Lower Jurasslc (Lias) ; Moselle, France.

LUDOVIOPSIS Saporta, 1868.

Ludoviopsis discerpta Saporta, 1868, p. 338 , pl. 4, fig. 3 ; leaf fragment, Pandanaceae; Eocene; Sézanne, France.

LUHEOPSIS Langeron, 1900.

Luheopsis dissymetra Langeron, 1900, p. 343 , pl. 1, fig. 5 ; pl. 2 , fig. 5 ; leaf, compared with Luhea; Eocene; Sezanne, France.

LUNZIA Krasser, 1918.

Lunzia austriaca Krasser, 1918, p. 492, pl. 1, figs. $1-3$; pl. 2 , flgs. $1-4$; pl. 3 ; pl. 4 , figs. $2-4$; cycadophyte microsporophyll ; Triassic; Pramelreith near Lunz, Austria.

LYCHNOPHORITES Martius, 1822.

Lychnophorites dichotomus (Sternberg) Martius, 1822, p. 144. For Lepidodendron dichotomum Sternberg, 1820 (1820-38), p. 23 , pls. $1-3$; pl. 63, fig. 1 ; Upper Carboniferous ; Swina, Bohemia.

LYCOPODIOLITES.

See Lycopodiolithes.

LYCOPODIOLITHES Schlotheim, 1820.

Lycopodiolithes arboreus Schlotheim, 1820 , p. 413 , pl. 22 , fig. 2 ; lycopod branchlets with foliage; Upper Carboniferous ; Waldenburg, silesia. Sternberg, 1825 (1820-38), Tentamen, p. 1x, adopts spelling Lycopodiolites.

LYCOPODIOPSIS Renault, 1890.

Lycopodiopsis derbyi Renault, 1890, p. 809; lycopod stem; Permian; San Paulo, Piracicaba, Brazil, see also White, David, 1908, p. 437, pl. 5, flg. 11.

LYCOPODITES Brongniart, 1822.

Lycopodites taxiformis Brongniart, 1822, p. 231, pl. 13, fig. 1. This is the first species described by Brongniart, but, according to Seward, it is a conifer. See discussion by Seward, 1910, p. 76. 
LYOOPOGENIA Read, 1936.

Lycopogenia callicyrta Read, $1936 \mathrm{~b}$, p. 227, figs. 1, 2 ; petrifled stem, Lepldodendrales; Devonian; near Junction City, Boyle County, Ky.

LYCOSPORA Schope, Wllson, and Bentall, 1944.

Lycospora micropapillata (Wilson and Coe) Schopf, Wilson, and Bentall, 1944, p. 54. For Oirratriradites micropapillatus L. R. Wilson and Coe, 1940, p. 184, fig. 6 ; spore; Des Moines group, Pennsylvanian; Iowa.

LYCOSTROBUS Nathorst, 1908.

Lycostrobus scotti Nathorst, 1908 b, p. 8, pl. 1 ; lycopod cone.

LYCOXYLON Srivastava, 1946.

Lycoxylon indicum Srivastava, 1946, p. 192, pl. 1 ; petrifled Lycopodium-like stele; Jurassic; Santal Pargana District, Behar, India. Brief description given earlier in Srivastava, 1937, p. 273.

LYGINODENDRON Gourlie, 1843.

Lyginodendron landsburgii Gourlie, 1843 p. 108 , pl. 2 ; stem cast of arborescent lycopod?; Carboniferous ; Stevenston, Ayrshire, Scotland.

LYGINOPTERIS Henry Potonie, 1899.

Lyginopteris oldhamiana (BInney) Henry Potonie, 1899, p. 170 ; pteridosperm stem; Upper Carboniferous; England. For Dadoxylon oldhamium Blnney, 1866, p. 115. According to Seward, 1917, p. 39, Binney's specimen was first figured by Arber, E. A. N., 1902. See also Willlamson, 1873, p. 377 ; Seward, 1917, p. 38 ; Walton, 1940 ; and Jongmans, 1930.

LYGINORACHIS Kidston, 1923.

Lyginorachis papilio Kidston, in Scott, 1923 , p. 57 ; pteridosperm petiole; Cementstone group, Calclferous Sandstone sertes; Lower Carboniferous; Northam Bridge, Tweed, Scotland. See Crookall, 1931 , p. 27 , pl. 1 , flg. 2 ; pl. 2, flgs. 4 , 5 ; pl. 3, figs. 6-8.

LYGODITES Schulze, 1887.

Lygodites cf. aneimiifolius (Debey and Ettingshausen) Schulze, 1887, p. 463. For Pteridoleimma aneimiifolius Debey and Ettingshausen, 1859 , p. 230, pl. 7, fig. 1 ; fern pinnule ; Cretaceous (Senonian) ; Aachen, Rhenish Prussia.

I,YONSIAEPHYLLUM Deane, 1907.

Lyonsiaephyllum duni Deane, 1907, p. 191, pl. 36, fig. 1; leaf, compared with Lyonsia and Alstonia (Apocynaceae); Tertiary; Warrumburgle Mts., New South Wales.

LYSSOXYLON Daugherty, 1941.

Ly8soxylon grigsbyi Daugherty, 1941, p. 71 , pls. 26-30 ; petrifled trunk fragment, Williamsoniaceae; upper Triassic; island in Rio Puerco, three-quarters of a mile southeast of Adamana, Ariz.

\section{$\mathbf{M}$}

MACOLINTOOKIA Heer, 1866

Macclintockia dentata Heer, 1866, p. 277. See also Heer, 1868, p. 115, pl. 15, figs. 3,4 ; leaf fragment, Proteaceae; Miocene ; Atanekerdluk, Greenland.

MACRALETHOPTERIS Jongmans and Gothan, 1935.

Jaarb. mijnwezen Nederlandlish-Indlë, 1930, Verh., boekdeel 59 , p. 130 , pl. 40 , figs. 2-5 ; pl. 41, fig. 1, 1935 (not seen). See also Gothan, 1942a, p. 131.

MACROGLOSSOPTERIS Sze, 1931.

Macroglos8opteris leeiana Sze, 1931, p. 5, pl. 3, fig. 1 ; pl. 4, flg. 1 ; Jurassic ; Pinghsiang, Kiangsi province, China.

MACROPORELLA PIa, 1912.

Macroporella dinavica Pia, 1912, p. 33, pl. 2, figs. 1-6; alga, Siphoneae Verticlllatae; Triassic; Dalmatia, AustriaHungary.

MACROPTERYGIUM Schimper, 1870.

Macropterygium bronnii (Schenk) Schimper, 1870 (1869-74), p. 132. For Pterophyllum bronnii Schenk, 1865-76, p. 168, pl. 40 , figs. 2,3 ; cycadophyte foliage; Carin'thia.

MAOROSPHENOPTERIS KIdston, 1887.

Macrosphenopteris lindsaeoides KIdston, 1887 b, p. 353 , pl. 27 , fig. 1 ; sphenopteridlike frond fragment; Upper Carbonlferous ; Radstock, England.

MACROSPORITES Renault, 1899

Macrosporites insignis Renault, 1899, p. 1072 ; spores ; Carboniferous ; Germany.

MACROCYSTITES Fucini, 1936.

Reference not seen ; cited in Gothan, $1942 b$, p. 131.

MACROSTACHYA Schimper, 1869.

Macrostachya infundibuliformis (Bronn) Schimper, 1869 (1869-74), p. 333, pl. 23, flgs. 15-17 ; articulate cone; Carboniferous ; Zwickau, Saxony.

MACROTAENIA. Frenguell, 1943.

Macrotaenia fertilis Frenguelli, $1943 \mathrm{~b}$, p. 401 , pls. 1-3 ; fertile fern frond, Marattlaceae; 'Triassic; Cacheuta, Mendoza, Argentina.

MACROTAENIOPTERIS Schimper, 1869.

Macrotaeniopteris major (Lindley and Hutton) Schimper, 1869 (1869-74), p. 610. For Taeniopteris major Lindley and Hutton, 1831-37, p. 31, pl. 92; cycadophyte foliage; Jurassic; Gristhorpe, Yorkshire, England.

MACROTORELLIA Kryshtofovitch, 1927.

Macrotorellia hoshayahiana Kryshtofovitch, 1927, p. 604, pl. 13, figs. 2-9; Cycadophyte leaflets?; Jurassic; North Caucasus.

MAFFEIJA Massalongo, 1857.

Maffeifa ceratophylloides Massalongo, $1857 b$, p. 777 ; nom. nud. 
MAGNOLIAEPHYLLUM (Krasser) Seward, 1926.

Magnoliaephyllum alternans (Heer) Seward, 1926, p. 120, fig. 25 ; leaf, Magnoliaceae ; Cretaceous ; Atanlkerdluk, Greenland. Generic name cited in Krasser, 1896 , p. 131 , pl. 17 , fig. 12.

MAGNOLIAESPERMUM Kirchheimer, 1934. Magnoliaespermum fliegeli Kirchheimer, $1934 a$, p. 770 , flg. 2 ; seed, Magnoliaceae; Tertiary; Germany. See also Kirchheimer, 1936a, p. 45, pl. 2, figs. 5a-i.

MAGNOLIAESTROBUS Seward and Conway, 1935.

Magnoliaestrobus gilmouri Seward and Conway, 1935, p. 22 ; pl. 4, fig. 20 ; Mag. nolia-like infructescence; Cretaceous; west Greenland.

MAGNOLILEPIS Conwentz, 1886.

Magnolilepis orussica Conwentz, 1886, p. 56, pl. 6 , figs. $6-8$; bud scale?, in amber, Magnoliaceae; Tertiary; West Prussia.

MAGNOLIOIDES Ettingshausen, 1885.

Magnolioides carniolica Ettingshausen. 1885 , p. 19, pl. 30 , flg. 22 ; leaf, Magnoliaceae; Miocene; Steinbruch.

MAGNOLIOPHYLLUM.

Error for Magnoliphyllum, in Dorf, 1938, p. 64 .

MAGNOLIPIYLLUM Conwentz, 1886.

Magnoliphyllum balticum Conwentz, 1886, p. 57 , pl. 6, fig. 9 ; leaf; in amber, Magnoliaceae; Tertiary; West Prussia.

MAGNOLITES Tuzson, 1909.

Magnolites silvatica Tuzson, 1909, p. 376 ; wood; Schotter beds, Tertiary; Lake Balaton, Hungary. See also Tuzson, 1911 , p. 44 , figs. 17-21. Placed in Dryoxylon by Edwards, 1931.

MAJANTHEMOPHYLLUM Weber, 1851.

Majanthemophyllum petiolatum Weber, 1851 , p. 156, pl. 18 , fig. 5 ; Ieaf, Smilaceae; Oligocene; Quegstein, Rhenish Prussia.

MALACOTEST'A Williamson, 1876.

Malacotesta oblonga Williamson, $1876 \mathrm{a}$, p. 71 ; seed ; Upper Carboniferous; Oldham, England. See also Williamson, 1877 , p. 268 , pl. 12 , fig. 89 ; pl. 13 , figs. 88, 90-93.

\section{MALPIGHIASTRUM Unger, 1850.}

Malpighiastrum proorustac Unger, 1850a, p. 453; Malpighiaceae; Eocene; Radoboj, Croatia. See also Unger, 1860 (1860-65), p. 30, p1. 13, figs. 4-7.

MALVACARPUS E. W. Berry, 1925.

Malvacarpus tertiarius E. W. Berry, $1025 b$, p. 217, pl. 3, fig. 6 ; fruit, Malvaceae; Miocene; Mirador Mesa, north or Rio shubut, chubut province, Argentina.
MILVOOARPON Hollick, 1928.

Malvocarpon clarum Hollick, 1928, p.214, pl. 75, fig. 6 ; fruit, compared with Abutilon, Malvaceae ; Tertiary ; Collazo River, Puerto Rico.

MAMILLARIA Brongniart, 1825.

Mamillaria desnoyersii Brongniart, 1825, p. 423 , pl. 19, figs. 9,10 ; incertae sedis; Jurassic ; Mamers, France.

MAMMAEITES Fliche, 1896.

Mammaeites francheti Fliche, 1896, p. 283, pl. 13, fig. 7 ; seed referred to Clusiaceae; Cretaceous ; Chaudefontaine near St. Menehould, France.

IANCHURIOPHYCUS Endo, 1933.

Manchuriophycus yamamotoi Endo, 1933, p. 47 , pl. 6 , fig. 3 ; pl. 7 , fig. 2 ; alga? ; Nanshan formation, pre-Cambrian ; near Chiao-tou Station, South Manchuria.

MANICARITES Bureau, 1896.

Manicarites danteseanus (Visiani). Bureau, 1896, p. 282. For Hemiphoenicetes dantesiana Visiani, 1864, p. 451, pl. 18, figs. A, B ; Oligocene; Verona, Italy.

MANIHOTITES E. W. Berry, 1910.

Manihotites georgiana $\mathrm{E}$. W. Berry, $1910 \mathrm{~b}$, p. 507, fig. 1 ; leaf, Euphorbiaceae ; Cretaceous; Georgia.

MANTELLIA (Brongniart) Bronn, 1837.

Mantellia megalophylla (Buckland) Bronn, 1837, p. 227, pl. 15, fig. 2. First citation of genus : Mantellia nidiformis Brongniart, $1828 \mathrm{~b}$, p. 96 ; nom. nud.

IIARANTOIDEA Jaeger, 1827.

Marantoidea arenacea Jaeger, 1827, p. 28 , pl. 5, fig. 5 ; Taeniopteris leaf fragment; Triassic (Keuper); Stuttgart. See also Sternberg, 1838 (1820-38), p. 139.

WARATTIOPSIS Schimper, 1874.

Schimper, 1874 (1869-74), suggests that the species which he formerly assigned to Angiopteridium (Schimper, 1869, p. 602 ) should all be transferred to Marattiopsis. Presumably type would be for Angiopteridum mïnsteri (Goeppert) Schimper, 1869, p. 603, pl. 38, fgs. 1-6; frond, Marattiaceae; Rhaetic; Bayreuth, Bavaria.

MARATTIOTHECA Schimper, 1879

Marattiotheca grand'euryi Schimper, in

Schimper and Schenk, 1879 (1879-90), p. 91, fig. 66; fertile fern pinnule, Marattiaceae ; Úpper Carboniferous.

MARATTITES Marion and Laurent, 1898. Marattites desideratus Marion and Laurent, 1898 , p. 189 , pl. 1 , fig. 1 ; fragment or fern pinnule; Cretaceous; Rabadeg, Rilmania. 
MARCHANTITES Brongniart, 1849. Marchantites sesannensis Brongntart, 1849, p. 61. First illustration for this species appears to be in Watelet, 1866 , p. 40, pl. 11, fig. 6. Apparently first illustrated species is Marchantites sinuatus Saporta, 1865, p. 68, pl. 1, flg. 2.

MARGARETIA Walcott, 1931.

Margaretia dorus Walcott, 1931, p. 2, pl. 1, figs. 1-6; compared with living alga Kallymenia ; Burgess shale, Middle Cambrian; British Columbia.

MARGARITOPTERIS Gothan, 1913.

Margaritopteris pseudocoemansi Gothan, 1913a, p. 169, pl. 34, figs. 6, 6a ; fernlike follage; Upper Carbontferous; Upper Silesia.

MARIMINNA Unger, 1843.

Mariminna meneghinii Unger, 1843 (184147 ), p. 58 , pl. 18 , fig. 5 ; incertae sedis ; Eocene ; Monte Bolca, Italy.

MARIOPTERIS Zelller, 1879.

Mariopteris nervosa (Brongnlart) Zeiller, 1879 , p. 69 , pl. 167 , figs. $1-4$; fernlike follage; Upper Carboniferous; Bassin du Bas-Boulonnais, France.

MAROESIA Jongmans and Gothan, 1935.

Mfaroesia rhomboidea Jongmans and Gothan, 1935, p. 91, pl. 18, figs. 1-3 ; lycopod stem impression; Upper CarbonIferous; Residentie Djambi, Maroes, Sumatra.

MARPOLIA Walcott, 1919.

Marpolia spissa Walcott, 1919, p. 234, pl. 52, figs. $1 \mathrm{a}, 1 \mathrm{~b}$; alga, Cyanophyceae; Burgess shale, Middle Cambrian ; northeast of Burgess Pass, British Columbia.

MARSILIDIUM Schenk, 1871.

Marsilidium speciosum Schenk, 1871, p. 225 , pl. 26, fig. 3 ; leaves, incertae sedis ; Wealden; Osterwald, Hannover, Germany.

MARTINIA Crie, 1889.

Martinia elegans Crie, 1889 b, p. 20 ; nom. nud. See note under Bottgeria.

MARTYIA Reld, 1924.

Martyia navioulaeformis Reid, 1924, p. 327, figs. 5a-c; seed, Leguminosae;

Lower Pllocene; Pont-de-Gail, France.

MARZARIA Zigno, 1865.

Marzaria paroliniana Zigno, 1865, p. 32 ; fertile fern frond fragment; Jurassic (Oolite); near Rovere di Velo, Italy. See also Zigno, 1867 (1.856-68), p. 170, pl. 19, figs. 3-7.

MASOULOSTROBUS Seward, 1911.

Masculostrobus zeilleri Seward 1911b, p. 686, fig. 11 ; male inflorescence, $\mathrm{Co}$ niferales; Jurassic ; const of Sutherland between Brora and Helmsale, Scotland.
MASSULITES Sahni and H. S. Rao, 1943. Massulites coelatus Sahnl and H. S. Rao, 1943 , p. 56, pl. 7, figs. 56-63 ; massulae of water fern; Intertrappean cherts, early Tertlary; Sausar 'Tensll, Chhindwāra district, Central Provinces, India.

MASTIXICARPUM Chandler, 1926.

Mastixicarpum cras8um.Chandler, 1926, p. 36 , pl. 6, figs. 5a-d i endocarp, Cornaceae; Upper Eocene; Hordle, Hampshire, England.

MASTIXIOPSIS Kirchheimer, 1935.

Mastixiopsis nyssoides K1rchhelmer, 1935, p. 293, fig. 17 ; seed, Cornacene; Tertiary (Braunkohle) ; Riestedt, Germany. See also Kirchhelmer, 1936c, p. 291, pl. 7 , figs. $5 \mathrm{a}-\mathrm{g}$.

MASTOOARPITES Trevisan, 1856.

Mastocarpites crucaeformis (Sternberg) Trevisan, in Zigno, 1856 (1856-68), p. 22. For Algacites crucaeformis Sternberg, 1820-38, p. 36, pl. 2, Ags. 5, 6.

MASTOPORA Eichwald, 1840.

Mastopora concava Elchwald, 1840, p. 204 ; alga ?; Silurian; Russia.

IATONIDIUM Schenk, 1871.

Matonidium goepperti (Ettingshausen) Schenk, 1871, p. 220 ; pl. 27, flg. 5 ; pl. 28 , figs. $1 a-d, 2$; pl. 30 , fig. 3 ; leaves, Matonlaceae; Wealden, Germany.

MATONIELLA. Hirmer and Hoerhammer, 1936.

Matoniella wiesneri (Krasser) Hirmer and Hoerhammer, 1936, p. 47, fig. 7 ; leaf, Matonlaceae; Cretaceous ; Kunstadt, Mähren, Germany.

MAUCHERIA Brolli, 1928.

Maucheria gemundensis Brolli, 1928, p. 191, pls. 1, 2 ; Lower Devonian; Gemunden, Germany.

KAUERITES Zalessky, 1933.

Reference not seen; cited in Gothan, $1942 b$, p. 132.

MAUPASIA (Munler-Chalmas) Morellet and Morellet, 1917.

Maupasia dumasi Morellet and Morellet, 1917 , p. 369 , pl. 14 , figs. 11,12 ; alga, Dasycladaceae; Tertlary ; Bretagne, France. [On p. 368 these authors note "Maupasia Mun.-Ch. = Maupasina Mun.Ch."]

KAUPASINA Munier-Chalmas, 1877.

In Munier-Chalmas, 1877 , p. 817 ; nom. nud. See Maupasia.

LAWSONELLA Chapman, 1927.

Mawsonella wooltanensis Chapman, 1927a, p. 124 , pl. 6 ; calcareous alga; Lower Cambrian ?; 9 miles west of Wooltana Head Station, South Australia.

YAYOGYNOPHYLLUM Krăusel, 1929.

Mayogynophyllum paıcinervinum Kräusel, 1929 , p. 21 , pl. 5, fig. 12 ; leaf, Anonaceae ; Tertlary (Upper Mtocene?) ; Anak Slingsing, South Sumatra. 
MAZOCARPON (Scott) Benson, 1918. Mazocarpon shorense Benson, 1918, p. 579, pl. 17, figs. 1-14; petrifled sigfllarian cone; Upper Carboniferous; Yorkshire, England. Generic name first cited by Scott, 1907 , p. 169 . For recent consideration, see Schopf, 1941.

MEDULLOPITYS Kräusel, 1928.

Medullopitys sclerotica (Gothan) Krăusel, 1928 , p. 22 , pl. 1 , fig. 11 ; pl. 2, figs. 2-6 ; pl. 3, figs. 1-5; petrifled cordaltean stem; Karroo beds, Permian; German Southwest Africa.

MEDULLOSA Cotta, 1832.

Medullosa stellata Cotta, 1932, p. 65, pl. 13 ; petrified stem with polycyclic stelar system ; Permian ; Chemnitz, Germany. Of the species described by Cotta this one is proposed as the type, for it is the flrst in order of description which clearly displays the characteristic stelar pattern. For later accounts, see Scott, 1899 ; Baxter, 1948 ; Andrews, 1945.

MEDULLOSITES Bureau, 1914.

Medullosites mammiger Bureau, 1914, p. 288, pl. 27, flg. 6 ; fern stem?; Carboniferous; Loire, France.

MEDULLOXYLON Hartig, 1848.

Hartig, 1848b, proposes this genus to include certain species placed in Dadoxylon Endlicher. Presumably he Intended Medulloxylon withamil (Lindley) Hartig.

$M E G A D E N D R O N$ Reichenbach, 1836.

Megadendron saxonicum Reichenbach, 1836 , p. 6 ; Permian; Hilbersdorf, near Chemnitz, Germany.

MEGALOMYELON Cribbs, 1940.

Megalomeylon myriodesmon Cribbs, 1940 , p. 596, figs. $1-3,7,10,12-15,18$; stem of pityean affinity; Reed Spring formation, Mississippian ; Missourt.

MEGALOPTERIS (Dawson) E. B. Andrews, 1875.

Megalopteris dacosoni (Hartt) E. B. Andrews, 1875 , p. 415 ; fern or pteridosperm foliage; Devonian?; St. John, New Brunswick, Canada. For Neuropteris dawsoni Hartt, in Dawson, 1868, p. 551, fig. 193.

MEGALOPTERIS Schenk, 1883.

Megalopteris nicotianaefolia Schenk, $1883 \mathrm{c}$, p. 238, pl. 32, figs. 6-8; pl. 33, flgs. 1-3 : Upper Carboniferous; Lui-pa-Kou, Hunan province, China.

MEGALORHACHIS Unger, 1845.

Megalorhachis elliptica Unger, 1856, p. 169. D1. 7. figs. 19-21 : petiole or craaoxylon?; Upper Deronian; Saalfeld, Thuringia. See also Seward, 1917, p. 204 ; and Posthumus, 1931; This binomial first cited in Unger, 1854 ; nom. nud.
MEGALOSPERMUM E. A. N. Arber, 1914. Megalospermum widlii (Kidston) D. A. N. Arber, 1914, p. 91, pl. 7, fig. 28 ; seed ; Carboniferous.

MEGALOXYLON Seward, 1899.

Megaloxylon scotti, Seward, 1899, p. 172, pls. 5-7; pteridosperm stem; Upper Carboniferous ; Lancashire, England.

MEGALOZAMIA Hosius and Marck, 1880.

Megalozamia falciformis Hosius and Marck, 1880 , p. 203, pl. 43, figs. 181183 ; cycadophyte petiole fragment; Lower Cretaceous; Westphalia.

MEGAPHYTON Artis, 1825.

Megaphyton frondosum Artis, 1825, p. 20, fig. 20 ; tree fern trunk showing vertical row of large leaf scars; Carboniferous; near Rowmarsh, Yorkshire, England.

MEGATHEOA H. N. Andrews, 1940.

Megatheca thomasii $\mathrm{B}$. N. Andrews, 1940, p. 597, figs. 1-3; large pteridosperm cupule, probably identical with Calathospermum Walton; Oil Shale group, Carciferous Sandstone series, LowerCarboniferous ; Broxburn, West Lothlan, Scotland.

MEIBOMITES Knowlton, 1926.

Meibomites lucens Knowlton, 1926, p. 44, pl. 28, fig. 10 ; leaf, Papilionacene ; Latah formation, Miocene; Spokane, Wash.

MELANCONITES Goeppert, 1852.

Melanconites serialis Goeppert, 1852c, p. 487, nom. nud.

MELANOSPHAERITES Gruss, 1928.

Melanosphaerites devonicus Gruss, 1928a, p. 353, figs. $16,19,24$; fungus; Devonian; Bear Island, Norway.

MELANOSPORITES Pampaloni, 1902.

Melanosporites stefanii Pampaloni, 1802, p. 127 , pl. 10, fig. 12 ; fungus perithecium; Mtocene; Melilli, Sicily.

MELASTOMACEOPHYLLUM (G в I e r) Kräusel, 1929.

First? species described : Melastomaceophyllum geyleri Kräusel, 1929, p. 36, pl. 7 , fig. 1 ; leaf, Melastomaceae; Tertiary (Upper Miocene?) ; Suban Pulut, South Sumatra. Genus first cited: Melastomaceophyllum sp. Geyler, 1887a, p. 503, p1. 35. fis. 6 .

MELASTOMITES Unger, 1850.

Melastomites druidum Unger, $1850 \mathrm{a}, \mathrm{p}$. 480, leaf, Melastomaceae; Eocene; Sotzka, Styria. See also Unger, 1851, v. 181. p1. 55, flgs. 1-o.

MELIACEAECARPOM Menzel, 1913.

Meliaceaecarpum ligniticum Menzel, 1913. p. $39, \mathrm{pl}, 4$, fig. 22 ; fruit, Meliacede ; Tertiary (Braunkohle) ; near Herzogenrath, Prussia. 
MELICARYA Reid and Chandler, 1933.

Melicarya variabilis Reid and Chandler, 1933, p. 280, pl. 11, flgs. 20-24 ; fruit, Meliaceae; London Clay, Eocene; Sheppey, Kent, England.

MELICYTEPHYLLITES Hector, 1880.

Melitoxylon ungeri Hartig, 1848a, p. 171 ; 49, nom, nud.

MELITOXYLON Hartig, 1848.

Melitoxylon ungeri Hartig, 1848a, p. 171; wood; Tertiary ; Germany.

MELOBESITES Massalongo, 1857.

Melobesites membranacea Massalongo, 1857b, p. 777 ; Eocene; Monte Bolca, Italy.

MELOPHYTUM Debey and Ettingshaugen, 1859.

Melophytum cyclostigma Debey and EttIngshausen, $1859 \mathrm{~b}$, p. 241 , pl. 7, flgs. 28-30; incertae sedis; Upper Carboniferous; Aaachen, Rhenish Prussia.

MEMBRANITES Fucini, 1938.

Reference not seen; cited in Gothan, $1942 \mathrm{~b}, \mathrm{p} .132$.

$M E M I N E L L A$. Morellet and Morellet, 1913.

Meminella larvarioides Morellet and Morellet, 1913, p. 13, pl. 1, figs. 41, 42 ; alga, Dasycladaceae ; Eocene ; Chaussy, Croix-Blanche near Gisors, France.

MEAGEA Conwentz, 1886.

Mengea palaeogena Conwentz, 1886, p. 102, pl. 10, figs. 13-16; flower, in amber, Rosaceae; Tertiary ; West Prussia.

MENIPHYLLOIDES E. W. Berry, 1916.

Meniphylloides ettingshauseni E. W. Berry, 1916b, p. 166, pl. 11, figs. 4-7 ; leaf, Polypodiaceae ; Grenada formation, lower Eocene; Grenada, Grenada County, Miss.

MENIPHYLLUM Ettingshausen, 1879.

Meniphyllum elegans Ettingshausen in Gardner and Ettingshausen, 1879, p. 36. pl. 3, figs. 10-14; fern leaf fragments, Aspldeae; Hocene; Bournemouth, England.

MENISPERMAOITES Ettingshausen, 1879. Minispermacites abutoides Ettingshausen, 1879 , p. 394 ; Eocene; Sheppey, England; nom. nud.

MENISPERMITES Lesquereux, 1874.

Menispermites obtusiloba Lesquereux. 1874 , p. 94 , pl. 25 , figs. 1,2 ; pl. 26 , fig. 3 ; leaf, dicotyledon; Cretaceous ; Nebraska?

MENISPERMOPHYLLUM Velenovsky, 1901.

Menispermophyllum celakovskit Velenovsky, in Fric and Bayer, 1901, p. 128, fig. 90 ; leaf, dicotyledon; Cretaceous (Cenomanian); Bohemia. First citatIon : Valenorsky, 1889, p. 54 ; nom. nud.

MENOPTERIS Stenzel, 1889.

Menopteris dubia (Cotta) Stenzel, 1889, p. 12 , pl. 3, flgs. $19-26$; fern stem; Fermian; Chemnitz, Germany.
MEN YPHYLLUM Ettingshausen, 1878.

Menyphyllum elegans Ettingshausen and Gardner, 1878, p. 227 ; fern, Aspidiaceae; Eocene; Bournemouth, England; nom. nud.

MEIISTOPHYLLUM Zalessky, 1937.

Meristophyllum sojanaeanum Zalessky, $1937 \mathrm{~b}$, p. 99 , fig. 76 ; leaf, incertae sedis ; Permian ; Russia.

MERISTOPTERIS Zalessky, 1937.

Meristopteris laciniata Zalessky, 1937e, p. 590, fig. 8 ; incertae sedis; Upper Devonian; near village of Styla, Donets Basin, Russia.

MEKTENSIDES Fontaine, 1883.

Mertensides bullatus (Bunbury) Fontalne, 1883 , p. 35 ; pl. 15 , figs. $2-5$; pl. 16 , figs. $1-3$; pl. 17 , flgs. 1,2 ; pl. 18 , flgs. 1,2 ; fertile fern foliage; Triassic; Carbon Hill, Va. Apparently no connection with Mertensites of Wanklyn.

MERTENSITES Wanklyn, 1869.

Mertensites hantoniensis Wanklyn, 1869, p. 11, pl. 1, figs. 1, 2 ; fertile fern follage, Gleicheniaceae; "Bournemouth leaf bed," Miocene; Bournemouth, England. Intended as subgenus of Gleicheniaf but is actually used as a generic designation.

MESEMBRIOXYLON Seward, 1919.

Mesembrioxylon woburncnse (Stopes) Seward, 1919, p. 207 ; coniferous wood; Lower Greensand, Cretaceous; Woburn, Bedfordshire, England. For Podocarpoxylon woburnense Stopes, 1915, p. 211, pl. 20, figs. 1, 2.

MESOCALAMITES Hirmer, 1927.

Mesocalamites roemeri (Goeppert) Hirmer, 1927, p. 382 ; Calamitaceae ; Lower Carboniferous; varlous localities. For Calamites roemeri Goeppert, 1850, p. 45, pl. 7, fig. 6.

MESOLONCHOPTERIS Koldzumi, 1936.

Mesolonchopteris reticulata (Fontaine) Koldzumi, 1936, p. 143. For Cladophlebis reticulata Fontaine, in Ward, 1900a, p. 235, pl. 21.

MESONEURASTER Sandberg, 1866.

Mesoneuraster cordatus (Goeppert) Sandberger, 1866, p. 76, pl. 5, figs. 1-3 ; neuropterid follage thought to bear sporangia ; Permian.

MESONEVRON Unger, 1856.

Mesonevron lygioides Unger, 1856, p. 172, pl. 8 , fig. 18 ; Incertae sedis ; Upper Devontan; Saalfeld, Thuringia. See also Mesoneuron in Posthumus, 1931.

MESOPHYLLUM Lemoine, 1930.

Mesophyllum austriacum Lemolne, 1930, p. 538, pl. 2, fig. 17a ; Upper Cretaceous (Danian); Bruderndorf near Vienna, Austria. 
MESOPITYS Zalessky, 1911.

Mesopitys tchihatcheffanus (Goeppert) Zalessky, 1911a, p. 28, pl. 1 ; pl. 2, figs. 1-5. For Araucarites tchichatcheffianus Goeppert, 1950, p. 235.

MESOSIGILLARIA (Grand'Eury) Weiss and Sterzel, 1893.

Mesosigillaria lepidodendrifolia (Brongniart) Weiss and Sterzel, 1893, p. 249. For Sigillaria lepidodendrifolia Brongniart, 1828 a-38, p. 426 , pl. 161.

MESOSTROBUS Watson, 1909.

Mesostrobus scottii Watson, 1909 , p. 390 , pl. 27 ; lycopodiaceous cone; Mountain 4-foot mine, Lower Coal Measures, Upper Carboniferous; Cloughfoot, Dulesgate, England.

MESOXYLOIDES Maslen, 1930.

Mesoxyloides platypodium Maslen, 1930, p. 515 , pl. 25 , figs. $4-6$; pl. 26 ; pl. 28 , fig. 19 ; cordaitean stem; Lower Coal Measures, Upper Carboniferous; Shore, Littleborough, Lancashire, England.

MESOXYLON Scott and Maslen, 1910.

Mesoxylon sutcliffi (Scott) Scott and Maslen, 1910, p. 237 ; cordaitean stem; Lower Coal Measures, Upper Carboniferous ; Lancashire, England. See Scott, 1909 , p. 511, fig. 184.

MESOXYLOPSIS Scott, 1919.

Mesoxylopsis arberae Scott, 1919, p. 17, pl. 1, figs. 7-9; pl. 2, figs. 11-14; cordaitean stem; Lower Coal Measures, Upper Carboniferous; Shore, Littleborough, Lancashire, England.

METACAENOXYLON Zalessky, 1935.

Metacaenoxylon carpentieri Zalessky, $1935 a$, p. 740 , pl. 1 , figs. $3-5$; pl. 2 , figs. 4-6; Permian; village of Dratchonina, Kuznets Basin, Russia.

METACALAMOSTACHYS Hirmer, 1927.

Metacalamostachys palaeacea (Stur) Hirmer, 1927 , p. 454, fig. 544 ; Upper Carboniferous; Loire, France.

METACEDROXYLON Holden, 1913.

Metacedroxylon araucarioides Holden, 1013 , p. $538, \mathrm{pl}, 40, \mathrm{fg}_{\mathrm{g}}, 17-21$; coniferous wood; Jurassic (Oolite) ; Whitby and Scarborough, England.

METACLEPSYDROPSIS Paul Bertrand, 1907.

Metaclepsydropsis duples (Williamson) Paul Bertrand, 1907, p. 776, coenopterid fern; Carbonlferous. See also Bertrand, Paul, 1908, p. 121, pl. 2, fig. 7 ; and Posthumus, 1931.

METACORDAITES Renault, 1896.

Metacordaites rigolloti Renault, $1896 \mathrm{~b}, \mathrm{p}$. 91, figs, $1-10$; cordaitean stem; CarbonIferous ; Autun, France.
METACUPRESSINOXYLON Torrey, 1923.

Metacupressinoxylon cedroides (Holden) Torrey, 1923, p. 84. For Paracupressinoxylon cedroides Holden, 1913, p. 537, pl. 39, figs. 11-14 ; Jurassic ; Yorkshire, England.

METASEQUOIA Miki, 1941.

Metasequoia disticha (Heer) Miki, 1941, p. 262 , pl. 5 ; cones and foliage, Taxodiaceae; lower Pliocene; central Hondo, Japan.

METASOLENOPORA Yabe, 1912.

Metasolenopora rothpletzi Yabe, 1912, p. 2, pl. 1, flgs. 2, 3; alga ; Upper Jurassic to Lower Cretaceous; Shikoku, Japan.

METROSIDEROPHYLLITES Hector, 1880. Metrosiderophyllites ovata Hector, 1880 , p. 49 ; nom. nud.

METZGERIITES Steere, 1946.

Metzgerites glebosus (Harris) Steere, 1946, p. 306 ; liverwort, Jungermanniales; Thaumatopteris zone, Lower Jurassic (Liassic); Nelll's Cliff, Scoresby Sound, east Greenland. For Hepaticites glebosus Harris, 1931b, p. 4, pl. 1, figs. 3, 4.

MEYENITES Unger, 1842.

Meyenites aequimontanus Unger, 1842, p. 102 ; wood ; Mlocene; Gleichenberg, Styria. See also Unger, 1854c, p. 183, pl. 7, figs. 4-6.

MIADESMIA C. E. Bertrand, 1895.

Miadesmia membranacea C. E. Bertrand, 1895 , p. 588 ; lycopod cone ; Carboniferous; Hough Hill, Staleybridge, Eng. land. See also Benson, 1908, pls. 30-37.

MIOHEEVIA Zalessky, 1930.

Micheevia uralica Zalessky, 1930a, p. 738, pl. 72, flgs. 1-4; pl. 73, flg. 2; lycopod stem impression ; Carboniferous ; Podossinino, Oural, Russia.

MICONIIPHYLLUM Dusen, 1908.

Miconiiphyllum australe Dusen, 1908, p. 2 pl. 1, fig. 14 ; leaf, dicotyledon; Tertiary ; Seymour Island, Antarctic Ocean.

MICROCHEIRIS Harris, 1935.

Michrocheiris enigma Harris, 1935, p. 118, pl. 8 ; seed-bearing organ, Caytoniales? ; Thaumatopteris zone, Rhaetic; Scoresby Sound, east Greenland.

\section{MICROOHORTON Reis, 1923 ?}

Microchorton claviger Reis, 1923, p. 109 pl. 3, flgs. 10-13; pl. 5, fig. 1 ; Tertiary ; Rhenish Prussia.

MTOROCOCOITES Meschinelli, 1898.

Micrococcites lepidophagus (Renault) Meschinelli, 1898, p. 62, p1. 18, Ag. 13. pl, 10, tgs, 5,6 ; Schizomycete, in coprolite ; Permian. 
MICROCOCCUS Renault, 1895.

Micrococcus quignarli Renault, 1895a, p. 218 ; bacterfa; Upper Carboniferous (Stephanien); Grand Croix, France. .See also Renault, 1895b, p. 450, fig. 10.

MICROCODIUAf Gluck, 1912.

Hicrocodium elegans Gluck, 1912, p. 4, pls. 1-4; Tertiary (Braunkohle); Baden, Germany.

MICRODICTYON Saporta, 1872.

Microdictyon rutenicum Saporta, 1872-73, p. 309 , pl. 33 , fìgs. $2-4$; pl. 35 , fig. 3 ; pl. 44, fig. 5 ; fern follage; Jurassic; Liquisse, France.

MICROLDPIDIUM Velenorsky, 1889.

Microlepidium striatulum Velenovsky, 1889 , p. 11, pl. 1 , figs. 25-27 ; cone, Coniferales; Upper Cretaceous ; Lipenec, Bohemia.

MICROPHYCUS Matthew, 1890.

Microphycus catenatus Matthew, $1890 \mathrm{n}, \mathrm{p}$. 146, pl. 5, flg. 6; alga?; Cambrian; Canada.

MICROPHYLLOPTERIS E. A. N. Arber, 1917.

Microphyllopteris pectinata (Hector) E. A. N. Arber, 1917 , p. 40 , pl. 7, figs. 3-6, 8-11; Lower Jurassic; Matuura Falls, New Zenland; and Cretaceous (Neocomian); Waikato Heads, New Zealand.

MICROPODIUM Saporta, 1861.

Micropodium oligospermum Saporta in Heer, 1861, p. 149 ; seed, Leguminosae? ; Eocene; Aix, Provence, France. See also Saporta, $1873 a$, p. 123, pl. 18, flg. 1.

MICRORRHAGION Ettingshausen, 1883.

Microrrhagion liversidgei Ettingshausen, 1883 , p. 112 , pl. 1 , figs. 7-11. ; monocotyledonous infructescence? ; Tertiary ; Wallerawang, New South Wales.

MICROSPERMOPTERIS Baxter, 1949.

Microspermopteris aphyllum Baxter, 1949, p. 289 , pls. $2-5$; petrified stem, Pteridospermae?; Des Moines group, Pennsylvanian; What Cheer, Iowa.

MICROSPERMUM E. A. N. Arber, 1914.

Microspermum samaroides (Carpenter) E. A. N. $A$ rber, 1914 , p. 106 , and 90 , pl. 7 ; seed ; Carbonfferous (Westphalian) ; northern France.

MICROSTROMIUM Reinsch, 1881.

Microstromium sp. Reinsch, 1881, p. 91, pl. 31a, figs. 1- 7 ; Upper Carboniferous ; Zwlekau, Saxony.

MICROTAENIA Knowlton, 1918.

Microtaenia variabilis Knowlton, 1918, p. 81 , pl. 29, figs. 1-4 ; fertile fern foliage, Polypodiaceae ; Frontier formation, Lipper Cretaceous; Cumberland (15 miles south of Kemmerer), Wyo.
MICROTESTA Chapman, 1927.

Miorotesta triassica Chapman, $1927 \mathrm{~b}$, p. 144, pl. 12, fig. 38 ; seed, incertae sedis; Triassic; Rald Hill, Bacchus Marsh, Victoria.

MICROTHYRIACITES Cookson, 1947.

Microthyriacites fimbriatus $\mathrm{Cook}$ son, 1947 b, p. 211 , pl. 13, fig. 17 ; ascomata, Microthyriaceae ; Oligocene-Mlocene ; 'Travalgon, Victoria.

KICROTHYRITES Pampaloni, 1902.

Microthyrites disodilis Pampaloni, 1902, p. 127, pl. 1.1, fig. 1 ; fungus perithecium?: Miocene; Melilli, Sicily.

UICROTINOMISCIUM Reid and Chandler, 1933.

Microtinomiscium foveolutum Reid and Chandler, 1933, p. 164, pl. 4, figs. 5, 6 ; fruit, Menispermaceae; London Clay, Eocene; Minster, Kent, England.

MICROZYGIA. Read, 1936.

Microzygia lacunosa Read, $1936 \mathrm{~b}$, p. 223, fig. 9 ; petrified petiole, Palaeopteridales: New Albany shale, Upper Devonian; Junction City, Boyle County, Ky.

IIILDRAEDIODENDRON Harms, 1920.

Mildraediodendron excelsum Harms, in Menzel, 1920, p. 26 ; Pleistocene ; Jonje, Dibundja, Africa.

WILLERIA Lang, 1926.

Milleria thomsoni lang, 1926, p. 790, pl. 1, ligs. 6-8 ; fertile "frond," compared with Aneurophyton germanicum; Old Red Siudstone, . Devonian; Yesknary, Orkney, Scotland.

UIMOSITES Bowerbank, 1840.

Mimosites browniana Bowerbank, 1840, p. 140 , pl. 17 , fig. 42 ; fruit, Leguminosae ; Eocene ; Isle of Sheppey, Kent, England.

MINSTEROCARPUM Reld and Chandler, 1933.

Minsterocarpum alatum Refd and Chand. ler, 1933 , p. 416 , pl. 21, flgs. 26-31; fiutt, Lythraceae; London Clay, Eocene ; Sheppey, Kent, England.

KIQUELITES Goeppert, 1854.

Miquelites elegans Goeppert, 1854, p. 56, pl. 1, figs. 6, 7 ; wood, incertae sedis; Tertiary ; Java.

KIRBELLITES Unger, 1845.

Mirbellites lesbius Unger, 1845, p. 242 ; wood; Tertiary?; Island of Losbos, Greece.

MITCHELDEANIA Wethered, 1886.

Mitcheldeania nicholsoni Wethered, 1886, p. 535 , pl. 14 , fig. 6 ; plant?; Lower Carboniferous; Mitcheldean, England.

MITROPICEA Debey, 1848.

Mitropicea decheni Debey, 1848, p. 120 ; nom. nud. 
MITROSPERMUM Arber, 1910.

Mitrospermum compressum (Williamson) Arber, 1910, p. 503, pls. 37-39 ; petrifled seed, Cordaitales?; Lower Coal Measures, Upper Carboniferous; Oldham, Lancashire, England.

MITSCHERLICHIA Lorenz, 1904.

Mitscherlichia chinensis Lorenz, 1904, p. 194 ; alga ; Cambrian ; Shantung, China.

MITTAGIA Lignier, 1913.

Mittagia seminiformis lignier, 1913, p. 65, pl. 8 ; sporangia ; Carboniferous (Westphalien) ; Ostrau, Silesia.

MIXONEURA C. E. Weiss, 1870.

Mixoneura obtusa (Brongniart) C. B. Weiss, $1870 \mathrm{a}$, p. 865 . For Odontopteris obtusa Brongniart, 1828-38, pl. 78, figs. 3, 4 ; fernlike foliage ; Carboniferous.

MIZZIA Schubert, 1908.

Mizzia velebitana Schubert, 1908, p. 382, fig. 5 ; pl. 16, figs. 8-12 ; Upper CarbonIferous; Dalmatia, Austria-Hungary.

MOELLERINA Ulrich, 1886.

Moellerina greenei Ulrich, 1886 , p. 34 , pl. 3, fig. 8 ; Devonian ; Ohio.

MOHLITES Unger, 1839.

Mohlites parenchymatosus Unger, 1839, p. 13. See Unger, 1854, p. 182, pl. 6, figs. 14-16.

MOKRAWIA Kंnopp, 1933.

Preuss. geol. Landesanst. Inst. Paläobotanik u. Petrographic Brennsteine Arb., Band 3, p. 158 (not seen, cited in Gothan, 1942b, p. 133).

MOLASPORA Schemel, 1950.

Molaspora rugosa Schemel, 1950, p. 753, fig. 12 ; spore?; Cretaceous; Plymouth County, Iowa.

MOLTENIA Du Toit, 1927.

Moltenia dentata Du Toit, 1927, p. 380, fig. 20 ; bennettitalean? leaf ; Molteno beds, Upper Triassic ; Waterfal, Upper Umkomas Valley, Natal.

MOMIPITES Wodehouse, 1933.

Momipites coryloides Wodehouse, 1933, p. 511, flg. 43 ; pollen, Ulmaceae ; Parachute Creek member, Green River formation, Eocene; Colorado and Utah.

MONEMITES Massalongo, 1850.

Monemites codioides Massalongo, 1850, p. 25 ; alga ; Eocene : Monte Bolca, Italy.

MONHEIMIA Debey and Ettingshausen, 1859.

Monheimia polypodioides Debey and Ettingshausen, $1859 \mathrm{~b}$, p. 211, pl. 3, figs. 34-36 ; pl. 4, figs. 1, 2, 21 ; fern frond fragment; Upper Cretaceous; Aachen, Rhenish Prussia.

MONILITES Pampaloni, 1902.

Monilites albida Pampaloni, 1902, p. 128, pl. 11, fig. 2 ; fungus mycellum with conidia ; Miocene; Melilli, Siclly.
MONIMIOPSIS Saporta, 1868.

Monimiopsis amboraefolia Saporta, 1868, p. 361, pl. 8, fig. 13 ; leaf, Monimiaceae ; Eocene; Sézanne, France.

MONOCARPELLITES Perkins, 1904.

Monocarpellites whitfleldii Perkins, 1804, p. 180 , pl. 76 , fig. 21 ; fruit ; Tertiary ; Brandon, $\nabla t$.

MONOCARPIA Jongmans and Gothan, 1935. Jaarb. mijnwezen Nederlandish-Indië, 1930, Verh., boekdeel 59, p. 97 (not seen, cited in Gothan, 1942b, p. 133).

MONOCERAS.

Apparently error for Monocerocarpus, in Gothan, 1909, p. 399.

MONOCEROCARPUS Raciborski, 1909.

Monocerocarpuş miocaenicus Raciborskt. 1909, p. 283 , fig. 4 ; Miocene ; Tjitatjap, Pupu Merak, Java.

MONOCOTYLOPHYLLUM Reid and Chandler, 1926.

Monocotylophyllum sp. Reid and Chandler, 1926, p. 87, pl. 5, fig. 12 ; monocotyledonous leaf, family uncertain; Bembridge marl, Oligocene; Isle of Wight, England.

MONODOROSPERMUM Warburg, 1897.

Monodorospermum bangkanum Warburg, 1897, p. 232, pl. 4, figs. 1-5 ; Pliocene; Bangka Island, Indonesia.

MONOLETES (Ibrahim, 1933) emended by Schopf, Wilson, and Bentall, 1944.

Monoletes ovatus Schopf, 1936, p. 108, fig. 7. See also Ibrahim, 1933, p. 39 ; Schopf, Wilson, and Bentall, 1944, p. 38.

MONOPHYLLITES Kuntze, 1904.

No species name assigned, in Post and Kuntze, 1904, p. 373.

MONOSPHENOPHYLLUM Lotsy, 1909.

No new combination actually made but intended as Monosphenophyllum daw. soni (Williamson) Lotsy, 1909, p. 525 , fig. 349 , III.

MONOSULCITES Erdtman, 1948.

Monosulcites magnolioides Erdtman, 1948. p. 269, fig. 11; pollen, aflnities with Magnolia?; Lower Jurassic (Llassic) ; Palsjo, Scania, Sweden.

MONTIELLA (MUnter-Chalmas) Morellet and Morellet, 1922.

Montiella munieri Morellet and Morellet, 1922 , p. 12, pl. 9, fgs. 31, 32; alga, Dasycladaceae; Eocene (Montieni; Mons, Belgium.

MORANIA Walcott, 1919.

Morania confluens Walcott, 1919, p. 226 , p1. 43, аgs. 1-6; p1. 14, Ags. 1-1i ; pr. 45, fig. 1 ; pl. 58, fig. 3 ; alga, Nostocaceae; Middle Cambrian; 1 mile northeast of Burgess Pass, above Field, British Columbia. 
MORANIA Seward and Sahni, 1820.

Morania oldhami (Zeiller) Seward and Sahni, 1920; coniferous shoot; Lower Gondwana," "Permo-Carboniferous" ; Moran Valley, India. Sce Moranocladus. For Araucarites oldhami Zelller, 1902, p. 36, pl. 7, fig. 6 .

MORANOCLADUS Seward and Sahni, 1926. Moranocladus oldhami (Zeiller) Seward and Sahni, 1926, p. 288. Change of name for Morania Seward and Sahni; see above.

MIOREAUIA Pomel, 1849.

Moreauia araucarina Pomel, 1849, p. 350.

MORELLETOPORA Varma, 1950.

Morelletopora nammalensis Varma, 1950, p. 207, 2 figs.; alga, Dasycladaceae; Paleocene; Nammal Gorge of the Punjab Salt Range, India.

MOREOPHYLLUM Geyler, 1887.

Moreophyllum sp. Geyler, 1887 a, p. 492 , pl. 34 , figs. 4,5 ; leaf fragments, Moracene; Eocene; Labuan, Borneo.

MORESNETIA Stockmans, 1946.

Moresnetia zalesskyi Stockmans, 1946a, p. 1, fig. 1 ; Upper Devonian; Belgium. For full account, see Stockmans, 1948, p. 55, pl. 9, figs. 1-7a.

MORICONIA Debey and Ettingshausen, 1859.

Moriconia cyclotoxon Debey and Ettlngshausen, 1859b, p. 239, pl. 7, figs. 23-27 ; fern follage; Upper Cretaceous; Aachen, Rhenish Prussia.

MORINDIDIUM Stiehler, 1861.

Morindidium brongniarti Stiehler, 1861, p. 124.

MORINIUM Ettingshausen, 1854.

Morinium populifolium Ettingshausen, in Reuss, 1854, p. 740 ; Cretaceous (Cenomanian) ; Moletein, Moravia ; nom. nud.

MOROSPORIUM Renualt and Roche, 1898.

Morosporium lignitum Renault and Roche. 1898 , p. 227 , pl. 13 , flgs. $1-3$; fungus mycelium with conidia; Eocene; Herault, France.

MUUEDITES Bertrand and Renault, 1896.

Mucedites stercoraria Bertrand and Renault, in Renault, 1896a, p. 443, figs. 91,92 ; fungus, in coprolites; Upper Carboniferous ; Igornay, France.

MUCORITES Meschinelli, 1898.

II ucorites combrensis (Renault) Meschinellf, 1898 , p. 9 , pl. 5 , flg. 13 ; fungus, Phycomycete, in lycopod macrospore; Carboniferous; Loire, France.

MUCORODIUM Zalessky, 1915.

Mucorodium paleomycotdes Zalessky, 1915, p. 57 ; pl. 4, figs. $1-4$; pl. 5 ; pl. 6 , figs. $1-3$; pl. 7 , figs. 1-6; mycelium, Mucoracene; Carboniferous; Russia.

MUCUNITES Heer, 1859.

Mucunites grepini Heer, 1859, p. 103, pl. 134, flgs. 0-12; Tertiary; Switzerland.
MUNDAPTERIS Teixeira, 1948.

Mundapteris delicata Telxeira, 1848, pl. 44 , figs. $1-9$; fernlike foliage; Cretaceous; Vila Verde de Tentugal, Portugal.

MUNIERIA Hantken, 1883.

Munieria baconica Hantken, in Deecke, 1883 , p. 9, pl. 1, flgs. 4-10; siphonaceous alga; Cretaceous; Bakony, Hungary.

MUNIERINA Viguier, 1907.

Mrunierina oecenica, Viguier, 1907a, p. 605 ; flower, Ranunculaceae?; Eocene; Sezanne, France.

MÜNSTERIA Sternberg, 1833.

Miinsteria vermicularis Sternberg, 1833 (1820-38), p. 32 , pl, 1, flg. 3 ; alga?; Jurassic ; Solenhofen, Bavarla.

MUROHISONITES Goeppert, 1859.

Murchisonites forbesii Goeppert, 1859, p. 441 , pl. 35, fig. 1 .

IIUSAEITES Presl, 1838.

Musaeites primaevus PresI, in Sternberg. 1838 (1820-38), p. 191, pl. 39, fig. 6; stem, incertae sedis; Carboniferous; Kruschowitz, Bohemia.

MUSCITES Brongniart, 1828.

Muscites tournalii Brongniart, 1828 $(1828 \mathrm{a}-38)$, p. 93 , pl. 10, flgs. 1, 2 ; moss; Tertiary; Armissan near Narbonne, France.

MUSTTES.

Mistake? for Muscites, in Pimenova, 1929, p. 192 .

MUSCOCARPUM (Brongniart) Grand'Eury, 1877.

Musocarpum prismaticum Brongniart, in Grand'Eury, 1877, p. 184, pl. 15, flg. 3 (plate is labelled Trigonocarpus?); seed ; Carbontferous ; Roche-la- Mollère, France. See Musocarpum prismaticum Brongniart, 1828b, p. 137 ; nom. nud. ; also Seward, 1917, p. 361.

MUSOPHYLLUM Goeppert, 1854.

Ifusophyllum truncatum Goeppert, 1854, p. 39 , pl. 7 , fig. 47 ; leaf fragment, referred to Musaceae; Eocene; Java. Earlier citation by Geoppert, 1853a, p. 434 ; nom. nud.

MUSOXYLON Meschinell and Squinabol, 1893.

Musoxylon antracotherit (Massalongo) Meschinelli and Squinabol, 1893, p. 194 ; Scitaminaceae ; Tertiary; Italy.

MYCOGEMMA Zalessky, 1915.

Mycogemma saccharomycoides Zalessky, 1915 , p. 64 ; pl. 10, figs. 5-7; pl. 11; mycellum, Ascomycete?; Carbonlferous ; Russia.

MYCORHIZONIUM F. E. Welss, 1904.

Mycorhizonium sp. F. E. Weiss, 1904a, p. 264 , pls. 18,19 ; mycorrhizal fungus; Hallfax Hard bed; Upper Carboniferous ; England. 
MYELOCALAMITES Grund'Eurs, 1877 Myelocalamites approximatus (Schlotheim) Grand'Eury, 1877, p. 510. For Calamites approximatus Schlotheim, 1820, p. 399 ; see also Artis, 1825, p. 4 , p1. 4 .

MYELOPHYOUS Ulrich, 1904.

Myelophycus curvatum Ulrich, 1904, p. $145, \mathrm{pl}, 13$, fig. 2 ; alga? ; Yakutat formation, Lower Jurassic (Jiassic); Woody Island, Kodiak. Alaska.

MYELOPITHYS Corra, 1845.

Myelopithys medullosa Corda, 1S45, p. 30, pl. 11, figs. 4-8; fragment of Medullosa stem?; Carboniferous ; Mïhlhausen, Bohemia.

MYELOPTERIS Renault, 1874.

Myelopteris radiata Renault, 1874, p. 259 , medullosun vetiole; Permian?; sutun, France.

MYELOXYLON Brongniart, 1849.

Myeloxylon elegans (Cotta) Brongniart, 1849 , p. 109 . For Medullosa elegans Cotta, 1832, p. 61, pl. 12, tigs. 1-5. These illustrations actually convey little information. The following is suggested as a more suitable type species: Hyeloxylon radiatum (Renault) Schenk, see Zeiller, 1890, p. 290, pl. 27, fig. 1 .

MYOPORIPHYLLUM Ettingshausen, 1891. Hyoporiphyllum angustum Ettingshausen, 1891 , p. 291. pl. 5, figs. 24, 25 ; leaf, Asperifoliaceae ; Miocene ; JolnanniStollen, Schoenegg, Styria.

$M Y R C I P H Y L L U M$ Engelhardt, 1891.

Myrciphyllum ambiguaeoides Engelhardt, 1891 , p. 681 , p1. 3, fig. 5 ; leaf fragment, Myrtaceae; Tertiary; Chile.

MTRIASPERMUM C. F. W. Braun, 1840.

Myriaspermum granum C. F. W. Braun, 1840 , p. 105 ; nom. nud.

MYRICAEPHYLLUM EOntaine, 1889.

Myricaephyllum dentatum Fontaine, 1889, p. 316 , pl. 156 , fig. 6 ; leaf, compared with Myrica; Potonac group, Lower Cretaceous; near Brooke, Va.

MYRICANTHIUM Velenovsky, 1889. Myricanthium amentaceum Velenovsky, 1889 , p. 16 , pl. 2 , figs, $24-26$; inflorescence, Myricaceae?; Cretaceous (Cenomanian); Vyserovic, Bohemia.

MYRIOIPHYLLUM Conwentz, 1886

Myriciphyllum oligocenicum Conventz, 1886 , p. 42 , pl, 4, figs. $14-16$; leaf, in amber, Myricacene ; Teltiary; West Prussia.

MYRICIPITES Wodehouse, 1933.

Myricipites aubius Wodehouse, 1933, p. 506, fig. 33 ; pollen, Myricaceae ; Parachute Creek member, Green River formation; Eocenr; Colorado and Utah.
MYRICOPHYLLCM Saporta, 1862.

Myricophyllum gracile Saporta, 1862, p. 255 ; pl. 10, fig. 1; leaf, Proteaceae; Tertiary ; Aix, Provence, France.

MYRIOPHYLLIT'ES Artis, 1825.

Myriophyllites gracilis Artis, 1825, p. 12, pl. 12 ; roots, incertae sedis; Carboniferous ; near Wentworth, Yorkshire, England.

UYRIOPHYLIOIDES Hick and Cash, 1881. Myriophylloides williamsoni Hick and 1881 , p. 404 , pl. 21 ; calamitean roots; Upper Carboniferous; Halifax. England. See also Sewald, 1898, p. 342 .

ITYRIOTHECA Zeiller, 1883.

Myriotheca desaillyi Zeiller, 1883, p. 187, pl. 9, figs. 18-20 ; fertile fern frond; Upper Carboniferous; Pas-de-Calais, France.

MYRISTICOPHYILLUM Geyler, 1387.

Myristicophyllum minus Geyler, 1887a, p. 498 , pl. 33, figs. 5, 6 ; leaf fragments, Myristicaceae; Eocene; Labuan, Borneo.

MYRISTICOXYLON Boureau, 1950.

Myristicoxylon princeps Boureau, 1950a, p. 523, pl. 1, figs. 1, 2 ; Oligo-Mlocene; Sahara Soudanese, Asstlar.

MYRMEKIOPORELLA Pia, 1925.

Myrmekioporella mosana Pia, 1925, p. 85, pl. 1 , fig. 8 ; alga, Siphoneae Verticillatue; Jurassic (Malm); St. Mihiel, France.

MYRSINITES Ettingshausen, 1868.

Myrsinites antiquus Ettingshausen, 1868a, p. 227 , pl. 37 , fig. 26 ; leaf, Myrsinaceae ; Miocene; Priesen, Bohemia.

MYRSINOPHYLLUM Velenovsky, 1889.

Myrsinophyllum varians Velenovsky, 1889, p. 25 , pl. 4, figs. 8,9 ; pl. 5 , fig. 12 ; pl. (i, figs. 10; 11; leaf, compared with Myrsine feruginea (Myrsinaceae); Upper Cretaceous (Cenomanian); Lidic, Bohemia.

MYRSINOPSIS Conwentz, 1886.

Myrsinopsis succinea Conwentz, 1886, p. 118 , pl. 11, figs. 21-23 ; flower, in amber, Mycsinaceac; Tertinry. West Prussia.

IT RTHOHYOPHYTON Massalongo, 1857.

Myrthomyophyton stephanophorus Massalongo, 1857b, p. 777; Focene; Monte Bolca, Italy; nom. nud.

MPRTIFOLIUM Unger, 1864.

Myrtifolium lingua Unger, 1804, p. 10, nl. 4, figs. 1, 2 ; leaf, Myrtaceae ; Tertiary ; Drury, near Auckland, New Zealand.

MYRTIPHYLLUM Dusen, 1899.

Myrtiphyllum bagualense Dusen, 1899, p. 103, pl. 11, figs. 7-9; leaves, compared with Eugenia (Myrtaceae); Oligocene; Baguales, Chile. 
MYRTOMIOPHYTON Massalongo, 1858. Myrtomiophyton stephanophorus Massalongo, 1858 b, p. 769 ; frult, Myrtaceae ; Tertiary. See also Massalongo, 1859a, p. 77, pl. 32 , fig. 1 .

MYRTONIUM Ettingshausen, 1887 .

Myrtonium obtusifolium Ettingshausen, 1887 a, p. 133 , pl. 14 , fig. 20 ; pl. 15 , figs. 14,15 ; leaf, Myrtaceae ; Eocene ; Vegetable Creek, near Emmaville, New South Wales.

MYRTOPHYLLUM Heer, 1869.

Myrtophyllum geinit $i$ Heer, 1869a, p. 22, pl. 11, figs. 3, 4; Upper Cretaceous (Cenomanian) ; Moletein, Moravia.

MYXOMYCETES Renault, 1895.

Myxomycetes mangini Renault, 1895d, p. 77, fig. 2 ; Upper Carboniferous ; Combres, France. Meschinelli, 1898, p. 71, changes the spelling to Myxomycites.

MYXOMYCITES.

See Myxomycetes, Renault.

\section{$\mathbf{N}$}

NAGEIOPSIS Fontaine, 1889.

Nagciopsis longifolia Fontaine, 1889, p. 195 , pl. 75 , fig. 1 ; pl. 76, figs. $2-6$; pl. 77 , figs. 1,2 ; pl. 78 , figs. $1-5$; cycadophyte? foliage; Potomac group, Lower Cretaceous; Fredericksburg, Va.

NAIADEA.

See Naiadita.

NAIADITA Buckman, 1850.

Naiadita lanceolata Buckman, 1850, p. 415, fig. 2 ; emended by Harris, 1938. Original citation appears in Murchison, 1845 , p. 52. Variously spelled as Naiudes, Najadita, Najadites, Naiadea. For comprehensive review and complete synonomy, see Harris, 1938, n. 17-18.

NAIADITES.

See Nuiadita.

NAJADITA.

See Naiadita.

NAJADITES.

See Naiadita.

NAJABONIUM Ettingshausen, 1872.

Najadonium longifolium Ettingshausen, 1872 , p. 173 , pl. 3, figs. 3-5 ; leaf, Najadacene?. Original citation : Ettingshausen, 1871 , p. 410 ; nom. nud.

NAJADOPSIS Heer, 1855.

Najadopsis dichotoma Heer, 1855, p. 104, pl. 48, figs. 1-6; stem fragments?; Najadaceae; Tertlary ; Oeningen, Switzerland.

NAKTONGIA Oishi, 1939.

Naktongia yabei Oishi, 1939, p. 310, pl. 35 , fig. 3 ; fertile fern foliage ; Naktong series, Upper Jurassic ; Korea.
NATHORSTIA Heer, 1880.

Nuthorstia angustifolia Heer, 1880b, p. 7, pl. 1, figs. 1-6; fertile fern pinnules: Cretaceous; Pattorfik, Greenland.

NATHORSTIA Seward, 1894.

Nathorstia valdensis Seward, 1894a, 1. 145, pl. 7, flg. 5 ; fernlike follage; Wealden.

NATHORSTIANA Richter, 1909.

Nathorstiana arturea Rlchter, 1909 (1906$09)$, p. 3 , pl. 8 , figs. $1-3,5,8,13$; pl. 10, figs. 11, 15 ; Lower Cretaceous; Quedlenburg, Prussian Saxony.

NAUCLEOXYLON Crie, 1888.

Naucleoxylon spectabile Crie, 1.888, p. 19, pl. 8, figs. 1, 2 ; Pliocene; Buitenzorg, Java.

NAVA.TOIA Wielund, 1928.

Navajoia magnifica Wleland, 1928, p. 391 ; petrified cycudean trunks; Chuska Mts., N. Mex.; nom. nud.

NECHALEA Dehey, 1848.

Nechalea serrata Debey, 1848, p. 1.15 ; nom. nut.

NECT'ANDROPHYLLUM Engelhardt, 1891.

Nectandrophyllum sp. Engelhardt, 1891, p. 654, pl. 4, fig. 6 ; pl. 9, fig. 12 ; leaf, Lauraceae; Tertiary ; Chile.

NEGUNDOIDES Lesquereux, 1868.

Negundoides acutifolia Lesquereux, 1868, p. 101; leaves, compared with Acer; Cretaceous; north of Fort Ellsworth, Nebr: See also Lesquereux, 1874, p. 97, pl. 21, fig. 5 .

NELUMBITES E. W. Berry, 1911.

Nelumbites virginiensis (Fontaine) E. W. Berry, 1911a, p. 463, pl. 82, figs. 3-5 ; leaf, Nymphaeaceae; Patapsco formation, Lower Cretaceous; Maryland and Virginia.

NFMACLADA John Smith, 1896.

Nemacladia alternata John Smith, 1896, p. 320 , pl. 7 , fig. 10 ; fragment of mycelium, in auber; Upper Carboniferous ; Annandale near Kilmarnock, Scotland.

NEMCALIONIT'ES Massalongo, 1851.

Nemalionites limacoides Massalongo, 1851 , p. 41 ; nom. nud.

NEMAPLANA John Smith, 1896.

Nemaplana filiforme John Smith, 1896, p. 320 , pl. 7, fig. 9 ; fragment of mycelium, in amber; Upper Carboniferous; Annandale near Kilmainock, Scotland.

NEICATOLITES Keeping, 1882.

Nematolites edwardsii Keeping, 1882, p. 489 , pl. 11, figs. 8-11; alga; various localities, central Wales.

NEMATOPHORA Gruss, 1924.

Nematophora fascigera Gruss, 1924. p. 8, pl. 5 , fig. $44 ;$ pl. 6 , figs. $1.0,10,13 ;$ Devonian; Magialena Bay, Spitzbergen. 


\section{INDEX OF GENERIC NAMES OF FOSSIL PLANTS 1820-1950}

NEMATOPHYCUS Carruthers, 1872.

Nematophycus logani (Dawson) Carruthers, 1872 , p. 160 , pls. 21,$22 ;$ a problematical alga?; Devonian; Gaspé, Canada. See Arnold, 1947, p. 52.

NEMATOPHYLLITES S. A. Miller, 1892.

Nematophyllites angustus (Fontaine and White) S. A. Miller, 1892, p. 665. For Nematophyllum angustum Fontaine and White, 1880 , p. 35 , pl. 2, figs. 1-5 ; Permian?; West Union, W. Va.

NEMATOPHYLLUM Fontaine and White, 1880.

Nematophyllum angustum Fontaine and White, 1880 , p. 35 , pl. 2 , figs. $1-5$; apparently close to Asterophyllites; Waynesburg Coal, Pennsylvanian or Permian(?); West Union, W. Vit. See Nematophyllites.

NEATATOPHYTON Dawson, $18 s 8$.

Nematophyton logani Dawson, 1888, p. 21 ; marine alga?; Devonian ; Gaspé, Canada. For Prototaxites logani Dawson, 1859, p. 484, figs. 4a-c. See Arnold, 1947, p. ร2.

NEMATORITES Gruss, 1928.

Nematorites oscillatoriaeformis Gruss, 1928 b, p. 506, pl, 41, figs. $19,20$.

NEMATOTHALLUS Lang, 1937.

Nematothallus pseudovasculosa Lang, 1937, p. 269 , pl. 11, figs. $56,60,61$, 64 ; pl. 12, figs. 70-82 ; incertae sedis ; Downtonian, Devonian ; Perton Quarry, Saltwells, South Pembrokeshire, England.

NEMATOXYLON Dawson, 1863.

Nematoxylon crassum Dawson, 1863a, p. 466 , pl. 19 , fig. 24 ; compared with Prototaxites but with larger cells and no "medullary rays"; Devonian; Gaspé, Canada.

NEOCALAMITES Halle, 1908.

Neocalamites hoerinsis (Schimper), Halle, 1908, p. 6, pls. 1, 2 ; calamitean stem; Lower Jurassic; Helsingborg, Bjuf, Skromberga, etc., Sweden.

NEOCALLIERGON Williams, 1930.

Neocalliergon integrifolium Willams, 1930 , p. 36 , pl. 5, figs. 8-11 ; moss, compared with Calliergon and Calliergonella; Pleistocene; Minneapolis ; Minn.

NEOCHONDRITES Saporta, 1893.

Neochondrites sn. Snnortn, 1903b, p. 121; nom. nud.

NEOGYROPORELLA Yabe and Toyama, 1949.

Neogyroporella elegans Yabe and Toyama, 1949 , p. 163, figs. 5-10; alga, Dasycladaceae; Torinosu limestone, Upper Jurassic ; Hanabata Togano-mura, Japan.

NEOZAMIA Pomel, 1846.

Neozamia joubertiana Pomel, 1846, p. 655. For Flabellaria borassifolia Sternberg, $1820-38$, p. 32 , pl. 18.
NEPHELITES Deane, 1902.

Nephelites equidentata Deane, 1902a; p. 61 , pl. 15 , fig. 3 ; leaf, compared with Quercus dampieri Ettingshausen; Tertiary; Wingello, New South Wales.

VEPHROPSIS Zalessky, 1912.

Nephropsis integerrima (Schmalhausen) Zalessky, 1912, p. 28. A name suggested by Zalessky for Ginkgo integerrima Schmalhausen, 1879 , p. 85 , pl. 16 , figs. 12-15 ; Ginkgo-like leaves ; Permian ; Lower Toungouska, Russia. See also Seward, 1919, p. 77.

VEPHROPTERIS Brongniart, 1849.

Nephropteris obliqua Brongniart, 1849, p. 65. For Cyclopteris obliqua Brongniart, 1828 a-38, p. 221 , pl. 61 , fig. 3 ; cyclopterid "stipule"; Carboniferous; Greenough, Yorkshire. England.

NEREOGRAPSUS Geinitz, 1864.

Nereograpsus jacksoni (Emmons) Geinitz, 1864, p. 6 , pl. 2 , fig. 4 ; plant?.

\section{VERIOPTERIS Newberry, 1873.}

Neriopteris lanceolata Newberry, 1873, p. 381 , pl. 45 ; fernlike foliage; Pennsylvanian; near Cuyahoga Falls, Summit County, Ohio.

VERITINIUM Unger, 1850.

Neritinium dubium Unger, 1850 b, p. 125, pl. 14, fig. 13 ; leaves, Apocynaceae ; Miocene; Radoboj, Croatia. Cited by Unger, 1845 (1841-47), p. 81; nom. nud.

VEURALETHOPTERIS Cremer, 1893.

Neuralethopteris schlehani (Stur) Cremer, 1893, p. 33. For Neuropteris schlehani Stur, 1877 , p. 183 , pl. 11, flgs. 7,8 ; Lower Carboniferous ; Witkowitz, Moravia.

.VEUROCALLIPTERIS Sterzel, 1895.

Neurocallipteris gleichenioides (Stur) Sterzel, 1895 , p. 285 , pl. 8 , fig. 6 ; pl. 9 , fig. 1.

VEUROCARDIOPTERIS Lutz, 1933.

Neurocardiopteris broilii Lutz, 1833, p. 138, pl. 18, figs. 1-10 ; Neuropteris-like follage; Carboniferous (Culm); Germany.

NEURODONTOPTERIS Henry Potonle, 1893.

Neurodontopteris auriculata (Brongniart) Fenry Fotonie, 1893a, p. 12. For Neuropteris auriculata Brongniart, 1830 (1828a-38), p. 236, pl. 66 ; Upper Carboniferous; St.-Etienne, France.

NEUROGANGAMOPTERIS Zalessky, 1918.

Neurogangamopteris cardiopteroides (Schmalhausen) Zalessky, 1918, p. 48, pl. 2 , fig. 1 ; pl. 2 , figs. $7,8,10,11,13$, 14 ; pl. 4, figs. 1,2 ; pinnule, said to combine characters of Neuropteris and Gangamopteris; Permian; Tarbagatai, Russia. 
NEUROPHYLLUM Kon'no, 1941.

Neurophyllum koreanicum Kon'no, 1941, p. 24 , pls. 1,2 ; follage and cones, compared with Phyllotheca and Asterocalamites; Jido series, Lower Permian ; Taedong, South Heiando, Korea.

NEUROPTERIDIUM Schimper, 1879.

Neuropteridium grandifolium Schimper, in Schimper and Schenk, 1879 (1879-90), p. 117 , fig. 90 ; neuropterid pinnule; Lower Triassic.

NEUROPTERIS (Brongniart) Sternberg, 1825.

Neuropteris heterophylla (Brongniart) Sternberg, 1825 (1820-38), Tentamen, p. xvil. For Filicites (Nevropteris) heterophyllus Brongniart, 1822, p. 233, pl. 2, fig. 6. [When flrst used (as a subgenus of Filicites), Brongniart spelled this name with a "v"; it was changed to a "u" (Neuropteris) by Sternberg who gave it generic rank for the first time.]

NEUROPTEROCARPUS (Grand'Eury) Seward, 1917.

Neuropterocarpus lidstoni (Arbor) Seward, 1917, p. 114, fig. 422 ; a name for seeds attached to Neuropteris foliage. See Nevropterocarpus sp. Grand'Eury, 1904, p. 785 (footnote).

NEUROPTEROMEDULLOSA Lotsy, 1909.

Neuropteromedullosa stellata (Cotta) Lotsy, 1909, p. 724, fig. 509. For Medullosa stellata Cotta, 1832, p. 60̃. See note under Pecopteromedullosa.

NEURORAPHD Reid and Chandler, 1933.

Neuroraphe obovatum Reid and Chandler, 1933, p. 491 , pl. 28 , flgs. $37-42$; seed, incertae sedis; London Clay, Eocene ; Minster, Kent, England.

NEUROSPERMUM E. A. N. Arber, 1914.

Neurospermum kidstoni E. A. N. Arber, 1914 , p. 93 , pl. 8 , fig. 47 ; seed (named for seeds previously shown by Kidston to be borne on foliage of Neuropteris heterophylla); Middle Coal Measures, Upper Carboniferous; Clays Croft, Cosely, South Staffordshire, England.

NEUROSPHENOPTERIS Zalessky, 1907.

Neurosphenopteris bohdanowiczi Zalessky, 1907, p. 69. For Sphenopteris bohdanowiczi Zalessky, 1907, p. 65, pl. 2, fig. 2 ; fernlike foliage ; Carbontferous ; Dombrowa, Russia.

NEUROSPORANGIUM Debey and Ettingshausen, 1859 .

Neurosporangium follaceum Debey Ettingshausen $1859 \mathrm{a}$, p. 190 , pl. 1, fig. 5 ; alga; Cretaceous; Aachen, Rhenish Prussia.

NEVROPTERIS.

See Neuropteris.
NEVROSPERMUM Paul Bertrand, 1913.

Nevrospermum heterophyllae Paul Bertrand, 1913, p. 124, flg. 2 ; Bertrand creates this genus for seeds borne on Neuropteris foliage; three species are recorded, the one cited here being the only one illustrated.

NEWBERRYANA E. W. Berry, 1910.

Newberryana rigida (Newberry) E. W. Berry, 1910c, p. 254, Raritan formation, Upper Cretaceous; New Jersey. For Hausmannia rigida Newberry, 1895, p. 35 , pl. 2, flgs. 2, 3, 5 .

NEWLANDIA Walcott, 1914.

Newlandia frondosa Walcott, 1914, p. 105, pl. 5 , flg. 4 ; pl. 6, flgs. 1-3 ; pl. 7, figs. 1,2 ; alga ; Beltian series, Algonkian ; 8 miles west of White Sulphur Springs, Meagher County, Mont.

NIAYSSI Zalessky, 1937.

Palaeophy tographica, Moskau-Leningrad, 1937 b, p. 18 (not seen, cited in Gothan, 1942 b, p. 135).

NIAZONARIA Radsechenko, 1933.

Acad. sci. U. R. S. S., Inst. geologique, 1933, Travaux, tome 3, p. 252 (not seen, cited in Gothan. 1942b, p. 135).

NICOLIA Unger, 1842.

Nicolia aegyptiaca Unger, 1842b, p. 177; wood ; Tertlary ; Egypt. See Unger, 1858 , p. 214 , pl. 1, figs. $1,2$.

NIDCLITES Salter, .1851.

Nidulites favus Salter, in Murchison, 1851, p. 174, pl. 9 , figs. 16,17 ; plant?; Silurian; Pembrokeshire. Wales.

NILSSONIA Brongniart, 1825.

Nilssonia brevis Brongniart, 1825, p. 218, pl. 12, figs. 4, 5 ; cycadophyte follage; Rhaetic ; Hoer, Sweden. For history of genus, see Nathorst, $1909 \mathrm{a}$; 8ee also Harris. 1941.

NILSSONIOPTERIS Nathorst, 1909.

Nilssoniopteris tenuinervis Nathorst, 1909 a, p. 29 , pl. 6 , flgs. $23-25$; pl. 7 , fig. 21; cycadophyte leaf; Jurassic ; Cloughton Wyke, Yorkshire, England.

NIPADITES Bowerbank, 1840.

Nipadites umbonatus Bowerbank, 1840, pl. 1 ; palm fruit; Eocene; Sheppey, Kent, England. See Reld and Chandler, 1933, p. 118.

NIFANIOPHYLLUM Sahni, 1948.

Nipaniophyllum raoi Sahni, 1948, p. 52, fig. 1 ; Taeniopteris-like leaves borne on Pentoxylon; Rajmahal serles, Jurassic ; Nipania, Rajmahal Hills, India.

NIPANIORUHA RaO, 1947.

Nipanioruha granthia Rao, 1947, p. 389 , pls. 1-6 ; petrifled conlferous shoots, affinities with Podocarpineae or Cupressineae; Rajmahal serles, Jurasslc ; Nipania, Rajmahal Hills, India. 
NIPANIOSTROBUS Rao, 1943.

Nipaniostrobus sahnii Rao, 1943a, p. 115, pls. 1-3, 5 ; petrifled seed-bearing cone, Fodocarpaceae?; Rajmahal series, Jurassic ; Nipania, Rajmahal Hills, India.

NIPANIOXYLON Srivastava, 1944.

Nipanioxylon guptai Srivastava, 1944, p. 75, pl. 2, fig. 14 ; petrified stem closely related or actually referable to Pentoxylon; Rajmahal series, Jurassic ; Nipanta, Rajmahal Hills, India. See also Srivastava, 1937 ; 1946, p. 207 ; Sahn1, 1948.

NIPONOPHYLLUM Stopes and Fujil, 1910. Niponophyllum cordaitiforme Stopes and Fujii, 1910, p. 16, pl. 3, figs. 14-16 ; petrified gymnospermous leaves; Upper Cretaceous; Hokkatdo, Japan.

NIPPONOPHYCUS Yabe and Toyama, 1928.

Nipponophycus ramosus Yabe and Toyama, 1928 , p. 142 , pl. 18 , figs. $1-6$; pl. 19 , figs. $1-4$; pl. 23 , figs. 2,3 ; alga, Rhodophyceae; Torinosu limestone, Mesozoic; Tosa, Japan.

NODOPHYCUS Herzer, 1901.

Nodophycus thallyformis Herzer, 1901, p. 26, pl. 1, fig. 2 ; marine alga ; Carboniferous; Marietta, Ohio.

NOEGGERATHIA Sternberg, 1822.

Noeggerathia foliosa Sternberg, 1822 (1820-38), p. 33 , pl. 20 ; fern or cycad frond (see Seward, 1910, p. 428) ; Upper Carboniferous ; Bohemia.

NOEGGERATHIAESTROBUS Ottokar Feistmantel, 1871.

Noeggerathiaestrobus bohemicus ottokar Feistmantel, 1871, p. 59 ; Upper Carboniferous; Radnitz, Bohemia. See also Feistmantel, 1876a, p. 270, pl. 61, fig. 5.

NOEGGERATHIOPSIS Ottokar Feistmantel, 1879.

Noeggerathiopsis hislopi. (Bunbury) Ottokar Feistmantel, 1879, p. 23, pl. 19, figs. 1-6; pl. 20, fig. 1 ; Karharbari beds, Lower Gondwana; Domahni, India.

NOEGGERATHIOSTROBUS Němejc, 1928.

Naeggerathiostrobus bohemicus Němejc, 1928 , p. 53, pl. 1, figs. 2-7; pl. 2, figs. 5-8; Carboniferous; central Bohemia.

NOEOPTERIS Janssen, 1940.

Nocopteris asymmetrica Janssen, 1940, p. 97 , pl. 25 , fig. 3 ; fern stem impression; Pennsylvanian; Mazon Creek, Ill.

NORDENSKIOLDIA Heer, 1870.

Nordenskioldia borealis Heer, 1870 , p. 65, nl. 7, figs. 1-13 ; fruit, Tiliaceae?; Miocene; Kings Bay, Spitzbergen.

NORIMBERGIA Gothan, 1914.

Norimbergia braunii (Goeppert) Gothan, 1914 , p. 19 , pl. 18 , figs. $6-8$; fertile fern frond, Schizneacene; Rhaetic ; Nürnberg, Germany.
NORINIA Halle, 1927.

Norinia cucullata Halle, 1927, p. 218, pl. 56, figs. 8-12 ; cupule?; Upper Shihhotse series, Paleozoic ; Ch'en-chia-yu, central Shansi, China.

NOSTOCITES Maslov, 1929.

Nostocites problematica Masiov, 1929, p. 122, pl. 70, fig. 8 ; Carboniferous ; Donets Basin, Russia.

NOTHOFAGOXYLON Gothan, 1908.

Nothofagoxylon scalariforme Gothan, 1908, p. 20, pl. 2, figs. 14-18; wood, compared with Nothofagus (Fagaceae); Tertiary; Seymour Island, Antarctic Ocean.

NOTHOP'ERIS C. F. W. Braun, 1847.

Nothopteris mysteriosa C. F. W. Braun, 1847 , p. 87 ; nom. nud.

NOTOSCHIZAEA Graham, 1934.

Notoschizaea robusta Graham, 1034, p. 453, flgs. 1-5; pl. 8, flg. 26 ; petrified sporangia, Zygopteridaceae; up per McLeansbolo group, Pennsylvanian; Calhoun coal mine, Richland County, Ill.

NOTOTHYRITES Cookson, 1947.

Notothyrites setiferus Cookson, $1947 \mathrm{~b}, \mathrm{p}$. 209 , pl. 11, figs. 1-6 ; ascomata, Microthyriaceae; late Oligocene; Kerguelen Island near Fort Jeanne d'Arc, South Indian Ocean.

NUBECULARITES Maslov, 1937.

Nubecularites polymorphus Maslov, $1937 \mathrm{~b}$, p. 345 , pl. 4, fig. 1 ; calcareous alga; Middle Cambrian; Vvedenskoye, Russia.

NUCELLANGIUM H. N. Andrews, 1949.

Nucellangium grabrum (Darrah) H. N. Andrews, 1949, p. 491, pls. 35-39 ; sporangia of uncertain affinities, some showing gemmá-type reproductive tissue ?; Des Molnes group, Pennsylvanian; Urbandale coal mine, Des Moines, Iowa.

NULLIPORITES Heer, 1865 ?

Nulliporites hechingensis (Quenstedt) Heer, 1865, p. 140, pl. 9, figs. 18, 19.

NUMMULOSPERMUM Walkom, 1921.

Nummulospermum bowense Walkom, 1921, p. 290 , pl. 21 ; seed, associated with Glossopteris, "Permo-Carbontferous"; Three-Mile Creek, Bowen, Queensland.

NYOTAGINITES E. W. Berry, 1938.

Nyctaginites ellipticus E. W. Berry, 1938. p. 72 , pl. 17, figs. 1, 2 ; leaf, Nyctaginacene; Tertiary; Río Plchileufu, Argentina.

NYCTOMYCES Unger, 1841.

Nyctomyces antediluvianus Unger, 1841 $(1841-47)$, p. 3 , pl. 1 , fig. 3 ; fungus mycelium; M l o c e n e : Gleichenberg, Stvria. 
NYGMITES Mägdefrau, 1937.

Nygmites solitarius (Hagenow) Mügdefrau, 1937, p. 56.

NYMPHAEITES Sternberg, 1825.

Nymphaeites arethusae (Brongniart) Sternberg, 1825 (1820-38), Tentamen, p. xxxix. For Nymphaea arethusae Brongniart, 1822, p. 332, pl. 6, fig. 9 ; Tertiary ; fruit, Nymphaeaceae; Tertiary ; Lonjumeau near Paris, France.

NYMPHAEOPSIS Krüusel, 1939.

Bayer. Akad. Wiss., Math.-naturwiss. Abh., 1939, Neue Folge, Band 47, p. 39 (not seen, cited in Gothan, 1942b, p. 136).

NYSSIDIUM Heer, 1870.

Nyssidium ekmani Heer, 1870, p. 62, pl. 1.5 , figs. $1-5,7$; fruit, Araleaceae; Miocene; Cape Staratschin, Spitzbergen.

NYSSITES Geyler, 1887.

Nyssites obovatus (Weber) Geyler, $1887 \mathrm{~b}$, p. 162. For Nyssa obovata Weber, 1851, p. 184 , pl. 20, fig. 11 ; Oligocene ; Friesdorf, Rhenish Prussia. See also Geyler and Kinkelin, 1887, p. 28, pl. 3, figs. 1-6.

NYSSOIDITES Thiergart ?, 1950.

Nyssoidites rodderensis Thlergart, in Potonie, Robert, Thomson, Paul W., and Thlergart, Frledrich, 1950 , p. 59 , pl. B, fig. 49 ; pollen, Nyssaceae?; Mlocene; Chatt-Aquitan, Germany.

NYSTROEMIA Halle, 1.927.

Nystroemia pectiniformis Halle, 1927, p. 221 , pl. 59 ; seed-bearing organ and $\mathrm{ml}$ crosporangia, Pteridospermae?; Upper Shihhotse series, Paleozolc; Ch'en-chiauy Valley, central Shansi, China.

\section{$\mathbf{0}$}

OCHROSELLA Reid and Chandler, 1933. Ochrosella ovalis Reld and Chandler, 1933, p. 480 , pl. 27, figs. 30,31 ; fruit, Apocynaceae; London Clay, Eocene ; Minster, Kent, England.

OCHROSOIDEA Reld and Chandler, 1933.

Ochrosoidea sheppeyensis Reld and Chandler, 1933, p. 477, pl. 27, figs. 15-29; fruit, Apocynaceae; London Clay, Eocene; Sheppey, Kent, England.

OCHTHODOCARYON Mueller, 1877.

Ochthodocaryon wilkinsonii Mueller, $1877 \mathrm{a}$ (1877-79), p. 178, fruit ; Tertiary ; New South Wales. See also Mueller, 1879 (1877-79), p. 171, pl. 4, figs. 1, 2.

OCOTEOXYLON Schuster, 1908.

Ocoteoxylon ligurinum Schuster, 1908, p. 149 , pl. 2, figs. 1-5 ; wood, Lauracere; Eocene; Tegern Lake, Bavarla.

ODONTOCARYON Mueller, 1873.

Odontocaryon macgregori Mueller, 1873 (1871-82), p. 41, pl. 6, figs. 5-8; Pllocene; Nintingbool, Victoria.
DDONTOPTERIS Brongnlart, 1825.

Odontopteris brardii Brongniart, in Sternberg, 1825 (1820-38), Tentamen, p. xxi. For Filicites brardii Brongniart, 1822, p. 234 , pl. 2, fig. 5. See also Brongniart, $1828 n-38$, p. 252 , pls. 75,76 . In the Sternberg reference the specific name is spelled berardi which is apparently a mistake, for Brongniart originally (1822) employed brardii and retains this in 1828-38.

ODONTOPTEROCARPUS Lubière, 1930.

Odontoptervcarpus oblongus Loublère, 1930, p. 323 ; seeds ; Carboniferous ; St.Etienne, France.

ODONTOSORITES Kobayashl and Yosida, 1944.

Odontosorites heerianus (Yokoyama) Yobayashi and Yosida, 1944, p. 267, 269, pl. 28 , figs. 6,7 ; fern follage, compared with Odontosoria; Jurassic; Ryokusin, Manchuria.

TIDITES Meschinelli, 1892.

Oidites moniliformis (Menge and Goeppert) Meschinell, in Saccardo, 1892, p. 789 ; fungus, Hyphomyceteae. \$ee also Meschinelli, 1898, p. 77.

IIDOSPORA Williamson, 1878.

Oidospora anomala Williamson, 1878, p. 364 , pl. 25, fig. 102 (flgured but not described) ; Carboniferous.

TLDHAMIA Forbes, 1854.

Oldhamia antiqua Forbes, in Murchison, 1854, p. 32 , flg. 1 ; plant?; Cambrian; Bray Head in Wicklow, Ireland.

)LEAECARPUM Menzel, 1913.

oleaecarpum germanicum Menzel, 1913, p. 60 , pl. 5, flgs. $\cdot 25,26$; fruit, Oleaceae; Tertiary (Braunkohle); Germany.

TLEANDRIDIUM Schimper, 1869.

oleandridium vittatum (Brongnlart) Schimper, 1869 (1869-74), p. 607. For Taeniopteris vittata Brongniart, 1828a38 , p. 263 , pl. 82 , figs. $1-4$; now believed to be follage of Williamsoniella. Seo Thomas, H. H., 1915.

OLEARIAPHYLLITES Hector, 1880 .

Oleariaphylltes whaurangi Hector, 1880 , p. 49 ; nom. nud.

TLEINITES Cookson, 1947.

Oleinites willisii Cookson, 1947a, p. 188, pl. 8, flgs. 1-5 ; mummified leaves, probably Oleaceae; Ollgocene-Mlocene; Yallourn. Victoria.

OLEIPHYLLUM Conwentz, 1886.

Oleiphyllum boreale Conwentz, 1886, p. 122, pl. 12, figs. 12-14; leaf, in amber, Oleaceae ; early Tertiary ; West Prussia.

TLERACITES Saporta, 1862.

Oleracites convolvuloides Saporta, 1862, p. 241, pl. 7, fig. 8 ; leaf, Oleaceae; Tertiary ; France. 
OLFERSITES Guembel, 1859 .

Olfersites dichotomus Guembel, $1859 \mathrm{~b}$, p. 161. For Schizeites dichotomus Guembel, 1859 , p. 101, pl. 8 , fig. 7 ; compared with Olfersia peltata; Permian (Rothliegenden) ; Erbendorf, Bavaria.

OLIGOCARPIA. Goeppert, 1841.

Oligocarpia gutbieri Goeppert, $1841 \mathrm{~b}, \mathrm{p}$. 57, pl. 4, figs. 1, 2; fertile fern frond, probably Gleicheniaceae ; Carboniferous ; Saxony.

OLIGOPORELLA PIa, 1912.

Oligoporella pilosa Pia, 1912, p. 42, pl. 4, figs. 1-8; alga, Siphoneae Verticillatae ; Triassic ; Dalmatia, Austria-Hungary.

OMMATOXYLON Hartig, 1848.

Ommatoxylon germari Hartig, 1848a, p. 172 ; wood ; Tertiary ; Germany.

OMPHALOMELA Germar, 1846.

Omphalomela scabra Germar, 1846, p. 29 , pl. 3, fig. a; incertae sedis; Triassic (Keuper) ; Badeleben, Thuringia.

OMPHALOPHLOIOS David White, 1898.

Omphalophloios cyclostigma David White, 1898 , p. 336 , pls. $20-23$; arborescent lycopod stem impression; Pennsylvanian; Clinton, Henry County, Mo.

ONCOBYRSELLA J. H. Johnson, 1937.

Oncobyrsella coloradensis J. H. Johnson, 1937, p. 1235 , pl. 2, figs. 3,4 ; compared with Oncolyrsa, Cyanophyceae ; Antero formation, Oligocene; Park County, Colo.

ONCODENDRON Eichwald, 1860.

Oncodendron mirabile Eichwald, 1860, p. 213 , pl. 16, figs. 7,$8 ;$; pl. 21, fig. 8 ; lycopod? stem; Upper Carboniferous; Bjelebi, Orenbourg, Russia. Earlier citation : Eichwald, in Mercklin, 1856, $p$. 80 ; nom. nud.

ONCOPTERIS Dormitzer, 1853.

Oncopteris nettwalli Dormitzer, in Krejci, 1853, p. 28, pl. 2; Cretaceous (Cenomanian); Kaunitz, Bohemia. See also Posthumus, 1931.

ONOYLOGONATUM König, 1827.

Oneplogonatum carbonarium König, in Murchison, 1827, p. 300, pl. 32 (1829); compared with Equisetum; Jurassic; Brora, Sutherlandshire, Scotland.

ONICHIOPSIS.

Apparently misprint for Onychiopsis, in Sze, 1945 , p. 46.

ONOCLEITES Jneger, 1827.

Onocleites lanceolatus Jaeger, 1827, p. 34, pl. 6 , fig. 8 ; fern? leaf fregmant ; Triassic (Keuper) ; Esslingen, Wirttemberg.

ONTHEANTHUS Ganju, 1944.

Ontheanthus polyandra Ganju, 1944, p. 77, pl, 2, figs. 17-20; male fructification, Bennettitales; Jurassic; Onthea, Rajmahal Hills, India.
ONTHEODENDRON Sahni and A. R. Rao, 1931.

Ontheodendron florini Sahni and Rao, 1931 , p. 200 , pls. 15, 16 ; cone, Coniferales; Jurassic; Rajmahal Hills, India.

ONTHEOSTROBUS Ganju, 1944.

Ontheostrobus sessilis Ganju, 1844, p. 77, pl. 3, figs. 21-24; gymnospermous seedbearing fructification, possibly related to Bennettitales; Jurassic ; Onthea, Rajmahal Hills, India.

ONYCHIOPSIS Yokoyama, 1889.

Onychiopsis elongata (Geyler) Yokoyama, 1889 , p. 27 , pl. 2 , figs. 1-3 ; pl. 3, fig. $6 d$; pl. 12, figs. 9,10 ; fern, Polypodiaceae?; Jurassic; Tetorigawa, Japan. See also Seward, 1894, p. 40.

OOCHYTRIUM Renault, 1895.

Oochytrium lepidodendri Renault, 1895c, p. 160 , pl. 154 , figs. 15,16 ; fungus spores; Carboniferous (Culm) ; Esnost, France.

OO'THECA Nathorst, 1914.

Ootheca nordenskioldii Nathorst, 1914, p. 19, pl. 15, fig. 83 ; pteridosperm sporangia?; Palaeozoic; Spitzbergen.

OPEGRAPHITES Debey, 1859.

Opegraphites striatopunctatus Debey, in Debey and Ettingshausen, 1859a, p. 211, pl. 3, flg. 7 ; lichen ? Lower Cretaceous ; Aachen, Rhenish Prussia.

OPHIOGLOSSITES Massalongo, 1850.

Ophioglossites eocena Massalongo, 1850, p. 50 ; fern; Eocene; Monte Bolca ; Italy.

OREODOXITES Goeppert, 1864.

Oreodoxites martianus Goeppert, 1864, p. 147, pl. 26, fig. 5; seed; Permian; Braunau, Bohemia.

ORESTOVIA Zalessky, 1931.

Acad. sci. U. R. S. S. Bull., 1931, p. 402 (not seen, cited in Gothan, 1942b, p. 136).

ORIOPORELLA (Munier-Chalmas) Morellet and Morellet, 1922.

ovioporella briardi Munier-Chalmas, in Morellet and Morellet, 1922, p. 28, pl. 10, $\mathrm{A}_{5 \mathrm{~F}} .47,48$; alga, Dasycladacene ; Eocene (Montien); Mons, Belgium. Generic name cited in Munier-Chalmas, 1877 , p. 817 ; nom. nud.

ORMOXYLON Dawson, 1871.

Ormoxylon erianum Dawson, 1871, p. 14, pl. 1, figs. 10-15; woody stem of cordaitean aflnities; Devonian; Schohari County, N. $Y$.

ORNOXYLON Felix, 1882.

Ornoxylon fraxinoides Felix, 1882a, p. 35 ; wood, dicotyledon.

ORPHANIDESITES Caspary, 1881.

Orphanidesites primaevus Caspary, 1881, p. 29 ; fruit ; Ericaceae; Tertiary. 
ORTHOGONIOPTERIS E. B. Andrews, 1875. Orthogoniopteris clara D. B. Andrews, 1875 , p. 419 , pl. 50 , fig. 1 ; foliage resembling Tacniopteris; Pennsylvantan ; near Rushville, Perry County, Ohio.

ORTHOPORITES Schleiden, 1855.

Orthoporites apeltianus Sclileiden, in Schmid and Schleiden, 1855, p. 27. Tertiary (Braunkohle); Haering, Tirol, Austria.

ORTONELLA Garwood, 1914.

Ortonella furcata Garwood, 1914, p. 266 pl. 20, figs. 1-4; alga?; Lower Carbonfferous; Eskrigg Wood near Summerlands, Westmoreland, England.

ORVILLEA Lang, 1945.

Orvillea brasiliensis (Dawson) Lang 1945, p. 546, pls. 22-25; Upper Devonian; Brazil. For Protosalvinia brasiliensis Dawson, in Chicago Acad. Sci. Bull., 1886, v. 1, p. 115, figs. 1, 8, 9.

OSAGIA Twenhofel, 1919.

Osagia incrustata Twenhofel, 1919, p. 352, fig. 5 ; alga ; Foraker limestone member ; Pennsylvanian; Ekler Canyon, Cowley County, Kans.

OSCILLATORIITES Zalessky, 1927.

Oscillatoriitcs bertrandi Zalessky, 1927a, p. 98, pl. 4, fig. 8 ; alga, compared witl Oscillatoria ; Carboniferous; Simbirsk, Russia.

OSMUNDIA R. M. Johnston, 1894 .

Osmundia tasmanica R. M. Johnston, 1894, p. 176, pl. 1, fig. 2 ; fern leaflets; lower Tertiary ; Glenora, Tasmania.

OSMUNDITES Jaeger, 1827.

Osmundites pectinatu\& Jaeger, 1827, p. 29, pl.: 7, figs. 1-5; cycadophyte foliage, name changed to Pterophyllum jaegeri by Brongniart, $1828 \mathrm{~b}$, p. 100.

OSMUNDITES Unger, 1854.

Osmundites schemnicensis Unger, 1854a, p. 143, pl. 1; petrified rhizome, Osmundaceae; 'Tertiary ; Illa, near Schemnitz, Hungary. See also Kidston and Gwynne-Vaughan, 1907-10 ; Posthumus, 1931.

OSMUNDOPHYLLUM Velenovsky, 1889.

Osmundophyllum cretaceum Velenovsky, 1.889 , p. 6, pl. 2, fig. 21 ; fern frond fragment; Upper Cretaceous; Lipenec, Bohemia.

OSMUNDOPSIS Harris, 1931.

Osmundopsis sturii (Raciborski) Harris, $1931 a$, p. 136 ; fertile pinnae compared with Osmunda; Jurassic; Cracow, Poland. For Osmunda sturii Raciborski, 1890 , p. 2, pl. 1, figs. 1-5. See also Harris, 1931b, p. 48.

\section{OSTERITES.}

Error for Zosterites, in Bronglart, Alexander, 1829 , p. 409
OTIDOPHYTON David White, 1905.

otidophyton hymenophylloides David White, in Smith and White, 1905, p. 47, pl. 2, fig. 3 ; fern leaf fragment; Upper Devonian; Perry, Maine.

OTOPTERIS Lindley and Hutton, 1834.

otopteris obtusa Lindley and Hutton, 1834 (1831-37), p. 128, pl. 128; cycadophyte leaf ; Lower Jurassic (Llas) ; Membury, near Axminster, England.

OTOP'TERIS Sauveur, 1848.

Otopteris cycloidea Sauveur, 1848, p. 1 , pl. 26, figs. 1, 2, no description given; cyclopterid leaflet; Upper Carboniferous : Belgium.

OTOZAMITES Braun, 1842.

The following is suggested as a type species: Otozamites obtusus (Lindley and Hutton) Brongniart, 1849 , p. 104 . For Otopteris obtusa Lindley and Hutton, 1.834 (1831-37), p. 129 , pl. 128 ; cycadophyte foliage; Jurassic; England. See also Seward, 1904, pl, 1, flgs. 1, $\mathbf{3}, 5$.

OT'TOKARIA Zelller, 1902.

ottolvaria bengalensis Zelller, 1902, addenda facing. p. $1 ;$ pl. 4, figs. $9,10$. For Feistmantelia bengalensis Zelller, 1902, p. 34 . See also Seward, 1917, p. 139 ; Seward and Sahni, 1920, p. 12 ; and Thomas, 1921, p. 285.

O'T'TONOSIA Twenhofel, 1919.

ottonosia laminata Twenhofel, 1919, p. 350, figs. 3, 4; alga; Crouse limestone member. Permian ; Osage County, Okla.

OUROSTROBUS Harris, 1935.

Onrostrobus nathorsti Harris, 1935, p. 116 , bls. 23.27 ; seed-bearing cone, incertae sedis; Thaumatopteris zone; Rhaetic Scoresby Sound, east Greenland.

OVALITES LOMax, 1911.

Ovalites resinosus Lomax, 1911, p. 126 pl. 5 , fig. 18 ; pl. 6 , fig. 21 ; pl. 7 , fig. 23 ; a name assigned to oval resinous bodies found in coal; Arley coal senm and others; Upper Carbontferous; Ath,erton, Lancashire, England.

OVOPTERIDIUM Behrend, 1909.

Ovopteridium schumanni (Stur) Behrend, 1909 , p. 677 , pl. 17, flg. 10 ; sphenopterid foliage; Upper Carbonlferous.

OVOPTERIS Henry Potonie, 1893.

Ovopteris cremeriana Henry Potonie, 1893 b, p. 39, pl. 3, fig. 1 ; sphenopterid foliage; Permian ; Ilmenau, Prussian Saxony.

OVULARITES Whitford, 1916.

Ovularites barbouri Whitford, 1916, p. S5, figs. 1-5; fungus; Cretaceous; Rose Creek, Jefferson County, Nebr. 
OVULITES Lamarck, 1816.

Ovulites margaritula Lamarck, 1816, p. 194 ; alga?; Eocene ; near Paris, France. First species described after 1820 appears to be: Ovulites pavantina (d'Archiac) d'Orbigny, 1850, p. 405. First species fllustrated after 1820 appears to be : 0 . elongata Lamarck, in Schwager, 1883 , p. 146 , pl. 29 , flg. 22. See discussion in Seward, 1898, p. 161; Hirmer, 1927, p. 60.

OXALIDITES Caspary, 1886.

Oxalidites brachysepalns Caspary, 1886, p. 7 ; fruit, Oxalidaceae ; Tertiary ; Samland, Baltic Prussia. First species illustrated: 0 . averrhoides Conwentz, 1886, p. T0, pl. 8, figs. 1-3.

OXYCARPIA Trautschold, 1874.

Oxycarpia bifaria Trautschold, 1874, p. 132, pl. 3 ; Tertiary ; Kamuschin, Russia.

\section{$\mathbf{P}$}

PACHYPHLOEUS Goeppert, 1836.

Pachyphloeus tetragonus Goeppert, 1838, p. 433 , pl. 43 ; aborescent lycopod stem impression ; Lower Carboniferous ; Landshut, Falkenberg, Silesia.

PACHYPHYLLUM Lesquereux, 1854.

Pachyphyllum fimbriatum Lesquereux, 1854 , p. 421 ; fernlike foliage ; Pennsylvanian; Pennsylvania. See Lesquereux, in Rogers, 1858, p. 863, pl. 8, flg. 2.

PACHYPTERIS Brongniart, 1829.

Paohypteris lanceolata Brongniart, 1829 (1828a-38), p. 167, pl. 45, flg. 1 . Generic name cited in Brongniart, 1828b, p. 50. See Seward, 1910, p. 550.

PACHYSPERUM Reid and Chandler, 1933.

Pachysperum quinqueloculare Reid and Chandler, 1933, p. 419, pl. 22, figs. 1-7; fruit, Lythraceae; London Clay, Eocene ; Sheppey, Kent, England.

PACHYSPORANGIUM Salter, 1880.

Pachysporangium pilula Salter, 1880, p. 463 : nom. nud.

PACHYTESTA Brongniart, 1874.

Pachytesta incrassata Brongniart, 1874, p. 262, pl. 22, fig. 4 ; sllicifled seed; Upper Carboniferous; St.-Etienne, France.

PACHYTHECA Hooker, 1861.

Pachytheca sphaerica Hooker, in Salter, 1861 , p. 162. Devonian; Malvern, Scotland. Previously described by Hooker, 1852, p. 12, but not named. See also Harris, w. H., 1884, p. 28-32, figs. 21-23; and Kidston and Lang, 1925.

PAGIOPHYLLITES Tuzson, 1811.

Pagiophyllites keuperianus (Unger) Tuzson, 1911 , p. 30 , fig. 5.
PAGIOPHYLLUM Heer, 1881.

Pagiophyllum circinicum (Saporta) Heer, 1881 , p. 11 , pl. 10 , fig. 6 ; coniferous twigs and foliage; Jurassic (Malm); Sierra de San Luiz, Portugal.

PAIKHOIA Zalessky, 1936.

Paikhoia tchernovi Zalessky, 1936b, p. 237, figs. 1-5; lycopod leaf bases ; Permain ; Russia.

PAIACKYA Crie, 1889.

Palackya philippinensis Crie, 1889a, p. 87, pl. 17, figs. 1, 2 ; wood, dicotyledon ; Pliocene; San Juan del Monte, Manila, Philippine Islands.

PALAEACHLYA Duncan, 1876.

Palaeachlya perforans Duncan, 1876, p. 210 , pl. 16 ; alga or fungus?, in coral; Silurian.

PALAEALECTRYON Reid and Chandler, 1933.

Palaealectryon spirale Reid and Chandler, 1933 , p. 363 , pl. 17, figs. 13-19 ; seed, Sapindaceae; London Clay, Eocene; Sheppey, Kent, England.

PALAEALLOPHYLLUS Reid and Chandler, 1933

Palaeallophyllus ovoideus Reld and Chandler, 1933, p. 360, pl. 17, flgs. 1-7; seed, Sapindaceae; London Clay, Eocene; Sheppes, Kent, England.

PALAEANTHUS Newberry, 1895.

Palaeanthus problematicus Newberry, 1895 , p. 125 , pl. 35 ; fructification, Bennettitales?; Amboy clay, Upper Cretaceous ; New Jersey.

PALAEEUCHARIDIUM Reld and Chandler, 1933.

Palaeeucharidium cellulare Reid and Chandler, 1933 , p. 426 , pl. 23, figs. 1-4; fruit, Onagraceae; London Clay, Eocene : Minster, Kent, England.

PALAEOALGITES Weyland and Budde, 1932.

Palaeoalgites kräuseli Weyland and Budde, 1932 , p. 272, figs. 20, 21 ; Devonian; near Douglastown, Gaspe, Canada.

PALAEOASTER Knowlton, 1917.

Palaeoaster inquirenda Knowlton, 1917, p. 278 , pl. 49 , figs. 5,6 ; incertae sedis : Vermejo formation, Cretaceous; Alkali Gap, Canon City, Colo.

PALAEOAVENA Ettingshausen, 1890.

Palaeoavena stipaeformis Nttingshausen, 1890 , p. 77 , pl. 2, figs. 1-12; inflo. rescence fragments, Gramineae; Miocene; Schoenegg, Styria.

PALAEOBROMELIA Ettingshausen, 1852. Palaeobromelia jugleri Ettingshausen, 1852b, p. 3, pl. 1, fig. 1 ; pl, 2, figs. 1-3; not a plant, see R. W. Brown, 1950. 
PALAEOCARYA Saporta, 1873.

Palaeocarya atavia Saporta, 1873a, p. 101, pl. 15, figs. 36-39; involucre, Juglandaceae; Eocene; Aix, Provence, France.

PALAEOCASSIA Ettingshausen, 1867.

Palaeocassia angustifolia Ettingshausen, 1867, p. 261, pl. 3, figs. 6, 7 ; leaf, Papilionaceae; Cretaceous (Cenomantan) ; Nlederschoena, Saxony.

FALAEOCEDRUS Unger, 1842.

Palaeocedrus exstinctus Unger, in Endlicher, 1842 , p. 26 ; abetinean cone; Tertiary. Brief generic description only. See also Goeppert, 1850, p. 210.

PALAEOCHARA Massalongo, 1851.

Palaeochara rigida Massalongo, 1851, n. 44 ; Characeae; Eocene; Monte Bolca, Italy. Apparently given as a new name for Chondrites rigidus Massalongo, 1850 , p. 36.

PALAEOCHARA Bell, 1922.

Palaeochara acadica Bell, 1922, p. 160, pl. 1, figs. 3-9; oogonium, Charophyte ; Pennsylvanian; St. Rose mine, Inverness County, Nova Scotia.

PALAEACHLYA Duncan, 1876.

Palaeachlya perforans Duncan, 1876, $n$. 210 , pl. 16; alga?, compared with Achlya and found in Silurian corals; Silurian; Canada.

PALAEOCHONDRITES (Schimper) Saporta, 1882.

Palaeochondrites fruiticulosus (Goeppert) Saporta, 1882, p. 35, pl. 5, figs. 2-3 ; alga; Sllurian?; GIanzy near Vallhan, France.

PALAEOCHORDA M'COY; 1848.

Palaeochorda minor $\mathrm{M}^{\prime} \mathrm{Coy}$, in Sedgewick, 1848 , p. 225 ; alga ; upper Silurian; Cumberland and Westmoreland, England.

PALAEOCLADUS Ettingshausen, 1887.

Palaeocladus cuneiformis Ettingshausen, 1887 a, p. 93 , pl. 8 , fig. 33 ; foliage shoot, Taxineae; Eocene; Vegetable Creek, near Emmaville, New South Wales.

PALAEOCLADUS PIa, 1920.

Palaeocladus mediterraneus Pia, 1920, p. 118, pl. 6, figs. 1-5; alga, Siphoneae Verticillatae; Jurassic; Monte Potina, Italy.

I'ALAEOCODIUM Chiarugl, 1947.

Palaeocodium saharianum Chiarugl, 1947, p. 129 , pl. 9 ; alga, Codiaceae; Lower Carboniferous; Uadl near Gebel Auenat, Lybian Desert, Egypt.

PALAEOCYCAS Florin, 1933.

Palaeocycas integer (Nathorst) Florin, .1933 , p. 32 , pl. 1 , figs. 1,2 ; pl. 2 , figs. 1-3 ; pl. 3, figs. 1-3 ; cycad megasporophyll ; Rhaetic.
PALAEOCYPARIS Saporta, 1872.

Palaeocyparis expansus (Sternberg) Saporta, 1872 , p. 1056 . For Thuites exnansus Sternberg, 1823 (1820-38), p. 39, pl. 38 ; Jurassic ; Stonesfield, England.

PALAEODASYCLADUS PIa, 1927.

Palaeodasycladus mediterraneus $\mathrm{Pia}$, in Hirmer, 1927, p. 79, flg. 62 ; alga, Dasycladaceae; Lower Jurassic (middle Lias).

PALAEODENDRON Saporta, 1862.

Palaeodendron gypsophilum Saporta, 1863, p. 250 , pl. 7 , fig. 9 ; leaf, Proteaceae : Tertiary ; St.-Zacharie, France.

PALEODICTYON Heer, 1865.

Palaeodictyon singulare Heer, 1865 (1864$65)$, p. 245, pl. 10, fig. 10 ; alga ?; Eocene; Switzerland.

PALAEODICTYOTA Whitfield, 1902.

Palaeodictyota ramulosa (Spencer) Whitfleld, 1902, p. 399, pl. 53 ; marine alga ; Niagara Group, Sllurian; Lockport, N. $\mathbf{Y}$.

PALAEOGLEICHENIA Leuthardt, 1901.

Palaeogleichenia gracilis (Heer) Leuthardt, 1901, p. 128. For Pecopteris gracilis Heer, 1865 (1864-65), p. 54, pl. 2, fig. 1 .

PALAEOGONIOPTERIS KoldzumI, 1036.

Palaeogoniopteris mengkarangensis (Go. than and Jongmans) Koidzumi, 1936, p. 134. For Gigantopteris mengkarangensis Gothan and Jongmans, 1935, Jaarb. mijnwezen Nederlandish-Indië, 1930 , Verh., boekdeel 59, p. 143, p. 47, figs. 2-4 ; Stephanian, Carboniferous ; Djambi, Sumatra.

PALAEOGREWIA Massalongo, 1851.

Palaeogrewia dejopeae Massalongo, 1851, p. 182 ; Tillaceae; Tertiary ; Italy.

PALAEOHALIDRYS Gardner, 1924.

Palaeohalidrys californica Gardner, 1924, p. 362, pl. 25; alga, compared with Halidrys (Fucaceae) ; Miocene' (in diatomaceous earth) ; Los Angeles (Bairdstown), Calif.

PALAEOHYPNUM Steere, 1946.

Palaeohypnum arnoldianum Steere, 1946, p. .315, pls. 1, 2 ; moss, Bryales Pleurocarpl; Miocene ; Carter Creek, near Finley McKenzle ranch, Malheur County, Oreg.

PALAEOKEURA Massulongo, 1853.

Palaeokeura pellegriniana Massalongo, 1853d, p. 206, pls. 1-4 ; Pandanaceae ; Tertiary ; Italy.

PALAEOLEPIS Saporta, 1894.

Palaeolepis bicornuta Sporta, 1894, p. 179, pl. 33 , fig. $4 \mathrm{c}$; cone scales, Coniferales; Cretaceous (Albian) ; Buarcos, Portugal. 
PALAEOLOBIUM Unger, 1850.

Palaeolobium haeringianum Unger, 1850a, p. 490 ; fruit, Leguminosae ; Eocene; Haering, Tirol, Austria. See also Unger, 1851, p. 186, pl. 62, figs. 8-10.

PALAEOMYCES Renault, 1896.

Palaeomyces gracilis Renault, 1896a, p. 439 , figs. 88,89 ; fungus ; Upper Carboniferous; Esnost, France. [Meschinelli, 1898, p. 9, cites the genus "Palaeomycites, Renault." This is Meschinelli's change in spelling and should not be attributed to Renault as such; the only species cited is "Palaeomycites gracilis (Renault) Meschinelli."']

PALAEOMYCITES.

See Palaeomyces Renault.

PALAEONITELLA. Pia, 1927.

Palaeonitella cranii (Kidston and I.ang) Pia, in Hirmer, 1927, p. 91. For Algites cranii Kidston and Lang, 1921, p. 876, pl. 9, figs. 98-104; alga, probably Characeae; Old Red Sandstone, Middle Devonian; Muir of Rhynie, Aberdeenshire, Scotland.

PALAEONYSSA Reid and Chandler, 1933.

Palaeonyssa multilocularis Reid and Chandler, 1933, p. 431, pl. 23, figs. 1115 ; endocarp, Nyssaceae ; London Clay, Eocene; Sheppey, Kent, England.

PALAEOPEDE Etheridge, 1899.

Palaeopede whiteleggei Etheridge, 1899a, p. 127, pl. 23, figs 1-4; Nostoc-like endophytic alga; "Permo-Carboniferous" ; New South Wales.

PALAEOPERONE Etheridge, 1891.

Palaeoperone endophytica Etheridge, 1891, p. 97, pl. 7 , fig. 2 ; spores?, found in coal ; "Permo-Carboniferous"; New South Wales.

PALAEOPHOENIX Saporta, 1878.

Palaeophoenix aymardi Saporta, 1878a, p. 25, pls. 1, 2 ; pl. 3, figs. 2-4 ; Eocene ; Brives near Puy-en-Velay, France.

PALAEOPHYCUS Hall, 1847.

Palaeophycus tubularis Hall, 1847, p. 7, pl, 2, figs. $1,2,4,5$; alga ?; Silurian; New York.

PALAEOPHXTOORENE Reid and Chandler, 1933.

Palaeophytocrene foveolata Reld and Chandler, 1933, p. 333 ; pl. 15, figs. 2432 ; endocarp, Ícacinaceae; London Clay, Eocene ; Sheppey, Kent, England.

PALAEOPICEOXYLON Krăusel, 1949.

Palaeopiceoxylon transiens (Shimakura) Kräusel, 1949, p. 127, 182; conlferous wood; Cretaceous; Japan. For Piceoxylon transiens Shimakura, 1937, p. 24, pl. 6, figs. 1-9.

PALAEOPITYS M'Nab, 1870.

Palaeopitys milleri $\mathrm{M}^{\prime} \mathrm{Nab}, 1870$, p. 314 ; Devonian. See also Kidston and Lang, 1923b.
PALAEOPORELLA Stolley, 1893.

Palaeoporella variabilis Stolley, 1893, p. 138, pl. 7, figs. 1-5 ; siphonaceous alga ?; Silurian; Holstein, Kiel, Prussia.

PALAEOPOTAMOGETON Knowlton, 1916. Palaeopotamogeton florissanti Knowlton, 1916 , p. 251 , pl. 16, fig. 1 ; pl. 17, fig. 3 ; stems with leaves and fruits, Potamogetonaceae?; Oligocene ; Florissant, Colo.

PALAEOPTERIDIUM Kidston, 1923.

Palaeopteridium renssi (Ettingshausen) Kidston, 1923a, p. 201, pl. 55, flgs. 1-3 ; follage similar to Archaeopteris ; Westphalian, Upper Carboniferous.

PALAEOPTERIS Geinitz, 1855.

Palaeopteris schnorriana Geinitz, 1855, p. 32 , pl. 35, fig. 8 ; fern? stem impression; Upper Carboniferous; Niedercainsdorf, Saxony. See also Posthumus, 1931.

PALAEOPTERIS Schimper, 1869.

Palaeopteris hibernica (Forbes) Schimper, $1869(1869-74)$, p. 475 , pl. 36 ; this genus changed to Archaeopteris (Dawson, 1871) because of the earlier use of Palaeopteris by Geinitz.

PAIAAEOPYRUM Schmalhausen, 1883.

Pálaeopyrum incertum Schmalhausen, 1883 , p. 293 , pl. 31 , figs. 3,4 ; fruits, Gramineae ; Eocene; Russia.

PALAEORAOHIS Saporta, 1889.

Palaeorachis subgracilis Saporta, 1889, p: 46 , p1. 8 , fig. 1 ; inflorescence (axis only) of Sabal?; Eocene; Aix, Provence, France.

PALAEORCHIS Massalongo, 1858.

Palaeorchis rhyzoma Massalongo, 1858b, p. 750, Tertlary; Italy.

PALAEORHODOMYRTUS Reid and Chandler, 1933.

Palaeorhodomyrtus subangulata (Bowerbank) Reld and Chandler, 1933, p. 436, pl. 23 ; figs. 21-31 ; fruit, Myrtaceae; London Clay, Eocene; Sheppey, Kent England.

PALAEOSORDARIA Sahni and H. S. Rao, 1943.

Palaeosordaria lagena Sahni and Rao, 1943, p. 46, pl. 3, figs. 22,23 ; perithecia, Sordariaceae; Intertrappean cherts, early Tertiary ; Chhindwāra district, Central Provinces, India.

PALAEOSPADIX Saporta, 1886-91.

Palaeospadix girardoti Saporta, 1886-91, p. 260 , pl. 270 , fig. 3 ; pl. 271 , fig. 9 ; palm spadix?; Jurassic; Chattelneuf, France.

PALAEOSPATHE Unger, 1845.

Palaeospathe sternbergit Unger, 1845 (1841-47), p. lxxi; wood, Aurantiaceae ; Carboniferous; Swina, Bohemla. For Spatha (Flabellaria) borassifoliae Sternberg, 1820-38, pl. 41.

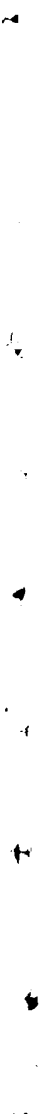


PALAEOSTAOHYA C. E. Weiss, 1876.

The following is suggested as the type in view of the clear-cut diagnostic characters displayed: Palaeostachya elongata (Presl) C. E. Weiss, 1876, p. 108, pl. 15 ; articulate cone; Upper Carboniferous; Swina near Radnitz, Bohemia.

PALAEOSTROBUS Renger, 1866.

Palaeostrobus mirabilis (Corda) Renger, 1866 , p. $13 \pi$, pl. 1 , flg. 1 .

PALAEOTAXODIOXYLON Frentzen, 1916 Palaeotaxodioxylon gruenuettersbachense Frentzen, 1916, p. 103, pl. 22 ; Triassic (Upper Bunter Sundstone) ; Gruenwettersbach, Baden.

PALAEOTAXUS Nathorst, 1908.

Palaeotaxus rediviva Nathorst, 1908a, p. 16, pl. 3, figs. 13-17 ; foliage and cone, Coniferales; Rhaetic; Skromberga, Sweden.

PALAEOTHALIA Squinabol, 1892.

Palaeothatia sanctaejustinae Squinabol, 1892 , p. 57, pl. 21, fig. 2 ; pl. 29, flg. 7 ; leáf, Scitamnieae; Tertiary; Santa Giustina, Italy.

PALAEOTHECIUM Saporta, 1888.

Palaeothecium ambiguum Saporta, 1888, p. 16, pl. 1 , fig. 15 ; sporophy te of moss? ; Eocene; Aix, Provence, France.

PALAEOTHRINAX Reid and Chandler, 1926.

Palaeothrinax mantelli Reid and Chandler, 1926 , p. 80 , pl. 5, figs. 1-5; palm leaf ; Bembridge Marl, Oligocene; Isle of Wight, England.

PALAEOVITIS Reid and Chandler, 1933.

Palaeovitis paradoxa Reid and Chandler, 1933, p. 388, pl. 19, figs. 20-27; seed, Vitaceae; London Clay, Eocene; Warden Point, Kent, England.

PALAEOVITTARIA. Ottokar Felstmạtel, 1876.

Palaeovittaria kurzi Ottokar Feistmantel, 1876 a, p. 368 , pl. 19 , figs. 3,4 ; fern leaf, compared with $\nabla$ ittaria (Polypodiaceae); Damuda series, Gondwana system ; Raniganj, India.

PALAEOWEICHSELIA Henry Potonte and Gothan, 1909.

Palaeoweichselia defrancei (Brongniart) Henry Potonie and Gothan, 1909, p. 4. For Pecopteris defrancei Brongniart, 1828a-38, p. 325, pl. 111 ; pl. 112, fig. 1.

PALAEOXYLON Hartig, 1848.

Palaeoxylon endlicher Hartig, 1848a, p. 172 ; wood; Tertiary; Riestadt, Germany.

PALAEOXYLON Brongniart, 1849.

Palaeoxylon withami (Lindley and Hutton) Brongniart, 1849, p. 126: For Pinites withami Lindley and Hutton, 1831 (1831-37), p. 9 , pl. 2 ; cordaitean wood ; Carboniferous ; Craigleith, Scotland.
PALAEOXYRIS Brongniart, 1828.

Not a plant; see Brown, R. W., 1950.

PALAEOZAMIA Endlicher, 1836.

Palaeozamia taxina (Lindley and Hutton) Endlicher, $1836(1836-40)$, p. 72? First speciflc reference in Endlicher is to Zamia taxina Lindley and Hutton, 1835 (1831-37), p. 67, pl. 175.

PALAMOPHYLLUM Zalessky, 1912.

Palamophyllum cuneifolium (Kutorga) Zalessky, 1912, p. 38. For Psygmophyllum cuneifolium (Kutorga) Schimper, 1870 (1869-74), p. 194. For Sphenopteris cuneifolia Kutorga, 1838, p. 32, pl. 7, fig. 3.

PALEODIOTYON Savi and Meneghini, 1851. Paleodictyon strozzi Savi and Meneghini, 1851 , p. 208 ; alga, affinities with $H y$ drodictyon?; Eocene; Tuscany, Italy. See also Silvestri, 1911; and Peruzzi, 1881, p. 7, pl. 1, fig. 8.

PALEOERIOCOMA Ellas, 1942.

Paleoeriocoma hitchcocki Elias, 1942, p. 100, pl. 15, flgs. 7,8 ; grass hull; Ash Hollow formation, middle Pliocene; Beecher Island Post Office, Yuma County, Colo.

PALEOHEPAT'ICA Raciborski, 1889.

Paleohepatica rostafinskii Raciborsk1, 1889 , p. 136 ; Jurassic ; Cracow, Poland. See Hirmer, 1927, p. 141, flgs. 135, 136.

PALEOHILLIA Knowiton, 1895.

Paleohillia arkansana Knowlton, 1895, p. 387 , figs. $1-3$; stem with epidermls preserved, incertae sedis; Trinity division, Lower Cretaceous ; 6 miles northeast of Center Point, Howard County, Ark.

PALEOMEANDRON Peruzzi, 1881.

Paleomeandron rude Peruzzi, 1881, p. 8 , pl. 1, fig. 4 ; incertae sedis; Eocene; Monte Fiesole, Mugnone, Italy.

PALEONELUMBO Knowlton, 1930.

Paleonelumbo macroloba Knowlton, 1930, p. 93 , pl. 39 , fig. 3 ; pl. 42 , figs. 3,4 ; leaf, Nymphaeaceae; Dawson arkose, Upper Cretaceous and Eocene(?); Colorado.

PALEONUPHAR Hollick, 1930.

Paleonuphar inopina Hollick, in Hollick and Martin, 1930 , p. 75, pl. 40, fig. 5 ; leaf, Nymphaeaceae; Upper Cretaceous; Yukon River, 6 miles above Nahochatilton, Alaska.

PALEOTAXITES David White, 1929.

Paleotaxites praecursor David White, 1929, p. 107 , pl. 49 , figs. 1,3 ; pl. 50, figs. 1,2 , 6 ; pl. 48 , fig. 3 ; coniferous twigs, Hermit shale, Permian; Hermit basin, near Yaki Trail, Ariz.

PALIBINIA Korovin, 1932.

Palibinia laxifolia Korovin, 1932, p. 517, pl. 1, Proteaceae; Tertiary; Turkistan. 
PALISSYA Endlicher, 1847.

Palissya braunii Endlicher, 1847, p. 306. For Cunninghamites sphenolepis Braun, in Münster, 1843 (1839-43), p. 24, pl. 13, figs. 19, 20.

PALIURITES Langeron, 1902.

Paliu'ites martyi Langeron, 1902, p. 94, pl. 6; fruit, compared with Paliurus (Rhamnaceae); Plio c e n e ; Cantal, France.

PALLIOPORIA Kirchheimer, 1934.

Pallioporia symplocoides Kirchhelmer, $1934 a$, p. 771 , fig. 8 ; fruit, Symplocaceae ; Teritary (Braunkohle) ; Germany. See also Kirchheimer, $1936 \mathrm{a}$, p. 68 , pl. 9, figs. $25 \mathrm{a}-\mathrm{v}$.

PALMACITES (Schlotheim) Brongniart, 1822.

Palmacites parisiensis Brongniart, 1822, p. 312 , pl. 16, fig. 1. [This genus created by Schlotheim, 1820, p. 393, and applied to arborescent lycopod trunk impressions; for example, his $P$. oculatus, p. 394, pl. 17, fig. 1 is clearly a Sigillaria and his $P$. quadrangulatus is a Lepidodendron. Palmacites as used by Brongniart clearly applies to palm leaves; later authors have applied it to supposed palm trunk and petiole impressions.]

PALMANTHIUM Schimper, 1870.

Palmanthium martii (Heer) Schimper, $1870(1869-74)$, p. 506 ; palm flower: Tertiary ; Berlingen, Canton Thurgovie. Switzerland. For Palmacites martii Heer, 1855, p. 97, pl. 41, figs. 2-4.

PALMATOPHYCUS Boucek, 1941.

Palmatophycus contractus Boucek, 1941, p. 1 ; alga; Sllurian; Beroum, Czechoslovakia.

PALMATOPTERIS Henry Potonie, 1893.

Palmatopteris furcata (Brongniart) Henry Potonle, 1893a, p. 1, pl. 1 ; figs. 1,5 ; sphenopterid foliage; Carboniferous.

PALMITES Hector, 1880.

Palmites pectinata Hector, 1880, p. 48 ; nom. nud.

PALMOCARPON Miquel, 1853.

Palmocarpon cretaceum Miquel, 1853, p. 51 , pl. 7 ; palm frult ; Upper Cretaceous (Senonian); Mt. St. Peter, Limburg, Belgium.

PALMOCARPON Lesquereux, 1878.

Palmocarpon compositum Lesquereux, 1878 a, p. 119, pl. 11, fig. 4 ; paim fruit? ; Tertiary ; Placiere Mtn., N. Mex.

PALMOGLOEITES Goeppert, 1869.

Palmogloeites adamantinus Goeppert, 1869, p. 64, pl. 1, fig. 7 .
PALMOPHYLLUM Conwentz, 1886.

Palmophyllum succineum Conwentz, 1886, p. 11, pl. 1, figs. 12, 13 ; leaf fragment in amber, Palmae; Tertiary ; West Prussia.

PALMOSPERMUM Reid and Chandler, 1933. Palmospermum jenkinsi Reid and Chandler, 1933, p. 110, pl. 1, flgs. 23, 24 ; seed ; Palmae; London Clay, Eocene; Herne Bay, Kent, England.

PAIMOXYLON Schenk, 1882.

Palmoxylon blanfordi Schenk, 1882, p. 355; palm stem; Pliocene; Nerbada River near Dschansi, Bandelkand, India. See also Schenk, in Zittel, 1890 (1879$90)$, p. 886 , fig. 427 .

PALOREODOXITES Knowlton, 1930.

Paloreodoxites plicatus (Lesquereux) Knowlton, 1930 , p. 41, pl. 11, figs. 1-4; leaves, Arecaceae; Denver formation, Upper Cretaceous and Eocene?; Golden, Colo.

PANAOITES Deane, 1902.

Panacites howitti Deane, 1902b, p. 18, pl. 1, fig. 8 ; Tertiary ; Pitfleld, Australia.

PANDANITES Tuzson, 1914.

Pandanites acutidens Tuzson, 1914, p. 245 , pl. 15, flg. 6; leaf fragment, monocotyledon; Cretaceous; Ruszkabanya, Krasso-Szorenz, Hungary.

PANDANITES Dorf, 1938.

Pandanites corconi Dorf, 1938, p. 46, pl. 3, fig. 4 ; leaf fragment, Pandanaceae; Upper Cretaceous; Corson Ranch, Wyo.

PANDANOCARPUM (Brongniart) żigno, 1873.

Pandanocarpum oolithicum (Carruthers) Zigno, 1873 (1873-85), p. 3. For Kaidacarpum oolithicum Carruthers, 1868, p. 153, pl, 9, figs. 1-6. Pandanocarpum oblongum cited in Brongnlart, 1828 b, p. 138 ; nom. nud.; the genus mentioned briefly in Brongniart, 1848, p. 137.

PANDANOPHYLLUM Kryshtoforich, 1029.

Pandanophyllum ahnertii Kryshtofovich, 1929 , p. 524, fig. 4 ; angiosperm leaf; Cretaceous; near town of NikolskUssurfysk, Ussurfland, Stberia.

PANESCOREA Saporta, 1882.

Panescorea glomerata Saporta, 1882, p. 25, pl. 5, fig. 1; alga? ; Permian; France.

PAPANINIA Fedin, 1943.

Papaninia involucrata Fedin, 1943, p. 365 ; cone, Coniferales ; age unknown; Franz Josef Land.

PAFAVERTTES Friedrich. 1883.

Papaverites sp. Frederich, 1883, p. 297, p1. 19, flg. 17; Ollgocene; Bornstedt, Saxony. 
PAPILIONITES E. W. Berry, 1924.

Papilionites erythrinaformis E. W. Berry, 1924a, p. 171, pl. 33, fig. 9 ; leaf, Papllionaceae; Fayette sandstone, Eocene; Mossy Creek, 3 miles southwest of Wellborn, Brazos County, Tex.

PARACALAMITES Zalessky, 1927.

Paracalamites striatus (Schmalhausen) Zalessky, 1827a, p. 51, pl. 40, flg. 5; calamite stem impression; Jurassic; Russia.

\section{PAILACALAMITINA.}

Apparently a mistake for Paracalamites, in Zalessky, 1934b, p. 242.

PARACALAMOSTAOHYS C. E. Weiss, 1884. Paracalamostachys polystachya (Sternberg) C. E. Welss, 1884 b, p. 190 , pl. 19, figs. 1,2 ; articulate cone, attached to stem ; Carboniferous.

PARACALLIPTERIS Richter, 1904.

Paracallipteris potoniei Richter, 1904, p. 17, pl. 1, fig. 13 ; leaf, incertae sedis; Upper Cretaceous; Hohlweg near Sternbrunnen, Saxony.

PARAOEDROXYLON Sinnott, 1909.

Paracedroxylon 8cituatense Sinnott, 1909, p. 171 , pls. 80,81 ; araucarian wood; Cretaceous; Second Cliff, Scltuate, Mass.

PARAOHAETE'WS Deninger, 1906.

Parachaetetes ternquisti Deninger, 1906, p. 65, pl. 6 tig. 6 ; alga; Mesozolc; Monte Zirra, Sardinia.

PARACREDNERIA Richter, 1905.

Paracredneria fritschii Richter, 1905, p. 15, pl. 2, fig. $14 ;$ pl. 3, fig. 9 ; Upper Cretaceous; Warustedt, Saxony.

I'ARACUPRESSINOXYLON Holden, 1913. Paracupressinoxylon cedroides Holden, 1913 , p. 537, pl. 39, figs. 11-14 ; coniferous wood; Jurassic ; Yorkshire, England.

PARADOXOCARPUS Nehring 1892.

Paradoxocarpus carinatus Nehring, 1892 p. 454, figs. 18-26 ; Pleistocene ; Klinge near Cottbus, Prussia.

PARADOXOPTERIS Hirmer, 1927.

Paradoxopteris stromeri Hirmer, 1927, p. 609, flgs. 733-736; Cretaceous (Cenomanlan) ; Baharije Oasis, Egypt.

PARAENGELHARDTIA Berry, 1916.

Paraengelhardtia eocenica Berry, 1916b, p. 186, pl. 17, figs. 2-5 ; fruit, Juglandaceae; Lagrange formation, Eocene ; Puryear, Henry County, Tenn.

PARAFAGUS W. R. B. Ollver, 1936.

Parafagus otakouia W. R. B. Oliver, 1936, p. 292 , figs. 8,9 ; leaf, Fagaceae ; Pliocene; Kalkorai Valley, Otago, New Zealand.

$296347-55-14$
PARAGONORRACHIS Grusd'Eury, 1877. Paragonorrachis gutbicriana (Presl) Grand'Eury, 1877, p. 381. For Rhodea gutbieriana Presl, in Sternberg, 182038 , p. 111.

PARANOCLADUS Florin, 1940.

Paranocladus dusenii Florin, 1940b, p. 320, pls. 165-166; leafy coniferous shoot; "Permo-Carbontferous" ; Iraty, Parana, Brazil.

PARANYMPHAEA E. W. Berry, 1935.

Paranymphaea crassefolia (Newberry) E. W. Berry, 1935, p. 39, pl. 7, flgs. 4, 5 ; pl. 9 ; leaf, Nymphaeaceae; Ravenscrag formation, uppermost Cretaceous?; Ravenscrag Butte, Saskatchewan, Canada.

PARAPECOPTERIS Grand'Eury, 1890. Parapecopteris nevropteriais Grand'Eury, 1890 , p. 288 , pl. 5, figs. 2-5.

PARAPHYLLANTHOXYLON Balley, 1924 Paraphyllanthoxylon arizonense Batley, 1924, p. 446, pl. 15 ; wood, dicotyledon ; Colorado group, Upper Cretaceous; Arizona.

PARAPHYLLOCLADOXYLON Holden, 1913. Paraphyllocladoxylon eboracense Holden, 1913 , p. 536 , pl. 39 , figs. 7-9 ; coniferous wood ; Oolite, Jurassic ; Scarborough, England.

PARAPITYS Zalessky, 1911.

Parapitys spenceri (Scott) Zalessky, $1911 a$, p. 28 . For Dadoxylon spenceri Scott, 1902, p. 357, pl. 2, figs. 12, 13 ; pl. 6, figs. 24, 25 .

PARARAUCARIA Wieland, 1935.

Pararaucaria patagonica Wieland, 1935, p. 21, pls. 2-5; petrified araucarian cone ; Triassic ?; Cerro Cuadrado, Santa Cruz, Argentina. See also Wleland, 1929 , p. 62.

PARASPORITES Schopf, 1938.

Parasporites maccabet Schopf, 1938a, p. 48 , pl. 1, flg. 6 ; pl. 7, figs. 1-3 ; spore ; No. 5 and No. 6 coal, Penusylvanian: Belleville, Ill.

PARATHINNFELDIA Richter, 1904.

Parathinnfeldia dubia Richter, 1904, p. 14, pl. 1, fig. 3 ; leaf fragment, Incertae sedis; Upper Cretaceous; Saxony.

PARENCHYMOPHYCUS Duden, 1897.

Parenchymophycus asphalticum Duden, 1897 , p. 118 , pl. 2 ; "fucoidal plants"; Genesee shale. Devonian; Indiana.

PARILINOPTERIS HIrmer, 1940.

Palaeontographica, 1940, Band 84, Abt. B, p. 188 (not seen, cited in Gothan, 1942b, p. 138)

PARINARIOXYLON HeUrn, 1928

K. Akad. Wetensch. Amsterdam Vers. 1928 Verh., Band 37, p. 470 (not seen, cited in Gothan, 1942b, p. 138). 
PARINEUROPTERIS Hirmer, 1940.

Palaeontographica, 1940, Band 84 Abt. $B$, p. 188 (not seen, cited in Gothan, 1942b, p. 138).

PARIPTERIS Gothan, 1941.

Palaont. Zeitschr., Band 22, p. 427 (not seen, cited in Gothan, 1942b, p. 139).

FARKA Fleming, 1857.

Parka decipiens Fleming, 1857, p. 448, fig. 121 ; Old Red Sandstone, Devonian; Scotland. See also Lang, 1937.

PARKERELLA (Munier-Chalmas) Morellet and Morellet, 1922.

Parkerella montensis Munier-Chalmas, in Morellet and Morellet, 1922, p. 15, pl. 1, figs. 56-60; alga, Dasycladaceae ; Eocene; Mons, France.

PARKERIOIDEA Renault, 1901.

Parkerioidea stephanensis Renault, 1901b, p. 350, fern fructification. See Renault, 1902, p. 104, pl. 6, fig. 4 ; pl. 7 bis.

PARNESINA Steinmann 1899.

Parnesina anuulus (Parker and Jones) Steinmann, 1899, p. 152 ; alga, Dasycladaceae; Miocene; Grignon, France. For Dactylopora annulus Parker and Jones, 1860 , p. 474 . See also Carventer, 1862 , p. 1.29 , pl. 10 , figs. 9-14.

PARTHENITES Saporta, 1861.

Parthenites priscus Saporta, in Heer, 1861, p. 146. See Saporta, 1862, p. 261, pl. 10 , fig. 4 .

PARTSCHIA Presl, 1838.

Partschia brongniartii Presl, in Sternberg, 1838 (1820-38), p. 116. For Pecopteris hemitelioides Brongniart, 1828a38 , p. 314 , pl. 108, figs. 1, 2 ; pecopterid foliage; Upper Carboniferous; Saarbruck.

PASIANOPSIS Saporta and Marion, 1878.

Pasianopsis retinervis Saporta and Malion, 1878 , p. 48 , pl. 7 , fig. 2 ; leaf, Fagacene; lower Eocene; Gelinden, Belgium.

PASINIA Massalongo, 1851.

Pasinia pyriformis Massalongo, 1851, p. 41. For Delesserites pyriformis Massalongo, 1850 , p. 48.

PASSALOSTROBUS Endlicher, 1847.

Passalostrobus tesselatus (Bowerbank) Endlicher, 1847, n. 278; conc, Con1rerales; Eocene; Sheppey, England. For Cupressinites tesselatus Bowerbank, 1840 , p. 63 , pl. 10 , figs. $26,27,30,31$. PASTILLUS Zalessky, 1928.

Pastillus cellulosius Zalessky, 1928, p. 3, pl. 2, fig. 3 ; Minoussinsk Basin, Siberia.

PATALOXYLON Sahni, 1920.

Pataloxylon scalariforme Sahni, 1920, p 29 , pl. 1 , fig. 6 ; pl. 2 , figs. $8-11$; wood, dicotyledon ; Tertiary ; Mt. Meerschaum, near Nerang, Queensland.
PAT'E'TE Hector, 1886

Patete scheffleri Hector, 1886, p. 61, fig. 24 ; Tertiary-Cretaceous; Pakawau, New England.

PATHEROTHECA Jongmans, 1929.

Reference not seen; cited in Gothan, $1942 b$, p. 139.

PATZEA Caspary, 1872.

Pat $\approx$ ea gnetoiles Caspary, 1872, p. 20 ; Tertiary; Prussia. First? species illustrated: $P$. johniana Conwentz, 1886, p. 136 , pl. 13, figs. 8-14.

PECOPTERIDIUM Fontaine and White, 1880.

- A suggested generic name, with no species assigned, to include fossils which Fontaine and White assign to Callipteridium unitum Fontaine and White, 1880, p. 61. Lacoe, 1884 , p. 10, lists the binomial Pecopteridium unitum F. and W.

PECOPTERIDIUM Picquenard, 1922.

Pecopteridium pluckenettii (Schlotheim) Picquenard, 1922, p. 347. For Pecopteris pluckenetii (Schlotheim) Sternberg, 1825 (1820-38), Tentamen, p. xix. [Note the following from Picquenard, 1922, p. 347 : "Je crois devoir réunir sous ce nom, dans un genre d'at. tente, les frondes filicoides faisant partie du groupe des Pecopteris Pluckenett Sternb., Essai Pl., monde prim., I, fasc. 4 , p. xix, et Sterseli, Zeiller que sont nom pas des fougeres comme les Pecopteris sensu stricto, mais des pteridospermées."]

PECOPTERIDIUM Kawasaki, 1934.

Pecopteridium manchuricum Kawasaki, 1934 (1927-34), p. 155, pl. 34, figs. $73-75$; pl. 40, flg. 97 ; fernllke foliage; Jido series, Carboniferous; Tae-dong, district, North Korea.

PECOPTERIS (Brongniart) Sternberg, 1825. Pecopteris pennaeformis (Brongniart) Sternberg, 1825 (1820-38), Tentamen, p. xvii. For Filicites pennaeformis Brongniart, 1822, p. 233, pl. 2, fig. 3; Carboniferous.

PECOPTEROMEDULLOSA Lotsy, 1909.

Pecopteromedultosa angita (Scott) Lotsy, 1909, p. 727, fig. 512. For Medullosa anglica Scott, 1899.

PECTINOPHYTON Hoeg, 1935.

Pectinophyton norvegicum Hoeg, 1935, p. 12 , pl. 4 ; Ag. 3 ; fertlle frond, compared with Barinophyton; Middle Devonian; Devonskardet, western Norway.

PEDIASTRITES Zalessky, 1927.

Pediastrites kidstoni Zalessky, 1927b, p. 97, pl. 5, figs. 1, 2; alga?; Chlorophyceae; Carboniferous ; Russia. 
PELLETIERIA Seward, 1913.

Pelletieria valdensis Seward, 1913, p. 91, pl. 12 , fig. 12 ; pl. 14, fig. 5 ; fertlle fern frond, Schlzaeaceae; Wealden; near Hastings, England.

PELOURDEA Seward, 1917.

Pelourdea vogesiaca (Schimper and Mougeor) Seward, 1917, p. 278, fig. 484 cordaitean leaves; Lower Triassic (Bunter sandstone); Vosges, France.

PELTANDRIPITES Wodehouse, 1933

Peltandripites davisii Wodehouse, 1933, p. 498, fig. 24 ; pollen, Araceae ; Parachute Creek member, Green River formation, Eocene; Colorado and Utah.

PELTASPERMUM Harris, 1937.

Peltaspermum rotula Harris, 1937, p. 34 ; peltate seed-bearing organ, Pteridospermae; Lepidopteris zone, Rhaetic; Scoresby Sound, east Greenland. See Harris, 1932a, p1. 6, figs. 3-6.

PELTASTROBUS Baxter, 1950.

Peltastrobus reedae Baxter, 1950, p. 175, figs. 1-6; petrifled articulate cone ; No. 5 coal, Pennsylvanian; Warrick County, Ind.

PELTOPHYLLUM Massalongo, 1854.

Peltophyllum nelumbioides Massalongo, 1854 , p. 22 ; leaf ; Eocene ; Monte Bolca, Italy. See Massalongo, 1859a, p. $1 \times x v$, pl. 28, flgs. 1, 2.

PEMPHIGALETES Zalessky, 1939.

Pemphigaletes, Zalessky, 1939a, p: 326 ; nom. nud.

PENHALLOWIA Kuntze, 1904.

Penhallowia Kuntze, in Post and Kuntze, \& 1904, p. 421.

PENIOILLITES Meschinelli, 1892.

Penicillites curtipes (Berkeley) Mesch1nelli, in Saccardo, 1892, p. 789 . See Meschinelli, 1898, p. 78, pl. 22, fig. 1.

PENIOILLOIDES Paul, 1938.

Reference not seen; cited in Gothan, 1942b, p. 189.

PENTACOILA Mueller, 1877.

Pentacoila gulgongensis Mueller, 1877 a (1877-79), p. 179 ; Pllocene ; Gulgong, Australia. See Mueller, 1883, p. 12, pl. 15, figs. 19, 20.

PENTEUNE Mueller, 1873.

Pentenne clarkei Mueller, 1873 (1871-82), p. 41, pl. 7 ; Pliocene; Smythes Creek, Victoria.

PENTOXYLON Srivastava, 1944.

Pentoxylon sahnii Srivastava, 1944, p. 74, pl. 1, flg. 11 ; polystelic stem, Pentoxyleae; Jurassic; Santal Parganas district, Behar, India. Brief description in Srivastava, 1937, p. 273. Full description in Srivastava, 1946, p. 196, pl. 2, figs. 6-17 ; pls. 3-5. For full consideration of Pentoxyleae, see Sahni, 1848.
PERFB BORITES Zalessky, 1934.

Pereborites rarinervis Zalessky, $1934 \mathrm{~b}, \mathrm{p}$. 268 , fig. 43 ; leaf fragment, Incertae sedis; Permian; Pechora basin, Russia.

PEREMOPTERIS Zalessky, 1937.

Peremopteris sylvaeana Zalessky, $1937 \mathrm{~b}$ p. 46, fig. 10 ; fernllke foliage ; Fermian; Tchekarda, Russia.

PERF'OSSUS Cotta, 1832.

Perfossus angularis Cotta, 1832, p. 52, pl. 10, fig. 1-3; petrified palm; Tertlary; Carlsbad, Bohemia.

PEKIASTRON Unger, 1856.

Periastron reticulatum Unger, 1856, p. 171, pl. 8, flgs. 13-15; petiole, Pterido. spermae?; Upper Devonian; Saalfeld, Thuringla. See also Scott and Jeffrey, 1914.

PERICHODERMA McLean, 1912.

Perichoderma asteroides (Williamson) McLean, 1912 , p. 508, fig. 4 ; spore or Radiolarian ?; Carbontferous.

PERICORDAITES Zalessky, 1927.

Pericordaites eugeniae Zalessky, 1927a, p. 45 , pl. 27, flgs. 1-7 ; cordaitean wood ; Permian ; Urals, Russia.

PEKIMNESTE Harris, 1939.

Perimneste horrida Harris, 1939, p. 54, pl. 14, figs. 2, 7, 9; Charophyta; Middle Purbeck, Jurassic, Dorset, England.

PERISPORIACITES Felix, 1894.

Perisporiacites larundae Felix, $1894 \mathrm{~b}$, p. 271 , pl. 19 , flg. 3 ; fungus peritheclum ?; Eocene; Perekeschkul near Baku. Meschinelli, 1898, p. 15, erroneously attributes this genus to Fries.

PERISPORITES Pampaloni, 1902.

Palaeontographia Italica, 1902, v. 8, p. 126 (not seen, cited in Gothan, 1942b, p. 139)

PERMOPHYLLUM Zalessky, 1937.

Permophyllum incisum Zalessky, $1937 \mathrm{~b}$, p. 70, fig. 34 ; follage fragment, Ginkgoales?; Permian; Matveyevo, USSR.

PEIRMOPORA Elfas, 1947.

Permopora keenae Dlias, 1947, p. 53, pl. 18, figs. 1-11; alga, Dasycladaceae; Childress dolomite, Permian; Childress and Cottle Counties, Tex.

PERMOPTERIDIUM Zalessky, 1939.

Permopteridium densinervum Zalessky, 1939 b, p. 353 , flg. 31 ; fern? frond fragment; Permian, Matveyevo, USSR.

PERMOSAMAROPSIS Kuntze, 1904.

Permosamaropsis Kuntze, in Post and Kuntze, 1904, p. 425.

PERMOSPERMA Zalessky, 1939.

Permosperma ornatum Zalessky, 1939b, p. 372, ; fig. 56; seed; Permlan; Matreyevo, USSR. 
PEKIMOTHEOA Zalessky, 1829.

Permotheca sardykensis Zalessky, 1929a, p. 688 , fig. 15 ; microsporangia ; Permian; village of Koullarovo; Arsk, Tatare, USSR.

PERONOSPORITES W. G. Smith, 1877.

Peronosporites antiquarius W. G. Smith, 1877 , p. 499 , figs. 97,98 ; fungus ; Carboniferous; England.

PERONOSPOROIDES John Smith, 1896.

Peronosporoides carbonifera John Smith, 1896 , p. 321 , pl. 7 , flgs. 17,18 ; fungus spores and mycelium, in amber; Upper Carbontferous; Annandale near Kilmarnock, Scotland.

PERONOSPOROIDES E. W. Berry, 1916

Peronosporoides palmi E. W. Berry, 1916c, p. 74, pl. 180 ; spores and mycelium, Peronosporaceae, in silicifled palm stem; Oligocene ; Bayou Plerre, Miss.

PERRANDOA. Squinabol, 1891.

Perrandoa protogaea Squinabol, 1891, p. 778, pl. 17, fig. 2 ; fragment of palm leaf ; lower Miocene; Ste.-Justine, Sassello, Italy.

PERSEOPHYLLUM Kurtz, 1902.

Perseophyllum hauthalianum Kurtz, 1902, p. 52; Lower Cretaceous; Cerro Guido, Patagonia.

PERSEOXYLON Felix, 1887.

Perseoxylon antiquum Felix, 1887b, p. 153, pl. 27a, figs. 1-4; dicotyledonous wood.

PERUPIOPHYLLUM Steinmann, 1929.

Peruviophyllum minutifolium Steinmann, 1929 , p. 105 , fig. 113 ; fern rachis?; Cretaceous; Huayanco, Peru.

PESTALOZZITES E. W. Berry, 1917.

Pestalozzites sabalana E. W. Berry, 1917, p. 46. pl. 8 , fig. 3 ; pl. 9 , fig. 9 ; leaf spot fungus, Melanconiaceae ; Alum Bluff formation, Miocene; Alum Bluff, Liberty County, Fla.

PFTCHERIA. Zalessky, 1934.

Petcheria elongata Zalessky, 1934b, p. 288,

figs. 74, 75; leaf fragment, incertae sedis; Permian; Pechora basin, Russia.

PETCHEROPTERTS Zalessky, 1931

Petcheropteris splendida Zalessky, 1931b. p. 705 , pls. 1,2 ; petrified stem, Osmundaceae: Permian ; Pechora, Russia.

PETROPHIJ OIDES Bowerbank, 1840.

Petrophiloides richardsonii Bowerbank, 1840 , p. 44, pl. 9, figs. 9--15; pl. 10, figs. 5-8; cone, Conlferales; London Clay, Eocene ; Herne Bay, Sheppey, Eng* land.

PETROPHYTON Yabe, 1912.

Petrophyton miyakoense Yabe, 1912, p. 6. pl. 2, figs. 1-8; alga; Cretaceous ; Rikuchü province, Japan.
PETROSPHAERIA Stopes and Fujit, 1910. Petrosphaeria japonica Stopes and Fujii, 1910 , p. 4, pl. 1, figs. 1-6 ; fungus hyphae; Upper Cretaceous; Hokkaido, Japan. Cited in Stopes and Fujil, 1909. p. 558 ; nom. nud.

PETZHOLDTIA Unger, 1842.

Petzholdtia tropica Unger, 1842b, p. 176, wood, incertae sedis ; Tertiary ; Antigua. West Indies.

PETZIA Zalessky, 1931.

Acad. sci. U. R. S. S. Bull., 1931, p. 402 (not seen, cited in Gothan, 1942b, p. 140).

PEUCE Lindley and Hutton, 1832.

Peuce withami Lindley and Hutton, 1832 (1831-37), p. 73, pl. 24; coniferous woor ; 4 miles northwest of Durham, England.

PEUCEDANITES Heer, 1859.

Peucedanites spectabilis Heer, 1859, p. 25. pl. 104 , fig. 20 ; fruit, Umbelliferae: Miocene ; Oentngen, Switzerland.

PEZIZITES Meschinelli, 1892.

Pezizites sylvaticus (Ludwig) Meschinelli, in Saccardo, 1892, p. 775. See also Meschinelli, 1898, p. 49, pl, 5, fig. 14; Discomycete: Snlzhausen, Germany.

PHACIDIOPSIS Geyler, 1887.

Phacidiopsis sp. Geyler, 1887a, p. 487, pl. 32 , fig. 2 ; fungus, compared with Phacilium coronatum; Labaun, Borneo.

PHACIDITES Meschinelli, 1892.

Phacidites sinuosus (Ludwig) Meschinelli, in Saccardo, 1892, p. 776. See also Meschinelli, 1898, p. 50, pl. 15, figs. 33-35 ; fungus, Discomycete ; Germany.

PHACITES Colla, 1829.

Phacites alpinus (Jacquin) Colla, in Borson, 1829, p. 182.

PHACOLEPIS Frenguelli, 1942.

Phacolepis mendozana Frenguell, 1942, p. 323 , pls. 1,2 ; cone scale, Contferales ; Triassic ; Argentina.

PHACOPLASMIUM Reinsch, 1881.

Phacoplasmium sp. Reinsch, 1881, p. 39 , pl. 8 b, figs. 6-8; Upper Carboniferous ; Zwickau, Saxony.

PHAETHUSA Koenig, 1825.

Phacthusa lachrymabunda Koenig, 1825, p. 2 , pl. 1, flg. 23.

PHANEROPHLEBITES Knowlton, 1922.

Phanerophlebites pealei Knowlton, 1822a, p. 110, pl. 3, fig. 5 ; leaf fragment, Poly. podiaceae; Laramie formation, Upper Cretaceous ; Lafayette, Colo.

PHASBOLITES Unger, 1850.

Phaseolites cassiaefolius Unger, 1850a, . 488 ; leaf, Leguminosae; Miocene: Radibij, Croatia. Cited in Unger, 1845 (1841-47), p. lxxxv ; nom. nud. First species lllustrated: $P$. orbicularise Unger, 1851, p. 184, pl. 40, figs. 3, 4. 
PHASEOLITES L. R. Wilson and Coe, 1940. Phaseolites desmoinensis L. R. Wilson and Coe, 1940 , p. 182 , pl. 1 , fig. 4 ; spore; Des Molnes group, Pennsylvanian ; What Cheer, Keokuk County, Iowa

PHEGONIUM Unger, 1839.

Phegonium vasculosum Unger, $1839 \mathrm{~b}, \mathrm{p}$. 14. See discussion under Fegonium Unger.

PHELLODENDRONOIDITES ThOmsOn, 1950.

Phellodendronoidites sp. Thomson, in Potonle, Robert, Thomson, Paul W., and Thiergart, Friedrich, 1950 , p. 58, pl. B, fig. 43 ; pollen compared with Phellodendron.

PHELLOMYCETES Renault, 1896.

Phellomycetes dubius Renault, $1896 \mathrm{a}, \mathrm{p}$. 421 , fig. 74 ; fungus; Upper Carboniferous; Autun, France. Meschinelli, 1898 , p. 97 cites this genus with the spelling changed to Phellomycites.

PHELLOMYCITES.

See Phellomycetes Renault.

PHELONITES Fresnius, 1861.

Phelonites lignitum Fresnius, 1861, p. 155, pl. 62, figs. 1-15; Miocene; Salzhausen, Hesse.

PHENACOCLADUS Cockerell, 1926.

Phenacocladus $h$ e $n$ derson $i$ Cockerell, $1926 \mathrm{~b}$, p. 111, fig. p. 112 ; alga, Khodomelaceae; Green River formation, Eocene; Kimball Creek, Roan Mtn., Colo.

'HENANTHERA Hollick, 1907.

Phenanthera petalifera Hollick, 1907, p. 182 , figs. 1,2 ; flower allied to Caryophyllaceae, Rosales or Myrtales; Miocene ; Florissant, Colo.

PHIALOPHLOIOS Horich, 1915.

Phialophloios quadratus Horich, 1915, p. 426 , figs. $1-3$; arborescent lycopod stem impression ; Upper Carboniferous.

PHIALOPTERIS Presl, 1838.

Phialopteris tenera Presl, in Sternberg, 1838 (1820-38), D. 114, pl. 32, ffg. 1; fertile fernlike foliage; Upper Triassic (Keuper); Steindorf near Bamberg, Bavaria.

PHILLIPSIA Presl, 1838.

Phillipsia harcourtii Presl, in Sternberg, 1838 (1820-38), p. 206. For Lepidodendron harcourtii Witham, 1833, p. 75, pls. $12,13$.

PHLEBOMERIS Saporta, 1894.

Phlebomeris spectanda Saporta, 1894, p. 168 , pl. 29, fig. 14 ; pl. 30, flg. 1 ; fern frond, Matoniaceae?; Cretaceous; Portugal.

PHLEBOPTERIS Brongniart, 1836.

Phlebopteris polypodioides Brongniart, $1836(1828 a-38)$, p. 372 , pl. 83, flg. 1 ; fern leaf, Matoniacene; Jurassic: Scarborough. Fingland.
PHLEBOXYLON Hartig, 1848.

Phleboxylon pannonica (Unger) Hartig, $1848 \mathrm{a}$, p. 138 ; coniferous wood; Tertiary (Braunkohle); Germany.

PHLOISBOLITHES Steger, 1883.

Phloisbolithes striatus Steger, 1883, p. 28 ; Miocene; Kokoschutz, Silesla.

PHOENICITES Brongniart, 1828.

Phoenicites pumila Brongniart, $1828 \mathrm{~b}$, p. 121 ; nom. nud. First valid description?: Phoenicites spectabilis Unger, in Heer, 1855 , p. 94 , pl. 39 ; palm leaf ; Tertiary; Lausanne, Switzerland.

PHOENICOCARPUS Massalongo, 1859.

Phoenicocarpus chiavonicus Massalongo, $1859 \mathrm{a}$, p. 125 ; nom. nud; Oligocene; Chiavon, Italy.

PHOENICOPSIS Heer, 1876.

Phoenicopsis angustifolia Heer, $1876 \mathrm{c}, \mathrm{p}$. 51 , pl. 1 , fig. $1 \mathrm{~d}$; pl. 2 , flg. $3 \mathrm{~b}$; cycadophyte? follage; Jurassic; Kajamundung, Siberin.

PHOENICOPTERIS.

Phoenicopteris croizeti Lapparent, 1883, p. 1045 ; error for Phoenicopsis?

PHOLIDOPHLOIOS Zalessky, 1934.

Pholidophloios calminisious Zalessky, 1934d, p. 1115, fig. 11 ; lycopod leaf base impression; Carboniferous; Donets, Russia.

PHOLIDOPHORUS Zigno, 1856.

Pholidophorus beggiatiànus Zigno, 1856b, p. 331, Jurassic (Oolite) ; Rotzo, Italy.

PHOLIDOPHYLLUM Zalessky, 1937.

Pholidophyllum ornatum Zalessky, 1937, p. 81 , fig. 47 ; incertae sedis ; Permlan; Matveyevo, U. S. S. R.

PHOMITES Fritel, 1910.

Phomites 'myricae Fritel, 1910, p. 14, pl. 20, fig. 13 ; fungus, compared with Phoma (Sphaerloldeaceae, Fungl Imperfecti) ; upper Paleocene ; Cessoy (Seineet-Marne), France.

PHORMIDIODEA Wieland; 1930.

Phormidiodea superba Wleland, 1930, p. 28, fig. 1b; reef-forming alga; Cloverly formation, Lower Cretaceous; 16 miles enst of Medicine Bow, Wyo.

PHRAGMOTHYRITES Edwards, 1922.

Phragmothyrites eocaenica Edwards, 1922, p. 69 , pl. 8 ; fungus, Microthyriaceae ; Eocene; Isle of Mull, Scotland.

PHTHINOPHYLLUM Stur, 1877.

Phthinophyllum debile (Sternberg) Stur, 1877, p. 187. For Pecopteris debile Sternberg, 1825 (1820-38), Tentamen, p. xvili, pl. 26, fig. 3 ; Upper Carbonlferous ; Radnitz, Bohemia.

PHYCODES Debey and Ettingshausen, 1859.

Phycodes sericens Debey and Ettingshausen, $1859 \mathrm{a}$, p. 200 ; alga, incertae sedis; Cretaceous; Aachen, Rhenish Prussia. 
PHYCOIDELLA Matthew, 1890.

Phycoidella stichidifera Matthew, 1890a, p. 144, pl. 5, figs. 5a-d ; alga ; Cambrian ; Hanford Brook, Nova Scotia, Canada.

PHYCOMYCITES Ellis, 1915.

Phycomycites frodinghamii Ellis, 1915, p. 111 , pl. 1 ; mycelium and sporangla, Phycomycete; Jurassic; Lincolnshire, England.

PHYCOPSIS Rothpletz, 1896.

Phycopsis affinis (Sternberg) Rothpletz, 1896 , p. 885 , pl. 22 , figs. 1,2 ; alga.

PHYCOSIPHON Fischer-Ooster, 1858.

Phycosiphon incertum Fischer-Ooster, 1858 , p. 59, pl. 15, flg. 4 ; alga ?; Cretaceous?; Gurnigel, Switzerland.

PHYCOSIPHON Massalongo, 1859.

In Massalongo and Scarabelli, 1859, p. 92 ; a suggested name change for Brachycladium thomasinum Berkeley, 1848, p. 382 , pl. 11, figs. 2a, 2b ; Miocene; Prussia.

PHYLLADODERAIA Zalessky, 1913.

Phylladoderma arberi Zalessky, 1913, p. 24 , pl. 1 , fig. 4 ; pl. 2 , figs. 7,9 ; pl. 3, $5-8,10,11$; cordaitean? leaf, cuticle preserved ; Permian ; Chome-chor, Mont Talbei, Russia.

PHYLLADODESME Zalessky, 1929.

Phylladodesme zeilleri Zalessky, 1929a, p. 196, pl. 18, figs. 1-4 ; ginkgophyte? leaf ; lower Westphalian, Carboniferous; near Rovenki, Donets Basin, Russia.

PHYLLANTHINIUM Ogura, 1932.

Phyllanthinium pseudohobashiraishi Ogura, $1932 a$, p. 189 , pl. 4 ; petrified wood, Euphorbiaceae ; Tertiary ("Palaeogene"); near Fukuoka City, Kiushu, Japan.

PHYLLERITES Meschinelli, 1892.

Phyllerites palaeocassiae (Ettingshausen) Meschinelli, in Saccardo, 1892, p. 805 . See also Meschinelli, 1898, p. 104, pl. 29, fig. 1 .

PHYLLITES Brongniart, 1822.

Phyllites populina Brongniart, 1822, p. 237 , pl. 14, fig. 4 ; leaf, dicotyledon M i o c e n e; Oeningen, Switzerland. Brongniart's genus is based on this species. However, incluaing as it does a miscellaneous assemblage of leaves of doubtful affinity, a type species has little or no real significance.

PHYLLOCANNITES Kuntze, 1904.

Phyllocannites Kuntze, in Post and Kuntze, 1904, p. 435.

PHYLLOCHORDA Schimper, 1879.

Phyllochorda sinuosa (Ludwig) Schimper, in Schimper and Schenk, 1879 (1879$90)$, p. 50, fig. 38, alga, Chordophyceae; Upper Devonian; Thuringla.
PHYLLOCLADOPITYS Kräusel, 1928.

Phyllocladopitys capensus Kräusel, in Kräusel and Range, 1928, p. 35, pl. f, figs. 5,6 ; pl. 7 , figs. $1-6$; coniferous stem; Karroo beds, Permian; German Southwest Africa.

PHYLLOCLADOPSIS Fonatine, 1889.

Phyllocladopsis heterophylla Fontaine, 1889 , p. 204 , pl. 84 , fig. 5 ; pl. 167 , fig. 4 ; foliage, compared with Phyllocladus (Podocarpaceae); Potomac group, Lower Cretaceous; Virginia.

I HYLLOCLADOXYLON Gothan, 1905.

Phyllocladoxylon mülleri (S c h e n k) Gothan, 1905, p. 55. For Phyllocladus miilleri Schenk, in Zittel, 1879-90, p. 873, fig. 424.

PHYLLOCLADITES Visiani, 1858.

Phyllocladites foliosa (Sternberg) Visiani, in Massalongo, 1858c, p. 816. For Noeggerathia foliosa Sternberg, 1820-38, $\mathrm{n}$. 33 , pl. 20.

PHYLLODERMIUM Miner, 1935.

Phyllodermium reinschii Miner, 1935, p. 594 , pl. 21 , figs. 72,73 ; angiosperm cuticle ; Upper Cretaceous ; Amisut, east coast Disco Island, Greenland.

PHYLLOPITYS Zalessky, 1918.

Phyllopitys heeri (Schmalhausen) Zalessky, 1918, p. 23 , pl. 15, fig. 7.

FHYLLOPTERIS Brongniart, 1849.

A name created by Brongniart for $\mathrm{Glos}_{\text {- }}$ sopteris phillipsii Brongniart, 1830 $(1828 \mathrm{a}-38)$, p. 225, pl. 61 bis, fig. 5 ; pl. 63 , fig. 2 ; a Sagenopteris leaflet; Jurassic; Gristhorpe Cliff, near Scarborough, Yorkshire, England.

FHYLLOSTROBUS Saporta, 1873.

Phyllostrobus lorteti Saporta, 1873b, n. 134 ; see also Saporta, 1884 (1876-84), p. $636, \mathrm{pl}, 221$, figs. 1,2 ; coniferous foliage and cones; Jurassic ; Orbagnoux, France. Generic name cited in Saporta, $1872 \mathrm{~b}$, p. 1056 .

PHYLLOTAENIA Saporta, 1 S94.

Phyllotaenia demersa Saporta, 1894, p. 216, pl. 38 , fig. 6 ; leaf fragment, monocotyledion; Upper Cretaceous; Padrao, Portugal.

PHYLLOTENIA Salfeld, 1909.

Phyllotenia longifolia Salfeld, 1909, p. 27, pl. 4 , figs. $3-5$; foliage and seeds, Ginkgoales?; Jurassic; Salzhemmendorf, Germany.

PHYLLOTHALLUS Rothplet\%, 1896.

Plyyllothallus lumbricarius (Münster) Rothpletz, 1896, p. 902. For Chondritos lumbricarius Münster, 1843 (1939$43)$, p. 79 , pl. 2 , fig. 1. 
PHYLLOTHECA Brongniart, 1828.

Phyllotheca australis Brongniart, $1828 \mathrm{~b}$, p. 150, articulate stem and foliage; Hawkesbury River, near Port Jackson, Australia. One of first illustrations in a reasonably accessible source appears to be Feistmantel, 1878 , p. 83 , pl. 6 , fig. 3 ; pl. 7, figs. 1,2 ; pl, 15, figs. $1,2$.

PHYMATOCARYON Mueller, 1871.

Phymatocaryon mackayi Mueller, 1871 (1871-82), p. 47, pl. 2; Pliocene; Smythe's Creek, Victoria.

PHYMATODERMA Brongniart, 1849.

Phymatoderma granulatum (Schlotheim) Brongniart, 1849, p. 59. For Algacites granulatus Schlotheim, 1822, p. 46, pl. 5, fig. 1 ; alga ?; Jurassic ; Württemberg.

PHYMATOLITHES Romanowski, 1890.

Phymatolithes algeformis Romanowsk1, 1890 , p. 142, pl. 21, fig. 5 ; Lower Jurassic; Thian-Schan, 'Turkistan, Asia.

PHYSAGENIA Heer, 1855.

Physagenia parlatorii Heer, 1855, p. 109. pl. 42, flgs. 2-17; incertae sedis; Ter tiary.

PHYSEMATOPITYS Goeppert, 1850.

Physematopitys salisburioides Goeppert 1850 , p. 242 , pl. 49 , figs. $1-3$; coniferous wood ; Tertiary (Braunkohle); Schwer ta, Lusatia, Germany.

PHYSOPHYOUS Schimper, 1869.

Physophycus marginatus (Lesquereux) Schimper, 1869 (1869-74), p. 207. For Caulerpites marginatus Lesquereux. 1869 , p. 314 , pl. 7 ; alga?; Carbonifer. ous; Württemberg, also Lawrence County, Pa.

PHYSOPHYLLUM Massalongo, 1858.

Physophyllum tococaefolium Massalongo. $1858 \mathrm{a}$, p. 122 ; leaf, Melastomaceae; Tertiary ; Italy. See Massalongo, 1859, p. 410 , pl. 8 , fig. 15 ; pl. 38 , fig. 23.

PHYSOPORELLA Steinmann, 1903.

Physoporella pauciforata (Gumbel) Steinmann, 1903, p. 17, fig. 7 ; alga, Dasycladaceae; Triassic (Keuper); South Tyrol.

PHYSOSTOMA Williamson, 1876.

Physostoma elegans Williamson, $1876 \mathrm{~b}, \mathbf{p}$. 160 ; petrified seed, Pteridospermae; Up per Carboniferous. For illustrations. see Williamson, 1877 , p. 262, pl. 11, flgs. 77, 78. [Name changed to Lagenostoma physoides in Willamson, $1876 \mathrm{a}, \mathrm{p} .70$, and again to Physostoma elegans, in Oliver, 1909 , p. 74.]

PHYTOOALYX Bornemann, 1886.

Phtocalyx antiquus Bornemann, 1886, p. 13, pl. 1, figs. 1-8; alga?; Cambrian ; Sardinia. Earlfer citation : Bornemenn, 1883 , p. 272 ; nom. nud.

\section{PHYTOLITHUS}

This name applied to a diverse assemblage of fossil plants by Martin, 1809. First citation after 1820 appears to be $P h y$ tolithus sulcatus Sternberg, 1825 (182038 ), p. 28, pl. 5, figs. 2-6.

PHYTOPSIS Hall, 1847.

Phytopsis tubulosum Hall, 1847, p. 38, pl. 8, figs. 1a-e; plant?; Lowville limestone (Birdseye limestone), Ordovician ; near Amsterdam, N. Y.

PHYTORADIOULARIA Hollick, 1930.

Phytoradicularia dubia Hollick, in Hollick and Martin, 1930, p. 116, pl. 2, fig. 10; incertae sedis; Upper Cretaceous; Herendeen Bay, Alaska Península.

PIAEA Florin, 1929.

Piaca punctata Florin, 1929a, p. 244, pl. 1., figs. 1-5 ; pl. 2, figs. 1-4; pl. 3, figs. 1.-6 ; alga, Dasycladacene?; Permian ; Oberhessen, Biidingen, Germany.

PIAELLA Fucini, 1.936.

Reference not secn ; cited in Gothan, 1942b, p. 141.

PICCOLOMINITES Unger, 1845.

Piccolominites sard118 Unger, 1845 (1841.47); p. xc; wood; Mlocene; Sardinia.

PICEITES Goeppert, 1850.

Piteites reucheanus (Goeppert and Berendt) Goeppert, 1850 , p. 209, pl. 30, tigs. 1, 2; cone, Coniferales ; Tertiary.

PICEOPHYLLUM Ogura, 1932.

Piceophyllum simplex Ogura, 1932b, p. 463 , pl. 22 , fig. 5 ; petrifled leaf, Abletineae, Contferales; Cretaceous ; Hokkaido, Japan.

PICEOXYLON Gothan, 1906.

Piceoxylon pseudotsugae Gothan, in Henry Potonie, 1906, no. 80, p. 1, fig. 1 ; coniferous wood; Tertiary; California.

PIETZSCHIA Gothan, 1927.

Pietzschia schulleri Gothan, 1927a, p. 5, pls. 1, 2; petrified stem, related to Cladoxylon; Wildenfels shale, Upper Devonian; Saxony.

PILA C. E. Bertrand and Renault, 1892.

Pila bibractensis C. E. Bertrand and Renault, 1892, p. 159, pl. 6 ; alga ?; Permian ; Autun, France.

PILODEA PIa, 1937.

Pilodea sp. Pla, 1937, p. 834 ; alga, Chaetangiaceae; Permian; Sumatra.

PILOPHOROSPERMA Thomas, 1933.

Pilophorosperma granulatum Thomas, 1933 , p. 207 , pl. 23 , fig. 58 ; pteridosperm inflorescence with seeds enclosed in cupules; Molteno beds, - Karroo system, Triassic; Upper Umkomas Valley, Natal.

PILULARITES Goeppert, 1837.

Pilularites braunii Goeppert, 1837, p. 439 ; Triassic (Keuper) ; Bayreuth, Bavaria. 
PIMPINELLITES Unger, 1839.

Pimpinellites zizioides Unger, 1839a, p. 104 ; fruit, Umbelliferae ; Miocene ; Radoboj, Croatia.

PINAKODENDRON C. E. Weiss, 1893.

Pinakodendron musivum C. E. Weiss, in Welss and Sterzel, 1893, p. 61, pl. 3, fig. 16 ; Upper Carboniferous ; near Wattenscheid, Westphalia.

PINIPHYLLUM Nathorst, 1886.

Piniphyllum Nathorst, 1886a, p. 53 ; nom. nud.

PINITES Lindley and Hutton, 1831.

Pinites brandlingi Lindley and Hutton, 1831 (1831-37), p. 1 , pl. 1 ; cordaltean petrified tree; Carboniferous; Wideopen, near Gosforth, 5 miles north of Newcastle-upon-Tyne. Described and flgured but not named by Witham, 1831 , p. 31 , pl. 4, flgs. $1-5$; later placed in Dadoxylon. See Seward, 1917, p. 254.

PINNULARIA Lindley and Hutton, 1832.

Pinnularia capillacea Lindley and Hutton, 1832 (1831-37), p. 81, pl. 111 ; probably calamitean roots; Carboniferous; England.

PINOSTROBUS (Feistmantel) Stopes, 1915 ?

Pinostrobus sussexiensis (Mantel) Stopes, 1915 , p. 123 , pl. 10 , figs. $2-4$; pl. 11 , fig. 3 ; abietinean cone ; Lower Greensand, Cretaceous; Selmeston; Sussex, England. Original citation: Pinostrobus vallidus Ottokar Feistmantel, 1875. p. 272 ; nom. nud. See also Stopes, 1915, p. 122.

PINOXYLON Knowlton, 1900.

Pinoxylon dacotense Knowlton, in Ward, 1900 a, p. 420 , pl. 179 ; wood, compared with Pinus but lacking large rays; Jurassic ; 3 miles west of Sturgis, $S$. Dak.

PINUXYLON Gothan, 1906?

Pinuxylon succiniferum (Goeppert and Berendt) Gothan, in Heinhold, 1906, p. 118. Cited originally as Pinuxylon sp. Gothan, 1905, p. 102. For l'inites su;cinifer Goeppert and Berendt, in Berendt, 1845, p. 89 . pl. 2, figs. 1-8.

PIPERITES Goeppert, 1853.

Piperites miquelianus Goeppert, 1853, p. 41, pl. 7 , figs, 48,49 , leaf, Pinerncene Tertiary ; Dorfe Tandjung, Java.

PIROCONITES Gothan, 1914.

Piroconites kusperti Gothan, 1914, p. 42, pl. 28 , fig. 4 ; portion of cone, Bennettitales; Rhaetic; Nurnberg, Germany.

PISONIAEPHYLLITES Hector, 1880.

Pisoniaephyllites novaezealandiae Hector, 1880 , p. 49 ; nom. nud.
PISTITES Hosius and Marck, 1880.

Pistites loriformis Hosius and Marck, 1880 , p. 182 , pl. 38 , flgs. 151, 152 ; leaves, Pistiacene; Upper Cretaceous Westphalia.

PITOXYLON Hartig, 1848.

Hartig, 1848b, p. 138, proposes this genus to include certain species formerly placed in Pence.

PITUS Witham, 1833.

Pitus antigua Witham, 1833, p. 37, p1. 8, figs. 1-3; wood, Cordaitales. Lower Carboniferous ; Lennel Braes, : Tweed Mill, Berwick, Scotland. Witham's name was corrected by later authors to Pity8; see Unger, 1842 (1841-47), p. 78 ; Seward, 1917, p. 285 ; Scott, 1923, p. 255.

PITYANTHUS (Nathorst) Seward, 1919.

Pityanthus granulatus (Heer) Seward, 1919 , p. 395. For Ophioglossum granulatum Heer, 1883, pl. 57, figs. 8, 9; abietinean microsporangiate cone; Cretaceous (Patoot); Greenland. Original citation of genus: Pityanthus sp. Nathorst, 1899 , p. 16 , pl. 2, fig. 7 .

PITYITES Seward, 1919.

Pityites solmsi Seward, 1919, p. 373, figs. 772, 773; coniferous shoots and cones, appear similar to Prepinus; Wealden; Sussex, England.

PITYOCLADUS (Nathorst) Seward, 1919.

Pityocladus longifolius (Nathorst) Seward, 1919 , p. 378 , figs. 775,776 ; foliage shoots, Coniferales; Rhaetic; Scania, Sweden. Originally applied as a subgenus of Pinites by Nathorst.

PITYOIDOLEPIS Hollick and Jeffrey, 1909. Pityoidolepis statenensis Hollick and Jeffrey, 1909, p. 53, pl. 9, figs. 13, 14 ; pl. 27, figs. 1-3; cone scale, Coniferales; Cretaceous; Kreischerville, Staten Island, N. Y.

PITYOPHYLLUM Nathorst, 1899.

Pityophyllum staratschini Nathorst, 1899, p. 19 , pl. 2, figs. 24,25 ; coniferous leaves; Jurassic ; Franz Josef Land.

PITYORADIX Chachloff, 1924.

Pityoradix irkutensis Chachloff, 1924, p. 29 ; pl. 10, figs. 62,67 ; Upper Jurassic ; Irkoutsk, Siberia.

PITYOSPERMOM Nathorst, 1899.

Pityospermum maakianum (Heer) Nathorst, 1899, p. 17, pl. 2, fig. 15; seed, affinlties with Tsuga?; uppermost Jurassic ; Franz. Josef Land.

PITYOSPORITES SEward, 1914.

Pityosporites antareticus Seward, 1914, p. $\mathbf{2 3}$, p1. 8, fig. 45 ; winged spores, Abie tineae ; supposedly derived from Beacon sandstone, not older than Rhaetic; Priestley Glacier, Antarctica. 
PITYOSTROBUS (Nathorst) Dutt, 1916.

Pityostrobus macrocephalus (Lindley and Hutton) Dutt, 1916, p. 529, pl. 15; cone, compared with Pinus excelsa Linnaeus; lower Eocene; Dover, England. Original generic citation: Pityostrobus sp. Nathorst, 1899, p. 17, pl. 2, figs. 9, 10.

PITYOXYLON Kraus, 1870.

Pityoxylon sandbergeri Kraus, in schimper, 1870 (1869-74), p. 378, pl. 79, fig. 8 ; Triassic (Keuper) ; Kitzingen, Bavaria.

PITYS.

See Pitus Witham.

PLAGIOPODOPSIS Britton and Hollick, 1915.

Plagiopodopsis scudderi Britton and Hollick, 1915, p. 10 , figs. 1,2 ; moss, compared with Plagiopus (Bartramiaceae); Miocene; Florissant, Colo. See later discussion by Steere, 1946, p. 313.

PLAGIOZAMITES Zelller, 1804.

Plagiozamites planchardi (Renault) Zeiller, 1894, p. 174, pl. 8, fig. 1 ; pl. 9, fig. 1 ; cycadophyte? leaf; Permian; Trienbach, Alsace.

PLAGIOZAMIOPSIS Sze, 1943.

Plagiozamiopsis podozamioides Sze, 1943, p. 511, figs. 1-10 ; cycadophyte foliage ; Permian.

PLANOXYLON Stopes, 1916.

Planoxylon hectori Stopes, 1916, p. 120. pl. 4, figs. 1-5; coniferous wood; Cretaceous; Amuri Bluff, New Zealand.

PLANTAGINOPSIS Fontaine, 1905.

Plantaginopsis marylandica Fontaine in Ward, 1005, p. 561, pl. 117, flg. 7 ; pl. 118 , figs. 1,2 ; leaf, dicotyledon ; Potomac group, Lower Cretaceous; Federal Hill, Baltimore, Md.

PLATAEANTHUS.

Error for Palaeanthis, in Knowlton, 1898, p. 168.

PLATANINIUM Unger, 1842.

Plataninium acerinum Unger, 1842b; p. 174. See Unger, $1847(1841-47)$, p. 138, pl. 47, figs. 8-10.

PLATANITES Forbes, 1851.

Platanites herbridicus Forbes, 1851, p. 103, pl. 4, fig. 1 ; leaf, compared with Platanus (Platanaceae); Tertiary; Isle of Mull, Scotland.

PLATANOIDITES Robert Potonie, 1950.

Platanoidites gertrudae Robert Potonie, in Potonie, Robert, Thomson, Paul W., and Thiergart, Friedrich, 1950 , p. 57, pl. B, fig. 40 ; pollen, Platanaceae?; Pliocene; Chatt-Aquitan, Germany.

PLATANOPHYLLUM Fontaine, 1889.

Platanophullum crossinerve Fontaine, 1889 , p. 316 , pl. 158 , fig. 5 ; leaf fragment, compared with Araliaephyllum and Hedera platanoides Lesquereux; Potomac group; Lower Cretaceous ; Virginia.
PLATYCERIPHYLIUM Velenovsky, 1889. Platyceriphyllum cretaceum Velenovsky, 1889, p. 29, pl. 5, tig. 16 . For Platyccrium cretaceum Velenovsky, 1889, p. 5 ; leaf fragment; Cretuceous (Cenomanian); Vyserovic, Bohemia.

PLATYCERITES Goeppert, 1854.

Platycerites wirthgenianus Goeppert, 1854, p. 98 ; nom. nud. ; Miocene; Niederrhein, Germany.

PLATYCOILA Mueller, 1874.

Platycoila sullivani Mueller, 1874, p. 23, pl. 9, figs. 5-9; angiospermous fruit; lower Pliocene; near Nintingbool, Victoria.

PLATYLEPIS Saporta, 1874.

Platylepis micromyela Saporta, 1874 $(1873 \mathrm{c}-75)$, p. 278 , pl. 120, figs. $1-3$; cycadophyte trunk; Jurassic (Lias); Tournay-sur-Odon, France.

PLATYMASTIXIA Kirchheimer, 1934.

platymastixia cacaoides (Zenker) Kirchheimer, $1934 \mathrm{~b}$, p. 790 , figs. 21 ; fruit, Cornaceae; Tertiary (Braunkohle): Altenburg, Germany.

PLATYPEUOE Menge, 1850.

Platypeuce alichotoma Menge, 1850 , p. 26, pl. 3, figs. 8-14; Tertiary (Braunkohle); Redlau near Danzig, Prussia.

PLATYPHYLLUM (Dawson) David White, 1905.

Platyphyllum brownianum Dawson, in Smith and White, 1905, p. 37, pl. 2, figs. 1, 2. [Dawson, 1881a, p. 11, proposed Platyphyllum for Oyclopterts brownii if the latter is found at a later date with a fructification. Dawson, 1888, p. 265, uses the binomlal Platyphisllum brownii but only in a list and without description. The above reference to White appears to be the first valid one.]

PIATYPTERYGIUM (Schimper) Ottokar Feistmantel, 1886.

Platynterygium balli Ottokar Felstmantel, 1886, p. 37, pl. 2A, figs. 4-8; pl. 3A, fig. 2 ; cycadophy te leaf ; Barakar group ; west of Gurtur, western Bengal, India.

PLA TYSOLENITES Quenstedt, 1867.

Platysolenites sp. Quenstedt, 1867, p. 842, pl. 80 , fig. 20 ; Tertiary ; Russia.

PLATYSPERMUM E. A. N. Arber, 1914.

Platyspermum sulcatum (Presl) E. A. N. Arber, 1914, p. 95, pl. 6, flg. 11; seed; Transition Coal Measures of South Staffordshire and Middle Coal Measures of Warwickshire and Yorkshire, England.

PLECTITES Reinsch, 1881.

Plectites sp. Reinsch, 1881, p. 72, pl. 16a, figs. $1-5 ;$ pl. $17 \mathrm{a}$, figs. $1-8$; Permian; Stockheim. Württemberg. 


\section{INDEX OF GENERIC NAMES OF FOSSIL PLANTS 1820-1950}

PLLIAGRON Mueller, 1877.

Pleiacron elachocarpum Mueller, 1877a (1877-79), p. 179; fruit ; Tertiary ; New South Wales. See also Mueller, 1883, p. 2, pl. 15, figs. 15-18.

PLEIOCLINIS Mueller, 1882.

Fleioclinis couchmanii Mueller, 1882 (1871-82), p. 43, pl. 19, figs. 1-11; Pliocene; Nintingbool and Haddon, Victoria.

PLEIOMERITES Ettingshausen, 1868.

Pleiomerites reticulatus Ettingshausen, $1868 \mathrm{a}$, p. 226 , pl. 38 , flg. 6 ; leaf, Myrsineae ; Tertiary.

PLEIOMEROPSIS Weyland, 1938.

Pleiomeropsis rottensis Weyland, $1938 \mathrm{~b}$, p. 161, pl. 23, figs. 1-7; inflorescence, Myrsinaceae; Tertiary ; Rott, Siebengebirge, Germany.

PLEOSPORITES Suzuki, 1910.

Pleosporites shirainus Suzuki, 1910, p. 191 , pl. 7 , fig. 6 ; fungus; Upper Cretaceous ; Hokkaido, Japan.

PLESIOCAPPARIS Mueller, 1871.

Plesiocapparis prisca Mueller, 1871 (187182), p. 40, pl. 4, figs. 9-11; Pllocene; Haddon, Victoria.

PLEURODIOTYTES Reinsch, 1881.

Pleurodictytes sp. Reinsch, 1881 , p. 89 , pl. 29, figs. 1-7 ; pl. 29a, figs 1-7 ; Permian ; Stockheim, Württemberg.

PLEUROMEIA Corda, 1852.

Pleuromeia sternbergi (Münster) Corda, in Germar, 1852, p. 184 (original spelling given by Corda is Pleuromeya). For Sigillaria sternbergi Münster, 1839 (1839-43), p. 47, pl. 3, fig. 10 ; Triassic (Bunter Sandstein) ; Magdeburg, Prussian Saxony.

PLEUROMEYA.

See Pleuromeia Corda, 1852.

PLLUROPLASMIUM Reinsch, 1881.

Pleuroplasmium sp. Reinsch, 1881, p. 24, pl. 1, flgs. 1-7; pl. 2, flgs. 1-6 ; Upper Carboniferous ; Zwickau, Saxony.

PLEUROSTROMIUM Reinsch, 1881.

Pleurostromium sp. Retnsch, 1881, p. 59, pl. 14a, figs. 1-4; Upper Carboniferous; Zwickau, Saxony.

PLEXXIPLICA Kirchheimer, 1935.

Plexiplica reidi Kirchheimer, 1935, p. 293, fig. 18 ; endocarp, Cornaceae; Oligocene (Braunkohle) ; Helene near Borna, Germany. See also Kirchheimer, 1936c, p. 292 , pl. 8, figs. 1a-e.

PLINTHIOTHEOA Zeiller, 1899.

Plinthiotheca anatolica Zelller, 1899, p. 54, pl. 4, figs. $18,18 \mathrm{a}$; incertae sedis; Carboniferous ; Bassin d'Heraclée, Asia Minor.

PLOCARITES Massalong0, 1851.

Plocarites polymorphus Massalongo, 1851, p. 63, alga ; Tertiary ; Italy.
PLOCHMOPELTINITES Cookson, 1947. Plochmopeltinites masoni Cookson, $1947 \mathrm{~b}$, p. 212 , pl. 13 ; figs. 14,15 ; ascomata, Micropeltaceae ; late Oligocene ; Kerguelen Island, South Indian Ocean.

PLUMALINA Hall, 1858.

Plumalina gracilis Hall, 1858, p. 175; probably not a plant; Chemung group, Devonian; Missouri. See also Miller, S. A., 1889, p. 134.

PLUMATOPTERIS Kidston, 1894.

Plumatopteris elegans Kidston, 1894, p. 259 , pl. 5, figs. $1,1 a$; sterile fern follage; Calderwood group, Carboniferous Limestone series, Lower Carboniferous; East Kilbride, Lanarkshire, Scotland.

PLUTONIA Velenovsky, 1889.

Plutonia cretaceae Velenovsky, 1889 , p. 11, pl. 2 , figs. $11-20$; pl. 3 , figs. 1,2 ; follage and cones, Coniferales ; Upper Cretaceous ; Lipenec, Bohemia.

POACITES Schlotheim, 1820.

It seems evident that Schlotheim proposed this genus to include supposed grass leaves. The species he described are Carboniferous in age and clearly not grasses. A variety of fossils have been assigned to the genus, for example: Poacites carinata Brongniart, 1822, p. 238 , pl. 14 , fig. 2 ; this species is apparently an arborescent lycopod leaf. Poacites cocoina Lindley and Hutton (see Seward, 1898, p. 366) ; is probably a calamite. Poacites firmus Heer, 1855, p. 70 , pl. 25 , fig. 11 ; the first well-1llustrated description of a fossil that bears good evidence of being a grass and is suggested as the type (Miocene; Lausanne, Switzerland).

POACORDAITES Grand'Eury, 1877.

Poacordaites latifolius (Goeppert) Grand'Eury, 1877, p. 224. For Noeggerathia palmaeformis Goeppert, 1852b, p. 216 , pl. 15 ; pl. 16, figs. 1-3; given earlier as Poacites latifolius Goeppert, 1844, p. 216.

POACORDAIXYLON Renuult, 1885.

Poacordaixylon stephanense Renault, 1885, p. 81, pl. 6, flgs. 20-23 ; cordaitean wood ; Upper Carboniferous ; Montmartre, st.Etienne, France.

PODALYRIOPHYLLUM Ettingshausen, 1895.

Podalyriophyllum brochidodromum Ettingshausen, 1895, p. 51, pl. 4, fig. 17 ; leaf, Leguminosae; Upper Cretaceous; between Warnagh and Oxley Station, Australia.

\section{PODOCARPITES Andrae, 1855.}

Podocarpites acicularis Andrae, 1855, p. 45, pl. 10, fig. 5 ; coniferous leaves?; Jurassic ; Hungary. 
PODOOARPOIDITES Robert Potonie, 1950. Podocarpoidites libellus. Robert Potonie, in Potonie, Robert, Thomson, Paul W., and Thiergart, Friedrich, 1950 , p. 49 , pl. C, fig. 6 ; pollen, Podocarpaceae?; lower Miocene; Niederalusitz, Germany.

PODOCARPOXLON Gothan, 1904.

Podocarpoxylon juniperoides Gothan, in Gagel, 1904, p. 272 ; coniferous wood ; Pleistocene; Elmshorn, Prussia. First? illustrated species: Podocarpoxylon aparenchymatosum Gothan, 1908, p. 8, pl. 1, figs. 9-1.. See also Gothan, 1905 ; Seward, 1919, p. 173 ; Kríusel, 1949.

PODOCARYA (Buckland) Goeppert, 1848.

Podocarya bucklandi Goeppert, in Bronn, 1848 , p. 1023 ; Lower Oolite, Jurassic ; Charmouth, England. Originally cited as Podocarya sp. Buckland, 1836, p. 505, pl. 43, figs. 2-10 ; petrified fruit referred to Pandanaceae.

PODOQONIUM Feer, 1859.

Podogonium knorrii Heer, 1859, p. 114, pl. 134, figs. $22-26$; pl. 135 ; pl. 136, figs. 1-9; leaves, Caesalpineae; Miocene, Switzerland.

PODOLOMA Ettingshausen, 1879.

Podoloma polypodioides Gardner and Ettingshausen, 1879 , p. 29, pl. 3, figs. 4-6, 9 ; leaf fragment, Polypodiaceae; Eocene ; Bournemouth, England.

PODOSPORITES Rao, 1943.

Podosporites tripakshi Rao, 1943b, p. 182, figs. 1-13; spores with three bladders, probably Podocarpineae; Jurassic; Nipania, Rajmahal Hills, Behar, India.

PODOSTEMON Unger, 1853.

Podostemon ceratophylloides Unger, in Massalongo, 1853b, p. 7 ; Eocene ; Monto Bolca, Italy.

PODOSTEMONOPSIS Weyland, 1938.

Podostemonopsis tertiaria Weyland, 1938 , p. 90 , pl. 11 , 6gs. $6-10$; Infructescence, Podostemonaceae ; Tertiary ; Rott, Siebengebirge, Germany.

PODOZAMITES (Brongniart) C. F. W. Braun, 1843.

Podozamites distans (Presl) C. F. W. Braun, in Münster, 1843 (1839-43), p. 28. For Zamites distans Presl, in Sternberg, 1820-38, p. 196, pl. 41, flg. 1 ; Jurassic (Lower Lias); Bayreuth, Bavaria.

POECILITOCAULON Fliche, 1910.

Poecilitocaulon dubium Fliche, 1910, p. 261 , pl, 26, flg. 3 ; stem impression, incertae sedis; Triassic; Meurthe-et-Moselle, France.

POECILITOSTACHYS Fliche, 1910.

Poecilitostachys hangi Fliche, 1910, p. 264 , pl. 26, fig. 4 ; pl. 27 , flg. 1 ; incertae sedis ; Triassic; Meurthe-et-Moselle, France.
POECILOXYLON Grand'Eury, 1877.

Poeciloxylon proprium Grand'Eury, 1877, p. 307 , wood, some comparison with Dadoxylon; Carboniferous; Loire, France.

POLIOEXOLOBUS E. W. Berry, 1938.

Polioexolobus prenuntius $\mathrm{E}$. W. Berry, 1938 , p. 128 , pl. 51 ; leaf, Asclepladaceae ; Rio Pichileufu, Argentina.

POLLENITES Robert Potonle, 1931.

Pollenites iliacus Robert Potonie, 1931a, p. 556, fig. 5 ; pollen; Miocene.

POLYCARPELLA Reld and Chandler, 1933.

Polycarpella caespitosa Reid and Chandler, 1933, p. 486, pl. 28, flgs. 13-21; Incertae sedis; London Clay, Eocene; Sheppey, Kent, England.

POLYGONITES Saporta, 1865.

Polygonites ulmaceus Saporta, 1865, p. 92 , pl. 3 , fig. 14 ; winged fruit, Polygonaceae ; Tertiary ; St.-Jean-de-Garguier, France.

POLYGONOCARPUM Weyland, 1938.

Polygonocarpum fimbriatum Weyland, 1938a, p. 87, pl. 11, figs. 1, 1a ; winged fruit, Polygonaceae; Tertiary ; Rott, Siebengebirge, Germany.

POLYGONOOARPUS (Zeiller) Zalessky, 1907.

Polygonocarpus caarnockit Zalessky, 1907, p. 68 , pl. 2, fig. 15 ; Upper Carboniferous ; Dombrowa, Russia.

POLYGONOSPHAERITES Ferdinand Roemer, 1880.

Polygonosphaerites tessellatus (Phillips) Ferdinand Roemer, 1880, p. 297. For Sphaeronites tessellatus Phillips, 1841, p. 135, pl. 59, fig. 49 ; Devonlan; Ply. mouth, England.

POLYLOPHOSPERMUM Brongniart, 1874.

Polylophosphermum stephanense Brongnfart, 1874 , p. 264 , pl. 23, flgs. 6-8; silicifled seed; Carboniferous; St.-Dt1enne, France.

POLYMORPHOCODIUM Derville, 1031.

Polymorphocodium lapparenti Derville, 1931 , p. 54, pl. 4, flgs. $12-14,16$; alga, Codlaceae; Carboniferous; Henirette, Bas-Boulonnais, France.

POLYPODISPORITES Robert Potonie, 1934.

Polypodiisporites favus Robert Potonie, 1934 , p. 38 , pl. 1 , figs. 19,20 ; spore, Poly podiaceae; Miocene.

POLYPODIOLITES Sternberg, 1823.

Polypodiolites pectiniformis Sternberg, 1823 (1820-38), pl. 33, fig. 1 ; cycadophyte frond; Jurassic; Stonesfleld, England. 
POLYPODITES Goeppert, 1836.

Polypodites mantellii (Brongniart) Goeppert, 1836, p. 341. For illustration, see Lonchopteris mantelli Brongniart, in Iindley and Hutton, 1837 (1831-37), p. 59, pl. 171; fern? foliage; Lower Cretaceous; near Wansford, Northamptonshire, England.

POLYPORITES Lindley and Hutton, 1833.

Polyporites bowmanni Lindley and Hutton, 1833 (1831-37), p. 181, pl. 65 ; fungus, Polyporaceae; Upper Carboniferous ; near Wrexham, Denbigh, Wales. Meschinelli, 1892, p. 746 , erroneously attributes this genus to Fries.

POLYPTEROCARPUS Grand'Eury, 1877.

Polypterocarpus caudatus Grand'Eury, 1877 , p. 506, pl. 15, figs. 7-11; winged seed; Carboniferous ; France.

POLYPTEROSPERMUM Brongniart, 1874.

Polypterospermum renaultii Brongniart, 1874 , p. 256 , pl. 23, flgs. 1-3; silicified s e e d ; Carboniferous; St.-Etienne, France.

POLYSIPHONIDES Schimper, 1869.

Polysiphonides koechlini (Heer) Schimper, 1869 (1869-74), p. 178, pl. 3, fig. 5; alga?; Mfocene; Bouxwiller, near Ferrette, France.

POLYSORITES Raciborskt, 1889.

Polysorites sp. Raciborski, 1889, p. 138.

POLYSPORIA Newberry, 1853.

Polysporia mirabilis Newberry, 1853a, p. 108 ; nom. nud.

POLYSTICHITES Presl, 1838.

Polystichites murrayana (Brongniart) Presl, 1838, in Sternberg, 1820-38, p. 117. For Pecopteris murrayana Brongniart, 1828a-38, pl. 126, figs. 1-5 ; fernlike foliage; Jurassic; Scarborough, England.

POLYSTIGMITES Meschinelli, 1892.

Polystigmites priscus (Massalongo) Meschinelli, in Saccardo, 1892, p. 770. See also Meschinelli, 1898, p. 43, pl. 14, fig. 14 ; fungus ; Miocene; Italy.

POLYTHECA Henry Potonie, 1900.

Polytheca desaillyi (Zeiller) Henry Potonie, 1900 , p. 447, fig. 251; fern sporangia ; Upper Carboniferous.

POLYTRICHITES Britton, 1926.

Polytrichites spokanensis Britton, in Knowlton, 1926, p. 24, pl. 8, figs. 3, 4 ; moss, Polytrichaceae ; Latah formation, Miocene; Deep Creek, northwest of Spokane, Wash.

POLYTRICHITES Yasui, 1928.

Polytrichites aichiense Yasui, 1928, p. 439, pl. 22, figs. 95-103; moss, compared with Polytrichum; upper Tertiary ; Aichi coalfield, Japan.
POLYT'RIPA Defrance, 1825.

Polytripa elongata Defrance, in Bronn, 1825 , p. 44, pl. 7 , fig. 15 ; Palaeocene; Paris, France.

POMADERRITES Ettingshausen, 1883.

Ponaderrites banksii Ettingshausen, 1883, p. 141 , pl. 6, fig. 4 ; leaf, Rhamnaceae; Eocene; Dalton near Gunning, Austràlia.

PONDICHERRIA. Sahni, 1933.

Pondicherria ebenaleoidea Sahni, 1933, p. 436, pl. 25; syncarpous multilocular fruit, compared with Achras (Sapotaceae) and Diospyros (Ebenaceae) ; probably Upper Cretaceous; Pondicherry, south India.

PONDICHERRIOIDEA.

Error for Pondicherria, in Sahni, 1933, $p$. 436.

PONTEDERITES Knowlton, 1922.

Pontederites hesperia Knowlton, 1922b, $\mathrm{n}$. 154 , pl. 36, fig. 6 ; leaf fragment, Pontederiaceae; Green River formation, Eo. cene; Greasewood Creek, Rio Blanco County, Colo.

POPULITES Viviani, 1833.

Populites phaetonis Viviani, 1833 , p. 133 , pl. 10, fig. 2?; leaf, dicotyledon; Tertiary ; near Pavia, Italy.

IOPULITES Geoppert, 1852.

Populites platyphyllus Goeppert, 1852a, p. 276 , pl. 35 , fig. 5, leaf, Salicineae ; Tertiary; Stroppen, Silesia.

POPULOCAULIS Stopes and Fujii, 1910.

Populocaulis yezoensis Stopes and Fujil, 1910 , p. 64 , pl. 8 , fig. 49 ; petrified stem, compared with Populus; Upper Cretaceous; Hokkaido, Japan.

FOPULOPHYLLUM Fontaine, 1889.

Populophyllum reniforme Fontaine, 1889, p. 31.1, pl. 155, fig. 9 : pl. 156, fig. 3 ; leaves, compared with Populus; Potomac group, Lower Cretaceous; Brooke, Va.

PORODENDRON (Nathorst) Zalessky, 1909.

Forodendron tenerrimum (Auerbach and Trautschold) Zalessky, 1909, p. 5, pl. 1, figs. 1-4; Carboniferous; Mugodzary, Russia.

POROSTROBOSPORITES Wicher, 1934.

Porostrobosporites bennholdi Wicher, 1934, p. 92 , pl. 6, figs. 10-12 ; Carboniferous ; Ruhr, Germany.

POHOSTROBUS Nathorst, 1914.

Porostrobus zeilleri Nathorst, 1914, p. 70, pl. 5, figs. 12-16; lycopod cone compression; Paleozolc; Pyramidenberg, Spitzbergen.

POKOSUS Cotta, 1832.

Porosus communis Cotta, 1832, p. 39, pI 8. figs. 1-3; medullosan? stem fragment : Permian; Rudigsdorf near Chemnitz, Germany. 
POROXYLON Renault, 1879.

Poroxylon boysseti. Renault, 1879, p. 278, pl. 13, figs. 5-13 ; pl. 14, figs. 1-8 ; sllicifled stem, Cordaitales; Permlan ; Autun, France.

PORTELIA, Boursault, 1889.

Portelia meunieri Boursault, 1889, p. 728, fig. 2 ; plant? remains; Upper Jurassic ; Fortel, Pas-de-Calals, France.

POTAMOCARPITES Ettingshausen, 1852.

Potamocarpites thalictroides (Brongniart) Ettingshausen, 1852a, p. 7. For Carpolithes thalictroides Brongniart, 1822, p. 319 , pl. 14, fig. 5 ; Eocene; Isle of Wight, England.

POT AMOGENITES Geoppert, 1848.

Potamogenites vivianii Geoppert, in Bronn, 1848, p. 1035 ; Eocene ; Stradella, Italy.

IOT'AMOGETOPHYLLUM Fontaine, 1905.

Potamogetophyllum vernonense Fontaine, in Ward, 1905 , p. 500 , pl. 109 , fig. 7 ; leaf fragment, compared with Potamogeton (Fotamogetonacene) ; Potomac group, Lower Cretaceous; Mt. Vernon, $\mathrm{Va}$.

POTAMOPHYLLITES Brongniart, 1828.

Potamophyllites multinervis Brongniart, 1828 b, p. 114 ; brief generic description only.

POTHOCITES Paterson, 1844.

Pothocites grantonii Paterson, 1844, p. 45, pl. 3 ; spadix compared with T'ypha (Typhaceae) and Pothos? (Araceae); Carbonfferous?; Granton, Scotland.

POTHOCITOPSIS Nathorst, 1914.

Pothocitopsis bertilii Nathorst, 1914, p. 78, pl. 3, figs. 5, 6; incertae sedis; Paleozolc; Pyramidenberg, Spitzbergen.

POTONIEA Zeiller, 1899.

Potoniea adiantiformis Zelller, 1899, p. 52, pl. 4, flg. 19 ; pteridosperm microsporangiate organ; Carboniferous; Bassin d'Heraclee, Asia Minor.

IOUTERLABATIA E. W. Berry, 1938.

Pouterlabatia lanceolata D. W. Berry, 1938 , p. 123 , pl. 46 , figs. 1,2 ; leaf, Sapotaceae; Tertiary; Rto Pichlleufu, Argentina.

PRAEDEPARIA Stur, 1921.

Praedeparia banatica Stur, in Krasser, $1921 a$, p. 347 ; Polypodlaceae; Jurassic (Lower Lias) ; Steierdorf, Austria.

PRAEENGELHARDTIA.

Error for Paraengelhardtia, in Knowlton, 1919 , p. 501.

PRAMELREUTHIA Krasser, 1918.

Pramelreuthia haberfelneri Krasser, 1918, p. 533 , pl. 1 , figs. 5,6 ; cycadophyte microsporangiate organ; Upper Triassic ; Pramelrelth, .Lunz, Austria.
PRATTIA d'Archiac, 1850.

Prattia glandulosa d'Archiac, 1850, p. 407 , pl. 8, figs. 20, 20a, 20b ; Eocene; Biarritz, France. Earlier citation : d'Archlac, 1847, p. 1010 ; nom. nud.

PREISSITES Knowlton, 1894.

Preissites wardi Knowlton, 1894, p. 458, pl. 219 ; liverwort, compared with Preis8ia; Fort Union formation, Eocene; Burn's Ranch, 30 miles south of Glendive, Mont.

PREISSLERIA Presl, 1838.

Preissleria antiqua Presl, in Sternberg, 1838 (1820-38), p. 192, pl. 33, figs. 5, 10 ; incertae sedis; Triassic (Keuper); Steindorf near Bamberg, Bavaria.

PREMNOPHYLLUM Velenovsky, 1884.

Premnophyllum trigonum Velenovsky, 1884 , p. 51 , pl. 3 , tig. 2 ; leaf, Verbenaceae; Upper Cretaceous; Vyserovic, Bohemia.

PREPECOPTERIS Grand'Eury, 1877.

Prepecopteris dentata (Brongniart) Grand'Eury, 1877, p. 63, pecopterid follage-bearing schizgeaceous sporangia ; Carbontferous; Polle, France. For Pecopteris dentata Brongniart, 1828a-38, p. 346, pls. 123, 124. Sec also Radforth, 1938, 1939.

PREPINUS Jeffrey, 1908.

Prepinus statenensis Jeftrey, 1908, p. 209, pl. 13 ; short shoots bearing many leares, Coniferales; Raritan formation, Upper Cretaceous; Krefscherville, Staten Island, N. Y.

PRIMICORALLINA Whitfield, 1894.

Primicorallina trentonensis Whitfleld, 1894 , p. 357 , pl. 11, figs. 14-17 ; marine alga ; Trenton limestone, Middle Ordovician; Middleville, N. $\mathbf{Y}$.

PRIONOTES Reinsch, 1881.

Prionotes sp. Reinsch, 1881, p. 52, pl. 9a, figs. 1-4; Upper Carboniferous; Zwickau, Saxony.

PRITCHARDIA Unger, 1842.

Pritchardla insignis Unger, 1842b, p. 177, wood, incertae sedis; Tertiary; St. Bartholomew Island, West Indies.

PRITCHARDITES Bureau, 1896.

Pritchardites wettinioides Bureau, 1896, p. 284 ; palm, compared with Pritch. ardia pacifica; Tertiary; Italy.

\section{PRITOPHYLLOOLADUS ?}

Pritophyllocladus subinterrifolius (Lesquereux). Berry; this name cited in a list of fossils in Reagen, 1932, p. 232.

PROARAUCARIA Wieland, 1935.

Proaraucaria mirabilis Wleland, 1935, p. 26 ; pl. 8 , fig. 1 ; pl. 9 , fig. 1 ; pl. 10 ; pl. 12 , figs. 1,2 ; petrifled araucarian cone; Triassic; Cerro Cuadrado, Santa Cruz, Argentina. See earlier prelim. inary account, without illustrations, by Wieland, 1929a. 
PROBLEMATOSPERMUM

Ketova, 1930.

Problematospermum ovale TurutanovaKetova, 1930, p. 160, pl. 4, figs. 30, 30a ; Jurassic ; southwest Turkistan.

PROCHONDRITES Fritsch, 1908.

Prochondrites bifidus Fritsch, 1908, p. 22, pl. 4, fig. 6 ; alga ?; Silurian; Bohemia.

PROLEPIDODENDRON Arnold, 1939.

Prolepidodendron breviinternodium Arnold, 1939, p. 278 , pl. 1, figs. 2, 4 ; lycopod branch bearing two-veined leaves; Upper Devonian; near Port Allegany, McKean County, Pa.

PROPALMOPHYLLUM LIgnier, 1895.

Propalmophyllum liasinum Lignier, 1895, p. 146 , pl. 7 , figs. 20,21 ; petiole fragments, incertae sedis; Lower Jurassic (Liassic) ; Ste. Honorine, France.

PROSPIRAXIS Williams, 1887.

This name proposed in footnote, Williams, 1887 , p. 86, for Spiraxis randalli Newberry, 1885, p. 217. The latter probably not a plant.

PROTALTINGIA Reid and Chandler, 1933.

Protaltingia europaea Reid and Chandler, 1933 , p. 247 , pl. 9, figs. 1-5 ; fruit, Hamamelidaceae ; London Clay, Eocene ; Sheppey, Kent, England.

PROTAMYRIS Unger, 1850.

Protamyris eocenica Unger, 1850a, p. 476 ; leaves, Burseraceae ; Eocene; Sotzka, Styria. See also Unger, 1851, p. 180, pl. 52, fig. 15.

PROTANNULARIA Dawson, 1880 ?

Protannularia harknessii (Nicholson) Dawson, 1880 b, fig. 83 , p. 91 ; no description; Annularia-like follage; Skiddaw series, Lower Sllurian. Only other species is: Protannularia .laxa (Dawson) Arber, 1921, p. 75, fig. 41.

PROTÁSOLANUS Hörich, 1920.

Protasolanus wieprechti Hörích, 1920, p. 434, pl. 16 ; partly decorticated lycopod stem; Lower Carboniferous (Culm); Germany.

PROTEACIDITES Cookson, 1950.

Proteacidites tuberculatus Cookson, 1950, p. 170, pl. 1, figs. 12-14 ; pollen, incertae sedis ; Tertiary ; Yallourn, Victoria.

PROTEACITES Caspary, 1882.

Preteacites pinnatipartitus Caspary, 1882, p. 25.

PROTEAEPHYLLUM Fontaine, 1889.

Proteaephyllum reniforme Fontaine, 1889. p. 282 , pl. 139 , fig. 3 ; pl. 156, fig. 4 ; pl. 160, figs. 1, 2; leaf, Proteaceae? ; Potomac group, Lower Cretaceous; Fredertcksburg, Va.

PROTEOIDES Heer, 1866.

Proteoides grevilliaeformis Heer, in Capellint and Heer, 1866, p. 17, pl. 4, fig. 11 ; Cretaceous; Sloux City, Iowa.
PROTEOPHYLLUM Friedrich, 1883.

Proteophyllum bipinnatum Friedrich, 1883 , p. 335 , pl. 28, figs. 1, 2 ; Oligocene ; Eiselben, Saxony.

PROTEOPHYLLUM Velenovsky, 1889.

Proteophyllum paucidentatum Velenovsky, 1889 , p. 18 , pl. 4 , figs. $7,10-13$; pl. 5, figs. $13-15$; pl. 6 , figs. 12-15; leaf, dicotyledon; Upper Cretaceous; Bohemia.

PROTEOPSIS Velenovsky, 1889.

Proteopsis proserpinae Velenovsky, 1889, p. 19 , pl. 1, figs. 6-9 ; fruit, dicotyledon ; Upper Cretaceous (Cenomanian) ; Vyserovic, Bohemia.

PROTEOTITES Kuntze, 1904.

Proteotites Kuntze, in Post and Kuntze, 1904, p. 461.

PROTEOXYLON Kräusel, 1939.

Bayer. Akad. Wiss., Math.-naturwiss. Abh., 1939, Neue Folge, 47, p. 36 (not seen, cited in Gothan, 1942b, p. 143).

PROTOBAROLAYA Reid and Chandler, 1933.

Protobarclaya eocenica Reid and Chandler, 1933 , p. 152, pl. 3, figs. 23-28; fruit, Nymphaeaceae ; London Clay, Eocene; Sheppey, Kent, England.

PROTOBLECHNUM Lesquereux, 1880.

Protoblechnum holdeni (Andrews) Lesquereux, 1880 , p. 188 ; fernlike foliage; Carboniferous; Rushville, Ohio. For Alethopteris holdeni E. B. Andrews, 1875 , p. 420 , pl. 51, figs. $1,2$.

PROTOBRACHYOXYLON Holden, 1913.

Protobrachyoxylon eboracense Holden,. 1913 , p. 541 , pl. 40 , figs. 29,30 ; coniferous wood; Jurassic (Oolite); Scarborough, England.

PROTOCALAMITES Goebel, 1906.

Protocalamites scrobiculatus (Schlotheim) Goebel, 1906, p. 242. For Calamites: scrobiculatus Schlotheim, 1820 , p. 402 , pl. 22, fig. 4; Upper Carboniferous; Zurich, Switzerland. [The origin of modern usage, as applied to petrified stems, originates in Lotsy, 1909 , p. 528. See also Scott, 1908, p. 37.]

PROTOCALAMOSTACHYS Walton, 1949.

Protocalamostachys arranensis Walton, $1949 a$, p. 729 , pl. 1 ; petrified Equisetalean cone; Calciferous Sandstone series, Lower Carboniferous; Isle of Arran, Scotland.

PROTOCEDROXYLON Gothan, 1910.

Protocedroxylon araucarioides Gothan. 1910, p. 27, pl. 5, flgs, 3-5, 7 ; pl. 6, fg. 1 ; coniferous wood; Dpper Jurassic; Green Harbour, Spitzbergen.

PRGTOCLADUS Ettingshausen, 1887.

Protocladus lingua Ettingshausen, $1887 \mathrm{~b}$. p. 147 ; nom. nud. 
PROTOCLEPSYDROPSIS Hirmer, 1927.

Protoclepsydropsis kidstoni (Bertrand) Hirmer, 1827, p. 519; petrifled stem, Clepsydraceae ; Califerous Sandstone series, Lower Carboniferous; Langton Burn, Berwickshire, Scotland. For Zygopteris kidstoni Bertrand, 1911, p. 55, fig. 9.

PROTOCOMMIPHORA Reid and Chandler, 1933.

Protocommiphora europaea Reid and Chandler, 1933, p. 273, pl. 11, figs. 1-7 ; endocarp, Burseraceae; London Clay, Eocene; Sheppey, Kent, England.

PROTOCUPRESSINOXYLON Eckhold, 1922.

Protocupressinoxylon oupressoides (Holden) Eckhold, 1922, p. 491. For Paracupressionowylon cupressoides Holden, 1913, p. 538, pl. 39, figs. 15, 16; coniferous wood; Jurassic; Yorkshire, England.

PROTOCYATHEA Ottokar Feistmantel, 1877.

Protocyathea trichinopoliensis Ottokar Feistmantel, 1877, p. 136, pl. 10, figs. 1, 2; Upper Cretaceous (Cenomanian) ; near Trichinopoly, India. See also Posthumus, 1931.

PROTODAMMARA Hollick and Jeffrey, 1906.

Protodammara speciosa Hollick and Jeffrey, 1906, p. 199, pl. 1 figs. 5-13; pl. 2, figs. 1-5; cone scales, Araucartaceae ; Raritan formation, Upper Cretaceous ; Kreischerville, Staten Island, N. Y.

PROTODAPHANE Saporta, 1865.

Protodaphne delessii Saporta, 1865, p. 47 ; leaf ; Tertiary ; Sézanne, France.

FROT:OFICUS Saporta, 1868.

Protoficus crenulata Saporta, 1868, p. 355, pl. 6, fig. 5 ; leaf, compared with Ficus alba; Eocene ; Sézanne, France.

PROTOJUNIPEROXYLON Eckhold, 1922.

Protojuniperoxylon maidstonense (Stopes) Eckhold, 1922, p. 491. For Cedroxylon maidstonense Stopes, 1915, p. 149, pl. 12, figs. 1,2 ; conlferous wood ; Lower Greensand, Cretaceous ; Iguanodon Quarry, Maldstone, England. Generic name cited by Eckhold, 1921, p. 2.

PROTOLARIX Saporta, 1876-84.

Protolarix lundgrent (Nathorst) Saporta, 1876-84, p. 469. For Pinus lundgrent Nathorst, $1878 \mathrm{c}$, p. 31 , pl. 14, flgs. $9 a$, 13-17; pl. 15, flgs. 1, 2.

PROTOLEPIDODENDRON Krejđ̄, 1880.

Protolepidodendron scharianum Krejcl, 1880 , p. 203; lycopod stems, follage Upper Silurian?; Hostin, Bohemia. First ? 1llustrated in Potonle, Henry, and Bernard, 1903, p. 40, flgs. 94-102.

PROTOLEPIDODENDROPSIS Gothan, 1937.

Preuss. geol. Landesanst. Jahrb., 1937, Band 57, p. 497 (not seen, cited in Gothan, 1942b, p. 143).
PROTOLOTUS Saporta, 1865.

Protolotus raincourtil Saporta, 1865, p. 52; leaf, Rhamnaceae; Tertlary; Sézanne, France.

PROI:OMYOITES Meschinelli, 1892.

Protomycites protogenes (Smith) Mesch1nelli, in Saccardo, 1892, p. 748. Phycomycete ; Carboniferous; England. For Protomyces protogenes Smith, 1884, p. 333, fig. 140.

PROTONYSSA Reld and Chandler, 1933.

Protonyssa bilocularis Reid and Chandler, 1933 , p. 429 , pl. 23 , figs. 5-10; endocarp, Nyssacene ; London Clay ; Eocene ; Sheppey, Kent, England.

PROT'OOSHUNDITES H. N. Andrews and Baxter, 1948.

Protoosmundites wilsonii H. N. Andrews and Baxter, 1948, p. 194, pls. 9, 10 ; probably a lycopod branch tip; Des Molnes group, Pennslvanian; coal mine of What Cheer Clay Products Co., What Cheer, Iowa.

PROTOPHYLLOCLADOXYLON Krüusel, 1939.

Bayer, Akad, Wiss., Math.-naturwiss. Abh., 1939, Neue Folge 47, p. 16, (not seen, cited in Gothan, 1942b, p. 143).

PROTOPHYLLOOLADUS E. W. Berry, 1903.

Protophyllocladus subintegrifolius (Lesquereux) E. W. Berry, 1903, p. 440 ; compared with Phyllocladus asplenifolia Hooker; Cretaceous to Tertiary. For Phyllocladus subintegrifolius Lesquereux, 1868, p. 92 , and 1874 , p. 54, pl. 1, ifg. 12

PROTOPHYLLUM Lesquereux, 1874.

Protophyllum sternbergit Lesquereux, 1874 , p. 101 , pl. 16 ; pl. 17 , flg. 2 ; leaf, dicotyledon; Cretaceous ; south of Fort Harker, Nebr.?

PROTOPICEOXYLON Gothan, 1907.

Protopiceoxylon extinctum Gothan, 1907, p. 32 , figs. 16,17 ; coniferous wood ; Tertlary ; King Karl's Land.

PROTOPINUXYLON Eckhold, 1922.

Protopinuxylon ruffordi (Seward) Eckhold, 1922, p. 491. For Pinites ruffordi Seward, 1895, p. 199, and 1896c, p. 417 , pls. 2,3 ; coniferous wood; Wealden; near Hastings, England.

PROTOPITYS Goeppert, 1850.

Protopitys buchiana Goeppert, 1850, p. 229 , pl. 37 , figs. $4-7$; pl. 38 , figs. 1,2 ; gymnospermous wood; Carboniferous ; Falkenberg, Silesia. See also Posthumus, 1931.

PROTOPODOCARPOXYLON Eckhold, 1922. Protopodocerpoxylon blevillense (Lignier) Eckhold, 1922, p. 491. For Cedroxylon vlevillense Lignier, 1907, p. 267, pl. 18, flgs. $15-17$; pl. 21 , fig. 66 ; pl. 22 , i g. 72 ; contferous wood; Lower Cretaceous (Gault) ; France. 
PROTOPTERIDIUM Krejei, 1880.

Protopteridium hostinense Krejci, 1880, p. 203 ; Upper Silurian?; Hostin, Bohemia.

PROTOPTERIS Sternberg, 1938.

Protopteris punctata Sternberg, 1838 (1820-38), p. 170, pl. 65, figs. 1-3 ; leaf base impression of tree fern; Lower Cretaceous; Bohemia. See also Corda, 1845 , p. 77 , pl. 48, fig. 1. Seward, 1910, p. 372 , notes: "The generic name Caulopteris is used by some authors in preference to Presl's genus ; but Protopteris is more conveniently restricted to Mesozolc Cyatheaceous stems and Caulopteris to Palaeozoic stems, with the internal structure of Psaronius." See also Posthumus, 1931.

PROTORAVENSARA Reid and Chandler, 1933.

Protoravensara sheppeyensis Reld and Chandler, 1933, p. 214, pl. 7, figs. 3-5 ; fruit Lauraceae; London Clay, Eocene; Herne Bay, Kent, England.

PROTORCHIS Massalongo, 1859.

Protorchis monorchis Massalongo, 1859a, p. 64, pl. 23 , fig. 3 ; orchidaceous plant? Eocene; Italy.

PROTORHIPIS Andrae, 1855.

Protorhipis buchii Andrae, 1855, p. 36, pl. 8, fig. 1; leaf fragment, incertae sedis; Lower Jurassic (Lias); Steierdorf, Austria.

PROTORNITHOPTERIS Reed, 1947.

Protornithopteris fremonti (Knowlton) Reed, 1947, p. 149 ; frond, Schizaeaceae; Frontier formation, Upper Cretaceous ; Cumberland, Wyo.

PROTOSALVINIA (Dawson) Clarke, 1885. Protosalvinia bilobata Clarke, 1885, p. 285 , fig. 6 ; water fern sporocarp ?; Devonian; Hopewell, Ontario County, N. Y.

PROTOSPIROXYLLON Lingelsheim, 1929.

Protospiroxylon lusaticum Lingelsheim, 1929 , p. 111, figs. 1-8 ; wood, Coniferales; lower Miocene; Niederlausitz.

PROTOSTIGMA Lesquereux, 1877.

Protostigma sigillarioides Lesquereux, 1877 , p. 169 , pl. 1 , figs. 7,8 ; lycopod? stem; Cincinnati group, Silurian; near Cincinnati, Ohio.

PROTOTAMUS Langeron, 1899.

Prototamus paucinervis Langeron, 1899, p. 439 , pl. 3 , fig. 3 ; leaf, compared with Tamus; Eocene; Sézanne, France.

PROTOTAXITES Dawson, 1859.

Prototaxites logani Dawson, 1859, p. 484 figs. 4a-c ; alga ; Devonlan; Gaspe, Canada. For usage of name, see Arnold, 1947 , p. 52.
PROTOTHAMNOPTERIS RIchard Beck, 1920.

Protothamnopteris baldaufl. Richard Beck, 1920, p. 511, figs. 1-6; coenopterid fern ; Permian (Middle Rothliegendes) ; Chemnitz, Germany. See Hirmer, 1927, p. 538.

PRUNINIUM Platen, 1908.

Pruninium gummosum Platen, 1908, p. 122, pl. 3, figs. 2-6 ; Miocene; Amethyst Mtn., Yellowstone Park, Wyo.

PRUNIPHYLLUM Weyland, 1948.

Pruniphyllum prinoides (Weber) Weyland, 1948, p. 129, leaf, Rosaceae; Tertiary.

PRUNOIDES Perkins, 1904.

Prunoides bursaeformis Perkins, 1904, p. 208 , pl. 80 , fig. 133 ; fruit, compared with Prunus; Tertiary ; Brandon, vt.

PSAMMOPTERIS Elchwald, 1861.

Psammopteris knorriaeformis Eichwald, 1861 , p. 304 . See also Eichwald, 1865 (1860-68), p. 25, pl. 1, fig. 3 ; pl. 5, figs. $3,4$.

PSARONIOCAULON Grand'Eury, 1877.

Psaroniocaulon sulcatum Grand'Eury, 1877 , p. 91 , pl. B ; arborescent fern stem apparently close to Psaronius; Carboniferous; France. See also Posthumus, 1931.

PSARONITES Williamson, 1875.

Psaronites renaulti Williamson, 1875, p. 453 ; Psaronius roots; Upper Carboniferous; Oldham, England.

PSARONIUS Cotta, 1932.

Psaronius helmintholithus (Sprengel) Cotta, 1832, p. 32, pl. 5, fig. 1; petrified stem, believed to be Marattiaceous; Chemnitz, Germany. This is suggested as the type, for the first (p. 29) is illustrated only by roots. See also Posthumus, 1931.

PSEUDADIANTITES Gothan, 1929.

Pseudadiantites sessilis (v. Röhl) Gothan, 1929 , p. 17 , pl. 14, figs. 1 , 1a ; fernlike follage; Carboniferous ; Ruhr, Germany.

PSEUDOALETHOPTERIS Achepohl, 1883.

$P_{8}$ eudoalethopteris sp. Achepohl, 1883 ; unnumbered page following p. 160 ; unnumbered plate following pl. 41 ; foliage, more contracted pinuule attachment than in Alethopteris; Upper Carboniferous ; Westphalia.

PSEUDOANNULARIA Grand'Eury, 1877.

Pseudoannularia laxa (Dawson) Grand' Eury, 1877, p. 370. For Annularia laxa Dawson, 1871, p. 31, pl, 6, figs. 64-73. PSEUDOARAUCARIA Fiche, 1896.

Pseudoaraucaria loppineti Fliche, 1896, p. 189, pl. 6, figs. 3, 4; petrified cone, Araucariaceae; Cretaceous (Albten); Clermont, and Vaubecourt, France. 
PSEUDOASPIDIOI'HYLLUM Hollick, 1930. Pseudoaspidiophyllum platanoides Hollick, in Hollick and Martin, 1930, p. 96, pl. 62 , figs. 1, 2 ; leaf, Platanaceae ; Upper Cretaceous; Yukon River, $11 / 2$ miles below Seventymile Creek, Alaska.

PSEUDOASTEROPHYLLITES Velenovsky, 1887.

Pseudoasterophyllites cretaceous (Feistmantel) Velenovsky, 1887, p. 643, figs. 19-25; Asterophyllites-like foliage with cone; Cretaceous; Bohemia.

PSEUDOBAIERA Matthew, 1906.

Psendobaiera mointoshi Matthew, 1906a, p. 395 , pl. 8, figs. 1-6; sphenopterid? foliage ; Devonian ; Duck Cove, Lancaster, New Brunswick, Canada.

PSEUDOBORNIA Nathorst, 1894.

Pseudobornia ursina Nathorst, 1S94, p. 57, pl. 12, flgs. $1-7$; pl. 13 ; pl. 14, flg. 5 ; calamitelike stem impression; Devonian; Bear Island, Norway.

PSEUDOCALLIPTERIS Grand'Eury, 1877.

Pseudocallipteris discreta (Weiss) Grand'Eury, 1877, p. 430 ; Carboniferous; France. For Callipteris discreta Weiss, 1870, p. 872 , pl. 20 , figs. $1,2$.

PSEUDOCHAETETES Peterhans, 1929?

Pseudochaetetes champagnensis Feterhans, 1929 , p. 10 , pls. 1,2 ; Jurassic ; Champagne, France.

PSEUDOCHONDRITES H. B. Geinitz, 1863.

Pseudochondrites sp. H. B. Geinitz, 1863, p. 530 ; alga ; Permian.

PSEUDOCORDAITES (Heer) Fritsch, 1900.

Pseudocordaites palmaeformis (Goeppert) Fritsch, in Beyschlag and Fritsch, 1900, p. 68 ; Upper Carboniferous ; Suxony. For Noeggerathia palmaeformis Goeppert, $1852 b$, p. 216 , pl. 15 ; pl. 16, figs. 1-3.

PSLUDOCOTYLEDON Saporta, 1893.

Preudocotyledon inquirendum. Saporta, $1893 a$, p. xxxiv, pl. 1 , fig. 10 ; leaf, compared with Cotyledon (Crassulaceae); Tertiary (Aquitanien); Bois-d'Asson, Aix, France.

I'SFUDOCTENIS Seward, 1911.

Pseudoctenis eathiensis (Richards) Seward, 1911 b, p. 692 , pl. 4, figs. 62,67 ; pl. 7 ; figs. 11,12 ; pl. 8 , fig. 32 ; cycadophyte frond fragment; Jurassic.

PSEUDOCYOAS Nathorst, 1907.

Pseudocycas insignis Nathorst, 1907, p. 4, pl. 1 , figs. $1-5$; pl. 2 , figs. $1-9$; pl. 3, fig. 1 ; cycadophyte foliage ; Lower Jurassic (Lias) ; Hör, Sweden.

PSEUDOCYCLOPTERIS Grand'Eury, 1877.

Pseudocyclopteris oblata (Lindley and Hutton) Grand'Eury, 1877, p. 430 ; Upper Carboniferous; France. For $C y$ clopteris oblata Lindley and Futton, 1837 (1831-37), p. 173, pl. 217.
PSEUDODANAEOPSIS Foutalne, 1883.

Pseudodanaeopsis seticulata Fontaine, 1883 , p. 59 , pl. 30 , figs. $1-4$; fern foliage; Triassic; Clover Hill, Va.

PSEUDOFRENELOPSIS Nathorst, 1893.

Pseudofrenelopsis felixi Nathorst, in Felix and Nathorst, 1893 , p. 52, figs. 6-9 ; Lower Cretaceous (Neocomian) ; Tlaxiaco, Mexico.

PSEUDOGEINITZIA Hollick and Jeffrey, 1909.

Preidogeinit $\approx$ ia sequoiiformis Hollick and Jeffrey, 1909 , p. 45 , pls. 10,25 ; cone scales, Coniferales; Cretaceous ; Krelscherville, Staten Island, N. Y.

PSEUDOCINKGO Velenovsky and Viniklar, 1926.

Pseudoginkgo bohemica Velenovsky and Viniklär, 1926, p. 35 , pl. 5, flgs. 1-15; U.p pe $\mathbf{r}$ Cretaceous (Cenomanian); Bohemia.

PSEUDOMANGROVIA Fucini,.1938.

Reference not seen; cited in Gothan, 1942 b, p. 144.

PSEUDONYSSA Kinkelin, 1900.

Pseudonyssa palmiformis Klnkelin, 1900, p. 130 ; fruit, compared with $N y 8 s a$; Upper Pliocene; Klarbecken near Niederrad, Hesse. See also Engelhardt and Kinkelin, 1908, p. 225, pl. 27, flg. 15.

PSLUDOODONTOPTERIS Grand'Eury, 1877.

Pseudoodontopteris nevropteroides (Roemer) Grand'Eury, 1877, p. 430. For Odontopteris neuropteroides Roemer, 1860, p. 31, pl. 7, fig. 2.

PSEUDOPECOPTERIS Grand'Eury, 1877.

Pseudopecopteris defrancii (Brongniart) Grand'Eury, 1877, p. 379 ; Carboniferous; France. For Pecopteris defrancii Brongniart, 1828a-38, p. 325, pl. 111.

PSEUDOPECOPTERIS Lesquereux, 1880.

Pseudopecopteris mazoniana Lesquereux, 1880 , p. 190 , pl. 32 , figs. $1-7$; fernlike foliage; Pennsylvanian; Mazon Creek, IIl.

PSEUDOPEZIZITES Fiore, 1932.

Soc. naturalisti Napoli Boll., 1932, v. 43, p. 154 (not seen, cited in Gothan, 1942b, p. 144).

PSEUDOPHRAGMITES Saporta, 1873.

Pseudophragmites arundinaceus Saporta, $1873 a$, p. 33 , pl. 4, fig. 1 ; rhizome?; Tertiary ; France.

PSEUDOPINUS Ettingshausen, 1887.

Pseudopinus willinsoni Ittingshausen, 1887 a, p. 90, pl. 8 , figs. $12-18$; follage shoot and cone, Abletineae; Eocene; Vegetable Creek, near Emmaville, New South Wales. 
PSEUDOPOLYPORUS Hollick, 1910.

Pseudopolyporus carbonicus Hollick, 1910, p. 93, figs. 1, 2 ; a stalked polyporaceous fungus?; Carboniferous; West Virginia.

PSEUDOPROTOPHYLLUM Hollick, 1930.

Pseudoprotophyllum marginatum Hollick, in Hollick and Martin, 1930, p. 92, pl. 52, fig. $2 a$; pl. 65 , fig. 3 ; leaf, Platanaceae; Upper Cretaceous; Yukon River, 6 miles above Nahochatilton, Alaska.

PSEUDOPTEROPHYLLUM Florin, 1933.

Pseudopterophyllum cteniforme (Nathorst) Florin, 1933, p. 81, pl. 9, figs. 1-7; cycadophyte leaf; Rhatic; Bjuv, Sweden.

PSEUDOPTILOPHYLLUM Krasser, 1918.

Pseudoptilophyllum titzei Krasser, 1918, p. 547 , pl. 4 , fig. 6 ; cycadophyte follage; Upper Triassic ; Pramelreith, Lunz, Austria.

PSEUDORHIPIDOPSIS P'an, 1937.

Pseudorhipidopsis brevicaulis (Kawasaki and Kon'no) P'an, 1937, p. 265, pl. 1; pl. 2 ; pl. 3 , figs. 4,5 ; compared with Rhipidopsis; Tafenkou series, Lower Permian; Yuhsien, Honan, China.

PSEUDOSAGENOPTERIS Henry Potonie, 1900.

Pseudosagenopteris elliptica (Fontaine) Henry Potonie, 1900, p. 503. For Sagenopteris elliptica Fontaine, 1889, p. 149, pl. 27, figs. 9, 11-17 ; leaf, Caytoniales?; Potomac formation, Cretaceous ; Maryland.

PSEUDOSALVINIA Piton, 1940.

Pseudosalvinia dubia Piton, 1940, p. 17, pl. 13, fig. 6 ; pinnule fragment, compared with Salvinia; Eocene; Menat, France.

PSEUDOSCLEROCARYA Reid and Chandler, 1933.

Pseudosclerocarya lentiformis Reid and Chandler, 1933 , p. 303 , pl. 13, figs. 2528 ; endocarp, Anacardiaceae ; London Clay, Eocene ; Sheppey, Kent, England.

PSEUDOSIGILLARIA Grand'Eury, 1877.

Pseudosigillaria monostigma (Lesquereux) Grand'Eury, 1877, p. 144. For Sigillaria monostigma Lesquereux, 1870, p. 449, pl. 42, figs. 1-5; ly copod stem impression; Upper Carboniferous; France.

PSEUDOSPHENOPTERIS Grand'E u r y, 1877 .

Pseudosphenopteris integra (Andrae)

Grand'Eury, 1877, p. 389. For Sphenopteris integra Andrae, in Germar, 184453 , p. 67, pl. 28, figs. 1-4.

PSEUDOSPOROCHNUS Henry Potonie and Bernard, 1903.

Pseudosporochnus krejiii Henry Potonie and Bernard, 1903, p. 25, figs. 54-81; Psilophytales; upper Middle Devonian; Bohemia.
PSEUDOSPOROGONITES Stockmans, 1948.

Pseudosporogonites hallei Stockmans, 1948 , p. 61 , pl. 11, figs. $18,18 a$; Upper Devonlan; Belgium.

PSEUDOSTROMATOPORA SImIOnescu, 1926.

Pseudostromatopora rumana Simionescu, 1926, p. 105, figs. 4-6; alga?; Cretaceous ; Cernavoda, Rumania.

PSEUDOSYRINGODENDRON Grand'Eury, 1890.

Pseudosyringodendion pachyderma (Brongniart) Grand'Eury, 1890, p. 246. For Sigillaria pachyderma Brongniart, 1828a-38, p. 452 , pl. 150 , fig. 1 .

PSEUDOTORELLIA Florin, 1936.

Pseudotorellia nordenskioldi (Nathorst). Florin, 1936b. For Feildenia nordenskioldi Nathorst, 1897, p. 56, pl. 3, figs. 16-27 ; pl. 6, figs. 33,34 ; Upper Jurassic; Advent Bay, Spitzbergen.

PSEUDOT'SUGOIDITES Robert Potonie, 1950.

Pseudotsugoidites sp. Robert Potonle, in Potonle, Robert, Thomson, Paul W., and Thiergart, Friedrich, 1950 , p. 48 ; nom. nud.

PSEUDOVOLTZIA Florin, 1927.

Pseudovoltzia liebeana (Geinitz) Florin, 1927, p. 5. See also Florin, 1929, p. 257, pl. 4, fig. 10; and 1944, p. 479. For Voltzia liebeana H. B. Gelnitz, 1880, p. 26, pl. 5, figs. 1, 2, 5-7 ; Upper Permian; Trebnitz, near Gera, Saxony.

I'SILODENDRION Hoeg, 1942.

Psilodendrion spinulosum Hoeg, 1942, p. 26, pls. 5-7; some resemblance to Psilophyton; Devonian; Spitzbergen.

PSILOPHYTON Dawson, 1859.

Psilophyton princeps Dawson, 1859, p. 478, figs. 1a-1 ; Psilophytales; Devonian; Gaspé, Canada:

PSILOTIPHYLLUM Henry Potonie, 1891?

Psilotiphyllum bifidum (Geinitz) Henry Potonie, 1891, p. 979.

PSILOTITES. (Braun) Münster, 1842.

Psilotites filiformis Münster, 1842, p. 108 , pl. 13, fig. 11 ; pl. 15, fig. 20 ; Permian: Daiting near Monhelm, Rhenish Prussia. Earliest citation: Psilotites robustus. Braun, 1840 , p. 98 ; nom. nud.

PSILOTOPSIS Heer, 1883.

Psilotopsis racemosa Heer, 1883, p. $55, \mathrm{pl}$. 100, figs. 6,7 ; incertae sedis; Tertiary ; Unartok, Greenland.

PSYCHOTRIPHYLLUM Dean, 1902.

Prychotriphyllum attenuatum Dean, 1902a, p. 60 , pl. 15, fig. 2 ; leaf, compared with. Psychotria loniceroides (Rublaceae); Tertiary; Wingello, New South Wales. 
PSYGMATOPTERIS Lesley, 1880.

Psygmatopteris grandis Lesley, 1880, p. 133 ; nom. nud.; Pennsylvanian ; West Virginia.

PSIGMOCARPUS Susta, 1932.

Přirod. spol. v Moravské .Ostravé Sborník, svazek 7, p. 155 (not seen, cited in Gothan, 1942b, p. 145).

PSYGMOCLADUS Susta, 1932.

Př̀rod. spol. v Moravské Ostravé Sborník, svazek 7, p: 155 (not seen, c1ted in Gothan, 1942b, p. 145).

PSYGMOPHYLLUM Schimper, 1870.

Psygmophyllum flabellatum (Lindley and Hutton) Schimper, 1870, (1869-74), p. 193. For Noeggerathia Aabellata Lindley and Hutton, 1832 (1831-37), p. 89, pls. 28, 29 ; large cuneate leaves, affinities uncertain; Upper Carboniferous; England.

PSYGMOSTROBOPHYLLUM Susta, 1932.

Prirod. spol. v Moravské Ostravé Sborník, svazek 7, p. 162 (not seen, cited in Gothan, 1942b, p. 145).

PSYGMOSTROBUS Susta, 1932.

Př́irod. spol. v Moravské Ostravé Sborník, svazek 7, p. 156 (not seen, cited in Gothan, 1942b, p. 145).

FTELEACARPUM Weyland, 1948.

Pteleacarpum bronni Weyland, 1948, p. 130, pl. 31, fig. 5; winged frult; Tertiary.

PTELEOIDITES Thomson, 1950.

Pteleoidites sp. Thomson, in Potonle, Robert, Thomson, Paul W., and Thiergart, Friedrich, 1950 , p. 58; nom. nud.; pollen compared with Ptelea; Miocene; Chatt-Aquitan, Germany.

PTENOSTROBUS Lesquereux, 1874.

Ptenostrobus nebrascensis Lesquereux, 1874 , p. 114 , pl. 24 , fig. 1 ; cone, Coniferales?; Cretaceous; near Winnebago, Nebr.

PTERALETES Zalessky, 1939.

Pteraletes Zalessky, 1939a, p. 326 ; nom. nud.

PTERIDITES Tuzson, 1914.

Pteridites staubii Tuzson, 1914, p. 236, pl. 13 , fig. 4 ; fern foliage; compared w1th Pteridium; Oligocene; Zsilvolgy Valley, Petrozseny, Hungary.

PTERIDOLEIMMA Debey and Ettingshausen, 1859.

Pteridoleimma elisabethae Debey and Ettingshausen, $1859 \mathrm{~b}$, p. 222, pl. 5, figs. 5-9; sterile fern foliage; Upper Cretaceous; Aachen, Rhenish Prussia.

PTERIDOPSIS HOWSe, 1890.

Pteridopsis plumosa Howse, 1890 , p. 85 . pl. 3 ; fernlike frond fragment, some similarity to Alethopteris; Upper Carboniferous; Jarrow-on-the-Tyne, England.
PTERIDORACHIS Nathorst, 1902.

Pteridorachis striata Nathorst, 1902a, p. 12, pl. 1, fig. 8 ; fern ? rachis fragment; Upper Devonian; Bear Island, Norway.

PTERIDOTHECA Scott, 1907.

Pteridotheca williamsoni Scott, 1907, p. 184, fig. 14; annulate fern sporangia, family uncertain; Upper Carboniferous ; England. See al so $_{8}$ Scott, 1920, p. 265,

PTERIDOZAMITES Corsin, 1929.

Pteridozamites zamioides (Bertrand) Corsin, 1929 , p. 230 , pls. 7-10 ; frond, male fructification and seeds, affinities with pteridosperms and cycadophytes; Westphalian, Carboniferous ; mines of Bruay, France.

PTERIGOPHYCOS Massalongo, 1858.

Pterigophycos spectabilis Massalongo, 1858b, p. 743. alga; Eocene; Monte Bolca, Italy.

PTERISPERMOSTROBUS Stopes, 1914.

Pterispermostrobus bifurcatus Stopes, 1914 , p. 74 , pl. 17 , fig. 45 ; pl. 25 , fig. 69 ; pteridosperm cupulate organ?; Pennsylvanian; Fern Ledges, Lancaster, New Brunswick, Canada.

PTERISPERMOTHECA Carpentier, 1919.

Pterispermotheca sp. Carpentier, 1919a, p. 89 ; pl. 3 , figs. $5-7$; microsporangia compared with Archaeopteris hitchocki; Lower Carboniferous ; France.

PTEROBALANUS E. W. Berry, 1922.

Pterobalanus texanus E. W. Berry, 1922c, p. 20 , pl. 15, figs. 1,2 ; winged frult, incertae sedis; Wilcox group, Eocene; half a mile west of Carrizo Springs, Dimmit County, Tex.

PTEROCARPITES Keferstein, 1834.

Pterocarpites antiquus Kefersteln, 1834, p. 862.

PTEROCYCADITES C. F. W. Braun, 1840.

Pterocycadites münsteri C. F. W. Braun, 1840 , p. 100 ; nom. nud.

PTERODICTYON Unger, 1856.

Pterodictyon annulatum Unger, 1856, p. 172 , pl. 8 , fig. 17 ; incertae sedis; Upper Devonian; Saalfeld, Thuringia.

PTEROPETALUM Menge, 1858.

Pteropetalum palaeogonum Menge, 1858 , p. 14, figs. 20-23; Tertlary; Baltic Prussia.

PTEROPHYCUS Herzer, 1902.

Pterophycus plicatus Herzer, 1902, p. 40, fig. 1; "fucoid," incertae sedis ; Carboniferous ; Marietta, Ohio.

PTEROPHYLLUM Brongniart, 1828.

Pterophyllum longifolium Brongniart, $1828 \mathrm{~b}$, p. 95. For Algacites filicoides Schlotheim, 1822, pl. 4, fig. 2. Problem of citing a type species here is noted by Seward, 1897, p. 548-550. See also Harris, 1932b, p. 20, 40. 
PTERORRACHIS Frenguelli, 1942.

Pterorrachis ambigua Frenguelli, 1942, p. 303 , pl. 1 , fig. 1 ; probably male organ of Zuberia (see Frenguelli, 1944); Triassic ; Argentina.

PTEROSPERMITES Heer, 1859.

Pterospermites vagans Heer, 1859 , p. 36 , pl. 109, figs. 1-5; winged seeds?; Tertlary ; Oeningen, Switzerland.

PTEROSPERMUM E. A. N. Arber, 1914.

Pterospermum anglicum E. A. N. Arber, 1914 , p. 94 , pl. 8 , figs. 51,52 ; seed ; Middle Coal Measures; Upper Carboniferous; Cosely, South Staftordshire, England.

PTEROTRILETES Zalessky, 1939.

Pterotriletes Zalessky, 1939a, p. 326 ; nom. nud.

PTEROZAMITES C. F. W. Braun, 1843.

Pterozamites scitamineus (Sternberg) C. F. W. Braun, in Münster, 1843, p. 29. For Taeniopteris scitaminea Presl, in Sternberg, 1820-38, p. 139. For illustrations, see Phyllites scitamineaeformis Sternberg, 1820-38, p. 39, pl. 37, fig. 2.

PTERUCHUS Thomas, 1933.

Pteruchus africanus Thomas, 1933, p. 234, pl. 24 , figs. 71,72 ; pteridosperm microsporangiate inflorescence; Molteno beds, base of Stormberg series, Triassic; Uyper Umkomas Valley, Natal.

PTERYGOPTERIS Johansson, 1922.

Pterygopteris angelini (Nathorst) Johansson, 1922, p. 2, pl. 1 ; fertile fern frond fragment, compared with Laccopteris; Rhaetic; Skromberga, Sweden.

PIILOCARPUS Lesquereux, 1870.

Ptilocarpus bicornutus Lesquereux, 1870, p. 493; winged seed; Carboniferous; Coshocton, Ohio.

\section{PTILOPHYLLUM Morris, 1840 ?}

Ptilophyllum acutifolium Morris, in Grant, 1840 , p. 327 , pl. 21 , figs. $1 . a-3$; cycadophyte leaf; "south of Charivar Range," East India. See also Seward, 1917, p. 512-522.

pTSLOPHYTON Dawson, 1878.

Ptilophyton thomasoni Dawson, 1878, p. 385 , pl. 4 ; lycopod ; Devonian ; Calthness, Scotland.

PTILORHACHIS Corda, 1845.

Ptilorhachis aubis Corda, 1845, p. 84, pl. 54, figs. 17-19.

\section{PTILOTITES Massalongo, 1859.}

In Massalongo and Scarabell, 1859, p. 92 ; a suggested name change for Chondrites penicillatus Kurr, 1845 , p. 15, pl. 3, fig. 7 ; Lower Llas; Bodelshausen, Württemberg.
PTILOZAMITES Nathorst, 1878.

Ptilozamites nilssoni Nathorst, $1878 \mathrm{~b}$, p. 23 ; pi. 3 , figs. $1-5 ; 8$; cycadophyte foliage; Rhaetic ; Höganäs, Sweden.

P'T'YHOCARPUS C. E. Weiss, 1.869.

Ptychocarpus hexastichus C. E. Weiss, 1869 , p. 95 , pl. 11 , fig. 2 ; fertlle fern compression; Upper Carboniferous ; Breitenbach, Rhenish Prussia.

\section{PTYCHOPHYLLUM.}

Error for Pychnophyllum, in Brongniart, 1849 , p. 138.

PTYCHOPTERIS Corda, 1845.

Ptychopteris macrodiscus (Brongniart) Corda, 1845, p. 76. See also Brongniart, 1828a-38, pl. 139 ; and Posthumus, 1931.

PTYCHOTESTA Brongniart, 1874.

Ptychotesta tenuis Brongniart, 1874, p. 263 , pl. 22, figs. 9-11; silicifled seed; Carboniterous ; St.-Etienne, France.

PTYCHOXYLON Renault, 1896.

Ptychoxylon levyi Renault, 1896a, p. 313, pl. 69, figs. 57-63; petrified cycadophyte stem; Upper Carboniferous ; Champ des Borgis, France.

PUCCINITES Ettingshausen, 1853.

Puccinites lanceolatus Ettingshausen, 1853 , p. 26, pl. 4, fig. 11 ; Puccinia-like rust?: Eocene ; Haering, Tirol, Austria.

PUNCTATASPORITES Ibrahim, 1938.

Punctatasporites sabulosus Ibrahim, 1933, p. 37, p1. 5, fig. 43 ; spore ; Carbontferous.

PUNCTATISPORITES Ibrahim, 1933.

Punctatisporites punctatus Ibrahim, 1933, p. 21 , pl. 2, fig. 18 ; spore ; Carbontferous.

PUNCTATOSPORITES Ibrahim, 1933.

Punctatosporites minutus Ibrahim, 1933, p. 40 , pl. 5, flg. 33 ; spore ; Carbonlferous.

PUNICITES Weber, 1855.

Punicites hesperidum Weber, in Wessel and Weber, 1855 , p. 157 , pl. 30 , flg. 11; calyx ; Tertiary ; Rott, Germany.

PURSONGIA Zalessky, 1937.

Pursongia amalitzkii Zalessky, 1937a, p. 13, fig. 1; Glossopteris-like leaf; Permian; near village of Koltehoumkina. Ourals, Russia.

PUSROULATEA Royle, 1840.

Pustalaria calatiana Royle, 1840 (183340), p. Xxix*; nom, nud.

PYCNOIS Stenzel, 1872.

Pycnois densa (Ünger) Stenzel 1872, p. 71. For Fasciculites densus Unger, 1850, p. 337 ; Tertiary ; India. 
PYCNOLOBIUM Saporta, 1861.

Pycnolobium tetraspermum Saporta, in Heer, 1861 , p. 162 ; fruit ; Leguminosae ; Miocene ; Manosque, France.

PYCNOPHYLLITES Tuzson, 1811.

Pycnophylltes brandlingt (Lindley and Hutton), Tuzson, 1911, p. 22. For Pinites brandlingi Lindley and Hutton, 1831-37, p. 1, pl. 2 ; Upper Carboniferous; Wideopen, near Newcrstle, England.

PYCHNOPHYLLUM Brongniart, 1849.

Pychnophyllum borassifolia (Sternberg) Brongniart, 1849, p. 114. For Flabellaria borassifolia Sternberg, 1825 (1820-38), p. 32, pl. 18 . See note under Cordaites.

PYCHNOPORIDIUA Yabe and Toyama, 1928.

Pychnoporidium lobatum Yabe and Toyama, 1928 , p. 146 , pl. 20 , fig. 3 ; pl. 21 , figs. $1-5$; pl, 22 , fig. 1 ; alga, compared with Solenoporella, Ortonella, etc.; Torinosa limestone; "Younger Mesozolc" ; Iwaki, Japan.

PYCNOXYLON Cribbs, 1938.

Pycnoxylon leptodesmon Cribbs, 1938, p. 321, pls. 1-4; petrifled stem, Cordaltales; Reeds Spring limestone, Mississippian ; Missourt.

PYTHITES Pampaloni, 1902.

Pythites disodilis Baccarinl, in Pampaloni, 1902, p. 124, pl. 10, figs. 5, 6 ; fungus mycelium and spores, Oomycete?; Miocene; Melllli, Sicily.

\section{$\mathbf{Q}$}

QUERCINIUM Unger, 1842.

Quercinium sabulosum Unger, 1842b, p. 173 ; wood; Tertiary ; Austria. First, species illustrated: $Q$. austriacum Unger, 1841-47, p. 107, pl. 29, figs. 4-6.

QUERCIP.HYLLUM Nathorst, 1888.

Querciphyllum lonchitis Nathorst, 1888 , p. 205 , pl. 18, fig. 8 ; leaf, compared with Quercus; Miocene; Yamakumadamura, Echigo province, Japan. Generic name cited in Nathorst, 1886, p. 53 ; nom. nud.

QUERCIPOLLENITES Wolf, 1934.

Quercipollenites callosus Wolff, 1934, p. 71, pl. 5, fig. 10 ; Pliocene ; Frelgericht mine near Dettingen, Bavaria.

QUERCITES Berger, 1832.

Quercites lobatus Berger, 1832, p. 22, pl. 4, figs. 1, 3-5; Lower Lias; Coburg, Germany.

QUERCOIDITES Robert Potonie, 1950.

Quercoidites henrici Robert Potonle, in Potonie, Robert, Thomson, Paul W., and Thiergart, Frledrich, 1950 , p. 54, pl. B, figs. 22,23 ; pollen, Fagaceae?; Miocene; Chatt-Aquitan, Germany.
QUERCOPHYLLUM. Fontaine, 1889.

Quercophyllum grossedentatum Fontaine, 1889 , p. 307 , pl. 156 , fig. 9 ; leaves, compared with Quercus; Potomac group, Lower Cretaceous; Brooke, Va.

QUERVAINIA T. M. Harris, 1932.

Quervainia spectabilis T. M. Harris, 1932a, p. 16, fig. 9 ; cycadophy te leaf? ; Stachyotaxus bed, Rhaetlc; Scoresby Sound, enst Greenland.

\section{$\mathbf{R}$}

RABDOTUS Presl, 1.838.

Rabdotus verrucosus Presl, in Sternberg, $1838(1820-38)$, p. 193 , pl. 13 ; incertae sedis ; Carboniferous ; Swina, Bohemia. RACHIOP'IERIS Williamson, 1874.

Rachiopteris aspera Williamson, $1874 \mathrm{a}$, p. 684, pls. 51, 52. See Posthumus, 1926.

RACOBLASTITES Reinsch, 1881.

Racoblastites sp. Reinsch, 1881, p. 80 , pl. 19 , figs. $1 . \overline{5}$; pl. 20 , figs. $1-6$; pl. 21 , flgs. 1-4; Upper Carboniferous ; Zwickau, Saxony.

RACOSTROMIUM Reinsch, 1881.

Racostromium sp. Reinsch, 1881, p. 53, pl. 12a, figs. 1-4; pl. 13a, flg. 6 ; Upper Triassic (Keuper); Basel, Switzerland.

RAIICITES Henry Potonie, 1893.

Radicites capillacca (Lindley and Hutton) Henry Potonie, 1893, p. 261, pl. 34, fig. 2.

RADICOPSIS Fucini, 1938.

Reference not seen; cited in Gothan, 1942b, p. 146.

RADICUIITES Lignier, 1906.

Radiculites reticulatus, Lignier, 1906, p. 193 , firs. 1-3; roots; described as of Sequoian afflnities; possibly Cordaitean (see Seward, 1917, p. 217) ; Carboniferous (Stephanien); Grand Croix near St.-ftienne, France.

R.A.DICULITES Zalessky, 1937.

Radiculites huganicus Zalessky, 1937d, p. 191 , figs. 40,41 ; roots?, incertae sedis ; Permian ; Russia.

RADIMSKYA Ettingshausen, 1890.

Radimskya trinervia Ettingshausen, 1890, p. 81 , pl. 2, fig. 22 ; flower, Alismaceae?; Miocene; Schoenegg, Styria.

RADIOPHYTON Meunier, 1887.

Radiophyton sixii Meunler, 1887, p. 59, fig. 1 ; Jurassic ; near Boulogne-sur-Mer, France.

RADIOSPERMUM E. A. N. Arber, 1914.

Radiospermum perpusillum (Lesquereux) D. A. N. Arber, 1914 , p. 102 , pl. 7, fig. 31 ; seed; Middle Coal Measures; Upper Carboniferous; Billingsley Colliery, Wyre Forest, Shropshire, England.

RADIX Fritsch, 1908.

Radiso corrugatus Fritsch, 1908, p. 8, pl. 6 , flg. 8 ; plant?; Silurian; Bohemia. 
RADSTOCKIA Kidston, 1.923.

Radstockia sphenopteroides $\mathrm{K}$ id t to $\mathrm{n}$, $1923 b$, p. 373 , pl. 75 , flgs. 3 , $3 a$; fertile Coenopterid? fern; Radstock group, Upper Carboniferous; Ranstock, Somerset, England.

RADULITES Sadebeck, 1886.

Radulites macrolobus Sadebeck, 1886, p. 121 ; moss ; Tertiary ; Prussia ; nom. nud.

RAISTRICKIA Schopf, Wilson, and Bentall, 1944.

Raistrickia grovensis Schopf, in Schopf, Wilson, and Bentall, 1944, p. 55, fig. 3 ; No. 6 coal, uppermost Carbondale formation, Pennsylvanian ; near Middle Grove, Fulton County, Ill.

RAJMAHALIA Sahni and Rao, 1934.

Rajmahalia paradowa Sahnl and Rao, 1934, p. 265 , pl. 36 , flgs. 12,13 ; top of Bennettitalean receptacle; Jurassic; Rajmahal Hills, India. See also Sahni and Rao, 1935.

RAMALINITES C. F. W. Braun, 1840.

Ramalinites lacerus C. F. W. Braun, 1840, p. 94 ; nom. nud.

RAMICALAMUS Matthew, 1906.

Ramicalamus dumosus Matthew, 1906a, p. 115 , pl. 8, figs. 2-5; articulate stem impression; Dadoxylon sandstone, Little River group, Devonian; Duck Cove, Lancaster, New Brunswick, Canada.

\section{RAMMLUS.}

See Ramulus.

RAMULARITES PIa, 1927.

Ramularites oblongisporus (Caspary) Pla, in Hirmer, 1927, p. 122 ; fungus, Mucedinaceae; Fungi Imperfecti ; Eocene. For Ramularia oblongispora Caspary, 1887, p. 8. See also Caspart, 1907, p. 15, pl. 1, figs. 11, 11 a.

RAMULUS.

Sze, 1930, p. 29, cites Rammlus oordaititides Schenk. The latter refers to Ramulus cordaitidis which was given by Schenk, 1883c, on page opposite pl. 44. It was evidently not intended as a binomial but rather as a descriptive phrase for a cordaitean branch.

RANUNGULITES Hector, 1880.

Ranunculites peltafolla Hector, $1880, \mathrm{p}$ 49 ; nom. nud.

RATHAELIA Debey and Ettingshausen, 1859.

Raphaelia neuropteroides Debey and Ettingshausen, $1859 \mathrm{~b}$, p. 220 , pl. 4, figs. 23-28; pl. 5, figs. 18-20; fern frond rragments; Upper Cretaceous; Anchen, Rhenish Prussia.

RARITANIA Hollick and Jeffrey, 1909.

Raritania gracilis (Newberry) Hollick and Jeffrey, 1909 , p. 26, pl. 6 ; coniferous leafy twig; Cretaceous ; Kreischerville, Staten Island, N. Y.
RAUMERIA Goeppert, 1853.

Raumeria sohulziana Goeppert, 1853c, p. 259 , pl. 7 , figs. $1-5$; pl. 8, figs. 1-3. Earlier citation : Goeppert, 1844, p. 217 ; nom. nud. See also Wieland, 1934.

RA.VENALOSPERMUM Saporta, 1894.

Ravenalospermum invertissimum Saporta, 1894 , p. 200 , pl. 36, figs. 13, 14 ; winged seeds?; referred to Musaceae or Bromeliadaceae; Cretaceous (Albien Superieur) ; Nazareth, Portugal.

RAZUMOVSKYA Vologdin, 1939.

Razumovskya uralica Vologdin, 1939, p. 251 , pl. 1 , figs. 1,2 ; pl. 5, figs. 3,4 ; calcareous alga; Middle Cambrian: South Urals.

RECEPTACULES Defrance, 1827?

Receptacules neptuni Defrance, 1827, p. 7.

REIMANNIA Arnold, 1935.

Reimannia aldenense Arnold, 1935, p. 5, pl. 1 , figs. 1, 6, 9 ; petrified psilophyte? stem; Ludlowville shale, Middle Devonian; Spring Creek, near Alden, Erie County, N. Y.

REINSOHIA C. E. Bertrand and Renault, 1893.

Reinschia australis C. E. Bertrand and Renault, 1893, p. 321, pls. 4-7 ; “PermoCarboniferous"; Australia. Earlier citation : Bertrand, C. E., and Renault, Bernard, 1892, p. 172; nom. nud.

REINSCHOSPORA Schopf, Wilson, and Bentall, 1944.

Rcinschospora bellitas Bentall, in Schopf, Wilson, and Bentall, 1944, p. 53, flg. 2; spore, Battle Creek coal seam, Pennsylvanian; north side of Sweden Cove, Marion County, Tenn.

RENAULTIA Stur, 1883.

Renaultia intermedia (Renault) Stur, 1883 , p. 759 , fig. 26 ; fertile fern pinnules, Marattiaceae?

RENAULTIA Zeiller, 1883.

Renaultia chaerophylloides (Brongniart) Zeiller, 1883, p. 208, 185 ; pl. 9, figs. 16, 17 ; fertile fern foliage; Carboniferous; France.

RESTIACITES Saporta, 1861.

Restiacites pleiocaulis Saporta, in Heer, 1861, p. 144 ; Eocene ; Provence, France.

RETICULATASPORITES Ibrahim, 1933.

Reticulatasporites facetus Ibrahim, 1933, p. 38 , pl. 5, fig. 36 ; spore; Carboniferous.

RETICULATISPORITES Ibrah1m, 1933.

Reticulatisporites reticulatus Ibrahim, 1.932, p. 33 ; epore: Carboniferous. For
Sporonites reticulatus Ibrahim, 1932, p. 447, pl. 14, fig. 3.

RETICULUM Stefani, 1879.

Reticulum textum (Heer) Stefani, 1879, p. 446. For Palaeodictyon textum Heer, 1876 , p. 118, pl. 43, figs. 18-20. 
RETINODENDRON Zenker, 1833.

Retinodendron pityodes Zenker, 1833, p. 3, pl. 1, figs. 1-3 ; coniferous wood ; Tertiary (Braunkohle); Altenburg, Germany.

RETINODENDRON Renault, 1892.

Retinodendron rigolloti Renault, $1892 \mathrm{a}$, p. 339 ; Carboniferous ; Autun, France. See also Renault, 1893, pl. 77, figs. 9-14.

RETINOMASTIXIA Kirchheimer, 1938.

Retinomastixia schultei Kirchheimer, 1938 b, p. 350 , pl. 7, figs. 7-13; seed, Cornucere : Oligocene; Germany.

RETINOSPORITES Holden, 1915.

Retinosporites indica (Felstmantel) Hol den, 1915 , p. 221 , pl. 11, figs. 1, 4, 9 ; coniferous twigs with cuticle of foliage preserved, some resemblance to Retinospora; Triassic; Rajmahal Hills, India.

RETINOXYLON Endlicher, 1847.

Retinoxylon pityoides (Zenker) Endlicher, 1847, p. 282 ; coniferous wood; Tertiary ?; Altenburg, Saxony. For Retinodenaron pityoides Zenker, 1833, p. 3, pl. 1, figs. A-D.

RETIOFUCUS Keeping, 1882.

Retiofucus extensus Keeping, 1882, p. 488, pl. 11, figs. 6, 7 ; alga ; Constitution Hill, Aberystwyth, Wales. Earlier citation : Keeping, 1881, p. 152 ; nom. nud.

RETIPHYOUS Ulrich, 1904.

Retiphycus hexagonale Ulrich, 1904, p. 139 , pl. 18 , fig. 5 ; plant?; Yakutat formation, Lower Jurassic?; Pogibshi Island, opposite village of Kadiak, Alaska.

REUSSIA Presl, 1838.

Reussia scolependrioides (Brongniart) Presl, in Sternberg, $1838 \cdot(1820-38)$, p. 125. For Filicites scolependrioides Brongniart, 1828a-38, p. 388 , pl, 137, figs. $2,3$.

RHABDOCARPOS Goeppert and Berger, 1848.

Rhabdocarpos tunicatus Goeppert and Berger, 1848, p. 20, pl. 1, ffg. 8; seed compression: Carboniferous; Charlottenbrunn, Silesia. The spelling Rhabdocarpus adopted by later writers.

RHABDOPORELLA. Stolley, 1893.

Rhabdoporella bacillum Stolley, 1893, p. 139 , pl. 7, figs. $7 a-c$; siphonaceous alga ; Silurian ; Holstein, Kiel.

RHABDOSPERMUM Seward, 1917.

Rhaddospermum cyclocaryon $\mathrm{S}$ e w a $\mathrm{r}$, 1917, p. 344, figs. 501C, 501E ; Carboniferous.

RHABDOTOCAULON Fllche, 1910.

Rhabdotocaulon zeilleri Fliche, 1910, p. 257, pl. 25, fig. 5 ; stem compression, incertae sedis; Triassic (Keuper) Suriauville, Vosges, France.

RHACHIOPTERIS Dawson, 1862.

Rhachiopteris pinnata Dawson, 1862, p. 323 , pl. 16, flg. 60 ; fragment of fern? rachis ; Devonian ; New York.
RHACOGLOSSUM Debey, 1848.

Rhacoglossum heterophyllum Debey, 1848, p. 117 ; nom. nud.

RHACOPHYLLUM Schimper, 1869.

Rhacophyllum lactuoa (Sternberg) Schimper, 1869 (1869-74), p. 684, pl. 46, flg. 1 ; pl. 47, figs. $1,2$.

RHACOPHYTON Mourlon, 1875.

Rhacophyton condrusorum (Crepin) Mourlon, 1875 , p. 658. For Psilophyton conarusorum Crepin, 1874, p. 358, pl. 1 ; Upper Devonian; Condruz, Belgium.

RHACOPTERIDUM Hirmer, 1940.

Palaeontographica, Supp. 9, 1840, p. 60 (not seen, clted in Gothan, 1942b, p. 147).

RHACOPTERIS Schimper, 1869.

Rhacopteris elegans (Ettlngshausen) Schimper, 1869 (1869-74), p. 482. For Asplenites elegans Ettingshausen, $\mathbf{K}$.-k. geol. Relchsanst. Abh., 1852, Band 1, p. 15, pl. 3, figs. 1-3.

RHAMNACINIUM Fellx, 1894.

Rhamnacinium affne Fellx, 1894a, p. 88, pl. 8, figs. 3a d wood; Rhamnaceae; Eocene; Apscheron, Transcaucasia.

RHAMNITES Forbes, 1851.

Rhamnites multinervatus Forbes, 1851, p. 103 , pl. 3, fig. 2 ; leaf; Miocene ; Isle of Mull, Scotland.

RHAMNOSPERMUM Chandler, 1925.

Rhamnospermum bilobatum Chandler, 1925 , p. 30 , pl. 5, figs. 1a-c; seed, Rhamnaceae?; upper Eocene; Hordle, Hampshire, Fngland.

RHETINANGIUM Gordon, 1912.

Rhetinangium arberi Gordon, 1912, p. 821, pls. $1-3$; petrified pteridosperm stem; Calciferous Sandstone series, Lower Carbonlferous; Pettycur, Fife, Scotland.

RHEXOXYLON Bancroft, 1913.

Rhexoxylon africanum Bancroft, 1913, p. 100 , pls. 10-11; petrified polystelfc stem; Triassic; southern Rhodesla. see also Walton, 1923.

RHINANTHEAEIDES Stiehler, 1861.

Rhinantheaeides geoppertana stiehler, 1861, p. 159.

RHINIPTERIS Harris, 1931.

Rhinipteris concinna (Presl) Harris, 1931 b, p. 58 , pls. 12 , 13 ; fertile leaf, Marattlaceae ; Lepldopterls zone, Rhatic; Scoresby Sound, east Greenland.

RHIPIDION Zalessky, 1937.

Rhipidion tyrganum Zalessky, 1937c, p. 136, fig. 19 ; leaf fragment; Incertae sedis; Permian; Russia.

RHIPIDOPSIS Schmalhausen, 1878.

Khipidopsis gingkoides Schmalhausen, 1879 , p. 50, pl. 8, figs. 3-12 ; pl. 6, ffg. 1 ; g Inkgophyte ? foliage; Permian; Petschoralandes, Russia. 
RHIPTOZAMITES Schmalhausen, 1879.

Rhiptozamites goepperti Schmalhansen. 1879 , p. 32 , pl. 4 , figs. $2-4$; cordiatean leaves?; Permian; Russia.

RHIZOALNOXYLON Conwentz, 1880. Rhizoalnoxylon inclusum Conwentz; 1880, p. 38, pl. 8, figs. 33-35 ; wood ; Tertlary ; Karlsdorf, Silesia.

RHITOCALAMOPITYS Solms Laubach, 1896. Rhizocalamopitys sp. Solms Laubach, 1896, p. 77; Lower Carbonlferous; Saalfeld, Prussian Saxony.

RHIZOCARPITES Heer, 1878.

Rhizocarpites singularis Heer, $1878 \mathrm{~b}$, p. 15, pl. 3, figs. 20, 21 ; Marsiliaceae?; Upper Jurassic ; Siberia.

RHIZOCAULON Saporta, 1861.

Rhizocaulon macrophyllum Saporta, in Heer, 1861, p. 135 ; Gramineae?; Eocene; France. See also Saporta, 1862, p. 198 , pl. 1, figs. $4 \mathrm{a}-\mathrm{e}$.

RHIZOCEDROXYLON Felix, 1882.

Rhizocedroxylon hoheneggeri Fellx, 1882a, p. 33, coniferous wood; Tertiary. See also Felix, 1882b, p. 268, pl. 2, fig. 6 .

RHIZOCORDAITES Grand'Eury, 1890.

Rhizocordaites sp. Grand'Eury, 1890, p. 314 , pl. 7 , fig. 12 ; cordaitean roots; Upper Carboniferous; Gard, France.

RHIZOCUPRESSINOXYLON C o n w $\mathrm{t} \mathrm{z}$, 1880.

Rhizocupressinoxylon uniradiatum (Goeppert) Conwentz, 1880, p. 25, pls. 1-7 ; wood ; Tertiary ; Germany.

RHIZODENDRON Goeppert, 1865.

Rhizodendron oppoliense Goeppert, 1865a, p. 399 ; tree fern ; Cretaceous. See also Stenzel, 1886, p. 5, pl. 1, figs. 1-3, 5-12 ; pl. 2 , figs. $13-19$; pl. 3, figs. $20-29$; and Posthumus, 1931.

RHIZOLITHES (C. W. Braun) Lesquereux, 1860.

First valid species appears to be: Rhizolithes palmatifiaus Lesquereux, 1860, p. 313, pl. 5, fig. 9 ; Pennsylvanian; Frog Bayou, Ark. Original citation : Rhizolithes cylindricus Braun, 1847, p. 86; nom. nud.

RHIZOMITES Geyler, 1887.

Rhizomites moenamus Geyler, in Geyler and Kinkelin, 1887, p. 38 , pl. 4, fig. 11. Pliocene ; Frankfurt am Main.

RHIZOMOPSIS Gothan and Sze, 1933.

Rhizomopsis gemmifera Gothan and Sze, 1933 , p. 26 , pl. 4 , fig. 6 ; rhizome?; Carboniferous; Kiangsu province, China.

RHIZOMOPTERIS Scnimper, 1869.

Rhizomopteris lycopodioides Schimper, 1869 (1869-74), p. 699, pl. 49, fig. 2; fern rhizome?; Carboniferous; near Dresden.

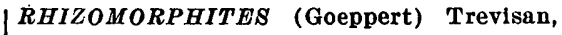
1856.

Rhizomorphites intertextus (Sternberg) Trevisan, in Zigno, 1856 (1856-68), p. 2. For Algacites intertextus Sternberg, 1820-38, p. 37, pl. 21, flg. 6. Earliest citation: Rhizomorphites geanthracis Goeppert, 1848, p. 1085 ; nom. nud. Meschinelli in Saccardo, 1892, p. 802, erroneously attributes this genus to Roth.

RHIZONIUM Corda, 1845.

Rhizonium orchideiforme Corda, 1845, p. 46 , pl. 27.

RHIZOPALMOXYLON Felix, 1883.

Rhizopalmoxylon sp. Fellx, 1883b, p. 27 ; palm root; Antigua, West Indies.

RHIZOPALMOXYLON Gothan, 1942.

Rhizopalmoxylon glaseli Gothan, 1942a, p. 13 , pl. 1 ; stump (root zone) of petrified palm ; Tertiary (Braunkohle) ; Bohlen, Germany.

RHIZOPHIDITES Daugherty, 1941.

Rhizophidites triassicus Daugherty, 1941, p. 43 , pl. 34, fig. 1 ; fungus, Chytridiales ; Triassic: Arizona.

RHIZOPHORITES Bayer, 1914.

Rhizophorites bornbacaceus Bayer, 1914, p. 56, fig. 28 ; leaf, Rhizophoracene; Bohemin.

\section{RHIZOPHOROCARPUS Velenovsky and} Viniklar, 1926.

Rhizophorocarpus dekapetalus Velenovsky and Viniklar, 1926, p. 51, pl. 1, fig. 19; fruit, Rhizophoraceae ; Cretaceous ; $\mathbf{\nabla y -}$ serovic, Bohemia.

RHIZOPTERODENDRON Goeppert, 1881.

Rhizopterodendron oppoliense Goeppert, 1881, p. 3 ; Cretaceous ; Oppeln, Silesia.

RHIZOSTAEMIS Reinsch, 1884.

Rhi ostaemis sp. Reinsch, 1884, p. 15, pl. 23 ; Carboniferous ; Russia.

RHIZOTAXODIOXYLON Fellx, 1882.

Rhizotaxodioxylon palustre Fellx, 1882b, p. 278 , pl. 2, figs. 2-4; coniferous wood ; Quarternary?

RHODDA Presl, 1838.

Rhodel trichomanoides (Brongntart) Presl, in Sternberg, 1838 (1820-38), p. 109. For sphenopteris trichomanoides Brongniart, 1828a-38, p. 182, pl. 48, fig. 3. See also Kidston, 1923, p. 223.

RHODEITES Němejc, 1937.

Rhodeites gutbieri (Ettingshausen) NY̌mejc, 1937, p. 6. For Sphenopteris gutbieri in Ettingshausen, Dle stein. kohlenflora von Radnitz, pl. 19, figs. 1 , 2 ; "Permo-Carboniferous"; Czechoslo. vakla. 
RHODOMELITES Sternberg, 1833.

Rhodomelites strictus (Agardh and Brongniart) Sternberg, $1833(1820-38)$, p. 25. For Fucoides strictus Agardh and Brongniart, in Brongniart, 1822, p. 237, 239, pl. 3, flg. 3 ; alga ; Lower Cretaceous ; Alx, near Rochelle, France.

RHODOMENI'TES Miquel, 1851.

Rhodomenites marginatus Miquel, 1851a, p. 268 ; alga ; Tertiary.

RHODYMENITES Trevisan, 1858.

Rhodymenites ciliatus (Sternberg) Trevisan, in Zigno, 1858 (1856-58), p. 35. For Sphaerococcites ciliatus Sternberg, 1820-38, p. 28, pl. 4, fig. 1.

RIIOIDIUM Unger, 1850.

Rhoidium juglandinum Unger, 1850a, p. 475 ; wood, Anacardiaceue ; Tertiary ; Hungary. First lllustrated specles : Rhoidium ungeri Mercklin, 1856, p. 21, pl. 1, figs. 1, 2 ; pl. 2.

RHOIPITES Wodehouse, 1033.

Rhoipites bradleyi Wodehouse, 1933, p. 513, fig. 45; pollen, Anacardiaceae ; Parachure Creek member, Green River formation, Eocene; Colorado and Utah.

RHOOIDITES Robert Potonie, 1950.

Rhooidites p8eudocingulum Robert Potonle, in Potonle, Robert, Thomson, Paul W., and Thiergart, Friedrich, 1950, p. 57, pl. B, Ags. 41-42 ; pl. C, flgs. 25, 26 ; pollen, Anacardlaceae?; Miocene and Pllocene.

RHOOPHYLLUM Dusen, 1899.

Rhoophyllum nordenskjoldi Dusen, 1899, p. 103 , pl. 11, fig. 1 ; leaf fragment, dicotyledon; Oligocene; Río de las Minas near Punta Arenas, Chile.

RHOPALOPHYLLUM Ettingshausen, 1888.

Rhopalophyllum acuminatum (Unger) Ettingshausen, 1888, p. 314, pl. 4, figs. 1619 ; leaf ; Miocene; Münzenberg, Austria.

RHOPALOSPERMITES Saporta, 1862.

Rhopalospermites strangeaeformis Saporta, 1862, p. 258 , pl. 8, flg. 7 ; seed, compared with Rhopala and strangea; Tertiary ; Alx, Provence, France.

RHPNCHOGONIOPSIS Neumann, 1907.

Rhynchogoniopsis neocomiensis Neumann, 1907 , p. 87 , pl. 1 , flg. 3 ; seed ?; Wealden ; Peru.

RHYNCHOGONIUM Heer, 1876.

Rhynchogonium crassirostre Heer, $1876 \mathrm{~b}$, p. 20, pl. 5 , flgs. 3,4 ; leaf fragment, incertae sedis; Carboniferous; Spitzbergen.

RHYNIA Kidston and Lang, 1917.

Rhynia groynne-vaughani Kidston and Lang, 1917, p. 780, pls. 1-10 ; Psllophytales; Old Red Sandstone, Devonian; Muir of Rhynie, Aberdeenshire, Scotland.
RHYSSOPHYCUS Eichwald, 1854.

Rhyssophycus embolıs Eichwald, 1854, p. 51, alga. See also Eichwald, 1860-68, p. 54, pl. 1a, fig. 4.

RHYTIDOCARYON Mueller, 1876.

Rhytidocaryon wilkinsonit Mueller, 1876, p. 124 , pl. 1, flgs. 1-3; truit, Menispermaceae; upper I'ertiary; Beneree, New South Wales.

RHYTIDODENDRON Boulay, 1876.

Rhytidodendron munitifolium Boulay, 1876 , p. 39 , pl. 3 , fig. 1 ; Upper Carboniferous; Fresnes, France.

RHYTIDOLEPIS Sternberg, 1822.

Rhytidolepis ocellata Sternberg, 1822 (1820-38), p. 32 , pl. 15 ; Sigillarian stem; Carbonlferous.

RHY'TDOPHLOYOS Corda, 1845.

Rhytidophloyos tenuis Corda, 1845, p. 30, pl. 9 , flg. 20 ; lycopod leaf base impression; Carboniferous; Radnitz, Bohemia.

RHYTIDOTHEOA Mueller, 1871.

Rhytidotheca lynchii Mueller, 1871 (187182 ), p. 39 , pl. 4, fig. 1-8; Pliocene ; Haddon, Victoria.

RHYTISMITES Meschinell, 1892.

Rhytismites palaeoucerinum (Engelhardt) Meschinelli, in Saccardo, 1892, p. 780. For Rhytisma palaeoacerinum Engelhardt, 1885, p. 310, pl. 8, flgs. 8a-c ; Miocene; Kundratitz, Bohemia.

RHY'TISMOPSIS Geyler, 1887.

Rhytismopsis sp. Geyler, 1887a, p. 488, pl. 32, fig. 4 ; fungus ; Eocene; Labuan, Borneo.

RHYZODENDRON Zalessky, 1037.

Rhyzodendron rossicum Zalessky, 1937d, p. 159, figs. 9,10 ; lycopod stem impression ; Permian; Russia.

RIENITSIA Walkom, 1932.

Rienitsia spathulata Walkom, 1932, p. 124, pl. 5, figs. 1, 2; fig. 1 . See also Jones and Jersey, 1947, p. 42.

RIMNOCLADON Zalessky, 1930.

Rimnocladon minutum Zalessky, 1930c, p. 227 , pl. 1, flgs. 7,8 ; lycopod ? stem impression; Lower Carboniferous; Urals, Russia.

RIVULARITES Fliche, 1905.

Rivularites repertus Fliche, $1905 a$, p. 47 , pl. 3 , fig. 4 ; alga, Cyanophyceae?; Triassic ; Gemmelaincourt, Vosges, France.

ROBERTIAE Choubert, 1932.

Robertiae katangae Choubert, in Hacquaert, 1932, p. 266; Devonian; Katanga, Belgian Congo.

ROBINIOXYLON Falqui, 1907.

Robiniowylon zuriensis Falqul, 1907, p. 11 ; wood; Ollgocene; Italy. 
RODEITES Sahni, 1943.

Rodeites dakshini Sahni, in Sahni, B1rbal, and Sitholey, R. V., 1943, p. 180, pl. 9, fig. 42 ; early Tertiary (probably Eocene) ; Mohgaon Kalan and Sausar, India.

ROEMERIA Unger, 1852.

Roemeria americana Unger, in Roemer, Ferdinand, 1852, p. 95; wood ; Cretaceous ; near Gonzales, Tex.

ROGERSIA Fontaine, 1889.

Rogersia longifolia Fontaine, 1889, p. 287, pl. 139 , fig. 6 ; pl. 144, fig. 2 ; pl. 150, fig. 1 ; leaf, Proteaceae ; Potomac group, Lower Cretaceous; Fredericksburg, Va.

ROHLFSIA Schenk, 1883.

Rohlfsia celastroides Schenk, 1883a, p. 9, pl. 4, fig. 12 ; wood, dicotyledon; Upper Cretaceous; Libya, North Africa.

ROMEROITES Spegazzini, 1924.

Romeroites argentinensis Spegazzini, 1924 b, p. 139 , figs. 5, 6 ; seed-bearing cone, Coniferales; Upper Cretaceous; Patagonla.

RONZOCARPON Marion, 1872.

Ronzocarpon hians Marion, 1872 , p. 358, pl. 23, figs. 28, 29 ; fruit, dicotyledon; Tertiary ; Ronzon, France.

ROSELLINITES Meschinelli, 1892.

Rósellinites congregatus (Beck) Meschnelli, in Saccardo, 1892, p. 750 ; Pyrenomycete; Oligocene; Saxony. For Rossellina congregata (Beck) Engelhardt, 1888, p. 33, pl..1, figs. 1-9.

ROSELLINITES Henry Potonie, 1893.

Rosellinites beyschlagii Henry" Potonie, $1893 \mathrm{~b}$; p. 27, pl. 1, flg. 8 ; fungus per1thecia ; Permian - (Rothliegendes); Manebach, Kammerberg, Germany.

ROSENBUSCHIA Sterzel, 1895.

Rosenbuschia schalchi Sterzel, 1895, p. 270, pl. 10, figs. 14-18; alga ? Permian ; Oppenau, Baden.

ROSSOVITES Zalessky, 1934.

Rossovites petchorensis Zalessky, $1934 \mathrm{~b}$, p. 289 , fig. 77 ; l leaf fragment, incertae sedis; Permian; Pechora basin, Russia.

ROSTHORNIA Unger, 1842.

Rosthornia carinthiaca Unger, 1842b, p. 175.

ROTHENBERGIA Cotta, 1843.

Rothenbergia hollebenii Cotta, 1843, p. 411 , pl. 2, fig. D ; incertae sedis; Saalfeld, East Prussia.

\section{ROTODONTIOSPERMUM Arnold and Steidt-} mann, 1937.

Rotodontiospermum illinoense Arnold and Steidtmann, 1937, p. 647, figs. 1, 11-14 ; petrified - seed, Pteridospermae; Leansboro formation, Pennsylvanian; Richland County, Ill.
ROTTIA Weyland, 1943.

Rottia incerta Weyland, 1943 , p. 108, pl. 19, figs. 3-7 ; leaf, dicotyledon ; Tertiary ; Rott, Siebergebirge, Germany.

ROTULARIA Sternberg, 1825.

Rotularia marsileaefolia Sternberg, 1825 (1820-38), Tentamen, p. xxxii ; $A n-$ nularia foliage; Carboniferous; Swina, Bohemia.

RUBIACEAECARPUM Menzel, 1913.

Rubiaceaecarpum multicarpellare Menzel, 1913 , p. 10 , pl. 1, figs. 20-24 ; fruit, Rubiaceae; Tertiary (Braunkohle).

RUBIACEOCARPUM Kräusel, 1939.

Bayer. Akad. Wiss., Math.-naturwiss. Abh., 1939, Neue Folge, 47, p. 108 (not seen, cited in Gothan, 1942b, p. 148).

RUBIACITES Weber, 1855.

Rubiacites asperuloides Weber, in Wessel and Weber, 1855, p. 149, pl. 26, flg. 12 ; Miocene; Rott, Germany.

RUBIAEPHYLLUM Bayer, 1893.

Rubiaephyllum gaylussaciae Bayer, in Fric, 1893, p. 131, fig. 192 ; Cretaceous (Senonian); Priesen, Bohemia.

$R U B I D G E A$ Tate, 1867.

Rubidgea mackayi Tate, 1867, p. 141, pl. 5, fig. 8; Glossopteris-like leaf ; Karroo beds, Carboniferous; Bloemkop, near Sunday's River, South Africa.

RUBIIPHYLLITES Hector, 1880.

Rubiiphyllites linearis Hector, 1880, p. 49 ; nom. nud.

RUBIOIDES Perkins, 1904.

Rubioides lignita Perkins, 1904, p. 193, pl. 78 , figs. 80,84 ; fruit, compared with Rubia tinctoria (Rubiaceae) ; Tertlary ; Brandon, Vt.

RUFFORDIA Seward, 1894.

Ruffordia goepperti Seward, 1894a, p. 76, pl. 3 , figs, 5,6 ; pl. 4 ; pl. 5, pl. 6, flg. 1 ; fertile fern follage, Schizaeaceae?; Wealden; England.

RUSOPHYCUS Hall, 1852.

Rusophycus clavatus Hall, 1852, p. 23, pl. 8. figs, 1a, 1b; plant?; Clinton group, Silurian; New Hartford, Oneida County, N. $\mathbf{Y}$.

RUTAECARPUS Velenovsky and Viniklar, 1926.

Rutaecarpus quadrilobus Velenovsky and Viniklar, 1926, p. 52, pl. 1, fig. 9 ; fruit, Rutaceae?; Cretaceous; Otruby, Bohemia.

RUTAPHYLLUM E. W. Berry, 1930.

Rutaphyllum trifoliatum E. W. Berry, 1930 , p. 92 , pl. 42 , fig. 3 ; leaf, Rutaceae ; Grenada formation, lower Eocene; 1 mile north of Somerville, Fayette County, Tenn. 
RUYSCHIOXYLON, Hermann Hofmann, 1844.

Ruyschiowylon sumatrense Hermann Hofmann, 1884b, p. 32 ; wood ; Tertiary ; Sumatra.

\section{$\mathbf{S}$}

SAARODISCITES Hirmer, 1940.

Palaeontographica, 1940, Supp. 9, p. 13 (not seen, cited in Gothan, 1942b, p. 148).

SAAROPTERIS HArmer, 1940.

Palaeontographica, 1940, Supp. 9, p. 37 (not seen, cited in Gothan, 1942b, p. 148).

SABALITES Saporta, 1865.

Sabalites oxyrhachis Saporta, 1865, p. 82, pl. 3, fig. 3 ; palm leaf fragment; Tertiary ; St.-Jean-de-Garguier, France.

SABALOIDITES Robert Potonie, 1950.

Sabaloidites areolatus Robert Potonle, in Potonle, Robert, Thomson, Paul W., and Thiergart, Friedrich, 1950, p. 49, pl. B, figs. 1,2 ; pl. C, fig. 15 ; pollen, Palmaceae; Miocene; Chatt-Aquitan, Germany.

SABIOCAULIS Stopes and Fujii, 1910.

Sabiocantis sakuraii Stopes and Fujti, 1910 , p. 66, pl. 8, fig. 54 ; pl. 9, figs. 5557 ; petrified stem, Sablaceae; Upper Cretaceous; Hokkaido, Japan.

SABIOXYLON Madler, 1939.

Sabioxylon francofurtense Madler, 1939 , p. 120, pl. 12, figs. 1-7 ; wood, Sabiaceae : Pliocene; near Nlederrad, Germany.

SABULIA Stopes, 1913.

O Sabulia scottii Stopes, 1913, p. 93, pl. 6, fig. 2 ; pl. 8 , fig. 9 ; wood, dicotyledon ; Lower Greensand, Lower Cretaceous; Woburn Sands, Bedfordshire, England.

SACCHAROMYCETES Gruss, 1928.

Preuss. geol. Landesanst. Jahrb., 1928, Band 49, p. 1046 (not seen, cited in Gothan, 1942a, p. 148).

SACCOPHYCUS U. P. James, 1879.

Saccophycus intortus U. P. James, 1879, p. 17 ; Lower Silurian; near Lebanon. Ohio.

SACCOPTERIS Stur, 1883.

Saccopteris essinghi (Andrae) Stur, 1883, p. 696 , fig. 18 ; fern?; sporangla.

SACHEOCLADUS Zalessky, 1937.

Palaeophy tographica, Moskau-Leningrad, 1937 , p. 21 (not seen, cited in Gothan, 1942 b, p. 148).

SACHERIA, Ettingshausen, 1852.

Sacheria asplenioides Ettingshausen, 1852a, p. 40 , pl. 20 , fig. 1 ; fertile fern folinge ; Radnitz, Bohemia.

SACHYOGYRUS Zalessky, 1939.

Sachyogyrus multifarius Zalessky, 1939b, p. 336, figs. 7,8 ; articulate cone ; Permian: Matveyevo, Krasnala Glinka, USSR.
SAGENARIA Brongniart, 1822.

Sagenaria coelata Brongniart, 1822, p. 224, pl. 12, fig. 6; a Lepidodendron stem impression ; Carboniferous.

SAGENOPTERIS Presl, 1838.

Sagenopteris nilssoniana (Brongniart) Ward; this species designated as the type by Harris, 1932b, p. 5. For Filicites nilsoniana Brongnlart, $1825 \mathrm{~b}$, p. 218, pl. 12, fig. 1. [First species designated is S. rhoifolia Presl, in Sternberg. 1838 (1820-38), p. 165, pl. 35, fig. 1.]

SAHNIANTHUS Shukla, 1944.

Sahnianthus parijai (Sahni) Shukla, 1944, p. 2, pls. 1-8; petrified flower, Lythraceae; base of Intertrappean series, Tertiary; Mohgaon Kalan, Chhindwāra district, Central Provinces, India.

SAKRISTROBUS K. Jacob, 1943.

Sakristrobus sahnii K. Jacob, in Sahni, Birbal, and Sitholey, R. V., 1943, p. 177, figs. 9, 10 ; Jurassic ; Sakrigalighat, India.

SALICINIUM Unger, 1850.

Salicinium populinum Unger, 1850a, p. 420 ; wood, Salicaceae. Only species lllustrated: S. messinianum Pampaloni, 1904, p. 545, flgs. 10, 11.

SALICINOXYLON Kaiser, 1880.

Salicinoxylon miocenicum Kaiser, $1880 \mathrm{~b}$, p. 511 ; wood, Salicaceae ; probably Miocene; Island of Sylt, Prussia.

SALICIN OXYLON Lingnier, 1907.

Salicinoxylon biradiatum Lignier, 1907, p. 272 , pl. 18 , flgs. $18-24$; wood, dicotyledon; Upper Cretaceous (Cenomanian) ; Hève, France.

SALICIPHYLLUM Conwentz, 1886.

Saliciphyllum 8uccineum Conwentz, 1886, pl. 4, figs. 17-19; leaf, in amber, Salicaceae; Tertiary ; West Prussia.

SALICIPHYLLUM Fontaine, 1889.

Saliciphyllum longifolium Fontaine, 1889, p. 302 , pl. 150 , flg. 12 ; leaves, compared with Salix; Potomac group, Lower Cretaceous; near Potomac Run, Va.

SALICITES Hisinger, 1837.

Salicites wahlbergii Hisinger, 1837, p. 112, pl. 34, fig. 9 ; leaf dicotyledon; Scania, Sweden.

SALICOIDITES Robert Potonie, 1950.

Salicoidites sp. Robert Potonie, in Potonie, Robert, Thomson, Paul W., and Thlergart, Friedrich, 1950 , p. 50 , pl. B, fig. 3 ; pollen, Sallcaceae?; upper Pliocene; Chatt-Aquitan, Germany.

SALICORNITES Principi, 1926.

Salicornites massalongoi Principi, 1926, p. 64 , pl. 2, figs. 8,9 . Earlier citation : Principi, 1821b, p. 90 ; nom. nud.

SALPINGOPORELLA Pla, 1918.

Salpingoporella muihlbergii (Lorenz) Pia, 1918 , p. 211, fig. $4 a$; alga, Dasycladaceae ; Eocene; Radstadt, Austria. 
SALPINGOSTOMA Gordon, 1941.

Salpingostoma dasu Gordon, 1941, p. 447, pls. 1-6 ; pteridosperm seed ; Cementstone group, lower part of Calciferous Sandstone series, Lower Carboniferous ; Oxroad Bay, Tantallon, East Lothian, Scotland.

SAMARAVECTIS Reid and Chandler, 1926. Samaravectis ovalis Reid and Chandler, 1926 , p. 142 , pl. 9, figs. 14-16; winged fruit, compared with fruits of Polygonaceae, Ulmaceae, Urticaceae; Bembridge marl, Oligocene ; Isle of Wight, England.

SAMAROPSIS Goeppert, 1864.

Samaropsis ulmiformis Goeppert, 1864, p. 177, pl. 28, figs. 10, 11; winged seed; Permian; Braunau, Bohemia.

SAMAROSPERMUM E. A. N. Arber, 1914.

Samarospermum moravicum (Helmhacker) E. A. N. Arber, 1914, p. 99, pl. 6, figs. 19, 20 ; winged seed ; Middle Coal Measures, Upper Carboniferous ; Kent coalfield, England.

SAMBUCOIDITES Thomson, 1950.

Sambucoidites sp. Thomson, in Potonie, Robert, Thomson, Paul W., and Thiergart, Friedrich, 1950 , p. 62, pl. B, fig. 63 ; pollen, Caprifoliaceae; Pliocene ; Chatt-Aquitan, Germany.

SANTALIAPHYLLITES Hector, 1880.

Santaliaphyllites maireoides Hector, 1880 , p. 49 ; nom. nud.

SAPINDIPHYLLUM Nathorst, 1888.

Sapindiphyllum dubium Nathorst, 1888, p. 212 , pl. 22 , fig. 5 ; leaf, compared with Sapindus (Sapindaceae); Tertiary ; Tanagori, Musashi province, Japan.

SAPINDOIDEA Kirchheimer, 1936.

Sapindoidea margaritifera (L $\mathrm{u} \mathrm{d} \mathbf{w} \mathrm{i} g$ ) Kirchheimer, 1936 b, p. 89, pl. 9, flgs. 1a-f ; seed, Sapindaceae; Tertlary (Braunkohle) ; Salzhausen, Germany.

SAPINDOIDES Perkins, 1904.

Sapindoides varius Perkins, 1904, p. 206, pl. 81, figs. $116,117,122$; fruit; Tertiary ; Brandon, $v t$.

SAPINDOPHYLLUM Ettingshausen, 1886.

sapindophyllum spinulosodentatum Et. tingshausen, 1886, p. 26, pl. 46, flg. 27 ; leaf, Sapindaceae; Miocene ; Kutschlin, Bohemia.

SAPINDOPSIS Fontaine, 1889.

Sapindopsis cordata Fontaine, 1889, p. 296, pl. 147, fig. 1 ; leaf fragment, compared with Sapindus (Sapindaceae) ; Potomac group, Lower Cretaceous; Fredericksburg, va.

SAPINDOSPERIIUM Reid and Chandler, 1933.

sapindospermum ovoideum Reid and Chandler, 1933, p. 371, pl. 18, figs. 1-5, seed, Sapindaceae; London Clay, Eocene ; Herne Bay, Kent, England.
SAPINDOSTROBUS Ettingshausen, 1887.

Sapindostrobus dubius Ettingshausen, $1887 \mathrm{a}$, p. 137 , pl. 15 , flg. 38 ; incertae sedis; Eocene; Vegetable Creek, near Emmaville, New South Wales.

SAPINDOXYLON Kriausel, 1922.

Sapindoxylon janssonii Kräusel, 1922, p. 124, pl. 1, fig. 9 ; pl. 2, fig. 3 ; pl. 3 , fig. 6 ; pl. 5 , fig. 5 ; wood, Sapindaceae ; Miocene; Sumatra.

SAPORTAEA Fontaine and White, 1880.

Saportaea salisburioides Fontaine and White, 1880 ; p. 102 , pl. 38, figs. 1-3 ; roof shale of Waynesburg coal; Pennsylvanian; Cassville, W. Va.

SAPORTAIA Seward, 1895.

See note under Withamia armata (Saporta) Seward.

SAPORTIA Squinabol, 1891.

Saportia striata Squinabol, 1891b, p. xx, pl. D, fig. 8; pl. E; alga ?; Tertiary ; Liguria, Italy.

SAPOTACITES Ettingshausen, 1853.

Sapotacites sideroxyloides Ettingshausen, 1853 , p. 61 , pl. 21 ; fig. 21 ; leaf, Sapotaceae ; Eocene; Haering, Tirol, Austria.

SAPOTAPHYLLITES Hector, 1880.

Sapotaphyllites linearis Hector, 1880, p. 49 ; nom. nud.

SAPOTEITES Andrae, 1855.

Sapoteites ackneri Andrae, 1855, p. 19, pl. 3, fig. 8; leaf, Sapotaceae ; Miocene; Szakadat, Transylvania.

SAPOTIOARPUM Reid and Chandler, 1933.

Sapoticarpum rotundatum Reid and Chandler, 1.933, p. 467, pl. 26, figs. 24-30; fruit, Sapotaceae; London Clay, Eocene; Sheppey, Kent, England.

SAPOTISPERMUM Reid and Chandler, 1933. Sapotispermum sheppeyense Reid and Chandler, 1933 , p. 471 , pl. 27, figs. 1 , 2 ; seed, Sapotaceae ; London Clay, Hocene ; Sheppey, Kent, England.

SAPOTOPHYLLUM Velenovsky, 1889.

Sapotophyllum obovatum Velenovsky, 1889 , p. 54. For Sapotacites obovata Velenovsky, 1884, p. 3, pl. 3, flg. 6; Upper Cretaceous ; Kuchelbad, Bohemia.

SAPOTOXYLON Felix, 1882,

Sapotoxyton gümbelii Felix, 1882a, p. 54 : wood, Sapotaceae?; Quarternary; wagenhofen near Neuberg. See atso Felix, 1883a, p. 67, pl. 2, figs. $5,8$.

SARCOPTERIS Renault, 1883.

Sarcoptcris bertrandi Renault, $1883 a$, p. 129, pl. 21, figs. 12-15 : petrified fertile pecopterid foliage; Upper Carboniferous.

\$ARCOSPERMUM Deevers, 1937.

Sarcospermum ovale Deevers, 1937, p. 580, figs. 27-36; petrified seed, Trigonocarpales ; Pennsylvanian ; Wilmington, Ill. 
SARCOSTROBILUS Fliche, 1900.

Sarcostrobilus paulini Fliche, 1900, p. 23, pl. 1, figs. 2-5 ; petrified cone, Araucariuceae; Cretaceous ; France.

SARCOTAXUS Brongniart, 1874.

Sarcotaxus angulosus Brongniart, 1874, p. 248 , pl. 21, fig. 16 ; silicified seed ; Carboniferous; St.-Etienne, France.

SARDOA Krasser, 1920.

Sardoa robitschelii Krasser, 1920, p. 21 ; Jurassic ; Sardinia.

SARDYKPHYLLUM Zalessky, 1929.

Sardykphyllum crassinervosum Zalessky, 1929c, p. 688, flg. 14 ; Sphenophyllum? leaf ; Permian; Bolchol Sardyk, Republic Tatare, Russia.

SARGASSITES Sternberg, 1833.

Sargassites septentrionalis (Agardh) Sternberg, 1833 (1820-38), p. 36. For Sargassum septentrionale Agardh, see Brongniart, 1828a-38, p. 50, pl. 2, fig. 24 ; alga?; Upper Carboniferous ; Hoganïs, Sweden.

SAROPTERIS Tschirkova, 1937.

Saropteris rossica Tschirkova, 1937, p. 244, fig. 12; sphenopteridlike fertlle foliage; Carboniferous; Bredy, Russia.

SAESAFROPHYLLUM Velenovsky, 1889.

Sassafrophyllum acutilobum (Lesquereux) Velenovsky, 1889, p. 58. For Sa8safras acutilobum Lesquereux, 1874, p. $79, \mathrm{pl}$. 14 ; Upper Cretaceous; Kansas.

SAURUROPSIS Stopes and Fujil, 1910.

Saururopsis niponensis Stopes and Fujil, 1910 , p. 58, pl. 7 , figs. $42-47$; stem, Saururaceae; Upper Cretaceous; Hokkaido, Japan.

SAUSAROSPERMUM Sahnl and Srivastava, 1940.

Sausarospermum fermori Sahnl and Srivastava, in Sahni, 1940, p. 14, pl. 3, fig. 12. See also Sahni and Srivastava, 1934 , p. 318 ; petrified seed; Deccan Intertrappean series; Tertiary; Sausar, India.

SAXEGOTHOPSIS Dusen, 1899.

Saxegothopsis fuegianus Dusen, 1899, p. 105, pl. 11, fig. 10 ; leaf, Podocarpaceae ; Oligocene; Barancas de Carmen Sylva, - Chile.

SAXIFRAGACEAECARPUM Menzel, 1913.

Suxifragaceaecarpum bifolliculare Menzel, 1913 , p. 32, pl. 4, figs. 7-10 ; frult, Saxifragaceae; Tertlary (Braunkohle); Germany.

SAXIFRAGISPERMUM Reid and Chandler, 1933.

Saxifragispermum spinosissimum Reid and Chandler, 1933 , p. 245 , pl. 8, figs. 3035 ; fruit, Saxifragaceae; London Clay, Eocene: Sheppey, Kent, England.
SAXIFRAGITES Ettingshausen, 1868.

Saxifragites crenulatus Ettingshausen, $1868 a$, p. 7 , pl. 41 ; figs. $1-3$; leaf, Saxifragaceae?; Miocene ; Kutschlin, Bohemia.

SBOROMIRSKIA Zalessky, 1936.

Sboromirskia asiatica Zalessky, 1936a, p. 234, fig. 18 ; coniferous? follage; Carboniferous; Russia.

SCALITES Reinsch, 1881.

Scalites sp. Reinsch, 1881 , p. 74 , pl. 17b, figs. 1-4; Upper Carboniferous ; Zwickau, Saxony.

SOAPANITES Gottsche, 1886.

Scapanites acutifolius Gottsche, 1886, p. 122 ; nom. nud.

SCAPANOPHYLLUM Zalessky, 1929.

Scapanophyllum sitzense Zalessky, 1929b, p. 133, fig. 14 ; fern ? pinnule ; Permian; Sitsa village near Vladivostock.

SCAPHIDOPTERIS Renault, 1883.

Sccphidopteris gilliotti Renault, 1883, p. 128, pl. 22, figs. 5-7 ; petrifled pinnules compared with Pecopteris; Upper Carboniferous ; Peronniere, France.

SCAFINA Pocta, 1889.

Sccupina cambrica Pocta, 1889, p. 429, fig. 10 [unnumbered plate]; Cambrian; Pribram, Bohemia.

SCHAFARZIKIA Tuzson, 1914.

Schafarzikia oligocaenica Tuzson, 1914, p. 251 , pl. 19, fig. 1 ; leaf fragment ; upper Oligocene; Zsil valley, near Petrozseny, Hungary.

SCHAFFERIA Fucini, 1938.

Palaeontographia Italica, 1938, app. 2, p. 133 (not seen, cited in Gothan, 1942b, p. 149).

SCHAFHAUTLIA. Naegell, 1863.

Schafhautlia teisenbergensis Naegell, in Schafhautl, 1863, p. 29, pl. 65, figs. 1, 2 ; wood, dicotyledon; Upper Cretaceous; Tiesenberg, South Bavaria.

SOHIDOLEPIUIS Heer, 1880.

Schidolepium gracile Heer, $1880 \mathrm{a}$, p. 27, pl. 8, figs. 6-12 ; cone, Coniferales; Jurassic; Siberia.

SCHILDERIA Daugherty, 1934.

Schilderia adamanica Daughtery, 1934, $n$. 363, pl. 5; petrified wood; Triassic ; Arizona.

SCHIMPERITES Schleiden, 1855.

Schimperites leptotichus Schlelden, in Schmid and Schleiden, 1855, p. 42 ; Tertiary; Libethen, Hungary; nom. nud.

SCHISTOSTACHYUM Schenk, 1864.

Schistostachyum thyrsoideum $\mathbf{S} \mathrm{ch}$ e $\mathbf{n}$, 1864 , p. 110 , pl. 6, figs. 3a, 3b ; Upper Triassic (Keuper); Estenfeld, Bavaria.

SCHIZAEITES Henry Potonie, 1893.

Schizacites angustus Henry Potonie, 1893a, p. 161 , pl. 20, fig. 4 ; fern leaf fragment ; Permian: Manebach, Prussian Saxony. 
SCHIZAEOPSIS E. W. Berry, 1911.

Schizaeopsis expansa (Fontaine) E. W. Berry, 1911c, p. 194, pl. 12 ; compared with Schizaea; Patuxent formation, Lower Cretaceous; Fredericksburg, Va.

SCHIZAEOPTERIS Stopes and Fujil, 1910.

Schizaeopteris mesozoica Stopes and Fujii, 1910 , p. 10, pl. 2 , fig. 1 ; sporangia, Schizaeaceae; Upper Cretaceous; Hokkaido, Japan.

SCHIZEITES Guembel, 1859.

Schizeites dichotomus Guembel, 1859a, p. 101, fig. 7 ; incertae sedis; Permian; Steinbruch, near Erbendorf, Bavaria.

SCHIZODENDRON Elchwald, 1860.

Schizodendron tuberculatum Eichwald, 1860 , p. 266, pl. 18, fig. 10 ; fern stem?; Permian; Bjelebei, Orenbourg, Russia. Generic name cited in Mercklin, 1856, p. 81 ; nom. nud.

SCHIZOLEPIDELLA Halle, 1913.

Schizolepidella gracilis Halle, 1913, p. 90, pl. 9, flgs. 18-21; liverwort?; Jurassic ; Hope Bay, Graham Land.

SCHIZOLEPIS C. F. W. Braun, 1847.

Schizolepis liasokeuperinus C. F. W. Braun, 1847 , p. 86 ; cone scales, Abietineae; Triassic. Later described as Schizolepis braunii Schenk, 1867 (1865-67), p. 179, pl. 44, figs. 1-8. See also Seward, 1919, p. 439 .

SCHIZONEURA Schimper and Mougeot, 1844.

Schizoneura paradoxa Schimper and Mougeot, 1844, p. 50, pls. 24-26; articula te stems and foliage; Mulhouse, Germany.

SCHIZONEUROPSIS Richter, 1906.

Schizoneuropsis posthuma Richter, 1906 (1906-09), p. 13, pl. 6, fig. 10 ; Lower Cretaceous; Quedlinburg, Prussian Saxony.

SCHIZONEUROPSIS Yabe and Shimakura, 1940.

Schizonewropsis tokuadi Yabe and Shimakura, 1940, p. 177, pl. 15 ; some similarity to Schizoneura; Permian; Huainan coal mines, Anhwei province, China.

SCHIZOPODIUM Morière, 1888.

Schizopodium renaulti Moriere, 1888, p. 133, pls. 1, 2; petrified cycadophyte trunk; Lower Jurassic (Lias); Montignu, France.

SCHIZOPODIUM Harris, 1929.

Schizopodium davidi Harris, 1929, p. 408 , pls. $91-93$; petrified stem intermediate in anatomy between Asteroxylon and Cladoxylon; Burdekin beds, Mictale Devonian; Burdekin basin, Queensland.

SCHIZOPTERIS Brongniart, 1828.

Schizopteris anomala Brongniart, 1828b, p. 63 ; fern frond compared with Schizea and certain Asplenium species; Carboniferous. See also Brongniart, 1828a-38, p. 384 , pl. 135.
SCHIZOSPERMUM E. A. N. Arber, 1914. Schizospermum noeggerathi (Sternberg) E. A. N. Arber, 1914 , p. 103, pl. 8, figs. 48-50 ; Upper Carboniferous; south Wales and south England.

SOHIZOSTACHYS Grand'Eury, 1877.

Schizostachys frondosus Grand'Eury, 1877, p. 201, pl. 17, flg. 3 ; coenopterid fer $n$ fructification; Carboniferous ; France. [The name Androstachys frondosus Grand'Eury appears on the plate.]

SCHIZOXYLON Unger, 1856.

Schizoxylon taeniatum Unger, 1856, p. 180, pl. 12, fig. 8 ; regarded as 1dentical with Cladoxylon (see discussion in Seward, 1917, p. 200) ; Upper Devonian; Saalfeld, Thuringia.

SCHLEIDENITES Unger, 1842.

Schleidenites compositus Unger, in Endlicher, 1842, p. 102 ; wood, incertae sedis; Tertiary ; Hungary.

SCHLOTHEIMIA Sternberg, 1822.

Schlotheimia arborescens Sternberg, 1822 (1820-38), p. 32 ; Asterophyllites follage; Carboniferous. For Casuarinites equisetiformis Schlotheim, 1820, pl. 2, fig. 3 ; pl. 1, fig. 1.

SCHMIDTTES Schleiden, 1855.

Schmidites vasculosus Schleiden, in Schmid and Schleiden, 1855, p. 39 ; wood, Leguminosae?; Tertiary (Braunkohle) ; Tapolesan, Hungary.

SCHMIEDELIOPSIS Fellx, 1882.

Schmiedeliopsis zirkelit Fellx, 1882a, p. 72 ; wood ; Antigua, West Indles. See also Felix, 1883, p. 16, pl. 2, figs. 6, 8 ; pl. 3 , fig. 9.

SCHOINOPHYTUM Jaeger, 1851.

Schoinophytum contortum Jaeger, in Stizenberger, 1851 , p. 43 ; nom. nud.; Jurassic ; Mundelfinger, Baden.

SCHOPFIA Janssen, 1940.

Schopfia deueli Janssen, 1940, p. 102, pl. 28 , figs. 5,6 ; incertae sedis; coal No. 2 , Pennsylvanfan; Mazon Creek, IIl.

SCHOPFIASTRUM Andrews, 1945.

Schophastrum decussatum Andrews, 1945. p. 334 , pl. 10 , figs. 17,18 ; pl. 11, figs. 20-22; pl. 15, fg. 36 ; petrified stem, tinangium; Des Moines group, Pennsylvanian; Urbandale coal mine, Des Moines, Iowa.

SCXYPFITES Kosanke, 1950.

Schopfltes dimorphus Tosanke, xo5o, p. 52, pl. 13, figs. 1-3; spore; No. 2 coal, Pennsylvanian; Franklin County, Ill.

SCHULZOSPORA Kosanke, 1950.

Schulz08pora rara Kosanke, 1950, p. 53, pl. 13, figs. 5-8; Battery Rock coal, Pennsylvanian; Hardin County, Ill. 
SCHUTZIA H. B. Geinitz, 1863.

Schutzia anomala H. B. Gelnitz, 1863, p. 525, pl. 6, figs. 1-3 ; inflorescence, Cordaitales; Carboniferous; Ottendorf, near Braunau, Bohemia.

SCIADIPTERIS Sternberg, 1838.

Sciadipteris radnicensis Sternberg, 1838 (1820-38), p. 118 , pl. 37 , flg. 1 ; fernlike foliage; Upper Carboniferous ; Brzas, near Radnitz, Bohemia.

SCIADISCA Zalessky, 1934.

Sciadisca petchorensis Zalessky, 1934b, p. 271, fig. 49 ; incertae sedis; Permian; Pechora basin, Russia.

SCIADOPHYTON Kräusel and Weyland, 1930.

Sciadophyton steinmanni Krïusel and Weyland, 1930, p. 220.

SCIADOPITYOXYLON Schmalhausen, 1879. Sciadopityoxylon vestuta Schmalhausen, 1879 , p. 40 ; wood, affinities with Sciadopity8 (Taxodiaceae) ; Jurassic ; Halbinsel, Mangyschlak, Russia. First? illustrated species: Sciadopityoxylon wettsteini Jurasky, 1928, p. 258, figs. 1-5.

SCIADOPITYTES Goeppert and Menge, 1883.

Sciadopitytes linearis Goeppert and Menge, 1883 , p. 36, pl. 13, figs. 117-119 ; Sciadopitys-like leaves; middle Miocene; Samland, Baltic Prussia.

SCIRPITIS Dusen, 1908.

Scirpitis sp. Dusen, 1908, p. 16; leaf fragment, compared with scirpus (Cyperaceae) ; Tertiary ; Seymour Island, Antarctic Ocean.

SCITAMINITES Sternberg, 1825.

Scitaminites musaeformis Sternberg, 1825 (1820-38), Tentamen, p. xxxvi, pl. 5, fig. 2 ; incertae sedis; Upper Carboniferous; Radnitz, Bohemia.

SCITAMINORPHYTON Massalongo, 1858.

Scitaminophyton meneghinianum Massalongo, $1858 \mathrm{~b}$, p. 783 ; leaf, Scitaminaceae?; Oligocene; Ronca, Italy.

SCLEROPHYLLINA Heer, 1864.

Sclerophyllina furcata Heer, 1864, p. 55, pl. 2, fig. 9 ; fern?; Upper Triassic (Keuper) ; Switzerland.

SCLEROPHYLLOIDES Heer, 1862.

Sclerophylloides furcatus Heer, in Muller, 1862 , p. 54 ; nom. nud.

SCLEROPTERIDIUM Heer, 1877.

Scleropteridium dahlianum Heer, 1877a, p. 12 , p1. 1 , fig. 1 ; fern ? foliage ; Jurassic ; Andö, Norway.

SCLEROPTERIS Saporta, 1872.

Scleropteris pomelii Saporta, 1872a-73, p. 370 , pl. 46 , flg. 1 ; pl. 47 , figs. 1,2 ; fern follage; Jurassic; near Verdun, France.
SCLEROPTERIS H. N. Andrews, 1942.

Scleropteris illinoiensis H. N. Andrews, 1942 , p. 3, pls. 1-3 ; rhizome, closely related to Botrychioxylon; coal No. 6, Pennsylvanian; Pyramid coal mine, Pinckneyville, Ill.

SCLEROTHAMNIUM A1roldi, 1936.

Sclerothamnium nitens Airoldi, 1936, p. 18, flgs. 1, 2, 4; alga ; Middle Triassic; northern Italy.

SCLEROTITES Meschinelli, 1892.

Sclerotites acericola (Heer) Meschinelli, in Saccardo, 1892, p. 803. See also Meschinelli, 1898, p. 98, pl. 26, flg. 10.

SCOLECOLITHUS Goeppert, 1852.

Scolecolithus linearis (Haldemann) Goeppert, 1852b, p. 101. For Skolithos linearis Haldemann, 1840, p. 3 ; Cambrian; Reading, $\mathrm{Pa}$. Goeppert refers to Paleontology of New York, Albany, 1847 , v. 1, p. 2, pl. 1, fig. 1 .

SCOLECOPTERIS Zenker, 1837.

Scolecopteris elegans Zenker, 1837, p. 509, pl. 10 ; fern foliage, fertile, Marattiaceae ; Permian; Chennitz, Germany.

SOOLITHUS Hall, 1847.

Scolithus linearis Hall, 1847, p. 2, pl. 1, flgs. 1a-c; plant?; Potsdam sandstone, Upper Cambrian; Adams, Mass., New Jersey, Pennsylvania, etc.

SCOLOPENDRITES Goeppert, 1836.

Scolopendrites jussieui Goeppert, 1836, p. 276 ; fertlle fern frond. For Filicites scolopendroides Brongniart, 1828d, p. 443 , pl. 18 , fig. 2 ; Triassic ; AlsaceLorraine. See also Brongniart, 1836 (1828a-38), p. 388 , pl. 137, flgs. 2, 3.

SCOLOPENDRITES Lesquereux, 1854.

Scolopendrites grossedentata Lesquereux, in Lesquereux and Rogers, 1854, p. 425 ; Pennsylvania. See also Rogers, 1858, p. 868 , pl. 8 , fig. 7 .

SCOIOPIOIDEA Langeron, 1899.

Scolopioidea palaeccenica Langeron, 1899. p. 454 , pl. 2 , fig. 4 ; leaf, compared with Scolopia, Bixaceae; Eocene; Sézanne, France.

SCORESBY.A Harris, 1932.

Scoresbya dentata Harris, $1932 a$, p. 38 , pls. 2, 3 ; leaf, related to Sagenopteris?; Thaumatopteris zone, Rhaetic ; Scoresby Sound, east Greenland.

SCOTTIELLA Schuster, 1931.

A generic name proposed for Medullosa anglica, $M$. pusilla, and $M$. centroflis. See Schuster, 1931, p. 235.

SCOUGOUPHYTON Fenri and Genevieve Termier, 1950.

Scougouphyton abdallahense Henri and Genevieve Termier, 1950 , p. 206,figs. 4952; Devonian; Dechra Ait Abdallah, Central Morocco. 
SCOYENIA David White, 1929.

Scoyenia gracilis David White, 1929, p. 115 , pl. 4, fig. 3 , pl. 5 ; probably not plant; lower Hermit shale, Permian; Arizona.

SCROPHULARINA Heer, 1859.

Scrophularina oblita Heer, 1859, p. 17, pl. 103 , fig. 17 ; calyx?, Scrophulariaceae ; Tertiary ; Oeningen, Switzerland.

SOUTOCORDAITES Renault and Zeiller, 1885.

Scutocordaites grand'euryi Renault and Zeiller, 1885 , p. 869 ; stem and follage, Cordaitales ; Upper Carbontferous ; Commentry, France. See also Renault and Zeiller, 1890 , p. 605 , pl. 63 , fig. 6 .

SCYTOPHYLLUM Bornemann, 1856.

Scytophyllum bergeri Bornemann, 1856, p. 75 , pl. 7, figs. 1-6; fernlike leaf fragment ; Keuper?; Mülhausen, Germany.

SEDGWICKIA Goeppert, 1848.

Sedgwickia yuccoides Goeppert, in Bronn, 1848, p. 1131. For Endogenites erosa Stokes and Webb, 1824, p. 423, pl. 46, figs. 1,2 ; pl. 47, figs. 5a, 5b ; Wealden ; I'ilgate Forest, Sussex, England. See also Read and Brown, 1937, p. 106.

SEDITES H. B. Geinitz, 1842.

Sedites rabenhorstii $\mathrm{H}$. B. Geinitz, 1842 (1839-42), p. 97, pl. 24, fig. 5; leaves and stem, compared with Sedum, Crassulaceae.

SELAGINELLITES Zeiller, 1906.

Selaginellites suissei Zeiller, 1906, p. 141, pl. 39 , figs. $1-5$; pl. 40 , figs. 1-10 ; pl. 41, figs. 4-6 ; fertile lycopod shoot ; Permian ; Blanzy, France.

SELAGINITES Brongniart, 1828.

Selaginites patens Brongniart, $1828 \mathrm{~b}$, p. 84; lycopod foliage shoots; Carboniferous. See also Brongniart, 1838 (1828a$38)$, p. 68, pl. 26.

SELENOCARPUS Schenk, 1866.

Selenocarpus münsterianus Schenk, 1866, p. 89 , pl. 22, flgs. 1-6; fertile fern, Gleicheniaceae; Rhaetic; Strullendorf and Reindorf, near Bamberg, Bavaria.

SELENOCHLAENA Corda, 1845.

Selenochlaena microrrhiza Corda, 1845, p. 81. For Tubicaulis dubius Cotta, 1832, p. 25 , pl. 1 , figs. 3,4 . See also Posthumus, 1931.

SELENOPTERIS Corda, 1845.

Selenopteris radnicensis Corda, 1845, p. 84 , pl. 52 ; coenopterid petlole?; Carboniferous; Radnitz, Bohemia. See also Posthumus, 1931.

SEMAPTERIS Unger, 1870.

Semapteris carinthiaca Unger, 1870, p. 788 , pl. 3, fig. 1; partly decorticated lycopod? stem; Upper Carboniferous; Carinthla, Austria-Hungary.
SEMECARPITES Fritel, 1912.

Semecarpites linearifolius Fritel, 1912, p. 643 , pl. 22 , fig. 1 ; leaf, compared with Semecarpus (Anacardiaceae); Oligocene (Aquitanien) ; Bois d'Asson, France.

$S E M E N$ Velenovsky and Viniklar, 1927.

Semen trigonum Velenovsky and Viniklar, 1927 , p. 43 , pl. 14, fig. 9 ; seed, incertae sedis; Cretaceous; Slivenec, Bohemia.

SENDELIA Goeppert and Berendt, 1845.

Sendelia rat ${ }^{2}$ burgeana Goeppert and Berendt, in Berendt, 1845, p. 81, pl. 5, figs. 18-20; staminate flower; Miocene; Prussia.

SENFTENBERGIA Corda, 1845.

Senftenbergia elegans Corda, 1845, p. 91, pl. 57, figs. 1-6. ; fertile foliage, Schizaeaceae; Carboniferous; Nachod, Bohemia. See also Radforth, 1938, 1939.

SEQUOIOIDITES Thiergart, 1950

Sequoioidites polyformosus Thiergart, in Potonie, Robert, Thomson, Paul W., and Thiergart, Friedrich, 1950 , p. 49, pl. A, figs. 20,21 ; pl. C, fig. 8 ; pollen, compared with Sequoia, Metasequoia; Miocene-Pliocene ; Chatt-Aquitan, Germany,

SEQUOIOPSIS Saporta, 1876-84.

Sequoiopsis buvignieri Saporta, 1876-84, p. 540, pl. 201, figs. 1-5 ; twigs, foliage, Coniferales; Jurassic; Creue, near St. Mihiel, France.

SEQUOIOXYLON Torrey, 1923.

Sequoioxylon montanense Torrey, 1923, p. 74, pl. 10, figs. 19-23 ; wood, Coniferales ; Laramie formation, Upper Cretaceous; bank of Missouri River, Culbertson, Mont.

SEQUOIOXYLON Yusui, 1928

Sequoioxylon hondoense Yusui, 1928, p. 420 , pl. 17, figs. 59-63; wood, compared with Sequoia; upper Tertiary; Alch! coalfield, central Japan.

SEQUOIIT'ES.

See Sequoites.

SEQUOTTES Brongniart, 1849.

Type species?: Sequoites taxiformis (Unger) Brongniart, 1849, p. 117. For Cupressites taxiformis Unger, 1842, (1841-47), p. 18, pls. 8, 9 ; foliage, cones Contferales; Haering. Spelling Sequoiites adopted by some authors.

SERENOPSIS Hollick, 1893.

Serenopsis kempii Hollick, 1893b, p. 169 , pl. 149; palm leaf ; Cretaceous; Glen Cove, Long Island, N. Y.

SESTROSPHAERA Pia, 1920.

Sestrosphaera liasina P1a, 1920, p. 138. pl. 7, figs. 27, 28; alga, Siphoneae Verticillatae; Jurassic; Italy. 
SETOSISPORITES Ibrahim, 1933.

Setosisporites subpilosus Ibrahim, 1933, p. 27, pl. 5, fig. 40 ; spore ; Carboniferous. [Ibrahim cites $S$. hirsutus as the type species, but I find no record of an il. lustration. The first species illustrated in his work is S. subpilosus.]

SEWARDIA Zeiller, 1900.

Sewardia latifolia Zeiller, 1900, p. 233, fig. 160. For Withamia saportae Seward, 1895 , p. 174 , pl. 2 , figs. 1,2 ; pl. 5 . fig. 1 ; cycadophyte frond fragment; Wealden; England. See also Seward, 1919, p. 103.

SEWARDIELLA Fucini, 1936.

Reference not seen ; cited in Gothan, 1942b, p. 150.

SEZANNELLA Viguier, 1908.

Sezannella major Viguier, 1908, p. 13, pl. $\overline{5}$, figs. 1, 4, 7, 10 ; flower, Sterculiaceae : Eocene (Thanetien); Sézanne, France. [In Viguier, $1907 \mathrm{~b}$, p. 1004 , a generic description is given and two species listed, 8. major and s. minor.]

SEZANNIA Saporta, 1865.

Sezannia credneriaeformis Saporta, 1865 , p. 45 ; leaf, dicotyledon (some resem. blance to Credneria); Tertiary; Sézanne, France.

SHERMANOPHYCUS J. H. Johnson, 1940. Shermanophyous gouldi J. H. Johnson. 1940 , p. 582, pl. 2, figs. 1, 2 ; alga. Cyanophyceae?; near top of Weber shale, Pennsylvanian; Park County, Colo.

SHIRAKIA Kawasaki, 1934.

Shirakia bilobifolia. Kawasaki, 1934 $(1927-34)$, p. 98 , pl. 22 , figs. 32,33 : fertile fernlike foliuge, compared with Eboracia lobifolia; Kobosan series, bed: I, H, G, Mesozoic ; Samch'ðk, South Korea.

SHIRAKIOPTERIS, Kon'no, 1950.

Shirakiopteris kawasaki Kon'no, 1950, p. 95, 5 figs.

SHOREOXYLON Berger, 1923.

Shoreoxylon palembangense (Kräusel) Berger, 1923, p. 145; wood, compared with Shorea; Tertlary; Sumatra. For Caesalpinioxylon palembangense Krïusel, 1922, p. 247, pl. 2, fig. 1 ; pl. 3, figs. 1,2 ; pl. 7, figs. $6,11$.

SHRUBSOLEA Reid and Chandler, 1933.

Shrubsolea jenkinsi Reid and Chandler, 1933 , p. 262 , pl. 10 , figs. 11,12 ; seed, Rutaceae; London Clay, Eocene; Herne Bay, Kent, England.

SIDERELLA Read, 1936.

Silerella scotti Read, 1936b, p. 226, figs. 12, 14-16; petrified stem, Siderellales, link between zygopterid ferns and Sphenophyllales?; Upper Devonian; Junction City, Boyle County, Ky.
SIGILLARIA Brongniart, 1822.

Sigillaria 8cutellata Brongniart, 1822, p. 222 , pl. 12, fig. 4 ; stem impression showing leaf hases; Carboniferous; France.

SIGILLARIOIDES Lesquereux, 1870.

Sigillarioides radicans Lesquereux, 1870, p. 449 , pl. 31, fig. 4 ; roots of sigillaria; Upper Carboniferous; Mazon Creek, IIl.

SIGILLARIOPHYLLUM Grand'Eury, 1877.

A generic name proposed for leaves which Grand'Eury reports having seen attached to Sigillaria. He cites as an example: Oypterites bicarinatus Lindley and Hutton, 1833 (1831-37), p. 123, pl. 43, figs. 1, 2.

SIGILLARIOPSIS Renault, 1879.

Sigillariopsis decaisnei Renault, 1879, p. 270 , pl. 12, figs. $15-19$; pl. 13, figs. $1-4$; petrifled lenves and small stems of sigillarian affinities; Carboniferous ; France.

SIGILLARIOSTROBUS (Schimper) Eugen Geinitz, 1873.

Sigillariostrobus bifidus Eugen Geinitz, 1873 , p. 70 , pl. 3 , figs. 5-7 ; terminally forked sporophylls with sporangia at base; Pelmian (lower Dyas); near Pillnitz, Saxony. Generic name glven by Schimper, 1870 (1869-74), p. 105, pl. 67, figs. 13-24.

SIGILLODENDRON C. E. Weiss, 1889.

Sigillodendron frondosum (Goeppert) C. $\mathrm{N}$. Weiss, 1889, p. 164, pl. 2, fig. 1.

SIGNACULARIA Zalessky, 1929.

Signacularia noinsliii Zalessky, 1929a, p. 192, pl. 17, figs. 1, 2 ; partly decorticated stem impression; Carboniferous; Donets, Russia.

SILESIOPTERIS Posthumus, 1924.

Silesiopteris sinuosa (Goeppert) Posthumus, 1924, p. 885 . For Gyropteris simuosa Goeppert, 1852 b, p. 138 , in part. See also Posthumus, 1931.

SILLIMANIA Unger, 1850.

Sillimania texana Unger, 1850a p. 524; wood, incertae sedis ; Cretaceous ; Texas.

SILPHIDIUM Massalongo, 1853.

Silphidium visianicum Mașsalongo, 1853a, p. 16. For illustration, see Massalongo, 1858 e, p. 122 , pl. 4 , flgs. $1-3$; pl. 5 , fig. 2.

SIMARUBITES E. W. Berry, 1930.

Simarubites eocenicus $\mathrm{D}$. W. Berry, 1930 , p. 94 , pl. 44 , flgs. 15, 16 ; winged fruit, Simarubaceae; Wilcox group, Lower Eocene; La Grange, Eayette County, Tenn.

SIMARUBINIUM Platen, 1908.

simarubinium crystallophorum Platen, 1908 , p. 54 ; Pliocene; Callstoga, Calle. 


\section{SINOCTENIS Sze, 1931.}

Sinoctenis grabauiana Sze, 1931, p. 14, pl. 2 , fig. 1 ; pl. 4 , fig. 2 ; cycadophyte foliage; Lower Jurassic (Lias) ; Pinghsiang, Kiangsi province, China.

SINUSIA Krestew, 1928.

Preuss. geol. Landesanst. Jahrb., 1928, Band 49, p. 574 (not seen, cited in Gothan, 1942b, p. 151).

SIPHODENDRON Saporta, 1884.

Siphodendron girardoti Saporta, 1884, p. 38, pl. 6, figs. 6, 7 ; Jurassic ; Chatelneuf, France.

SIPIIODICTYTES Reinsch, 1881.

siphodictytes sp. Reinsch, 1881, p. 75, pl. 18a, figs. $1-4$; pl. $18 \mathrm{~b}$, figs. 9-11 ; Permian (Dyas) ; Stockheim, Württemberg.

SIPIIONEMA Bornemann, 1886.

Siphonema incrustans Bornemann, 1886, p. 18, pl. 2, figs. 1, 2; alga ?; Cambrian; Sardinia.

SIPHONITES Saporta, 1872.

Siphonites heberti Saporta, 1872-73, p. 111 , pl. 22, figs. 1, 2 ; alga?; Jurassic ; Chalindrey, France.

SIPHONOTHALLUS Rothpletz, 1896.

Siphonothallus taeniatus Rothpletz, 1896, p. 896 , pl. 22 , fig. 10 ; alga ; upper Oligocene; Wernleite, near Siegsdorf, Bavaria.

SIRODESMITES Pia, 1927.

Sirodesmites subgranulosus (Renault) Pla, in Hirmer, 1927, p. 123 ; fungus, Dematiaceae, Fungi Imperfecti ; Oligocene. For Sirodesmium subgranulosum Renault, 1899 , p. 980 , pl. 17 , fig. 18 .

SITZIA Zalessky, 1930.

Sitzia klocki Zalessky, 1930f, p. 929, fig. 9; fern frond fragment; Permian; Pechora basin, Russia.

SITZOPTERIS Zalessky, 1930.

Sitzopteris superba Zalessky, 1930f, p. 929, fig. 10 ; fern frond fragment; Permian ; Pechora basin, Russia.

SJöGRENIA Felix, 1894.

Sjügrenia crystallophora Felix, 1894a, p. 93 , pl. 9 , figs. 1, 2 ; wood, dicotyledon; Eocene; Apscheron, Transcaucasia.

SLOANEOPSIS Kuntze, 1904.

Sloaneopsis Kuntze, in Post and Kuntze, 1004, p. 522.

SMEYSTERSIA Fraipont, 1921..

Smeystersia minuta Fraipont, 1921, j. M51; male cone, Coniferales; Wealden; Belgium. Pollen grains only figured; other illustrations and specimens destroyed during German invasion of Liége in August 1914.

SMILACIPITES wodehouse, 1933.

Smilacipites molloides Wodehouse, 1933, p. 500, fig. 25 ; pollen, Liliaceae ; Parachute Creek member, Green River formation, Eocene ; Colorado and Utah.
SMILACITES Brongniart, 1828.

Smilacites hastata Brongniart, 1828c, p. 45, pl. 3, fig. 8 ; Tertiary ; Armissan, France.

SOLANITES Saporta, 1862.

Solanites brongniartii Saporta, 1862, p. 262 , pl. 11, fig. 2 ; flower, Solanaceae ; Tertiary ; Aix, Provence, France.

SOLENIOPSIS Massalongo, 1851.

Soleniopsis linzoides Massalongo, 1851, p. 67 ; alga ; Tertiary ; Italy.

SOLENITES Lindley and Hutton, 1834.

Solenites murrayana Lindley and Hutton, 1834 (1831-37), p. 105, pl. 121 ; foliage, Ginkgoales; Jurassic; Gristhorpe Bay, near Scarborough, England. See also Seward, 1919, p. 64.

SOLENOPHYLLUM Maslov, 1935.

Solenophyllum paleozoicum Maslov, V. P. This reference not checked; it was reported by J. H. Johnson, 1943, as : Inst. Econ. Mineralogy Moskva Trans., 1935, v. 72, p. 1-28.

SOLENOPLASMIUM Reinsch, 1881.

Solenoplasmium sp. Reinsch, 1881, p. 27, pl. 4, figs. 1-6; pl. 5, figs. 1-5; pl. 6, figs. 1-3 ; Upper Carboniferous; Zwickau, Saxony.

SOLENOPORA Dybowski, 1877.

Solenopora spongioides Dybowski, 1877, p. 124 , pl. 2 , figs. 11a, 11.b ; Ordovician ; Herrküll, Russia.

SOLENOPORELLA Rothpletz, 1908.

Solenoporella jurassica (Brown) Rothpletz, 1908, p. 10, pl. 2, figs. 5, 6.

SOLENOSTELOPTERIS Kershaw, 1910.

Solenostelopteris japonica Kershaw, 1910 , p. 689 , pl. 58 ; petrified fern rhizome; Upper Cretaceous; Hokkaido, Japan. See also Posthumus, 1931.

SOLENOSTROBUंS Endlicher, 1847.

Solenostrobus subangulatus (Bowerbank) Endlicher, 1847, p. 272. For Cupressinites subangulatus Bowerbank, 1840, p. 60 , pl. 10 , figs. 24,25 ; Eocene ; Isle of Sheppey, England.

SOLENOULA Wood, 1861.

Solenoula psilophloeus Wood, 1861b, p. 238 , pl. 4 , fig. 3 ; stem impression, incertae sedis; Pennsylvanian; St. Clair, Schuylkill County, Pa.

SOMPHOSPONGIA Beede, 1899.

Somphospongia multiformis Beede, 1899, p. 128, pl. 38, figs. 1-10; described as a sponge but believed by later workers to be an alga (Cyanophyta); Burlingame limestone, upper Pennsylvanian; Kansas. See Johnson, J. H., 1946, p. 1104. SOPHORITES Kuntze, 1904.

Sophorites Kuntze, in Post and Kuntze, 1904, p: 524. 
SORITHAMNION Heydrich, 1900.

See Heydrich, 1900a, p. 82. A new genus erected to include species previously assigned to other genera, the flrst listed being Nullipora ramosissima Reuss, 1848 , p. 29 , pl. 3 , figs. $10,11$.

SOROOLADUS Lesquereux, 1880.

Sorocladus stellatus Lesquereux, 1880, p. 328 , pl. 48 , text fig. 8 ; fertile fern frond fragment?; Carboniferous; Arkansas.

SOROSACCUS Harris, 1935.

Sorosaccus gracilis Harris, 1935, p. 145, pls. 24, 28 ; cone, Thaumatopteris zone, Rhatic; Scoresby Sound, east Greenland.

SOROTHECA Stur, 1883.

Sorotheca orepini Stur, 1883, p. 807 ; flg. $3 a$; Upper Carboniferous; Belgium.

SPAKGANILITHES Woodward, 1879.

Sparganilithes gemmatus Woodward, 1879, p. 391 , pl. 10 , flg. 4 ; compared with infructescence of Sparganium (Sparganiaceae) ; Eocene; Sumatra.

SPARGANIOCARPUS Velenovsky and Viniklar, 1929.

Sparganiooarpus terminalis Velenovsky and Vintklar, 1929 , p. 29 , pl. 21, figs. 17-19; inflorescence, Sparganiaceae? ; Cretaceous ; Slivenec, Bohemia.

Sl'ARGANIOIDITES Robert Potonie, 1950.

Sparganioidites sp. Robert Potonie, in Potonie, Robert, Thomson, Paul W., and Thiergart, Friederich, 1950 , p. 50, pl. C, fig. 11, no description given; pollen, Typhaceae ; lower Miocene; Senftenberg, Bohemia.

SPARGANOFILIX Kuntze, 1904.

Sparganofilix Kuntze, in Post and Kuntze, 1904 , p. 525.

SPARGANUM Unger, 1856.

Sparganum maximum Unger, 1856, p. 167, pl. 8 , fig. 1 ; fibrous cortical strands; Upper Devonian; Saalfeld, Thuringia.

SPARTHOPHYOOS Massalongo, 1859.

Sparthophycos funalis Massalongo, in Massalongo and Scarabelli, 1859 , p. 92 (footnote). For Cylindrites funalis Massalongo, 1856, pls. 1, 2 ; pl. 3, flg. 1 ; Eocene; Monte Spilecco, Italy.

SPATHITES Stanton and Knowlton, 1897.

Spathites sp. Stanton and Knowlton, 1897 , p. 140 ; nom. nud. ; Laramle formation; Upper Cretaceous.

SPATHULOPTERIS Kidston, 1923.

Spathulopteris obovata (Lindley and Hutton) Kldston, 1923a, p. 173, pl. 42, figs. 1-7; pl. 44, fig. 1 ; sphenopteriduke foliage; Calciferous Sandstone sertes, Lower Carboniferous; various locallties in Midlothian, Dumfriesshire, Linlithgowshire, Scotland.
SPEGAZZINITES Fellx, 1894.

Spegazzinites cruciformis Felix, 1894a, p. 279 , pl. 19, fig. 8 ; spores, compared with Spegazzinia ornata; Pleistocene; Meck lenburg, Germany. See also Meschinelli, 1898 , p. 82.

SPEIROCARPUS Stur, 1888.

Speirocarpus bartoneci Stur, $1888 \mathrm{~b}, \mathrm{p}$ 107. Genus cited earlier in Stur, 1885, p. 97 ; nom. nud.

SPENCERITES Scott, 1897.

Spencerites insignis (Williamson) Scott, $1897 \mathrm{a}$, p. 167 ; petriffed lycopodiaceous cone ; Lower Coal Measures, Upper Carboniferous ; near Hallfax, England. For full description, see Scott, 1898a, p. 86, pls. 14,15 .

SPERMATITES Miner, 1935.

Spermatites elongatus Miner, 1935, p. 597, pl. 19, figs. 30-36, 38; Upper Cretaceous; Skansen, Disko Island, Green. land.

SPERMATOCODON Thomas, 1933.

Spermatocadon sewardi Thomas, 1933, p. 225 , pl. 24 , fig. 66 ; inflorescence of cupulate seeds; Molteno beds, Karroo system, Triassic; Upper Umkomas Val. lay, Natal.

SPERMATOSTROBUS Velenovsky and V1niklar, 1927.

Spermatostrobus suspectus Velenovsky and Viniklar, 1927, p. 30, pl. 11, figs. 7-9 cone, Coniferales; Cretaceous; Vyserovic, Bohemia.

SPERMITES Saporta, 1889

Spermites semialatus Saporta, 1889, $p$ 142 , pl, 20, figs. 27, 28; winged seed; Tertiary ; Aix, Provence, France

SPERMOLITHUS Thomas Johnson, 1917.

Spermolithus devonicus Thomas Johnson, 1917 , p. 249 , pl.. 11, figs. 4-6; pl. 12 figs. 1,2 ; isolated microsporangla and seeds, Pteridospermae?; Upper Devonian; Kiltorcan, County Kilkenny, Ireland.

SPHAOIDIUM

Error for Phacidium, in Ettingshausen. 1869, p. 74.

SPHAENOPHORA Massalongo, 1851.

- Sphaenophora crassa Massalongo, 1851, p. 95, Tertiary; Italy. See also Massalongo, 1858 b, p. 179 , pl. 3 , fig. 2 ; pl. 7 , fig. 1.

SPHAEREDA Lindley and Hutton, 1837.

Sphaereda paradoxa Lindley and Hutton, 1837 (1831-37), p. 17, pl. 159 ; Jurassic; Gristhorpe, Yorkshire, England.

SPHAERIODES Reid and Chandler, 1933.

Sphaeriodes ventricosa (Bowerbank) Reid and Chandler, 1933 , p. 331 , pl. 15, figs. 18-23; endocarp, Icaclnaceae; London Clay, Eocene; Sheppey, Kent, England. 
SPHAERIOPSIS Geyler, 1887.

Sphaeriopsis sp. Geyler, 1887 , p. 488 , pl. 32, fig. 3 ; fungus ; Eocene; Labuan, Borneo.

SPHAERITES Unger, 1850.

Sphaerites punctiformis Unger, 1850a, p. 37 ; Miocene; Parschlug, Styria. Cited as nom. nud. in Unger, 1848 , p. 53. See also Engelhardt, 1895 , p. 9. pl. 1, fig. 1. Meschinelli, 1892, p. 751, erroneously attributes this genus to Hallier.

SPHAEOCOCCIDES Schimper, 1869.

Sphaeococcides cartilagineus (Unger) Schimper, 1869 (1869-74), p. 163, pl. 4, fig. 6 ,

SPHAEROCOCCITES Sternberg, 1833.

Sphaerococcites ciliatus Sternberg, 1833 (1820-38), p. 28 , pl. 4, fig. 1 ; alga?; Jurassic ; Solenhofen, Bavaria.

SPHAEROCODIUM Rothpletz, 1890.

Sphaerocodium bornemanni Rothpletz, 1890 , p. 9 ; siphonaceous alga. See also Rothpletz, 1891, p. 299, pl. 15, figs. 2-9, $11-13$; pl. 16 , figs. $3,5,6$.

SPHAERONITES Hisinger, 1828.

Sphaeronites pomum (Wahlenberg) Hisinger, 1828, p. 240, pl. 5, figs. 2-4.

SPFAEROSPERMUM Renault, 1907.

Sphaerospermum sp. Renault, in Bertrand, C. E., 1907, p. 223.

SPHAEROSTOMA Benson, 1909.

Sphaerostoma ovale (Williamson) Benson, 1909 , p. 239 ; petrified seed, Pteridospermae; thought to be seed of Heterangium grievii; Calciferous Sandstone series, Lower Carboniferous; Pettycur, Fifeshire, Scotland. For full treatment, see Benson, 1914, p. 2, pls. 1, 2.

SPHAEROSTROBUS Harris, 1935.

Sphacrostrobus clandestinus Harris, 1935, p. 143 , pl. 29 ; isolated male cone, possibly belonging to Podozamites; Lepidopteris zone, Rhaetic; Scoresby Sound, east Greenland.

SPHAERANEMA John Smith, 1896.

Sphaeranema curta John Smith, 1896, p. 319 , pl. 7 , fig. 1 ; fungus mycellum ?, in amber: Upper Carboniferous: Annandale, near Kilmarnock, Scotland.

SPHALLOPTERIS.

See Sphalmopteris Corda, in Posthumus, 1931.

SPHALHOPTERIS Corda, 1845.

Sphalmopteris mougeotii (Brongniart) Corda, 1845, p. 76. For Anomopteris mougcotii Brongniart, 1828a-38, p. 258, pl. 80 . Brongniart originally based this species on fern follage and a stem ulthough there apparently was no evidence of organic connection; therefore Corda removed the stem to his new genus Sphalmopteris. [Eichwald, 1860, p. 92, believing that Sphalmopteris contained a typographical error, changed tt to Sphallopteris.]
SPHEGOPHYLLUM Zalessky, 1939.

Sphegophyllum striatum Zalessky, 1939b, p. 372 , fig. 54 ; leaf fragment, incertae sedis; Permian; Matreyevo, Kroutaia Katouchka, USSR.

SPHEGOPTERIS Zalessky, 1939.

Sphegopteris rugosa Zalessky, 1939b, p. 358, fig. 36 ; fernlike follage; Permian ; Matveyevo, USSR.

$S P H E N A S P I S$ Hollick and Jeffrey, 1909.

Sphenaspis statenensis Hollick and Jeffrey, 1909 , p. 51 , pls. 10,26 ; cone scales, Coniferales; Cretaceous ; Kreischerville, Staten Island, N. $\mathbf{Y}$.

SPHENASTROPHYLLITES Sterzel, 1907. Sphenastrophyllites diersburgensis SterZel, 1907, p. 694, pl. 56, flgs. 1-3; Upper Carboniferous ; Offenburg, Baden.

SPHENOBAIERA Florin, 1936.

Sphenobaiera spectabilis (Nathor st) Florin, 1936b, p. 38, pl. 5, figs. 1-4; ginkgophyte; Jurassic; Franz Joseph Land. See also Florin, 1936a, p. 108.

SPHENOCALLIPTERIS Zeiller, 1898.

Sphenocallipteris sp. Zeiller, 1898, p. 19.

SPHENOCYCLOPTERIDIUM Stockmans, 1948.

Sphenocyclopteridium belgicum Stockmans, 1948, p. 47, pl. 7, figs. 1-9a ; Upper Devonian; Belgium.

\section{SPHENOGLOSSUM Emmons, 1856.}

Sphenoglossum quadrifolium Emmons, 1856 , p. 335 , pl. 1, fig. 2 ; Triassic ; Haywood, Chatham County, N. C.

SPHENOLEPIDIUM Heer, 1881.

Sphenolepidium sternbergianum (Dunker) Heer, 1881 , p. 19 , pl. 13, figs. 1a, 2-9; pl. 14 ; twigs, follage, Coniferales ; Cretaceous; Valle de Lobos, Portugal.

SPHENOLEPIS Schenk, 1871.

Sphenolepis sternbergiana (Dunker) Schenk, 1871, p. 243, pl. 37, figs. 3, 4; p1. 38, figs. 3-13; foliage and cones, Coniferales; Wealden; Minden, Prussia, etc.

ERIINOFITGUS Ruedemann, 1012. Sphenophyous latifolius (Hall) Ruedemann, 1912, p. 74 , pl. 1 ; pl. 2, figs. 114 ; alga?; Schenectady beds, Silurian ; near Schenectady, N. $\mathbf{Y}$.

SPHENOPHYLLITES Brongniart, 1822.

Sphenophyllites emarginatus Brongniart. 1522 , p. 234 , pl. 13, flg. 8 ; sphenophyllaceous foliage; Carboniferous.

SPHENOPHYLLOSTACHYS Seward, 1896.

Sphenophyllostachys dawsonl (Williamson) Seward, 1896b, p. 436; a generic name created by seward for cones believed to have been borne by Sphenophyllum. For Volkmannia danosoni Williamson, 1871 b, p. 29 , pls. 1-3. See also Hoskins and Cross, 1943. 
SPHENOPHYLLOSTROBUS Carpentier, 1919.

Sphenophyllostrobus sp. Carpentier, 1919b, p. 247 , pl. 3 , fig. 7 ; no description; Carboniferous ; France.

SPHENOPHYLLUM Koenig, 1825.

Sphenophyllum emarginatum (Brongniart) Koenig, 1825, pl. 12, flg. 149. For Sphenophyllites emarginatus Brongniart, 1822 p. 234 , pl. 13, fig. 8 .

SPHENOPTERIDIUM Schimper, 1874.

Sphenopteridium dissectum (Goeppert) Schimper, 1874 (1869-74), p. 488, pl. 107, fig. 12 ; fernlike follage, compared with Triphyllopteris and Aneimites; Carboniferous; near Hausdorf, Silesia. For Cyclopteris dissecta Goeppert, 1852b, p. 161, pl. 14, flgs. 3, 4.

SPHENOPTERIS (Brongniart) Sternberg, 1825.

Sphenopteris elegans (Brongniart) Sternberg, $1825(1820-38)$, p. 15 . For Filicites elegans Brongnlart, 1822, pl. 2 , flg. 2 ; fernlike follage ; Carboniferous; Silesia. [When raised to generic rank by Sternberg, the name was spelled Sphaenopteris although Brongniart's usage as a subgenus was Sphenopteris, and the latter has been followed by later writers.]

SPHENOSTROBUS Levittan and Barghoorn, 1948.

Sphenostrobus thompsonii Levittan and Barghoorn, 1948, p. 353, figs. 1-12 ; petrifled strobilus of sphenopsid affinitles; Des Moines group, Pennsylvanian ; Shuler mine, Dallas County, Iowa.

SPHENOTHALLUS Hall, 1847.

Sphenothallus angustifolius Hall, 1847, p. 261 , pl. 68, fig. 1 ; alga?; Silurian ; between Canajoharie and Schoharie, N. Y.

SPHENOTHECA Kirchleimer, 1934.

Sphenotheca incurva Kircheimer, 1934b, p. 789 , fig. 19 ; fruit, Symplocaeae ; Tertiary (Braunkohle); Elfriede, near Gohra, Germany. See also Kirchbelmer, 1936, p. 71 , pl. 10 , flgs. $27 \mathrm{a}-1$.

SPHENOZAMIA (Pomel) Zwanziger, 1872.

Sphenozamia augustae Zwanziger, 1872, p. 337 ; Triassic (Keuper); Klagenfurt, Carinthia.

SPHENOZAMITES (Brongninrt) Miquel, 1851.

Sphenozamites beani (IAndley and Hutton) Miquel, $1851 \mathrm{~b}$, p. 210 . For $C y$ clopteris beani Lindley and Hutton, 1832 (1831-37), p. 127, pl. 44; cycadophyte leaf ; Jurassic ; Gristhorpe Bay, Yorkshire, England. Cited as subgenus of Otozamites in Brongniart, 1849, p. 61.

SPHERITES Dijkstra, 1949.

Spherites spinosus Dijkstra, 1949, p. 27, pl. 2, fig. 12 ; Hystrichosphaertdae; Senonian; South Limburg, Netherlands.
SPHINXIA Reid and Chandler, 1933.

Sphinxia ovalis Reid and Chandler, 1933, p. 397 , pl. 20 , flgs. 12-23 ; fruit, sterculiaceae; London Clay, Eocene ; Sheppey, Kent, England.

SPHYGMIUM Debey, 1881.

Sphygmium paradoxum Debey, in Mourlon, 1881 , p. 133 ; nom. nud.

SPHYROPTERIS Stur, 1883.

Sphyropteris crepini Stur, 1883, p. 656, fig. 6c; fertile fern pinnule; Upper Carboniferous ; Belgium.

SPILOSPHADRITES Massalongo, 1857.

Spilosphaerites maculans Massalongo, in Massalongo and Scarabelli, 1857, p. 8. See also Massalongo and Scarabellt, 1859 , pl. 1 , figs. $2,3,13,14$; fungus ; Miocene ; Sinigaglia, Italy.

SPIRALIA Toula, 1900.

Spiralia neudorfensis Toula, 1900, p. 11 ; nom. nud.

SPIRANGIUM Schimper, 1870.

Spirangium carbonaria Schimper, 1870 (1869-74), p. 516. Not a plant; for recent discussion of this and related fossils, see Brown, R. W., 1950.

SPIRAXIS Newberry, 1885.

Spiraxis major Newberry, 1885, p. 33 . Not a plant ; for recent discussion of this and related fossils, see Brown, R. W., 1950.

SPIREMATOSPERMUM Chandler, 1925.

Spirematospermum $w$ et $z l e r i$ (Heer) Chandler, 1925, p. 17, pl. 1, figs. 8a-c; fruit, Zingiberaceae; upper Focene; Hordle, Hampshire, England.

SPIROCHORDA Schimper, 1879.

Spirochorda Schimper, in Schimper and Schenk, 1879 (1879-90), p. 51. No species designated but intended for Dictyota spiralia Ludwig; alga ; Chordophyceac.

SPIROPHY'TON Hall, 1863.

Spirophyton typum Hall, 1863, p. 80, pl. 2, figs. 1-3; Devonian; Otsego, N. Y.

SPIROPTERIS Schiniper, 1869.

Spiropteris miltoni (Brongniart) Schimper, 1874 (1869-74), p. 19, pl. 49, tig. 4. See also Schimper, 1869 , p. 688-690. Figure 4 is designated as type, because it conforms most closely with generally accepted usage.

SPIRORAMMA Massalongo, 1859.

Spiroramma spiralis Massalongo, in Massalongo and Scarabelli, 1859, p. 92. For Münsteria spiratis Massalongo, $1857 \mathrm{a}$, p. 778 ; nom. nud.

SPIROXYLON Hartig, 1848.

Spiroxylon ratzeburgii Hartig, 1848a, p. 172 ; wood ; Tertiary; north Germany.

SPIROXYLON Walton, 1925.

Spiroxylon africanum Walton, 1925b, p. 18 , pl. 2 , fig. 12 ; pl. 3 , figs. 15,16 ; coniferous wood; horizon unknown; Harmsfontein, South Africa. 
SPONDIAECARPON Langeron, 1899.

Spondiaecarpon dubium Langeron, 1899, p. 454, pl. 3, figs. 2 , 4 ; fruit, compared with Spondias (Anacardiaceae); Eocene; Sezanne, France. Menzel, 1913, p. 6, gives spelling as Spondiaecarpum.

\section{SPONDIAECARPUM.}

See Spondiaecarpon.

SPONDICARYA Reid and Chandler, 1933. Spondicarya trilocularis Reld and Chandler, 1933 , p. 306 , pl. 13, figs. 35,36 ; endocarp, Anacardiaceae; London Clay, Eocene; Minster, Kent, England.

SPONDIOCARPUS Warburg, 1897.

Spondiocarpus verbeekii Warburg, 1897, p. 229, pl. 4, figs. 6-15; Pliocene; Bangka Island, Malay [Indonesia].

SPONDYLPHYTON Schultes and Dorf, 1938. Spondylophyton hyenioides Schultes and Dorf, 1938, p. 21, figs. 1, 2 ; sphenopsid ; Lower Devonian; Beartooth Butte, Wyo.

SPONDYLOSTROBUS Müller, 1870.

Spondylostrobus smythii Müller, in Müller and Smyth, 1870, p. 610 ; cone fragment, Coniferales; Haddon, near Smythesdale, Victoria. See also Müller, 1871 (1871$82)$, p. 48, pl. 1.

SPONGELIOMORPHA. Saporta, 1887.

Spongeliomorpha iberica Saporta, 1887, p. 299 , pl. 6, figs. 2,3 ; incertae sedis ; Miocene; Alcoy, France.

SPONGILLOPSIS H. B. Geinitz, 1862.

Spongillopsis dyatica $\mathrm{H}$. B. Gelnitz, 1862, p. 132 , pl. 24 , flgs. 2,3 ; incertae sedis, probably not a plant; Permian ; Saxony and Bohemia.

SPONGIOSTRQMA Gurich, 1906.

Spongiostroma maeandrinum Gurich, 1906, p. 41, pl. 7 , fig. 1 ; alga ?; placed in Rivulariaceae in Hirmer, 1927, p. 36 ; Carboniferous?; Namur, Belgium.

aporangIostrobUS Bode, 1928.

Sporangiostrobus orzeschensis Bode, 1928, p. 247, pl. 22, fig. 2 ; Upper Carboniferous ; Upper Silesia.

SPORANGITES Dawson, 1863.

Sporangites papillata Dawson, $1863 \mathrm{~b}$, p. 454 ; generic name proposed "for spores or spore cases of Lepidodendron, Calamites and similar plants, not referred to the species to which they belong" ; Carboniferous ; Nova Scotia. See also Dawson, 1866 , p. 165 , pl. 12 , fig. 80 .

SPORITES Henry Potonie, emended by Schopf, 1938.

Sporites plicatus Schopf, 1938a, p. 51, pl. 7, figs. 7-9.

SPORLEDERIA Stiehler, 1860.

Sporlederia carbonaria (Schimper) Stiehler, 1860 , p. 8, pl. 1. [For Palaeoxyris, not a plant, see Brown, R. W., 1950.]
SPOROCARPON Williamson, 1878.

Sporocarpon cellulosum Williamson, 1878 , p. 347 (footnote); pl. 23, figs. 75, 75a, $75 \mathrm{~b}$; problematical reproductive organs. Several specimens are described and figured, and, judging from a later contribution (Williamson, 1880, p. 507), the figures cited above are intended to illustrate $S$. cellulosum. Another species, S. ornatus (Williamson, 1879, p. 511), is reported by Seward, 1917, p. 309 as being Physostoma elegans; Upper Carboniferous.

SPOROCYSTIS Lesquereux, 1880.

Sporocystis. planus Lesquereux, 1880, p. 458 , pl. 69 , fig. 15 ; spores ? Carboniferous; Pittston, $\mathrm{Pa}$.

SPOROGONITES Halle, 1916.

Sporogonites exuberans Halle, 1916b, p. 79 ; compared with sporogonium of moss ; Devonlan ; Röragen, Norway. See also Halle, 1916, 1936.

SPOROLITHES Eichwald, 1853.

Sporolithes cordatus Eichwald, in Mercklin, 1853, p. 304 ; nom. nud.

SPORONITES Robert Potonie, 1931.

Sporonites neddeni Robert Potonie, 1931b, p. 332. See also Potonie, Robert, 1931, 1932 ; Potonie, Robert, and Gelletich, J., 1933.

SPOROPOLLENITES Thiergart, 1949 ?

Sporopollenites rostratus Thiergart, 1949, p. 7, pl. 1, flg. 7; spore; Triassic (Keuper).

SPOROTRICHITES Goeppert and Berendt, 1845.

Sporotrichites heterospermus Goeppert and Berendt, in Berendt, 1845, p. 116, pl. 6, figs. 42-46; fungus on insect, in amber ; Miocene; Prussia. Meschinelli, 1892 , p. 790 , and 1898 , p. 79 , erroneously attributes this genus to Link.

SQUAMA Renault, 1885.

Squama taxinoides Renault, 1885, p. 82, p1. 5, figs. 11, 12 ; petrified microsporophylls, Coniferales?; Carboniferous; Grand Croix, near Rive-de-Gier, France. This seems to be the first use of this name in a generic sense; see discussion under Squamae.

SQUAMAT.

"Squamae cycadearum," Nathorst, 1876, pl. 12, figs. 14-17; apparently cycadophyte bracts; Rhaetic; Palyo, Sweden. This is evidently not intended as a generic name. The term squamae (Latin, scales) has been used by other authors, for example, Feistmantel, 1881, p. 119 , as a general term to describe gymnosperm scales.

SQUAMOPSIS Fucini, 1938.

Palaeontgraphia Italia, 1038, app. 2, p. 182 (not seen, cited in Gothan, 1942 b, p. 152). 
SQUAMULARIA Rothpletz, 1896 ?

Squamularia cicatricosa (Heer) Rothpletz, 1896, p. 893, pl. 22, fig. 5 .

STACHANNULARIA C. E. Welss, 1876.

Stachannularia tuberculata (Sternberg) C. E. Welss, 1876 , p. 17, pl. 1, flgs. $2-4$; pl. 2, figs. $1-3,5$; pl. 3 , figs. 3-10, 12 ; articulate cone; pl. 2 , fig. 1 shows attachment to calamitean? stem ; Carboniferous.

STACHYOARPITES Ogura, 1932.

Stachycarpites projectus Ogura, 1932b, p. 458 , pl. 23, flgs. $8-10$; petrifled seed, Coniferales ; Cretaceous; Hokkatdo, Japan.

STACHYCARPUS Meunier, 1898.

Stachycarpus eocenica Meunier, 1898, 17, fig. p. 17 ; Infructescence, Phytolacaceae?; Eocene; Beuvry, Bethune, France.

STAOHYOPITYS Schenk, 1867.

Stachyopitys preslii Schenk, 1867, p. 185, pl. 44, flgs. 9-12; microsporangiate cone?; Rhaetic ; Strullendorf, near Bamberg, Bavaria.

STACHYOTAXUS Nathorst, 1886.

Stachyotaxus septentrionalis (Agardh) Nathorst, 1886 c, p. 98 , pl. 22 , figs. $20-$ $23,33,34$; pl. 23 , fig. 6 ; pl. 25 , flg. 9 ; twigs, follage, Coniferales; Rhaetic ; Bjuf, Sweden.

STACHYPTERIS Pomel, 1849.

Stachypteris spicans Pomel, 1849, p. 336 ; fern ; Jurassic ; St. Mihiel, France. Apparently first illustrated species is $\boldsymbol{S}$. litophylla Saporta, 1872 (1872-73), p. 387, pl. 50, figs. 1-5. See also Thomas, 1912.

STACHYURA Velenovsky and Viniklar, 1927 ?

Stachyura spicata Velenovsky and Viniklar, 1927 , p. 41 , pl. 9 , fig. 2 ; pl. 12, flgs. $3-6$; pl. 14, fig. 8 ; Cretaceous ; Slivenec, Bohemia.

STANGERITES.

See Strangerites.

STAPHIDIOPHORA Harris, 1935.

Staphidiophora secunda Harris, 1935, p. 114, pl. 8; seed-bearing fructiflcation, ginkgophyte ?; Lepidopteris zone, Rhaetic ; Scoresby Sound, east Greenland.

STAPHIDOIDES Perkins, 1906.

Staphidoides venosus (Lesquereux) Perkins, 1906 , p. 223 , pl. 58, flg. 1 ; fruit ; Tertiary ; Brandon, Vt.

STAPHYLOPTERIS Presl, 1838.

Staphylopteris polybotrya (Brongniart) Presl, 1838, in Sternberg, 1820-38, p. 174. For Filicites polybotrya Brongníart, $1828-38$ p. 390 , pl. 137 , fig. 6 ; fernlike follage; Tertlary; Armissan, near Narbon, France.
STATZIA, Weyland, 1938.

Statzia divaricata (Wessel and Weber) Weyland, 1938 a, p. 101 ; pl. 12 ; figs. 1-13; inflorescence with male flowers, family uncertain; Tertiary; Rott, Siebengebirge, Germany.

STAUBIA Felix, 1884.

Staubia eriodendroides Fellx, 1884, p. 29, pl. 2, figs. 2, 4-6, 8 ; wood, dicotyledon ; Mlocene; Medgyazo, Hungary.

STAUROPHYTON Meunier, 1891.

Staurophyton bagnolensis Meunler, 1891, p. 134, fig. 1 ; incertae sedis.

STAUROPTERIS BInney, 1872.

Stauropteris oldhamia Binney, 1872b, p. 69 ; very briefly cited. Anatomy of frond described and fllustrated by Williamson, 1874. More fully treated in Scott, 1905b, p. 114, figs. 1, 2 ; petrified coenopterid fern ; Upper Carboniferous ; England. See also Posthumus, 1931.

STEFFENSIA Goeppert, 1836.

Steffensia davallioides Goeppert, 1836, p. 269 , pl. 11, figs. 3,4 ; fertlle fern follage ; Carboniferous; Waldenburg, Sllesia.

STEGITES Meschinelli, 1892.

Stegites poacitum (Alexander Braun) Meschinelli, in Saccardo, 1892, p. 779. See also Meschinelli, 1898, p. 55, pl. 16, flg. 20; Discomycete; Tertiary ; Oenigen, Switzerland.

STEINHAUERA Presl, 1838.

Steinhauera subglobosa Presl, in Sternberg, 1838 (1820-38), p. 202, pl. 49, flg. 4 ; pl. 57, figs. 1-4; cone, Coniferales?; Miocene ; Alsattel, Bohemia.

STELEOPTERIS Goeppert, 1865.

Steleopteris angiopteriodes Geoppert, 1865a, p 267, pl. 61, figs. 7, 8 ; Permian. See also Posthumus, 1931.

S'TELOXYLON Solms-Laubauch, 1897.

Steloxylon ludwigii (Geoppert and Leuckart) Solms-Laubach, 1897, p. 198 . For Medullosa ludwigii Geoppert and Leuckart, in Geoppert and Stenzel, 1881, p. 126, pl. 17.

STEMMATOPTERIS Corda, 1867.

Siemmatopteris peltigera (Brongniart) Corda, 1867 , p. 76. For Sigillaria peltigera Brongniart, 1828-38, pl. 138. See also Posthumus, 1931.

STENIXYS Harris, 1938.

Stenixys cosmarioides Harris, 1938, p. 15, pl. 5, fig. 4; desmld?; Naiadita Bed, upper Rhaetic; Bristol, England. Generic name cited in Kellaway, 1937. p. 226 ; nom. nud.

STENOCARPITES Brongniart, 1861.

Stenocarpites anisolobus Brongniart, 1861, p. 1237; leaf, Proteaceae; Tertlary ; near Koumi, Greece. 
STENOGRAMMITES Kretschetovitsch, 1936. Stenogrammites pseudocostata Kretschetovitsch, 1936, p. 261, figs. 1-6; red alga ; Jurassic ; Gor'kia district, Russia.

STENOMISCHUS Harris, 1935.

Stenomischus athrous Harris, 1935, p. 144, pl. 24 ; male cone possibly related to Cunninghamia; Thaumatopteris zone, Rhaetic; Scoresby Sound, east Greenland.

STENOMYELON Kidston, 1909.

Stenomyelon tuedianum Kidston, in Scott, 1909 , p. 498 ; stem, Pteridospermae ; Calciferous Sandstone Series, Lower Carboniferous; Norham Bridge, Berwickshire, Scotland. For detalled account, see Kidston and GwynneVaughan, 1912.

STENONIA Endlicher, 1847.

Stenonia ungeri Endlicher, 1847, p. 290. See also Geoppert, 1850 , p. 228 , pl. 37 , figs. 1-3.

STENOPHRAGMIUM Reinsch, 1881.

Stenophragmium sp. Reinsch, 1881, p. 104, pl. 46, figs. 1-8; Upper Carboniferous; Newcastle, England.

STENOPHYCUS Fenton, 1943.

Stenophycus teichertii Fenton, 1943, p. 112, fig. 1 ; alga ; Upper Goniatite Beds ; Devonian; 2 miles west of Mt. Pierre, Kimberley Division, western Australia.

STENOPHYLLUM Zalessky, 1937.

Stenophyllum uninervium Zalessky, 1937c, p. 139 , fig. 23 ; leaf fragment, incertae sedis ; Permian ; Russia.

STENOPORIDIUM Yabe and Toyamra, 1928. Stenoporidium chaetetiformis Yabe and Toyama, 1928, p. 150, pl. 22, figs. 2-4; alga ?; Hiraiga sandstone, Lower Cretaceous; Rikuchū province, Japan.

STENOPTERIS Saporta, 1872.

Stenopteris desmomera Saporta, 1872-73, p. 292 , pl. 32 , figs. 1,2 ; pl. 33, fig. 1 ; foliage, Pteridospermae?; Jurassic (Kimmeridglen) ; Morestel, near Lyon, France.

STENORHACHIS Saporta, 1879.

Stenorhachis ponseleti (Nathorgt) Saporta, 1879, p. 193, 6g. 22; cone of Podozamites?; Lower Jurassic. Various spellings employed by later writers as Stenorachis, Stenorrachis.

STENZELIA Goeppert, 1864.

Stenzclia elegans (Cotta) Goeppert, 1864, p. 218 , pls. 38,39 ; medullosan petiole; Permian: Chemnitz, Germany. ses also Seward, 1917, p. 106.

STEPHANIDA Unger, 1856.

Stephanida gracilis Unger, 1856, p. 170 pl. 8, fig. 11; Devonian; Saalfeld, Thuringia. Earlier citation: Unger, 1854 b, p. 599 ; nom. nud. See also Posthumus, 1931.
STEPHANOFILIX Kuntze, 1904.

Stephanofilix Kuntze, in Post and Kuntze, 1904, p. 536.

STEPHANOPHYLLUM Florin, 1936.

Stephanophyllum solmsi (Seward) Florin, 1936 b, p. 82 , pl. 11 , figs. $7-10$; pls. $12-$ 16 ; structurally preserved ginkgophyte foliage; Jurassic; Franz Joseph Land.

STEPHANOSPERMUM Brongniart, 1874.

Stephanospermum achenioides Brongniart, 1874 , p. 260 , pl. 23 , figs. $13-15$; petrifled s e e $d$ Carboniferous; . St.- Stienne, France.

STEPHANOSTEMON Caspary, 1881.

Stenhanostemon brachyandra Caspary, 1881 , p. 29 ; flower, Saxifragaceae ; Miocene; Samland, Baltic Prussia. First illustrated species: A. helmi Conwentz, 1886 , p. 89 , pl. 9 , figs. 4-7.

STEPHANOXYLON Felix, 1882.

Stephanoxylon dubium Felix, 1882a, p. 43 ; wood, dicotyledon.

ऽTERCULIOCARPUS E. W. Berry, 1916.

Sterculiocarpus eocenicus E. W. Berry, 1916b, p. 288, pl. 74, flgs. 1-3; large capsular fruit, Sterculiaceae; Wilcox group, Eocene; Frierson Mill, De Soto Parish, La.

STERCULIPHYLLUM Nathorst, 1886.

Sterculiphyllum limbatum (Velenovsky) Nathorst, 1886a, p. 52. For Sterculia limbata Velenovsky, 1883, p. 21, pl. 5, figs. $2-5$; pl. 6 , figs. 1 .

STERCULITES Dawson, 1888.

Sterculites vetustula Dawson, 1888, p. 193, leaf, Malvaceae?; Kootenai formation, Lower Cretaceous; Rocky Mts. For Sterculia vetustula Dawson, 1885, p. 10 . pl. 3, fig. 2.

STEREOPTERIS Scott and Jeffrey, 1914.

Stereopteris annularis Scott and Jeffrey, 1914 , p. 341 , pl. 32 , fig. 42 ; pl. 33, flgs. 45-48 petiole, Zygopterideae; Mississippian; Kentucky.

STERNBERGIA Artis, 1825.

Sternbergia transversa Artis, 1825, p. 8, pl. 8 ; stem cast; Upper Carboniferous ; England.

STERZELIA Gothan, 1928.

Sterzelia nindeli Gothan, 1928a, p. 4, pl. 3 ; compared with Bothrodendron; Carbonlferous; Flöha, Saxony.

STICHOPORELLA PIa, 1927.

Stichoporella oylinarica (Lignier) Pla, in Hirmer, 1927 , p. 69 ; alga, Dasycladacene : Middle Jurassic (Dogger) ; France. For Gondolina cylinarica Lignier, 1913, p. 70, fig. 1.

STICHOPTERIS H. B. Geinitz, 1858.

Stichopteris ottonis (Gutbier) H. B. Geinitz, 1858, p. 14. For Pecopteris ottonis Gutbier, in Geinitz H. B., and Gutbier, $1849 \cdot(1848-49)$, p. 15, pl. 9, fig. 1. 
STICHOSTROMIUM Reinsch, 1881.

Stichostromium sp. Reinsch, 1881, p. 56, pl. 12a, flgs. 5-8; Upper Carboniferous; Zwickau, Saxony.

STICHUS Etheridge, 1904.

Stichus mermisoides Etheridge, 1904, p. 255 , pls. 30,31 ; fungus? ; Cretaceous ; Australia.

STICTODICTYTES Reinsch, 1881.

Stictodictytes sp. Reinsch, 1881, p. 74, pl. 18, flgs. 1-5; pl. 18b, figs. 1-8; Upper Carboniferous ; Zwickau, Saxony.

STICTOPLASMIUM Reinsch, 1881.

Stictoplasmium sp. Reinsch, 1881, p. 43, pl. 9, figs. 1-7 ; Upper Carboniferous ; Zwickau, Saxony.

STIGMARIA Brongniart, 1822.

Stigmaria ficoides (Sternberg) Brongniart, 1822 , p. 228 , pl. 12 , fig. 7 ; lycopod "rootstock" cast; Carboniferous.

STIGMARIOCARPUM Achepohl, 1883.

Stigmariocarpum sp. Achepohl, 1883, p. 50 , pl. 13 ; incertae sedis; Upper Carbonfferous ; Westphalia.

STIGIIARIOIDES Lesquereux, 1870.

Stigmarioides truncatus Lesquereux, 1870 , p. 453 , pl. 29 , fig. 4 ; said to differ from Stigmaria in lack of regularity of appendage arrangement; Pennsylvanian; Mazon Creek, Ill.

STIGMARIOPSIS Grand'Eury, 1877.

Stigmariopsis inaequalis Grand'Eury, 1877 , p. 173 ; compared with Stigmaria; Carboniferous; France. First species illustrated: Stigmariopsis eveni (Lesquereux) Grand'Eury, 1890, p. 243, pl. 13, figs. 7,13 .

STIGMARITES Fliche, 1903.

Stigmarites nicklesi Fliche, 1903a, p. 908 ; rhizome?; Triassic ; Meurthe-et-Moselle; France. See also Fliche, 1905a p. 138 , pl. 13, fig. 2.

STIGMATIOPHYLLUM Guembel, 1859.

Stigmatiophyllum lepidophylloides Guembel, 1859a, p. 106, pl. 8 , fig. 13 ; Permian ; Erbendorf, Bavaria.

STIGMATOCANNA Goeppert, 1852.

Stigmatocanna volkmanniana Goeppert, $1852 a$, p. 126 , pls. 8,9 ; stem casts; Landeshut, Silesia.

STIGMATODENDRON Eichwald, 1860.

Stigmatodendron ledebourii Eichwald, 1860 , p. 208 , pl. 18 , fig. 5 ; pl. 19 , figs. 7 , 8; Carboniferous; Artinsk, Russia. First citation: Mercklin, 1856, p. 81; nom. nud.

STIGAOPHYTON Kräusel and Weyland, 1933.

Stigmophyton sturi Kräusel and Weyland, 1933 , p. 40 , pl. 3, fig. 6 ; vascular plant, incertae sedis; Middle Devonian; Bohemia. First citation: Kräusel and Weyland, 1932, p. 189 (nom. nud.?).
STILBITES Pia, 1927.

Stilbites succini (Caspary) Pla, in Hlrmer, 1927, p. 124, fig. 117 ; fungus, Stllbaceae; Eocene; Samland, Baltic Prussia. For Stilbum succini Caspary, 1887 , p. 7 .

STIPITOPTERIS Grand'Eury, 1877.

Stipitopteris aequalis Grand'Eury, 1877, p. 81 , pl. 13 , fig. 2 ; rachis of an arborescent fern ; Carboniferous ; France. See also Posthumus, 1931.

STIPTOSTROMIUM Reinsch, 1881.

Stiptostromium sp. Reinsch; 1881, p. 57, pl. 14b, figs. 1-5 ; Upper Carboniferous ; Mittelbexback, Bavaria.

STIZOCARYA Reld and Chandler, 1933.

Stizocarya communis Reld and Chandler, 1933 , p. 336 , pl. 15 , figs. $35-42$; endocarp, Icacinaceae; London Clay, Eocene ; Sheppey, Kent, England.

STOLIDERMIUM Reinsch, 1884.

Stolldermium sp. Reinsch, 1884, p. 34, pls. 84-85D; Upper Carbonlferous ; M.etschowk, Russia.

STOLIPLASMIUM Relnsch, 1881.

Stoliplasmium sp. Reinsch, 1881, p. 42, pl. 10b, figs. 2-6; pl. 10c, flg. 1 ; pl. 29a, fig. 5 ; Upper Carboniferous; Zwickau, Saxony.

STOLISPHAERITES Reinsch, 1881.

Stolisphaerites sp. ReInsch, 1881, p. 30, pl. 7c, figs. 13-17 ; Upper Carboniferous ; England.

STOLITES Reinsch, 1881.

Stolites sp. Reinsch, 1881, p. 119, pl. 52a, figs. 4-7; Upper Carboniferous; Zwickau, Saxony.

STOLLEYA Schubert, 1907.

Stolleya sp. Schubert, 1907, p. 212.

STCLLEYELLA Schubert, 1908.

Stolleyella velebitans Schubert, 1908, p. 383 , pl. 16, figs. 8, 10, 12 ; Upper Carboniferous; Dalmatia, Yugoslavia.

STORGAARDIA Harris, 1935.

Siorgaardia spectabilis Harris, 1935, p. 58, pls. $11,12,16$; coniferous follage; Rhatic; Scoresby Sound, east Greenland.

STORMBERGIA. Seward, 1911.

Stormbergia gardneri Seward, 1911a, p. 299, pl. 14; Cladophlebis type follage; Stormberg series; Cyphergat, Cape Colony.

STRAELINIPTERIS Stockmans, 1936

Straelenipteris eocenica Stockmans, 1936 , p. 15, pl. 1 ; petrifled fern rhizome; Eocene; Brussels.

STRANGERITES Bornemann, 1856.

Strangerites vittatus (Brongniart) Bornemann, 1856, p. 60. For Taeniopteris vittata Brongniart, 1828-38, p. 263, pl. 82 , flgs. $1-4$. 
STRATIOTITES Heer, 1855.

Siratiotites najadum Heer, 1855, p. 106, pl. 46, flgs. 9-11; flower, Hydrocharideae; Tertiary ; Oeningen, Switzerland.

STREPHOPTERIS Presl, 1838.

Strephopteris ambigua Presl, in Sternberg, 1838 (1820-38), p. 120, pl. 50, figs. 2a, $2 \mathrm{~b}$; fernlike foliage; Carboniferous; near Plass, Bohemia.

STREPTOTRICHITES Meschinelli, 1892. Streptotrichites spiralis (Berkeley) Meschinelli, in Saccardo, 1892, p. 790. See also Meschinelli, 1898, p. 81, pl. 21, flg. 11 ; pl. 22, fig. 7.

STRIAESTROBUS Velenovsky and Viniklar, 1926.

Striaestrobus bohemicus Velenovsky and Viniklar, 1926, p. 43, pl. 1, fig. 4 ; seedbearing cone, compared with Picea; Cretaceous; Berovice, Bohemia.

STRICKLANDIA Buckman, 1845.

Stricklandia acuminata Buckman, in Murchison, 1845, p. 94, pl. 2, fig. 2 ; leaf ; Stonesfield slate; Sevenhampton Common, England.

STR.OBILANTHUS Velenovsky and Vinlklar, 1929.

Strobilanthus cretaceous Velenovsky and Viniklar, 1929, p. 13, pl. 21, flgs. 14-16 ; inflorescence, related to Myrica; Cretaceous; Slivenec, Bohemia.

STROBILITES Lindley and Hutton, 1833.

Strobilites elongata Lindley and Hutton, $1833(1831-37)$, p. 23 , pl. 89 ; cone, Coniferales?; Lower Jurassic (Blue Lias) ; Lyme, Dorsetshire, England.

STROBILOSTROBUS Bayer, 1914.

Archiv Pŕŕod. Výzkum Cech, svazek 15, p. 29 (not seen, cited in Gothan, 1942b, p. 153).

STROBILUS Hildreth, 1837.

Strobilus caryophyllus Hildreth, 1837, p. 32 , fig. 8 ; incertae sedis ; New York.

STROMATOCERIUM Seely, 1904.

Stromatocerium rugosum Seely, 1904, p. 144 , pl. 70 ; coral or alga ?; Black River limestone, Ordovician; Isle La Motte, ve.

STRZELECKIA Johnston, 1896

strzeteclica gangamopteroides Johnston, 1896, p. 58, figs. 5-7 ; leaves, compared with Gangamopteris but lacks anastomosed veins; upper Mresozoic : Mt. Nicholas, Tasmania.

STURIA Nrmejc, 1934.

Sturia amoena (Stur) Nemejc, 1934, p. 2, figs. 1-6 [unnumbered plate] ; sphenopterid follage bearing sporangia; Carboniferous; central Bohemia.
STURIELLA C. E. Weiss, 1885.

Sturiella intermedia (Renault) C. E. Weiss, 1885 a, p. 492 . For Pecopteris intermedia Renault, 1883, p. 122, pl. 22, figs. 8-11.

STURIELLA Kräusel, 1948.

s'turiella langeri Kräusel, 1948, p. 141, figs. 1-7 ; inflorescence, Bennettitales ; Triassic ; Lunz, Austria.

STYCHITES Reinsch, 1881.

istychites sp. Reinsch, 1881, p. 66, pl. 15c, figs. 1-6; Upper Triassic (Keuper); Mittelbronn, Württemberg.

STYLOCALAMITES C. E. Weiss, 1884.

Stylocalamites arborescens (Sternberg) C. E. Welss, 1884 a, p. 206, pl. 2, fig. 2 ; pl. 3 , fig. 1 ; pl. 8, fig. 3 ; Upper Carboniferous; Swina, Bohemia. For Volkmannia arborescens Sternberg, 1833 (1820-38), p. 52.

STYLOCODIUM Derville, 1931.

Stylocodium rhopaloides Derville, 1931, p. 106, pl. 14, figs. $48-51$; pl. 15, figs. 52-56 ; alga, Codiaceae ; Carboniferous ; Bas-Boulonnais, France.

STYLOPHYCUS J. H. Johnson, 1940.

Stylophycus carbonarius J. H. Johnson, 1940 , p. 587 , pl. 4 , fig. 2 ; calcareous alga, probably Cyanophyceae; Weber formation, Pennsylvanian; P a r k County, Colo.

SUBLEPIDODENDRON Hirmer, 1927 ?

Sublepidodendron mirabile (Nathorst) Hirmer, 1927, p. 204.

SUBLEPIDOPHLOIOS Sterzel, 1907.

Sublepidophloios hagenbachensis Sterzel, 1907 , p. 728 , pl. 61 , figs. $1-3$; pl. 62 , figs. 1-4; arborescent lycopod stem impression; Upper Carboniferous ; Hagenbach, Baden.

SUBTETRAPEDIA Renault, 1899.

Subtetrapedia russiana Renault, 1899, p. 1036 ; alga?; Carboniferous ; Alexandrewski, Kourakino, Russia.

SUEVIOXYLON Kräusel, 1928.

Suevioxylon zonatum Krüusel, 1928, p. 253, figs. 5-8; wood, dicotyledon; Jurassic; Heubach, Germany.

SULOOCARPOLITHES Kuntze, 1904.

Sulcocarpolithes Kuntze, in Post and

Kuntze, 1904, p. 543.

SULCODIPTERTS Kuntze, 1904.

Sulcodipteris Kuntze, in Post and Kuntze, 1904, p. 543.

SUMATROXYLON Ber'ger, 1923.

Sumatroxylon mollii (Kräusel) Berger, 1923 , p. 145 ; wood, Burseraceae ; Tertiary; Sumatra. For Anacardioxylon mollii Kräusel, 1922, p. 252, pl. 2, fig. 5 ; pl. 5, figs. 4,5 ; pl. 5, figs. 2-4. 
SUPAIA David White, 1929.

Supaia thinnfeldioides David White, 1929, p. 62 , pl. 14 ; pl. 15 , figs. $1-3$; pl. 16 , figs. 2,3 ; frond, compared with $D a$ naeopsis and Protoblechnum; lower part of Hermit shale, Permian; Hermit basin, 7.5 miles west of Grand Canyon station, Arizona.

SUTCLIFFIA Scott, 1906.

Sutcliffa insignis Scott, 1906b, p. 62, pls. 7-10; petrified stem, Medulloseae; Lower Coal Measures, Upper Carboniferous; Shore, Iittleborough, Lancashire, England.

SUVUNDUKIA Zalessky, 1948.

Suvundukia aciculata Zalessky, 1948, p. 42, 7 figs.

SVALBARDIA Foeg, 1942.

Svalbardia polymorpha Hoeg, 1942, p. 70 , pls. 20-31; psilophyte; Deronian; Spitzbergen.

SWEDENBORGIA Nathorst, 1876.

Swedenborgia cryptomerides Nathorst, 1876 , p. 66 , pl. 16 , figs. $6-12$; cones, Coniferales?; Lower Jurassic (Hörssandstein, Iias) ; Palsjo, Sweden.

SIVIETENIOXYLON Hermann Hoffmann, 1883.

Swietenioxylon sternbergense Hermann Hoffmann, 1883 , p. 105 ; Tertiary ; Mecklenburg, Germany.

SYCIDIUM Sandberger, 1849.

Sycidium reticulatum Sandberger, 1849 , p. 672 , pl. 8b, flgs. 1a-d ; Devonian; Eifel, Rhenish Prussia.

SYCOPHYLLUM Schulze, 1887.

Sycophyllum dentatum Schulze, 1887, p. 464; Upper Cretaceous (Senonian); Heimberg, Sivitzerland.

SYLVELLA Zalessky, 1937.

Sylvella alata Zalessky, $1937 \mathrm{~b}$, p. 86 , figs. 53-55; winged seed ; Permian; Matveyevo, USSR.

SYLVIA Zalessky, 1937.

Sylvia striata Zalessky, 1937b, p. 66, fig. 28 ; fernlike foliage; Permian; Matveyevo, USSR.

SYLVOPTERIS Zalessky, 1937.

Sylvopteris conspicua Zalessky, $1937 \mathrm{~b}$, p. 52, flg. 17, fernlike foliage; Permian; bank of river Sylva near river Tchekarda, Russia.

SYMPHONIOXYLON Chiarugi, 1933.

Symphonioxylon stefaninii Chiarugi, 1933, p. 118 , p1. 15, figs. 1, 2 ; Cretaceous ; Scec-Gure, southern Italian East Africa (Soma!jland).

SYMPHOROOARPOPHYLLUM Daw $\mathrm{DO}$, 1886.

Symphorocarpophyllum albertum Dawson, 1886 , p. 30 , pl, 2, fig. 17 ; leaf, dicotyledon ; upper Laramie, Upper Cretaceous ; Great Valley, Canada.
SYMPHYOPLASMIUM Reinsch, 1881.

Symphyoplasmium sp. Reinsch, 1881, p. 44 , pl. 7 , figs. 1,2 ; pl. 31 a, figs. $1-7$; Algonkian, pre-Cambrian; Thiersheim, Bavaria, etc.

SYMPLOCOIDES Chandler, 1926.

Symplocoides glandulosa Chandler, 1926, p. 41 , pl. 7 , fig. 5 ; endocarp, Symplocaceae?; upper Eocene; Hordle, Hampshire, England.

SYNACARPITES.

Mistake for Syncarpites, in Pimenova, 1929, p. 187.

SYNCARDIA Unger, 1856.

Syncardia pusilla Unger, 1856, p. 171, pl. 8, fig. 16 ; petiole of Cladoxylon?; Upper Devonian; Saalfeld, Thuringia. See also Posthumus, 1931.

SYNCARPITES Schmalhausen, 1883.

Syncarpites ovalis Schmalhausen, 1883, p. 321 , pl. 38 , figs. $16-20$; fruit, compared with Syncarpia; Oligocene; Magelno in Wolhynien, Russia.

SYNIA Zalessky, 1934.

Synia perelegan8 Zalessky, 1934b, p. 252, fig. 21 ; fernlike foliage; Permian; Pechora basin, Russia.

SYNIOPTERIS Zalessky, 1929.

Syniopteris nesterenkoi Zalessky, 1929a, p. 729 , figs. $1-3$; foliage, compared with Callipteris; Upper Permian; Pechora basin, Russia.

SYRINGODENDRON Sternberg, 1820.

Syringodendron organum Sternberg, 1820 $(1820-38)$, p. 24 , pl. 13 , fig. 1 ; decorticated sigillarian stem.

SYRINGOMORPHA Nathorst, 1886.

Syringomorpha nilssoni (Torell) Nathorst, 1886 b, p. 47 , fig. 22 .

SYRINGOXYLON Dawson, 1862.

Syringoxylon mirabile Dawson, 1862, p. 305 , pl. 12 , figs. 1-5; wood, incertae sedis ; Hamilton group, Devonian ; Eighteen-mile Creek, Lake Erie.

\section{$\mathrm{T}$}

\section{TABERNAEMONTANOPHYLLUM Geyler,} 1887.

Tabernaemontanophyllum sp. Geyler, 1887 a, p. 496 , pl. 33 , flg. 8 ; leaf fragment, Apocynaceae; Eocene; Labuan, Borneo.

TAENIDIUM Heer, 1877.

Taeniditm serpentinum Heer, 1877a,. 117 , pl. 45, flgs. 9, 10 ; plant?; Lower Jurassic (Lias) ; Ganei, Switzerland.

TAENIOCRADA David White, 1902.

Taeniocrada lesquereuxi David White, 1902 , p. 603. For Hallserites dechenianus Goeppert as described and 11lustrated by Penhallow, 1893b, p. 109, pl. 10, flg. 6 ; Catskill beds, Devonian: Factoryville, $\mathrm{Pa}$. 
DIMORPHOSIPHON Hoeg, 1927.

Dimorphosiphon rectangulare Hoeg, 1927, p. 4 , pls. $1-3$; petrified alga, Codiaceae ; Middle Ordovician; south of Bergviken. Island of Helgöen, Norway.

DIMORPHOSTROMA Reis, 1921.

Dimorphostroma varians Reis, 1921, p. 313 ; Tertiary ; Rhenish Bavaria. See also Reis, 1923, pl. 4, fig. 12.

DINEURON Renault, 1896.

Dineuron pteroides Renault, 1896a, p. 22, fig. 19 ; coenopterid fern petiole; Esnost, France. See also Posthumus, 1931.

DIOONIPITES Wodehouse, 1933.

Dioonipites sp. Wodehouse, 1933, p. 484, figs. 4, 5; cycad pollen; Parachute Creek member, Green River formation. Eocene; Colorado and Utah.

DIOONITES Miquel, 1851.

Designation of a type species is problematical. Dioonites feneonis (Brongniart) Miquel, 1851b. For Zamia feneonis Brongniart, 1828b, p. 99, illustrated in Miller, 1857 , p. 69 , fig. 36 . Other species described by Emmons, 1856, 1857 ; and Schenk, 1871.

DIOONITOCARPIDIUM Lilienstern, 1928.

Dioonitocarpidium pennaeforme (Schenk) Lilienstern, 1928, p. 103, pls. 5, 6 ; fig. 1 ; cycadophyte megasporophyll ; Upper Triassic (Keuper); Estenfeld, Bavaria. For Dioonites pennaeformis Schenk, $1864 \mathrm{~b}$.

DIOONOPTERIS Goeppert, 1864.

Dioonopteris permica Goeppert, 1864, p. 126, pl. 13, figs. 3,4 ; leaf fragment; Permian; Braunau, Bohemia.

DIOSCORITES Saporta, 1863.

Dioscorites resurgens Saporta, 1863 , p. 42 , pl. 4 , fig. 5 ; leaf, Dioscoreae ; Tertiary ; France.

DIOSCOROIDES Fritel, 1904.

Dioscoroides lyelli (Watelet) Fritel, 1904, p. 233, figs. 1, 2 ; Eocene ; Belleu, Paris, France.

DIOSPYROPHYLLUM Velenovsky, 1889.

Diospyrophyllum provectum Velenovsky, 1889 , p. 50. For Diospyros provecta Velenovsky, 1884 , p. 49 , pl. 8, fig. 1-5, 10 ; Upper Cretaceous; Melnik, near Liebenau, Bohemia.

DIPHYLLITES Heer, 1883.

Diphyllites membranaceus Heer, 1883, p. 45 , pl. 60 , fig. $4 a$; leaf fragment, Leguminosae; Upper Cretaceous; Patoot, Greenland.

DIPLASIOPHYLLUM Frenguelli, 1943.

Diplasiophyllum hughesi (Feistmantel) Frenguelli, 1943a, p. 299, figs. 23, 24 ; sterile fern? frond; Rhaetic to Keuper; China, India, South Africa.
DIPLASTEROTHECA. Hirmer, 1927.

Diplasterotheca exigua (Renault) Hirmer, 1927, p. 585 ; fertile pecopterid foliage; Permian ; Autun, France. For Pecopteris exigua Renault, 1883, p. 115, pl. 19, figs. 13-18. Hirmer refers to $\mathrm{Re}-$ nault in Zeiller, 1890, p. 70-72.

DIPLAZITES Goeppert, 1836.

Diplazites emarginatus Goeppert, 1836, $\mathrm{p}$. 274, p1. 16, fig. 12 ; fern pinnules ; Carboniferous.

DIPLOCYMA Steinmann and Elberskirch, 1929.

Diplocyma elberskirchianum Steinmann and Elberskirch, 1929 , p. C57, fig. 21; Lower Devonian; Wahnbachtals near Sieburg, Germany.

DIPLODENDRON Eichwald, 1846.

Diplodendron hastatum Eichwald, 1846, p. 456. See also Eichwald, 1860, p. 225, pl. 17 , figs. 3,4 ; fern or cycadophyte stem ?; upper Paleozoic; mines of Kloutschewsk, Orenbourg, Russia.

DIPLODICTYCM Braun, 1843.

Diplodictyum obtusilobum Braun, in Münster, 1843 (1839-43), p. 14, pl. 13, flgs. 11, 12 ; Jurassic; Bayreuth, $\mathrm{Ba}-$ varla.

DIPLOLABIS Renault, 1896.

Diplolabis forensis Renault, 1896a, p. 14, figs. 6-10 ; coenopterid fern.

DIPLOMASTIXIA Kirchheimer, 1934.

Diplomastixia carinat Kirchheimer, 1934 b, p. 789 , fig. 17 ; fruit, Cornaceae ; Tertiary (Braunkohle); Germany.

DIPLOPHACELUS Corda, 1845.

Diplophacelus arboreus Corda, 1845, p. 87, pl. 55 ; fern petiole ; Upper Carboniferous ; Radnitz, Bohemia.

DIPLOPHRAGMIUM Reinsch, 1881.

Diplophragmium sp. Reinsch, 1881, p. 102 ; pl. 41 , fig. 6 ; pl. 42 , figs. $1-5$; pl. 43 , figs. 1-5; Pennsylvanian; Swickau, Saxony.

DIPLOPHYLLUM Velenovsky and Viniklar, 1929.

Diplophyllum cretaceum Velenovsky and Viniklar, 1929 , p. 25 , pl. 17 , fig. 10 ; pl. 19 , fig. 10 ; pl. 20 , fig. 5 ; leaf, Leguminosae?; Cretaceous; Cernikov, Bohemia.

DIPLOPORA Schafhautl, 1863.

Diplopora annulata Schafhautl, 1863, p. 324 , pl. 65e, fig. 6 ; alga, Dasycladaceae.

DIPLOPTERIDIUM Walton, 1931.

Diplopteridium teilianum (Kidston) Walton, 1931 , p. 349 , pl. 23 ; sphenopterid foliage, probably bore Telangium-like fructifications; Lower Carboniferous ; Gwaenysgor, Flintshire, England. 
TCHERNOVIA Zalessky, 1929.

Tchernovia synensis Zalessky, 1929a, p. 189 , pl. 16, figs. 4,5 ; incertae sedis; Carboniferous; Donets, Russia.

TCHIRKOVIELLA Zalessky, 1930.

Acad. sci. U. R. S. S. Bull., 1930, p. 924 (not seen, cited in Gothan, 1942b, p. 154).

T'ECT'OCARYA Kirchleivier, 1934.

Tectocarya lusatica Kirchhelmer, 1934a, p. 773, fig. 15, fruit, Cornaceae; Tertiary (Braunkohle); Germany. See also Kirchheimer, $1936 \mathrm{a}$, p. 62, pl. 7, figs. 22a-n.

TEICHOSPERMA Renner, 1907.

T'eichosperma spadiciflorum. Renner, 1907, p. 219, figs. 1-6; Lower Oligocene; Egypt.

TEILHARDIA Seward, 1913

T'eilhardia valdensis Seward, 1913, p. 96, pl. 11, figs. 7a-9b; fern foliage; Fairlight Clay, Wealden; Ecclesbourne, near Hastings, England.

TELANGIUM Benson, 1904.

Telangium scotti Benson, 1904, p. 162, pl. 11; microsporaugiate organ, Pteridospermae ; Gannister beds, Upper Carboniferous ; Dulesgate and Hough Hill, Englaud.

TELEPHRAGMOXYLON 'IOTHE, 1921.

Telephragmoxylon brachyphylloides 'Torrey, 1921 , p. 74 , pl. 3 ; wood, Coniferales ; Lower Cretaceous ; Texas.

TELEUTOSPORA Renault, $18: 4$.

Teleutospora milloti Renault, 1894, p. 171 ; Carboniferous (Culm); Combres near Rigny, France. Sec also Renault, 1896, p. 427 , fig. 80 .

TELEUTOSPORITES Meschínell, 1898.

T'eleutosporites milloti (Renault) Meschinelli, 1898, p. 13, pl. 5, fig. 13 ; fungus, in Lepidodendron megaspore; CarbonIferous; Loire, France.

TEMPSKYA Corda, 1845.

Tempskya pulchra Corda, 1845, p. 81, pl. 58 , figs. 1-5; fern trunk composed of numierous siphonostelic stems; Upper Cretaceous; Germany. See also Andrews and Kern, 1947; Read, 1939 ; Read and Brown, 1937; Posthumus, 1931.

TENUICUTITES C. E. Bertrand, 1898.

I'enuicutites chytridiaeformis C. E. Bertrand, 1898, p. 188, pl. 10, fig. 118; pl. 11, fig. 140 ; Chytrideaceae ; Upper Carboniferous.

TERMINALIOPHYLLUM Geyler, 1887.

Terminaliophyllum sp. Geyler, 1887, p. 502, pl. 34, fig. 1 ; Eocene; Labuan, Borneo.
TERMINALIOXYLON Georg Schonfeld, 1947.

Terminalioxylon naranjo Georg Schonfeld, 1947 , p. 36 , pl. 5, figs. 1-3; wood, Combretaceae; Tertiary ; Colombia.

TERMINALIPHYLLUM Velenovsky, 1889.

Terminaliphyllum rectinerve Velenovsky, 1889 , p. 54. For Terminalia rectinervis Velenovsky, 1884, p. 5, pl. 5, figs. 1, 2 ; Combretaceae; Upper Cretaceous; Kaunic, Bohemia.

TERNITHRIX Reis, 1921.

Ternithrio compressa Reis, 1821, p. 313. See also Reis, 1923, p. 105, pl. 4, figs. 1, 2 ; Miocene ; Bavaria.

TERNSTROEMIOXYLON Eric Schonfeld, 1930.

Ternstroemioxylon kräuseli Eric Schonfeld, 1930, p. 119, figs. 10-18; wood, dicotyledon; Mioceue; Vogelsberg, Germany.

TERNSTROEMIPHYLLUMI Velenovsky, 1889.

Ternstroemiphyllum crassipcs Velenovsky, 1889 , p. 54 . For Ternatroemia crassipes Velenovsky, 1884, p. 7, pl. 3, flgs. 3, 4 ; Upper Cretaceous; Vyserovic, Bohemia.

TEIX STROEMITES E. W. Berry, 1916.

Ternstroemites coligniticus E. W. Berry, 1916b, p. 294, pl. 76, figs. 1,2 ; pl. 78, fig. 5 ; leaf, Ternstroemlaceae ; Lagrange formation, lower Bocene; Puryear, Henry County, Tenn.

TERNSTROMIAOINIUM Felix, 1894.

Tcrnstromiacinium euryoides Felix, 1894a p. $99, \mathrm{pl}, 10$, fig. 4 ; wood, Ternstromiaceae.; Eocene; Apscheron, Transcaucasta. See also Schonfeld, Eric, 1930, p. 119.

TEKQUEMELLA (Munier-Cbalmas) Morellet and Morellet, 1913.

Terquemella parisiensis Munfer-Chalmas, in Morellet and Morellet, 1913, p. 25, pl. 3, flg. 11 ; Eocene ; Orme, France. Clted in Munier-Chalmas, 1877 , p. 817 ; nom. nud.

T'ESCHTA Reld and Reld, 1915.

Teschia crassicarpa Reid and Reld, 1915 , p. 108 , pl. 10 , figs. $22 a, 22 b$; frult, Anacardiaceae; Plocene (Reuverian); Reuver, Swalmen, Netherlands.

TESSELLARIA Eichwald, 1860

I'cssellaria antiqua Eichwald, 1860, p. 221, pl. 17, fig. 5 ; cycadophyte? stem; Permian?; Bjelebel, Orenbourg, Russia. Cited as Tessellaria Schimper and Mougeot, in Mercklin, 1856 , p. 81 ; nom. nud.

T'ET'ONOPHYOUS Fenton and Fenton, 1939. I'etonophycus blackwelderii Fenton and Fenton, 1939 , p. 99 , pl. 4, figs. 1, 2 calcareous alga; Housetop Mtn., Grand Teton Park, Wyo. 
TETRACENTRONITES Mathiesen, 1932

Tetracentronites hartzi Mathiesen, 1932, p. 5, figs. 1-3; wood, compared with Tetracentron; early Tertiary; Cape Dalton, east Greenland.

TETRAGONIS Bichwald, 1842.

Tetragonis murchisoni Eichwald, 1842, p. 81 , pl. 3 , flg. 18 ; Upper Silurian; Russia.

TET'RAMERIDIUM Gothan, 1913.

Tetrameridium caducum Gothan, 1913a, p. 132, pl. 27, figs. 1, 2; sphenopterid foliage; Upper Carboniferous; Upper Silesia.

TETRAN'THROIDEA Langeron, 1899.

Tetrantheroidea polita Langeron, 1899, p 445 , pl. 4, fig. 3 ; leaf, compared with T'etranthera; Eocene ; Sézanne, France.

TETRAPLOPORELLA Steinmann, 1903.

Tetraploporella remesi Steinmann, 1903, p. 45, fig. 11; alga, Dasycladaceae ; Cretaceous ; Stramberg, Moravia.

TETRAPTILON Frenguelli, 1950.

Tetraptilon heteromerum Frenguelli, 1950, p. 15, figs. 1, 2 ; fern? frond; Upper Jurassic; between Villa Union and Guandacal, La Rioja, Argentina.

TETRASPHENOPHYLLUM Lotsy, 1909.

Tetrasphenophyllum majus (Kidston) Lotsy, 1909, p. 526, fig. 350.

TET'RASPORITES Fliche, 1886.

Tetrasporites alsaticus Fliche, 1886, p. 350 ; Oligocene ; near Mulhouse, AlsaceLorraine.

TETRASTICHIA Gordon, 1938.

Tetrastichia bupatides Gordon, 1938, p. 362 , pls. 1-6 ; pteridosperm stem ; Calciferous Sandstone series, Lower Carboniferous ; Oxroad Bay, east of Tantallon Castle, East Lothian, Scotland.

TEUTLOPORELLA PIa, 1912.

Teutloporella herculea (Stoppani) Pia, 1912 , p. 37 , pl. 2 , fig. 27 ; pl. 3 , figs. 1 . 2 ; alga, Siphoneae Verticillatae; Triassic ; Rohrback, Austria.

THALASSOCHARIS Debey, 1853.

Thalassocharis bosqueti Debey, in Miquel, 1853, p. 51, pl. 6, fig. 1. Cited in Debey, 1848 , p. 119 ; nom. nud.

THALICTROIDES Mantell, 1844.

Thalictroides parisensis Mantell, 1844, p. 190 , fig. 1 ; seed?; illustration only; Tertiary ; Paris.

T'HALLITES Walton, 1925.

Thallites erectus (Leckenby) Walton, $1925 a$, p. 564 ; for thalloid liverworts of doubtful familial affinities. For Marchantitcs erectus reckenby, 1864. p. 74, pl, 1, figs. $2 a, 2 b$.

THALLOMIA Heard and Jones, 1931.

Thallomia llandyfriensis Heard and Jones, 1931 , p. 557 , pls. $43-46$; a liverwortlike plant but with spirally thickened elements ; Lower Downtonian, Devonian; Carmarthenshire. A striking example of the liberality that is occasionally taken with paleobotanical taxonomy. The fossil was originally described as Eohepatica dyfriensis (British Assoc. Adv. Scl. Rept., 1930 , p. 330-331 [1931]), a name which conveyed to some botanists that it was a liverwort rather than liverwortlike, and as "This name has not prover to be a very happy on's," it was changed to T'hallomia.

THAMNITES Reinscl, 1881.

Thamnites sv. Reiusch, 1881. p. 60, pl. 13a, fig. 4; Upper Carboniferous; Zwickau, Saxony.

THAMNOCLADUS Dr.vid White. 1902.

Thamnocladus clarkei David White, 1902, p. 596 , pl. 3 , fig. 1 ; pl. 4, figs. 1,2 ; alga ; Chemung f(irmation, Upper Devonian; East Windsor, N. Y.

T'HAMNOPTERIS Brongniart, 1849.

Thamnopteris schlechtendali (Eichwald) Brongniart, 1849, p. 85. For Anomopteris schlechtendali Eichwald, 1842, p. 180 , pl. 4, figs. 3-5; petrified stem, Osmundaceae; Permian; Kamskowothin, Russia. See also Kidston and Gwynne-Vaughan, 1909 ; Posthumus, 1931.

THAUMASIODENDRON Bureau, 1905.

Thaumasiodendron andegavense Bureau, 1905 , p. 157, figs. p. 150, 152, 154, 156.

THAUMATOPORELLA Pia, 1927.

Thaumatoporella parvovesiculifera (Raineri) Pia, in Hirmer, 1927, p. 69, alga, Dasycladaceae ; U p p er Cretaceous ; Libia. For Gyroporella parvovesiculifera Raineri, 1922, p. 83, pl. 3, igs. 17, 18.

THAUMATOPTERIS Goeppert, 1841.

Thaumatopteris münsteri Goeppert, 1841a, p. 33, pls. 1-3 ; fertile frond, Dipteridaceae ; Rhatic ; Bayreuth, Bavaria.

THECOPHYLLUM Massalong0, 1858

Thecophyllum fabellatum Massalongo, 1858 b, p. 815 ; nom. nud.

THECOPTERIS Miner, 1935.

Thecopteris major Miner, 1935, p. 591, pl. 18, figs. 11-15 ; fern sporangia?; Upper Cretaceous; Skansen, east coast Disco Island, Greenland.

THEOBALDIA Heer, 1877.

Theovalaia raetica $\mathrm{Bcer}, 1877 \mathrm{n}, \mathrm{p} .114, \mathrm{pl}$ 44, figs. 1-3, 15b; alga ?; Lower Jurassic (Lias) : Ganei, Switzerland.

THESIANTHIUM Conwentz, 1886.

Thesianthium inclusum Conwentz, 1886, p. 132, pl. 13, flgs. 1-5 ; llower, in amber, Santalaceae; early Tertiary; West Prussia. 
THINNFELDIA Ettingshausen, 1852.

Thinnfeldia rhomboidalis Ettingshausen, 1852a, p. 2, pl. 1, figs. 4-7 ; pteridosperm? foliage; Lower Jurassic (Llas) ; Stelerdorf, Hungary.

THOMASIODENDRON.

Error for Thaumasiodendron, in Bureau and Bureau, 1908, p. 653.

THOREITES Massalongo, 1850.

Thoreites brongniartii Massalongo, 1850, p. 21 ; alga ; Eocene ; Monte Bolca, Italy.

THOUINOPSIS MacGinitie, 1941.

Thouinopsis myricaefolia MacGinitie, 1941 , p. 144 , pl. 36 , figs. 2 , 4 ; pl. 37 , figs. $6-9$; pl. 45 , fig. 9 ; leaves and winged fruits, Sapindaceae; Chalk Bluffs flora, middle Eocene; near You Bet, Nevada County, Calif.

THUIOXYLON.

See Thuyoxylum.

THUITES Sternberg, 1825.

Thuites alienus Sternberg, 1825 (182038), Tentamen, p. xxxvili, pl. 45, fig. 1 ; coniferous foliage twigs; Cretaceous; Smetschna, Bohemia.

THUJOXYLON.

See Thuyoxylum.

THUOXYLON.

See Thuyoxylum.

THURSOPHYTON Nathorst, 1915.

Thursophyton milleri Nathorst, 1915, p. 17 , pl. 5, figs. $3-9$; pl. 6 , flgs. $1-5$; pl. 7 , fig. 1 ; lycopod stem impression; Midde Devonian; Roeragen, Norway.

THCYOXYLON.

See Thuyoxylum.

THUYOXYLUM Unger, 1842.

Thuyoxylum juniperinum Unger, 1842 (1841-47), p. 31 . See also Unger, 1854, p. 172 , pl. 1, figs. 1-3. Varlous later spellings as : Thuoxylon (Unger, 1854); T'huioxylon (Unger, 1852) ; Thuoxylum (Unger, 1854) ; Thuyoxylon (Roemer, 1852) ; Thujoxylon (Hartig, 1848).

THYLAX Renault, 1896.

Thylax brittannicus Renault, 1896a, p. 549, fig. 144, alga, in boghead coal; Carboniferous ; Autun, France.

THYLLOXYLON Gothan, 1910.

Thylloxylon irregulare Gothan, 1910, p. 34 , pl. 6, figs. $2-8$; coniferous wood; Upper Jurassic; Green Harbour, Spitzbergen.

THYRSOPORELLA Guembel, 1871.

Thyrsoporella cancellata Guembel, 1871, p. 266 , pl. Di, figs. $14 \mathrm{a}, 14 \mathrm{~b}$; Miocene; Parnes, Greece.

THYSANOSPERMA Zalessky, 1937.

Thysanosperma ovatum Zalessky, 1937, p. 87, fig. 57, winged seed; Permian; Matveyevo, USSR.
THYSANOTESTA Nathorst, 1914.

Thysanotesta 8agittula Nathorst, 1914, p. 33, pl. 15, figs. 69, 70 ; seed; Paleo\%olc ; Spitzbergen.

TIETEA Solms-Laubach, 1913.

Tietea singularis Solms-Laubach, 1913, p. 673 , pls. 6,7 ; petrified fern stem ; near São Paulo, Brazil. See also Posthumus, 1931.

T'IGILLI'ES Rouault, 1850.

Tigillites dufrenoyi Rouault, 1850 , p. 740 ; plant?; Silurian; Gahard, Brittany, France. See also Lebesconte, 1883, p. 68 , pl, 20, figs. 21-22.

TILIAEPHYLLUAI Newberry, 1895.

Tiliaephyllum dubium Newberry, 1895, p. 109, pl. 15, fig. 5 ; leaf, Tiliacene ; Amboy clays, Cretaceous; New Jersey.

TILIAEPOLLENITES Robert Potonie, 1934. Tiliaepollenites instructus Robert Potonie, in Potonie, Robert, and Venitz, H., 1934 , p. 37 , pl. 4, figs. $1.09-110$; pollen, Tiliaceae; Miocene; Oberlausit», Germany.

TILOXYLON Hartig, 1848.

A new generic name proposed for Peuce lindleyana Witham, 1833, p. 70 , pl. 9 , figs. 1-5. See Hartig, 1.848b, p. 137.

T'INGIA Halle, 1925.

Tingia carbonica (Schenk) Halle, 1925, p. 5, pl. 1, figs. 1-4; compared with Noeggerathia; Permian; China.

TINGIOSTACHYA Kon'no, 1929.

Tingiostachya tetralocularis Kon'no, 1929, p. 145 , pl. 23 , fig. 5 ; pl. 24 , figs. 4,5 ; pl. 27, flgs. 1-5; cone of Tingia; Jido and Lower Kobosan series, Permian-Triasic; northern Korea.

TINOMISCOIDEA Reid and Chandler, 1933.

Tinomiscoidea scaphiformis Reld and Chandler, 1933 , p. 162, pl. 4, figs. 1-4; fruit, Menispermaceae; London Clay, Eocene; Sheppey, Kent, England.

TINPAHARIA K. Jacob, 1943.

Tinpaharia sinuosa K. Jacob, in Sahni, Birbal, and Sitholey, R. V., 1943, p. 175, fig. 8 ; Jurassic ; Tinpahar, India.

TITANOPHYLLUM Renault, 189.0.

Titanophyllum grand'euryi Renault, in Renault and Zeiller, 1890, p. 623, pl. 69, figs. 1-14; leaves, probably Cordaitales; Carboniferous ; Commentry, France.

TITHYMALITES Presl, 1838.

Tithymalites biformis Presl, in Sternberg, 1838 (1820-38), p. 205, pl. 53 figs. 1-6; cordaitean pith cast.

TMEMATOSTROBUS Harris, 1935.

Tmematostrobus eremus Harris, 1935, p. 119 , pls. 23,28 ; cone, incertae sedis; Lepidopteris zone, Rhaetic; Scoresby Sound, east Greenland. 
TOBLERIA Jongmans and Gothan, 1925. T'obleria biscuspis Jongmans and Gothan, 1925 , p. 294 , pl. 2, figs. 8,9 ; seeds?; Upper Carboniferous; Soengel Garing and Soengi Menkarang, Sumatra.

TODEOPSIS Renault, 1896.

Todeopsis primaeva Renault, 1896a, p. 21, fig. 18 ; sporangia, compared with Todea, Osmundaceae; Lower Carboniferous (Culm) ; Esnost, France.

TODITES Seward, 1900.

Todites williamsoni (Brongniart) Seward, 1900 , p. 87 , pl. 14 , figs. $2,5,7$; pl. 15 , figs. $1-3$; pl. 21 , fig. 6 ; follage, compared with Todea, Osmundaceae ; Jurassic.

TOMIPHYTON Zalessky, 1937.

Palaeophytographica, p. 27: Moskvau, Akad. Nauk SSSR (not seen, cited in Gothan, 1942b, p. 156).

TOMISTACHYS Zalessky, 1934.

Tomistachys thyrsiculus Zalessky, 1934c, p. 772, fig. 37 ; fructification, incertae sedis; Permian; Ivanovka, Kuznets, Russia.

TORELLIA Heer, 1870.

Torellia rigida Heer, 1870 , p. 44 , pl. 6, figs. 3-12; pl. 16, fig. 1b ; leaf, Taxaceae; Miocene; Cape Staratschin, Spitzbergen. See also Florin, 1936a.

TORREYITES Seward, 1919.

Torreyites carolianus (Berry) Seward, 1919 , p. 420 ; coniferous foliage ; middle Cretaceous; North Carolina. For Tumion carolianum Berry, 1908, p. 383, figs. 1-3.

TORULITES Pia, 1927.

Tomulites conventzi (Fellx) Pia, in Hirmer, 1927, p. 124, fig. 116; fungus, Dematiaceae, Fungi Imperfecti; Upper Cretaceous.

TRACHEOTHECA F. W. Oliver, 1904.

Tracheotheca sp. F. W. Oliver, 1904, p. 395 (footnote); sporangium ; Upper Carboniferous?; Grand Croix, France. Described but not named in Oliver, 1902, D. 60-67.

TRACHYPHYTON Gothan, 1928.

Trachyphyton neglegibile Gothan, $1928 \mathrm{~b}$, p. 296 , pl. 14 , figs. 3,4 ; stem cast ; Carboniferous; Peru.

TRAMETITES Meschinelli, 1892.

T'rametites pini (Brotero) Meschinelli, in Saccardo, 1892, p. 747. See also Mesch1nelli, 1898, p. 6, pl. 4, fig. 5 ; fungus in pine? wood; Upper Cretaceous ; Ryedal, Sweden.

TRAPOPHYLTUM Massalongo, 1858.

Trapophylum europaeum Massalongo, 1858b, p. 768 ; Tertiary ; Italy.
TRAQUAIRIA (Carruthers) Rina Scott, 1911.

Traquairia carruthersii Rina Scott, 1911, p. 463 , pl. 39 , figs. $5-7$; pl. 40 , fig. 14 ; spores or radiolarians?; Lower Coal Measures, Upper Carboniferous ; Lancashire and Yorkshire, England. The generic name proposed by Carruthers, 1873, p. 126. See also Williamson, 1880 , p. 511.

TREAATOCARYON Mueller, 1871.

Trematocaryon mclellani Mueller, 1871 (1871-82), p. 48, pl. 3 ; Pliocene ; Haddọn Goldfield, Nintingbool, Australia.

TREMATOSPHAERITES Meschinelli, 1892.

Trematosphaerites lignitum (Heer) Meschinelli, in Saccardo, 1892, p. 751. See also Meschinelli, 1898, p. 17, pl. 9, figs. 24-26; fungus, in Sequoia couttsiae; Bovey Tracey, Devon, England.

TREMATOSPHAERITES Gruess, 1924.

Trematosphaerites intercellularis Gruess, 1924, p. 77 , pl. 6, figs. 17-19 ; fungus, Devonian; Magdalena Bay, Spitzbergen.

TREMATOXYLON Hartig, 1848.

Trematoxylon leunisii Hartig, 1848c. p. 187 ; contferous wood ; Tertiary (Braunkohle) ; Germany.

TREVISANIA Zigno, 1856.

Trevisania furcellata Zigno, 1856a, (185668 ), p. 23 , pl. 1 , fig. 4 ; incertae sedis ; Middle Jurassic (Lower Oolite); Val d'Assa near Rotzo, Italy.

TRIANTHERA Conventz, 1886.

Trianthera eusideroxyloides Conwentz, 1886 , p. 50 , pl. 5 , figs. $1-5$; flower, in amber, Lauraceae ; early Tertiary ; West Prussia.

TRICALTCITES Newberry, 1895.

Tricalycites papyraceus Newberry, 1895, p. 132 , pl. 46, figs. $30-38$; incertae sedis; Cretaceous (Amboy clay) ; Woodbridge, N. J.

TRICARPEILITES Bowerbank, 1840.

Tricarpellites communis Bowerbank, 1840, p. 79. pl. 11, figs. 25-31; London Clay, Locene; Sheppey, Kent, England.

TRICHIOBLASTES Reinsch, 1881.

Trichioblastes op. Reinsch, 1881, p. 37, pl. 8, figs. 3, 6-8; Middle Triassic (Muschelkalk); Rotheriburg, Franconia.

TRICHOBLASTIUAT Reinsch, 1881.

Trichoblastium sp. Reinsch, 1881, p. 107, pl. $46 \mathrm{a}$, figs. 1-9 ; Upper Carboniferous; Zwickau, Saxony.

TRICHODES Reinsch, 1881

Trichodes sp. Reinsch, 1881, p. 88, pl. 28, figs. 1-5: pl. 28a. figs. 1-5; Upper Carboniferous ; Zwickau, Saxony.

TRTCHOTDNS Harkness, 1855.

Trichoides amoiguus Harkness, 1850, p. 474 ; alga ; Silurian; Scotland. 
TRICHOMANIDES Tenison-Woods, 1884.

Trichomanides laxum 'ienison-Woods, 1884 , p. 95 , pl. 10 , fig. 2 ; "this fossil cannot be distinguished from Trichomanes"; age uncertain; Ipswich, New South Wales.

TRICHOMANITES Goeppert, 1836.

Trichomanites myriophyllum Geoppert, $1836, p .263$; fern of supposed hymenophyllaceous affinities. See also Brongniart, 1828-38, pl. 55.

TRICHOPELTINITES Cookson, 1947.

I'richopeltinites pulcher Cookson, $1947 \mathrm{~b}$, p. 211, pl. 14 , figs. 22,23 ; Trichopeltaceae; Oligocene-Miocene; Yallourn and Hazelwood, Victoria.

TKICHOPHRAGMIUM Reinsch, 1881.

Trichophragmium sp. Reinsch, 1881, p. 1.05, pl. 44, fig. 206 ; Upper Carboniferous; Zwickau, Saxony.

IRICHOPHYOUS Miller and Dyer, 1878.

Trichophyous lanosus Miller and Dyer, 1878 , p. 25 , pl. 1 , figs. 3,4 ; plant?; Upper Ordovician; Warren County, Ohio.

T'RICHOPHYLLUM.

Trichophyllum heteromorpha. Mistake? for Trichopitys heteromorpha Saporta, in Grand-Eury, 1877, p. 274.

TRIOHOPITYS Saporta, 1875.

Trichopitys heteromorpha Saporta, 1875b, p. 1020 ; foliage, Contferales; Permlan; Lodeve, France. See also Renault, 1885, p. 64, pl. 3 , fig. 2 .

T'RICHOPLASMIUM Reinsch, 1881.

T'richoplasmium sp. Reinsch, 1881, p. 26, pl. 10, figs. 1-4; pl. 10a, fig. 5; Upper Carboniferous ; Zwickau, Saxony.

TRICHOPTERIS Hall, 1845.

Trichopteris flamentosa Hall, in Fremont, 1845 , p. 306 , pl. 2 , fig. 6 ; fragment of fern frond; probably from Frontier formation, Upper Cretaceous ; Cumberland, Wyo.

TRYCHOSPORITES Felix, 1894.

Trichosporites conventzi Felix, 1894a, p. 273; conidia, compared with Trichosporium; Upper Cretaceous; Ryedal, Sweden. This genus erroneously attributed to Saccardo in Meschinelli, 1898, p. 80 , pl. 22 , fig. 5. See also Stopes, 1913, p. 270 , fig. 25.

TRICOOOITES Rode, 1933.

T'ricoocites trigonum Rode, 1933, p. 172, figs. 1-3 ; petrified fruit, probably Palmaceae; Intertrappan beds, Tertiary ; Mahgaon Kalan, Chhindwāra district, Central Provinces, India. See also Sahnl and Rode, 1937, p. 167.

TRIOOILOCARYON Mueller, 1878.

I'ricoilooaryon barnardi Mueller, 1878 (1871-82), p. 35, pl. 14, Pliocene; Gulgong, Australia.
TRICOLPITES Erdtman, 1948.

Tricolpites troeds8onii Erdtman, 1948, p. 267, figs. 5-10; pollen, dicotyledon ; Lower Jurassic (Liassic); Palsjo, Scanla, Sweden.

TRIGONOCARPOLITHUS Arnold, 1948.

Trigonocarpolithus typicus Arnold, 1948, p. 139, figs. 2, 13-16; seed cuticle, Trigonocarpales; Saginaw formation, lower Pennsylvanian; Big Chlef No. 8 mine, St. Charles; Saginaw County, Mich.

TRIGONOCARPON.

See Trigonocarpus.

TRIGONOOARPUM.

See Trigonocarpus.

TRIGONOCARPUS Brongniart, 1828.

Trigonocarpus parkinsoni Brongnlart, $1828 \mathrm{~b}$, p. 137 ; Brongniart refers to Parkinson, 1804, pl. 7, flgs. 6-8. Apparently first described and fllustrated in full in Geinitz, H. B., 1855, p. 43, pl. 22, figs. $17-20$; see also Scott and Maslen, 1907. Name originally given as Trigonocarpon by Brongniart although he adopted Trigonocarpus in 1881 , p. 39 , and this usage has been followed by most later writers, as Seward, 1917 ; Scott, 1923 ; Arnold, 1947.

TRILETES Reinsch, 1881.

Triletes reinschi (Ibrahim) Schopf, 1936b, 173, figs. 1, 2 ; Pennsylvanian.

TRILOBIUM Saporta, 1861.

Trilobium ungeri Saporta, in Heer, 1861, p. 148; flower calyx, Anacardiaceae; Eocene; Provence, France. See also Saporta, 1862, p. 279, pl. 13, flg. 6.

TRIMATOPTERIS Corda, 1845.

Trimatopteris speciosa Corda, 1845, p. 106 ; cited as synonym for Psaronius speciosus Corda, 1845, p. 106, pl. 44, figs. 1-4.

TRINOCLADUS Raineri, 1922.

Trinocladus tripolitanus Raineri, 1922, p. 79, pl. 3, flgs. 15, 16; siphonaceous alga ; Cretaceous (Cenomanian); Uadi Msaaba, Libia.

TRIOOLEPIS Zeiller, 1903.

Trioolepis lecleret Zeiller, 1903, p. 208, pl. 50 , fig. 15 ; cone, some resemblance to Picea; Rhaetic; Tonkin. See also Seward, 1919, p. 424.

TRIORITES Cookson, 1950.

Triorites magnificus Cookson, 1950, p. 175, pl. 3, figs. 32-35; pollen, Proteaceae? ; Oligocene-Miocene; Moorlands, South Australia.

TRIPHYLLOPTERIS Schimper, 1869.

Triphyllopteris collombiana Schimper, 1869 (1869-74), p. 479 , pl. 107, flg. 13 ; sphenopteridlike follage. 
TRIPLICARPUS Velenovsky and Viniklar, 1926.

Triplicarpus purkynei Velenovsky and Viniklar, 1926, p. 52, pl. 2, fig. 5 ; fruit, family uncertain; Cretaceous; Vyserovic, Bohemia.

TRIPLOPORELLA Steinmann, 1880.

Triploporella fraasi Steinmann, 1880 , p. 136, pl. 5, flgs. 1-8; siphonaceous alga ; Cretaceous,

TRIPLOSPORITES Unger, 1850.

Triplosporites brownii Unger, 1850a, p. 270 ; lycopod cone?; Carboniferous. See also Brown, Robert, 1851 , p. 473 , pls. 23, 24.

TRIPTEROOARPUS Grand'Eury, 1877.

Tripterocarpus sp. Grand'Eury, 1877, p. 519 ; nom. nud.

TRIPTEROSPERMUM Brongniart, 1874.

Tripterospermum rostratum Brongniart, 1874 , p. 262 , pl. 22 , figs. $6-8$; petrified seed, compared with Trigonocarpus; U p per Carboniferous; St.-Etienne, France.

TRIQUITRITES L. R. Wilson and Coe, 1940. Triquitrites arculatus L. R. Wilson and Coe, 1940 , p. 185 , pl. 1 , flg. 8 ; spore; Des Moines group, Pennsylvanian; Green County coal mine, Franklin Township, Green County, Iowa.

TRISTACHYA Lilpop, 1937.

Tristachya raciborskii Lilpop, 1937, p. 2, pl. 1; articulate, cones and foliage; Karnlowice limestone, "Permo-Carboniferous"; Karniowice, $35 \mathrm{~km}$ west of Cracow, Poland.

TRISTANITES Saporta, 1865.

Tristanites cloeziaeformis Saporta, 1865, p. $217, \mathrm{pl}, 13$, fig. 3 ; infructescence, Myrtaceae; Tertiary ; Armissan, France.

TRISTANITES Deane, 1902.

T'ristanites angustifolia Deane, 1902a, p. 23 , pl. 3, fig. 1 ; pl. 6, fig. 7 ; Tertiary ?: Berwick, Australla.

TRITICOIDES De Stefani, 1948.

Triticoides bianchii De Stefani, 1948; grass; Tertiary ; Italy.

TRIZYGIA Royle, 1840.

Trizygia speciosa Royle, 1840 (18331840 ), p. xxix*, pl. 2, fig. 8 ; Sphenophyllum-like foliage.

TROCHILISCUS Karpinsky, 1906.

Trochiliscus ingricus Karpinsky, 1906, p. 112, pl. 2, figs. 23-28; oogonium, Characeae; Devonian; Pawlowsk, Russia.

TROCHODENDROIDES E. W. Berry, 1922

Trochodenaroides rhomboideus (Lesquereux) E. W. Berry, 1922b, p. 166, pl. 36, fig. 6; leaf; Trochodendraceae ; Woodbine formation, Upper Cretaceous ; Arthus Blüff, Tex.
TROCHODENDROMAGNOLIA Zander, 1923. Braunkohle, 1923, Band 22, p. 41, (not seen, cited in Gothan, $1942 \mathrm{~b}$, p. 157).

TROCHOPHYLLUM Wood, 1861.

Trochophyllum fertilis (Sternberg) Wood, $1861 \mathrm{~b}$, p. 438 . This is a proposed name change for Annularia fertilis Sternberg on the grounds that Annularia had been used for a mollusk. Wood gives the spelling Trocophyllum in 1861, p. 522 .

TROCOPHYLLUM.

See Trochophyllum.

TRYPTEROCARPUS Grand'Eury, 1890.

Trypterocarpus arcuatus Grand'Eury, 1890 , p. 310 , pl. 4, fig. 14 ; seed impression; Upper Carboniferous; Traquette, France.

TSUGAEPOLLENITES Robert Potonie, 1934.

Tsugaepollenites igniculus Robert Potonie, in Potonie, Robert, and Venitz, H., 1934, p. 17 , pl. 1 , fig. 8 ; pollen, compare.1 with Tsuga; Miocene; Oberlausitz, Germany.

TSUGITES Fliche, 1896.

Trugites magnus Flfche, 1896, p. 211, pl. 9, fig. 2 ; petrified cone, Coniferales ; Lower Cretaceous (Albien); Clermont, France.

TUBERCOLARITES Arcangell, 1903.

Tubercolarites iani Arcangeli, in Barsanti, 1903 , p. 12 ; fungus; Upper Carboniferous; Iano, Italy.

TUBERCULATISPORITES Ibrahim, 1933.

Tuberculatisporites tuberosus Ibrahim, 1933 , p. 22 , pl. 3, fig. 27 ; spore ; Carboniferous,

TUBICAULIS Cotta, 1832.

Tubicaulis solenites (Sprengel) Cotta, 1832 , p. 22, pl. 2, figs. 1, 3; petrified fern, Tubicaulidaceae (Hirmer, 1927, p. 540) ; Permian ; Chemnitz, Germany.

TUBICULITES Grand'Eury, 1877.

Tubiculites relaxatomaximum Grand'Eury, 1877, p. 102; apparently a Psaronills stem; no speciflc designations are given with figures; Upper Carboniferous; France.

TUMULISTIGMA Bayer, 1914.

Tumulistigma furculorum Bayer, 1914, p. 64 ; Cretaceous; Ober-Haatz, Bohemia. TUSSILAGITES Grüss, 1927.

Tussilagites tertiaria Grüss, 1927, p. 205, figs. 1-3; Tertiary; Preschen: near Bilin, Bohemia.

TUZSONIA Andreanszky, 1949.

Tuzsonia hungarica Andreanszky, 1949, $p$. 31, illustrated; Palmaceae; Tertiary; Hungary.

TYCHTOPTERIS Zalessky, 1930.

Tychtopteris cuneata (Schmalnausen) Zalessky, 1930f; p. 926; Permian; Pechora basin, Russia!.$\therefore$. $\because, "$ 
IYLODENDRON C. E. Weiss, 1870.

Tylodendron speciosum C. E. Weiss, $1870 \mathrm{~b}$, p. 47 ; Upper Carbonlferous; Otzenhausen, Prussia.

TYLOPHORA Hick, 1892.

Tylophora radiculosa Hick, 1892a, p. 101, pls. 16, 17; stigmarian "rootstock"; Upper Carboniferous ; Clnder Hills, near Halifax, England. This name later withdrawn ; see Xenophyton.

TYMPANOPHORA Lindley and Hutton, 1837.

Tympanophora simplex Lindley and Hutton, 1837 (1831-37), p. 57, pl. 170; fertile frond, Cyatheaceae; Jurassic (Oolitic); Cloughton Wyke, Scarborough, England. See also Seward, 1910 , p. 367.

TYPHACITES Saporta, 1890.

Typhacites rugosus Saporta, 1890, p. 3, pl. 13, fig. 4 ; leaf fragment, Typhaceae?; Cretaceous; Fuveau, Provence, France.

TYPHAELOIPUM Unger, 1845.

Typhaeloipum lacustre Unger, 1845 (184147 ), p. Ixix ; leaf fragment of Typhalike plant: Miocene; Radoboj. Illustrated in Unger, 1852 , p. 90 , pl. 30, figs. $6-8 ;$ pl. 28 , figs. 6,7 .

TYRGAEINA Zalessky, 1944.

Tyrgaeina mamillaris Zalessky, 1944, p. 250.

T'YSONIA Fontaine, 1889.

T'ysonia marylandica Fontaine, 1889 , p. 193, pls. 174-180 ; petrified trunk, Bennettitales; Potomac group, Lower Cretaceous; Maryland.

\section{$\mathrm{U}$}

ULAARIA Zalessky, 1937.

Palaeophytographica, p. 10 : Moskva, Akad. Nauk SSSR, 1937 (not seen, cited in Gothan, 1942b, p. 157).

ULLMANNIA Goeppert, 1850.

Ullmannia bronnii Goeppert, 1850, p. 185, pl. 20, figs, 1-26; cones and follage; Permian (Zechstein), Frankenberg, Saxony.

ULLMANNITES Tuzson, 1911.

Ullmannites beinertianus Tuzson, 1911, p. 24, fig. 2.

(Goeppert)

ULMACITES Caspary, 1886.

Ulmacites succineus Caspary, in Conwentz, 1886 , p. 47 ; leaf, in amber, compared with Ulmus; Tertiary.

ULMINIUM Unger, 1842.

Ulminium diluviale Unger, 1842b, p. 174; wood; Tertiary; Bohemia. See also Unger, $1841-48$, p. 97 , pl. 25, figs. 6-9.

ULMIPHYLLUM Fontaine, 1889.

Ulmiphyllum brookense Fontaine, 1889, p. 312 , pl. 155 , fig. 8 ; pl. 163 , fig. 7 ; leaves, compared with Ulmus; Potomac group, Lower Cretaceous ; Brooke, Va.
ULMIPOLLENITES Wolff.

Ulmipollenites undulosus Wolff, 1934, p. 75, pl. 5, fig. 25 ; Pliocene; Freigericht mine near Dettingen, Bavaria.

ULMITES Dawson, 1890.

Ulmites pusillus Dawson, 1890, p. 88 , fig. 24 ; leaf ; Tertiary ; British Columbia.

ULMOPHYLLUM Ettingshausen, 1887.

Vlmophyllum oblongum Ettingshausen, 1887 a, p. 104 , pl. 10 , figs, $12,12 \mathrm{a}$; leaf., Ulmaceae; Vegetable Creek, near Emmaville, New South Wales.

ULMOXYLON Kaiser, 1879.

Ulmoxylon lapidariorum (Unger) Kaiser, 1879 , p. 100. For Cottaites lapidariorum Unger, $1842 \mathrm{~b}$, p. 176 . See also Unger, 1854, p. 182, pl. 7, figs 1-3.

ULODENDRON Lindley and Hutton, 1831. Ulodendron majus Lindley and Hutton, 1831 (1831-37), p. 22, pl. 5; lycopod stem impression; Carboniferous ; Jarrow Colliery, near Newcastle-upon-Tyne. England.

ULODENDROSTROBUS Renier, 1931?

Ulodendrostrobus squarrosus Renier, 1931, p. 276 ; Westphalien, Upper Carboniferous; coal basin of Charleroi, Belgium.

CLLOSPERMUM Pomel, 1849.

Ulospermum conicum (Lindley and Hutton) Pomel, 1849 , p. 346. For Carpolithes conicus Lindley and Hutton, 1836 (1831-37), p. 101, pl. 189, figs. 1, 2, 4; Jurassic; Malton, England.

ULVITES Reinsch, 1881.

Ulvites sp. Reinsch, 1881, p. 60, pl. 13, figs. 1-5; Upper Carboniferous; Zwickau, Saxony.

ULTOPTERIS Schuster, 1908.

Ulvopteris ammonis Schuster, 1908, p. 184, fig. 2 facing p. 192 ; Upper Carboniferous ; Germany.

UMBELLIFERITES Engelhardt and Kinkelin, 1908.

C'mbelliferites sp. Engelhardt and Kinkelin, 1908 , p. 249 , pl. 32 , fig. 12 ; Upper Pliocene; Klïrbecken near Ntederrad, Hesse.

UMBELLIFEROSPERMUM IT. W, Berry, 1929.

Umuelliferospermum latahense $\mathrm{E}$. W. Berry, 1929c, p. 261, pl. 64, flgs. 10-12 ; fruit, Umbelliferae; Latah formation, Miocene; brickyard at Spokane, Wash.

UMKOMASIA Thomas, 1933.

Umkomasia macleani Thomas, 1933, p. 203, pl. 23, fig. 56 ; figs. 1-4; pteridosperm inflorescence bearing cupulate seeds; Molteno beds, Karroo system, 'Triassic; Upper Umkomas Valley, Natal. Cited briefly in Thomas, 1931, p. 663.

UNATHECA Kidston, 1891.

Unatheca oblongus Kidston, 1891, p. 32, pl. 3, fig. 33 ; fertile coenopterid? frond; Radstock series, Upper Carboniferous ; Camerton, Somerset, England. 
UNOINULITES Pampaloni, 1902.

Uncinulites baccarinii Pampaloni, 1902, p. 125, pl. 10, flg. 7 ; fungus perithecia; Miocene?; Sicily.

UNGERIA Salfeld, 1908.

Ungeria 8olnhofensis Salfeld, 1908, p. 385, fig. p. 385 ; fern frond ; Jurassic ; Solenhofen, Bavaria.

UNGERITES Schleiden, 1855.

Ungerites tropicus Schlefden, in Schmid and Schleiden, 1855 , p. 37 ; wood, Leguminosea?; Oligocene; Koistenblatt, Bohemia.

UPHANTENIA Vanuxem, 1842.

Uphantenia chemungensis Vanuxem, 1842, p. 184, fig. 50 ; plant?; Chemung group, Upper Devonian; New York.

URALIDIUM Zalessky, 1939.

Cralidium singulare Zalessky, 1939 b, 373, fig. 57; incertae sedis; Permian; Matveyevo, USSR.

URA.LO'BAIERA Zalessky, 1939.

Uralobaiera bairmica Zalessky, 1939b, p: 361, fig. 41 ; incertae sedis; Permian; Matveyevo, USSR.

URALODENDRON Zalessky, 1939.

Uralodendron verticillatum $\mathrm{Z}$ a l e s $\mathrm{k} \mathrm{y}$, 1939 b, p. 368, flg. 50 ; follage twig, Coniferales?; Permian; Matveyevo, USSR.

URALOPHYLLUM Krystofowitsch and Prynada, 1933.

United Geol. Prosp. Service USSR, 1933, Trans. 346, p. 25 (not seen), cited in Gothan, 1942b, p. 157.

URALOPTERIS Zalessky, 1939.

Uralopteris valida Zalessky, 1939b, p. 355 , fig. 34 ; fern? pinnule fragment; Permian; Mikhallovskole, USSR.

URALOSPERMA Zalessky, 1939.

Uralosperma insigne Zalessky, 1939b, p. 372, fig. 55; seed; Permian ; Sivkova, USSR.

URANIOPHYLLITES SUVi, 1843.

Uraniophyllites spathulata Sayi, 1843, p. 75 , pl. 1 fig. 8 ; Miocene; Monte Bam boli, Italy.

UREDINITES Velenovsky, 1889.

Credinites cretaceous Velenovsky, 1889, p. 29, pl. 3, fig. 14; Upper Cretaceous (Cenomanian); Vyserovic, Bohemia.

URNATOPTERIS Kidston, 1884.

Urnatopteris tenella (Brongniart) Kidston, 1884, p. 594 ; fertlle sphenopterld foliage; Upper Carboniferous ; various localities, scotland; England. For Sphenopteris tenella Brongniart, 1828a38 , p. 186, pl. 49, fig. 1 .

UROHELMINTHOIDA Sacco, 1888.

Urohelminthoida dertonensis Sacco, 1888, p. 184 , pl. 2, figs. 8,16 ; probably not a plant ; Eocene ; Lombardy, Italy.
UROMYCETITES C. F. W. Braun, 1840.

Uromycetites concentricus C. F. W. Braun, 1840, p. 93 ; nom. nud ; Triassic ; Eckersdorf, Bavaria.

UROPHLYCTITES Magnus, 1903.

Urophlyctites oliverianus Magnus, 1903, $\mathrm{n}$. 249 ; fungus ; Carboniferous. Apparently first lllustrated species: Urophylyotites stigmariae Weiss, $1904 \mathrm{~b}$, p. 68 , figs. $66,67$.

UROPLASMIUM Reinsch, 1881.

Uroplasmium sp. Reinsch, 1881, p. 46, pl. 6, figs. 4-8; Upper Cárboniferous ; Zwickau, Saxony.

URSATOPTERIS.

Error for Urnatopteris; in Kidston, 1884b, p. 295.

URTICICARPUM Reid and Chandler, 1933.

Urticicarpum scutellum Reid and Chandler, 1933, p. 146, pl. 3, fig. 14 ; fruit, Urticaceae?; London Clay, Eocene, Minster, Kent, England.

UTERIA Michelin, 1847.

Uteria encrinella Michelin, 1845 (184047 ), p. 177 , pl. 46 , fig. 26 ; alga ?; Upper Cretaceous; Cuise-la-Motte, France.

UTRICULARITES Massalongo, 1857.

Utricularites protogaeus Massalongo, in Massalongo and Scarabelli, 1857, p. 11 ; for illustrations, see Massalongo and Scarabelli, 1859, pls. 3, 4; incertae sedis ; Miocene ; Sinigaglia, Italy.

\section{V}

VACCINOPHYLLUM Dawson, 1890.

Vaccinophyllum quaestum Dawson, 1890 , p. 88 , fig. 23 ; leaf ; Tertiary ; Similkameen River, British Columbia.

VALERIANELLITES Saporta, 1862.

Valerianellites capitatus Saporta, 1862, p. 260 , pl. 10 , fig. 3 ; inflorescence Rubiaceae?; Tertiary; Aix, Province, France.

VALIDOPTERIS Paul Bertrand, 1932.

Reference not seen ; cited in Gothan, 1942b, p. 157.

VALLISNERITES Heer, 1878.

Vallisnerites jurassicus Hecr, $1878 \mathrm{~b}$, p. 8, pl. 1, figs. 22-27; grasslike leaves; Jurassic ; Ust-Balei, Siberia.

VALONITES Sordell1, 1873.

Valonites utriculosus Sordelli, 1873, p. 367, fig. C; incertae sedis; Pliocene; Lombardy, Italy.

VALVISPORITES Ibrahim, 1933.

Valvisporites trilobus Ibrahim, 1933, p. 33, p1. 4, fig. 30 ; spore; Carboniferous.

VARDEKLOEFTIA Harris, 1932.

Vardekloeftia sulcata Harris, 1932b, p. 109 , pls. $15,17,18$; female portion of cone (gynaecium), Bennettitales; Lepidopteris zone, Rhaetic; Scoresby Sound, east Greenland. 
VARIOLARIA Sternberg, 1820.

Variolaria ficoides Sternberg, 1820 (1820$38)$, p. 22, pl. 12, figs. 1, 2 ; Stigmaria; Carboniferous.

VEOTIA Stopes, 1915.

Vectia luccombensis Stopes, 1915, p. 247, pls. 23-25; petrified phloem; Lower Greensand, Cretaceous; Isle of Wight, England.

V.BRBENOPHYLLUM Ettingshausen, 1858. Verbenophyllum aculeatum Ettingshausen, 1858 , p. 749 , pl. 3, fig. 11 ; Mlocene; Koeflach, Styria.

VERMICULITES Rouault, 1850.

Vermiculites panteri Rouault, 1850, p. 744 ; plant?; Silurian; Guichen, Brittany, France.

TERMIPORELLA Stolley, 1893.

Vermiporella fragilis Stolley, 1893, p. 140, pl, 8, figs. 7-11; siphonaceous alga ; Silurian.

VERONICITES Heer, 1859.

Veronicites oeningensis Heer, 1859, p. 191, pl. 153, fig. 54 ; seeds, Labiatae?; Miocene; Oeningen, Switzerland.

VERRUCANIA Fucini, 1936.

Reference not seen; cited in Gothan, $1942 \mathrm{~b}$, p. 157.

VERRUCARITES Goeppert, 1844.

Verrucarites geanthracis Goeppert, 1844, p. 195 ; nom. nud.

VERRUCOSISPORITES Ibrahim, 1933.

Verrucosisporites verrucosus Ibrahim, 1933 , p. 25 , pl. 2, fig. 17 ; spore ; Carboniferous.

VERTEBRARIA Royle, 1840.

Vertebraria indica Royle, 1840 (1833-40), p. $\Sigma x_{i x}$, pl: 2 , figs. $1-3$; stem, possibly of Glossopteris ; shales of Ranigunj and Chinnakooree, India ; "Permo-Carboniferous." See also Walton and Wilson, 1932.

VESQUIA C. E. Bertrand, 1883.

Vesquia tournaisii C. E. Bertrand, 1883, p. 1382 ; seeds, Taxaceae?; Cretaceous?; Tournai, France.

VETACAPSULA.

See discussion by Brown, R. W., 1950.

$\nabla E X I L L U M$ Rouault, 1850.

Vexillum labechei Rouault, 1850, p. 734 ; Silurian; Brittany, France.

VIATCHESLAVIA Zalessky, 1936.

Viatcheslavia vorcutensis Zalessky, $1936 \mathrm{~b}$. p. 240, figs. 6, 7 ; lycopod leaf bases; Permian ; Russia.

VIBURNIPHYLLUM Nathorst, 1886.

Viburniphyllum giganteum (Saporta) Nathorst, $1886 \mathrm{a}$, p. 52. For Viburnum giganteum Saporta, 1868, p. 370, pl. 30, figs. 1, 2.
VIBURNITES Lesquereux, 1892.

Viburnites crassus Lesquereux, 1892, p. 124, pl. 45, figs. 1-4 ; leaf, Caprifoliaceae; Cretaceous; 10 miles northeast of Delphos, Kans.

VIBURNOIDITES Robert Potonie, 1950.

Viburnoidites sp. Robert Potonle, in Potonie, Robert, Thomson, Paul W., and Thlergart, Frledrich, 1950, p. 62.

VILLARSITES Mïnster, 1842.

Villarsites ungeri Münster, 1842 (183943 ), p. 109 , pl. 4 , flg. 5 .

VILLERSIA Stockman8, 1948.

Villersia radians Stockmans, 1948 , p. 69 , pl. 10, figs. 6,7 ; Upper Devonian; Belglum.

VIRACARPON Sahni, 1934.

Viracarpon hexaspermum Sahni, 1934, p. 318 ; fruit, monocotyledon; Intertrappean serfes, Tertiary; India. See also Sahni, 1940, pl. 3, fig. 13 ; and Sahni, 1944, p. 81, pl. 3, flgs. 25-28.

VISCOPHYLLUM Knoll, 1904.

Piscophyllum morloti (Unger) Knoll, 1904, p. 67 , pl. 4 ; leaf, Loranthaceae ; Miocene; Kumber, Styria.

VITICOCARPUM Menzel, 1913.

Viticocarpum pusillum Menzel, 1913, p. 62 , pl. 5, fig. 36 ; fruit, Verbenaceae; Tertiary (Braunkohle); Germany.

VITIGENE Saporta, 1865.

Vitigene cissoides Saporta, 1865 , p. 48 ; leaf, compared with Cissus adnata; Tertiars ; France.

VITIPHYLLUM Nathorst, 1888.

Vitiphyllum raumanni Nathorst, 1888, p. 211 , pl. 22, fig. 2 ; leaf, compared with Vitis; Tertiary; Sakugorl, Shimano, province, Japan.

VITIPHYLLUM Fontaine, 1889.

Vitiphyllum crassifolium Fontalne, 1889, p. 308 , leaves, compared with Vitis; Potomac group, Lower Cretaceous ; near Potomac Run; Va.

VITIPITES Wodehouse, 1933.

Vitipites dubius Wodehouse, 1933, p. 514, fig. 47 ; pollen, Vitaceae; Parachute Creek member, Green River formation, Eocene; Colorado and Utah.

VITOXYLON Schuster, 1911.

Vitoxylon coheni Schuster, 1911a, p. 541, pl. 20 ; wood, Vitaceae; early Tertiary

VOLKELIA Solms-Laubach, 1896.

Volkelia refracta (Goeppert) Solms-Laubach, 1896 , p. 58. For Sphenopteris refracta Goeppert, 1852, p. 141, pl. 12; Lower Carboniferous; Falkenberg, S1lesia.

VOLKMANNIA Sternberg, 1825

Volkmannia distachya Sternberg, 1825 (1820-38), Tentamen, p. xxx, pl, 48, figs. $3 \mathrm{a}, 3 \mathrm{~b}$; articulate stem and cone impressinn; Carboniferous; Bohemla. 
VOLNOVAKHIA Zalessky, 1931.

Acad. Sci. U. S. S. R. Bull., 1931, p. 582 (not seen, cited in Gothan, 1942b, p. 158).

VOLTZIA Brongniart, 1828.

Voltzia brevifolia Brongniart, 1828d, p. 449 , pl. 15 ; pl. 16 , figs. 1,2 . See also Florin, 1944, p. 492.

VOLTZIOPSIS Henry Potonie, 1899.

Voltziopsis coburgensis (Schauroth) Henry Potonie, 1899, p. 304. For Voltzia coburgensis Schauroth, 1852, p. 540, fig. p. 539 ; Triassic (middle Keuper); Coburg, Prussian Saxony.

DOLTZIOXYLON Torrey, 1923.

Volt zioxylon dockumense Torrey, 1923, p. 64, pl. 8, figs. 1,2 ; wood, Coniferales; Dockum group, Triassic; Spur, Tex.

VOLTZITES Tuzson, 1911.

Voltzites hungarica (Heer) Tuzson, 1911, p. 36. For Voltzia hungarica Heer, 1876, K. Ungarischen geol. Anst. Jahrb., Band 5, p. 12 , pl. 22 , figs. $1-5$; pl. 23 , figs. 1-4, Budapest.

VOLUBILITES Liburnau, 1901.

Volubilites praecarbonicus (Gümbel) Liburnau, 1901, p. 566. For Taenidium praecarbonicum Gümbel, 1879, p. 535; Carboniferous (Lower Culm): Wurzbach, Prussian Saxony.

\section{W}

WAHPIA Walcott, 1919

Wahpia insolens Walcott, 1919, p. 239, pl. 57 , fig. 1 ; alga, Rhodomelaceae ; Stephen formation, Middle Cambrian; great "fossil bed" on northwest slope of Mt. Stephen, above Field, British Columbia.

WALCHIA Sternberg, 1825.

Walchia filiciformis (Schlotheim) Sternberg, 1825 (1820-38), Tentamen, p. xxii. For Lycopodiolites filiciformis Schlotheim, 1820, pl. 24 ; coniferous foliage twigs; Wettin, Germany. See also Florin, 1951, p. 316.

WALCHIANTHUS Florin, 1940.

Walchianthus cylindraceus Florin, $1940 \mathrm{~b}$, p. 269 , pls. 155,156 , figs. $11-21$; cones, Coniferales; Lower Permian; Ottendorf, near Braunau, Germany. Florin notes that, because this is an artificial genus, no type species is designated. The above is the first one described.

WALCHIOPREMNON Florin; 1940.

Walchiopremnon (Lebachia) valdajolense (Mougeot) Florin, 1940b, p. 277, pls. 157,158 , figs. $18-20$; pls. 159,160 , figs. 1-23 ; petrified stem, Coniferales; Lower Permian; Faymont (Val-d'Ajol), France. Florin (p. 273) notes that, because this is an artificial genus, no type species is designated; valdajolense is the only species described.
WALCHTOSTROBUS Florin, 1940.

Walchiostrobus (Lebachia!) gothanii Florin, $1940 \mathrm{~b}$, pls. 151 , 152, figs. 47-52 ; pls. 153,154 , figs. $1-10$; cone, Coniferales; Lower Permian; Thüringer Wald, Germany. Florin (p. 261) notes that, because this is an artificial genus, no type species is designated. The species above in the first one described.

WALDENBURGIA Gothan, 1950.

Waldenburgia corynepteroides Gothan, 1950 , pl. 1 ; fertlle fern frond, possibly primitive Schizaeaceae ; lowermost Carboniferous; Waldenburg.

WALKOMIA Schuster, 1931.

Walkomia Schuster, 1931, p. 256.

WALKOMIA Florin, 1940.

Walkomia australis (Feistmantel) Florin, $1940 a$, p. 8 , pls. 1-4; foliage shoots, Coniferales; Newcastle series, Upper Permian ; Bowenfels, near Lithgow, New South Wales.

WALKOMIELLA Florin, 1944.

Walkomiella a u \& $t r a l i s$ (Feistmantel) Florin, 1944, p. 370. For Walkomia australis (Feistmantel) Elorin; see above.

WAPUTIKIA Walcott, 1919.

Waputikia ramosa Walcott, 1919, p. 236, pl. 54, fig. 2 ; alga, Rhodomelaceae ; Burgess shale, Stephen formation, Middle Cambrian; above Field, British Columbia.

WARDIA David White, 1904

Wardia fertilis David White, 1904, p. 329, pl. 48 ; this name given to seeds borne by foliage described as Aneimites fertilis Ward; Thurmond formation, lower Pottsville, Pennsylvanian; near Nuttall, W. Va.

WEEDIA Walcott, 1914.

Weedia tuberosa Walcott, 1914, p. 108, pl. 11, figs. 1, 2 ; alga, Cyanophycene? ; Siyeh limestone, Algonkian ; above Lake McDonald, Glacier National Park, Mont.

WEICHSELIA Stiehler, 1857.

Weichselia ludovicae Stiehler, 1857, p. 73, pls. 12, 13 ; Upper Cretaceous ; Quedlinburgh, Prussian Saxony.

WEISSITES Goeppert, 1836.

Weissites vescicularis Goeppert, 1836, p. xiv. For Neuropteris conferta Sternberg, 1833 (1820-38), p. 75, pl. 22, fig. 5.

WELTRICHIA V. F. W. Braun, 1847.

Weltrichia mirabilis C. F. W. Braun, 1847, p. 86. See also Braun, C. F. W., 1849, p. 710 , pl. 2, figs. 1-3.

WESTERSHEIMIA Krasser, 1918.

Westersheimia pramelreuthensis Krasser, 1918 , p. 549; cycadophyte stem frag. ment; Upper Triassic; Pramelreith, Lunz, Austria. 
WETHERELLIA Bowerbank, 1840.

Wetherellia variabilis Bowerbank, 1840, p. 89 , pl. 12 , figs. $1-40$; fruits, Linaceae ; London Clay, Eocene ; Sheppey, Kent, England.

WHITTLESEYA Newberry, 1853.

Whittleseya elegans Newberry, 1853a, p. 106 ; microsporangiate organ, Pteridospermae; Pennsylvanian; Cuyahoga Falls and Poland, Ohlo. See also Newberry, 1853 b, fig. p. 116 ; Halle, 1.933 ; Schopf, 1948 .

WIDDRINGTONITES Endlicher, 1847.

Widdringtonites ungeri Endlicher, 1847, p. 271. For Juniperites baccifera Unger, $1843(1841-47)$, p. 80, pl. 21, figs. 1-3.

WIDDRINGTONOXYLON Penny, 1947.

Widdringtonoxylon borealis Penny, 1947, p, 287, figs. $13,15,16$; wood, Coniferales ; Magothy formation, Upper Cretaceous; Deep Cut, west of Summit Bridge, Del.

WIELANDIA Nathorst, 1909.

Wielandia angustifolia Nathorst, 1909a, p. 22 , pls. 5,6 ; cycadophyte cones and follage; Rhatic; Bjuf, Sweden. See Wielandiella.

TIIELANDIELLA Nathorst, 1910.

Wielandiella angustifolia Nathorist, 1910. A. name that Nathorst substituted for Wielandia; it appears on errata slip (dated Jan. 7, 1910) to title page of Nathorst, 1909a.

WILKINSONIA. Mueller, 1879.

Wilkinsonia flaminata Mueller, 1879 (1877-79), p. 170, pl. 3, fig. 4 ; Pllocene ; Gulgong, Australia.

WILLIAMSONIA Carruthers, 1870.

Williamsonia gigas (Lindley and Hutton) Carruthers, 1870, p. 693. As treated by Carruthers, $W$. gigas consists of a combination of the foliage described by Lindley and Hutton as Zamia gigas and fructifications originally figured, but not named, by Young and Bird, 1822, p 183, pl. 2, figs. 2, 6. Williamson, 1870. gave an exhaustive description of the latter. For additional information, sce Seward, 1917, p. 421-423 ; Sahni, 1932d.

WILLIAMSONIELLA Thomas, 1915.

Williamsoniella coronata Thomas, 1915 , p. 115, pls. 12-14; strobilus, Bennettitales; Gristhorpe plant bed, Jurassic ; Yorkshire, England.

WILSONIA Kosanke, 1950.

Wilsonia'vesicatus Kosanke, 1950, p. 54, pl. 14, flgs. 1-3 ; spore; LaSalle coal bed, Pennsylvanian; Bureau County, IIl.

WINCHELLIA Lesquereux, 1893.

Winchellia triphylla Lesquereux, 1898, p. 209 , pl. 8 ; leaf, Berberidaceae ; Cretaceous; Yellowstone River near mouth of Powder Kiver.
WINCHELLINA Herzer, 1893.

Winchellina fascina Herzer, 1893c, p. 286, pl. 6; apparently a Psaronius trunk; Upper Carboniferous; Monroe County, Ohio.

WINDWARDIA F'lorin, 1936.

Winduardia crookallii Florin, 1936b, p. 91 , pl. 21 , figs, $1-10$; pls. $17-20$; structurally preserved follage, Ginkgonles: Jurassic ; Franz Joseph Land.

WITHAMIA Unger, 1842.

Withamia styriaca Unger, 1842b, p. 177 ; wood, incertae sedis; Tertiary; Styria.

WITHAMIA Seward, 1895.

Withamia armata (Saporta) Seward, 1895, p. 174 , pl. 2 , figs. 1,2 ; pl. 5, fig. 1 ; cycadophyte frond fragment?; Wealden; Eccleshourne, England. This is an especially confusing case. On page 174 the name is given as Withamia $8 a$ portae although Seward states that he is transferring Saporta's Cycadorachis armata to the new genus Withamia. The plates bear the generic name Saportaia, but the captions opposite the plates bear the footnote that the name Saportaia was abandoned (after the plates wele engraved) in view of its closeness to Saportaea, a previously established genus. 'Withamia, itself being in alid, was later changed to Sewardia ,by Zeiller.

WOBURNIA Stopes, 19.12.

Woburnia porosa stopes, 1912, p. 92, pl. 7 , fig. 7 ; pl. 8 , fig. 8 ; wood, dicotyledon; Lower Greensand, Aptian, Lower Cretaceous; Woburn Sands, Bedfordshire, Ingland.

WONNACOTTIA Harris, 1942.

Wonnacottia crispa Harris, 1942b, p. 577, figs. 1-3; microsporophyll, Bennettitules; Middle Estuarine, Jurassic ; Cayton Bay, Yorkshire, England.

WOODWORTHIA .Tefrey, 1910.

Woodworthia arizonica Jeffrey, 1910, p. 330, pls. 31, 32 ; wood, Araucariaceae; Triassic ; Arizona

\section{$\mathbf{x}$}

XANTHOLITHUS (Ward) Cockerell, 1926 Xantholithus hastatiformis Cockerell, .1926a, p. 11. For Ophioglossum hastatiforme Cockerell, 1924, p. 10, flg. p. 10, Incertae sedis; Eocene; Tipperary, Wyo. [The binomial Xantholithus propheticus created by Ward, 1915, p. 150 ; nom. nud.]

XANTHOXYLUM.

Error for Zanthoxylum, in Yabe and Endo, 1930 , p. 600 .

XWNOCLADIA Arnold, 1940.

Xenocladia medullosina Arnold, 1940, p. 61, figs. 4, 6, 7 ; Tully limestone, Middle Devonian; Erle County; N. Y. 
XENOPHYTON Hick, 1892.

Xenophyton radioulosa Hick, 1892a, p. 216. For Tylophora radiculosa Hick, $1892 \mathrm{~b}$, p. 101 , pls. $16 ; 17$.

XENOPTERIS C. E. Weiss, 1870.

Xenopteris brardi (Brongniart) C. E. Weiss, 1870 a, p. 765. For Odontopteris brardi Brongniart, 1828a-38, pls. 75, 76 ; fernlike follage ; Carboniferous.

XENOTHECA E. A. N. Arber and Goode, 1915.

Xenotheca devonica F. A. N. Arber and Goode, 1915, p. 96, pl. 4, figs. 1-7; pteridosperm cupule?; Devonian ; Devon, England.

XENOXYLON Gothan, 1905.

Xenoxylon latiporosum (Cramer) Gothan, 1905, p. 38. For Pinites latiporosus Cramer, in Heer, 1868 , p. 176, pl. 40 , flgs. 1-8. See also Gothan, 1910, p. 23, pl. 4, figs. 7-11; pl. 5, figs. $1,2$.

XIPHOPHYLLUM Zalessky, 1930.

Xiphophyllum kuliki Zalessky, 19301, p. 917; nom. nud.; Permian; Pechora basin, Russia.

XULINOSPRIONITES Bowerbank, 1840.

Xulinosprionites latus Bowerbank, 1940, p. 143, pl. 17, figs. 43,44 ; fruit, incertae sedis; London Clay, Eocene; Sheppey, Kent, England.

XYLOCARYA Reid and Chandler, 1933.

$X$ ylocarya tricularis Reid and Chandler, 1933 , p. 312 , pl. 14, figs. 9-12 ; endocarp, Anacardiaceae; London Clay, Eocene; Sheppey, Kent, England.

XYLOCARYON Mueller, 1875.

$J_{y}, l o c a r y o n$ lockii Mueller, 1875 (1871$82)$, p. 41 , pl. 11 ; Pliocene; Nintingbool, Victoria.

$X Y L O I S$ Stenzel, 1872.

Xylois antiquensis (Unger) Stenzel, 1872, p. 71. For Fasciculites antiquensis Unger, in Martius, 1846, p. lviii, pl. 2 . figs. 5-7.

XYLOLITHES Debey, 1848.

$X$ ylolithes sp. Debey, 1848, p. 124: nom. nud.

XYLOMASTIXIA. Kirchheimer, 1938.

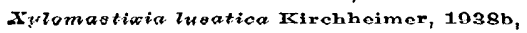
p. 348 , pl. 7, figs, 1-6; endocarp, Cornaceae ; Oligocene ; Germany.

XYI.OMIDES (Unger) Schimper, 1869.

Xylomides umbilicatus (Unger) Schimper, 1869 , p. 138 , pl. 1 , fig. 8 ; fungus ; Tertiary; Radobof, Croatia.

XYLOMITES Unger, 1841.

Xylomites umbillicatus Unger, 1841 (184147 ), p. 3, pl. 1, fig. 2 ; fungus ; Tertiary ; Radobof, Crotia. This genus erroneously attributed to Persoon in Meschinelli, 1892, p. 791.
XY LOPHYLLITES Massalongo, 1858.

Xylophyllites pelasgica Massalongo, 1858a, p. 114 ; for illustration, see Massalongo and Scarabelli, 1859, pl. 35, flgs. 18a, 18b; leaf Euphorblaceae; Mlocene; Sinigaglia, Italy.

XYLOPSARONIUS Pohlig, 1910.

Xylopsaronius cottai (Corda) Pohlig, 1910, p. 335, figs. 1-3. See also Posthumus, 1931.

XYLOPTERIS Franguelli, 1943.

Xylopteris elongata (Carruthers) Fren. guelli, 1943a, p. 324, figs. 30, 31; pteridosperm frond?; Upper Triassic; Queensland, Tasmania, Natal, etc.

$X Y L O P H Y L L U M$ Zalessky, 1927.

Xylophyllum kuliki Zalessky, 1927a, p. 52, pl. 44, flg. 6 ; cordaitellke leaf ; Jurassic ; Pechora basin, Russia.

\section{Y}

YABEIELLA Oishi, 1931.

Yabeiella brachebuschiana (Kurtz) Oish1, 1931a, pl. 26, figs. 4-6 ; taeniopterid foliage; Rhaetic; Argentina.

YAKIA David White, 1929.

Yakia heterophylla David White, 1929, p. 86 , pl. 39 , flgs. $1-8$; pteridosperm ? follage, associated fructifications compared with Ullmannia bronni; Hermit shale, Permian; near Bright Angel Trail, below El Tovar, Ariz.

YARRAVIA Lang and Cookson, 1935.

Yarravia oblonga Lang and Cookson, 1935, p. 437 , pl. 32 , figs. $37-41$; terminal synangial fructification; Silurian (Lower Ludlow); Victoria, Australia.

YATESIA Carruthers, 1874.

Yatesia morrisii (Morris and Carruthers) Carruthers, 1874 , p. 688 , pl. 55, figs. 3-6; cycadophyte trunk; Lower Greensand, Cretaceous; Leighton-Buzzard, Potton, Bedfordshire, England. Name cited by Carruthers, 1868 , p. 80 ; nom. nud.

YEZONIA Stopes and Fujii, 1910.

Yezonia vulgaris Stopes and Fujii, 1910, p. 23 , pl. 2 , figs. $5-8 ;$ pl. 3 , fig. $9 ;$ pl. 4 fig. 19 ; petrified gymnosperm stem; Upper Cretaceous; Hokkaido, Japan.

YEZOSTROBUS Stopes and Fujii, 1910

Yexostrobus oliverii Stopes and Fuj11, 1910, p. 33 , figs. $12-14$; pl. 1 , fig. 8 ; pl. 3 , figs. 10-13; cone, Coniferales; Upper Cretaceous; Hokkaido, Japan. Name cited in Stopes and Fujil, 1909, p. 558; nom. nud.

YORKIA Wanner, 1900

Yorkia gramineoides Ward, 1900, p. 254, pl. 34, figs. 4-6 ; grasslike leaves ; Trias. sic; York Haven, York County, Pa [The generic description is given by Atreus Wanner and the description for the type specles by Lester Ward.] 
YUBARIA Ogura, 1932.

Yubaria invaginata Ogura, 1932b, p. 476, pl. 24, figs. 14-17; petrified petiole, dicotyledon ; Cretaceous ; Hokkaido, Japan.

YUCCITES Martius, 1822.

Yuccites microlepis Martius, 1822, p. 136. YUCCITES Schimper and Mougeot, 1844.

Yuccites vogesiacus Schlmper and Mougeot, 1844, p. 42 , pl. 21 ; incertae sedis ; Triassic; Soulz-les-Bains, Alsace-Lorraine.

YUKNESSIA Walcott, 1919.

Yuknessia simplex Walcott, 1919, p. 235, pl. 54, fig. 1 ; alga, Chlorophyceae; Stephen formation, Middle Cambrian; Burgess pass fossil quarry, above Field, British Columbia.

\section{$\mathbf{Z}$}

ZALESSKYA Kldston and Gwynne-Vaughan, 1908.

Zalesskya gracilis Kidston and GwynneVaughan, 1908, p. 220, pl. 1, figs. 1-3 ; pl. 2 , figs. $4,5,8$; pl. 3 , flgs. 9,10 ; petrifled stem, Osmundaceae; Upper Permian; Bjelebel district, Orenburg, Russia. See also Posthumus, 1931.

ZALESSKYELLA Tschirkova, 1939.

Zalesskyella bifurcata Tschirkova, in Zalessky, 1939 b, p. 355 , fig. 33 ; fern? frond fragment; Permian; Tchekarda. USSR.

ZAMIOIDEA Schuster, 1931.

Zamioidea macrozamioides Schuster, 1931, p. 188. For Cycadocarpidium macrozamioides Schuster, 1911, Svenska vetansk. akad. Handl., band 51, p. 5, fig. 11.

ZAMIOLEPIS Pomel, 1846.

Zamiolepis dissecta Pomel, 1846, p. 653; nom. nud.; Jurassic; Moselle, France.

ZAMIOPHYLLUM Nathorst, 1890.

Zamiophyllum buchianum (Ettingshausen) Nathorst, 1890 , p. 46 , pl. 2, figs. 1,2 ; pl. 3 , pl. 5, flg. 2 ; cycadophyte leaf; Mesozole; Togodani, Tosa province, Japan.

ZAMIOPSIS Fontaine, 1889.

Zamiopsis pinnaflda Fontaine, 1889 , p. 161 , pl. 61 , flg. 7 ; pl. 62 , fig. 5 ; pl. 64 , fig. 2 ; fern? follage; Potomac group, Lower Cretaceous ; Fredericksburg, Va.

ZAMIOPTERIS Schmalhausen, 1879.

Zamiopteris glossopteroides Schmalhausen, 1879 , p. 80 , pl. 14, figs. 1-3 ; Glossopteris-llke leaf; Permian; Ssuka, Russia.

ZAMIOSTROBUS Endlicher, 1836.

Zamiostrobus macrocephala (Lindley and Hutton) Endlicher, 1836 (1836-40), p. 72. For Zamia macrophylla Lindley and Hutton, 1834 (1831-37), p. 117, pl. 125, cone, Coniferales?; Cretaceous ; England. See also Seward, 1917, p. 503.
ZAMIPHYLLUM Caspary and Klebs, 1907.

Zamiphyllum sambiense (Caspary) Caspary and Klebs, 1907 , p. 63 , pl. 8 , flg. 51 ; Tertiary ; Baltic Prussia.

ZAMITES Brongniart, 1828.

Owing to innumerable name changes in the cycadophyte leaf genera, it is extremely difficult to cite type species, especially for Zamites. The following is rather arbitrarily suggested : Zamites gigas (Lindley and Hutton) Morris, 1843 , p. 24 . For Zamia gigas Lindley and Hutton, 1835 (1831-37), p. 45, pl. 165 ; cycadophyte leaf ; Jurassic ; Scarborough, England. See discussion in Seward, 1917, p. 529-532.

ZANICHELLIOPSIS Massalongo, 1851.

Zanichelliopsis repens Massalongo, 1851, p. 46 ; nom. nud ; Eocene ; Monte Bolca, Italy. Later changed to Halochloris repens (Massalongo) Stiehler, 1869.

ZEARAMOSUS Webster, 1920.

Zearamosus elleria Webster, 1920, p. 286 ; marine alga; Devontan; Bloody Run, Iowa.

\section{ZEILLERIA KIdston, 1884.}

Zeilleria delicatula (Sternberg) Kidston, 1884a, p. 592, pl. 25 ; Pteridospermae ; Upper Carbonlferous; Forest of Wyre, Worcestershire, England. See also Kid. ston, 1924, p. 427.

ZEILLEROPTERIS Koidzumi, 1936.

Zeilleropteris yunnanensis Koldzumi, 1936, p. 135. For Gigantopteris nicotinaefolia Zeiller, 1907, Annales mines, ser $10^{\circ}$, tome 11 , p. 480 , pl. 14 , fig. $15,15 a$; Sinesi-Kou, Yunnan, China.

ZEITES Caspary, 1874.

Zeites succineus Caspary, 1872, p. 17 ; nom. nud.

ZELKOVOIDITES Thlergart?, 1950.

Zelkovoidites sp. in Potonie, Robert, Thomson, Paul W., and Thiergart, Friederich, 1950 , p. 57, pl. C, fig. 24 ; pollen ; Pliocene ; Chatt-Aquitan, Germany.

ZEUGOPHYLLITES Brongniart, 1828.

Zeugophyllites calamoides Brongniart, $1828 \mathrm{~b}$, p. 121, leaf ; Carboniferous. First fully described species appears to be $Z$. elongatus Morris, in Strzeleck1, 1845 , p. 250 , pl. 6, fig. 5 .

ZIMMERMANNIA Gothan and Zimmerman 1932.

Zimmermannia eleutherophylloides Gothan and Zimmerman, 1932 , p. 113, pl. 13, fig. 4 ; Upper Devonian; Upper Bögendorf, Silesla.

ZINGIBERITES Heer, 1859.

Zingiberites multinervis Heer, 1859 , p. 172 , pl. 148, flgs. 13-15; leaf fragments, Scitamineae?; Miocene ; Rossberg, Rhenish Prussia. 
ZIPPEA Corda, 1845.

Zippea disticha Corda, 1845, p. 76, p1. 26 ; incertae sedis; Carboniferous. See also Posthumus, 1931.

ZITTELIA Felix, 1882.

Zittelia elegans Felix, 1882a, p. 73, fig. 2; wood, Leguminosae?

ZITTELINA (Munier-Chalmas) Morellet and Morellet, 1913.

Zittelina elegans Morellet and Morellet, 1913, p. 27, pl. 3, figs. 5, 6 ; alga, Bortellées; Eocene; Grignon, France.

ZIZYPHITES Kuntze, 1904.

Zizyphites Kuntze, in Post and Kuntzè, 1.1904, p. 600 .

ZIZYPHOIDES Seward and Conway, 1935.

Zizyphoides colombi (Heer) Seward and Conway, 1935 b, p. 23 , fig. 8 ; leaf fragment, Rhamuaceae; Mesozoic; Kagdlungauk, west Greenland.

ZONALASPORITES Ibrahim, 1933.

Zonalasporites ulughbeki Ibrahim, 1933, p. 38, pl. 1, fig. 11 ; spore ; Carboniferous.

ZONALESSPORITES Ibrahim, 1933.

Zonalessporites saturnoides Ibrahim, 1933, p. 27, pl. 3, fig. 26; spore; Carboniferous.

ZONALOSPORITES Ibrahim, 1933.

Zonalosporites vittatus Ibrahim, 1933, 'p. 41, pl. 6, fig. 45 ; spore ; Carboniferous.

ZONARIDES Schimper, 1869.

Zonarides digitutus (Brongniart) Schimper, 1869 (1869-74), p. 186, pl. 3, fig. 2 ; described as alga; shows some resemblance to ginkgophyte leaf?; Permian; Mansfield, Prussian Saxony.

ZONARITES Sternberg, 1833.

Zonarites flabellaris (Brongniart) Sternberg, 1833 (1820-38), p. 34. For Fucoides flabellaris Brongniart, $1828 \mathrm{a}-38$, p. 67, pl. 8, fig. 5 ; alga ?; Tertiary ; Monte Bolca, near Verona, Italy.

ZONOPLEURA Massalongo, 1859.

Zonopleura hampeana (Stiehler) Massalongo, 1859. For Delesserites hampeana Stiehler, 1857, p. 56, pl. 11, fig. 12 .

ZONOPTERIS Debey and Ettingshausen, 1859.

Zonopteris goepperti Debey and Ettingshausen, 1859 b, p. 213 , pl. 4, figs. 11-20; portion of fertile fern frond ; Upper Cretaceous; Aachen, Rhenish Prussia.

ZONOTRICHITES Bornemann, 1887.

Zonotrichites lissaviensis Bornemann, 1887, p. 126, pl. 5, figs. 1,2 ; pl. 6, figs. 1, 2; Rbaetic; Silesia.

ZONOTRILETES Waltz, 1935

Feference not scen; cited in Gothan, 1942 b, p. 160.
ZOOGLEITES C. E. Bertrand; 1898.

Zoogleites elaverensis C. E. Bertrand, 1898 , p. 184 , pl. 10 , fig. 107 ; pl. 11 , figs. 133, 134; bacteria?; Permian; France.

ZOOPHYCOS Massalongo, 1855.

Zoophycos caputmedusae Massalongo, 1855 , p. 48 , pl. 1 , fig. 1 ; figure suggests Isoetes?; Eocene; Monte Bolca, Italy.

ZOSTERITES Brongniart, 1823.

Zosterites orbigniana Brongniart, 1823, p. 317 , pl. 21 ; leaf, monocotyledon ; Lower Cretaceous (Neocomian); Isle of Aix, France.

ZOSTERITES C. F. W. Braun, 1840.

Zosterites lignitarum C. F. W. Braun, 1840 , p. 99 ; nom. nud.

zosteropHYLLUM Pomel, 1847.

Zosterophyllum articulatum Pomel, in Graves, 1847, p. 708 ; nom. nud.

ZOSTEROPHYLLUM Penhallow, 1892.

Zosterophyllum myretonianum Penhallow, 1892 , p. 9 , pl. 1 , fig. 1 ; pl. 2 , figs. $1-3$; psilophyte ; Devonian; Myreton, Scotland.

ZUBERIA Frenguelli, 1943.

Zuberia zuberi (Szajnocha) Frenguelli, $1943 a$; p. 308 ; fronds, cupulate seeds and microsporangiate organs ; Triassic ; Argentina. See Frenguelli, 1944a, p. 9, pls. 4-11, for full account.

ZYGOPHILLITES Keferstein, 1834.

Zygophillites calamoides (Brongniart) Keferstein, 1834, p. 876. For Zeugophyllites calamoides Brongniart, $1828 \mathrm{~b}$, p. 123.

ZYGOPHYLLOCARPUM Weyland, 1938.

Zygophyllocarpum rottense Weyland 1938 b, p. 153 , pl. 22, figs. 1, 2 ; winged fruit, Zygophyllaceae ; Tertiary : Rott, Siebengebirge, Germany.

ZYGOPTERIS Corda, 1845.

Zygopteris primaeva (Cotta) Corda, 1845, p. 81 ; coenopterid fern ; Carboniferous. For Tubicaulis primarius Cotta, 1932, p. 20, pl. 1, figs. 1, 2. See also Sahni, 1932e; Posthumus, 1931.

ZYGOSPORITES Williamson, 1880.

Zygosporites brevips Williamson, 1880 , p. 516 , pl. 19 , figs. $51,53,55,56$; spore; Carboniferous ; England.

ZYGOSPORITES McLean, 1912.

Zygosporites brevipes MeLean, 1912, p. 509 , fig. 5a ; spore?; Upper Carboniferous; Dulesgate, England.

ZYMPANOPHORA.

Error for Tympanophora, in Hectol, 1880, p. 47. 